\title{
Synthesis of 5-(Fluoroalkyl)isoxazole Building Blocks by Regioselective Reactions of Functionalized Halogenoximes
}

Bohdan A. Chalyk, Kateryna V. Hrebeniuk, Yulia V. Fil, Dr. Konstantin S. Gavrilenko, Dr. Alexander B. Rozhenko, Bohdan V. Vashchenko, Dr. Oleksandr V. Borysov, Dr. Angelina V. Biitseva, Dr. Pavlo S. Lebed, Iulia Bakanovych, Yurii S. Moroz, Dr. Oleksandr O. Grygorenko*

Table of contents

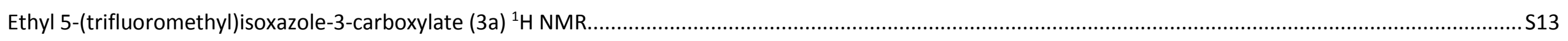

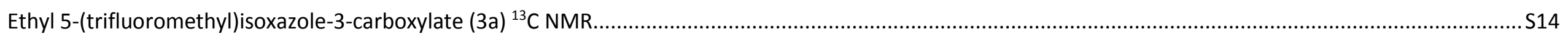

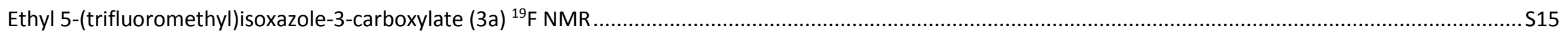

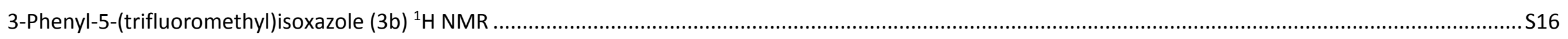

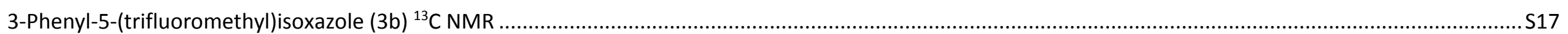

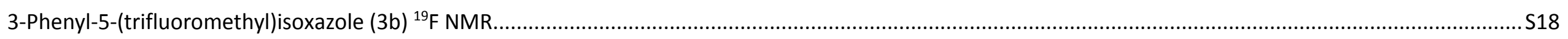

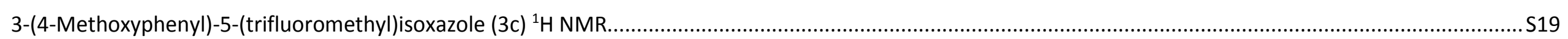

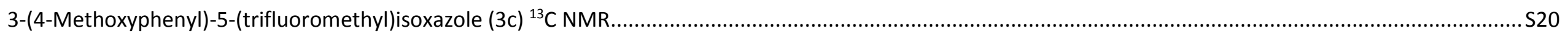

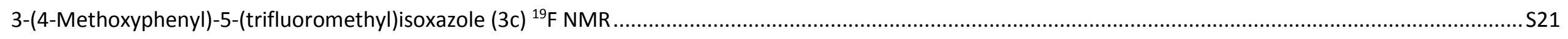

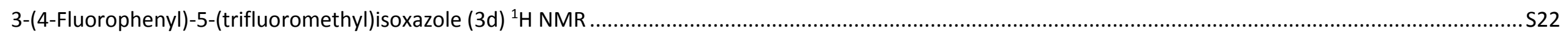

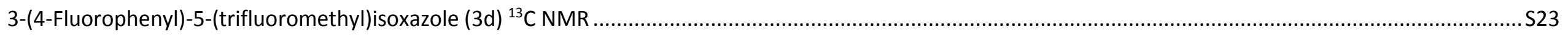

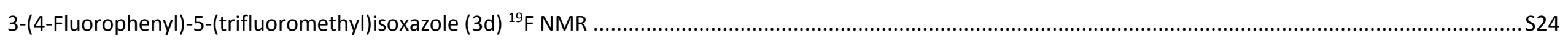

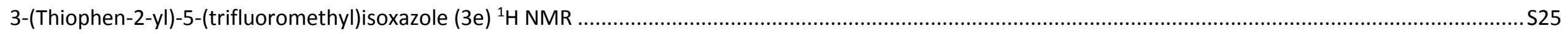

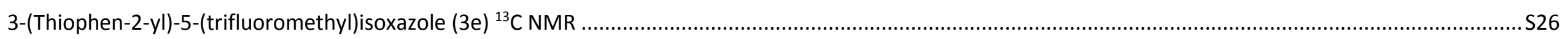

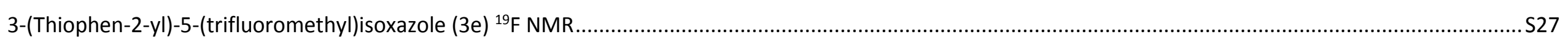

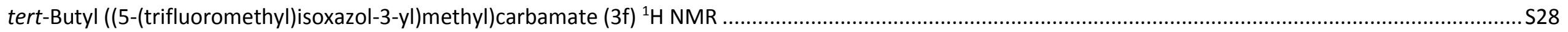


tert-Butyl ((5-(trifluoromethyl)isoxazol-3-yl)methyl)carbamate (3f) ${ }^{13} \mathrm{C} \mathrm{NMR}$

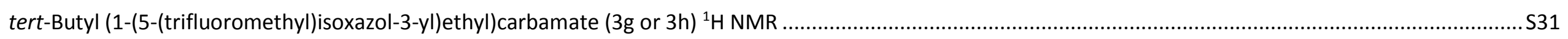

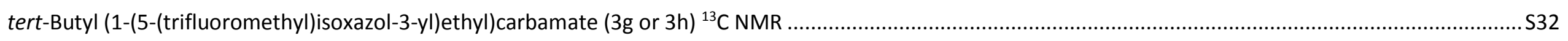

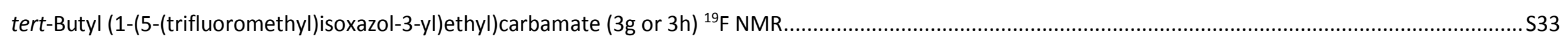

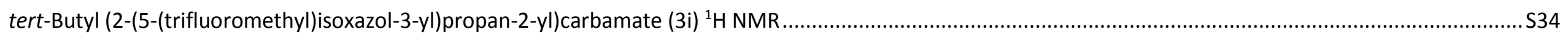

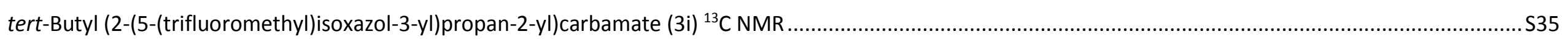

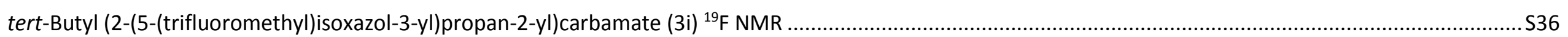

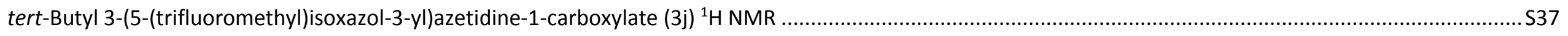

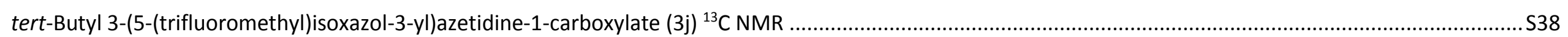

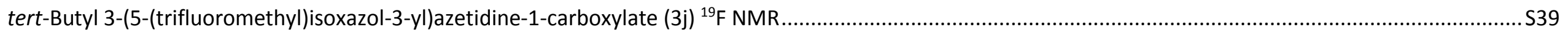

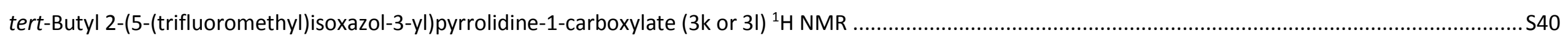

tert-Butyl 2-(5-(trifluoromethyl)isoxazol-3-yl)pyrrolidine-1-carboxylate (3k or 3I) ${ }^{13} \mathrm{C}$ NMR ................................................................................................. S41

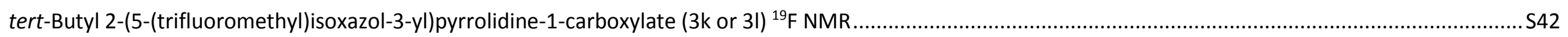

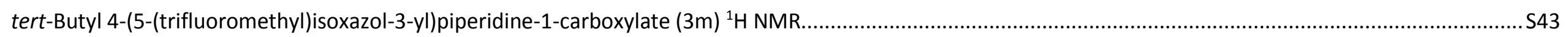

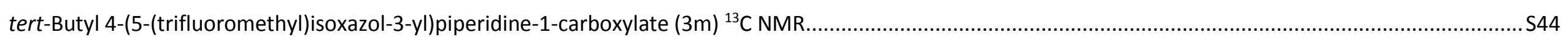

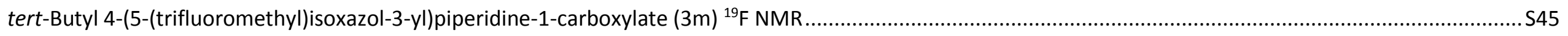

tert-Butyl 2,2-dimethyl-4-(5-(trifluoromethyl)isoxazol-3-yl)oxazolidine-3-carboxylate (3n or 30) ${ }^{1} \mathrm{H}$ NMR................................................................................. S46

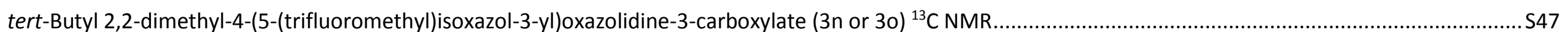

tert-Butyl 2,2-dimethyl-4-(5-(trifluoromethyl)isoxazol-3-yl)oxazolidine-3-carboxylate (3n or 30) ${ }^{19} \mathrm{~F} \mathrm{NMR} \mathrm{............................................................................} \mathrm{S48}$

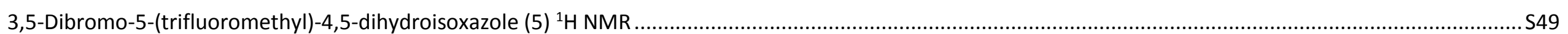

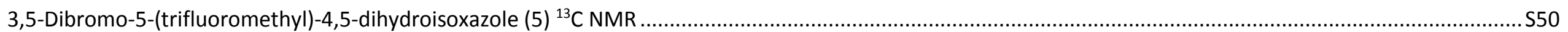




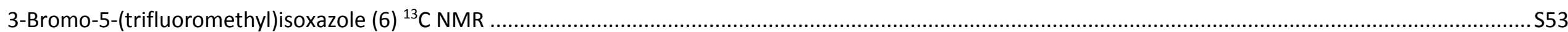

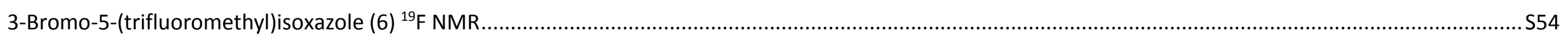

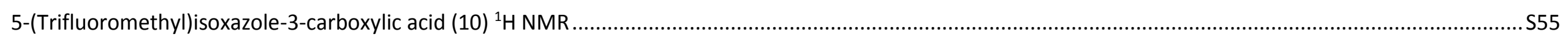

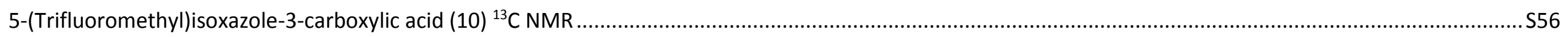

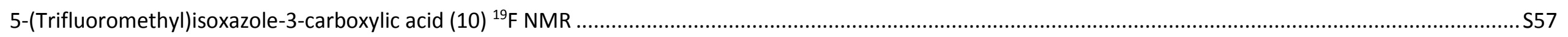

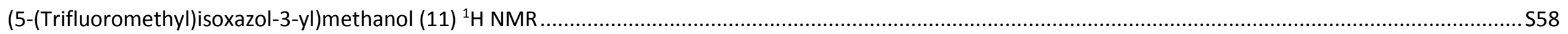

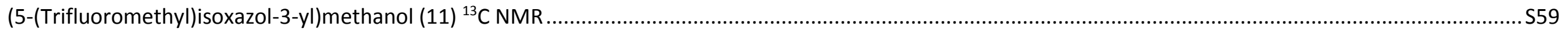

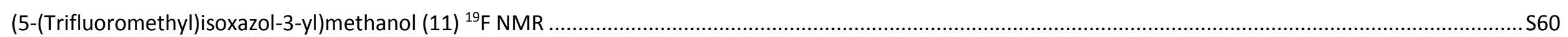

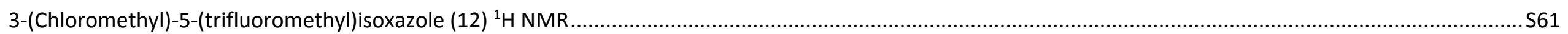

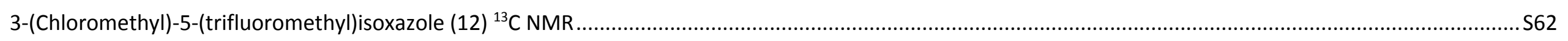

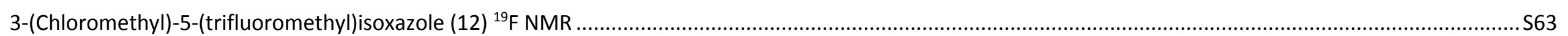

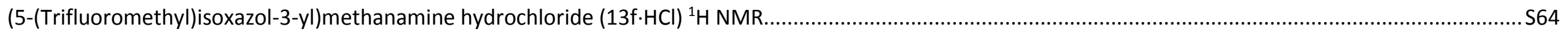

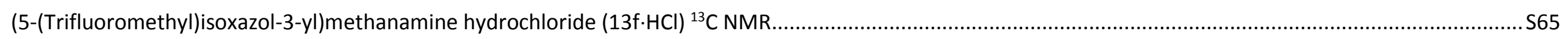

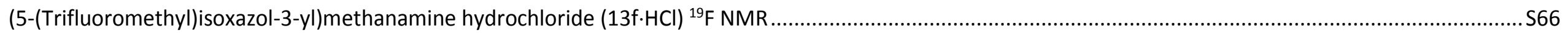

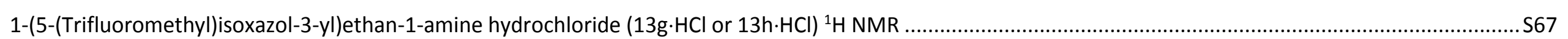

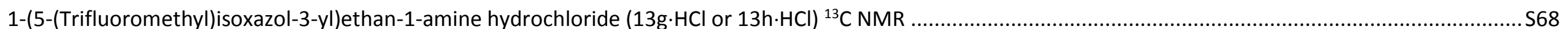

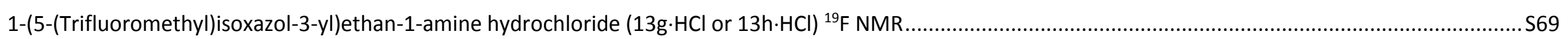

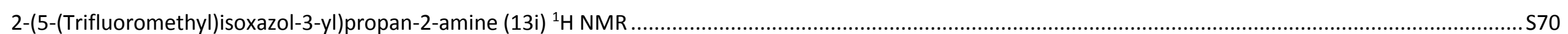

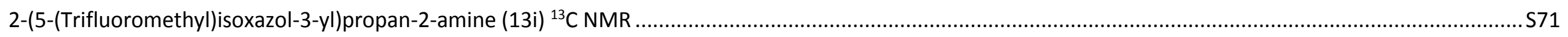

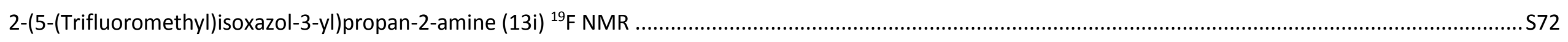


3-(Azetidin-3-yl)-5-(trifluoromethyl)isoxazole hydrochloride $(13 \mathrm{j} \cdot \mathrm{HCl}){ }^{1} \mathrm{H} \mathrm{NMR}$

3-(Azetidin-3-yl)-5-(trifluoromethyl)isoxazole hydrochloride $(13 \mathrm{j} \cdot \mathrm{HCl}){ }^{13} \mathrm{C} \mathrm{NMR}$

3-(Azetidin-3-yl)-5-(trifluoromethyl)isoxazole hydrochloride $(13 \mathrm{j} \cdot \mathrm{HCl}){ }^{19} \mathrm{~F} \mathrm{NMR}$

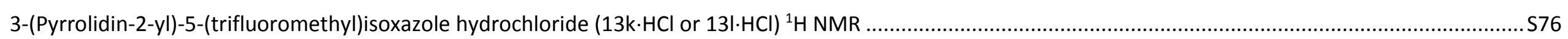

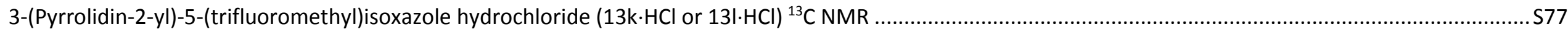

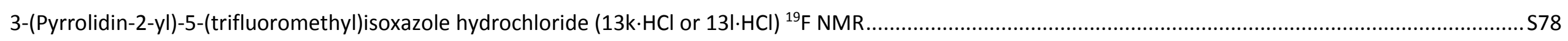

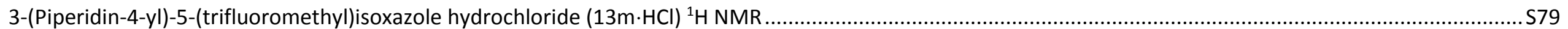

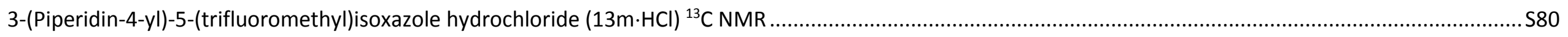

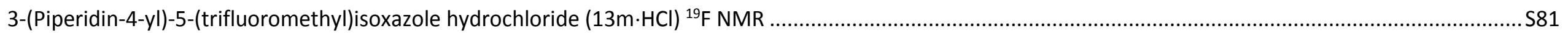

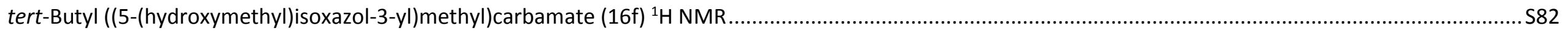

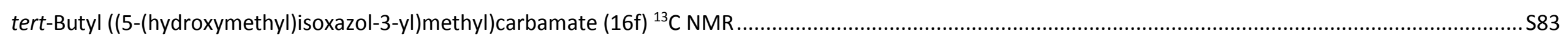

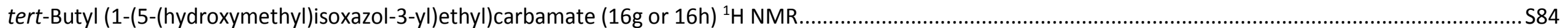

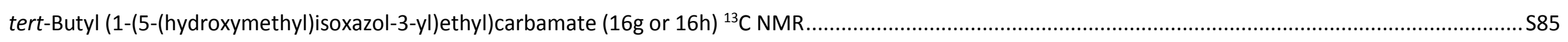

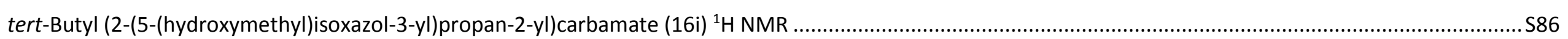

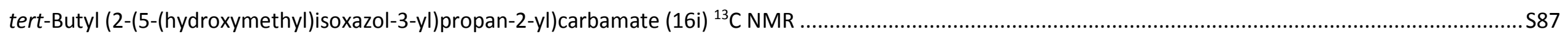

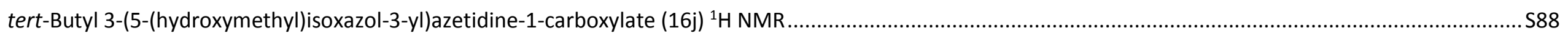

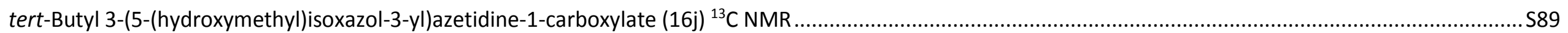

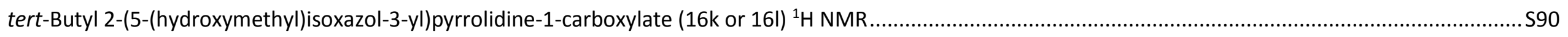

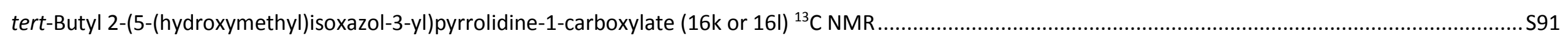

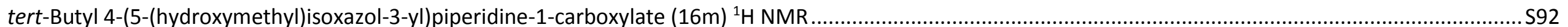

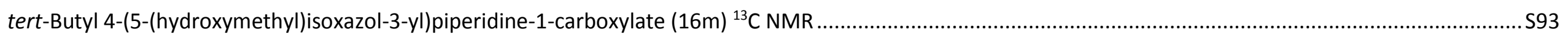


tert-Butyl (1-(5-formylisoxazol-3-yl)ethyl)carbamate (17g or $17 \mathrm{~h}){ }^{1} \mathrm{H}$ NMR

tert-Butyl (1-(5-formylisoxazol-3-yl)ethyl)carbamate (17g or $17 \mathrm{~h}){ }^{13} \mathrm{C}$ NMR

tert-Butyl (2-(5-formylisoxazol-3-yl)propan-2-yl)carbamate (17i) ${ }^{1} \mathrm{H}$ NMR ........

tert-Butyl (2-(5-formylisoxazol-3-yl)propan-2-yl)carbamate (17i) ${ }^{13} \mathrm{C}$ NMR.

tert-Butyl 3-(5-formylisoxazol-3-yl)azetidine-1-carboxylate (17j) ${ }^{1} \mathrm{H}$ NMR

tert-Butyl 3-(5-formylisoxazol-3-yl)azetidine-1-carboxylate $(17 \mathrm{j}){ }^{13} \mathrm{C}$ NMR

tert-Butyl 2-(5-formylisoxazol-3-yl)pyrrolidine-1-carboxylate (17k or $17 \mathrm{l}){ }^{1} \mathrm{H}$ NMR ......

tert-Butyl 2-(5-formylisoxazol-3-yl)pyrrolidine-1-carboxylate (17k or 17l) ${ }^{13} \mathrm{C} \mathrm{NMR}$

tert-Butyl 4-(5-formylisoxazol-3-yl)piperidine-1-carboxylate $(17 \mathrm{~m}){ }^{1} \mathrm{H}$ NMR

tert-Butyl 4-(5-formylisoxazol-3-yl)piperidine-1-carboxylate (17m) ${ }^{13} \mathrm{C}$ NMR .

Ethyl 5-(fluoromethyl)isoxazole-3-carboxylate (18a) ${ }^{1} \mathrm{H}$ NMR

Ethyl 5-(fluoromethyl)isoxazole-3-carboxylate (18a) ${ }^{13} \mathrm{C}$ NMR

Ethyl 5-(fluoromethyl)isoxazole-3-carboxylate (18a) ${ }^{19}$ F NMR

tert-Butyl ((5-(fluoromethyl)isoxazol-3-yl)methyl)carbamate (18f) ${ }^{1} \mathrm{H}$ NMR

tert-Butyl ((5-(fluoromethyl)isoxazol-3-yl)methyl)carbamate $(18 f){ }^{13} \mathrm{C} N M R$

tert-Butyl ((5-(fluoromethyl)isoxazol-3-yl)methyl)carbamate (18f) ${ }^{19} \mathrm{~F}$ NMR

tert-Butyl (1-(5-(fluoromethyl)isoxazol-3-yl)ethyl)carbamate (18g or $18 \mathrm{~h}){ }^{1} \mathrm{H}$ NMR

tert-Butyl (1-(5-(fluoromethyl)isoxazol-3-yl)ethyl)carbamate (18g or $18 \mathrm{~h}){ }^{13} \mathrm{C}$ NMR

tert-Butyl (1-(5-(fluoromethyl)isoxazol-3-yl)ethyl)carbamate (18g or $18 \mathrm{~h}){ }^{19} \mathrm{~F}$ NMR

tert-Butyl (2-(5-(fluoromethyl)isoxazol-3-yl)propan-2-yl)carbamate (18i) ${ }^{13} \mathrm{C}$ NMR. 
tert-Butyl 3-(5-(fluoromethyl)isoxazol-3-yl)azetidine-1-carboxylate (18j) ${ }^{1} \mathrm{H}$ NMR

tert-Butyl 3-(5-(fluoromethyl)isoxazol-3-yl)azetidine-1-carboxylate (18j) ${ }^{13} \mathrm{C}$ NMR

tert-Butyl 3-(5-(fluoromethyl)isoxazol-3-yl)azetidine-1-carboxylate (18j) ${ }^{19} \mathrm{~F}$ NMR

tert-Butyl 2-(5-(fluoromethyl)isoxazol-3-yl)pyrrolidine-1-carboxylate (18k or 18I) ${ }^{1} \mathrm{H}$ NMR

tert-Butyl 2-(5-(fluoromethyl)isoxazol-3-yl)pyrrolidine-1-carboxylate (18k or $18 \mathrm{I}){ }^{13} \mathrm{C}$ NMR

tert-Butyl 2-(5-(fluoromethyl)isoxazol-3-yl)pyrrolidine-1-carboxylate (18k or 18I) ${ }^{19} \mathrm{~F} \mathrm{NMR}$

tert-Butyl (1-(5-(difluoromethyl)isoxazol-3-yl)ethyl)carbamate (19g or 19h) ${ }^{1} \mathrm{H}$ NMR....

tert-Butyl (1-(5-(difluoromethyl)isoxazol-3-yl)ethyl)carbamate (19g or $19 \mathrm{~h}){ }^{13} \mathrm{C}$ NMR

tert-Butyl (1-(5-(difluoromethyl)isoxazol-3-yl)ethyl)carbamate (19g or 19h) ${ }^{19} \mathrm{~F}$ NMR

tert-Butyl (2-(5-(difluoromethyl)isoxazol-3-yl)propan-2-yl)carbamate (19i) ${ }^{1} \mathrm{H}$ NMR

tert-Butyl (2-(5-(difluoromethyl)isoxazol-3-yl)propan-2-yl)carbamate (19i) ${ }^{13} \mathrm{C}$ NMR.

tert-Butyl (2-(5-(difluoromethyl)isoxazol-3-yl)propan-2-yl)carbamate (19i) ${ }^{19} \mathrm{~F}$ NMR .

tert-Butyl 3-(5-(difluoromethyl)isoxazol-3-yl)azetidine-1-carboxylate (19j) ${ }^{1} \mathrm{H}$ NMR

tert-Butyl 3-(5-(difluoromethyl)isoxazol-3-yl)azetidine-1-carboxylate (19j) ${ }^{13} \mathrm{C}$ NMR

tert-Butyl 3-(5-(difluoromethyl)isoxazol-3-yl)azetidine-1-carboxylate (19j) ${ }^{19} \mathrm{~F}$ NMR

tert-Butyl 2-(5-(difluoromethyl)isoxazol-3-yl)pyrrolidine-1-carboxylate (19k or 19l) ${ }^{1} \mathrm{H}$ NMR

tert-Butyl 2-(5-(difluoromethyl)isoxazol-3-yl)pyrrolidine-1-carboxylate (19k or 19l) ${ }^{13} \mathrm{C} \mathrm{NMR}$

tert-Butyl 2-(5-(difluoromethyl)isoxazol-3-yl)pyrrolidine-1-carboxylate (19k or 19l) ${ }^{19} \mathrm{~F}$ NMR

2-(5-(Fluoromethyl)isoxazol-3-yl)propan-2-amine hydrochloride $(20 \mathrm{i} \cdot \mathrm{HCl})^{1} \mathrm{H} \mathrm{NMR}$

2-(5-(Fluoromethyl)isoxazol-3-yl)propan-2-amine hydrochloride $(20 \mathrm{i} \cdot \mathrm{HCl}){ }^{13} \mathrm{C} \mathrm{NMR}$

2-(5-(Fluoromethyl)isoxazol-3-yl)propan-2-amine hydrochloride $(20 \mathrm{i} \cdot \mathrm{HCl}){ }^{19} \mathrm{~F} \mathrm{NMR}$ S136

5-(Fluoromethyl)-3-(pyrrolidin-2-yl)isoxazole hydrochloride $(20 \mathrm{k} \cdot \mathrm{HCl}$ or $20 \mathrm{O} \cdot \mathrm{HCl}){ }^{1} \mathrm{H} \mathrm{NMR}$ 
(S)-5-(Fluoromethyl)-3-(pyrrolidin-2-yl)isoxazole hydrochloride $(20 \mathrm{k} \cdot \mathrm{HCl}$ or $20 \mathrm{O} \cdot \mathrm{HCl}){ }^{13} \mathrm{C} \mathrm{NMR}$

(S)-5-(Fluoromethyl)-3-(pyrrolidin-2-yl)isoxazole hydrochloride $(20 \mathrm{k} \cdot \mathrm{HCl}$ or $201 \cdot \mathrm{HCl}){ }^{19} \mathrm{~F} \mathrm{NMR}$

1-(5-(Difluoromethyl)isoxazol-3-yl)ethanamine hydrochloride $(21 \mathrm{~g} \cdot \mathrm{HCl}$ or $21 \mathrm{~h} \cdot \mathrm{HCl}){ }^{1} \mathrm{H} N M R$.

1-(5-(Difluoromethyl)isoxazol-3-yl)ethanamine hydrochloride $(21 \mathrm{~g} \cdot \mathrm{HCl}$ or $21 \mathrm{~h} \cdot \mathrm{HCl}){ }^{13} \mathrm{C} \mathrm{NMR}$

1-(5-(Difluoromethyl)isoxazol-3-yl)ethanamine hydrochloride $(21 \mathrm{~g} \cdot \mathrm{HCl}$ or $21 \mathrm{~h} \cdot \mathrm{HCl}){ }^{19} \mathrm{~F} \mathrm{NMR}$

2-(5-(Difluoromethyl)isoxazol-3-yl)propan-2-amine hydrochloride $(21 \mathrm{i} \cdot \mathrm{HCl}){ }^{1} \mathrm{H} N \mathrm{NR}$

2-(5-(Difluoromethyl)isoxazol-3-yl)propan-2-amine hydrochloride $(21 \mathrm{i} \cdot \mathrm{HCl}){ }^{13} \mathrm{C} \mathrm{NMR}$..

2-(5-(Difluoromethyl)isoxazol-3-yl)propan-2-amine hydrochloride $(21 \mathrm{i} \cdot \mathrm{HCl}){ }^{19} \mathrm{~F} \mathrm{NMR}$

3-(Azetidin-3-yl)-5-(difluoromethyl)isoxazole hydrochloride (21j·HCl) ${ }^{1} \mathrm{H}$ NMR .

3-(Azetidin-3-yl)-5-(difluoromethyl)isoxazole hydrochloride (21j·HCl) ${ }^{19} \mathrm{~F} \mathrm{NMR}$

5-(Difluoromethyl)-3-(pyrrolidin-2-yl)isoxazole hydrochloride $(21 \mathrm{k} \cdot \mathrm{HCl}$ or $21 \mathrm{l} \cdot \mathrm{HCl}){ }^{1} \mathrm{H}$ NMR

5-(Difluoromethyl)-3-(pyrrolidin-2-yl)isoxazole hydrochloride $(21 \mathrm{k} \cdot \mathrm{HCl}$ or $211 \cdot \mathrm{HCl}){ }^{13} \mathrm{C} \mathrm{NMR}$.

5-(Difluoromethyl)-3-(pyrrolidin-2-yl)isoxazole hydrochloride $(21 \mathrm{k} \cdot \mathrm{HCl}$ or $211 \cdot \mathrm{HCl}){ }^{19} \mathrm{~F} \mathrm{NMR}$.

Ethyl 5-(bromomethyl)isoxazole-3-carboxylate (22a) ${ }^{1} \mathrm{H}$ NMR

Ethyl 5-(bromomethyl)isoxazole-3-carboxylate (22a) ${ }^{13} \mathrm{C}$ NMR .

(R)-tert-Butyl (1-(5-(bromomethyl)isoxazol-3-yl)ethyl)carbamate (22g or 22h) ${ }^{1} \mathrm{H}$ NMR ...

(R)-tert-Butyl (1-(5-(bromomethyl)isoxazol-3-yl)ethyl)carbamate (22g or $22 \mathrm{~h}){ }^{13} \mathrm{C}$ NMR . ...S157

tert-Butyl 3-(5-(bromomethyl)isoxazol-3-yl)azetidine-1-carboxylate (22j) ${ }^{1} \mathrm{H}$ NMR . 
tert-Butyl 3-(5-(bromomethyl)isoxazol-3-yl)azetidine-1-carboxylate (22j) ${ }^{13} \mathrm{C}$ NMR

tert-Butyl 4-(5-(bromomethyl)isoxazol-3-yl)piperidine-1-carboxylate $(22 \mathrm{~m}){ }^{1} \mathrm{H}$ NMR

tert-Butyl 4-(5-(bromomethyl)isoxazol-3-yl)piperidine-1-carboxylate $(22 \mathrm{~m}){ }^{13} \mathrm{C} N M R$

Ethyl 5-(hydroxymethyl)isoxazole-3-carboxylate (23) ${ }^{1} \mathrm{H}$ NMR

Ethyl 5-(hydroxymethyl)isoxazole-3-carboxylate (23) ${ }^{13} \mathrm{C}$ NMR.

(3-Bromoisoxazol-5-yl)methanol (24) ${ }^{1} \mathrm{H}$ NMR

(3-Bromoisoxazol-5-yl)methanol (24) ${ }^{13}$ C NMR

Ethyl 5-formylisoxazole-3-carboxylate (25) ${ }^{1} \mathrm{H}$ NMR

Ethyl 5-formylisoxazole-3-carboxylate (25) ${ }^{13} \mathrm{C}$ NMR .

Ethyl 5-(difluoromethyl)isoxazole-3-carboxylate (27) ${ }^{1} \mathrm{H}$ NMR

Ethyl 5-(difluoromethyl)isoxazole-3-carboxylate (27) ${ }^{13}$ C NMR

Ethyl 5-(difluoromethyl)isoxazole-3-carboxylate (27) ${ }^{19} \mathrm{~F}$ NMR .

3-Bromo-5-(difluoromethyl)isoxazole (28) ${ }^{1} \mathrm{H}$ NMR

3-Bromo-5-(difluoromethyl)isoxazole $(28){ }^{13} \mathrm{C}$ NMR

3-Bromo-5-(difluoromethyl)isoxazole (28) ${ }^{19} \mathrm{~F} \mathrm{NMR}$

5-(Difluoromethyl)isoxazole-3-carboxylic acid (29) ${ }^{1} \mathrm{H}$ NMR

5-(Difluoromethyl)isoxazole-3-carboxylic acid (29) ${ }^{13}$ C NMR

5-(Difluoromethyl)isoxazole-3-carboxylic acid (29) ${ }^{19}$ F NMR

Ethyl 5-acetylisoxazole-3-carboxylate (31) ${ }^{1} \mathrm{H}$ NMR S177

Ethyl 5-acetylisoxazole-3-carboxylate (31) ${ }^{13}$ C NMR S178

Ethyl 5-(1,1-difluoroethyl)isoxazole-3-carboxylate (32) ${ }^{1} \mathrm{H}$ NMR S179

Ethyl 5-(1,1-difluoroethyl)isoxazole-3-carboxylate (32) ${ }^{13} \mathrm{C}$ NMR 
Ethyl 5-(1,1-difluoroethyl)isoxazole-3-carboxylate (32) ${ }^{19} \mathrm{~F}$ NMR .

5-(1,1-Difluoroethyl)isoxazole-3-carboxylic acid (33) ${ }^{1} \mathrm{H}$ NMR.

5-(1,1-Difluoroethyl)isoxazole-3-carboxylic acid (33) ${ }^{13} \mathrm{C}$ NMR

5-(1,1-Difluoroethyl)isoxazole-3-carboxylic acid (33) ${ }^{19}$ F NMR

5-(1,1-Difluoroethyl)isoxazol-3-amine (34) ${ }^{1} \mathrm{H}$ NMR

5-(1,1-Difluoroethyl)isoxazol-3-amine (34) ${ }^{13} \mathrm{C}$ NMR .

5-(1,1-Difluoroethyl)isoxazol-3-amine (34) ${ }^{19} \mathrm{~F}$ NMR..

(3-(Chloromethyl)isoxazol-5-yl)methanol (36) ${ }^{1} \mathrm{H}$ NMR ....

(3-(Chloromethyl)isoxazol-5-yl)methanol (36) ${ }^{13}$ C NMR

3-(Chloromethyl)isoxazole-5-carbaldehyde (37) ${ }^{1} \mathrm{H}$ NMR

3-(Chloromethyl)isoxazole-5-carbaldehyde (37) ${ }^{13}$ C NMR

3-(Chloromethyl)-5-(difluoromethyl)isoxazole (38) ${ }^{1} \mathrm{H}$ NMR

3-(Chloromethyl)-5-(difluoromethyl)isoxazole (38) ${ }^{13} \mathrm{C}$ NMR

3-(Chloromethyl)-5-(difluoromethyl)isoxazole (38) ${ }^{19}$ F NMR

tert-Butyl (1-(5-(2,2,2-trifluoro-1-hydroxyethyl)isoxazol-3-yl)ethyl)carbamate (39g or 39h) ${ }^{1} \mathrm{H}$ NMR.

tert-Butyl (1-(5-(2,2,2-trifluoro-1-hydroxyethyl)isoxazol-3-yl)ethyl)carbamate (39g or 39h) ${ }^{13} \mathrm{C}$ NMR

tert-Butyl (1-(5-(2,2,2-trifluoro-1-hydroxyethyl)isoxazol-3-yl)ethyl)carbamate $(39 \mathrm{~g}$ or $39 \mathrm{~h}){ }^{19} \mathrm{~F}$ NMR

tert-Butyl 2-(5-(2,2,2-trifluoro-1-hydroxyethyl)isoxazol-3-yl)pyrrolidine-1-carboxylate (39k or 39l) ${ }^{1} \mathrm{H}$ NMR

tert-Butyl 2-(5-(2,2,2-trifluoro-1-hydroxyethyl)isoxazol-3-yl)pyrrolidine-1-carboxylate (39k or 39I) ${ }^{13} \mathrm{C} \mathrm{NMR}$ S199

tert-Butyl 2-(5-(2,2,2-trifluoro-1-hydroxyethyl)isoxazol-3-yl)pyrrolidine-1-carboxylate (39k or 39I) ${ }^{19} \mathrm{~F} \mathrm{NMR}$

tert-Butyl 4-(5-(2,2,2-trifluoro-1-hydroxyethyl)isoxazol-3-yl)piperidine-1-carboxylate (39m) ${ }^{1} \mathrm{H}$ NMR S201

tert-Butyl 4-(5-(2,2,2-trifluoro-1-hydroxyethyl)isoxazol-3-yl)piperidine-1-carboxylate (39m) ${ }^{13} \mathrm{C}$ NMR 
tert-Butyl 4-(5-(2,2,2-trifluoro-1-hydroxyethyl)isoxazol-3-yl)piperidine-1-carboxylate $(39 \mathrm{~m}){ }^{19} \mathrm{~F}$ NMR .

tert-Butyl 2-(5-(2,2,2-trifluoro-1,1-dihydroxyethyl)isoxazol-3-yl)pyrrolidine-1-carboxylate (40k or 40I) ${ }^{1} \mathrm{H}$ NMR...

tert-Butyl 2-(5-(2,2,2-trifluoro-1,1-dihydroxyethyl)isoxazol-3-yl)pyrrolidine-1-carboxylate (40k or $40 \mathrm{l}){ }^{13} \mathrm{C} \mathrm{NMR}$.....

tert-Butyl 2-(5-(2,2,2-trifluoro-1,1-dihydroxyethyl)isoxazol-3-yl)pyrrolidine-1-carboxylate (40k or 40I) ${ }^{19} \mathrm{~F} \mathrm{NMR} \mathrm{....}$

tert-Butyl 2-(4-(methoxy(methyl)carbamoyl)isoxazol-3-yl)pyrrolidine-1-carboxylate (42a or 42b) ${ }^{1} \mathrm{H} N M R$

tert-Butyl 2-(4-(methoxy(methyl)carbamoyl)isoxazol-3-yl)pyrrolidine-1-carboxylate (42a or $42 \mathrm{~b}$ ) ${ }^{13} \mathrm{C} \mathrm{NMR}$

tert-Butyl 2-(4-(2,2,2-trifluoroacetyl)isoxazol-3-yl)pyrrolidine-1-carboxylate (43a or 43b) ${ }^{1} \mathrm{H}$ NMR.

tert-Butyl 2-(4-(2,2,2-trifluoroacetyl)isoxazol-3-yl)pyrrolidine-1-carboxylate (43a or 43 b) ${ }^{13} \mathrm{C}$ NMR.

tert-Butyl 2-(4-(2,2,2-trifluoroacetyl)isoxazol-3-yl)pyrrolidine-1-carboxylate (43a or 43b) ${ }^{19} \mathrm{~F}$ NMR

Ethyl 3-(((tert-butoxycarbonyl)amino)methyl)-5-(difluoromethyl)-isoxazole-4-carboxylate (48f) ${ }^{1} \mathrm{H}$ NMR

Ethyl 3-(((tert-butoxycarbonyl)amino)methyl)-5-(difluoromethyl)-isoxazole-4-carboxylate (48f) ${ }^{13} \mathrm{C} N M R$

Ethyl 3-(((tert-butoxycarbonyl)amino)methyl)-5-(difluoromethyl)-isoxazole-4-carboxylate (48f) ${ }^{19} \mathrm{~F} \mathrm{NMR}$

Ethyl 3-(((tert-butoxycarbonyl)amino)methyl)-5-(difluoromethyl)-isoxazole-4-carboxylate (48f) HMBC

Ethyl 3-(((tert-butoxycarbonyl)amino)methyl)-5-(difluoromethyl)-isoxazole-4-carboxylate (48f) HSQC.

Ethyl 3-(((tert-butoxycarbonyl)amino)methyl)-5-(difluoromethyl)-isoxazole-4-carboxylate (48f) SELNOESY

tert-Butyl 3-(5-(2-ethoxy-2-oxoethyl)-5-(trifluoromethyl)-1,4,2-dioxazol-3-yl)azetidine-1-carboxylate (50j) ${ }^{1} \mathrm{H}$ NMR.

tert-Butyl 3-(5-(2-ethoxy-2-oxoethyl)-5-(trifluoromethyl)-1,4,2-dioxazol-3-yl)azetidine-1-carboxylate $(50 \mathrm{j}){ }^{13} \mathrm{C}$ NMR

tert-Butyl 3-(5-(2-ethoxy-2-oxoethyl)-5-(trifluoromethyl)-1,4,2-dioxazol-3-yl)azetidine-1-carboxylate (50j) ${ }^{19} \mathrm{~F} \mathrm{NMR}$

tert-Butyl 2-(5-(2-ethoxy-2-oxoethyl)-5-(trifluoromethyl)-1,4,2-dioxazol-3-yl)pyrrolidine-1-carboxylate (50k or 50I) ${ }^{1} \mathrm{H}$ NMR

S221

tert-Butyl 2-(5-(2-ethoxy-2-oxoethyl)-5-(trifluoromethyl)-1,4,2-dioxazol-3-yl)pyrrolidine-1-carboxylate (50k or 50I) ${ }^{13} \mathrm{C} \mathrm{NMR}$

tert-Butyl 2-(5-(2-ethoxy-2-oxoethyl)-5-(trifluoromethyl)-1,4,2-dioxazol-3-yl)pyrrolidine-1-carboxylate (50k or 50I) ${ }^{19} \mathrm{~F}$ NMR 
Ethyl 2-(3-(((tert-butoxycarbonyl)amino)methyl)-5-(difluoromethyl)-1,4,2-dioxazol-5-yl)acetate (51f) ${ }^{13} \mathrm{C}$ NMR.....

Ethyl 2-(3-(((tert-butoxycarbonyl)amino)methyl)-5-(difluoromethyl)-1,4,2-dioxazol-5-yl)acetate (51f) ${ }^{19} \mathrm{~F}$ NMR

Ethyl 2-(3-(1-((tert-butoxycarbonyl)amino)ethyl)-5-(difluoromethyl)-1,4,2-dioxazol-5-yl)acetate (51g or 51h) ${ }^{1} \mathrm{H}$ NMR

Ethyl 2-(3-(1-((tert-butoxycarbonyl)amino)ethyl)-5-(difluoromethyl)-1,4,2-dioxazol-5-yl)acetate (51g or 51h) ${ }^{13} \mathrm{C} \mathrm{NMR}$

Ethyl 2-(3-(1-((tert-butoxycarbonyl)amino)ethyl)-5-(difluoromethyl)-1,4,2-dioxazol-5-yl)acetate (51g or 51h) ${ }^{19} \mathrm{~F} \mathrm{NMR}$

tert-Butyl 3-(5-(difluoromethyl)-5-(2-ethoxy-2-oxoethyl)-1,4,2-dioxazol-3-yl)azetidine-1-carboxylate (51j) ${ }^{1} \mathrm{H}$ NMR .

tert-Butyl 3-(5-(difluoromethyl)-5-(2-ethoxy-2-oxoethyl)-1,4,2-dioxazol-3-yl)azetidine-1-carboxylate (51j) ${ }^{13} \mathrm{C} \mathrm{NMR}$

tert-Butyl 3-(5-(difluoromethyl)-5-(2-ethoxy-2-oxoethyl)-1,4,2-dioxazol-3-yl)azetidine-1-carboxylate (51j) ${ }^{19} \mathrm{~F}$ NMR.

tert-Butyl 2-(5-(difluoromethyl)-5-(2-ethoxy-2-oxoethyl)-1,4,2-dioxazol-3-yl)pyrrolidine-1-carboxylate (51k or 51l) ${ }^{1} \mathrm{H}$ NMR

tert-Butyl 2-(5-(difluoromethyl)-5-(2-ethoxy-2-oxoethyl)-1,4,2-dioxazol-3-yl)pyrrolidine-1-carboxylate (51k or 51l) ${ }^{13} \mathrm{C} \mathrm{NMR}$

tert-Butyl 2-(5-(difluoromethyl)-5-(2-ethoxy-2-oxoethyl)-1,4,2-dioxazol-3-yl)pyrrolidine-1-carboxylate (51k or 51l) ${ }^{19} \mathrm{~F}$ NMR

S235

tert-Butyl 3-(5-(bromomethyl)-5-(trifluoromethyl)-1,4,2-dioxazol-3-yl)azetidine-1-carboxylate (52j) ${ }^{1} \mathrm{H}$ NMR

tert-Butyl 3-(5-(bromomethyl)-5-(trifluoromethyl)-1,4,2-dioxazol-3-yl)azetidine-1-carboxylate (52j) ${ }^{13} \mathrm{C} \mathrm{NMR}$

tert-Butyl 3-(5-(bromomethyl)-5-(trifluoromethyl)-1,4,2-dioxazol-3-yl)azetidine-1-carboxylate (52j) ${ }^{19} \mathrm{~F}$ NMR

tert-Butyl 3-(5-(bromomethyl)-5-(difluoromethyl)-1,4,2-dioxazol-3-yl)azetidine-1-carboxylate (53j) ${ }^{13} \mathrm{C}$ NMR

3-(1-Methylpyrrolidin-2-yl)-5-(trifluoromethyl)isoxazole (56k or 56l) ${ }^{1} \mathrm{H}$ NMR

3-(1-Methylpyrrolidin-2-yl)-5-(trifluoromethyl)isoxazole (56k or 56l) ${ }^{13} \mathrm{C} \mathrm{NMR}$...

3-(1-Methylpyrrolidin-2-yl)-5-(trifluoromethyl)isoxazole (56k or 56l) ${ }^{19} \mathrm{~F}$ NMR

$N^{\prime}$-(3-Chlorophenyl)-2-oxo-2-(5-(trifluoromethyl)isoxazol-3-yl)aceto-hydrazonoyl cyanide (57) ${ }^{1} \mathrm{H}$ NMR. 
X-Ray diffraction studies of $3 \mathrm{~h}$

Computational part

Scheme S1. [3+2] cycloaddition reaction .....

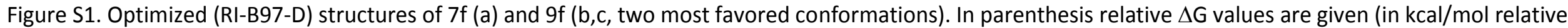
to the free reactants).

Figure S2. Optimized (RI-B97-D) structures of $7 \mathrm{~b}(\mathrm{a})$ and $9 \mathrm{~b}(\mathrm{~b})$. In parenthesis relative $\Delta \mathrm{G}$ values are given (in kcal/mol relative to the free reactants). S251

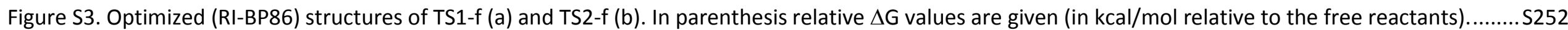

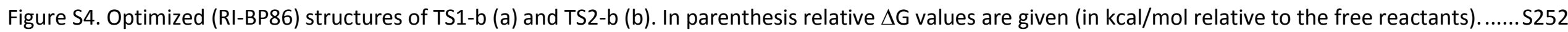
Table S1. Calculated (RI-B97-D/TZVP) total energy values (E), zero-point energy corrections (ZPE), thermal corrections to Gibbs free energy (TCGFE), corrected energy magnitudes ( $E+Z P E$ and $E+T C G F E)$, reaction energies (DE and DG) and lowest vibration frequencies ( $\Delta \Delta \delta$ for the local minima structures)

Table S2. Calculated (RI-BP86/TZVP) total energy values (E), zero-point energy corrections (ZPE), thermal corrections to Gibbs free energy (TCGFE), corrected energy magnitudes (E+ZPE and E+TCGFE), reaction energies (DE and DG) and lowest vibration frequencies ( $\Delta \Delta \delta$ for the local minima and transition state structures)

Table S3. Atom coordinates for the optimized [RI-BP86 (left) and RI-B97-D (right)] structures. 

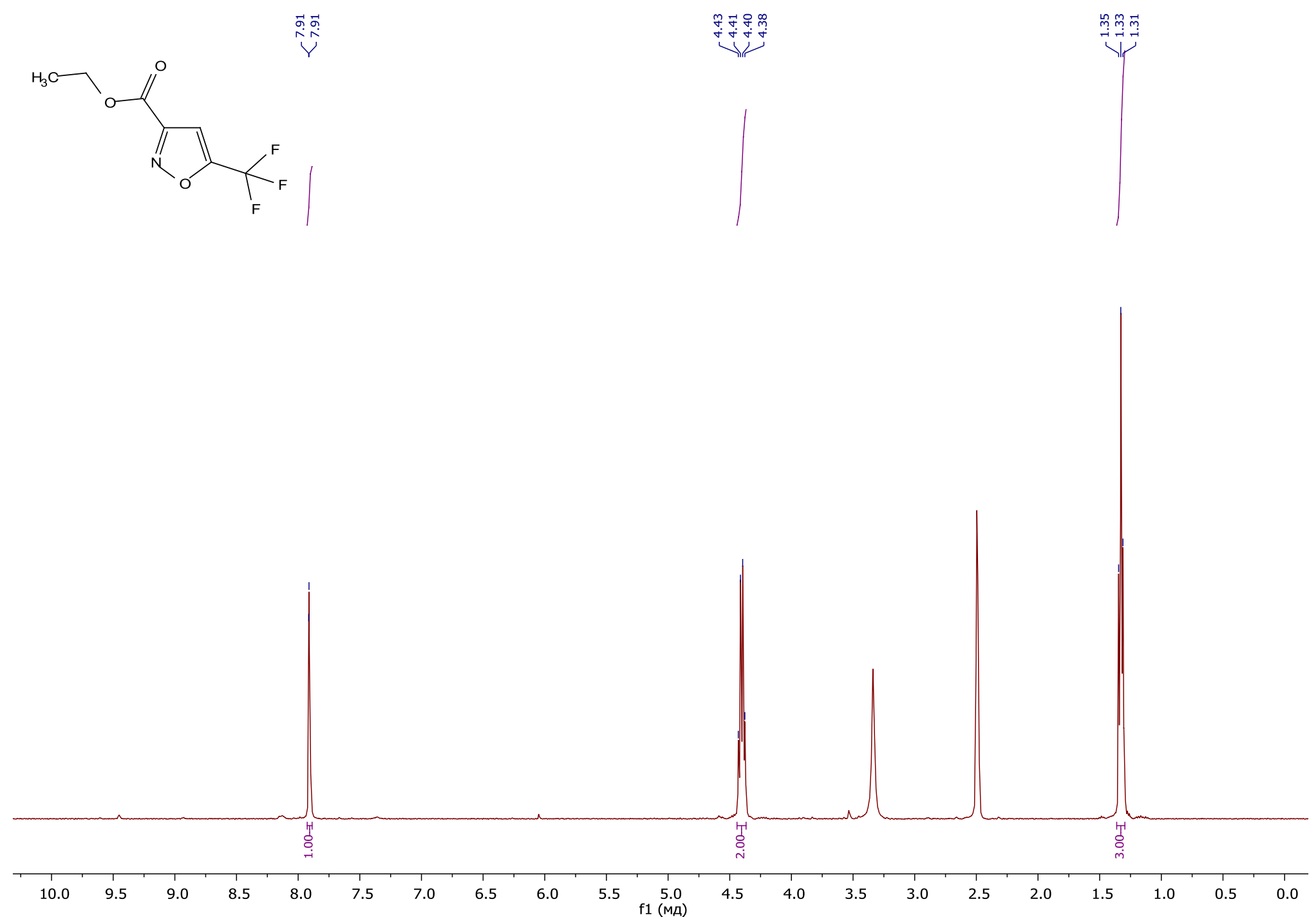

Ethyl 5-(trifluoromethyl)isoxazole-3-carboxylate (3a) ${ }^{1}$ H NMR 


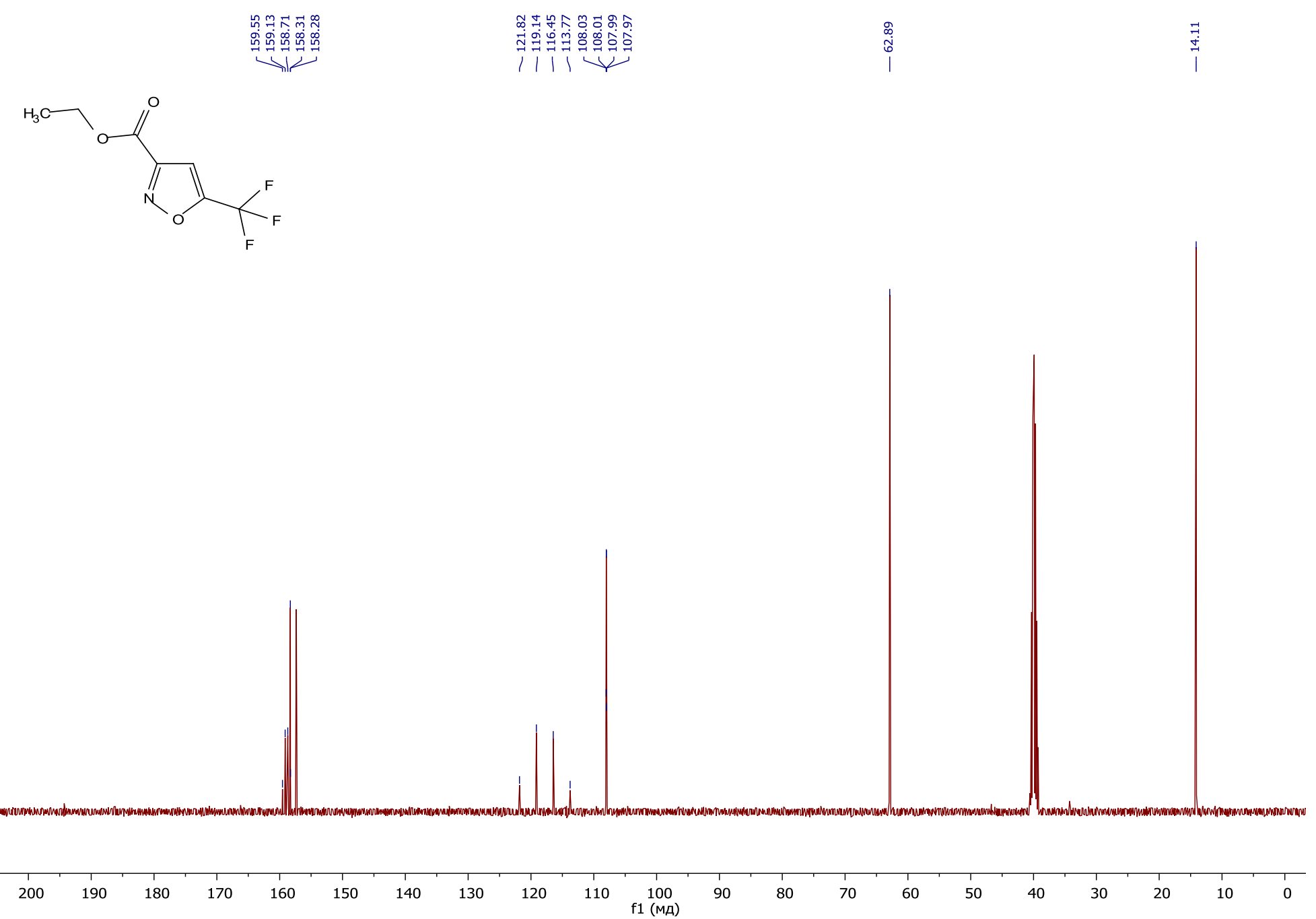

Ethyl 5-(trifluoromethyl)isoxazole-3-carboxylate (3a) ${ }^{13}$ C NMR 

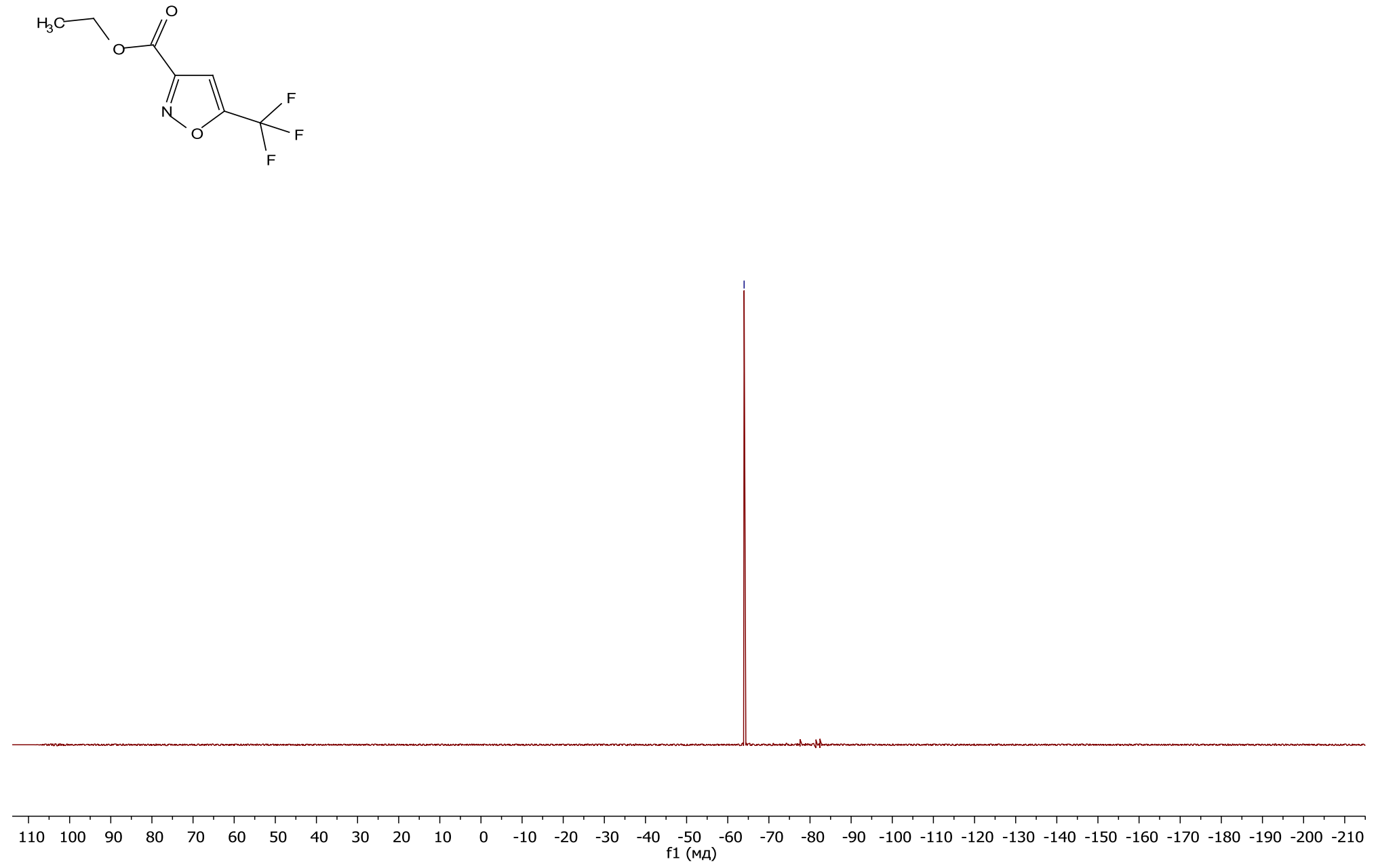

Ethyl 5-(trifluoromethyl)isoxazole-3-carboxylate (3a) ${ }^{19}$ F NMR 

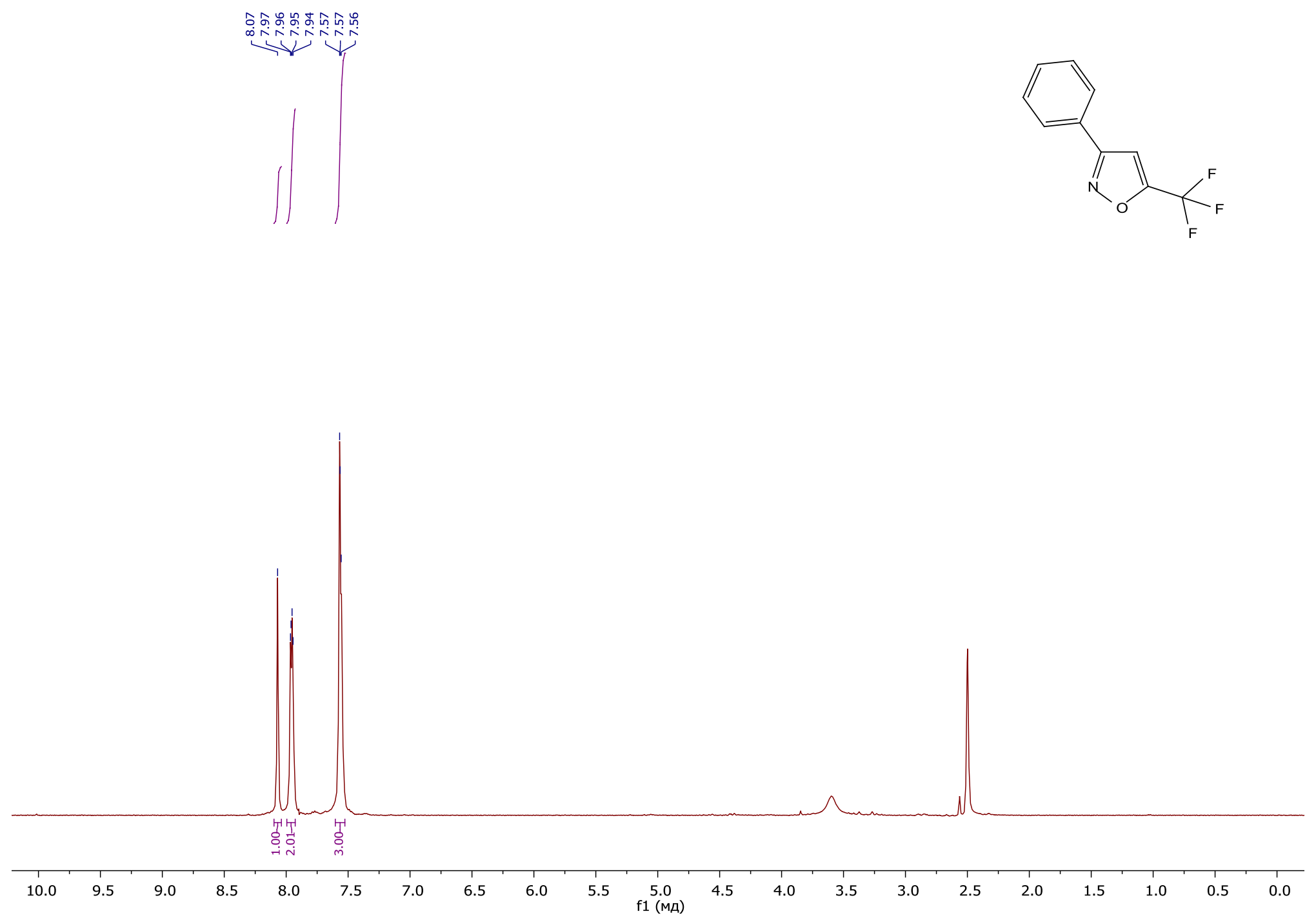

3-Phenyl-5-(trifluoromethyl)isoxazole (3b) ${ }^{1}$ H NMR 


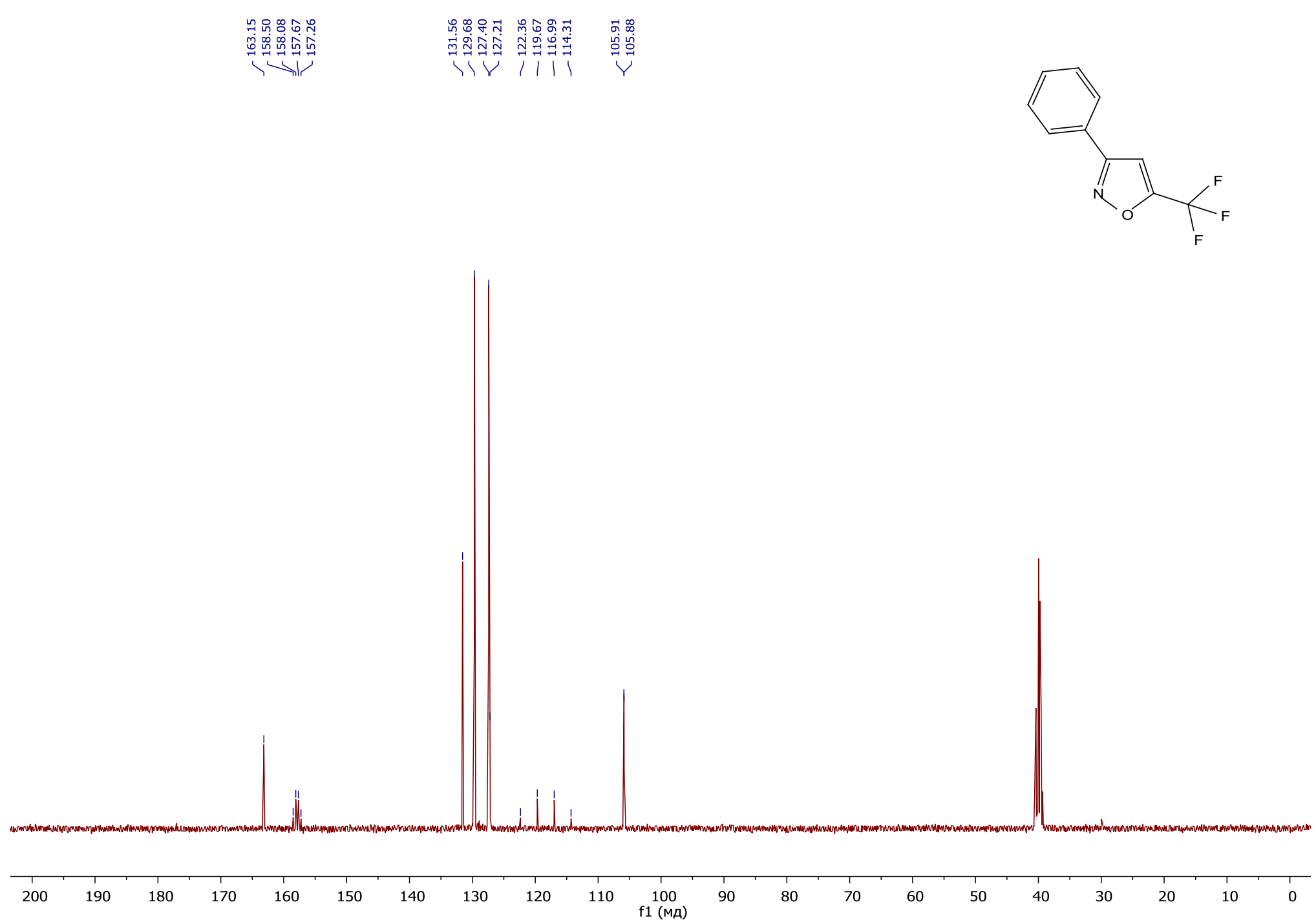

3-Phenyl-5-(trifluoromethyl)isoxazole (3b) ${ }^{13} \mathrm{C}$ NMR 

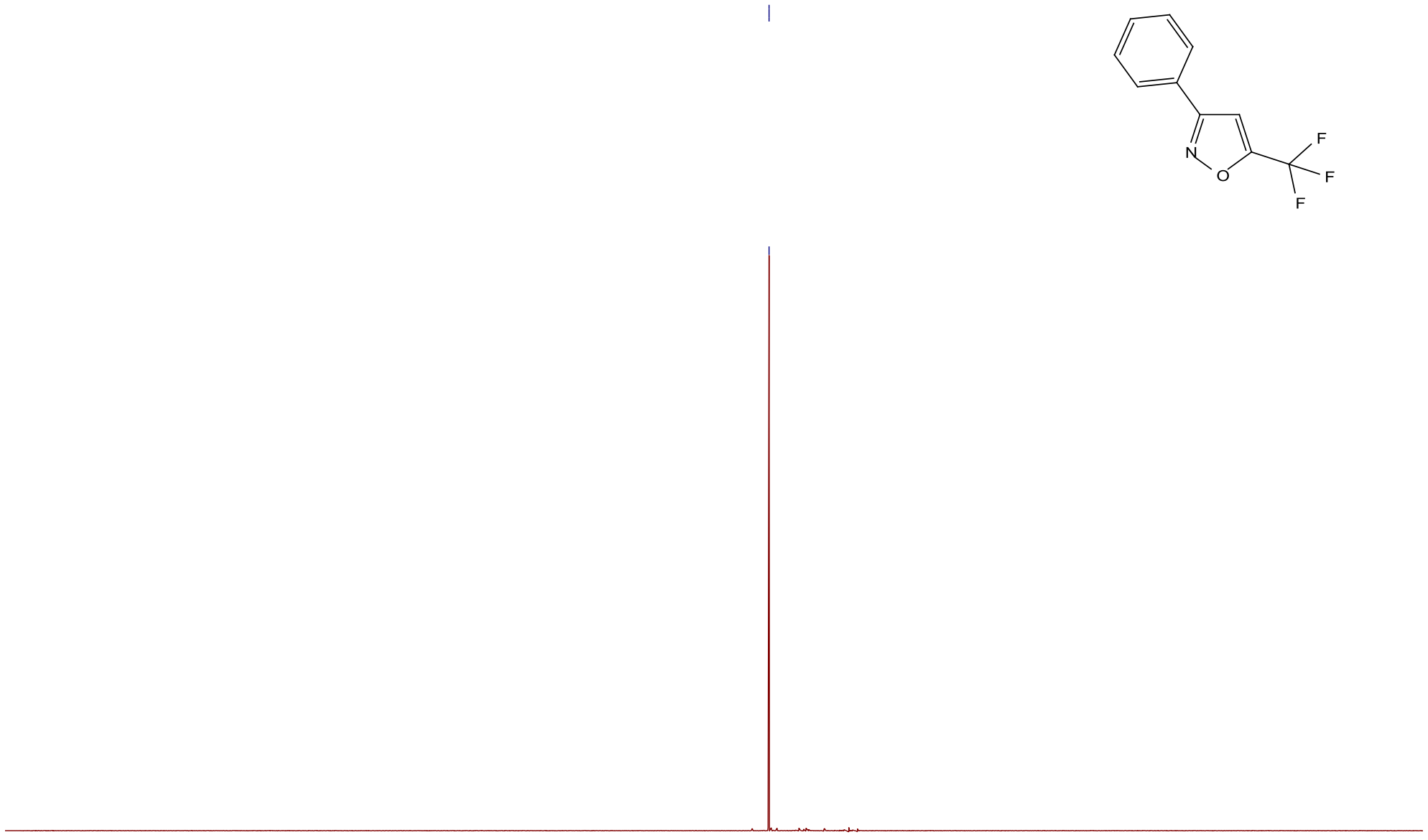

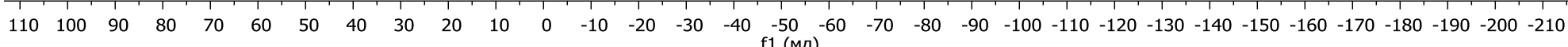

3-Phenyl-5-(trifluoromethyl)isoxazole (3b) ${ }^{19}$ F NMR 


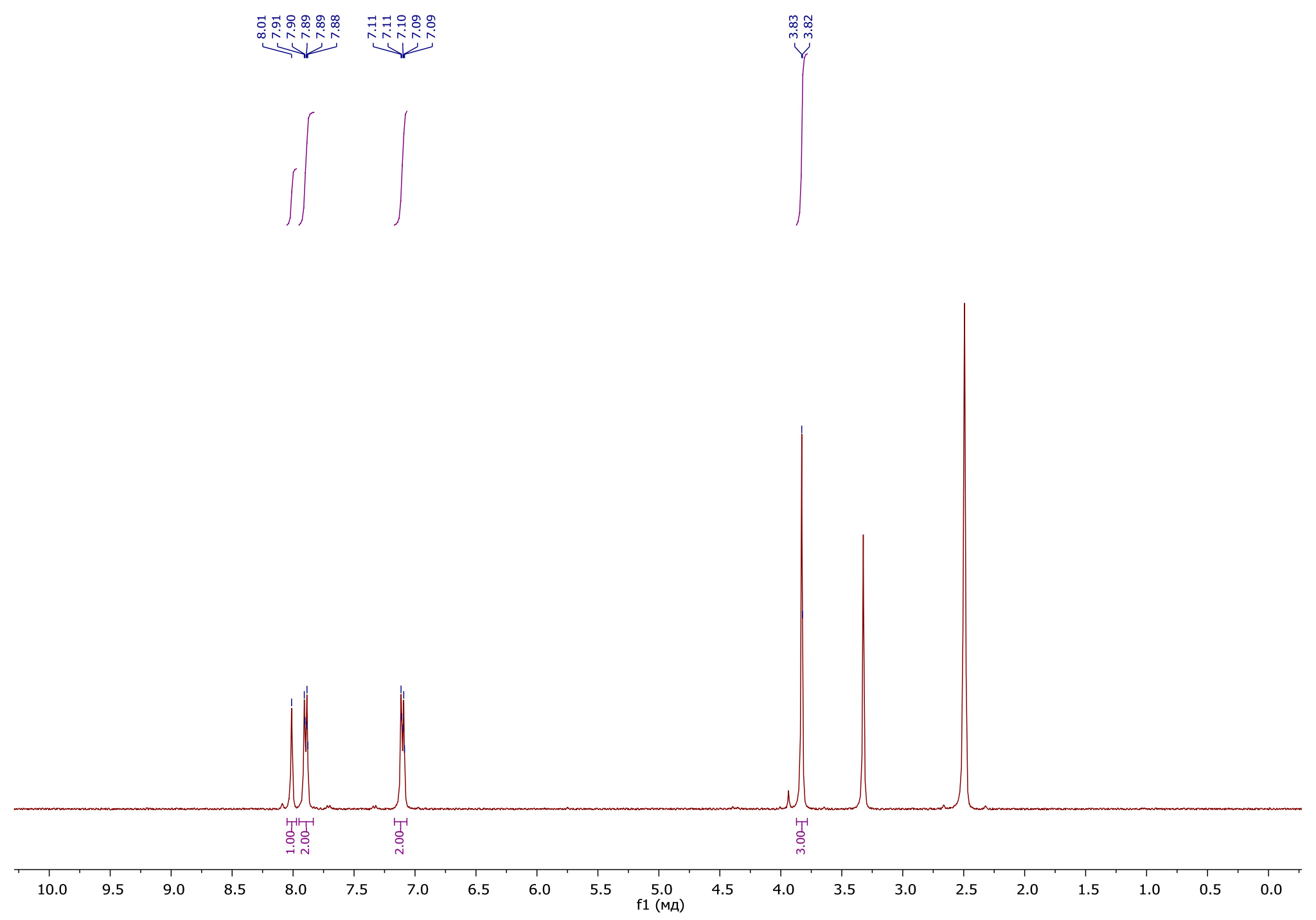

3-(4-Methoxyphenyl)-5-(trifluoromethyl)isoxazole (3c) ${ }^{1}$ H NMR 


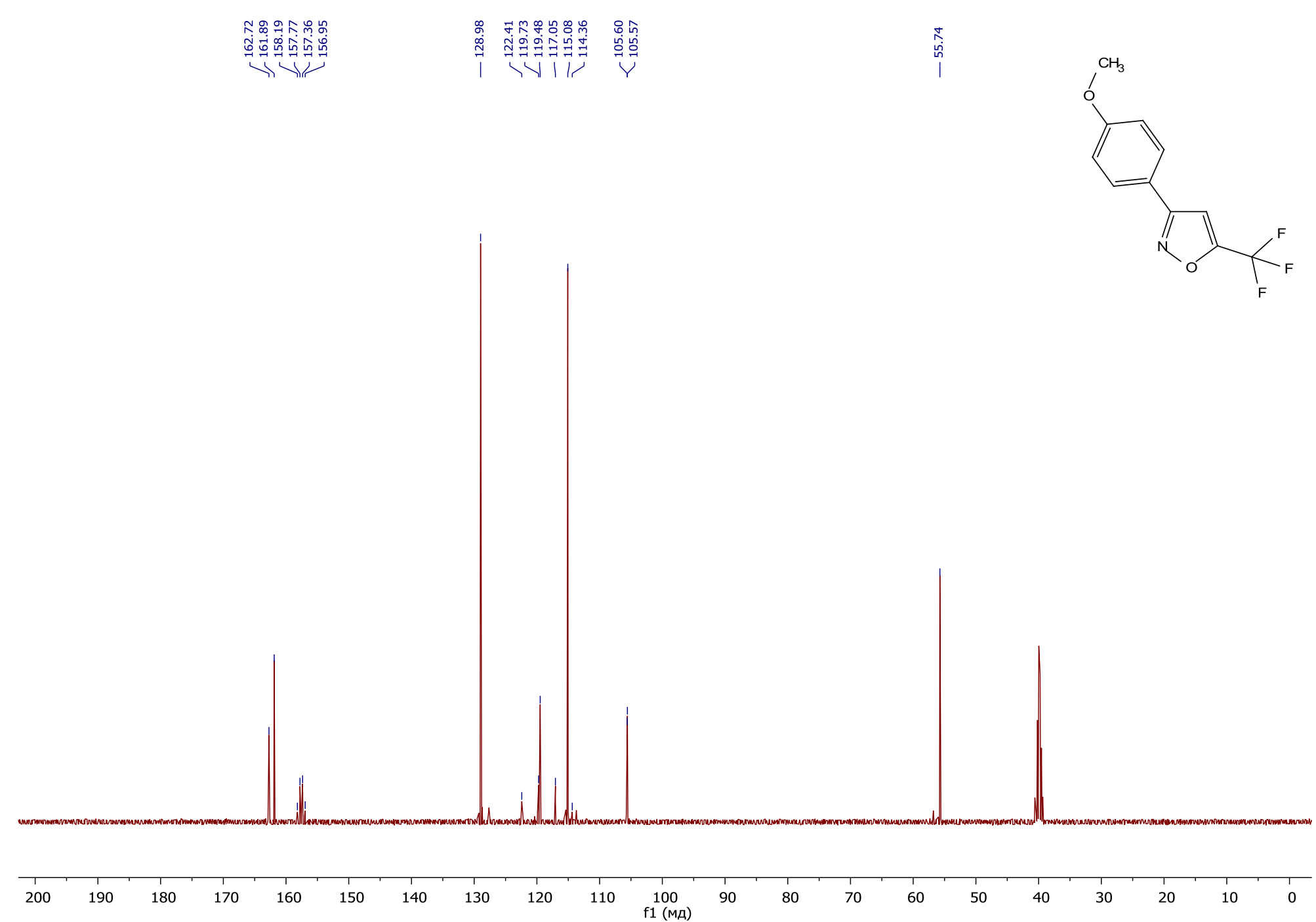

3-(4-Methoxyphenyl)-5-(trifluoromethyl)isoxazole (3c) ${ }^{13} \mathrm{C}$ NMR 

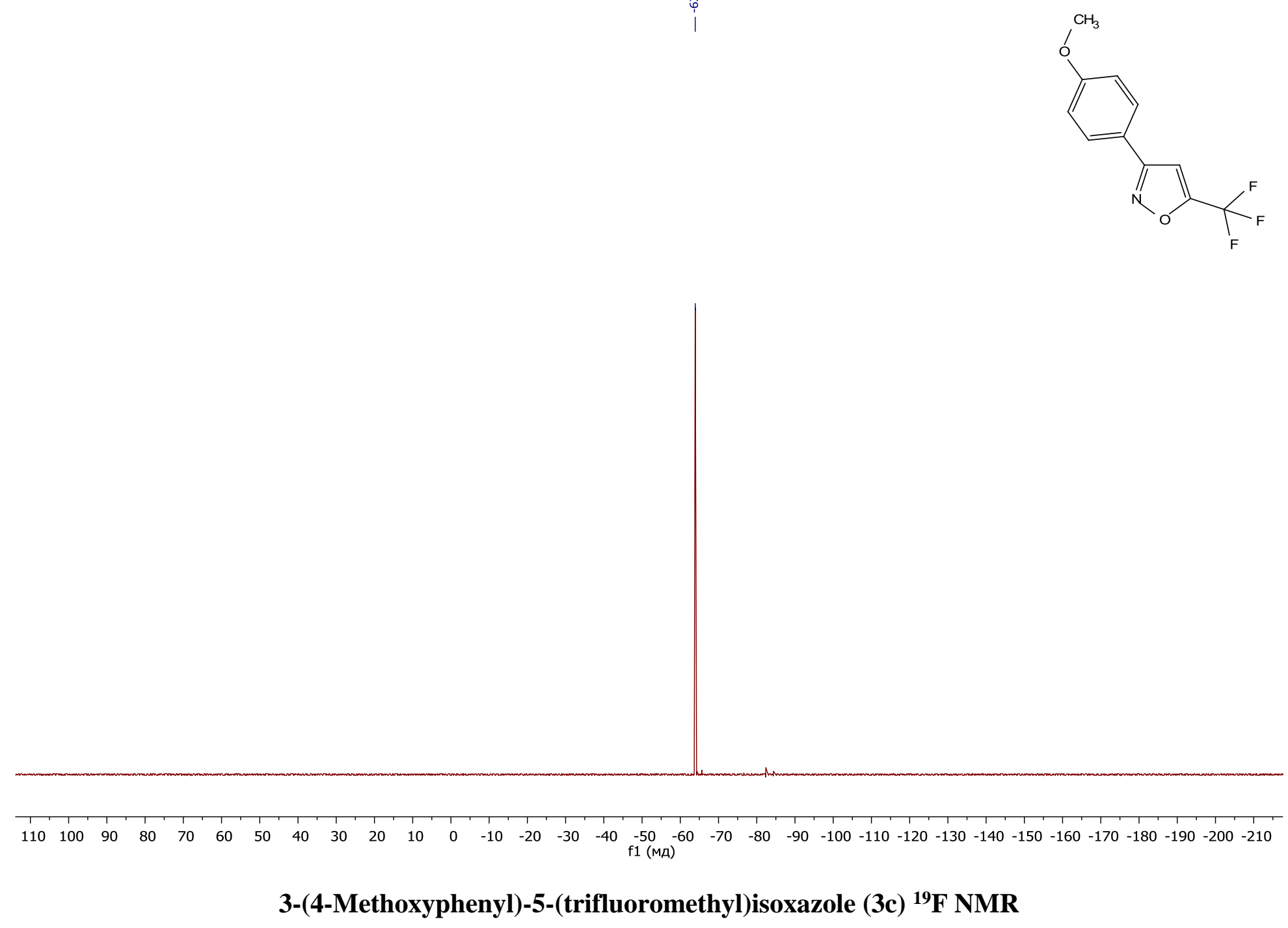

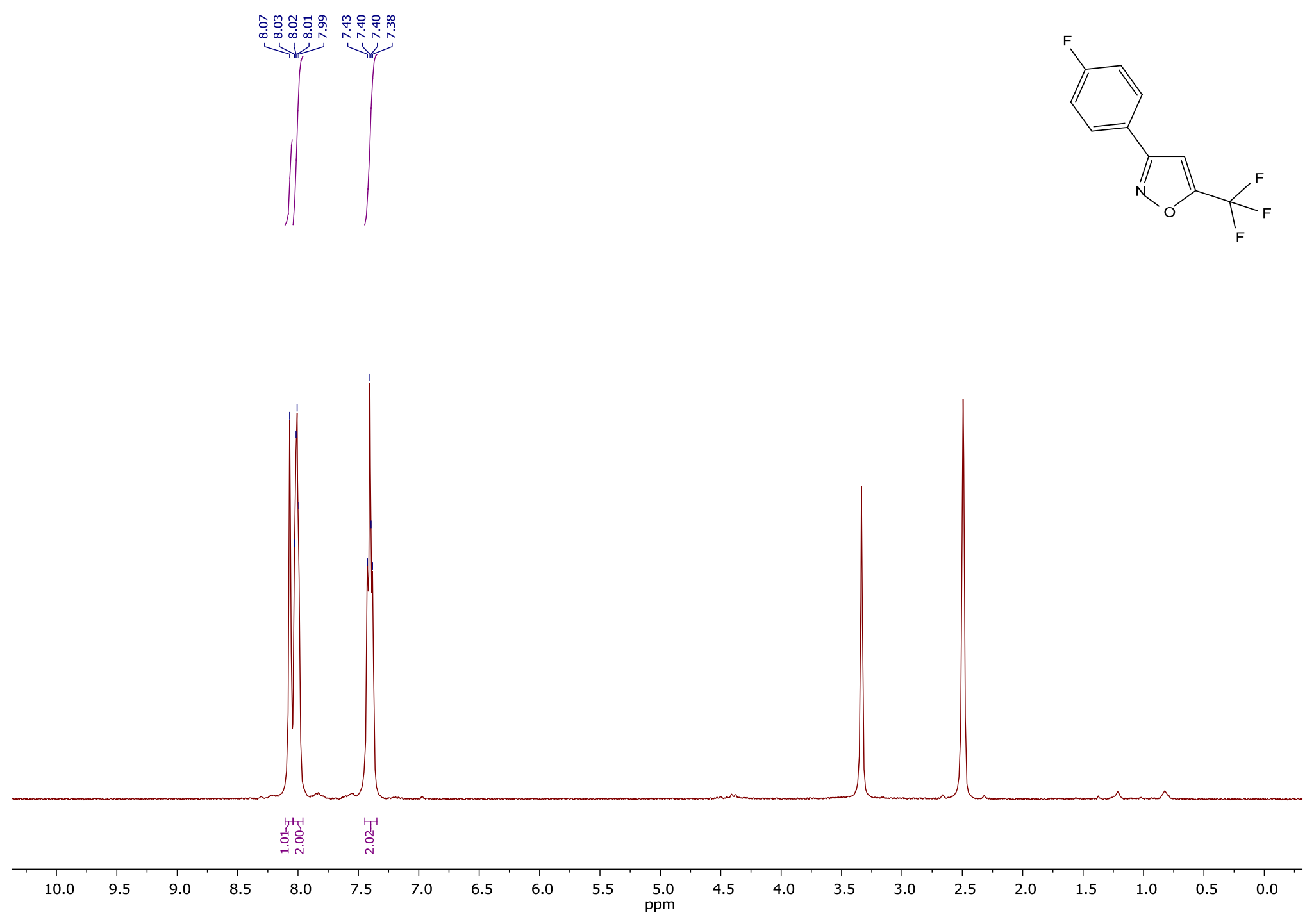

3-(4-Fluorophenyl)-5-(trifluoromethyl)isoxazole (3d) ${ }^{1}$ H NMR 


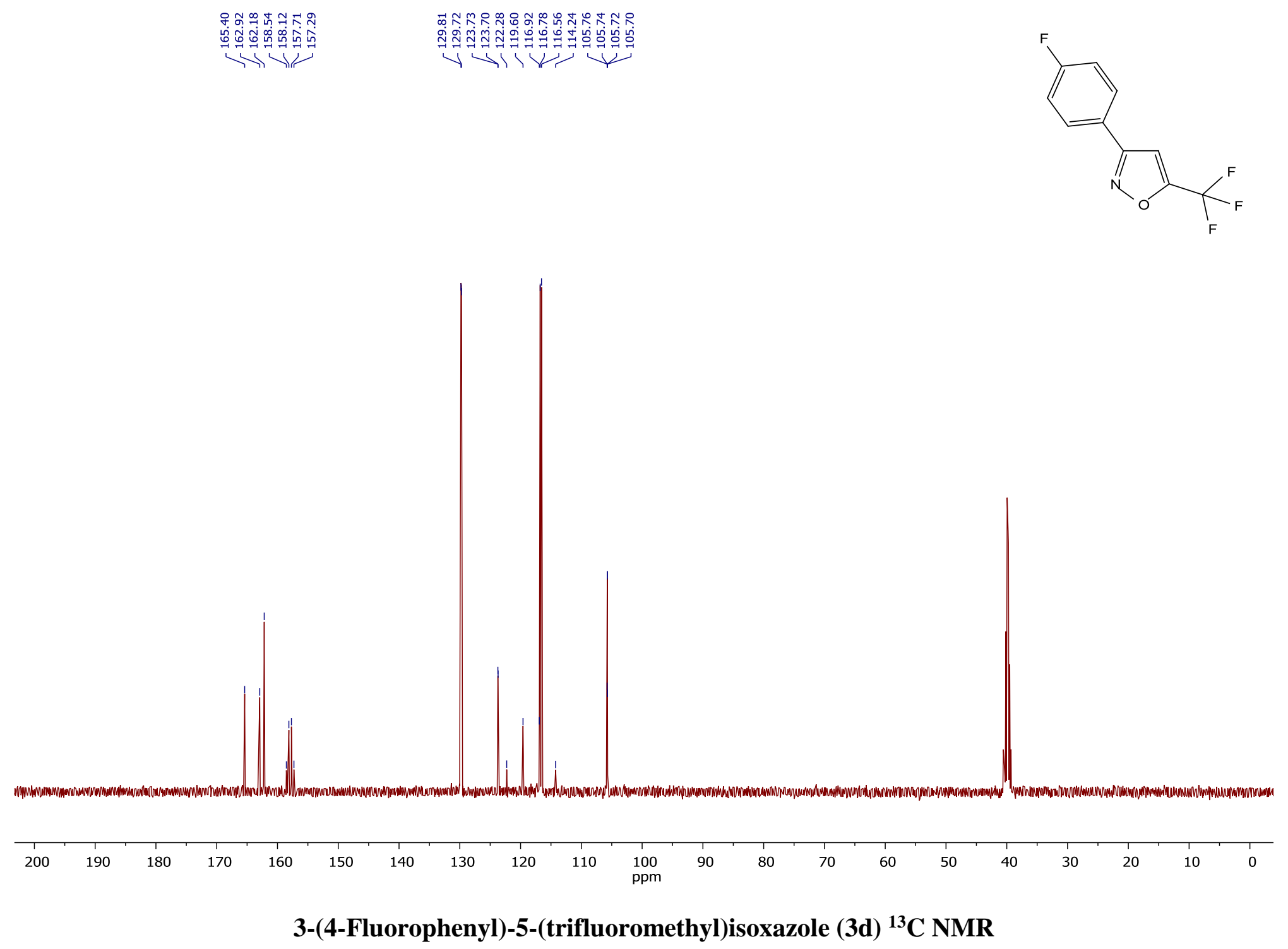



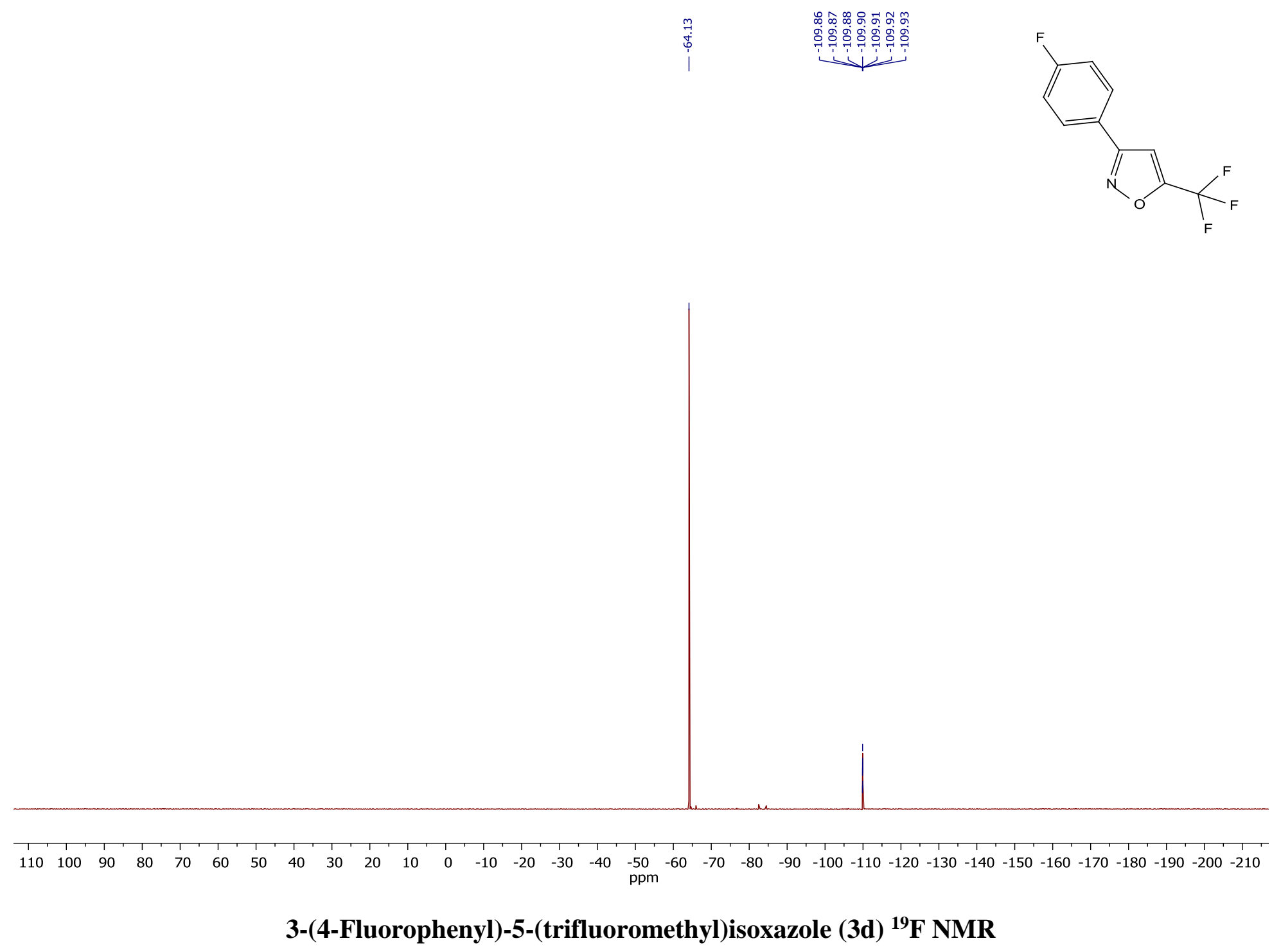


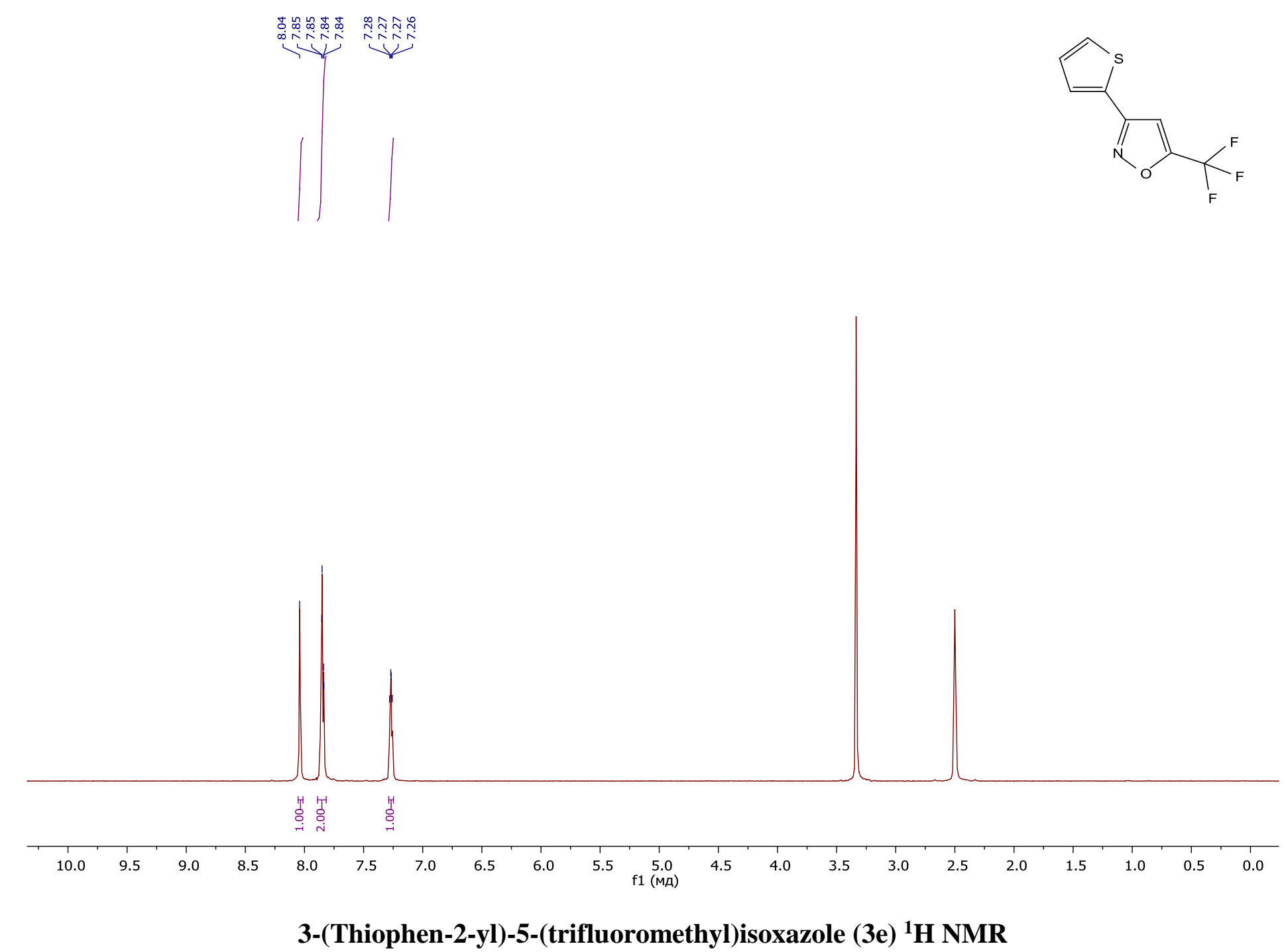




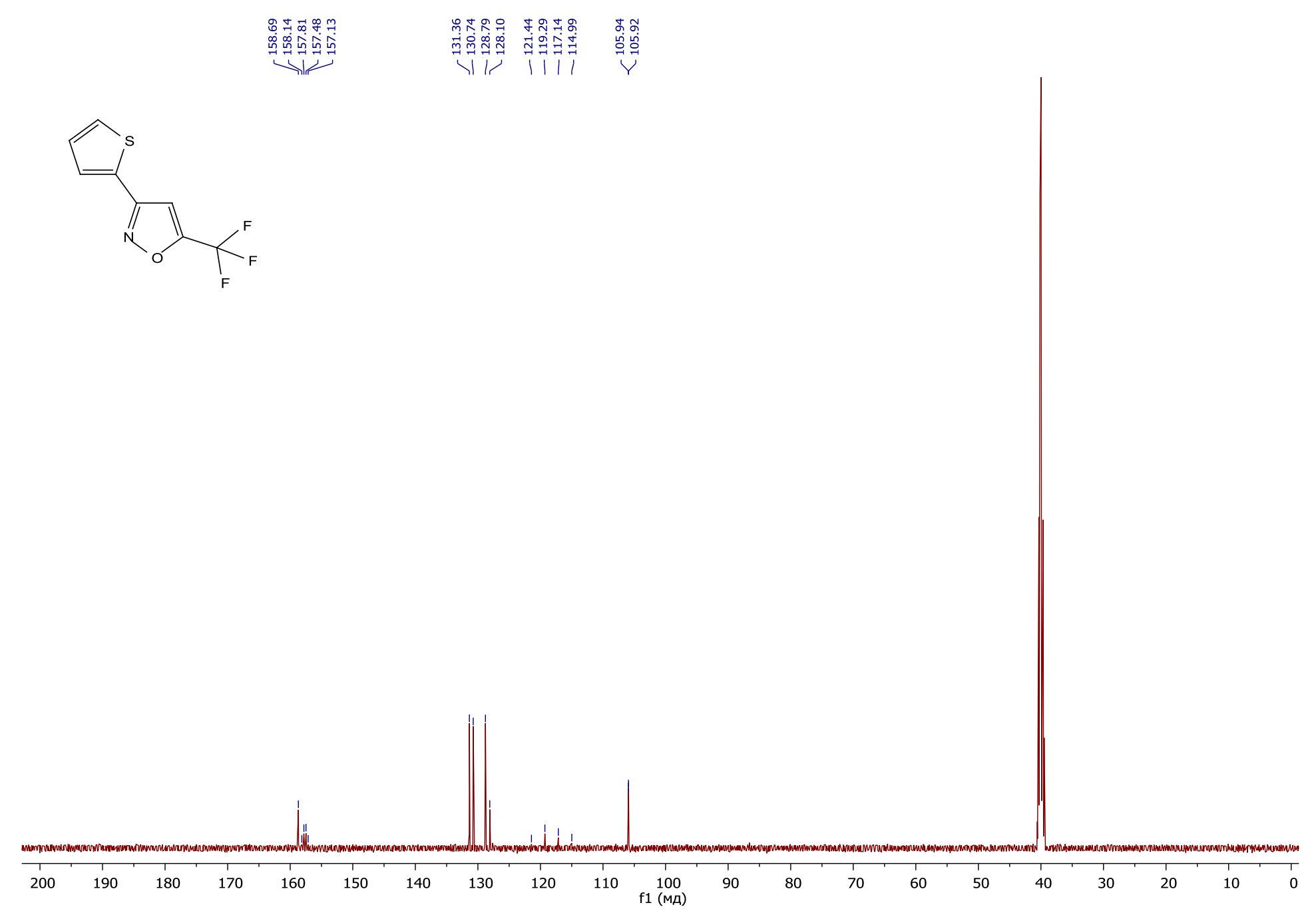

3-(Thiophen-2-yl)-5-(trifluoromethyl)isoxazole (3e) ${ }^{13}$ C NMR 

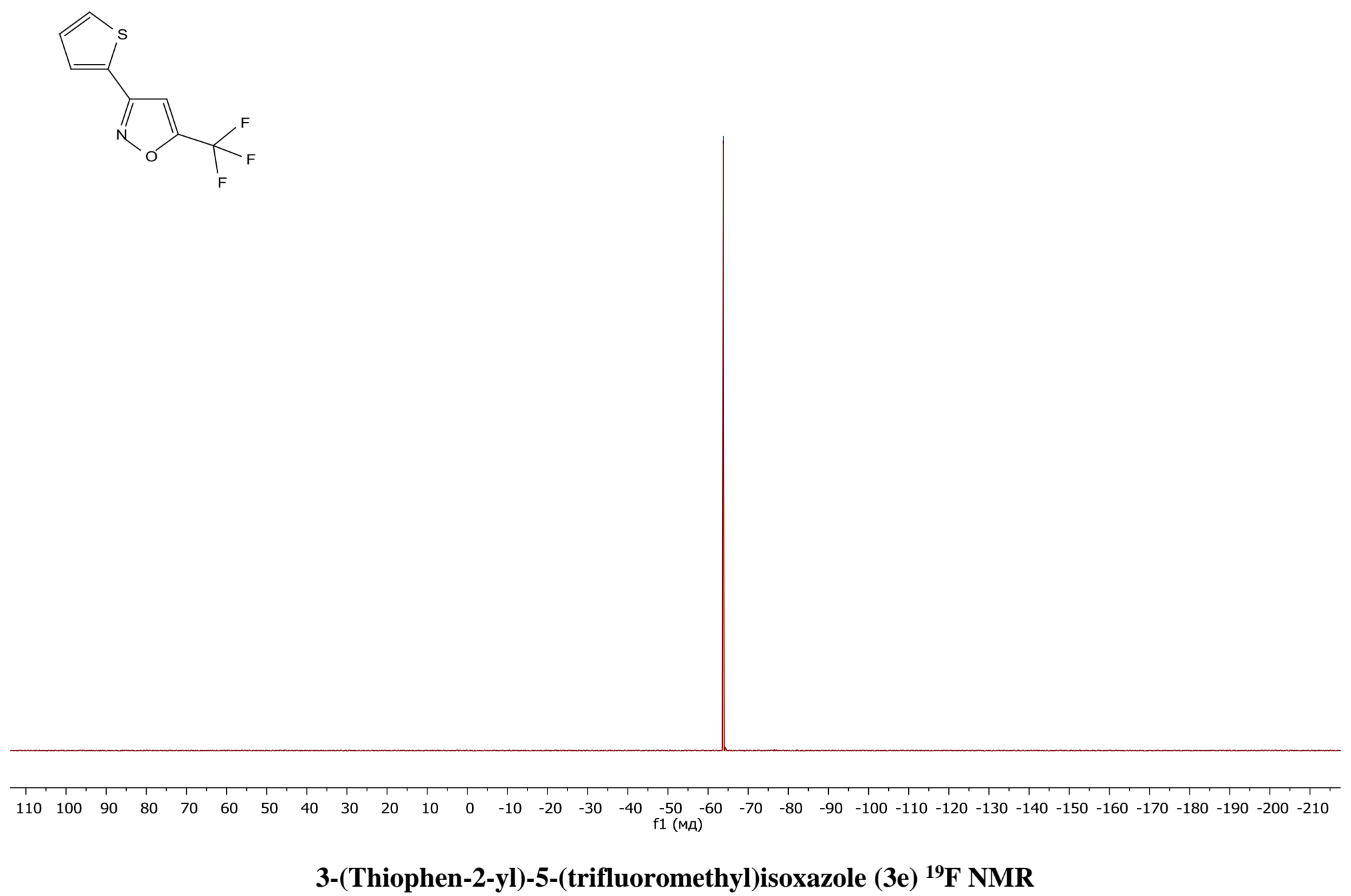


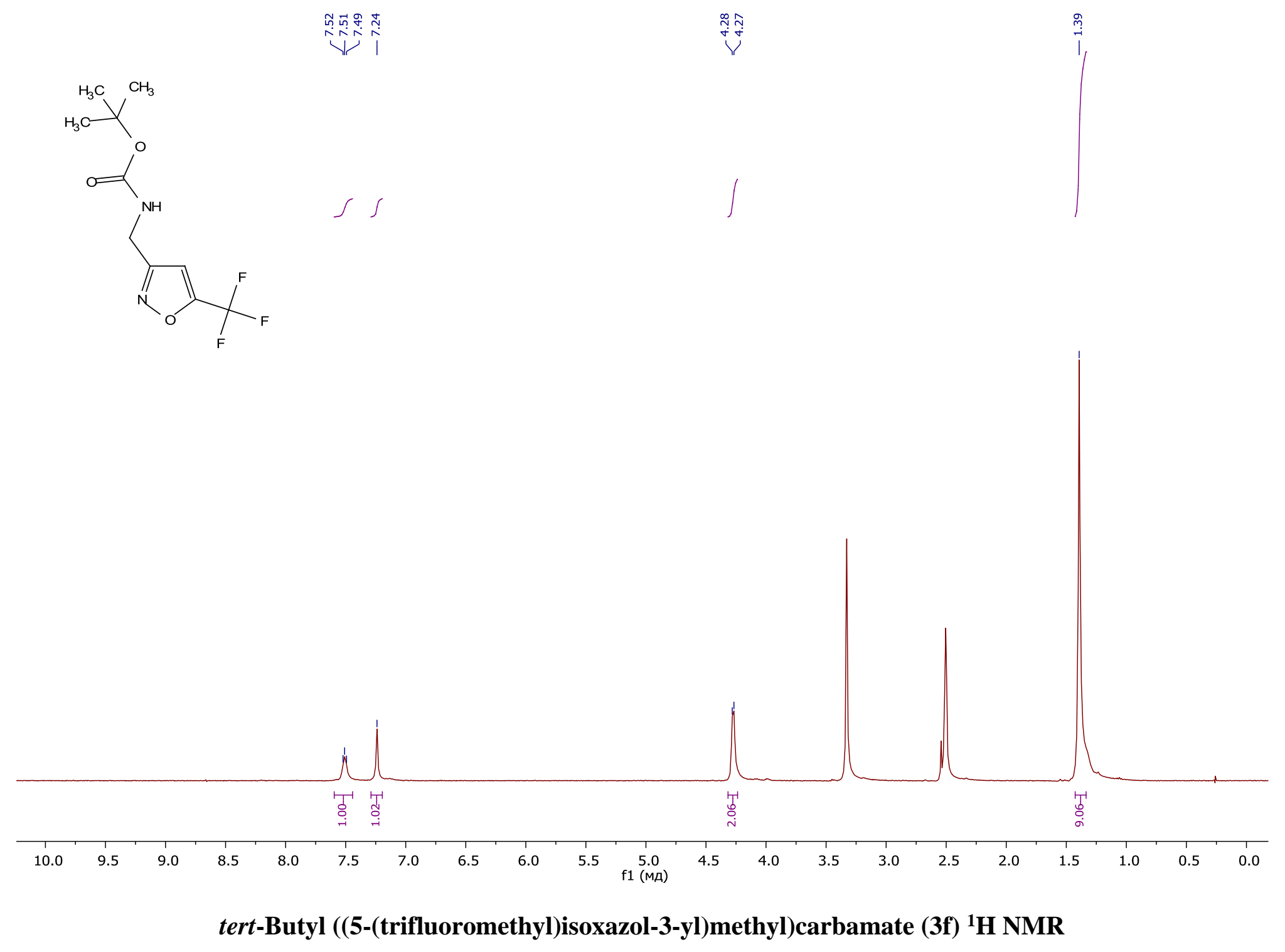




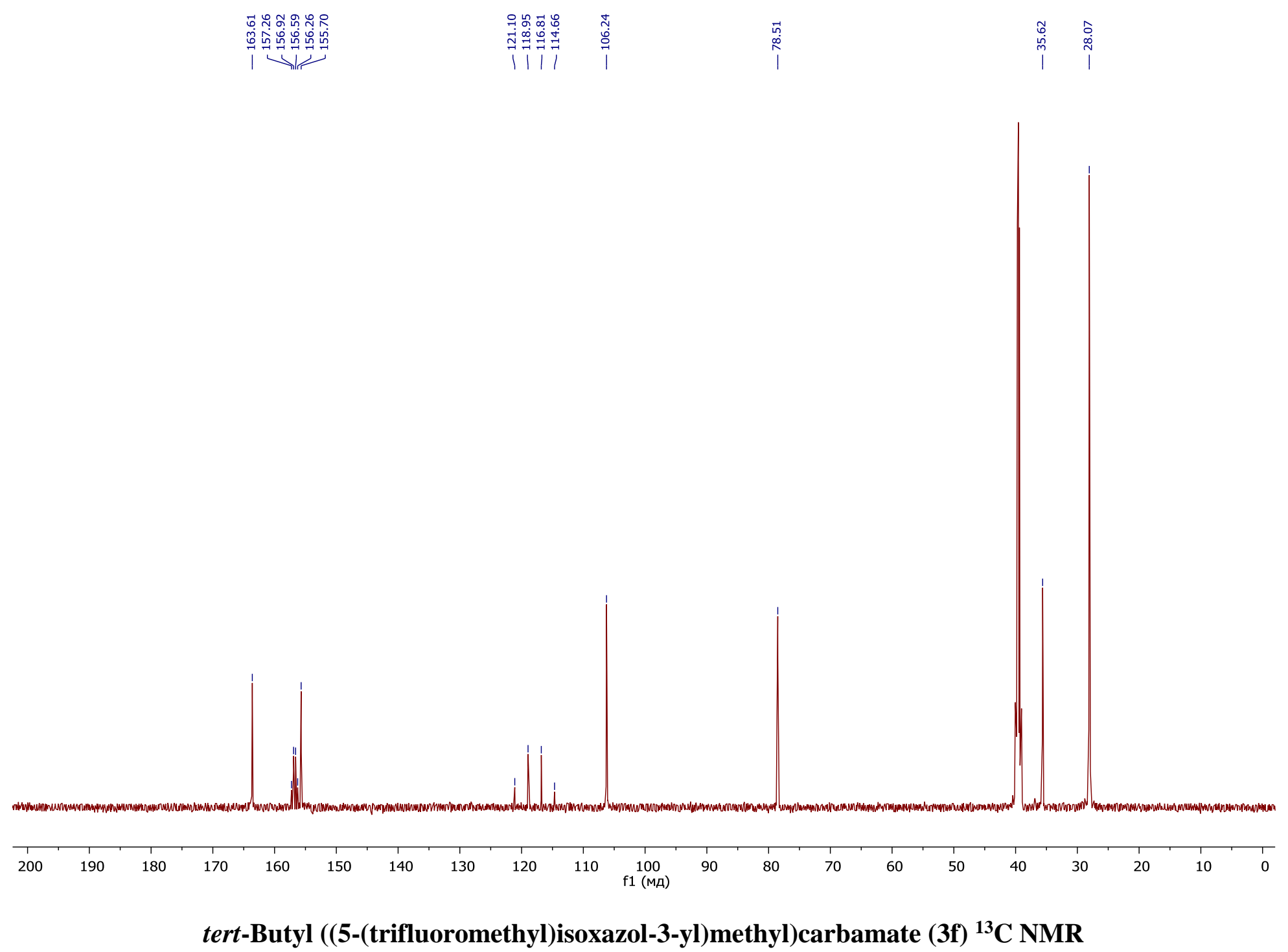



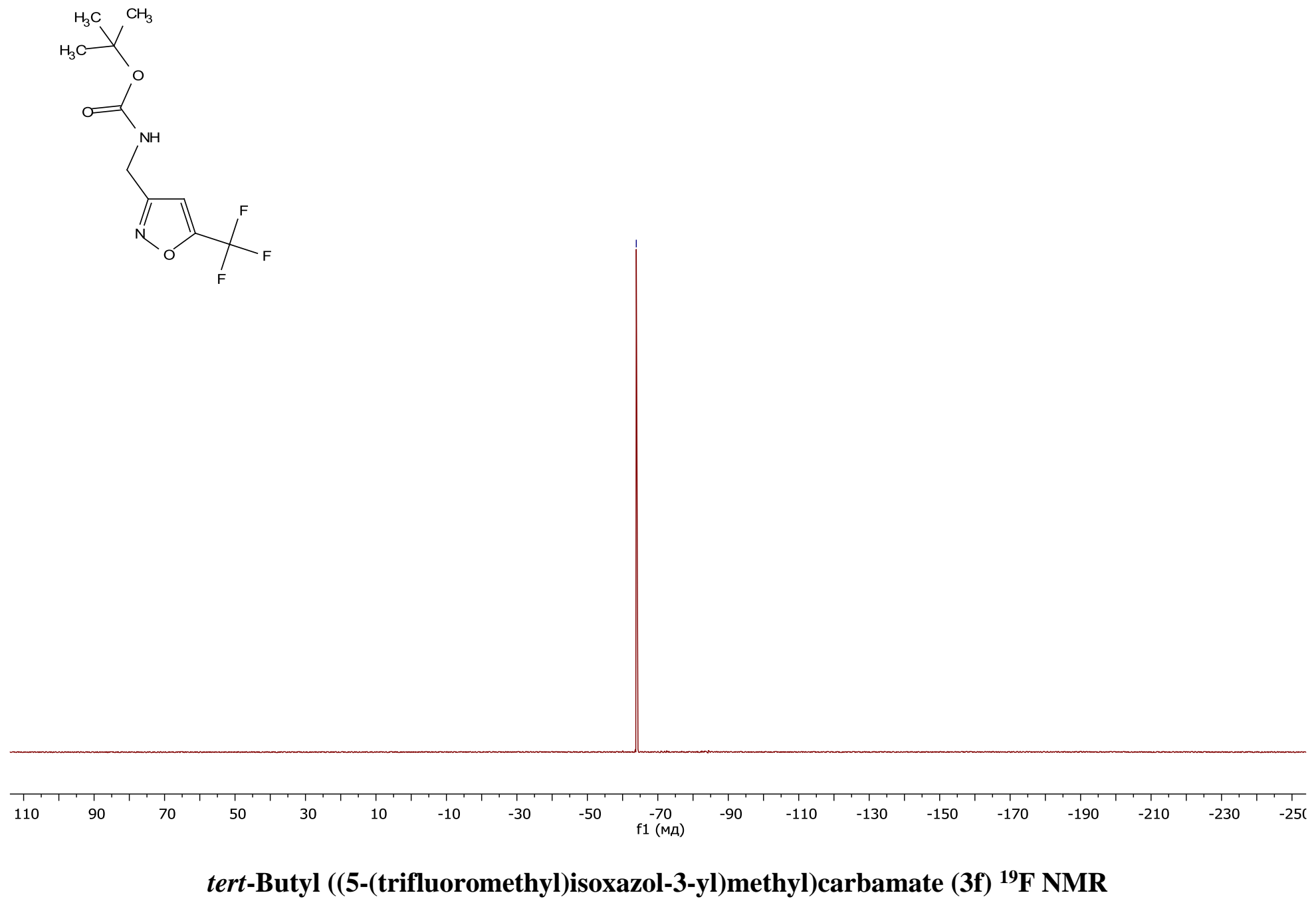

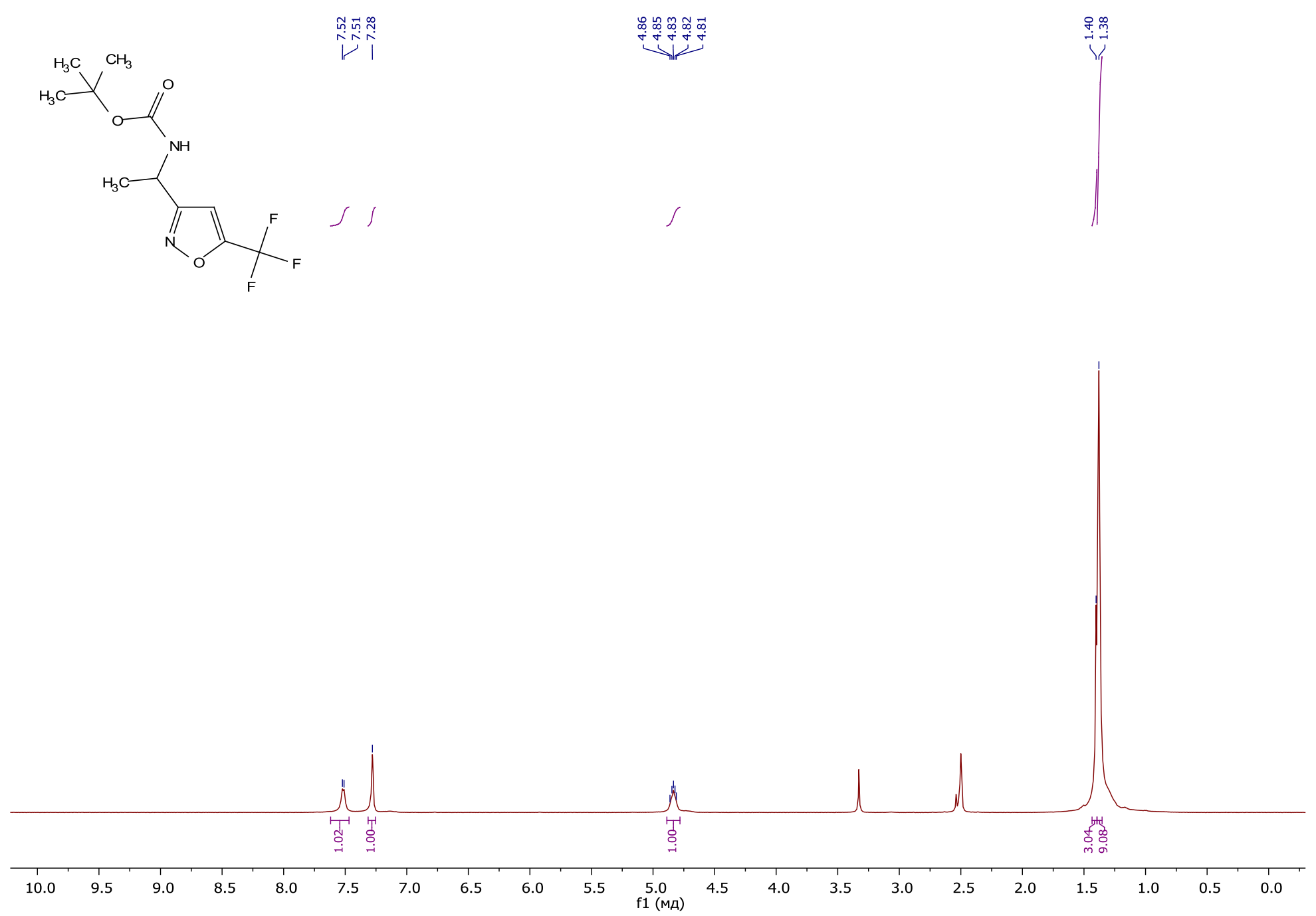

tert-Butyl (1-(5-(trifluoromethyl)isoxazol-3-yl)ethyl)carbamate (3g or 3h) ${ }^{1} \mathrm{H}$ NMR 


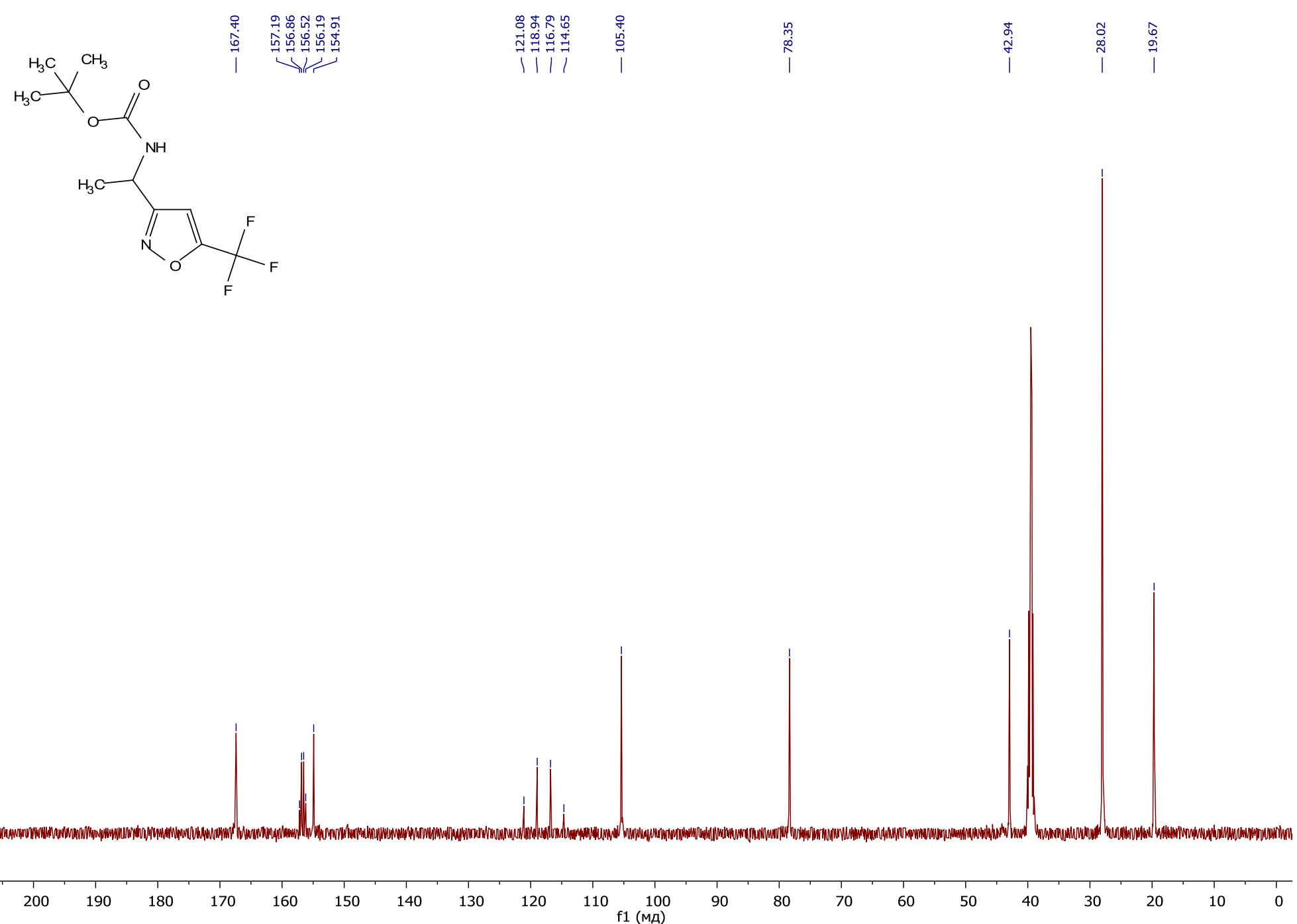

tert-Butyl (1-(5-(trifluoromethyl)isoxazol-3-yl)ethyl)carbamate (3g or 3h) ${ }^{13} \mathrm{C}$ NMR 


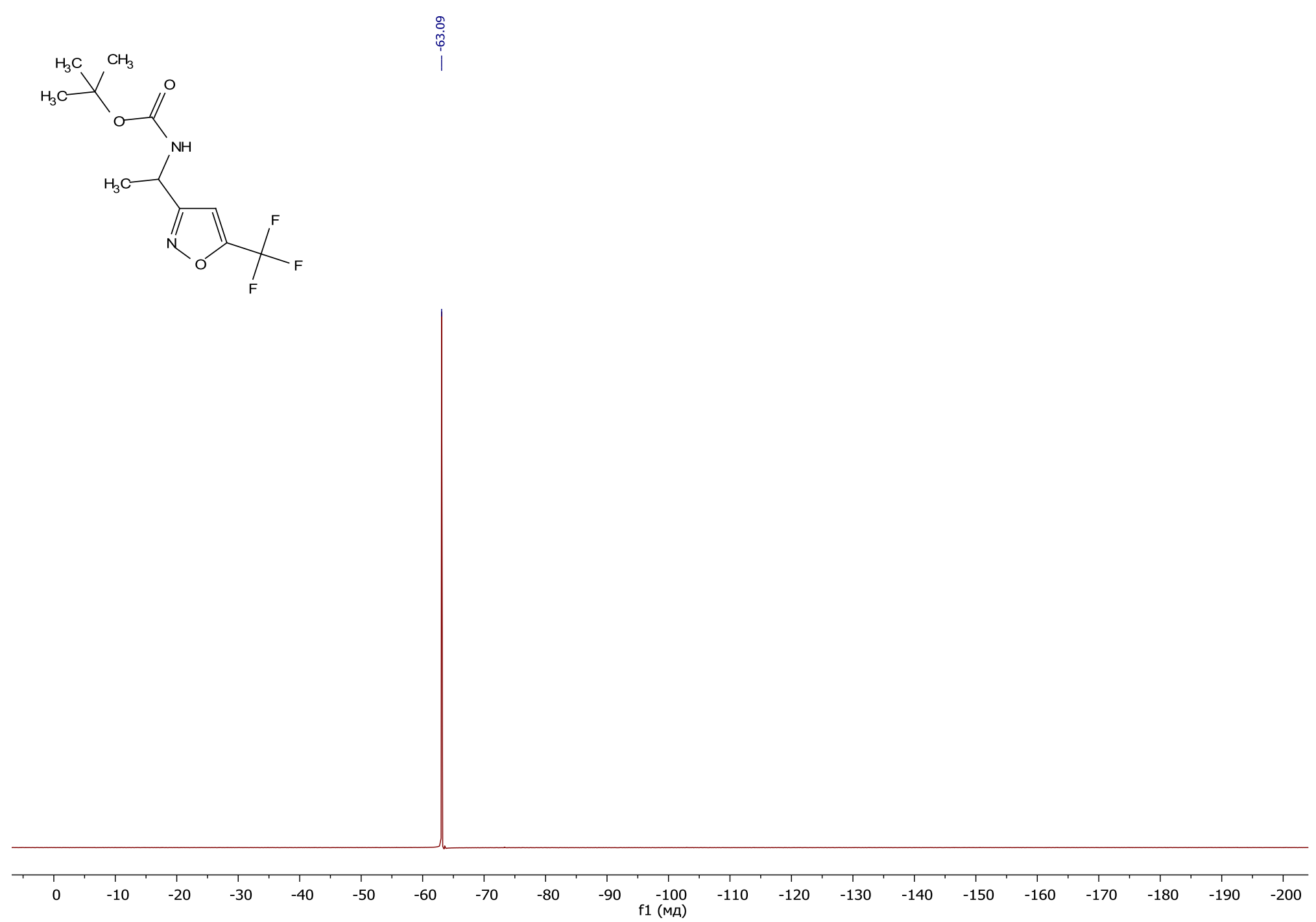

tert-Butyl (1-(5-(trifluoromethyl)isoxazol-3-yl)ethyl)carbamate (3g or 3h) ${ }^{19}$ F NMR 

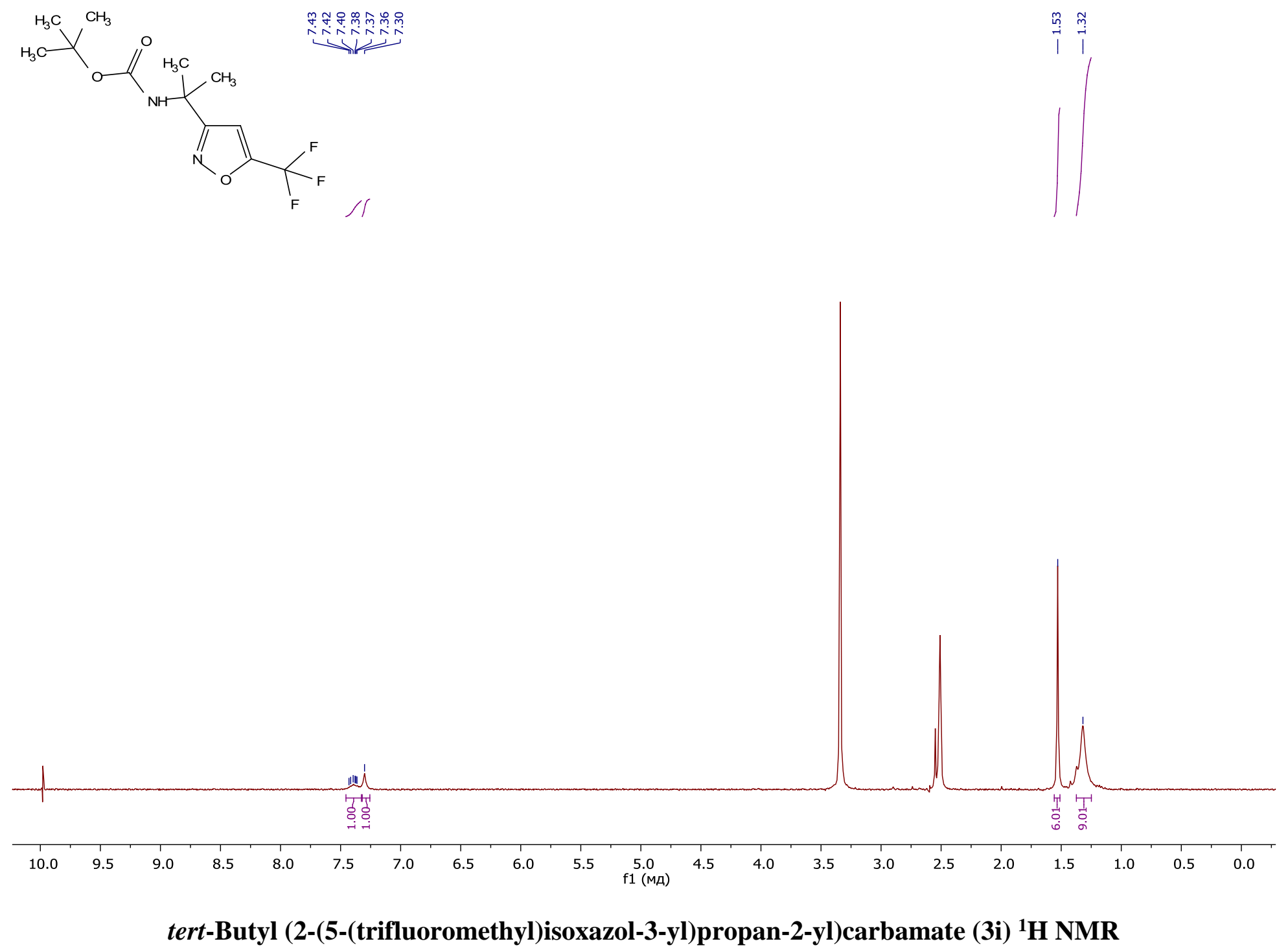


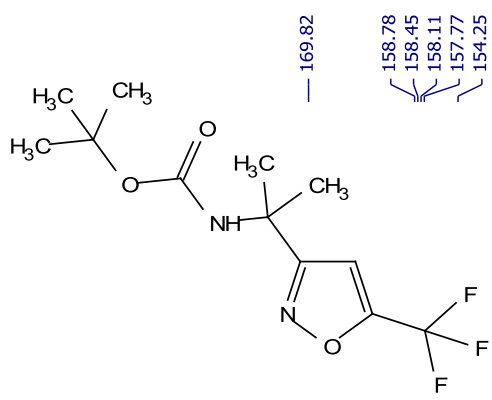

|

$\hat{0}$
$\dot{\infty}$
$\mid$
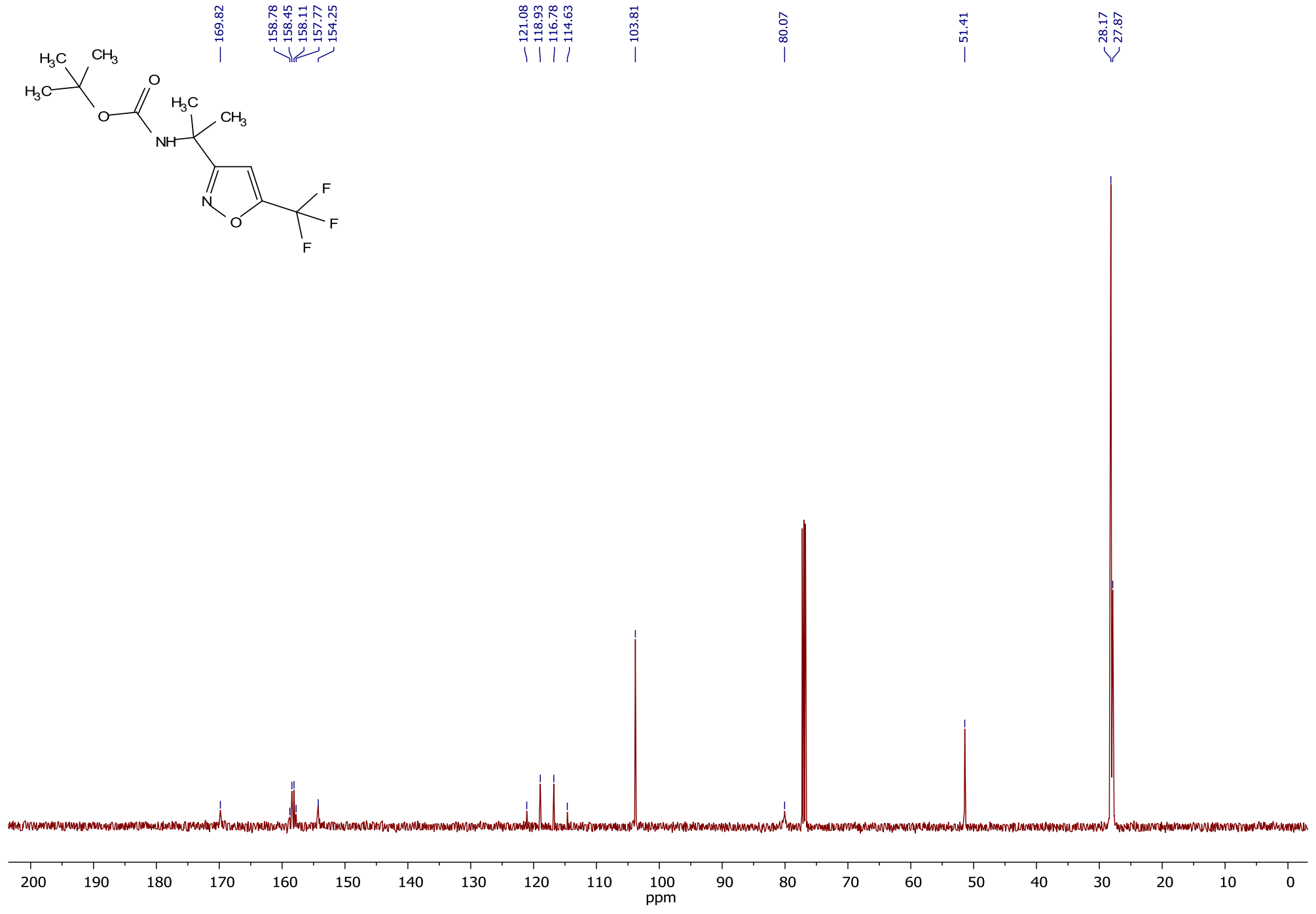

tert-Butyl (2-(5-(trifluoromethyl)isoxazol-3-yl)propan-2-yl)carbamate (3i) ${ }^{13} \mathrm{C}$ NMR

S35 


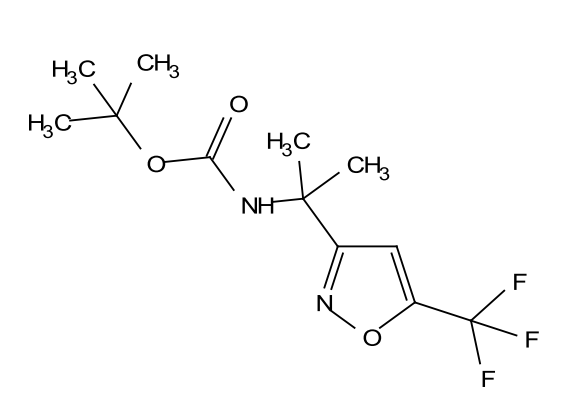

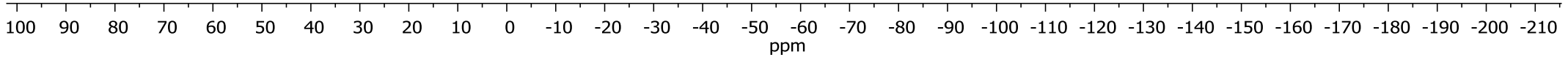 tert-Butyl (2-(5-(trifluoromethyl)isoxazol-3-yl)propan-2-yl)carbamate (3i) ${ }^{19}$ F NMR}



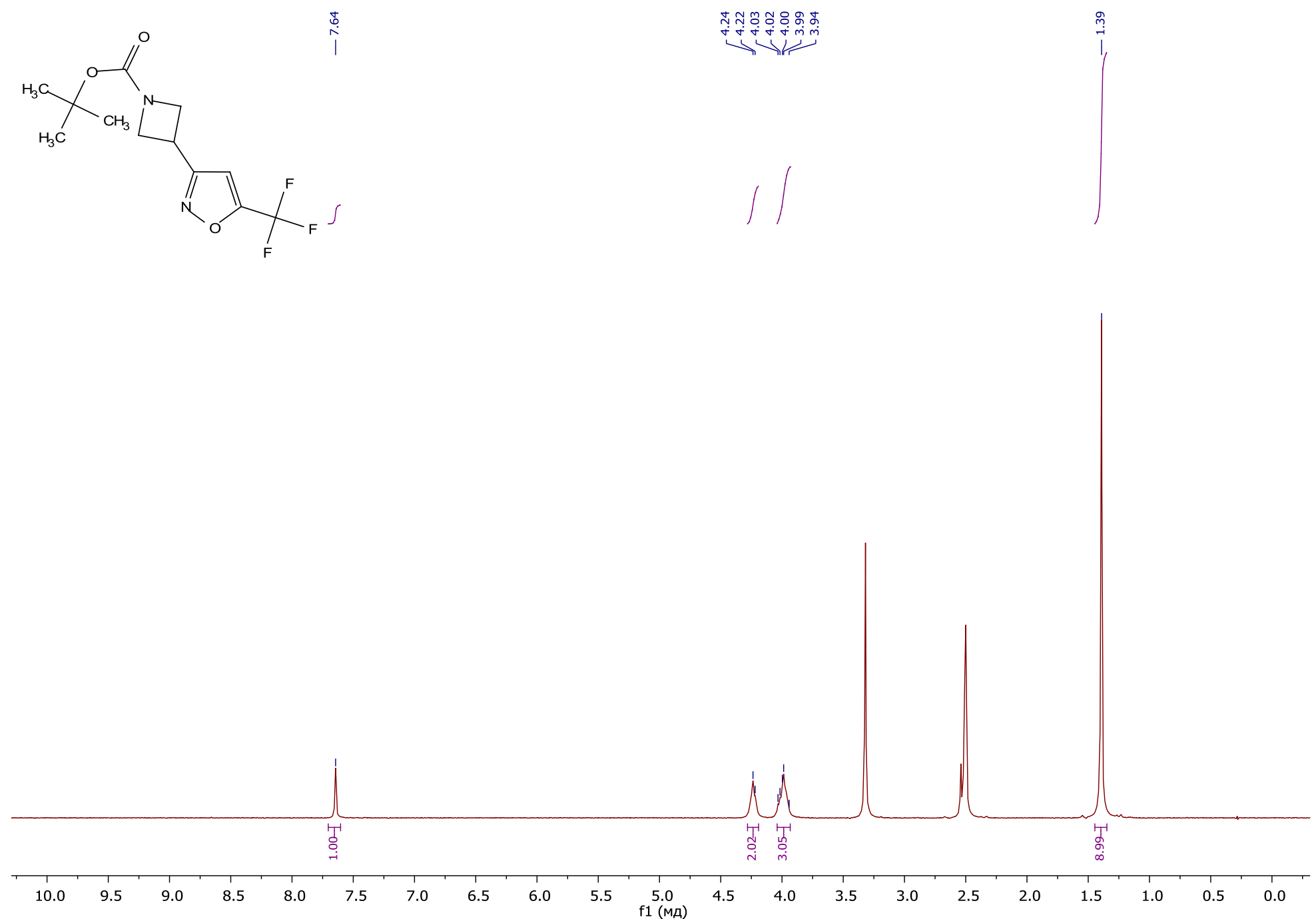

tert-Butyl 3-(5-(trifluoromethyl)isoxazol-3-yl)azetidine-1-carboxylate (3j) ${ }^{1} \mathrm{H}$ NMR 


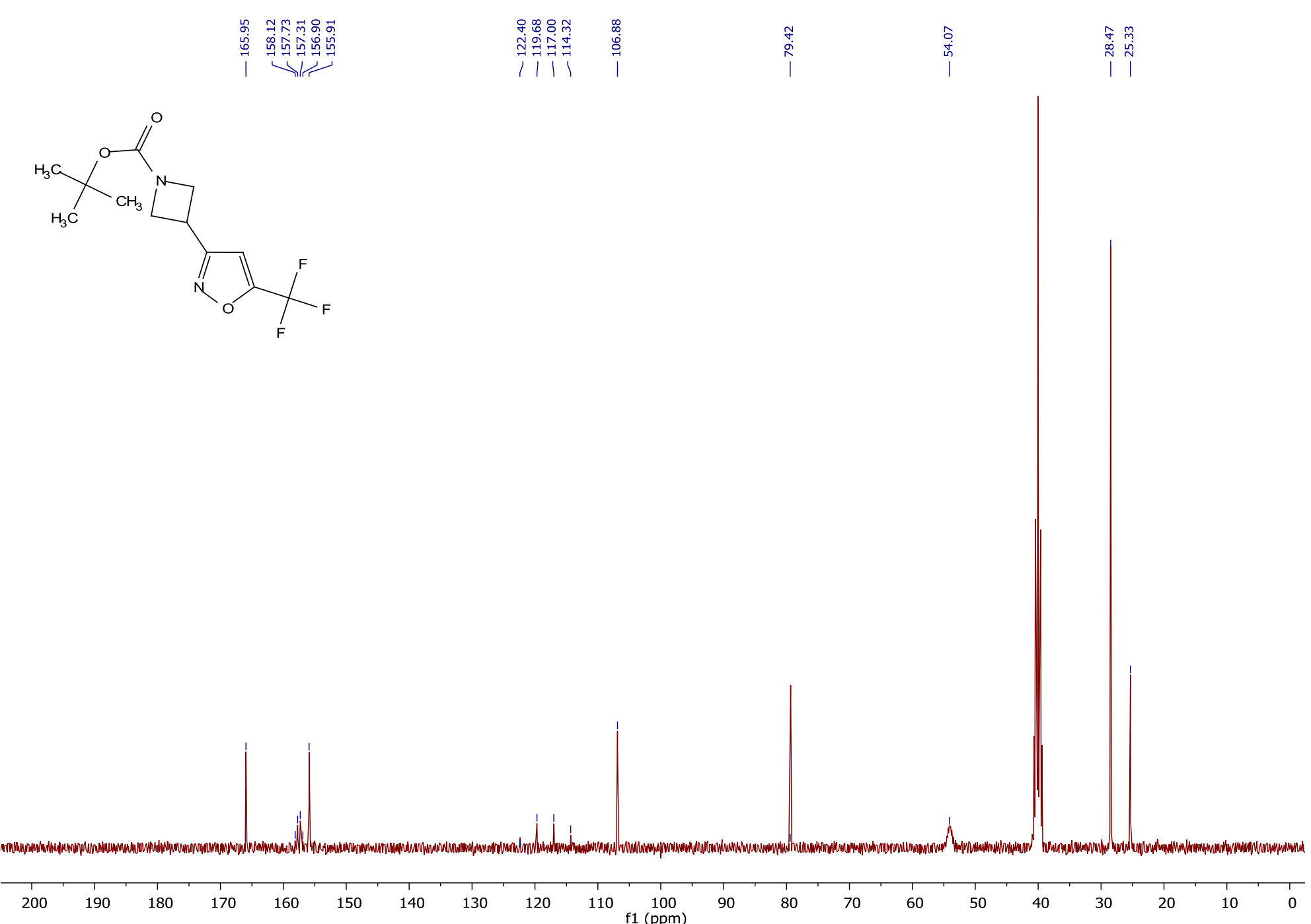

tert-Butyl 3-(5-(trifluoromethyl)isoxazol-3-yl)azetidine-1-carboxylate (3j) ${ }^{13} \mathrm{C}$ NMR 


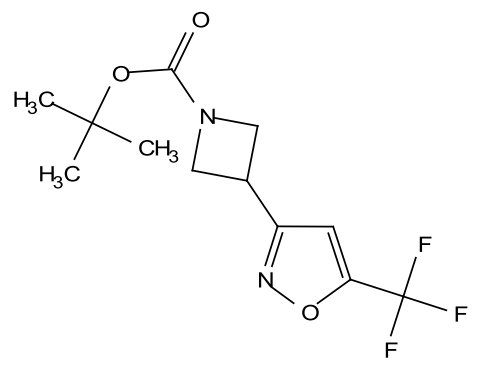

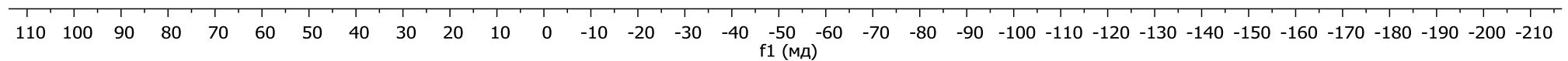
tert-Butyl 3-(5-(trifluoromethyl)isoxazol-3-yl)azetidine-1-carboxylate (3j) ${ }^{19}$ F NMR 

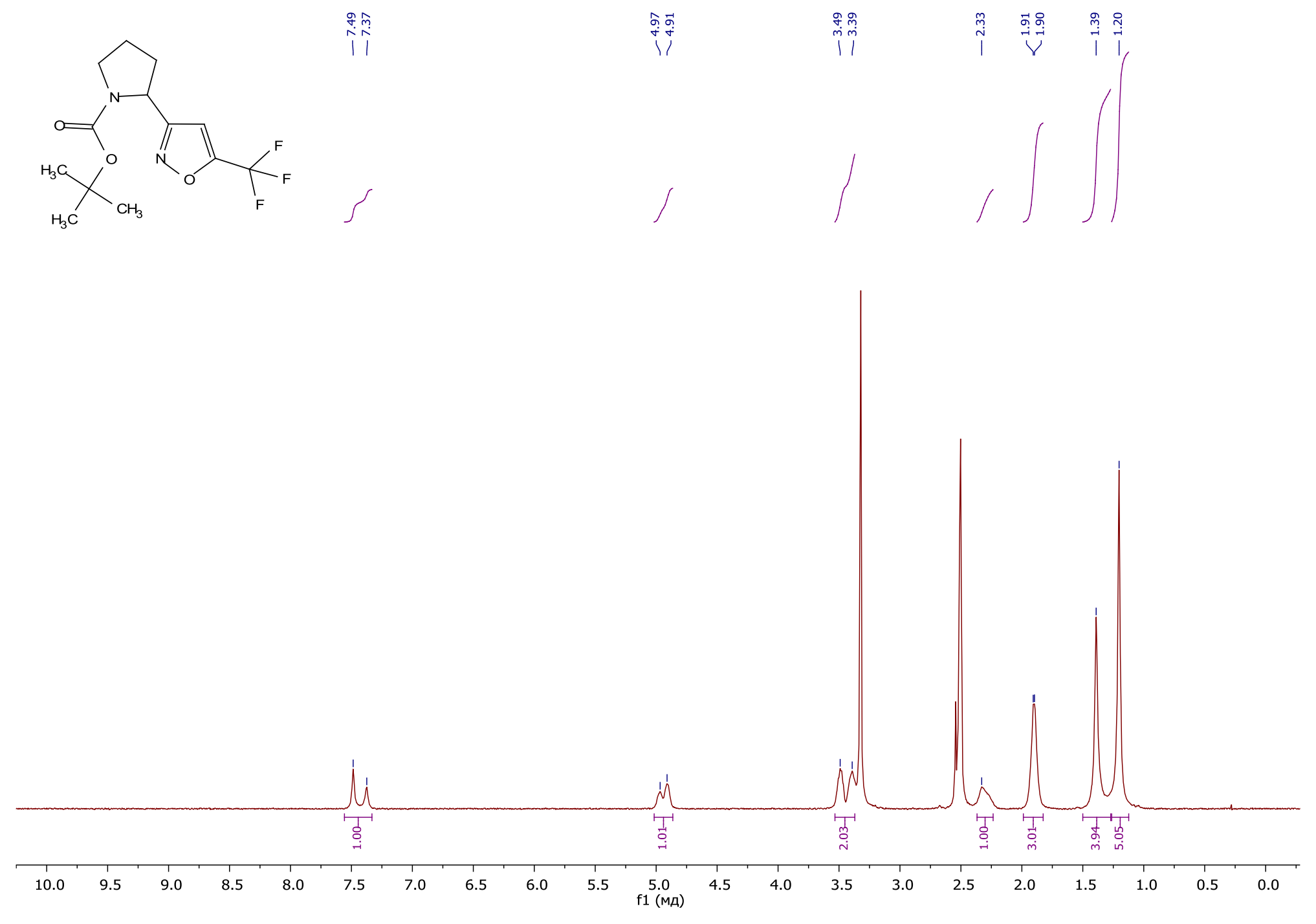

tert-Butyl 2-(5-(trifluoromethyl)isoxazol-3-yl)pyrrolidine-1-carboxylate (3k or 3l) ${ }^{1} \mathrm{H}$ NMR 


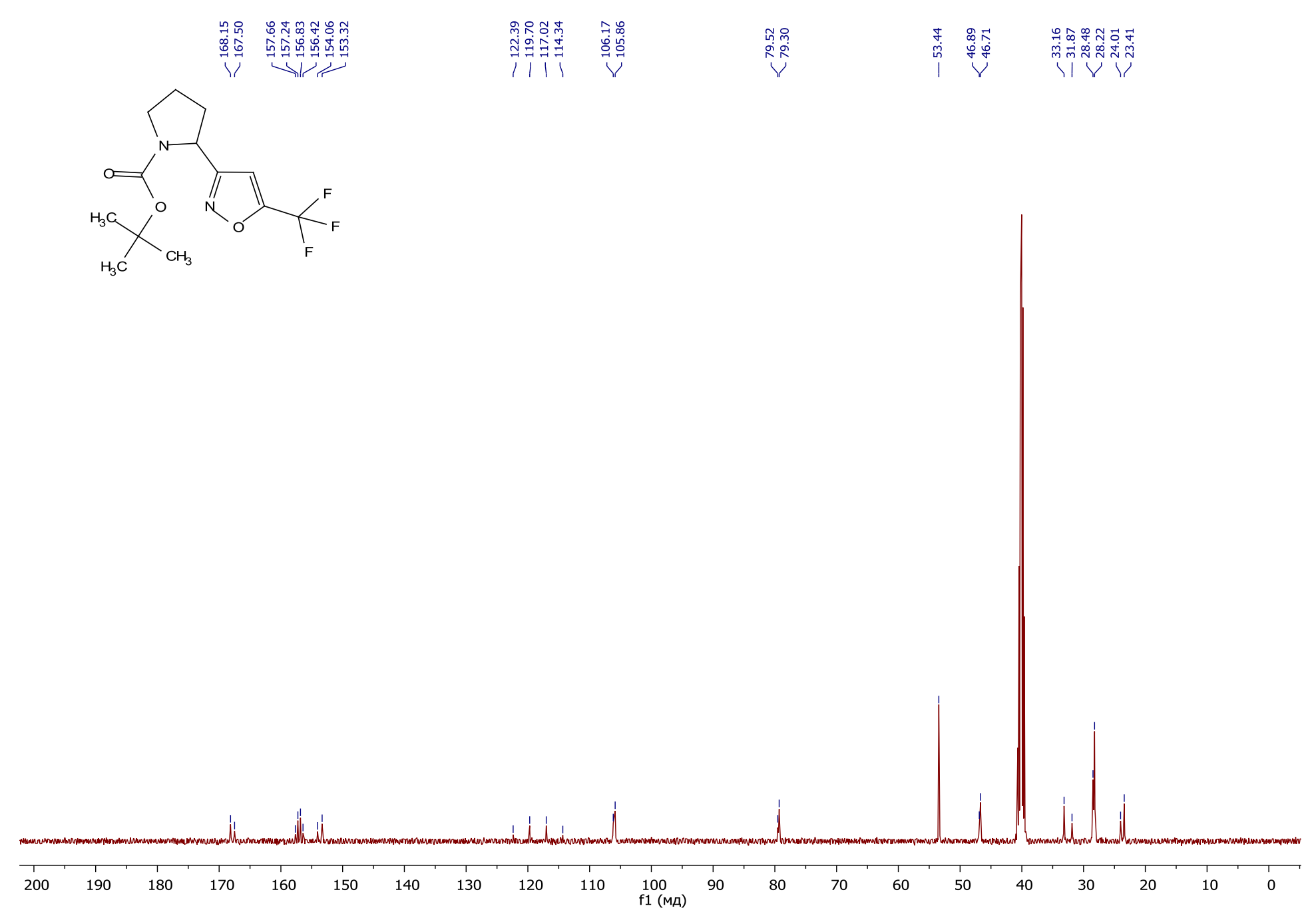

tert-Butyl 2-(5-(trifluoromethyl)isoxazol-3-yl)pyrrolidine-1-carboxylate (3k or 3l) ${ }^{13} \mathrm{C} \mathrm{NMR}$ 

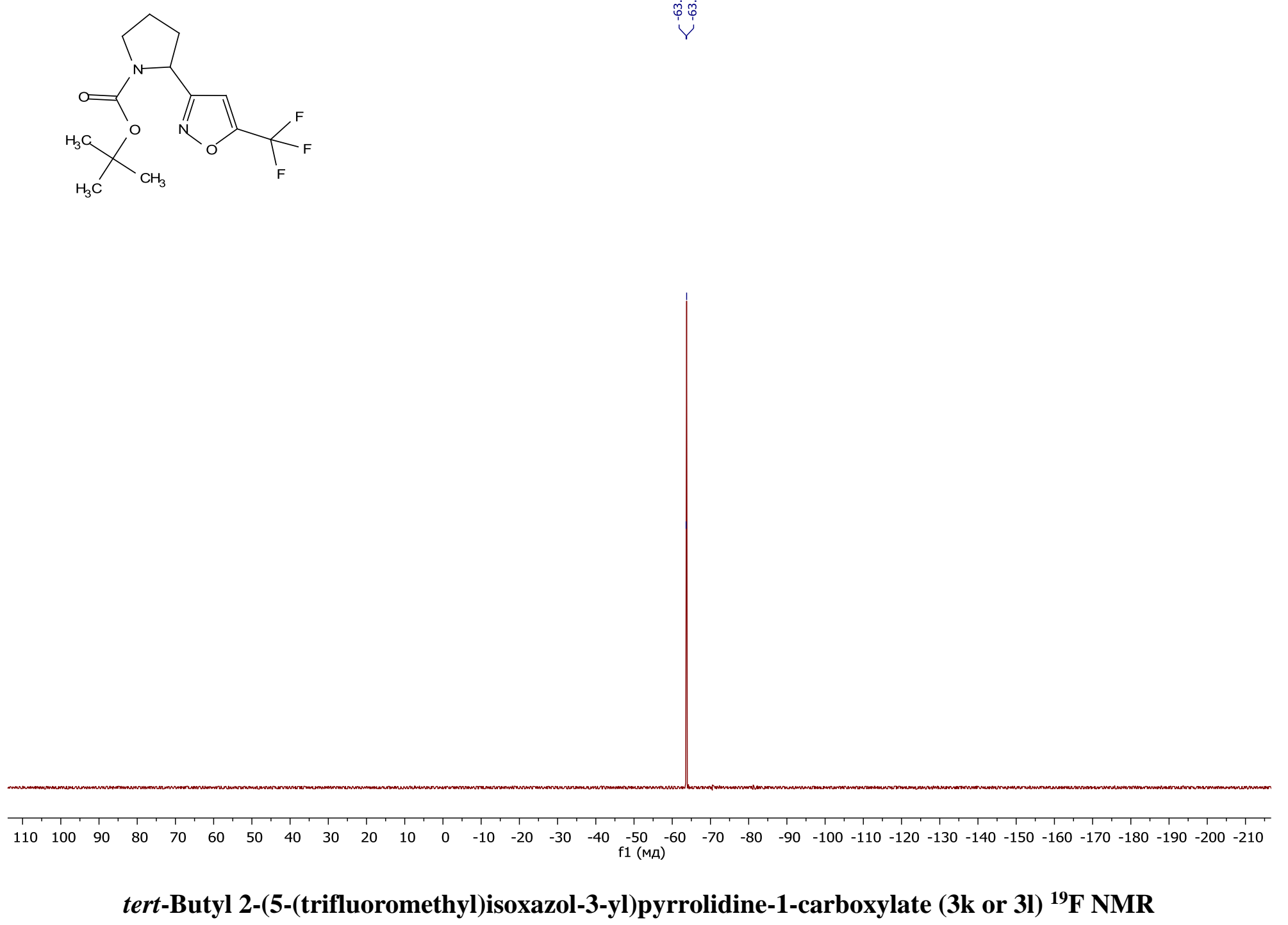

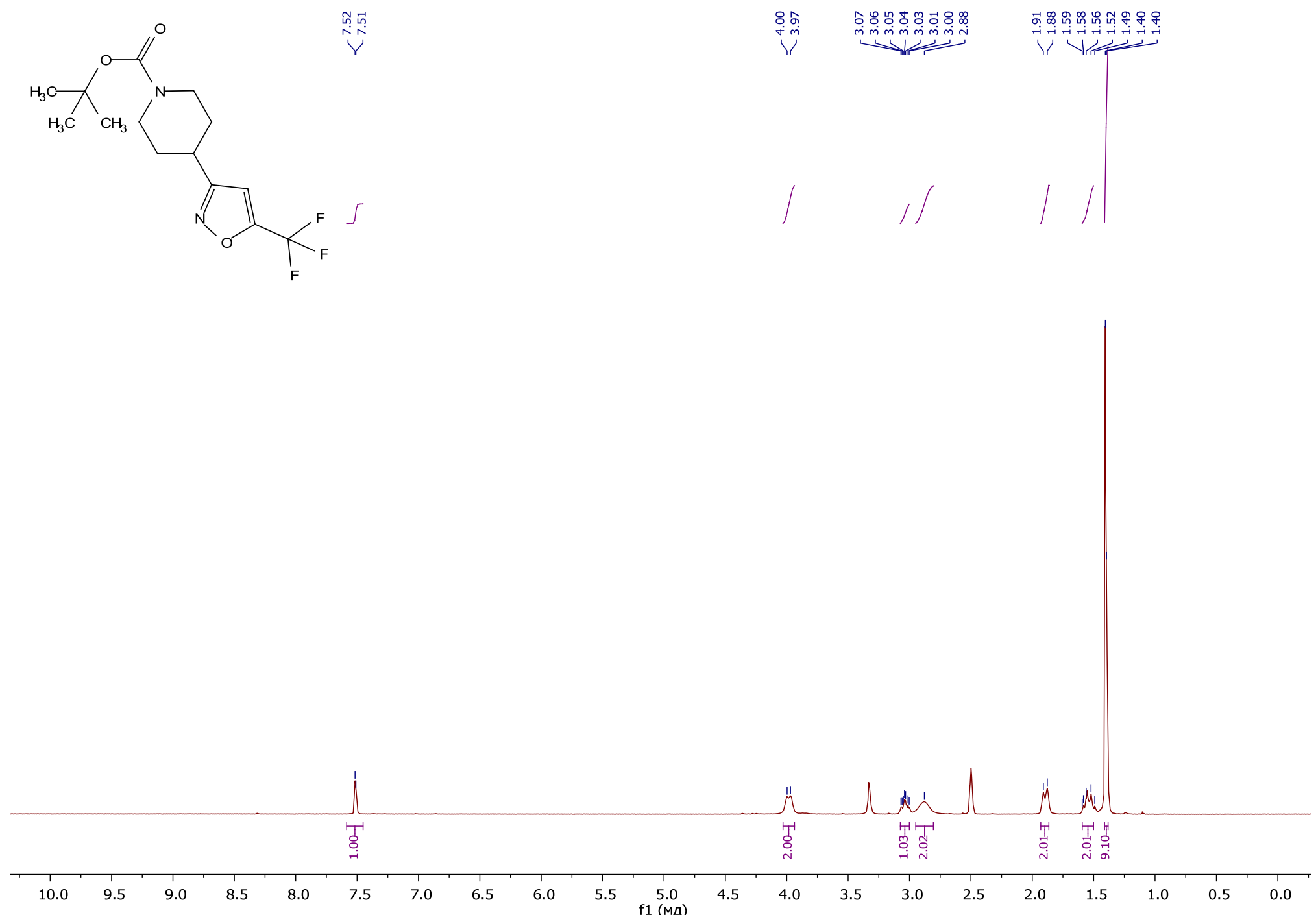

tert-Butyl 4-(5-(trifluoromethyl)isoxazol-3-yl)piperidine-1-carboxylate (3m) ${ }^{1}$ H NMR 


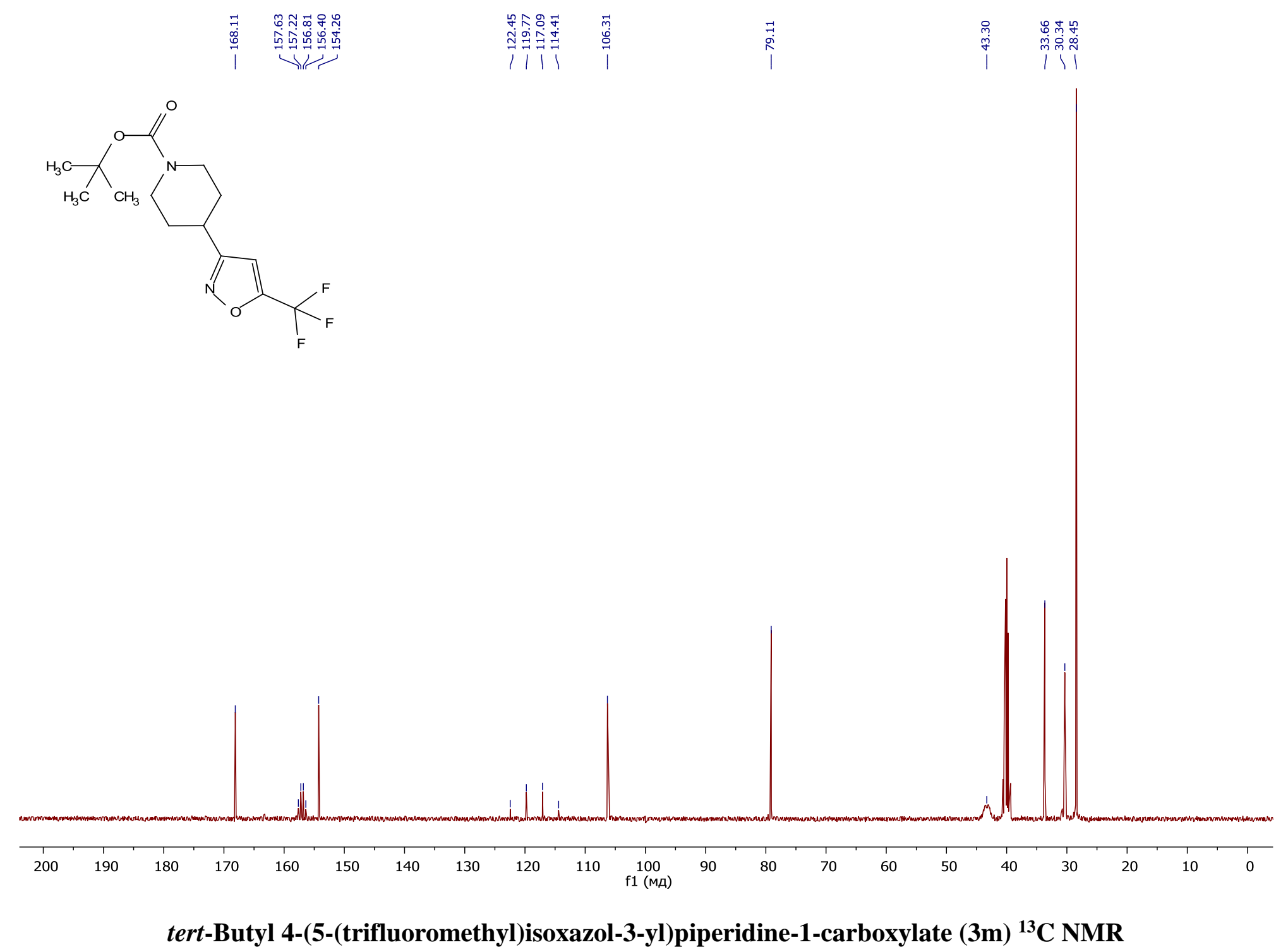



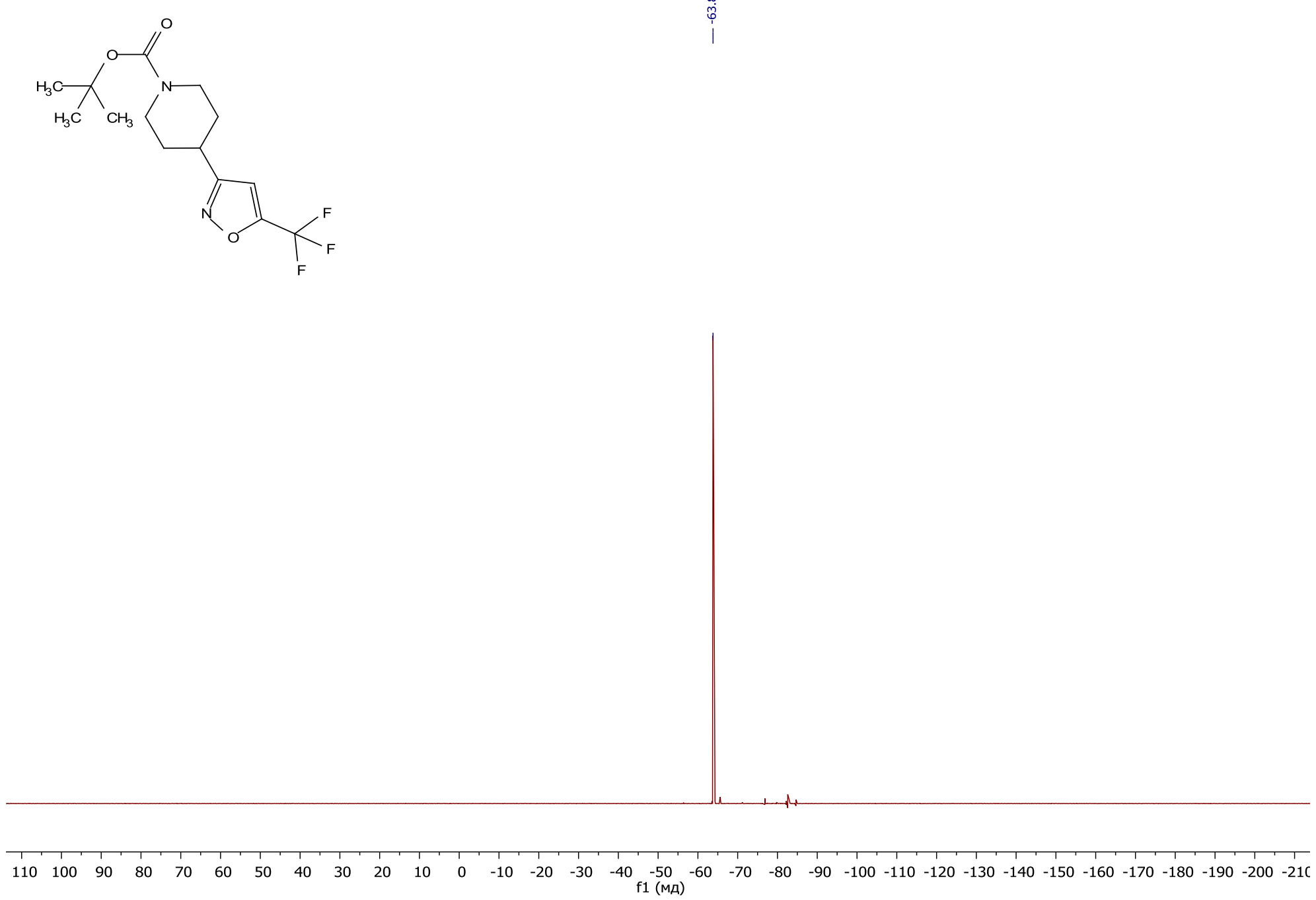

tert-Butyl 4-(5-(trifluoromethyl)isoxazol-3-yl)piperidine-1-carboxylate (3m) ${ }^{19}$ F NMR 

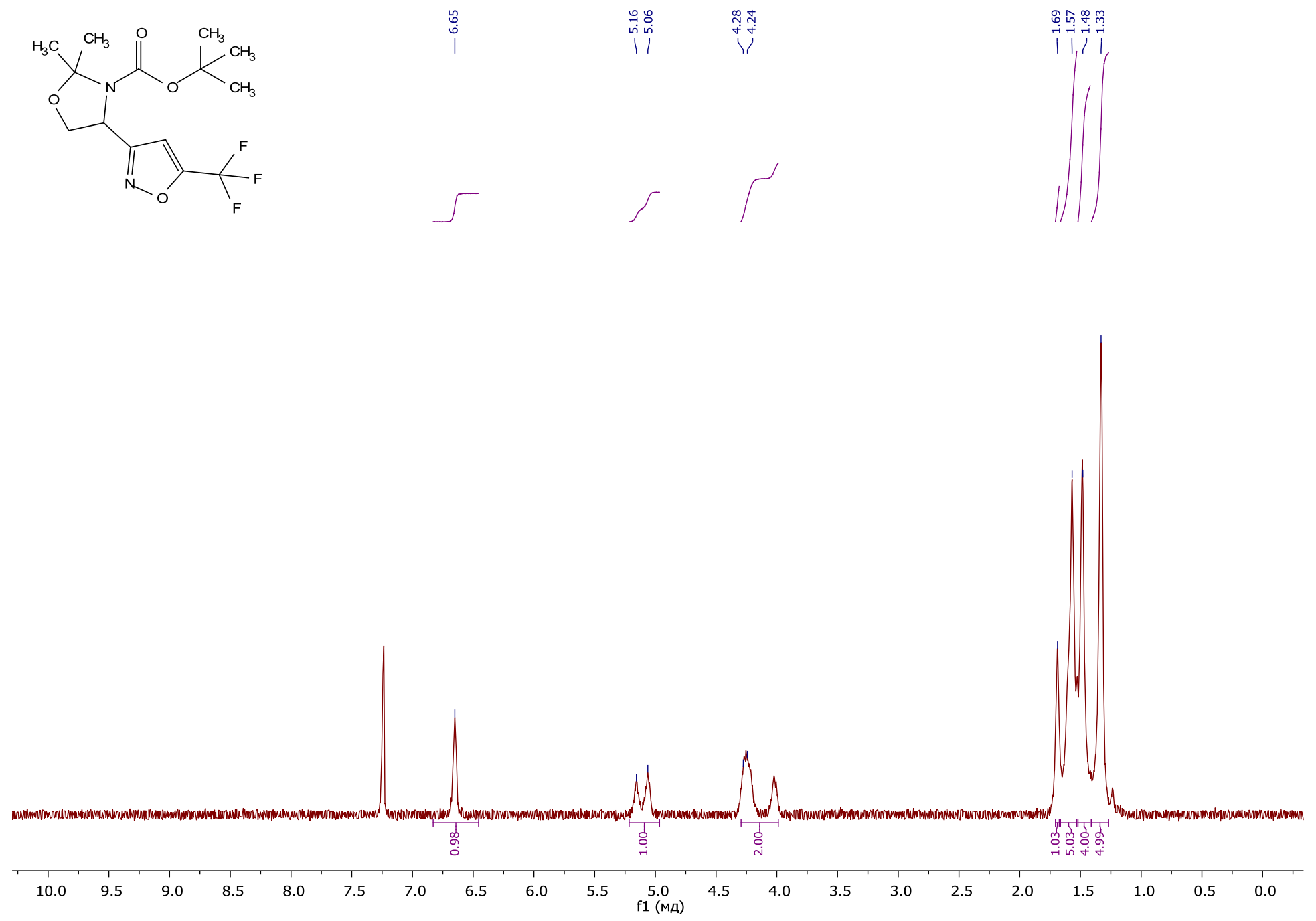

tert-Butyl 2,2-dimethyl-4-(5-(trifluoromethyl)isoxazol-3-yl)oxazolidine-3-carboxylate (3n or 3o) ${ }^{1}$ H NMR 


ل每
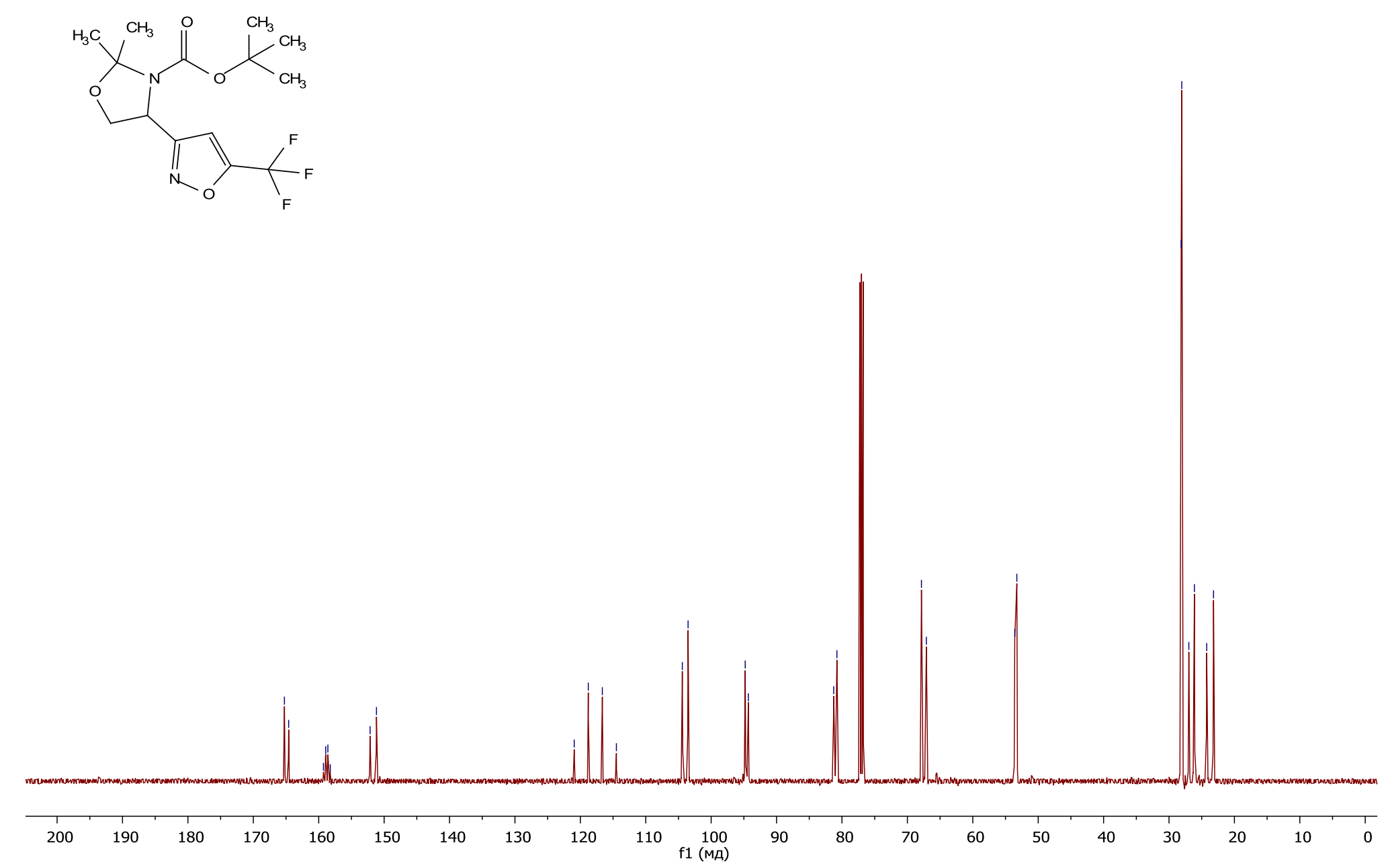

tert-Butyl 2,2-dimethyl-4-(5-(trifluoromethyl)isoxazol-3-yl)oxazolidine-3-carboxylate (3n or 3o) ${ }^{13}$ C NMR 

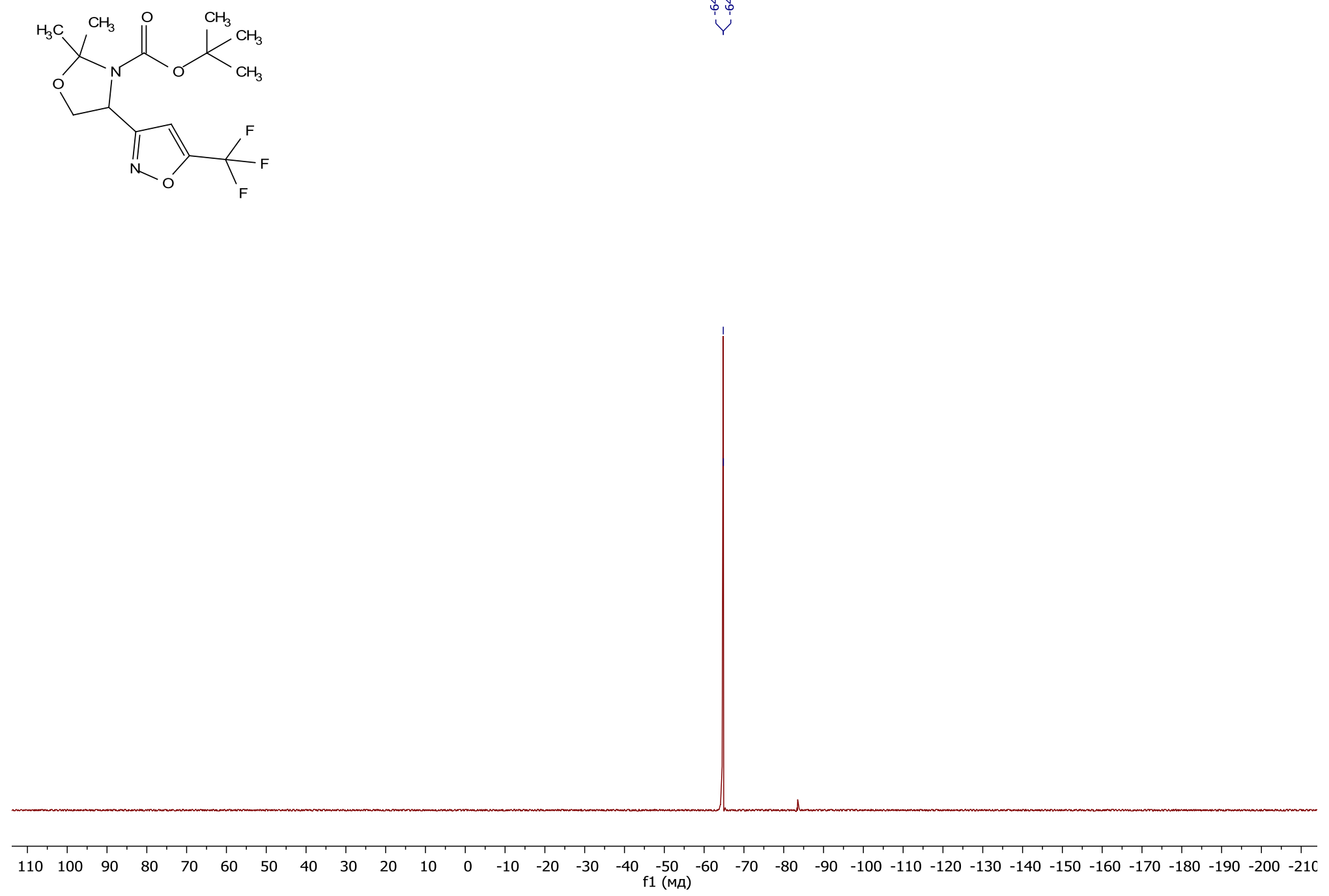

tert-Butyl 2,2-dimethyl-4-(5-(trifluoromethyl)isoxazol-3-yl)oxazolidine-3-carboxylate (3n or 3o) ${ }^{19}$ F NMR 

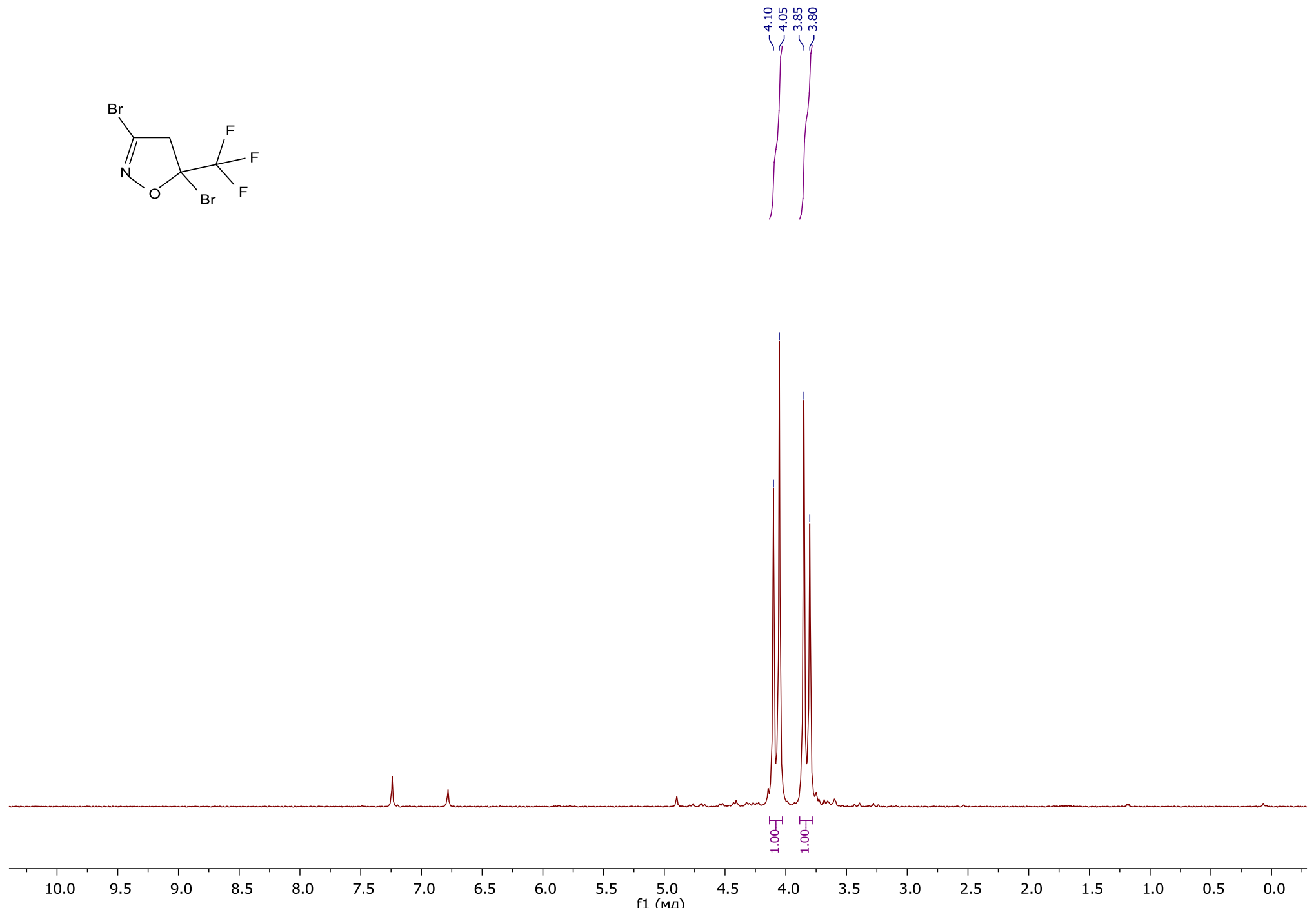

3,5-Dibromo-5-(trifluoromethyl)-4,5-dihydroisoxazole (5) ${ }^{1} \mathrm{H}$ NMR 

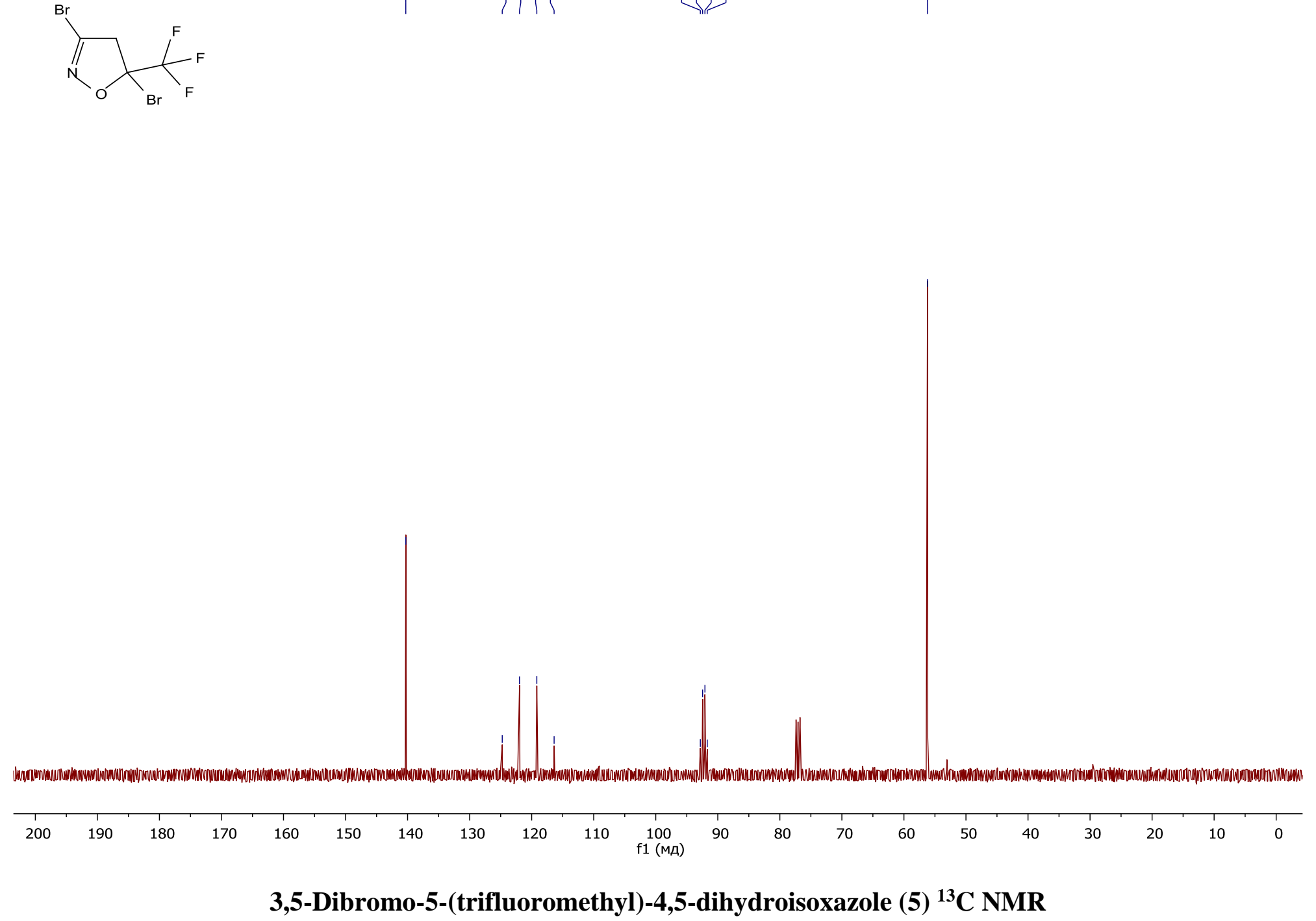

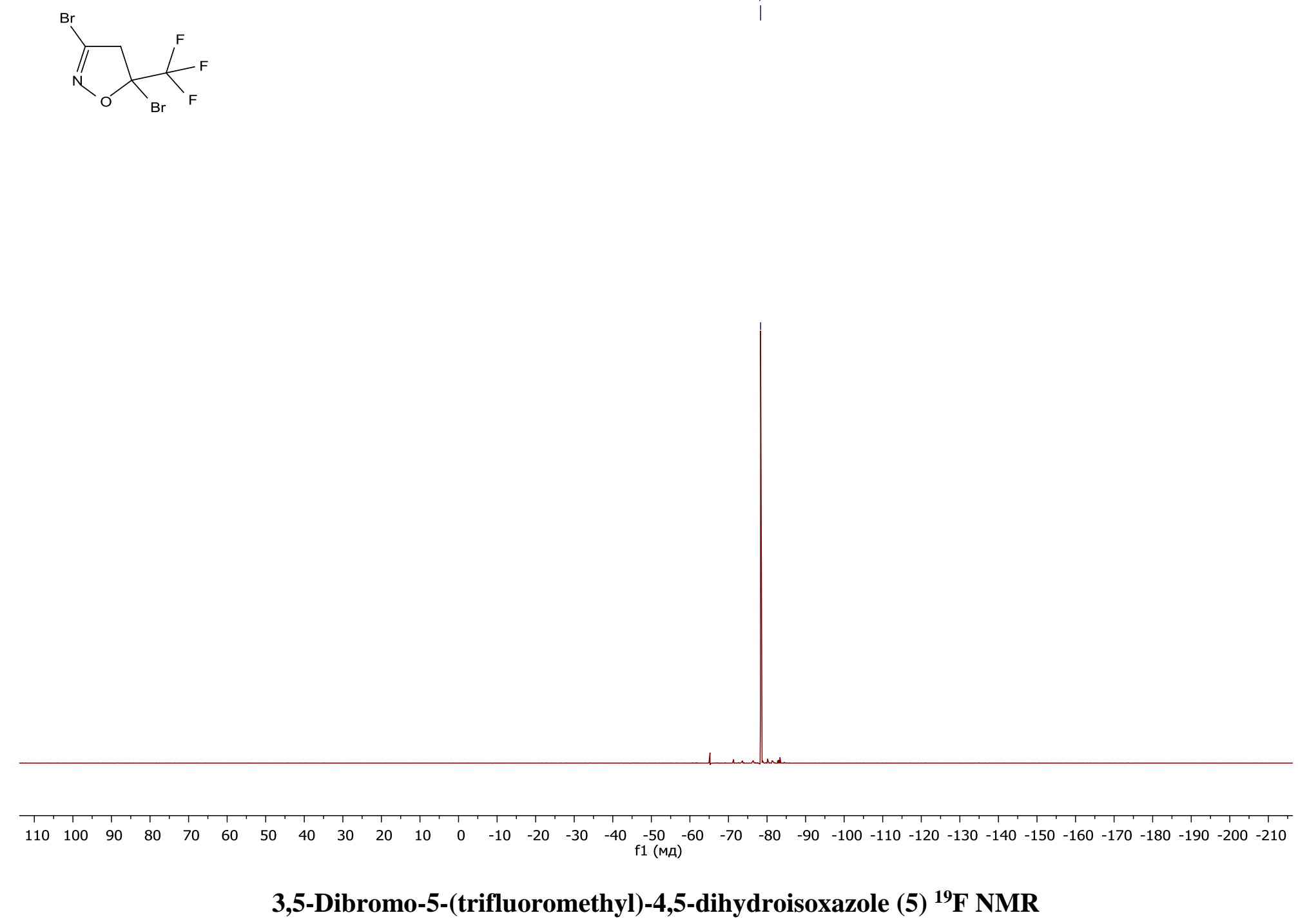

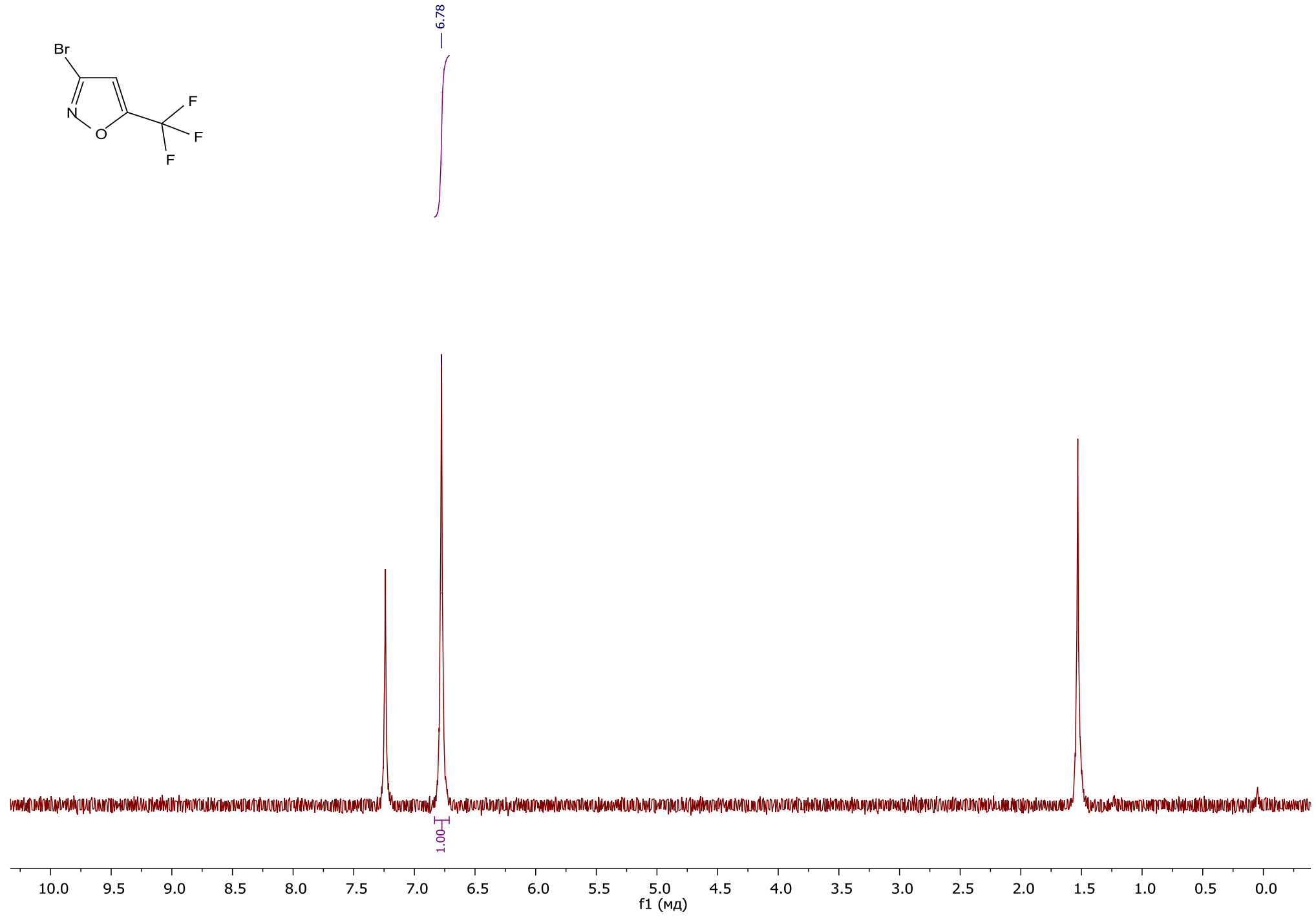

3-Bromo-5-(trifluoromethyl)isoxazole (6) ${ }^{1}$ H NMR 

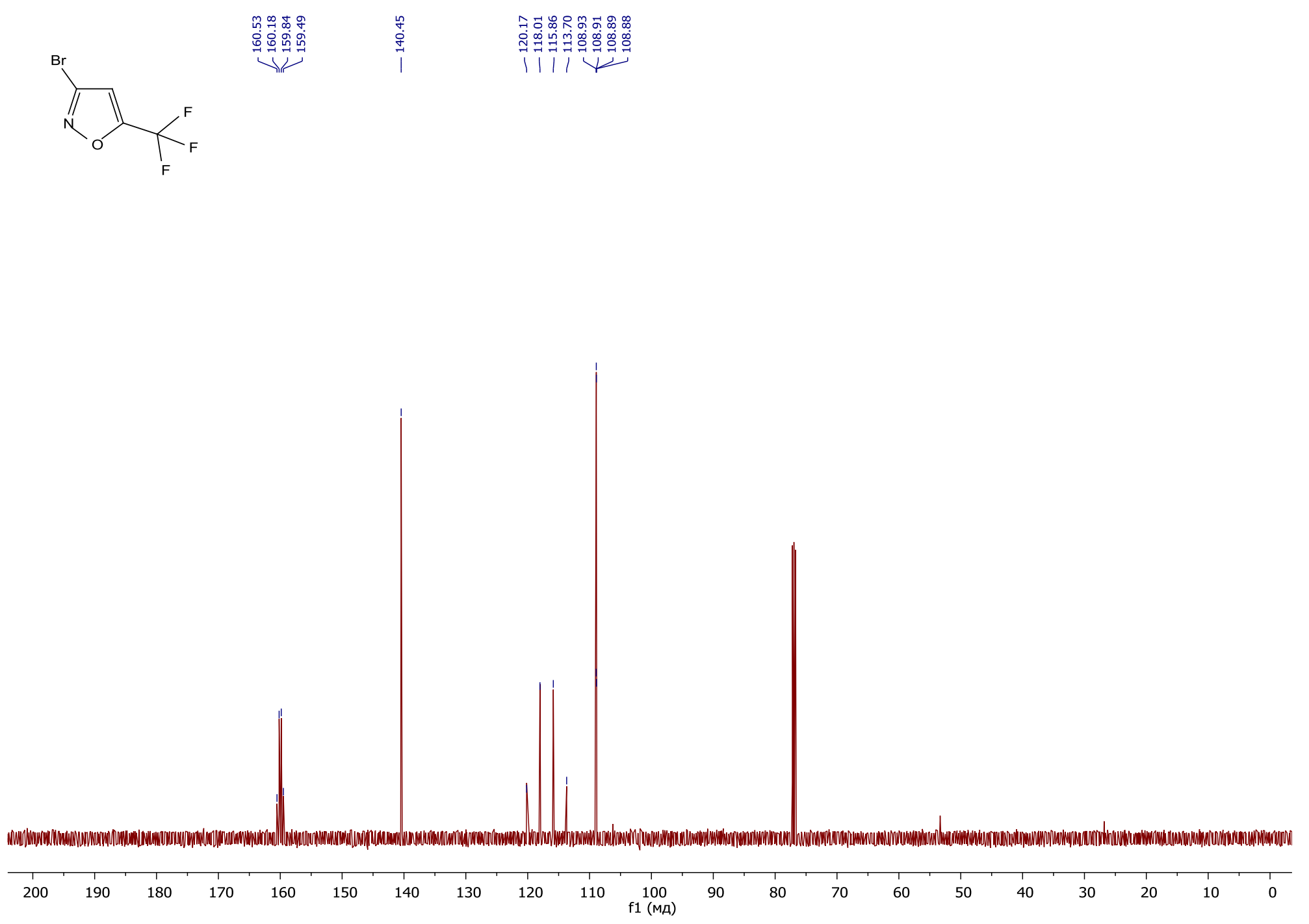

3-Bromo-5-(trifluoromethyl)isoxazole (6) ${ }^{13} \mathrm{C}$ NMR 

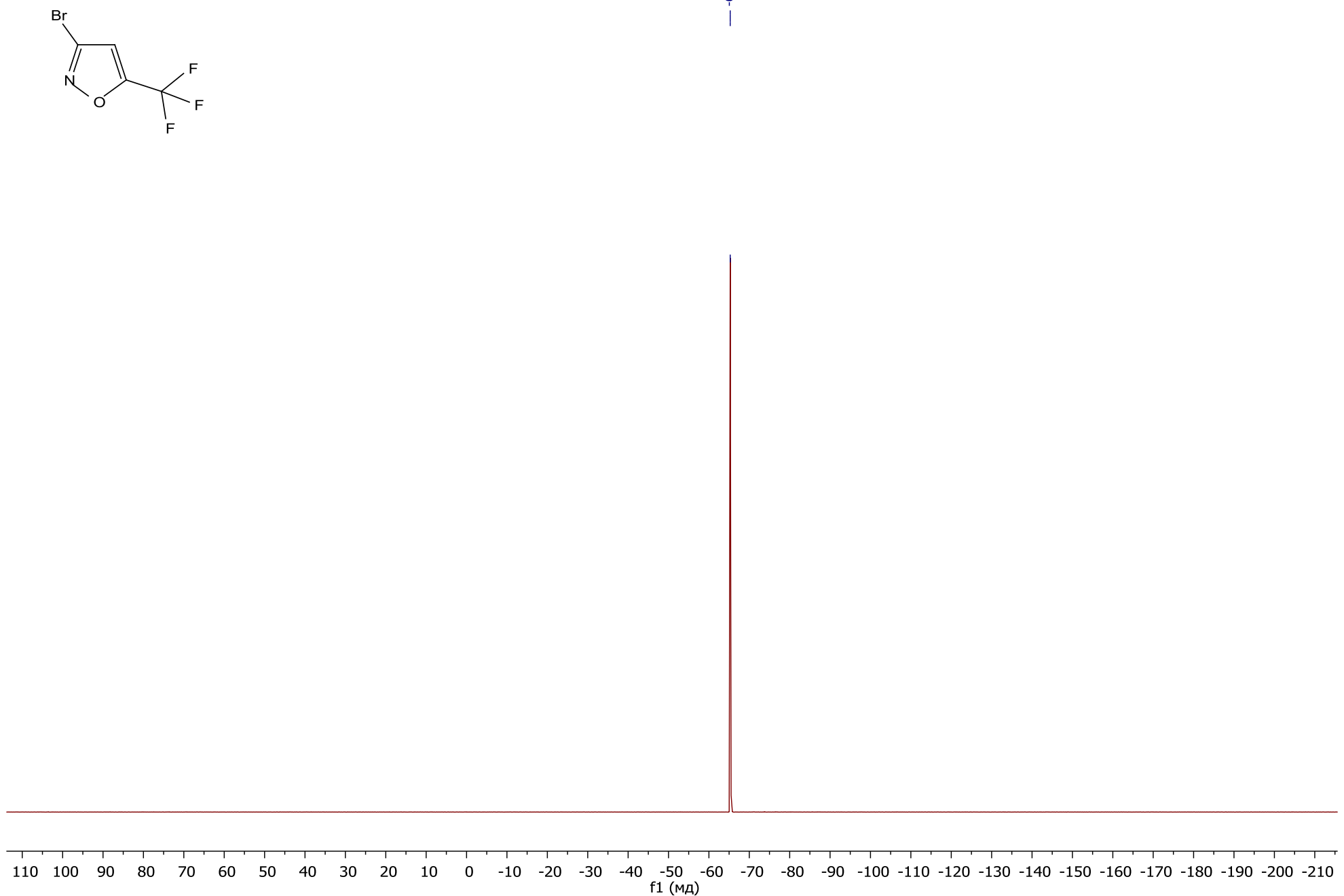

3-Bromo-5-(trifluoromethyl)isoxazole (6) ${ }^{19}$ F NMR 


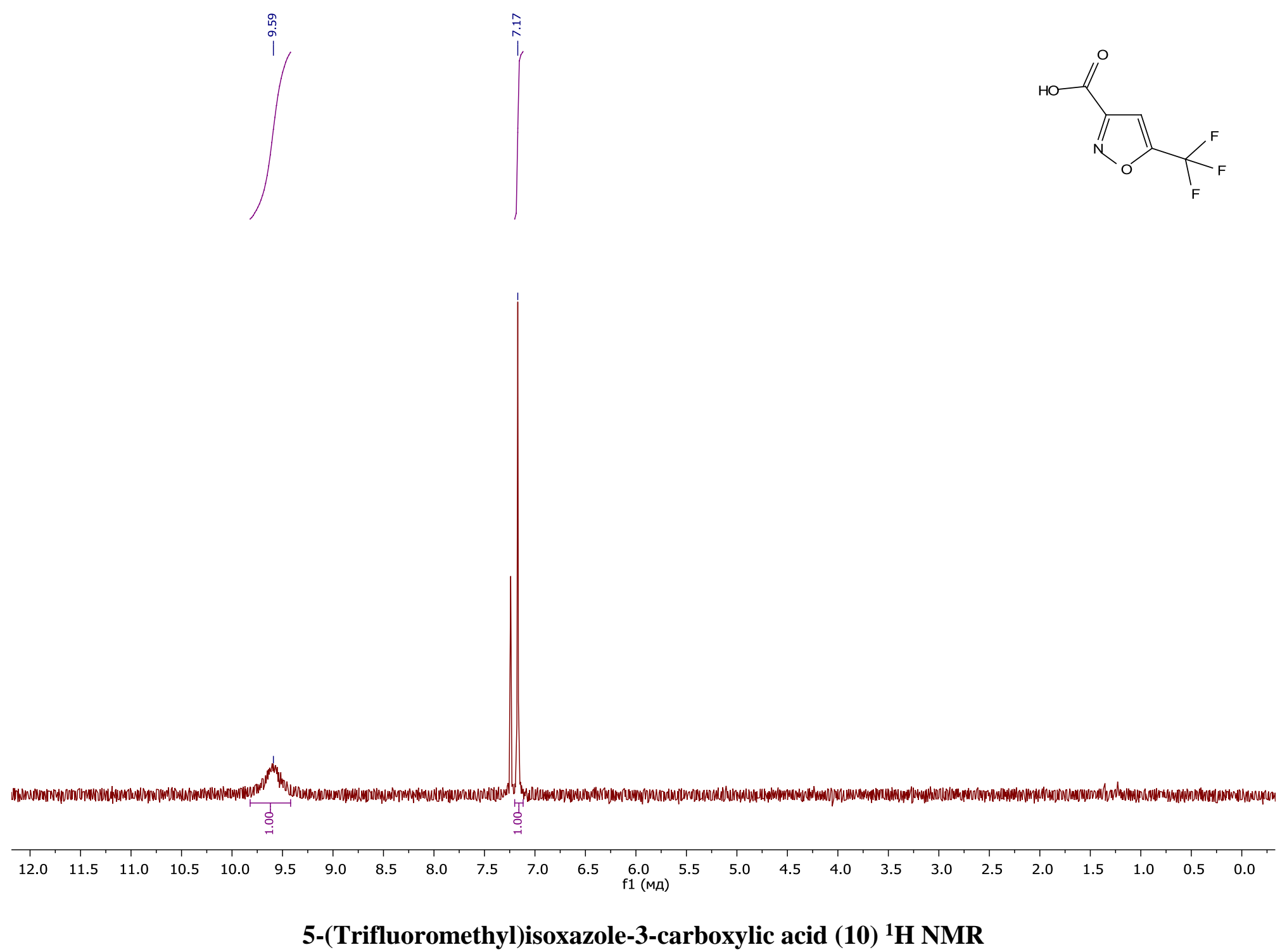




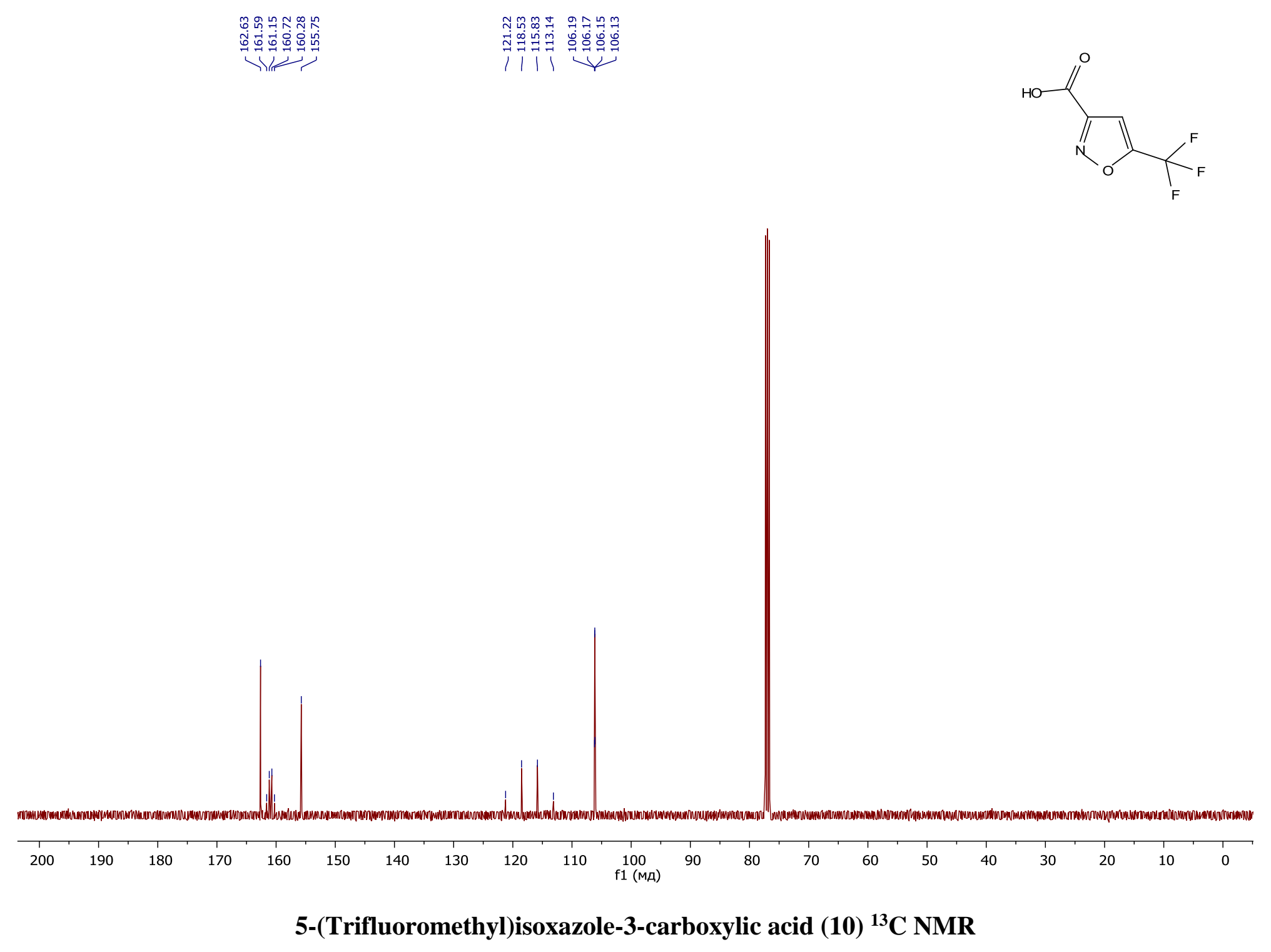



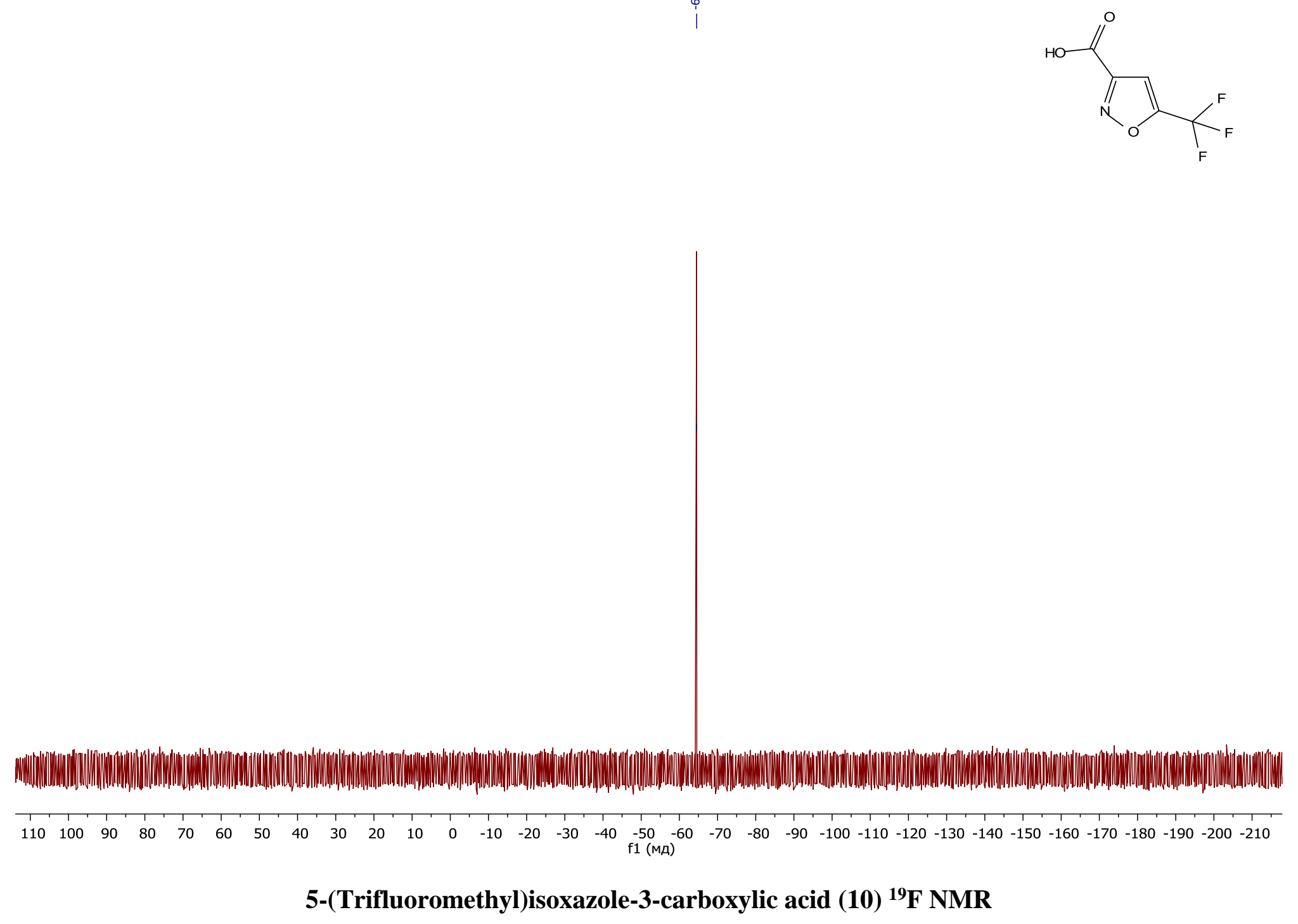

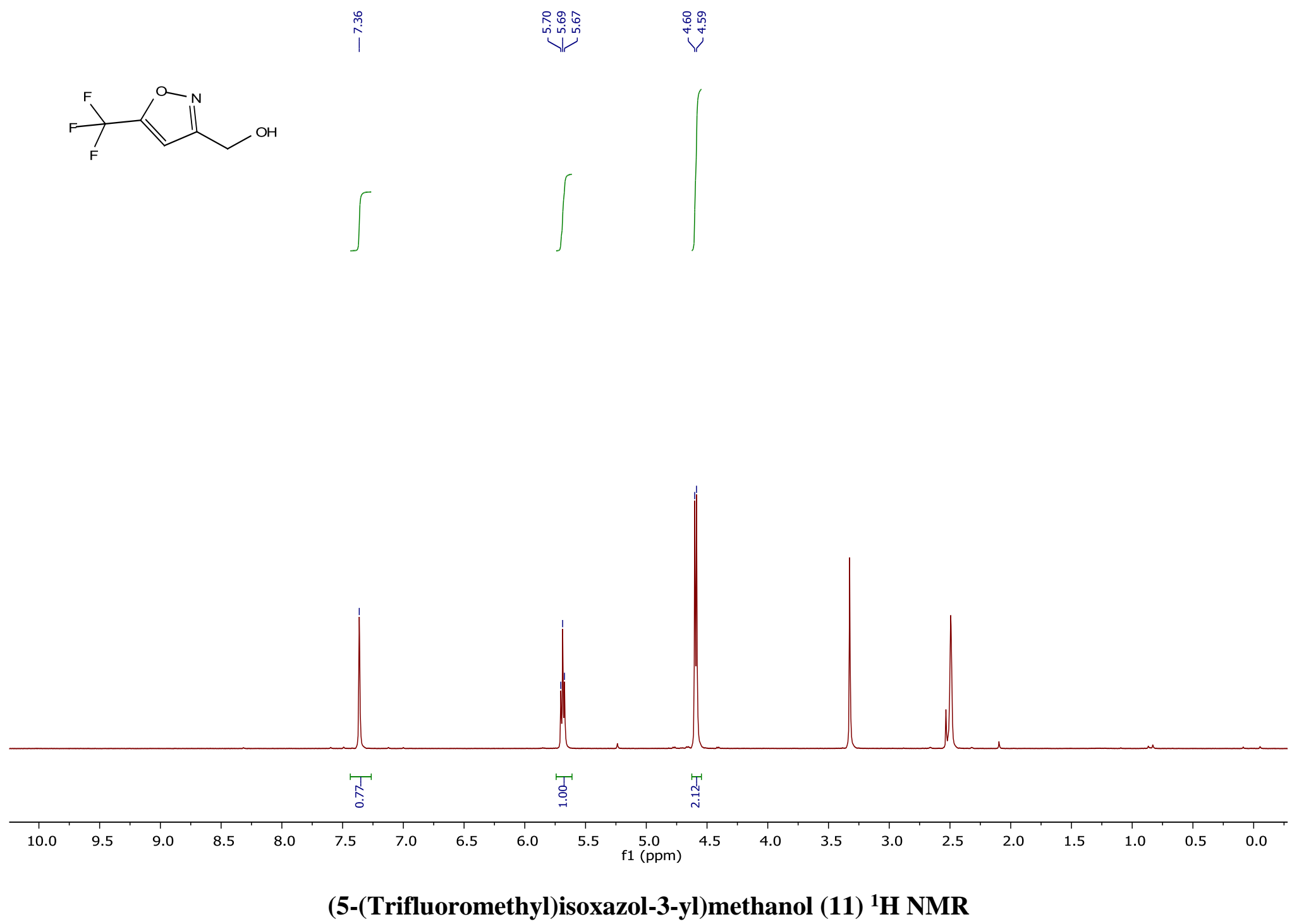

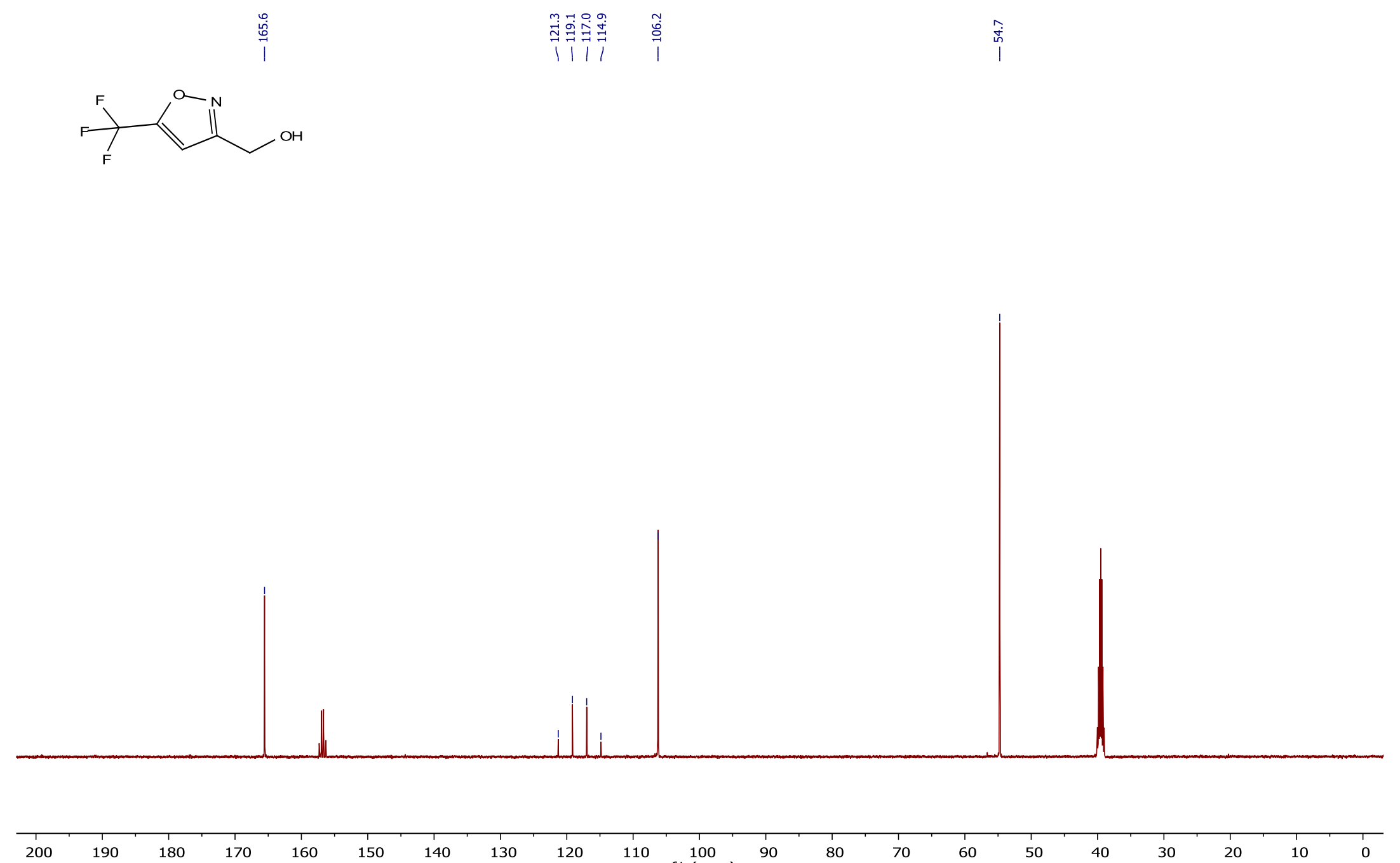

f1 $\stackrel{100}{(\mathrm{ppm})}$

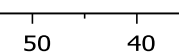

(5-(Trifluoromethyl)isoxazol-3-yl)methanol (11) ${ }^{13} \mathrm{C}$ NMR 

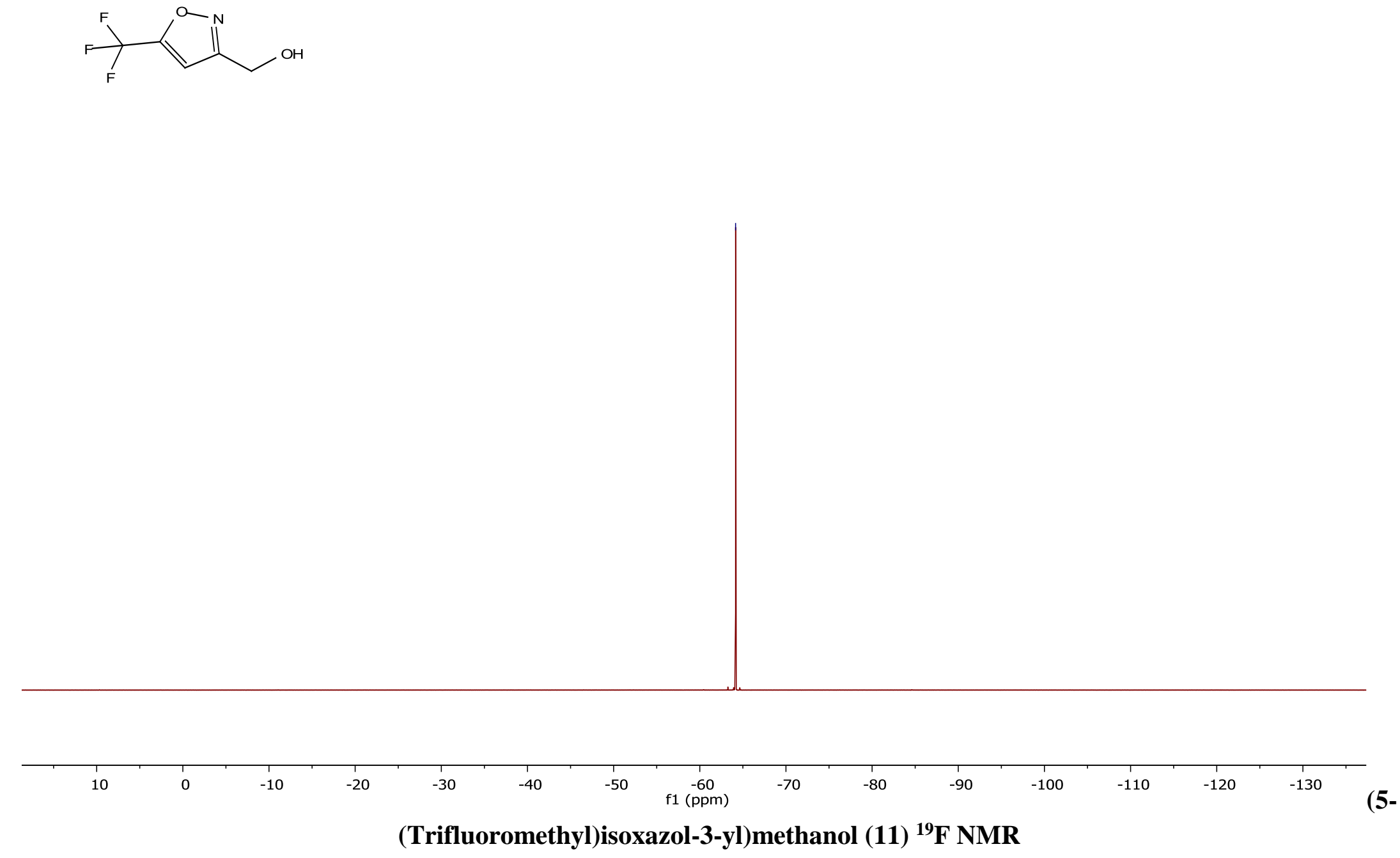


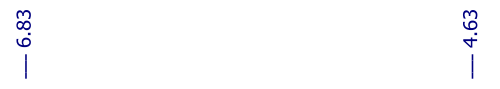
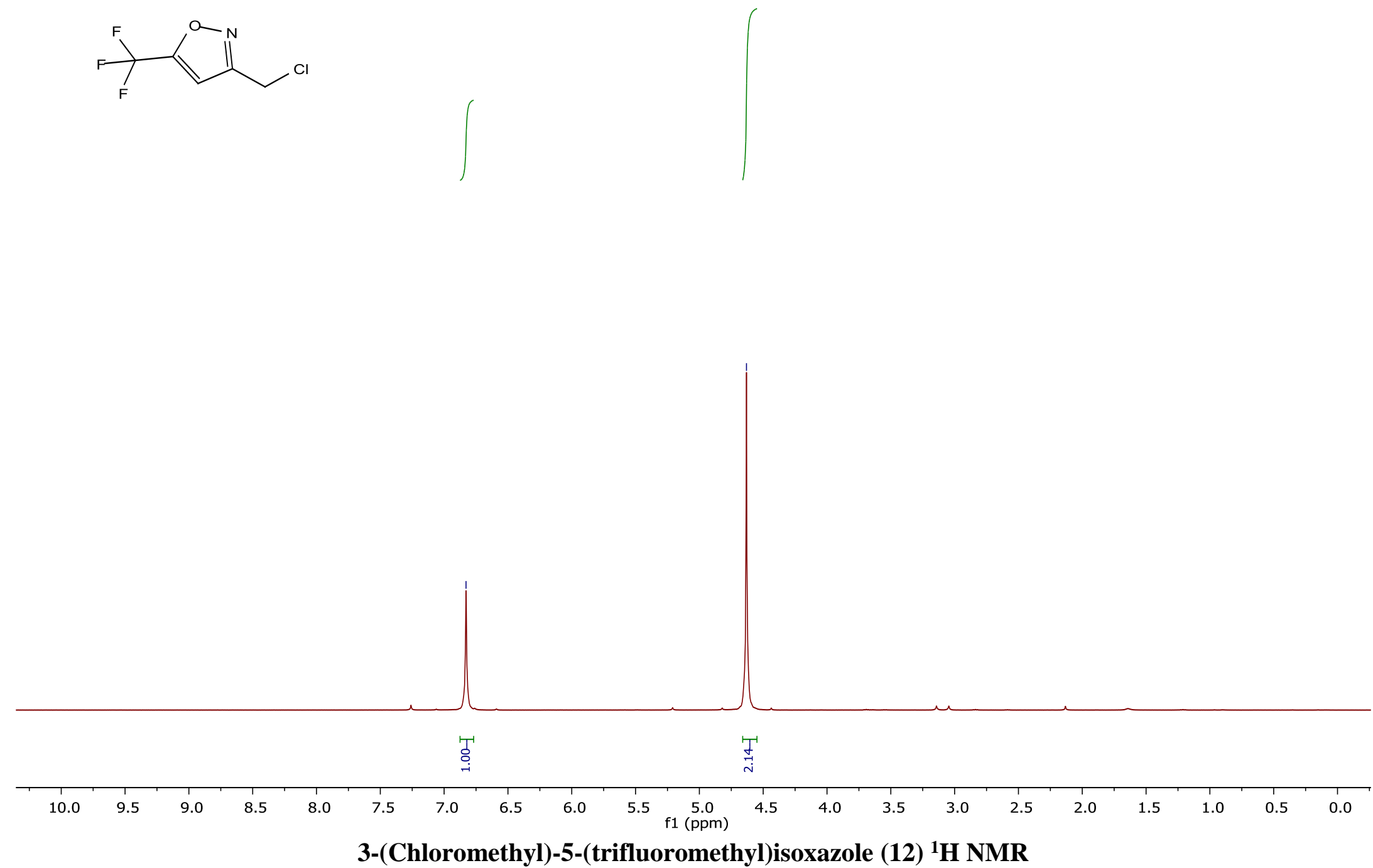


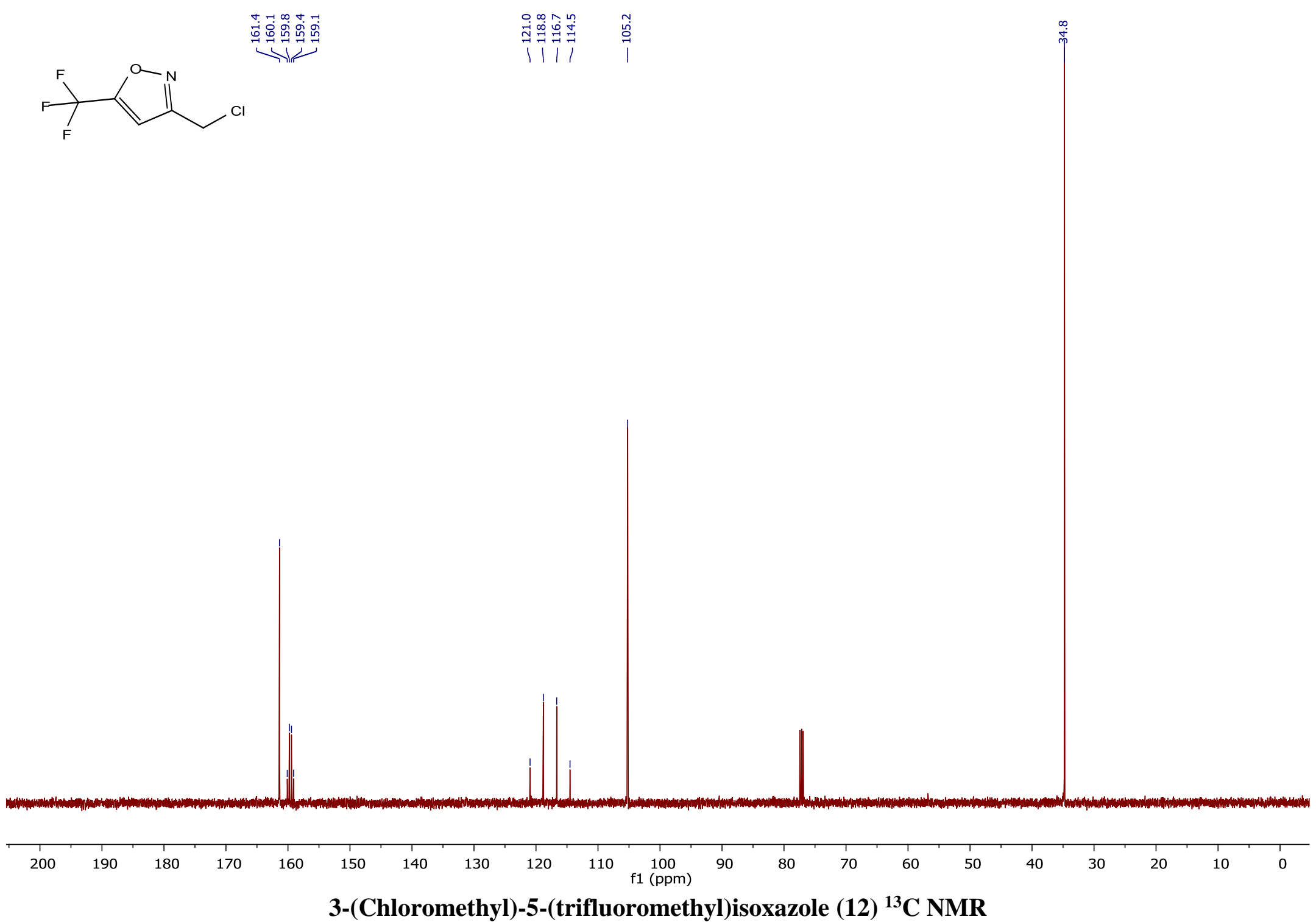



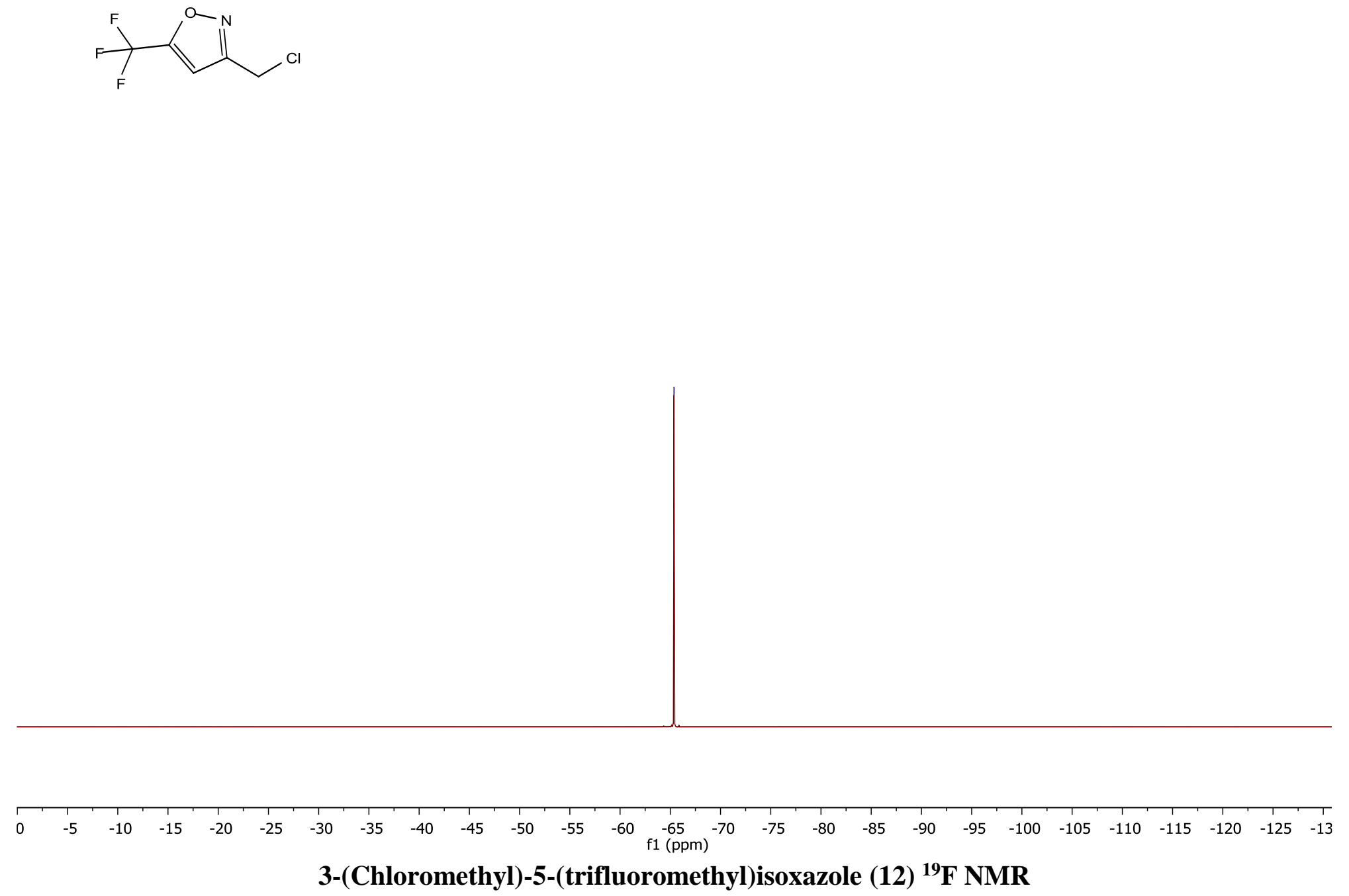

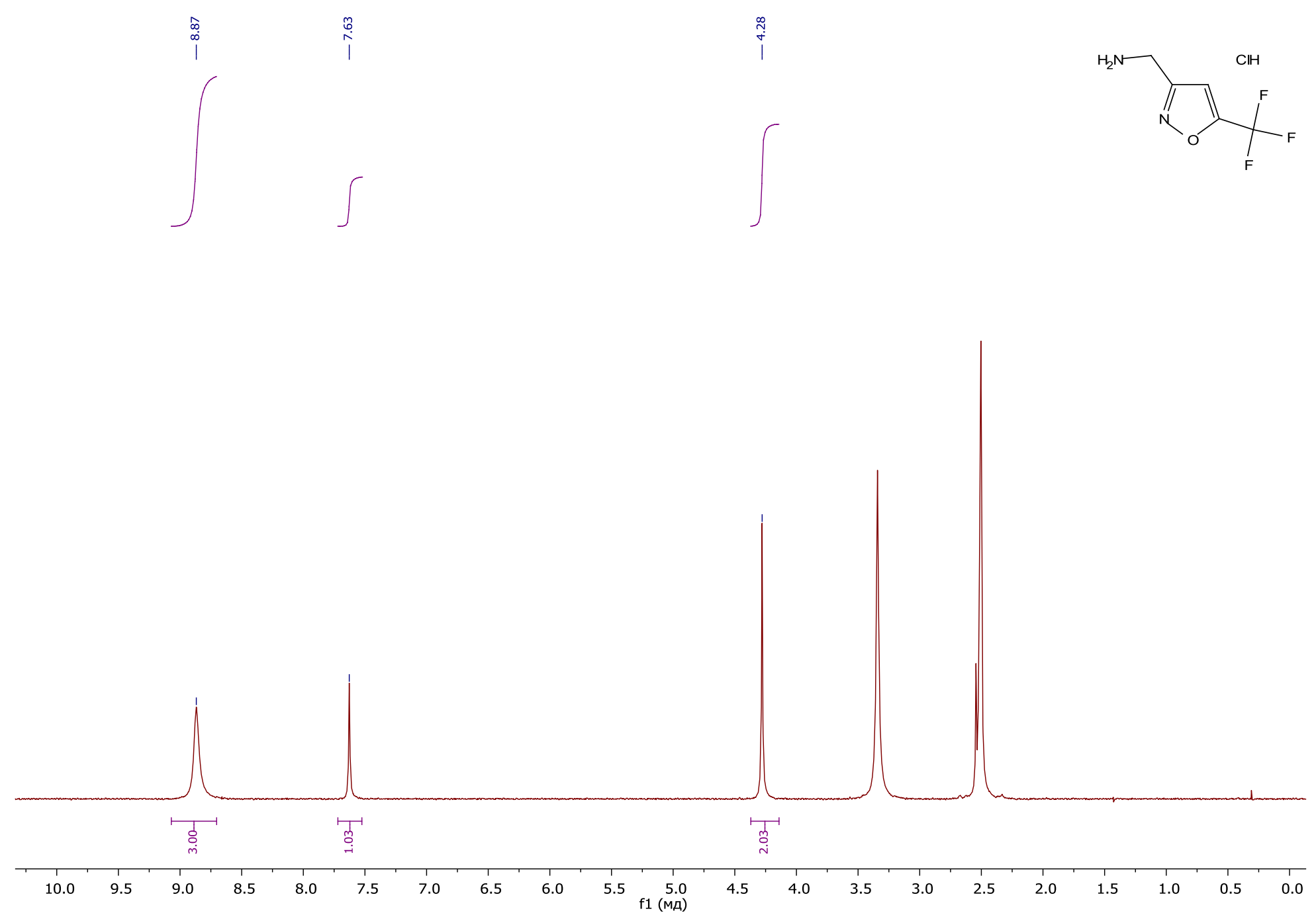

(5-(Trifluoromethyl)isoxazol-3-yl)methanamine hydrochloride (13f·HCl) ${ }^{1} \mathrm{H}$ NMR 

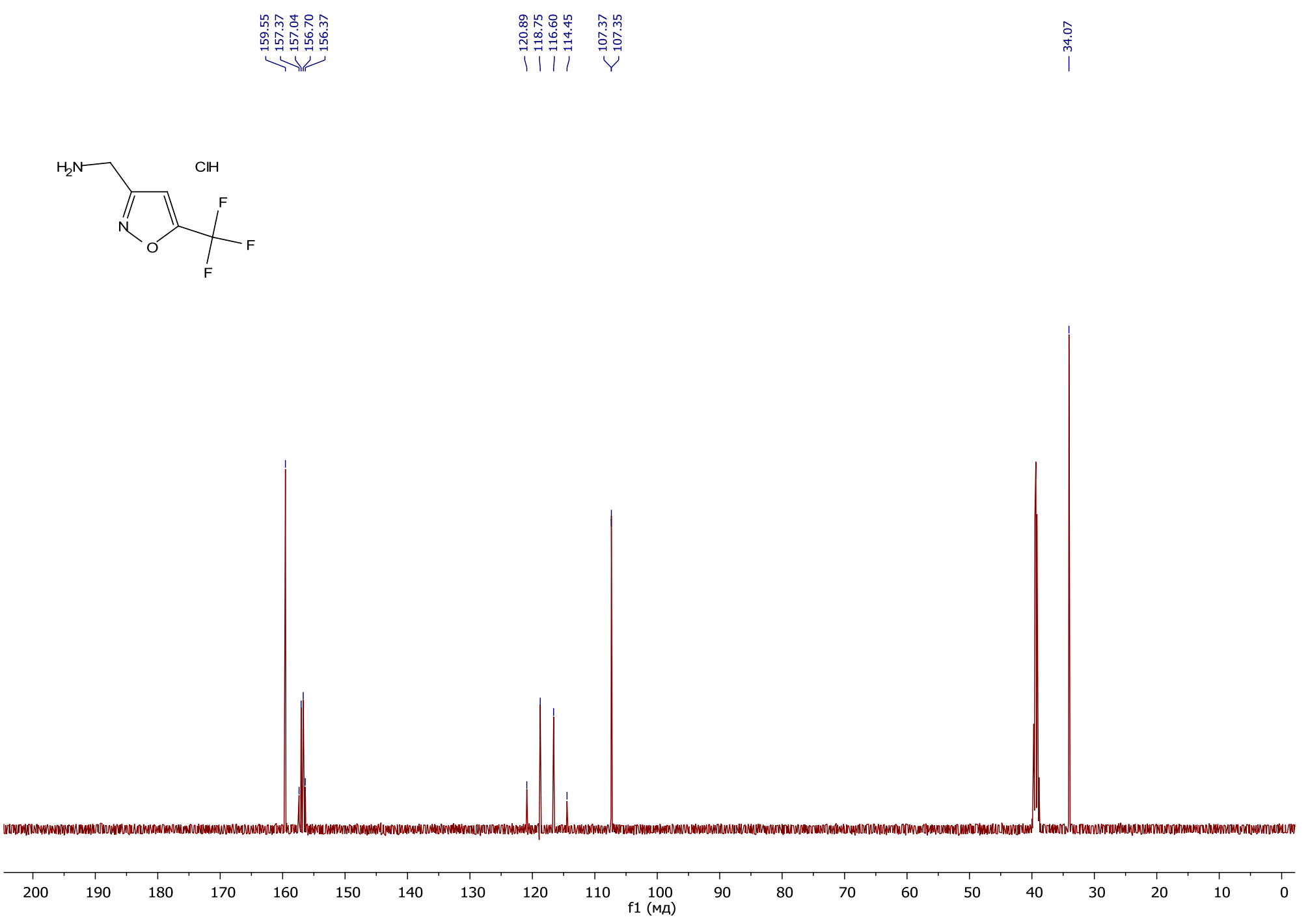

(5-(Trifluoromethyl)isoxazol-3-yl)methanamine hydrochloride $(13 \mathrm{f} \cdot \mathrm{HCl}){ }^{13} \mathrm{C} \mathrm{NMR}$ 

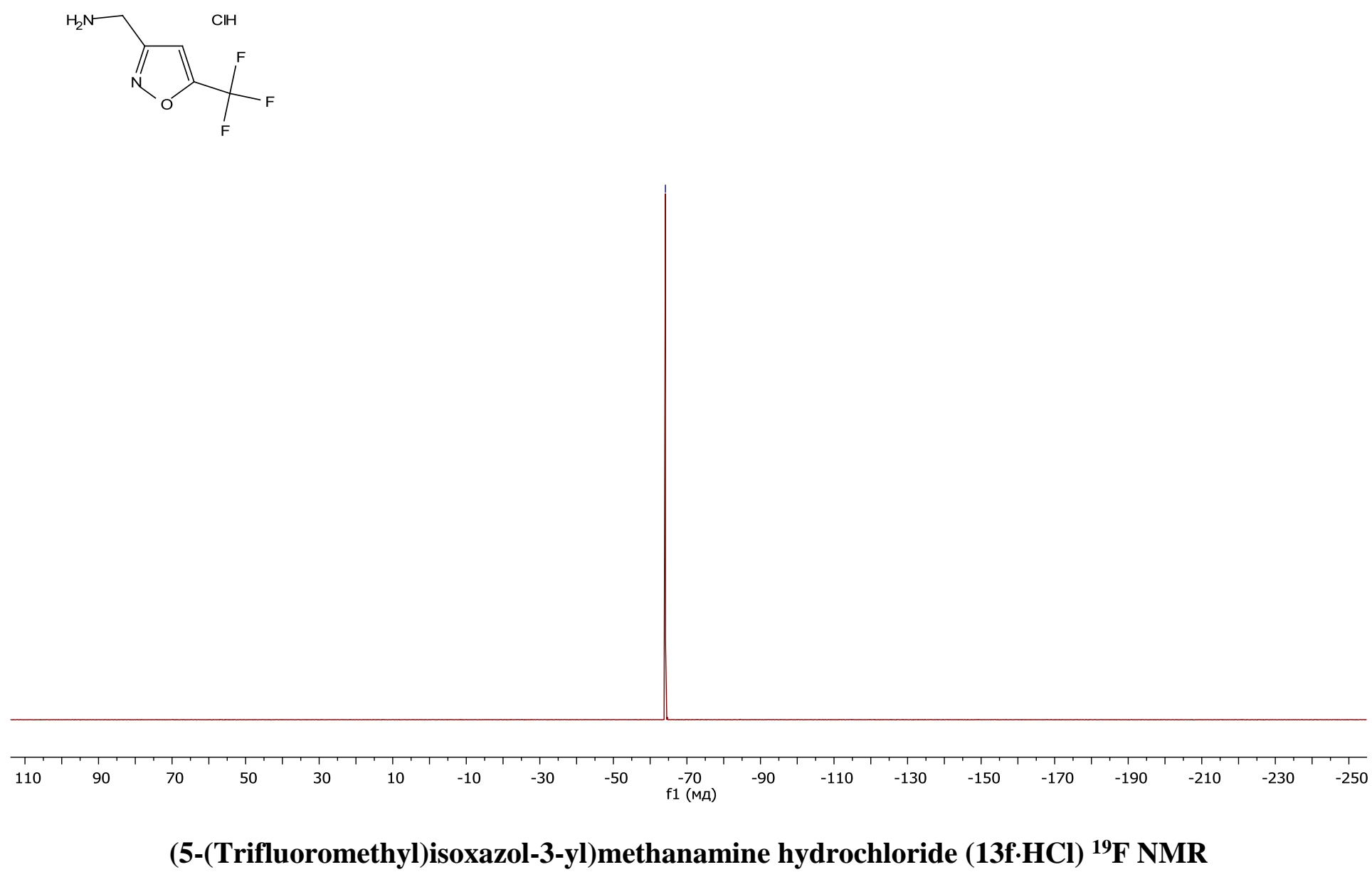

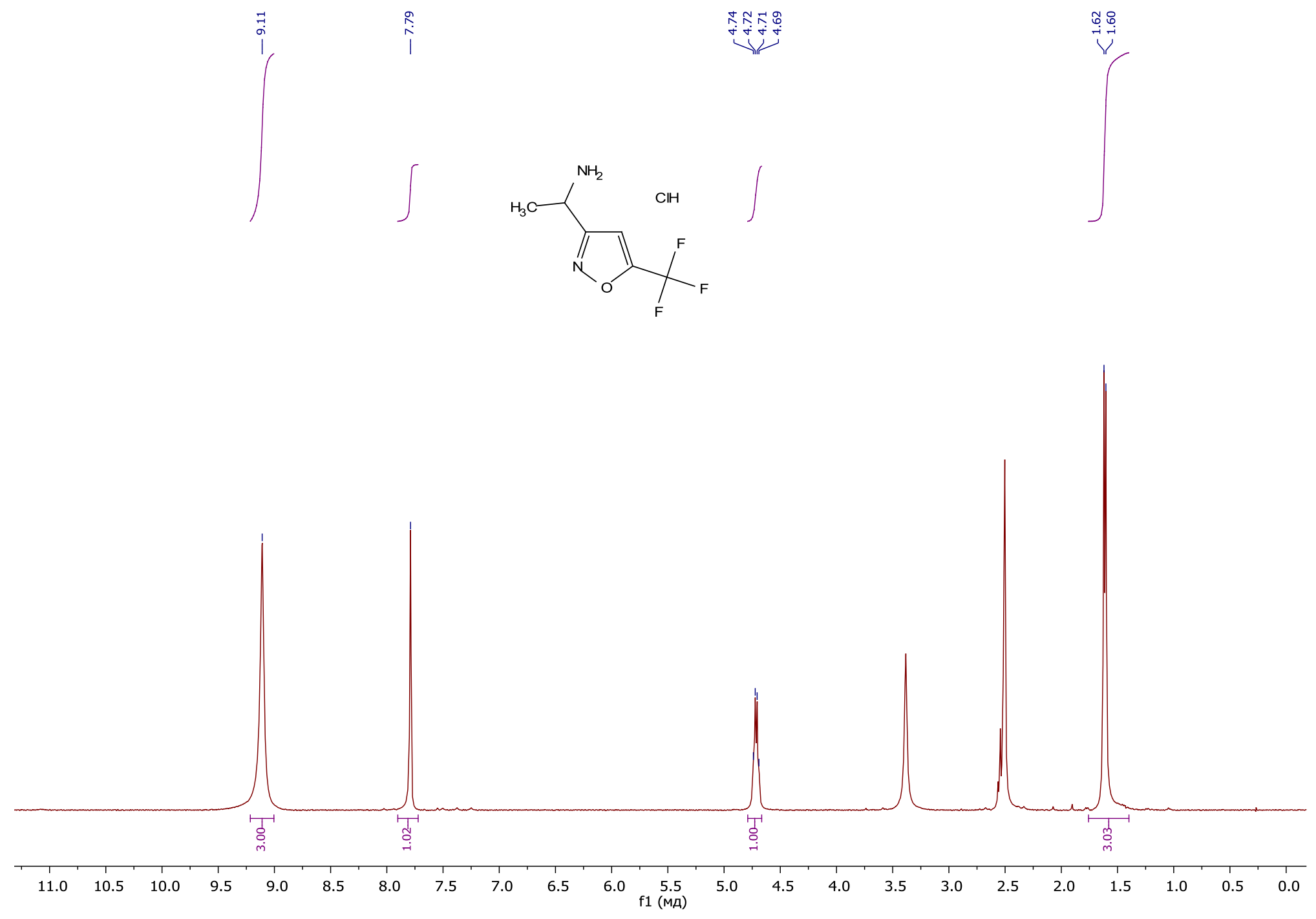

1-(5-(Trifluoromethyl)isoxazol-3-yl)ethan-1-amine hydrochloride $(13 \mathrm{~g} \cdot \mathrm{HCl}$ or $13 \mathrm{~h} \cdot \mathrm{HCl}){ }^{1} \mathrm{H}$ NMR 


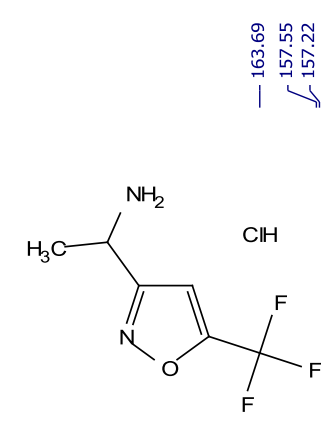

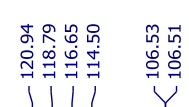

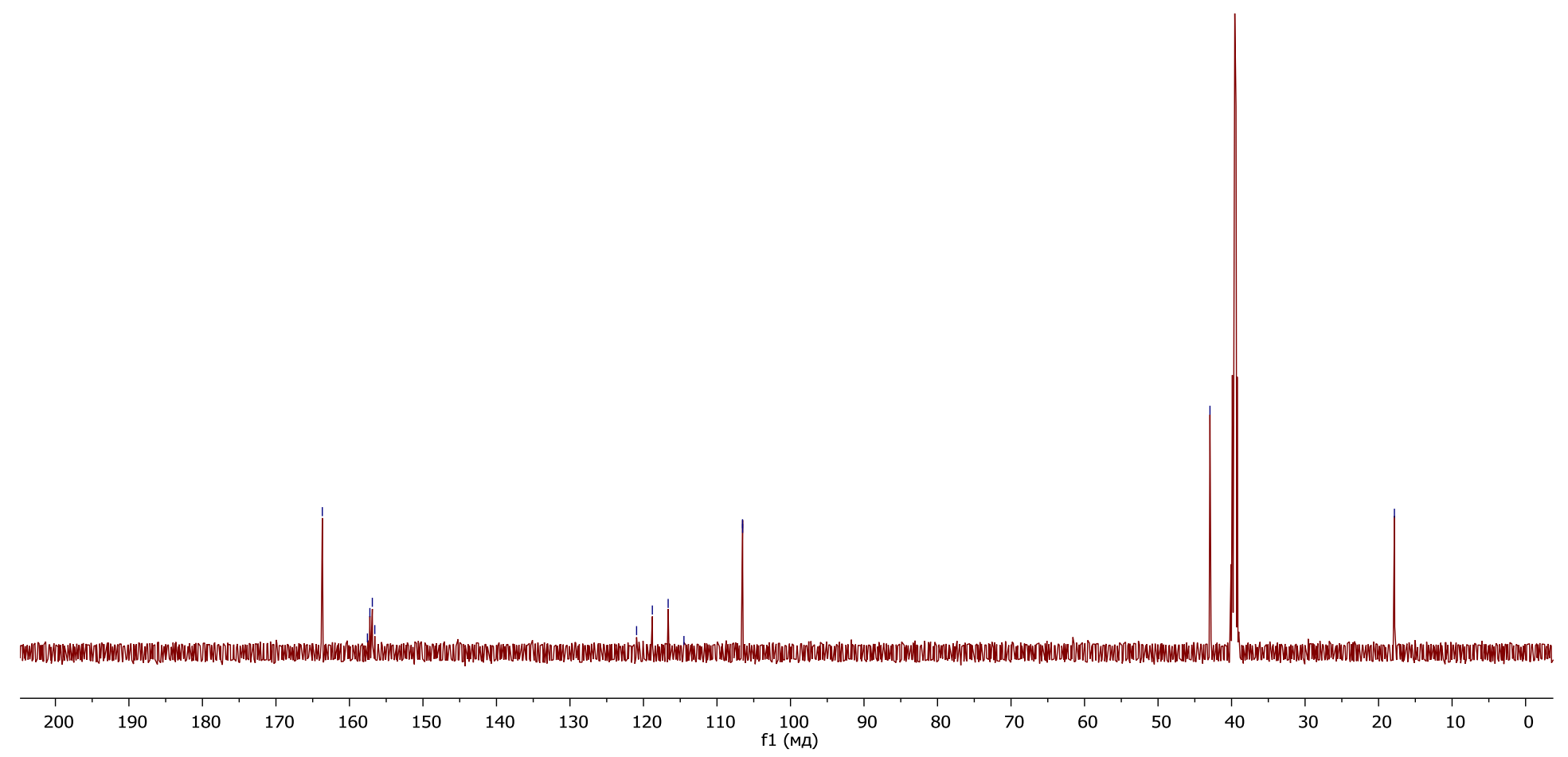

1-(5-(Trifluoromethyl)isoxazol-3-yl)ethan-1-amine hydrochloride (13g.HCl or $13 \mathrm{~h} \cdot \mathrm{HCl}){ }^{13} \mathrm{C} \mathrm{NMR}$ 

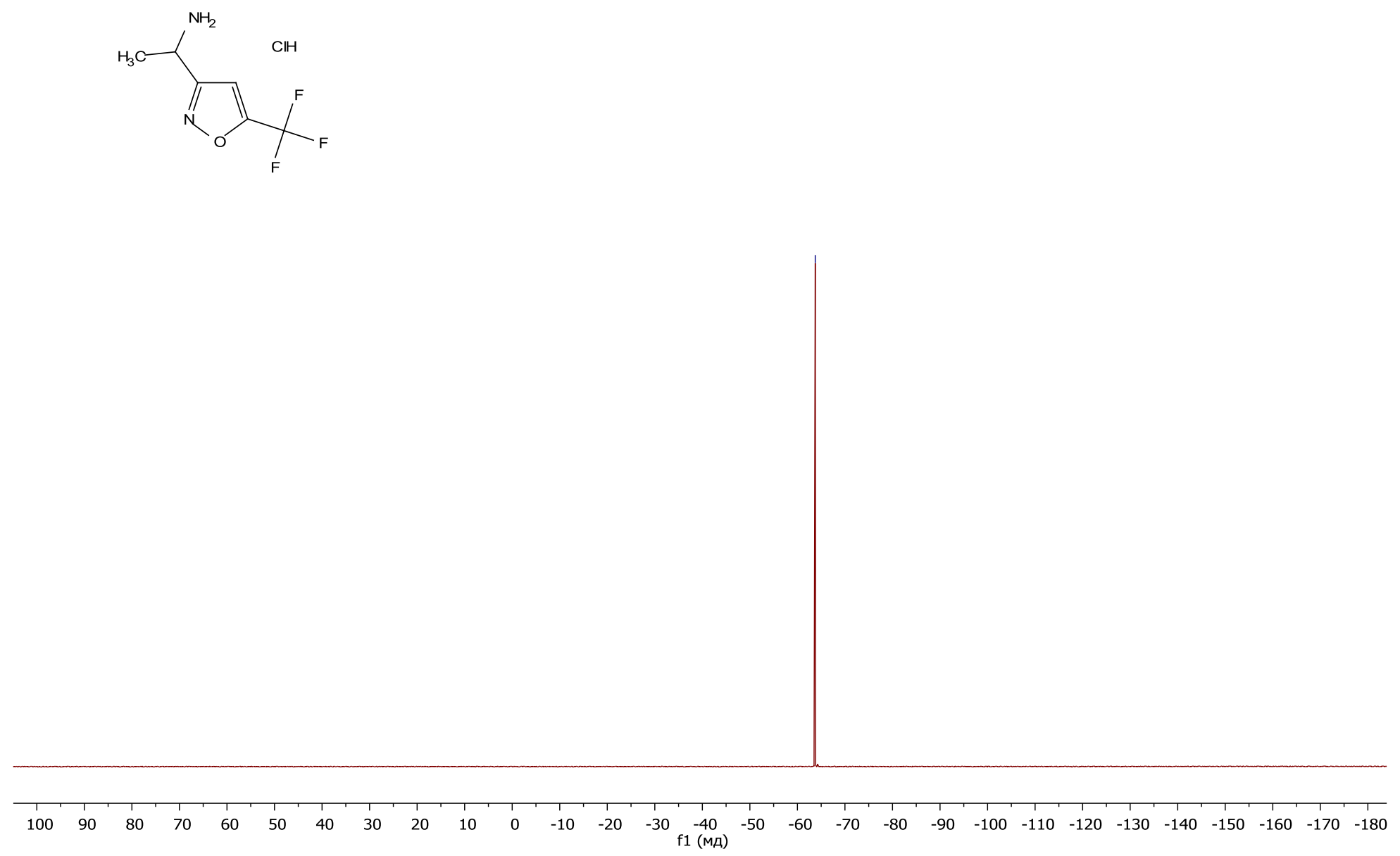

1-(5-(Trifluoromethyl)isoxazol-3-yl)ethan-1-amine hydrochloride (13g-HCl or $13 \mathrm{~h} \cdot \mathrm{HCl}){ }^{19}$ F NMR 

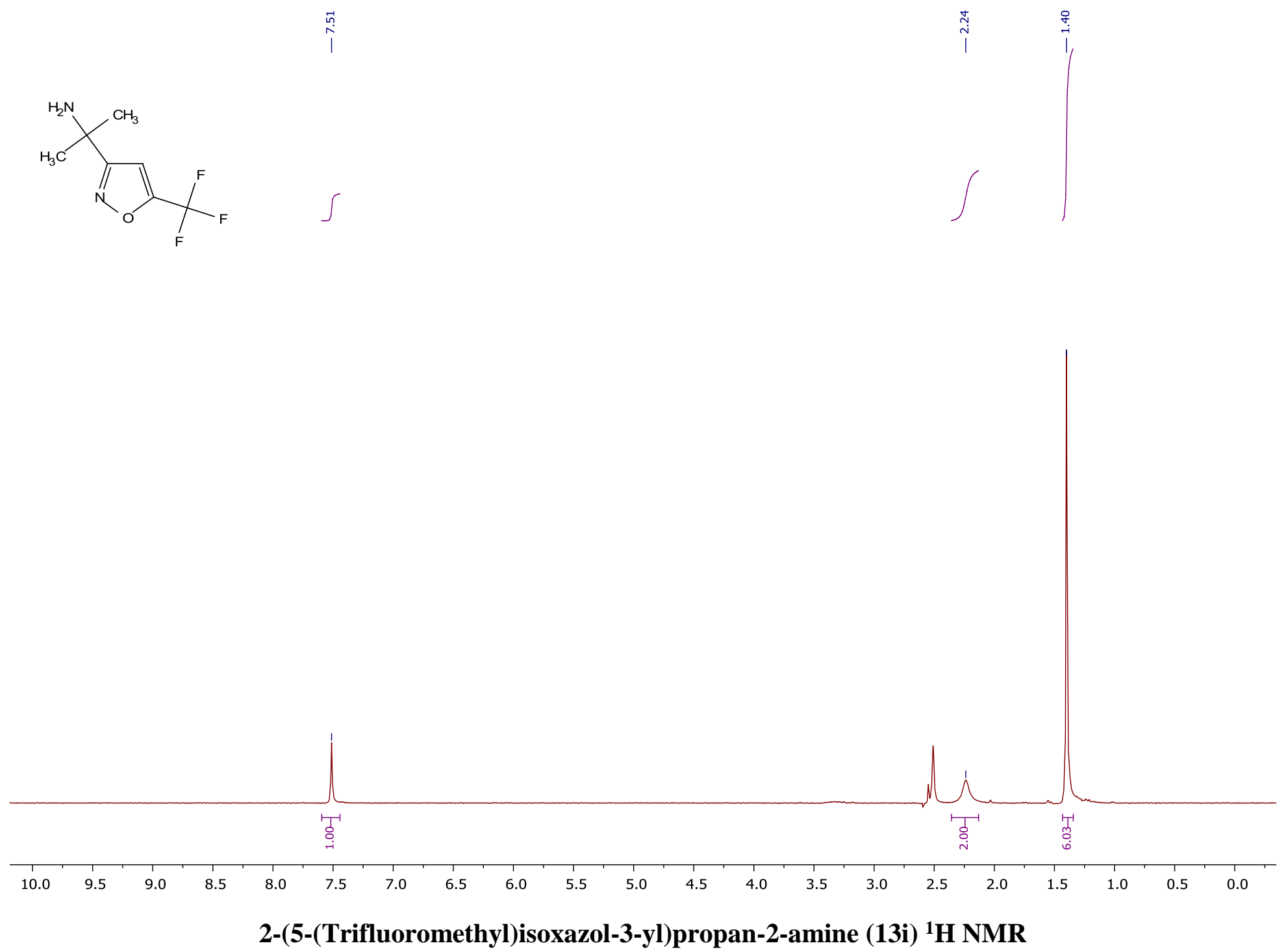

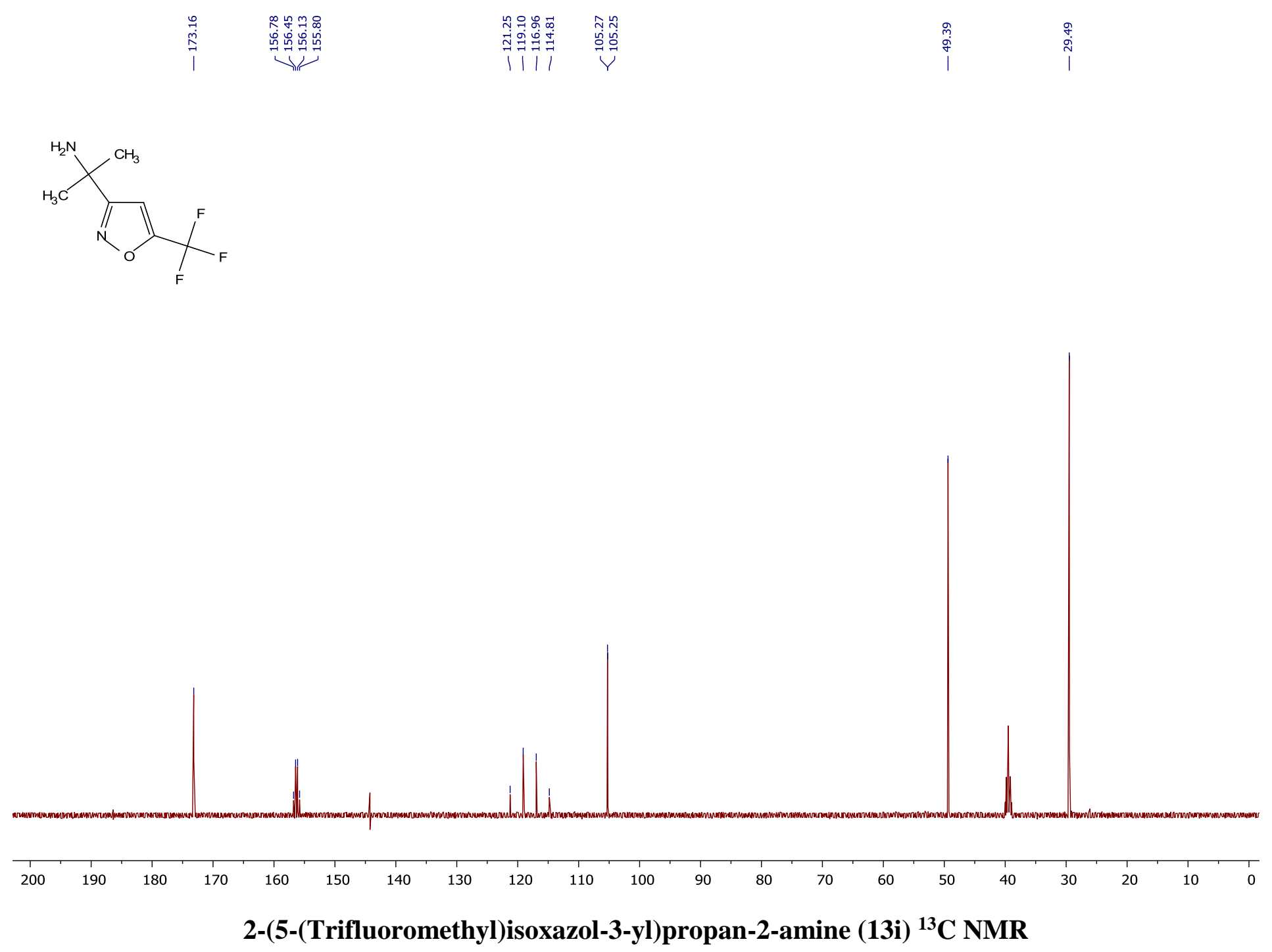

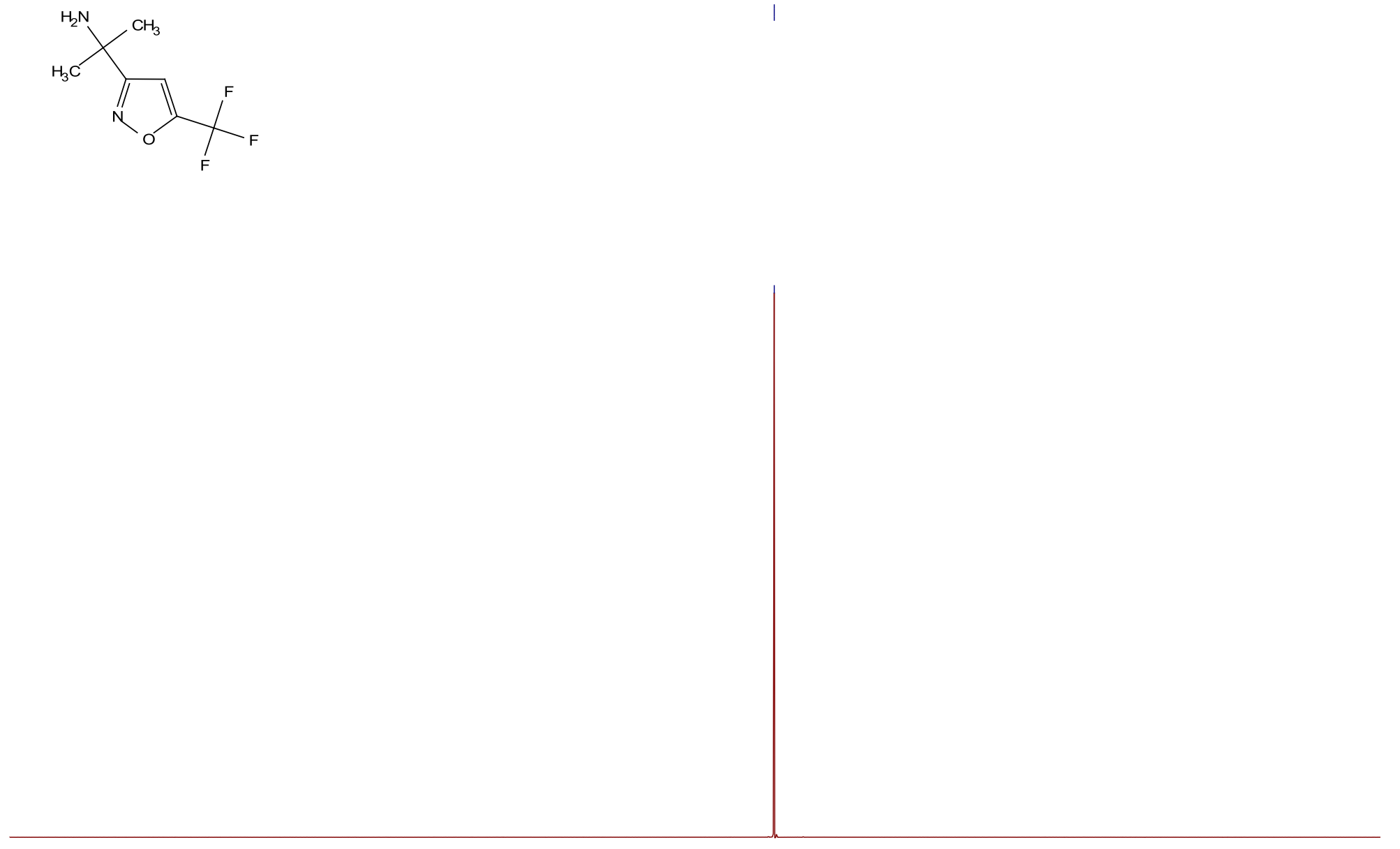

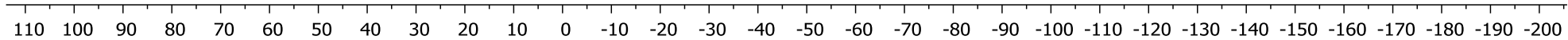

2-(5-(Trifluoromethyl)isoxazol-3-yl)propan-2-amine (13i) ${ }^{19}$ F NMR 


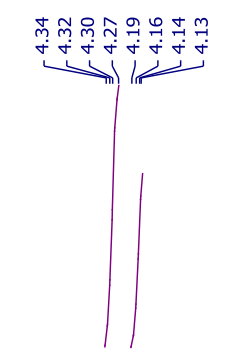

$\mathrm{CH}$
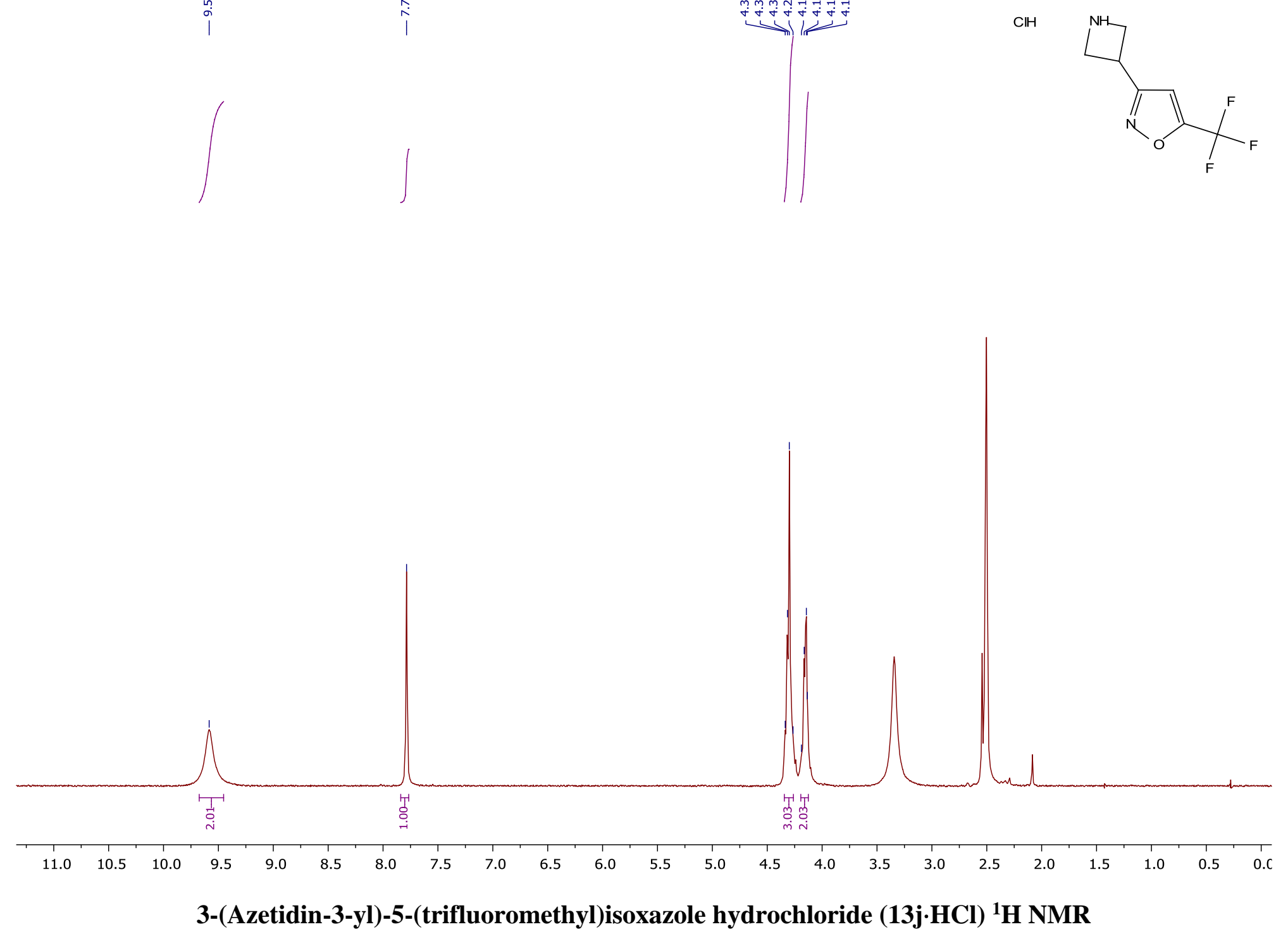


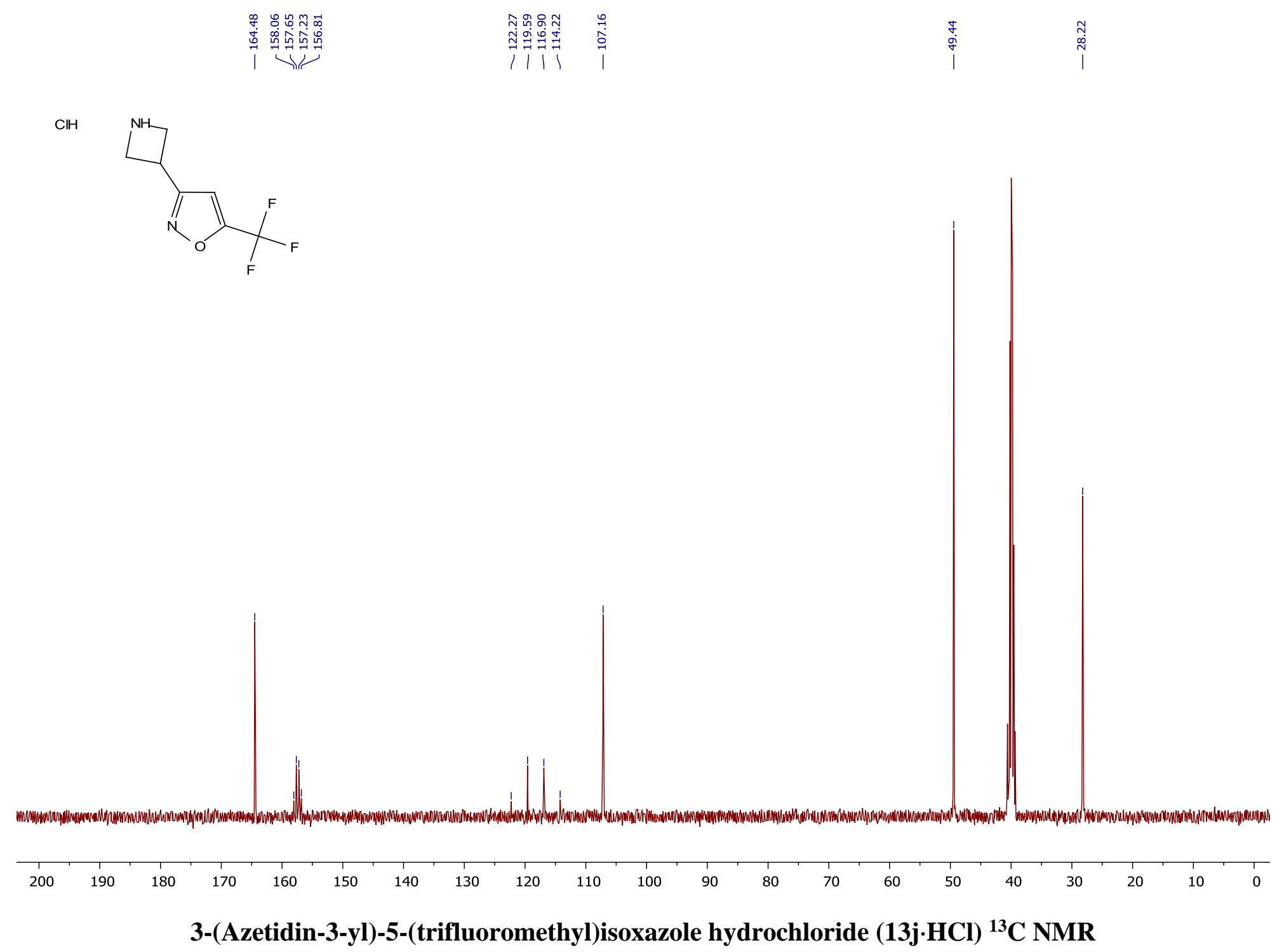


$\mathrm{ClH}$

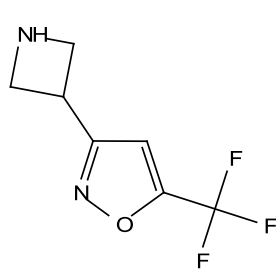

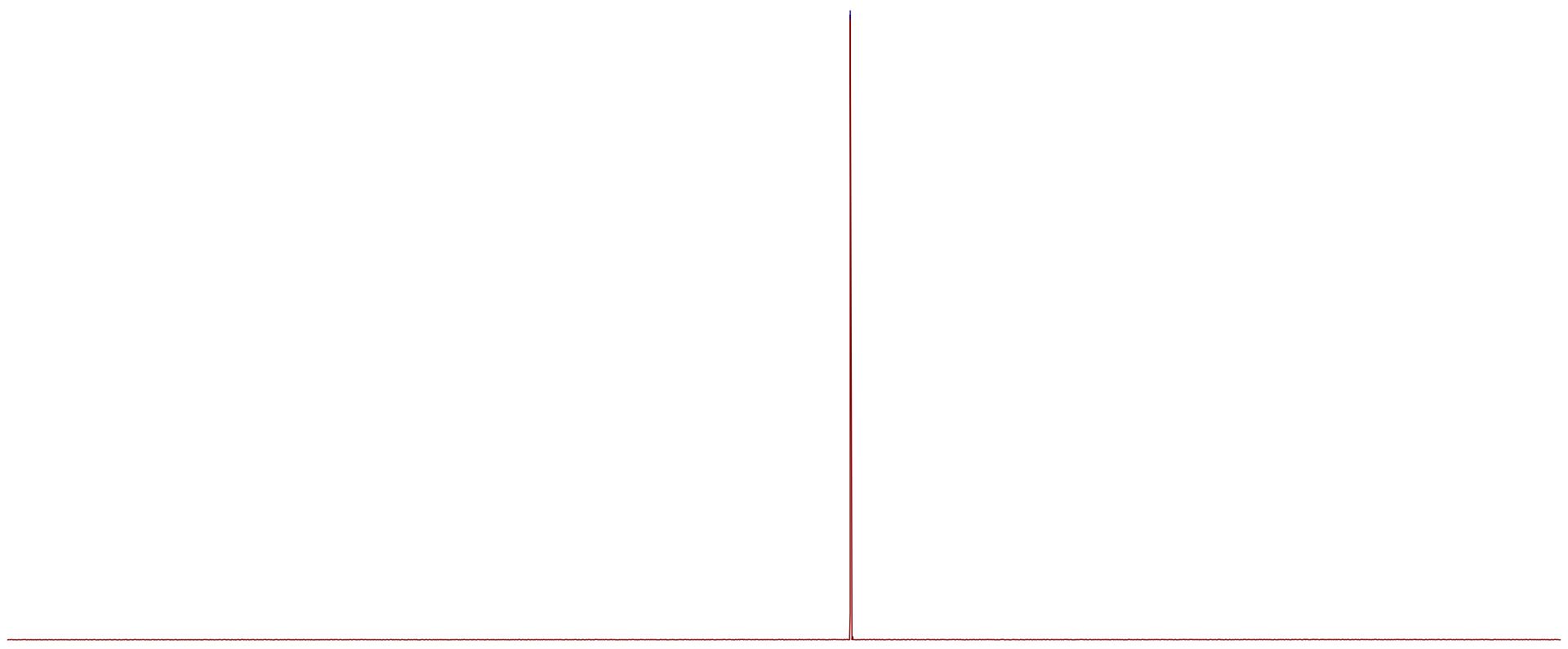

$\begin{array}{llllllllllllllllllllllllllllllllll}110 & 100 & 90 & 80 & 70 & 60 & 50 & 40 & 30 & 20 & 10 & 0 & -10 & -20 & -30 & -40 & -50 & -60 & -70 & -80 & -90 & -100 & -110 & -120 & -130 & -140 & -150 & -160 & -170 & -180 & -190 & -200 & -210\end{array}$

3-(Azetidin-3-yl)-5-(trifluoromethyl)isoxazole hydrochloride (13j·HCl) ${ }^{19}$ F NMR 

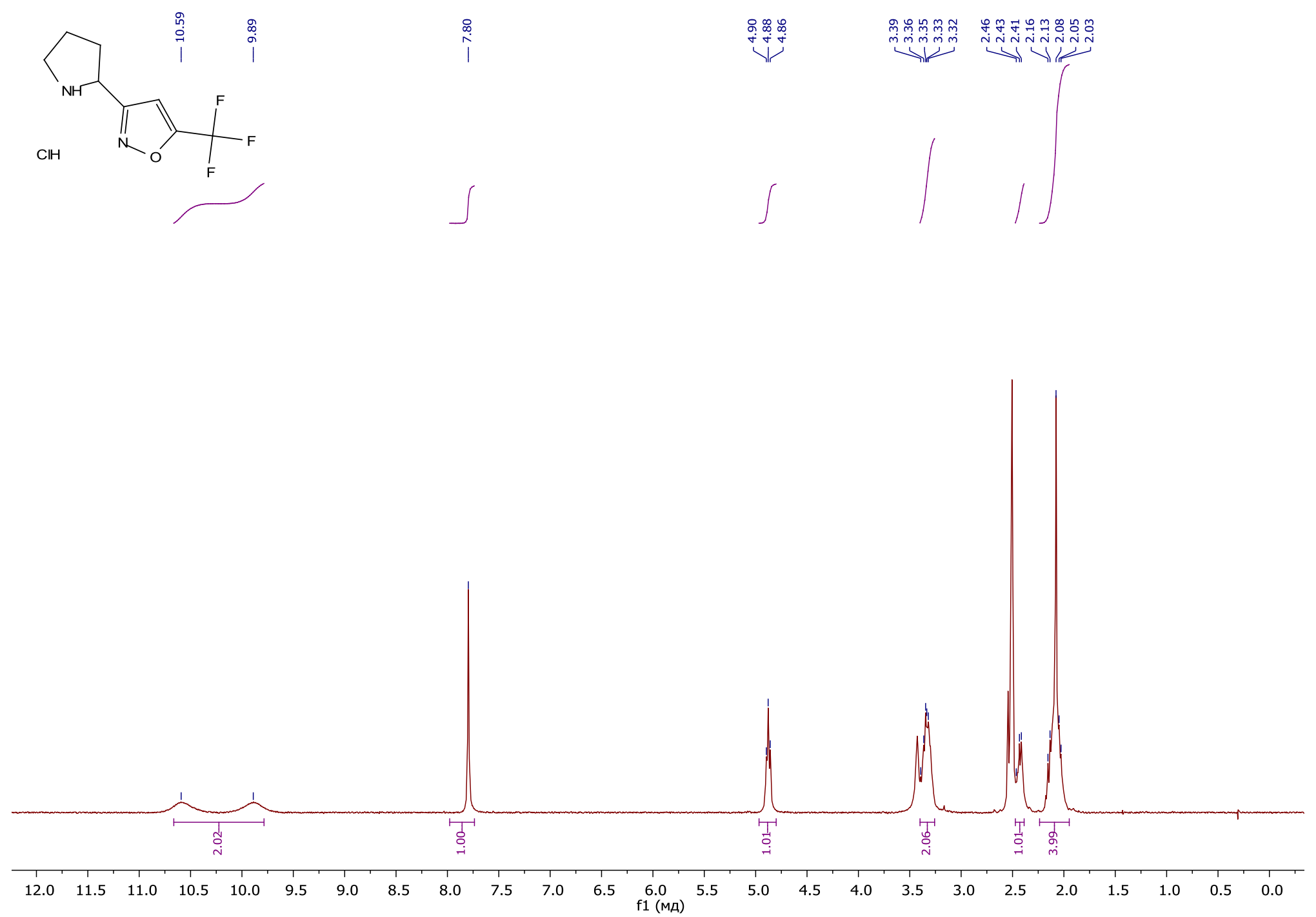

3-(Pyrrolidin-2-yl)-5-(trifluoromethyl)isoxazole hydrochloride (13k·HCl or $13 \mathrm{l} \cdot \mathrm{HCl}){ }^{1} \mathrm{H}$ NMR 


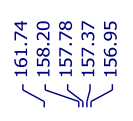

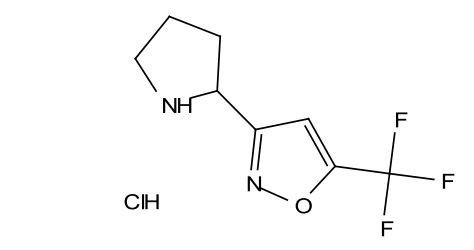

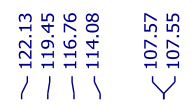

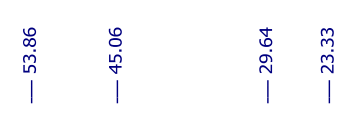

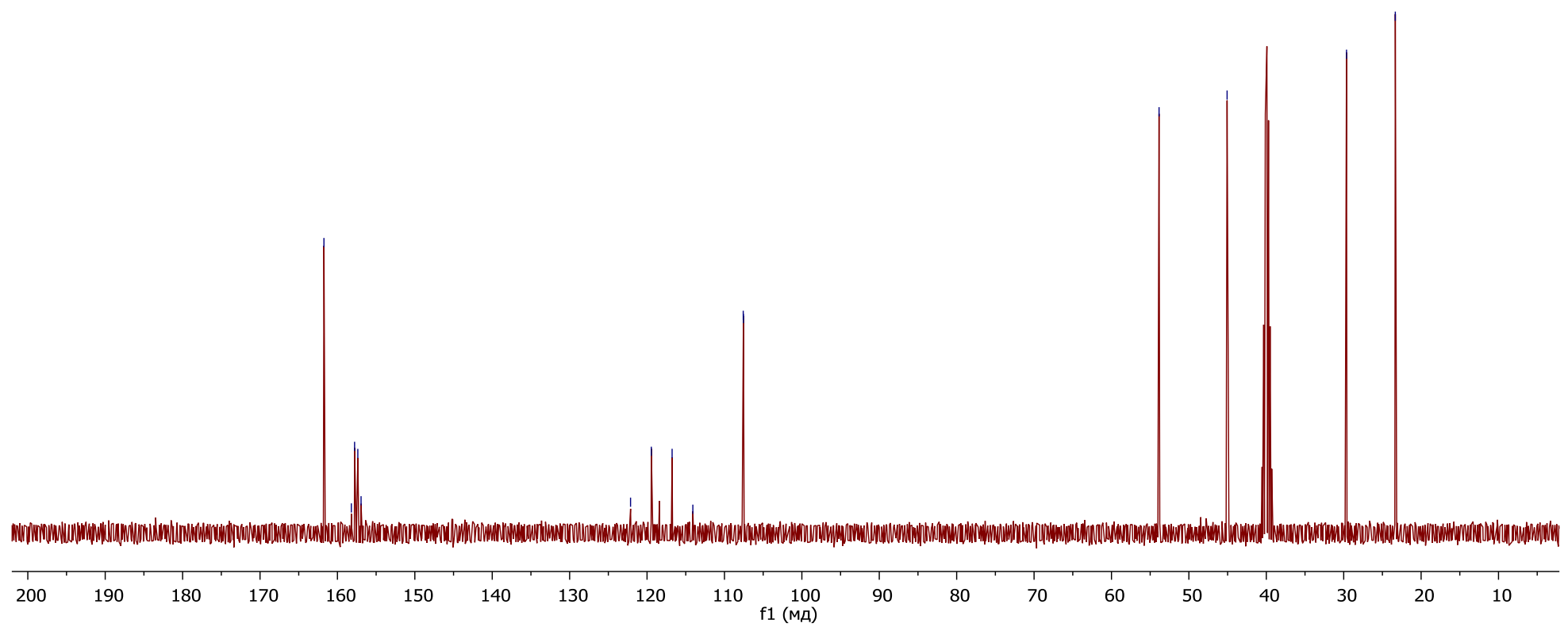

3-(Pyrrolidin-2-yl)-5-(trifluoromethyl)isoxazole hydrochloride $(13 \mathrm{k} \cdot \mathrm{HCl}$ or $13 \mathrm{l} \cdot \mathrm{HCl}){ }^{13} \mathrm{C} \mathrm{NMR}$

S77 

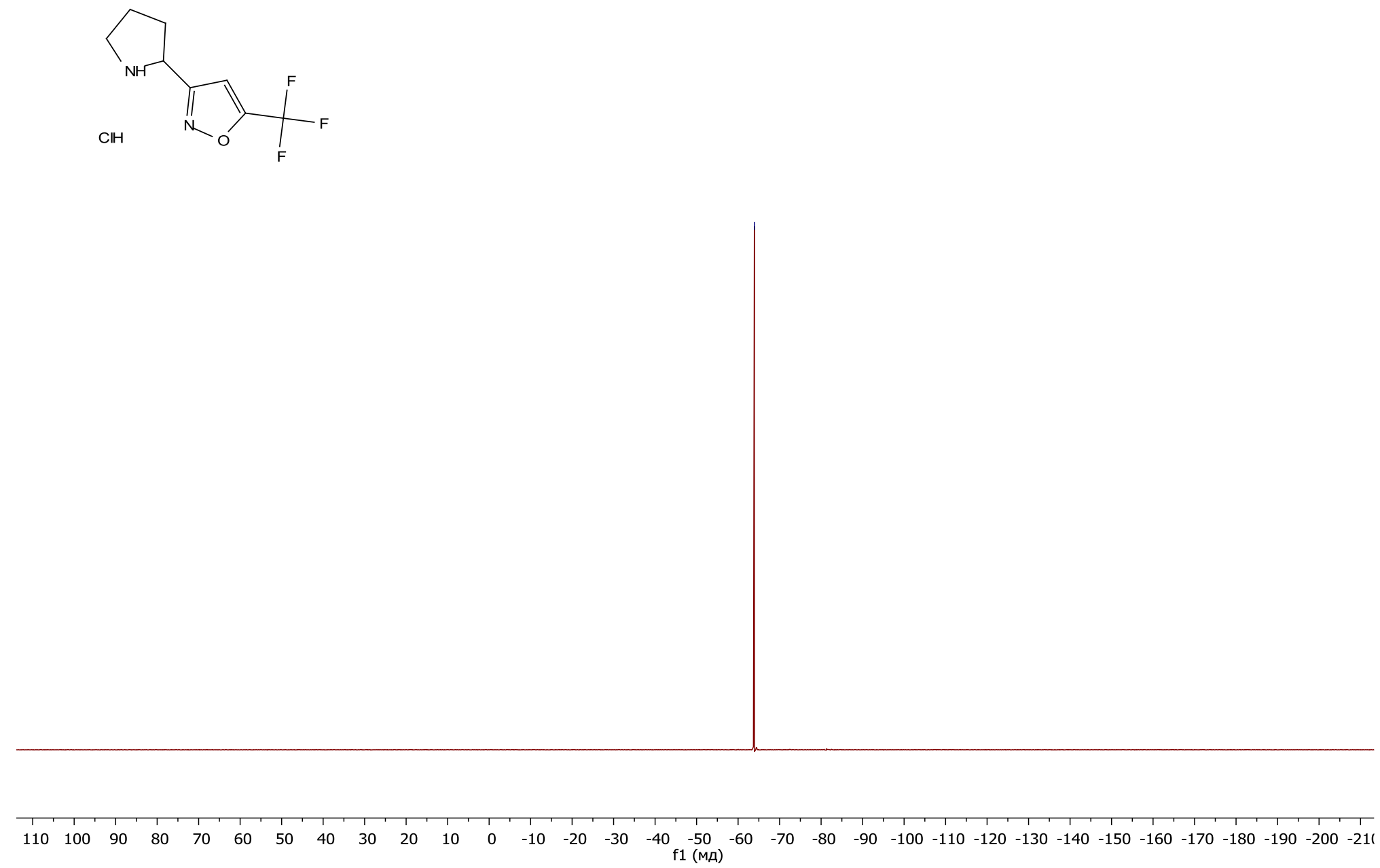

3-(Pyrrolidin-2-yl)-5-(trifluoromethyl)isoxazole hydrochloride (13k·HCl or $13 \mathrm{l} \cdot \mathrm{HCl}){ }^{19} \mathrm{~F}$ NMR 

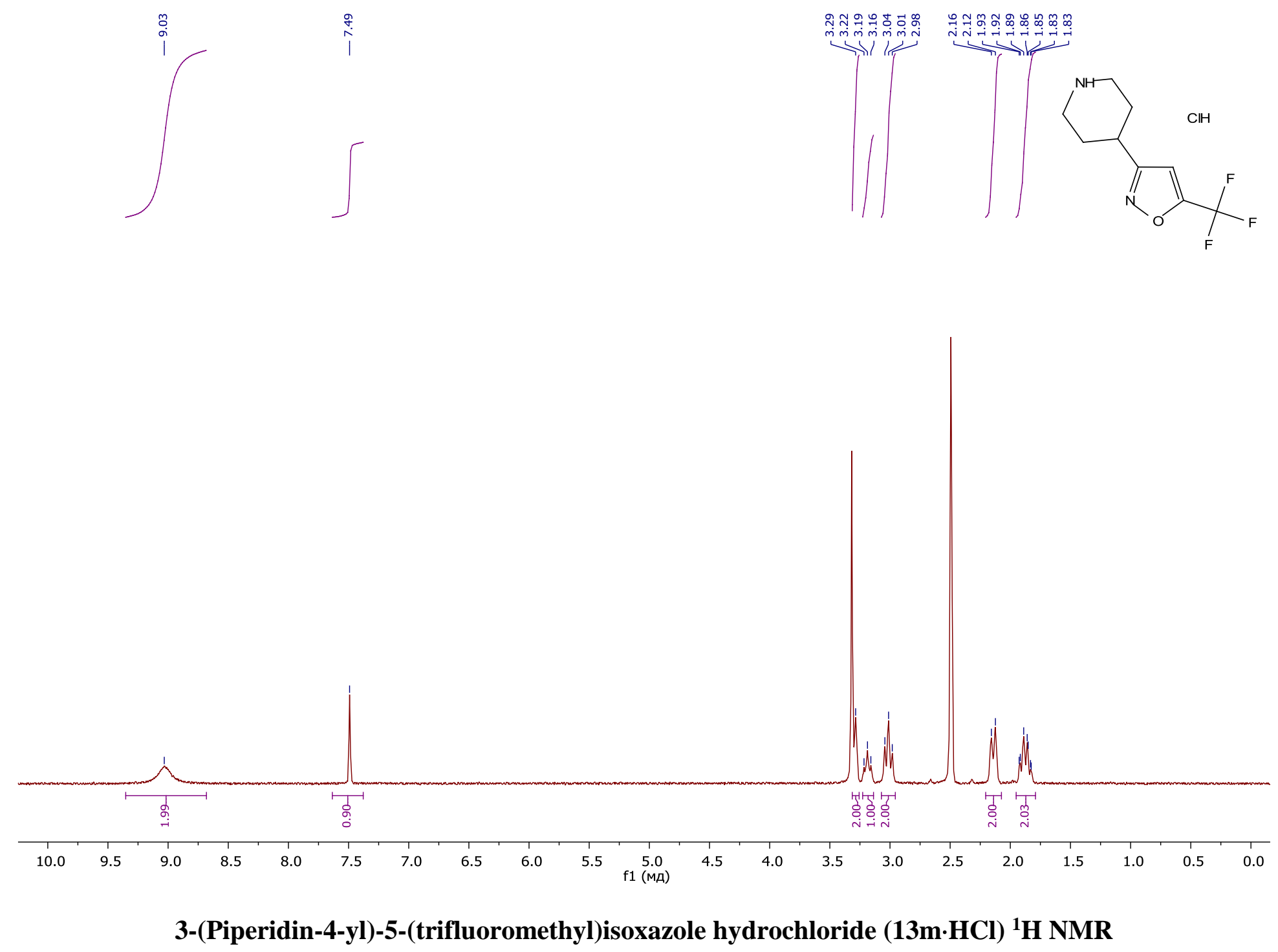

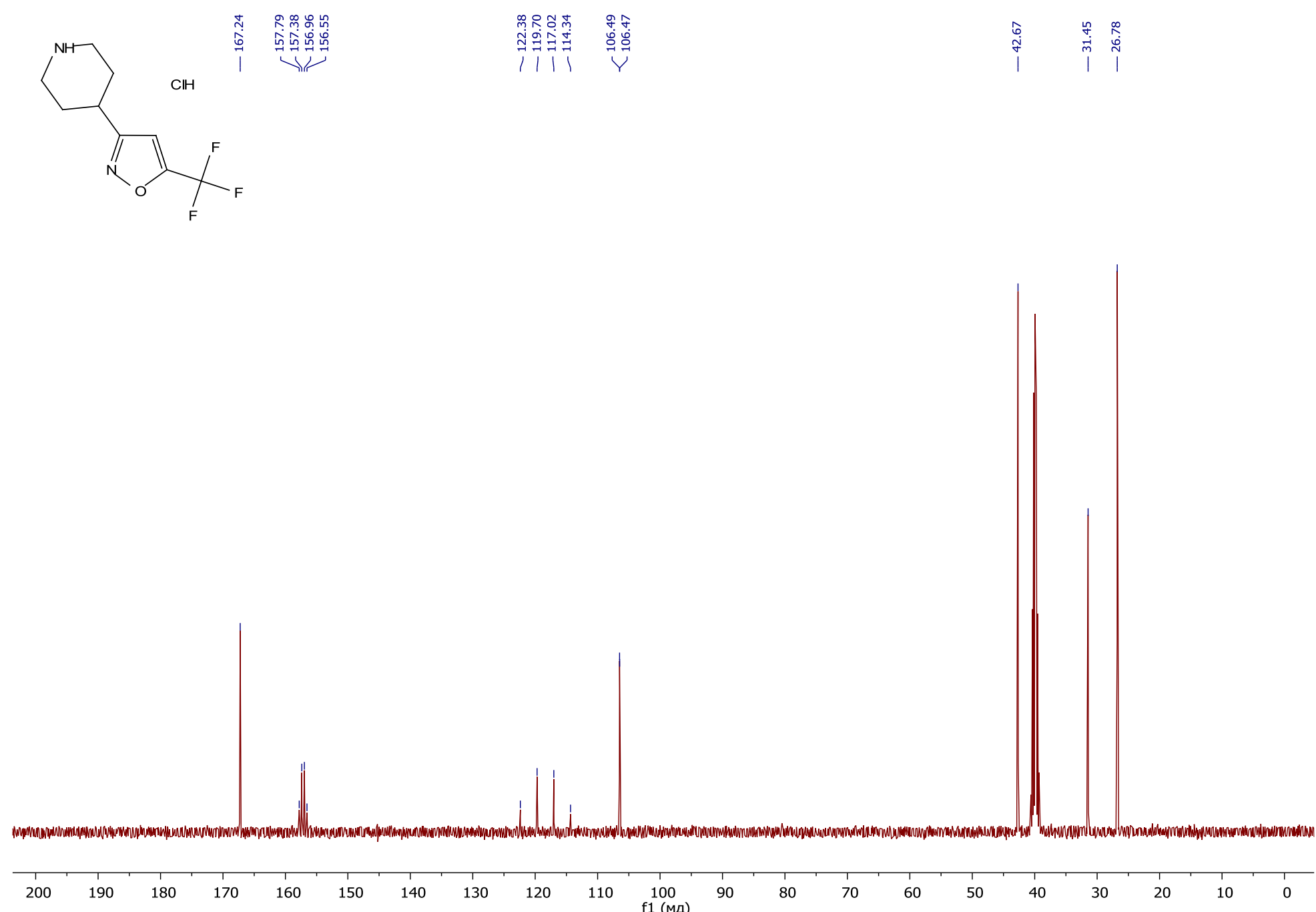

3-(Piperidin-4-yl)-5-(trifluoromethyl)isoxazole hydrochloride $(13 \mathrm{~m} \cdot \mathrm{HCl}){ }^{13} \mathrm{C}$ NMR 

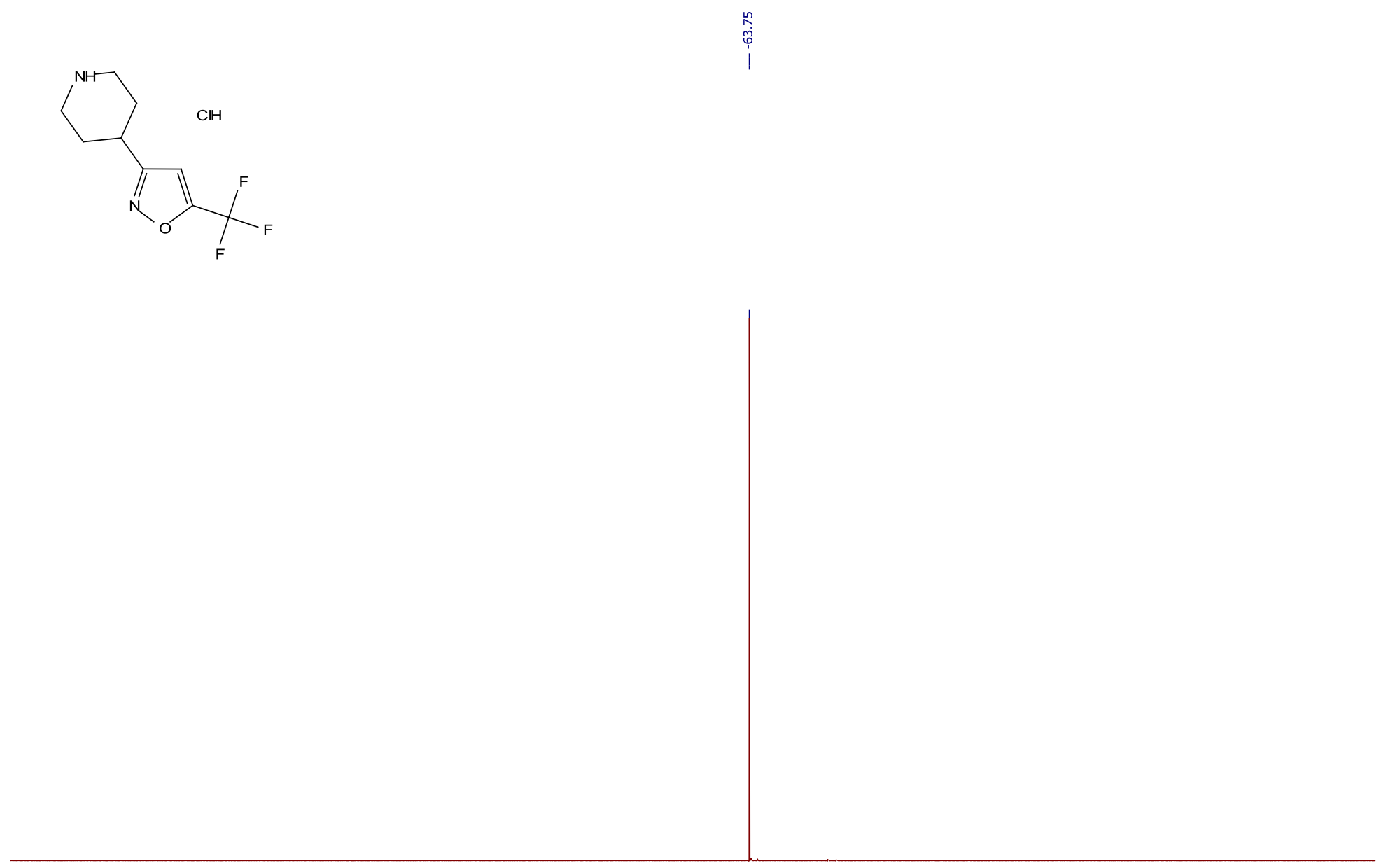

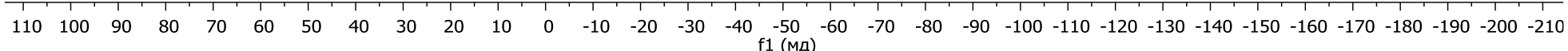

3-(Piperidin-4-yl)-5-(trifluoromethyl)isoxazole hydrochloride $(13 \mathrm{~m} \cdot \mathrm{HCl}){ }^{19} \mathrm{~F}$ NMR 


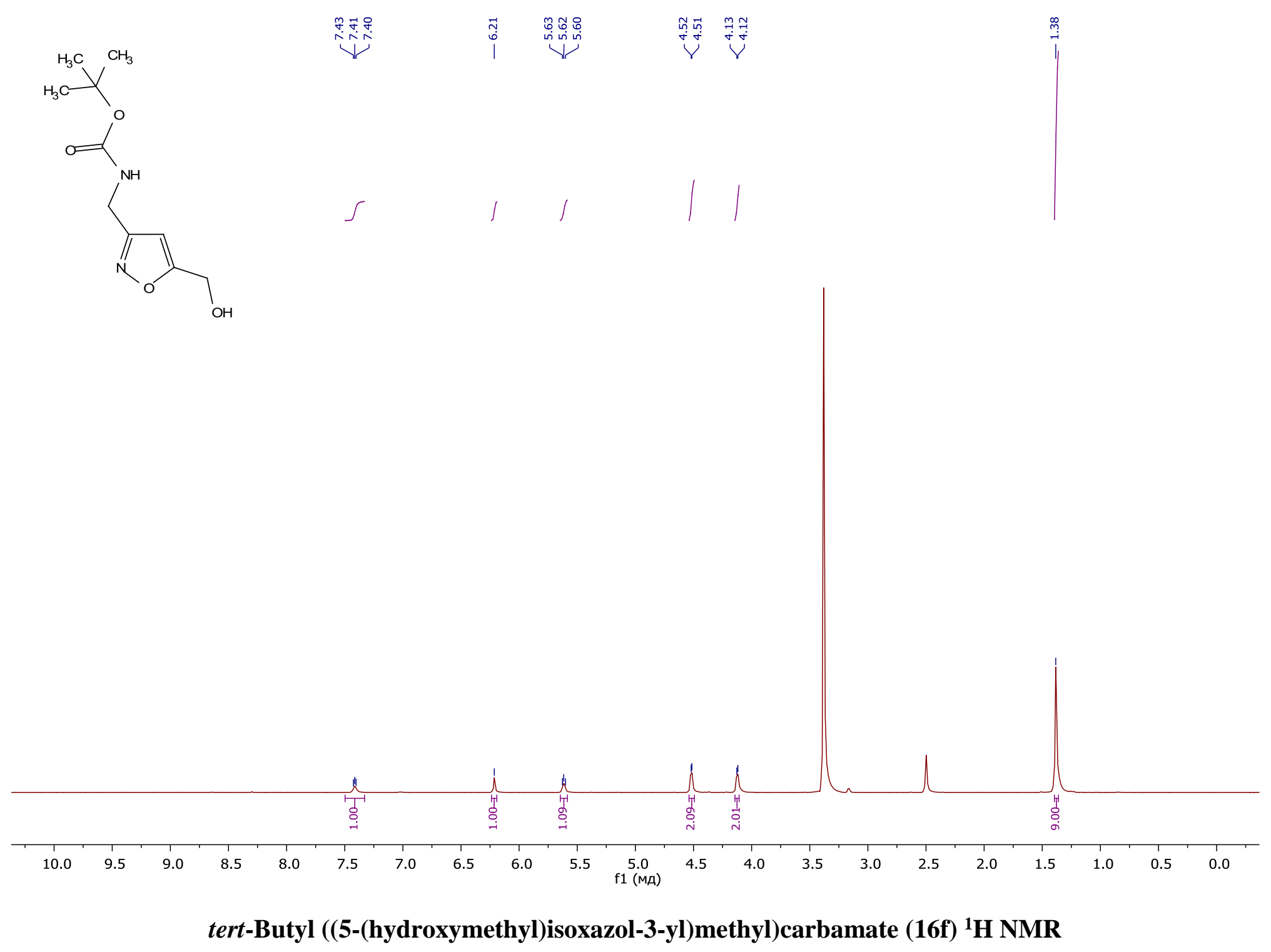




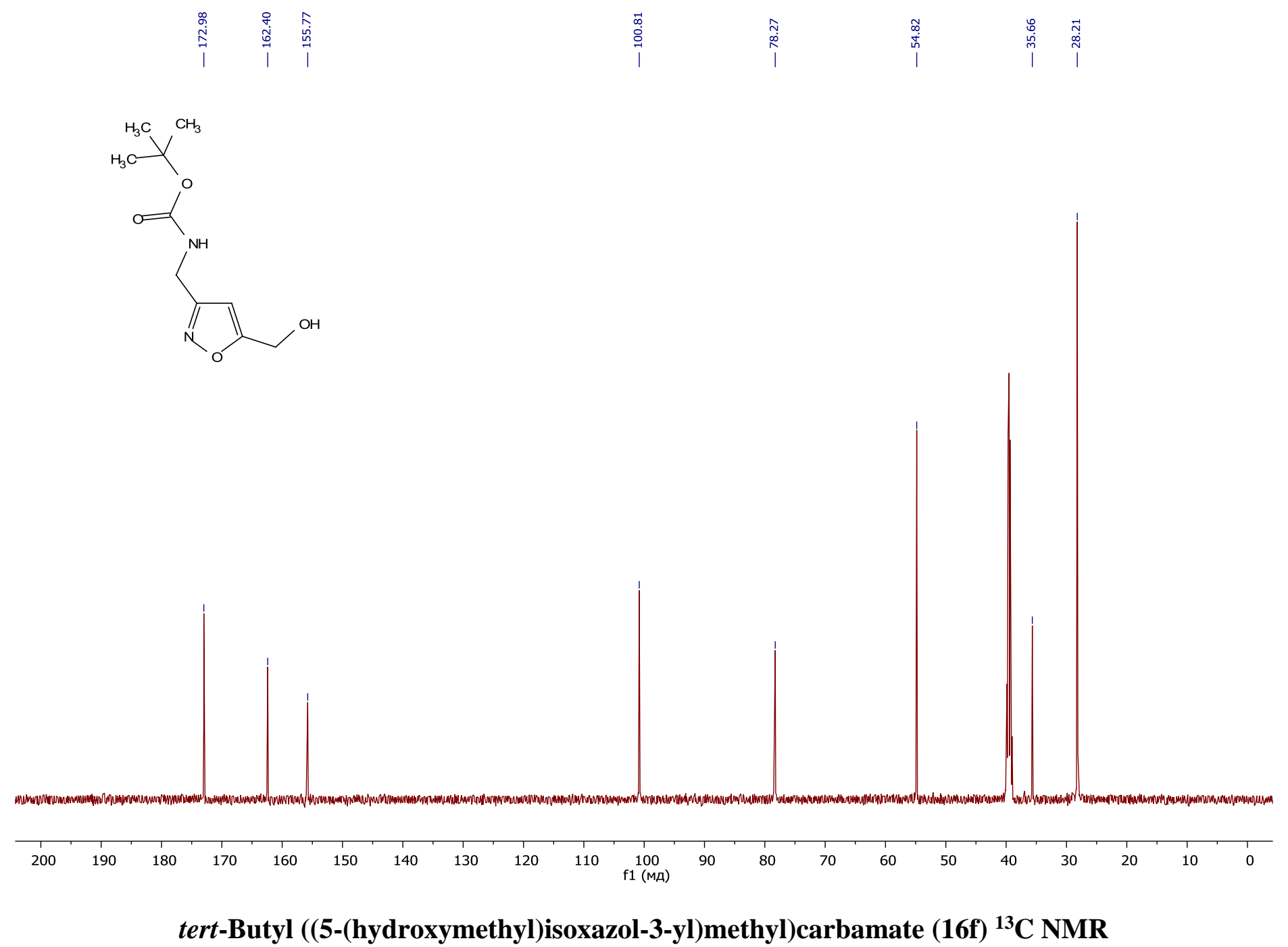



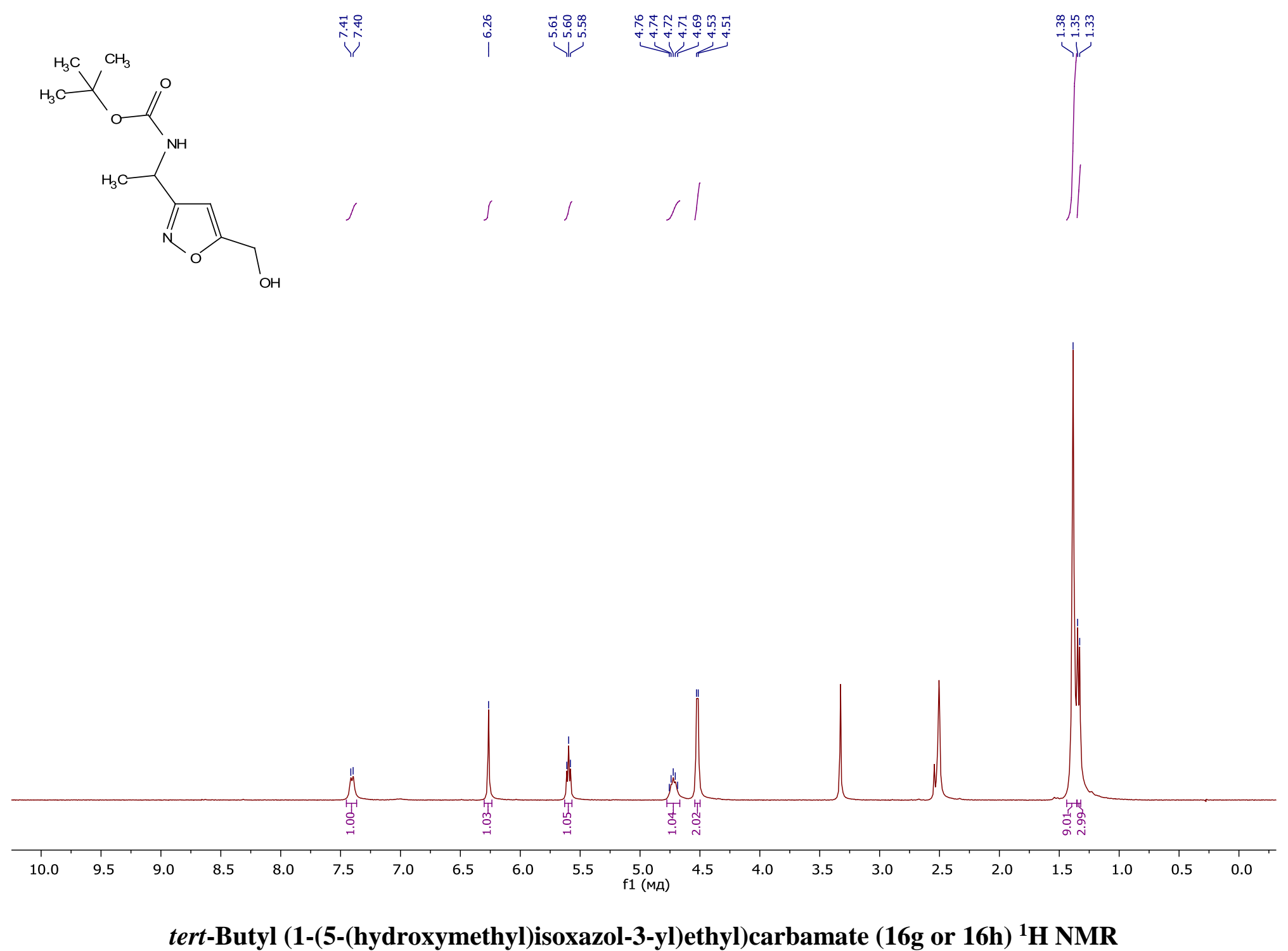


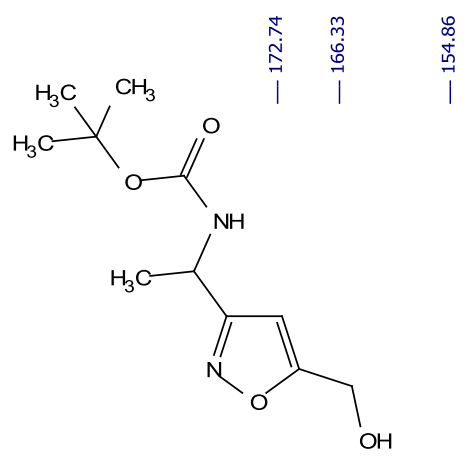

$\overrightarrow{0}$
0
0
0
0
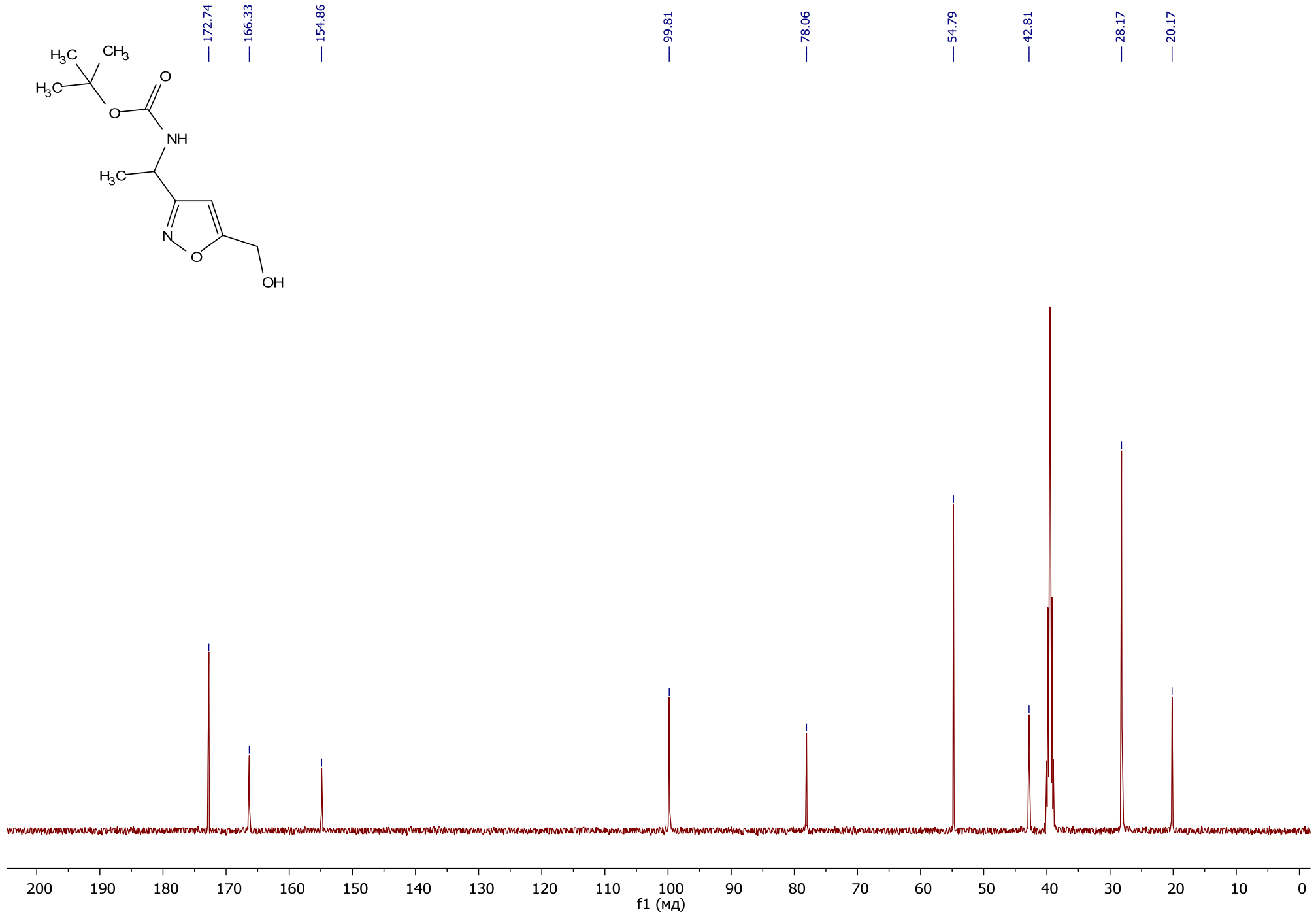

tert-Butyl (1-(5-(hydroxymethyl)isoxazol-3-yl)ethyl)carbamate (16g or 16h) ${ }^{13} \mathrm{C}$ NMR

S85 

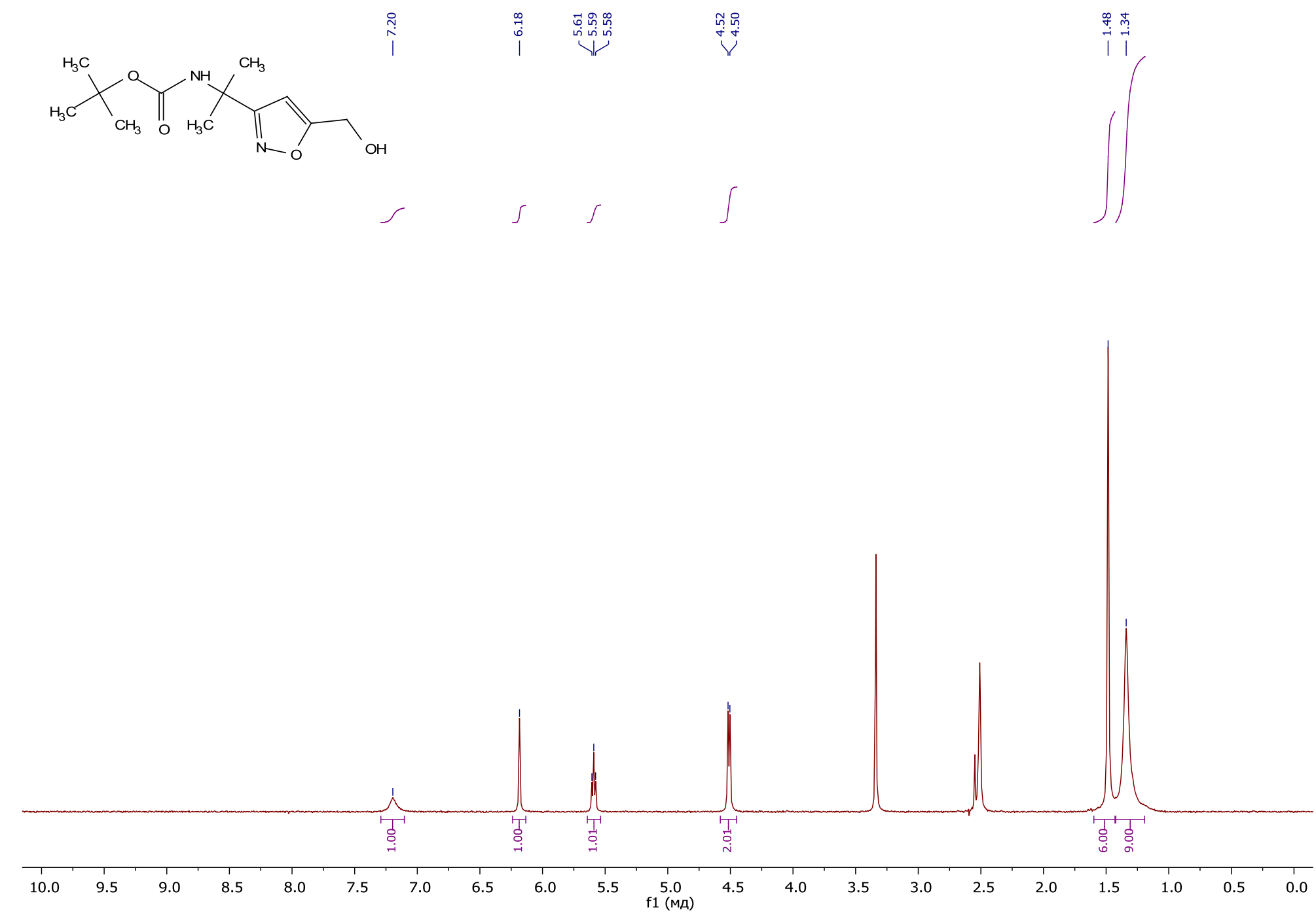

tert-Butyl (2-(5-(hydroxymethyl)isoxazol-3-yl)propan-2-yl)carbamate (16i) ${ }^{1} \mathrm{H}$ NMR 

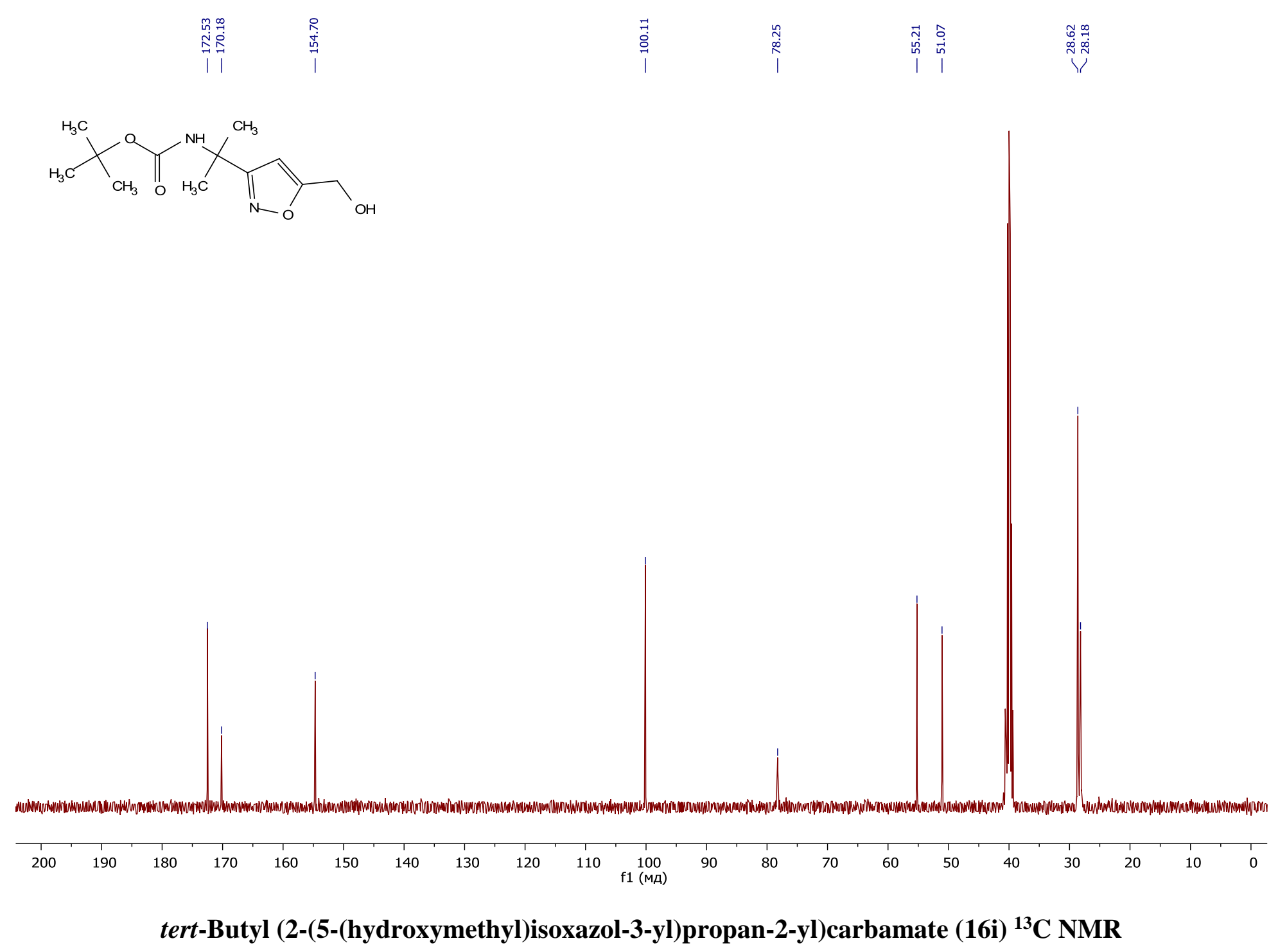

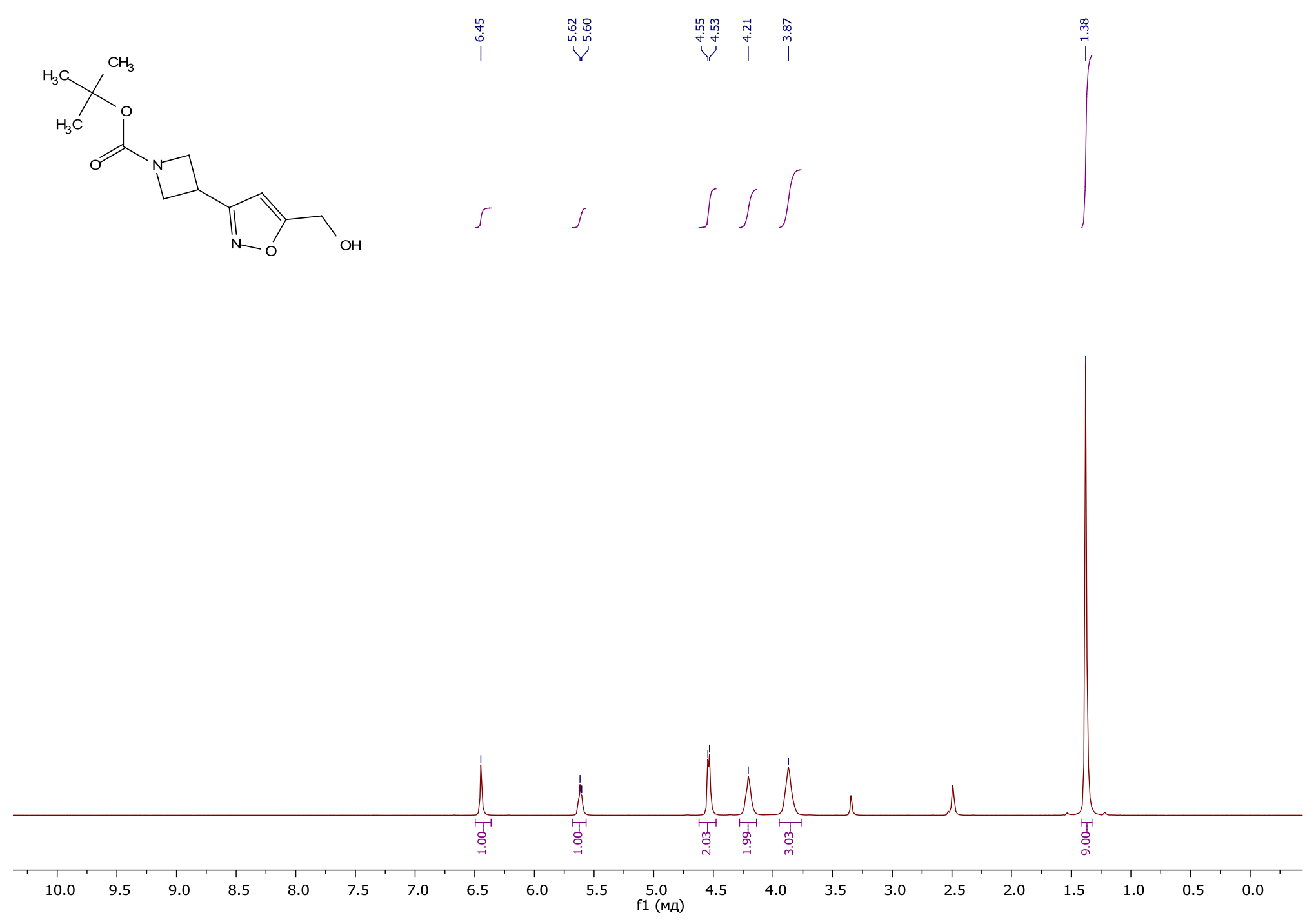

tert-Butyl 3-(5-(hydroxymethyl)isoxazol-3-yl)azetidine-1-carboxylate (16j) ${ }^{1} \mathrm{H}$ NMR 


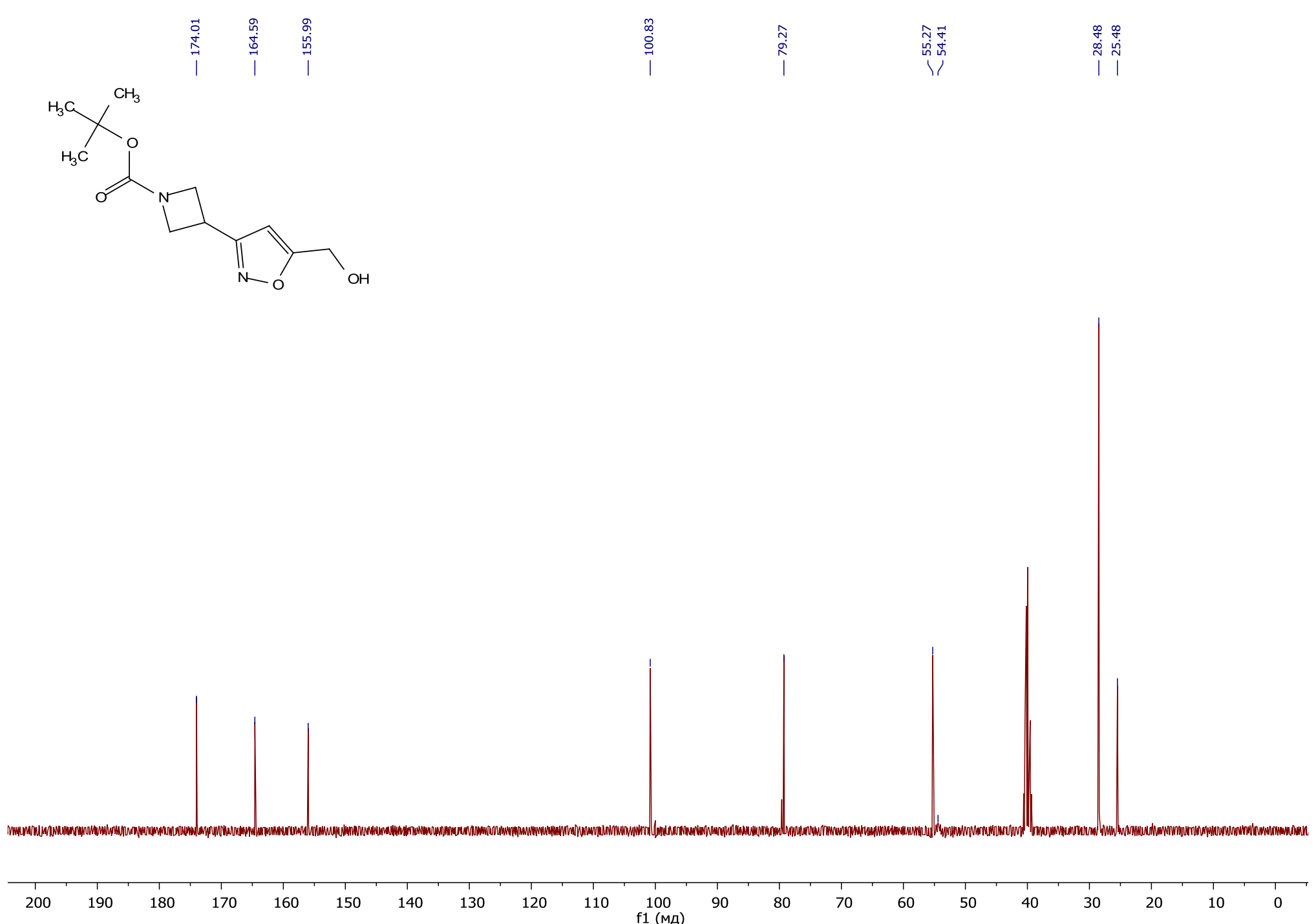

tert-Butyl 3-(5-(hydroxymethyl)isoxazol-3-yl)azetidine-1-carboxylate (16j) ${ }^{13} \mathrm{C}$ NMR 

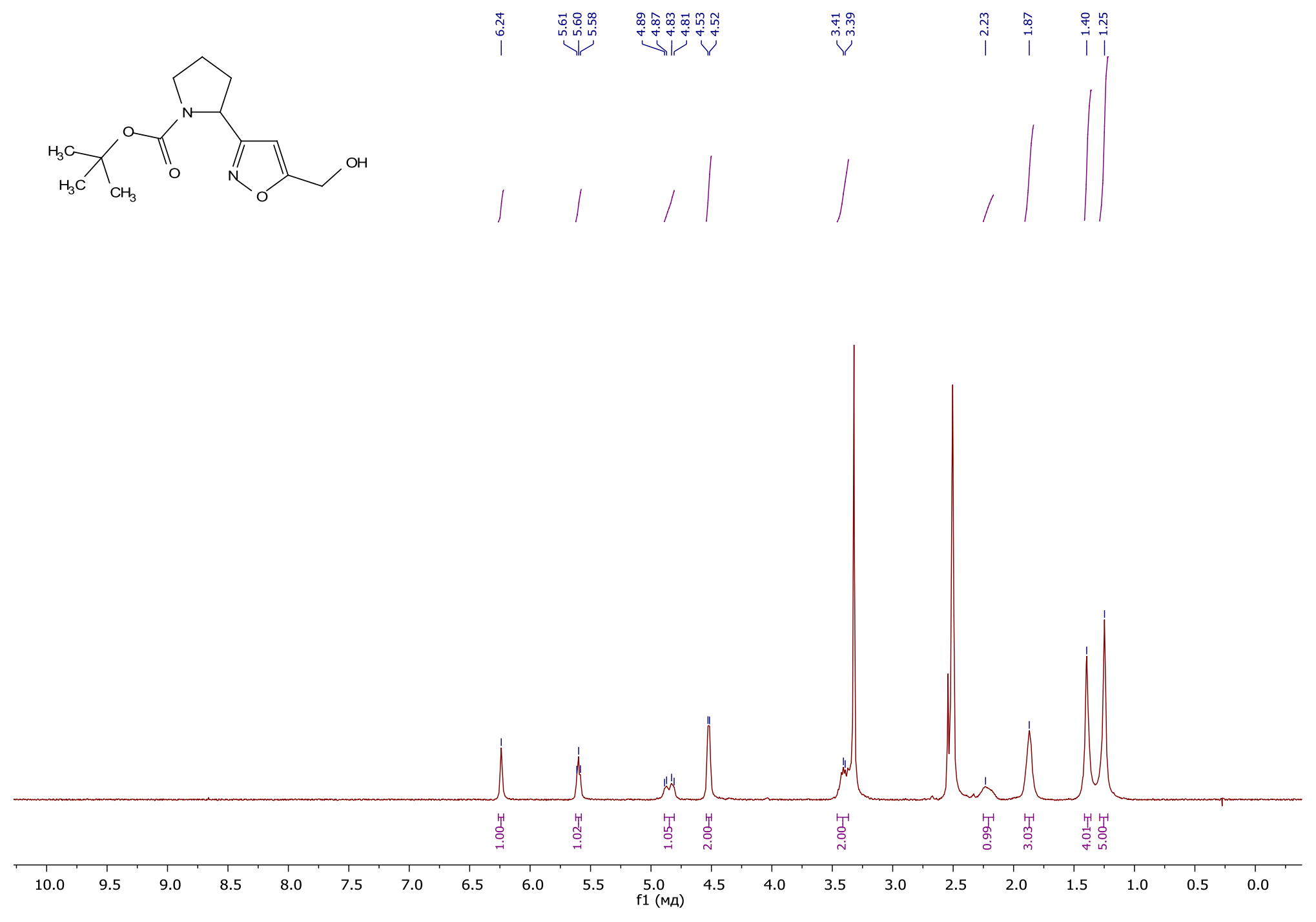

tert-Butyl 2-(5-(hydroxymethyl)isoxazol-3-yl)pyrrolidine-1-carboxylate (16k or 16l) ${ }^{1} \mathrm{H}$ NMR 


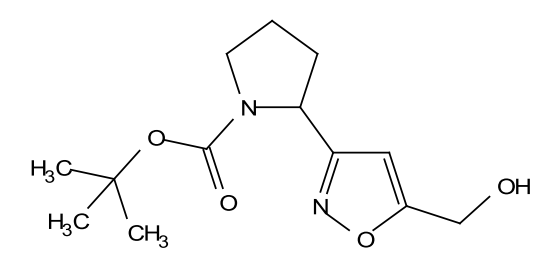

V)

$\mathrm{H}_{3} \mathrm{C} \mathrm{CH}_{3}$

o

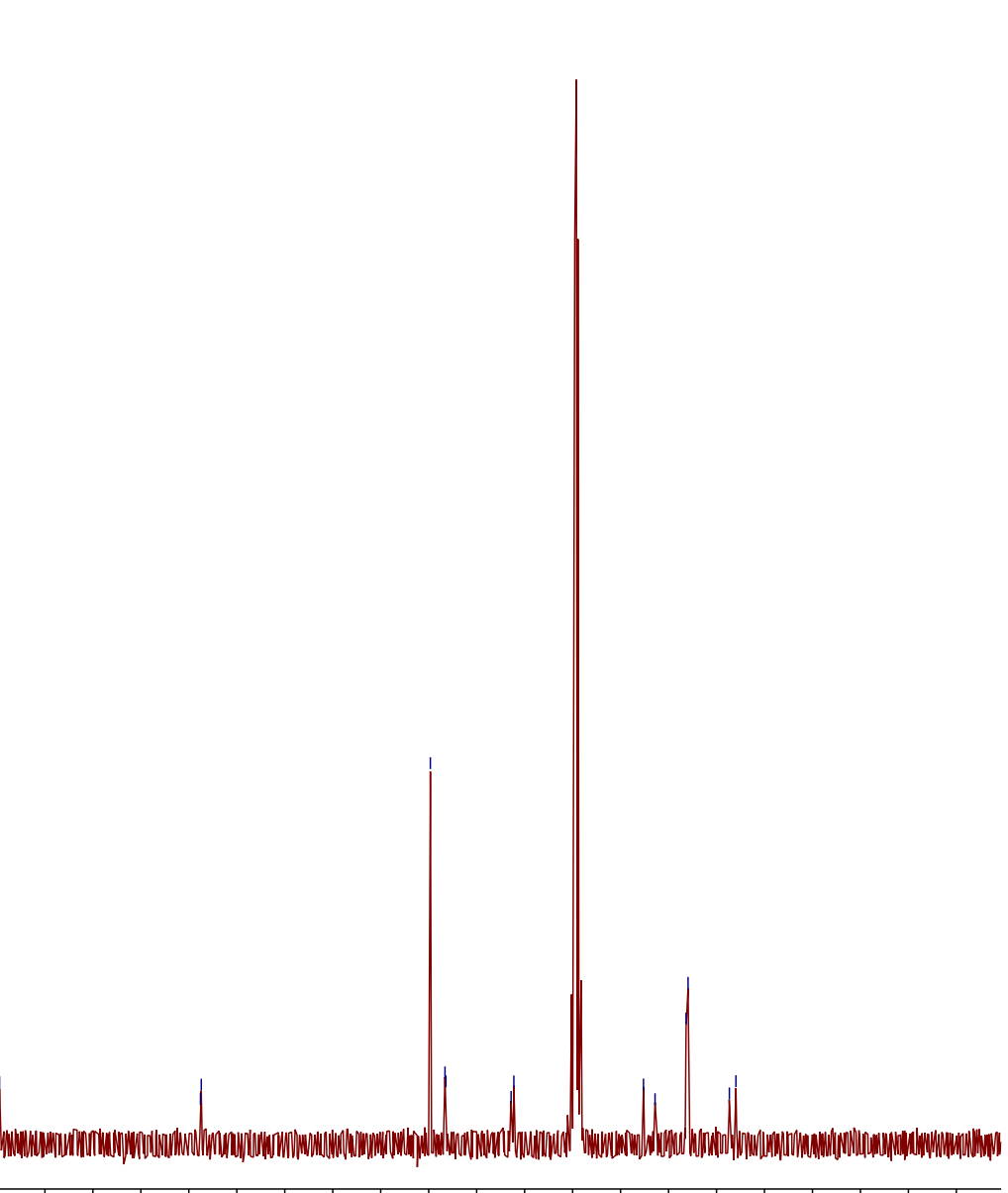

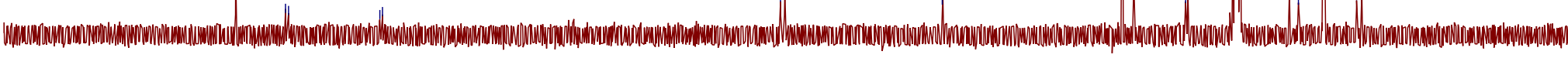

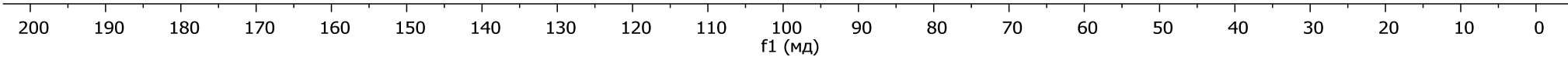

tert-Butyl 2-(5-(hydroxymethyl)isoxazol-3-yl)pyrrolidine-1-carboxylate (16k or 161) ${ }^{13} \mathrm{C}$ NMR 

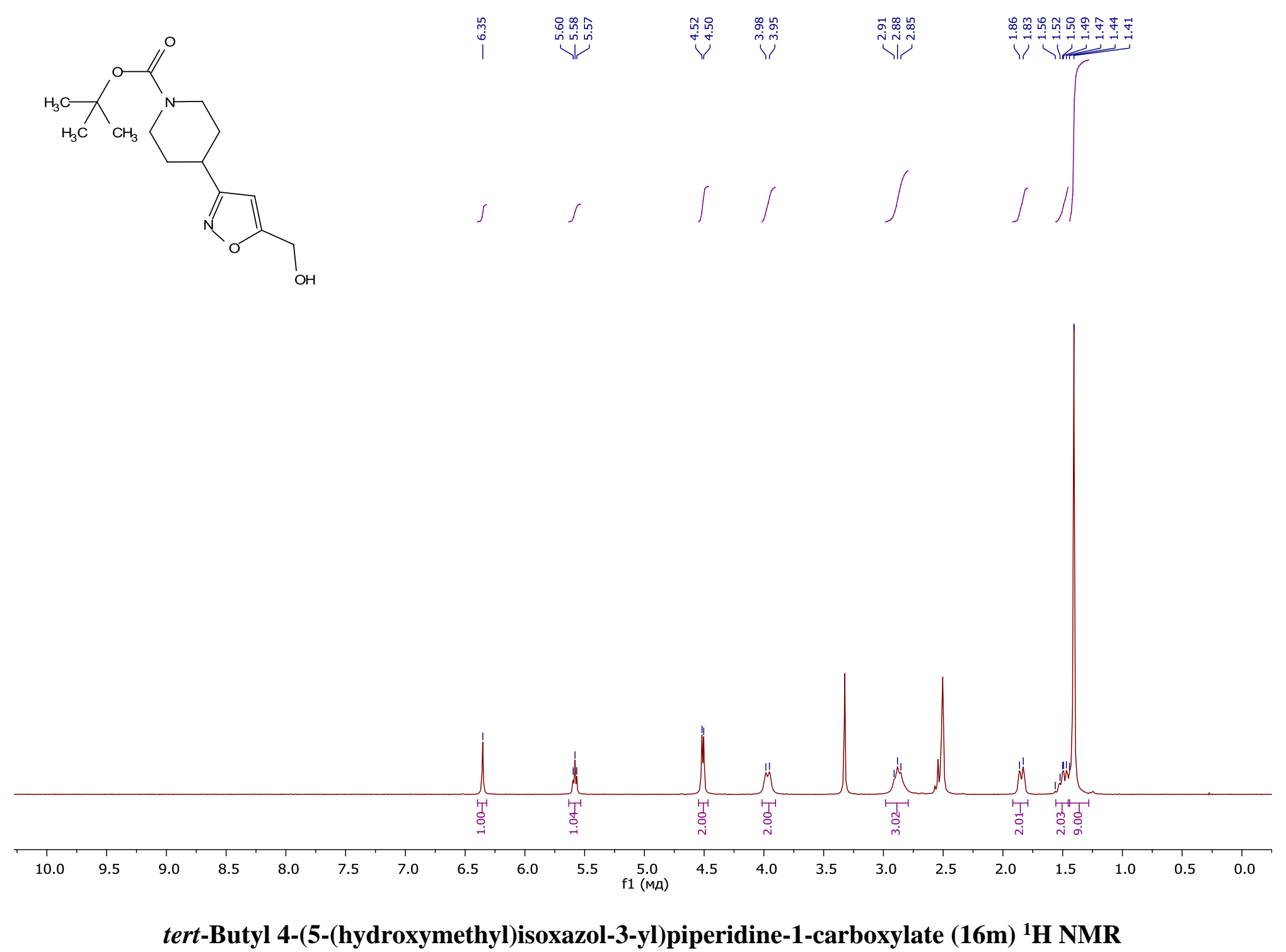

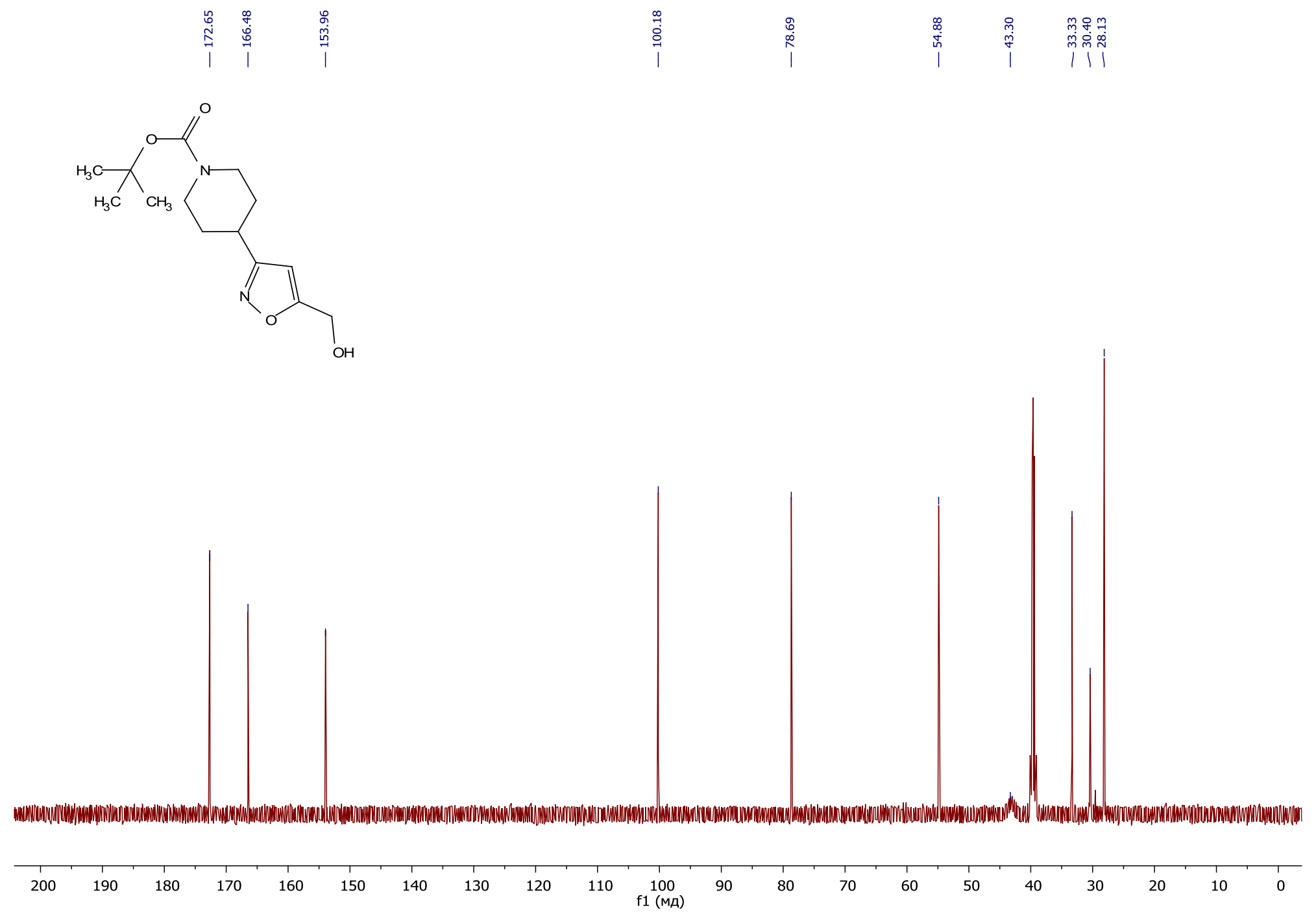

tert-Butyl 4-(5-(hydroxymethyl)isoxazol-3-yl)piperidine-1-carboxylate (16m) ${ }^{13} \mathrm{C}$ NMR 


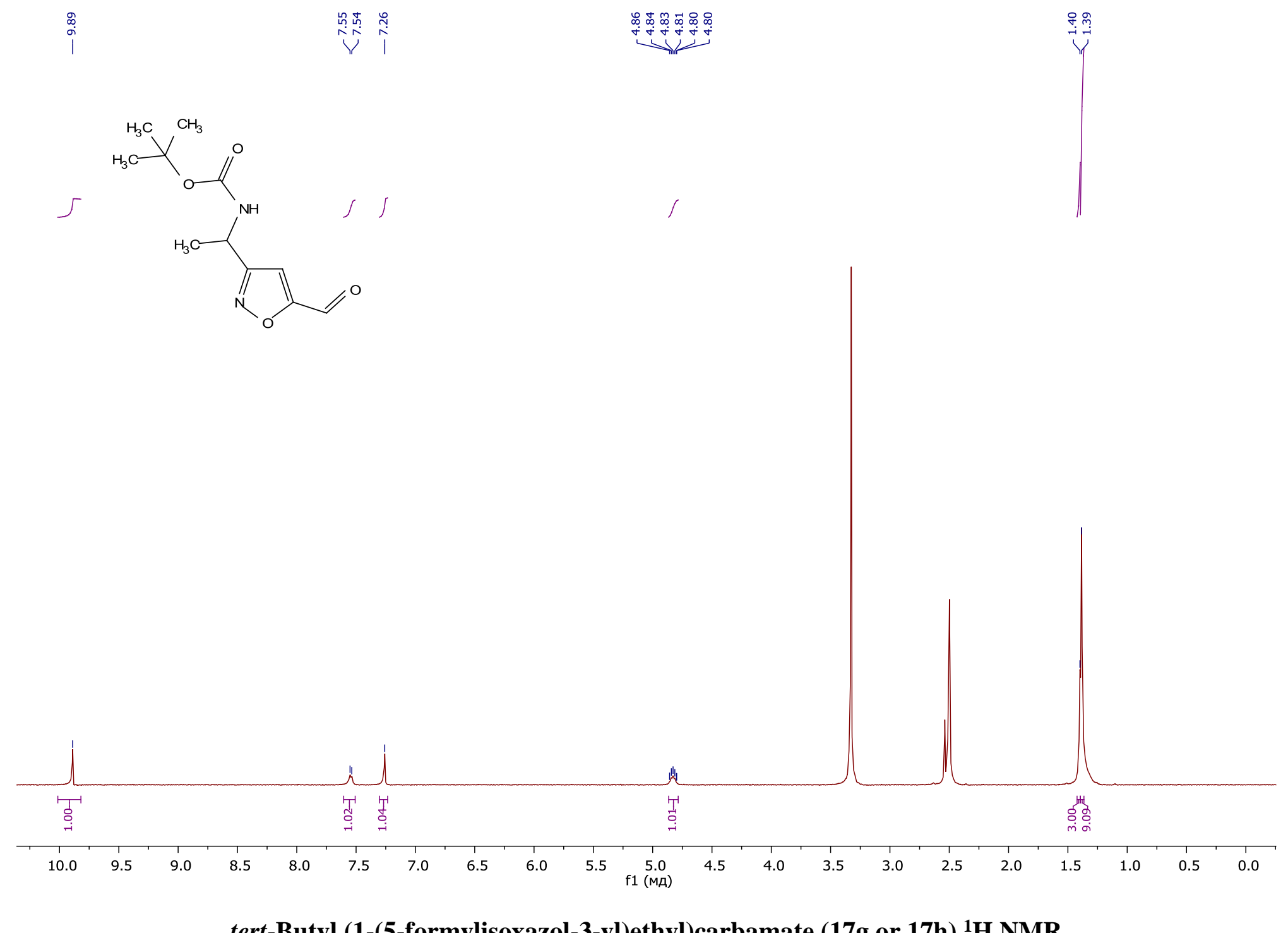

tert-Butyl (1-(5-formylisoxazol-3-yl)ethyl)carbamate (17g or 17h) ${ }^{1} \mathrm{H}$ NMR 


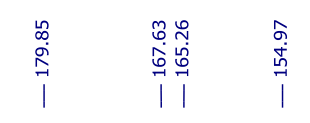

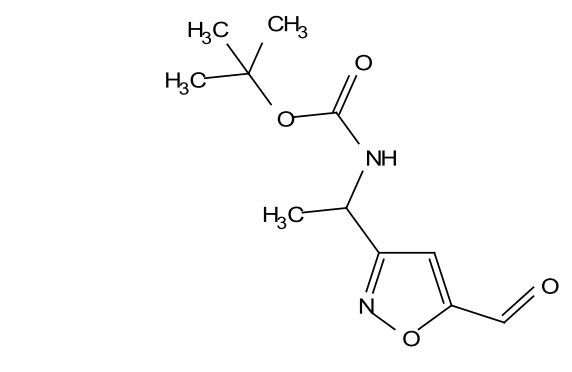

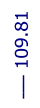

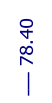

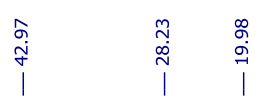

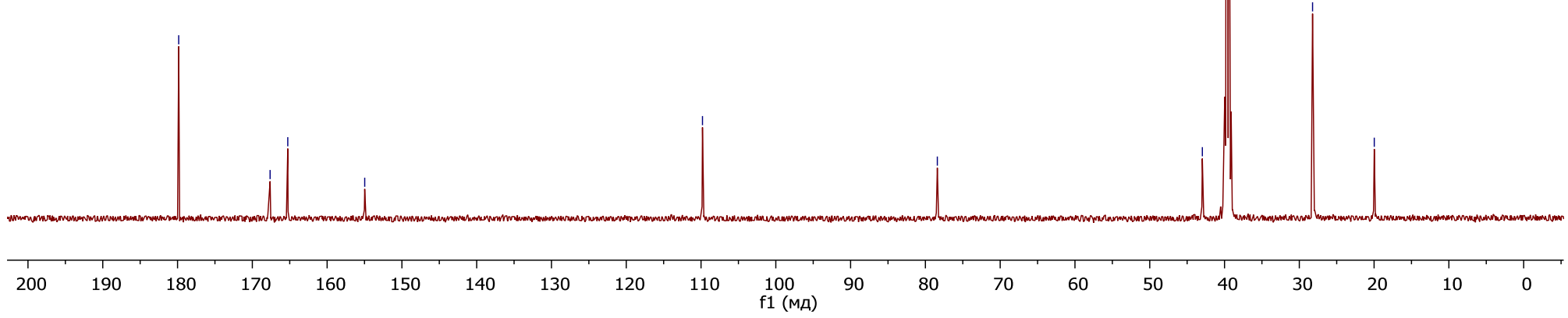

tert-Butyl (1-(5-formylisoxazol-3-yl)ethyl)carbamate $(17 \mathrm{~g}$ or $17 \mathrm{~h}){ }^{13} \mathrm{C}$ NMR

S95 


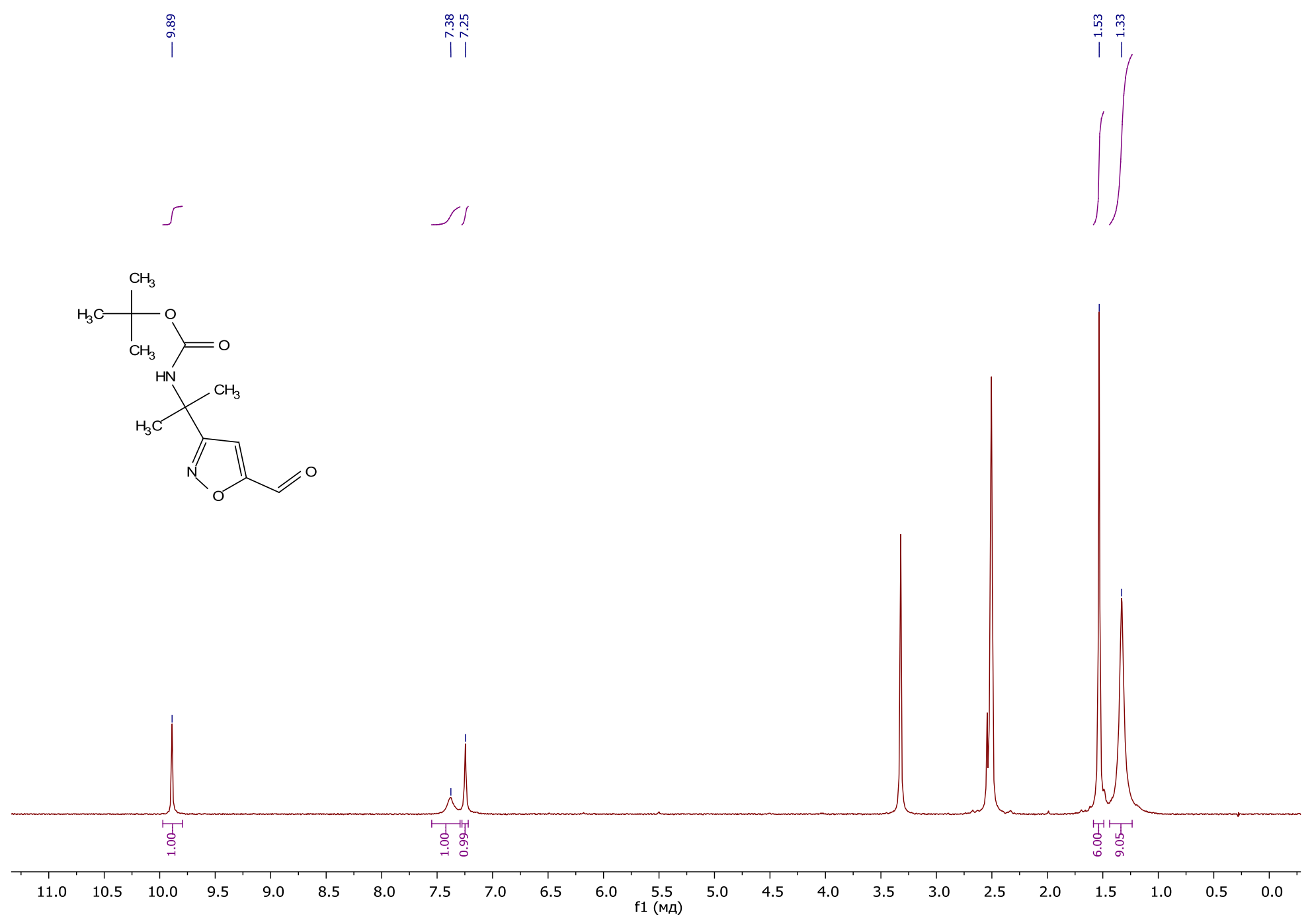

tert-Butyl (2-(5-formylisoxazol-3-yl)propan-2-yl)carbamate (17i) ${ }^{1} \mathrm{H}$ NMR 


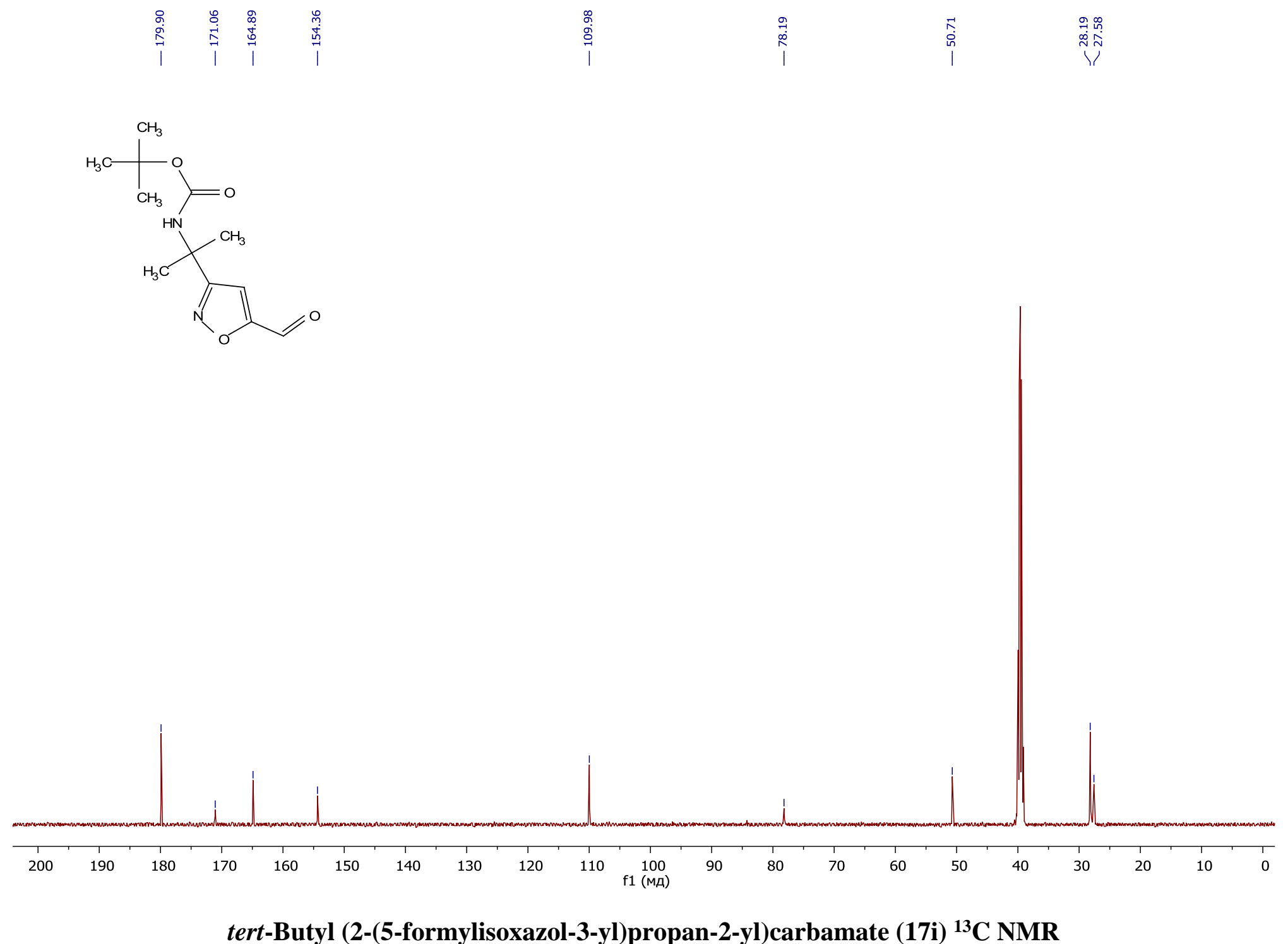




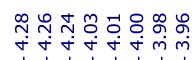

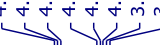
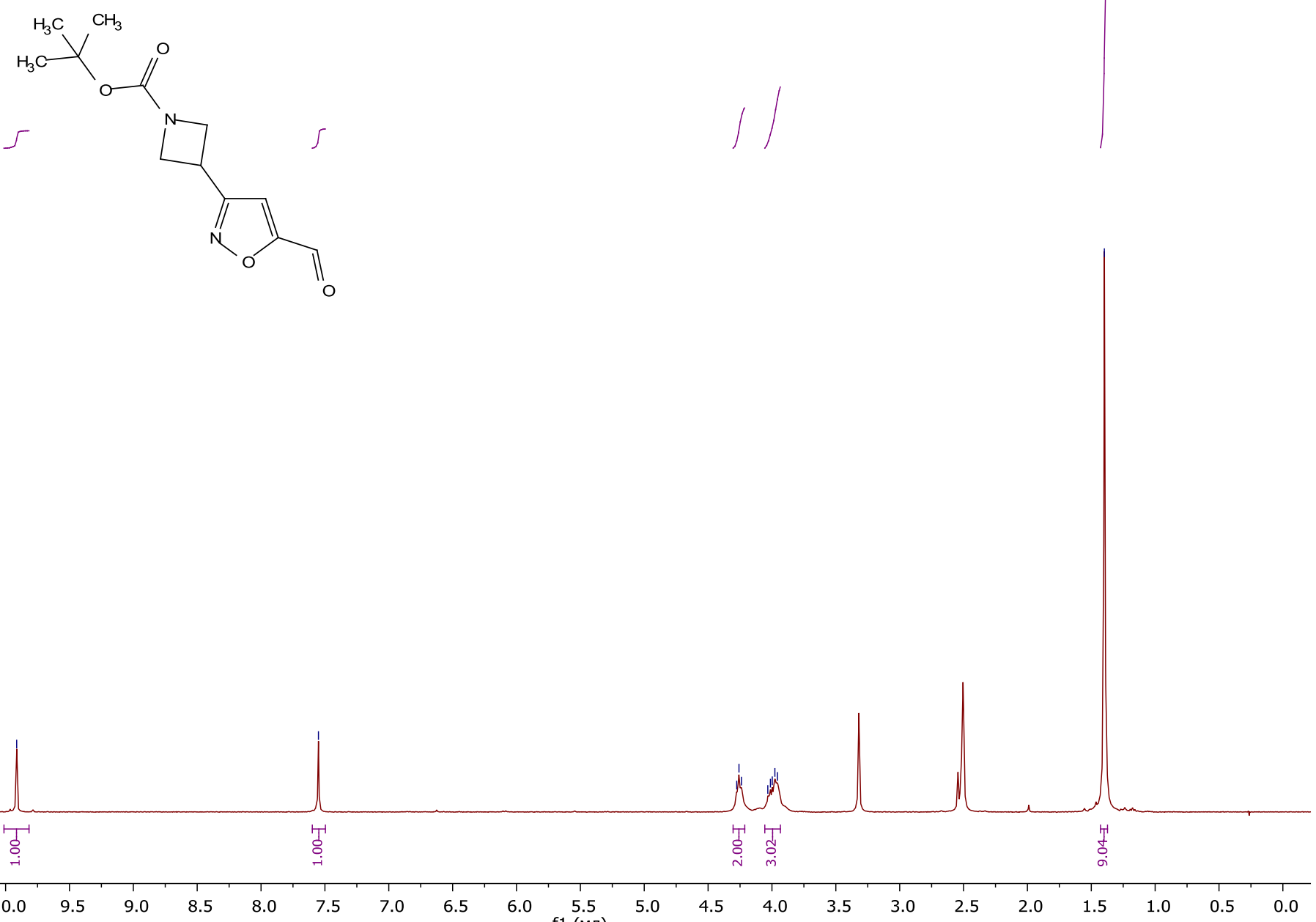

tert-Butyl 3-(5-formylisoxazol-3-yl)azetidine-1-carboxylate (17j) ${ }^{1} \mathrm{H}$ NMR 
品
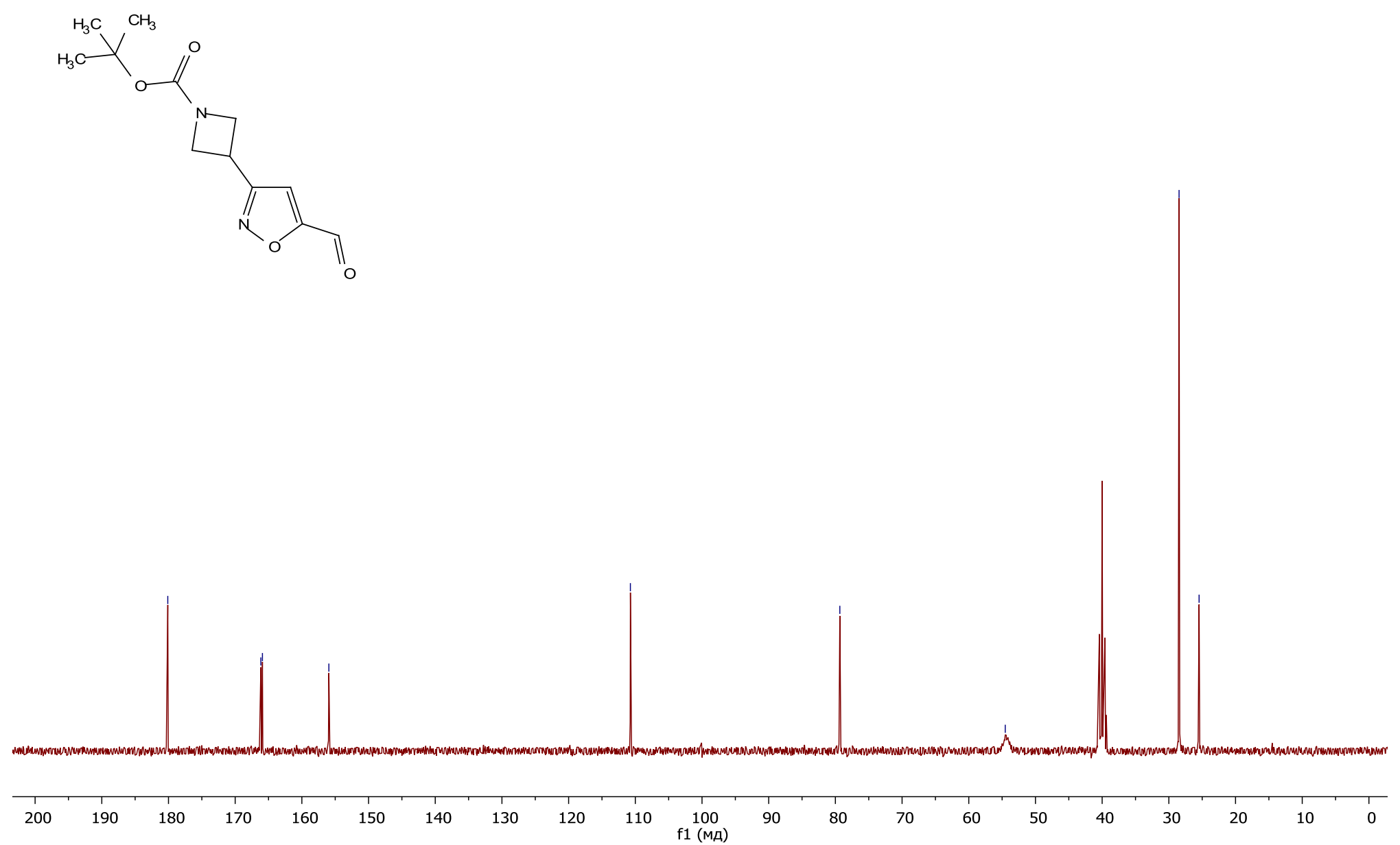

tert-Butyl 3-(5-formylisoxazol-3-yl)azetidine-1-carboxylate (17j) ${ }^{13} \mathrm{C}$ NMR 

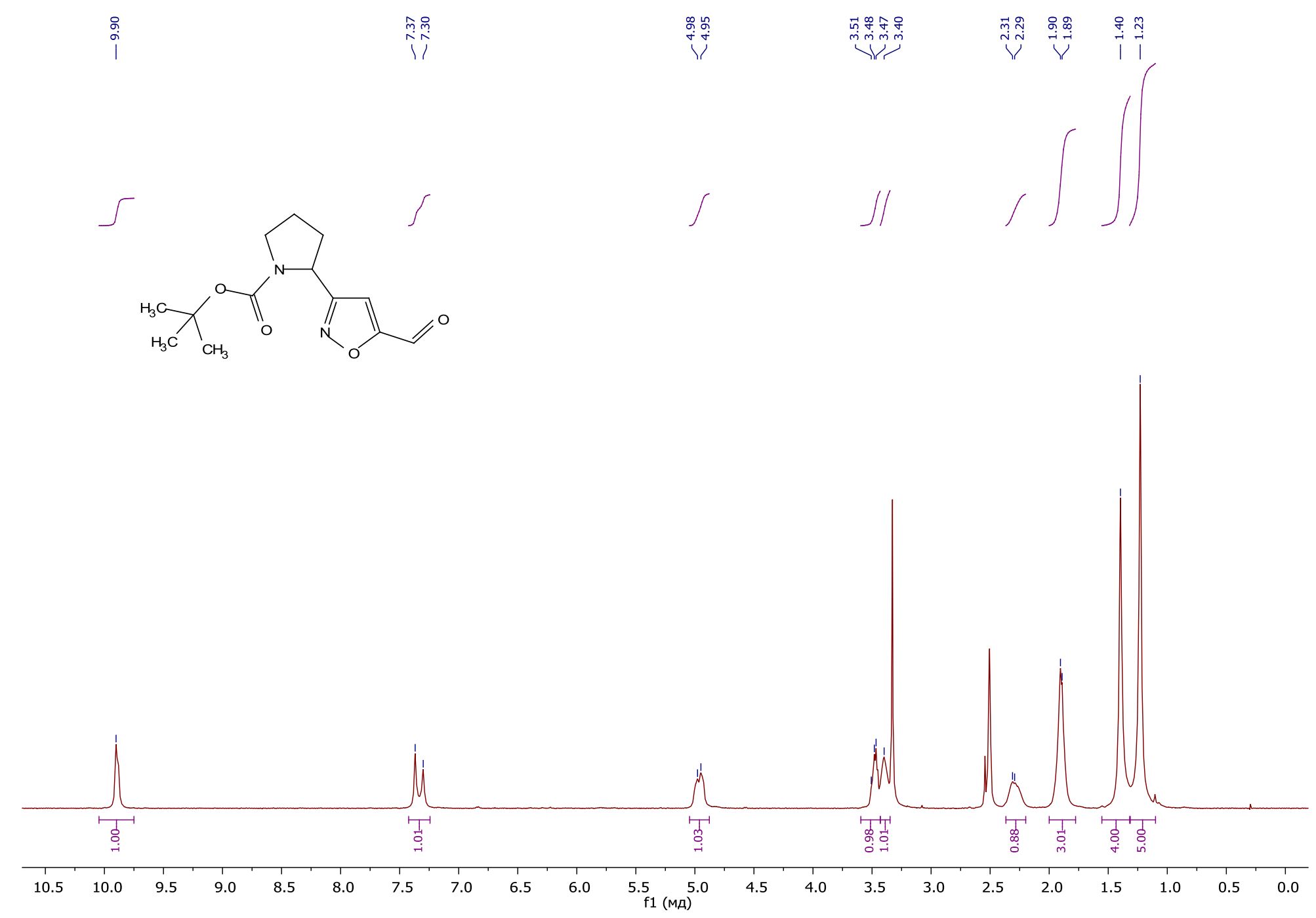

tert-Butyl 2-(5-formylisoxazol-3-yl)pyrrolidine-1-carboxylate (17k or 17l) ${ }^{1} \mathrm{H}$ NMR 

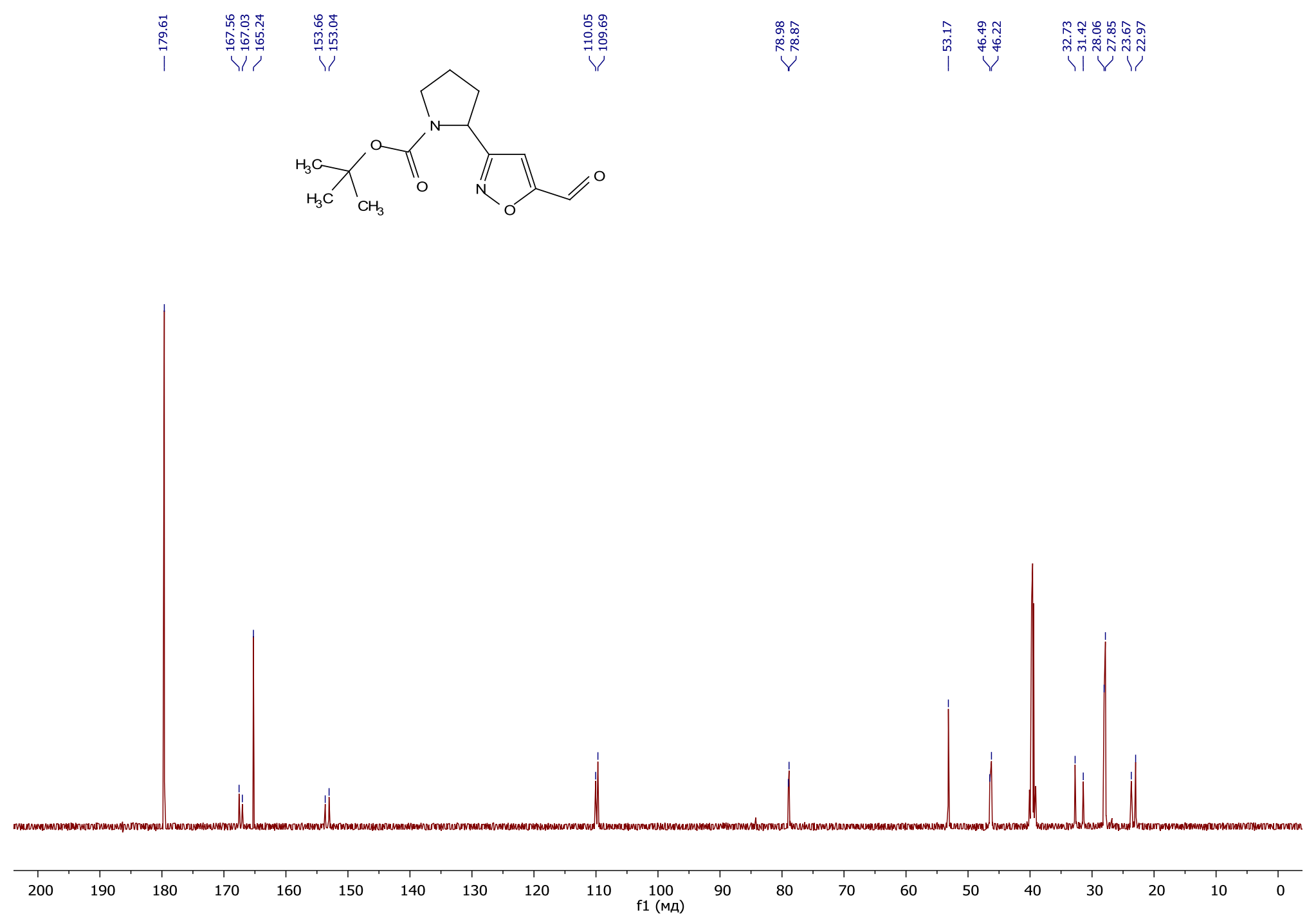

tert-Butyl 2-(5-formylisoxazol-3-yl)pyrrolidine-1-carboxylate (17k or 17l) ${ }^{13} \mathrm{C}$ NMR 


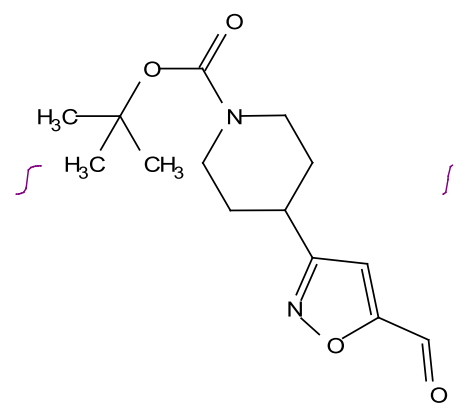

ب
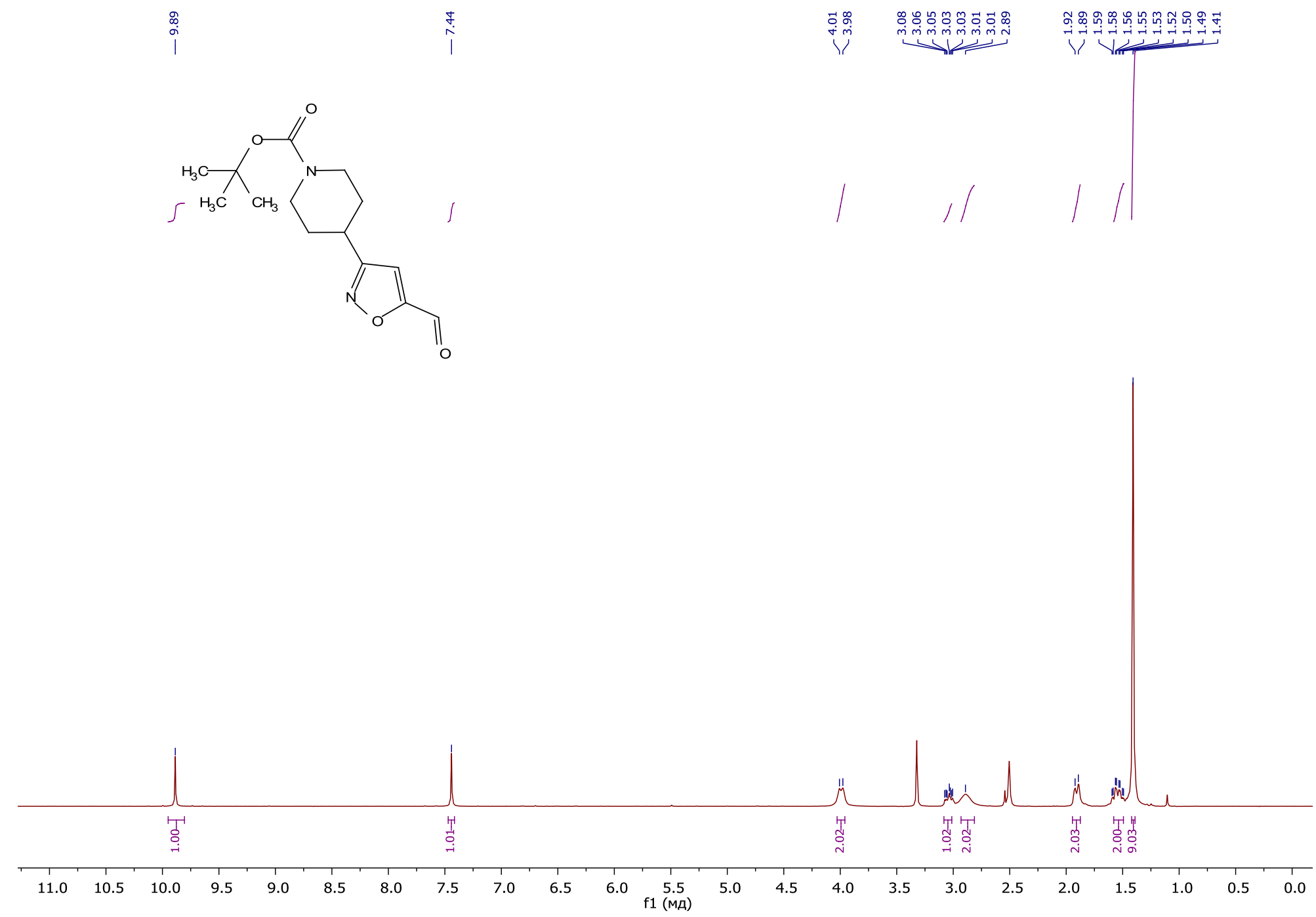

tert-Butyl 4-(5-formylisoxazol-3-yl)piperidine-1-carboxylate (17m) ${ }^{1} \mathrm{H}$ NMR 


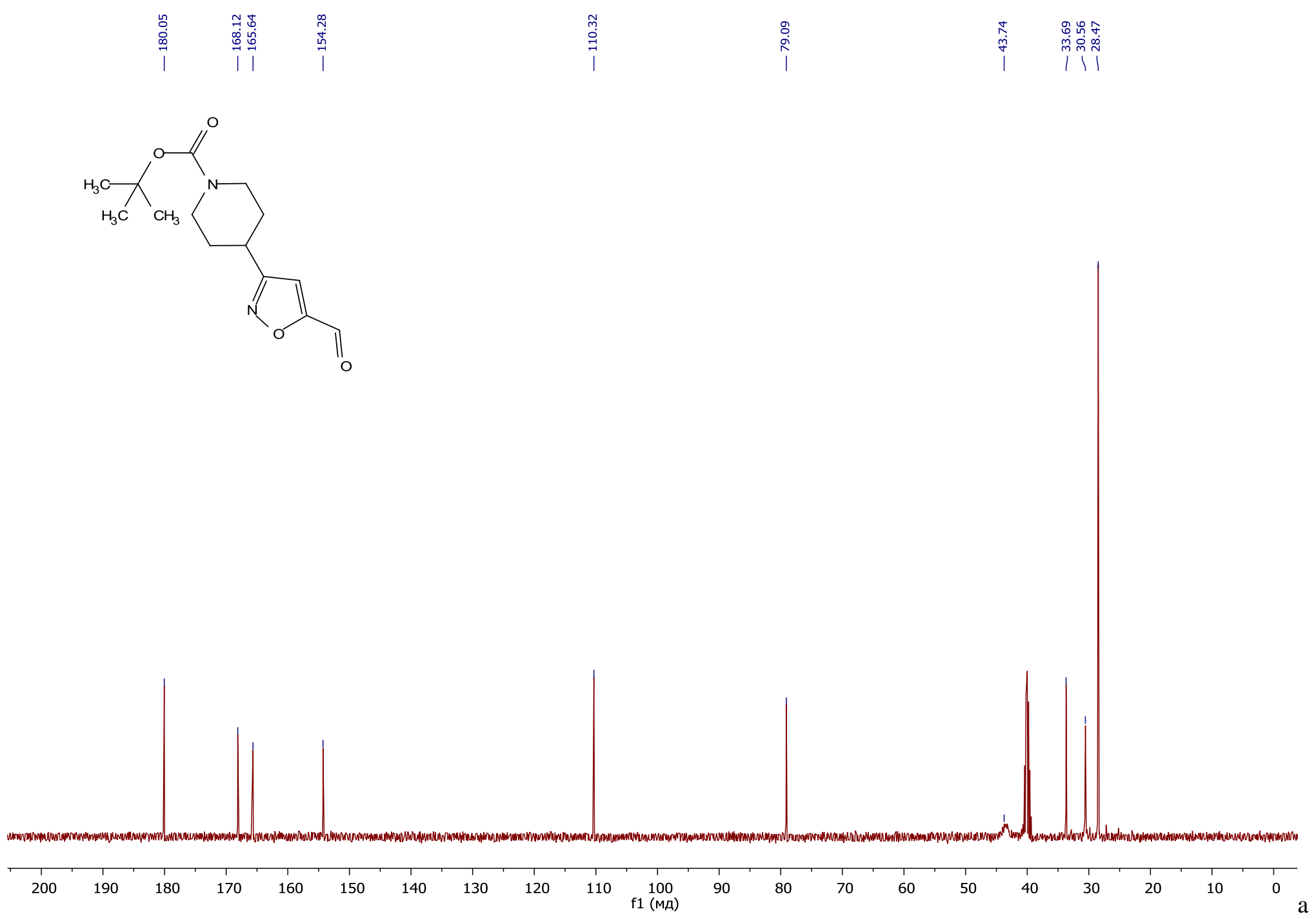

tert-Butyl 4-(5-formylisoxazol-3-yl)piperidine-1-carboxylate (17m) ${ }^{13} \mathrm{C}$ NMR 

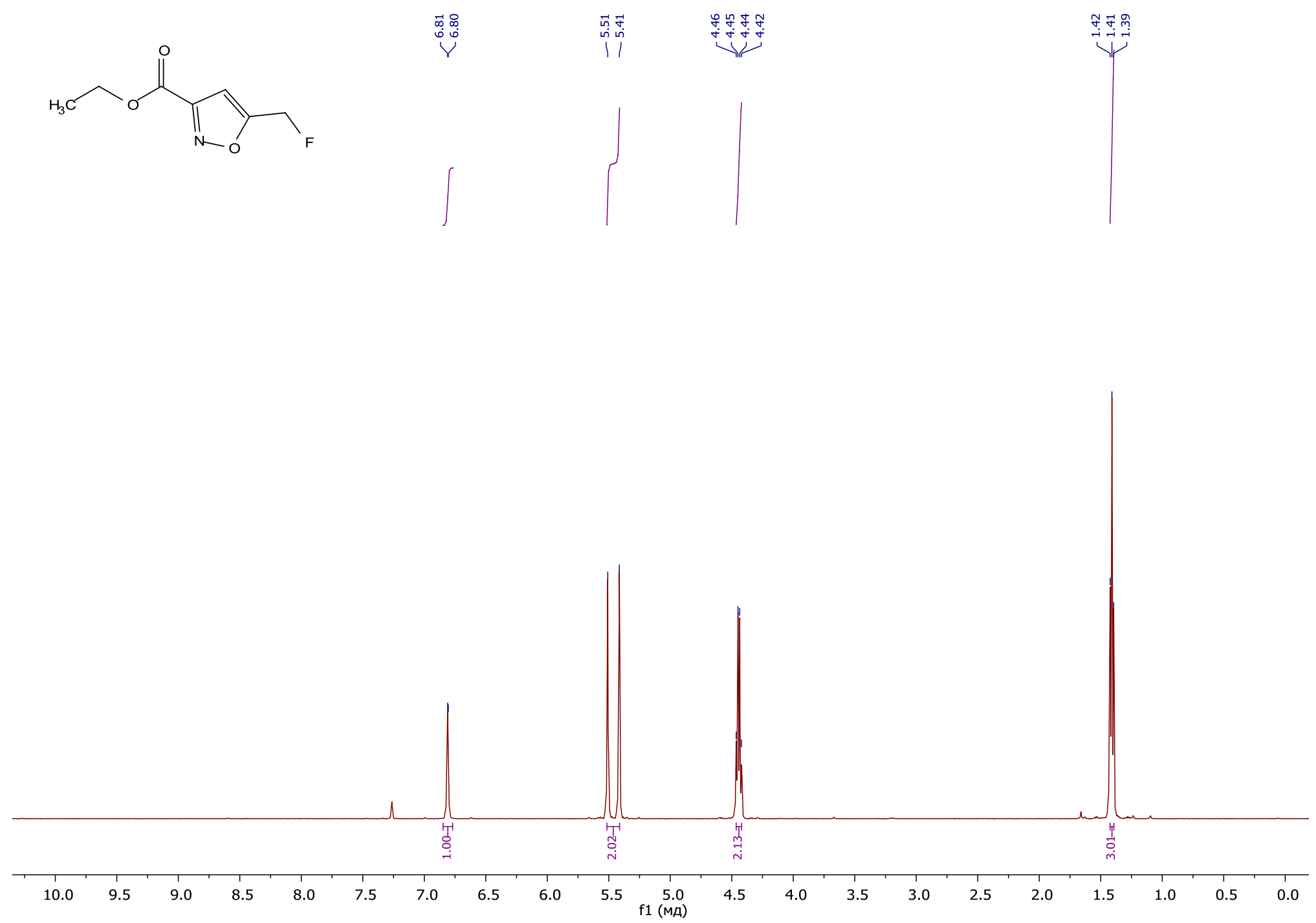

Ethyl 5-(fluoromethyl)isoxazole-3-carboxylate (18a) ${ }^{1}$ H NMR 

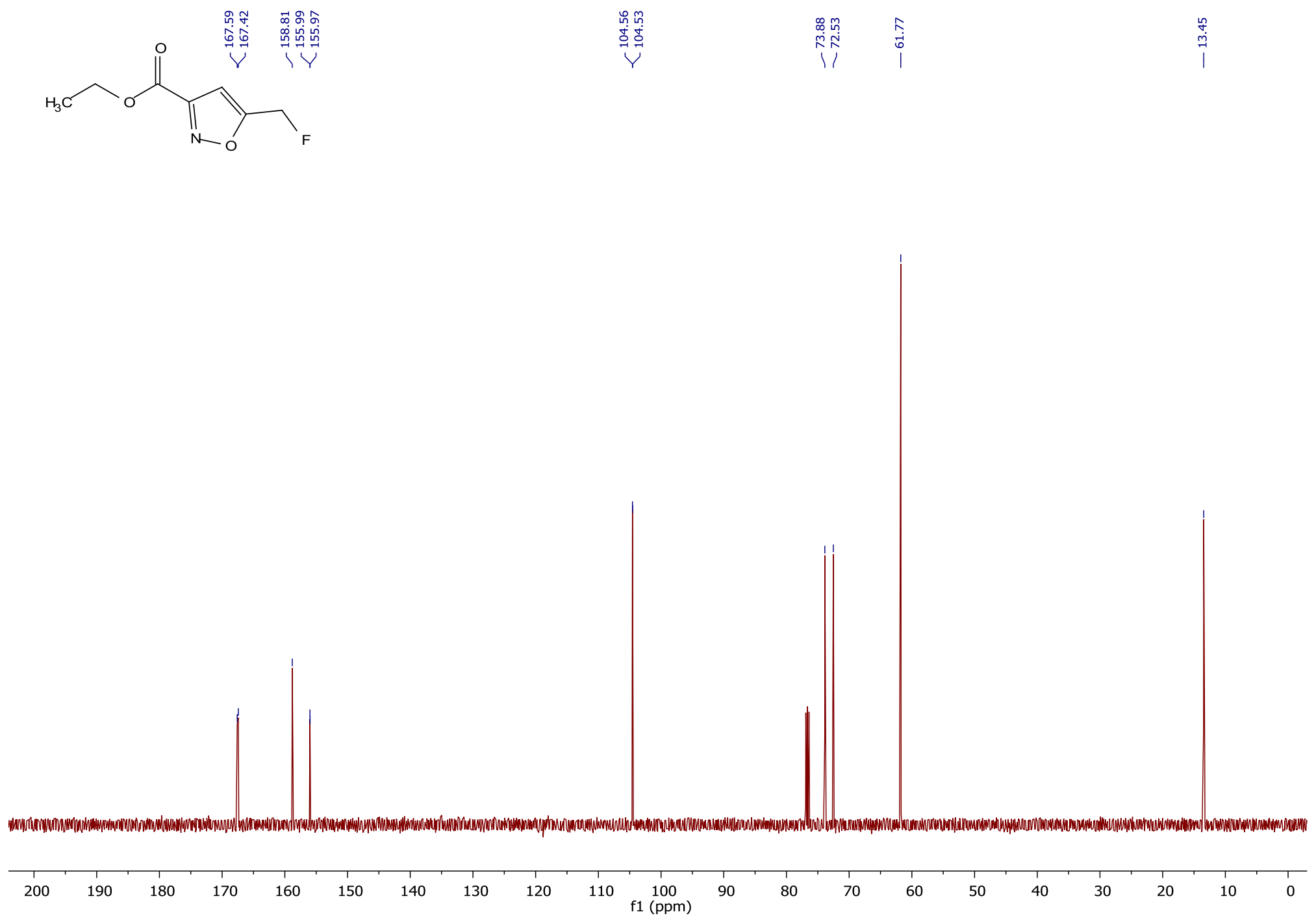

Ethyl 5-(fluoromethyl)isoxazole-3-carboxylate (18a) ${ }^{13} \mathrm{C}$ NMR 

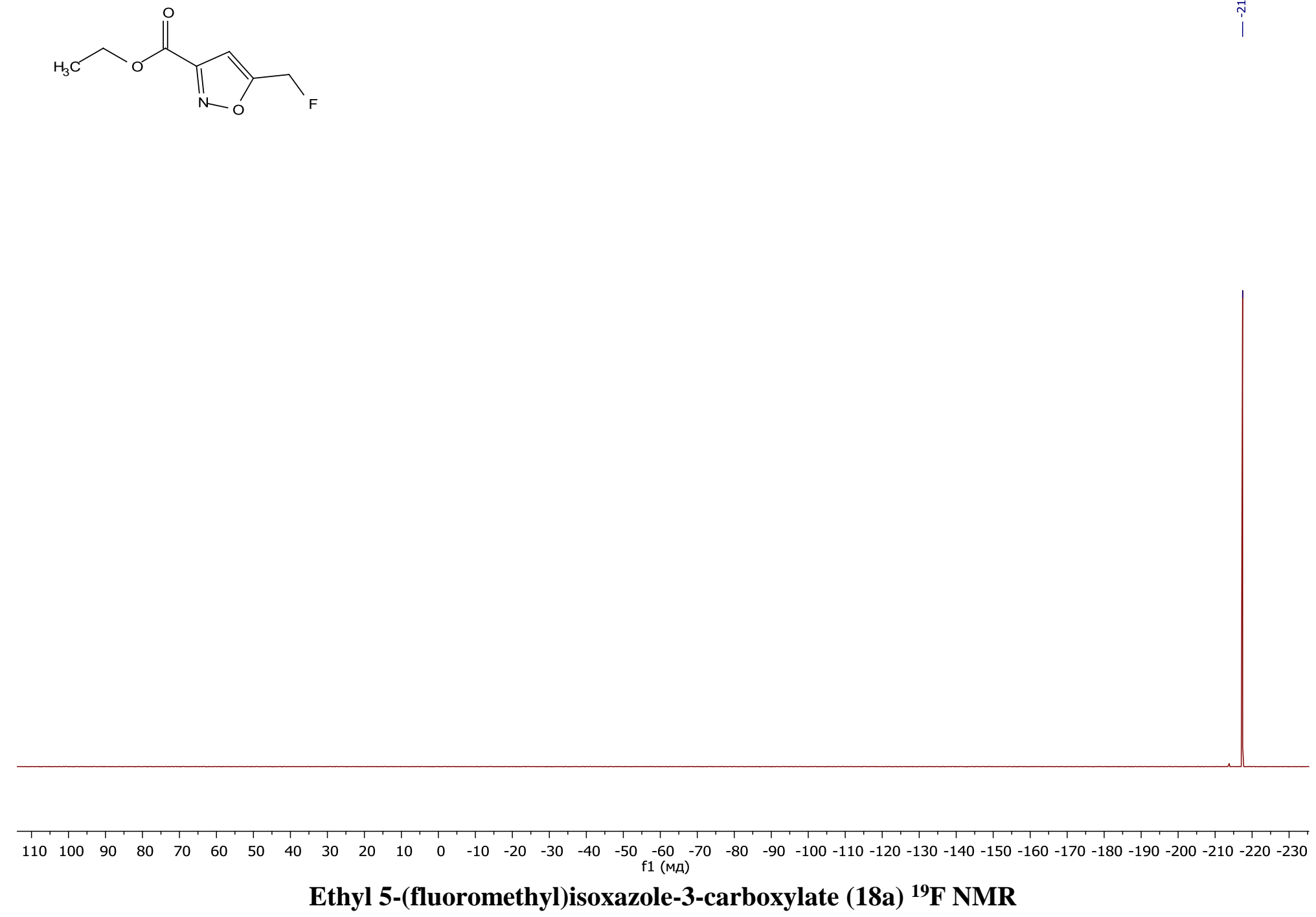


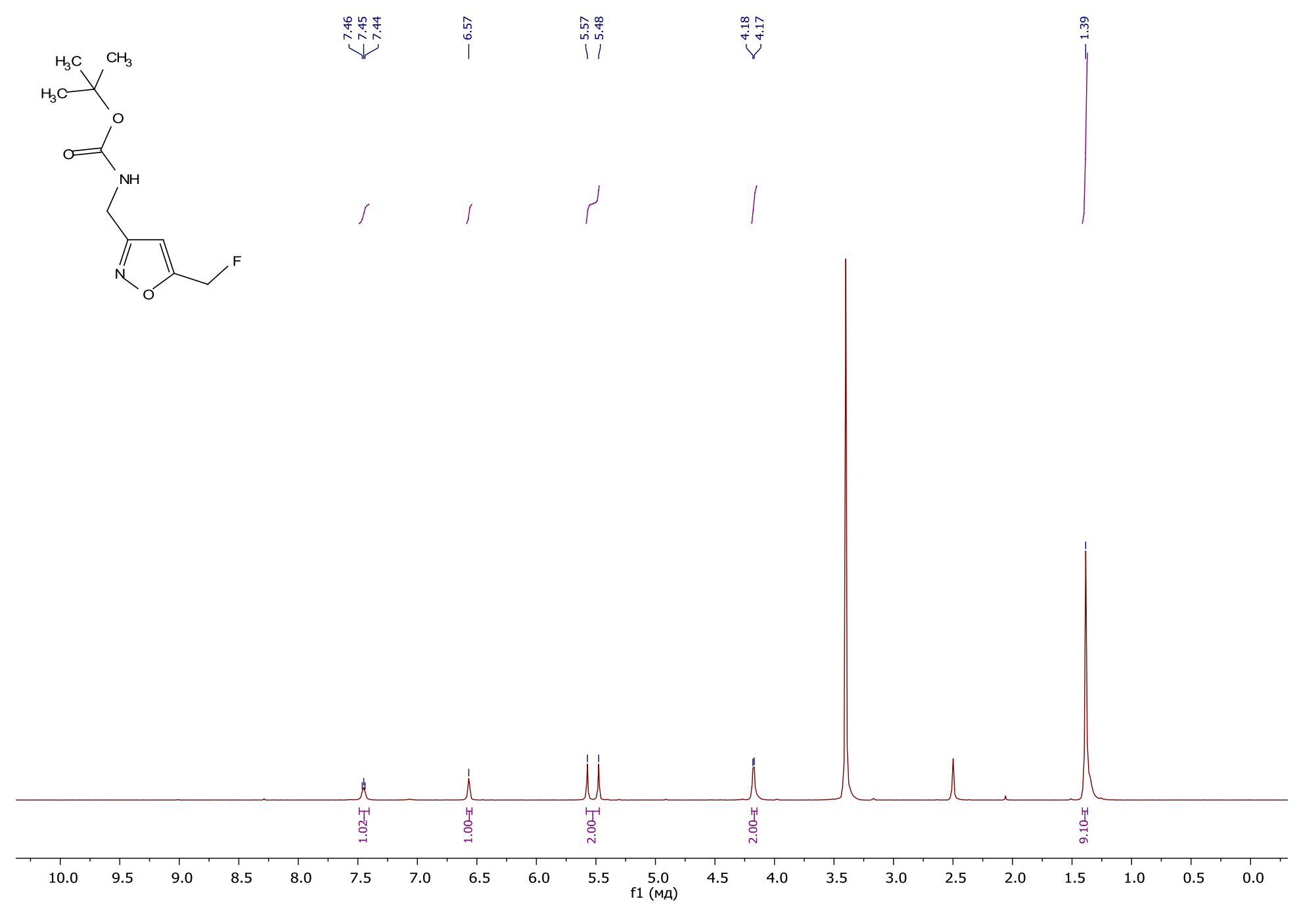

tert-Butyl ((5-(fluoromethyl)isoxazol-3-yl)methyl)carbamate (18f) ${ }^{1}$ H NMR 


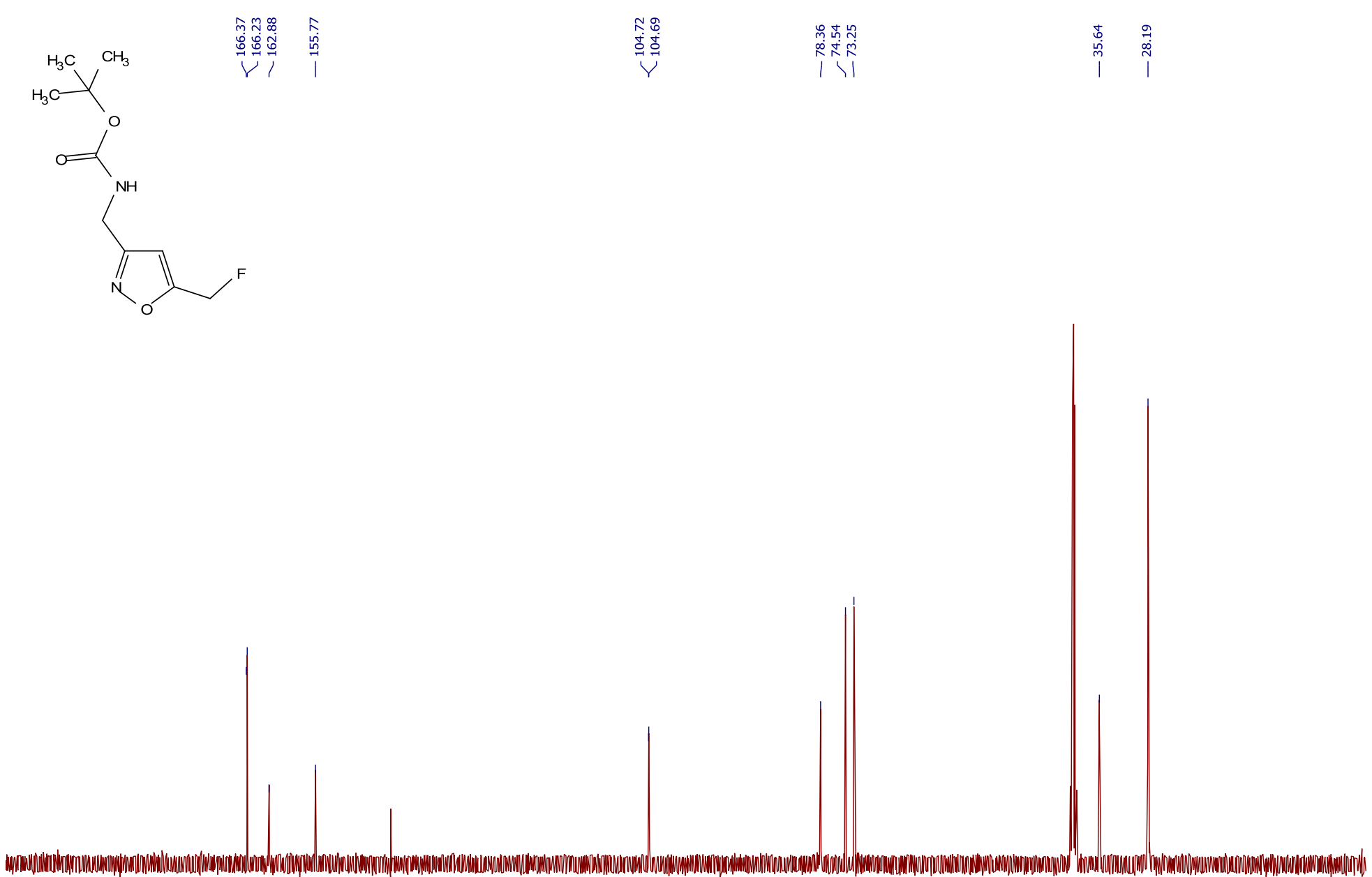
$\begin{array}{llllllll}170 & 160 & 150 & 140 & 130 & 120 & 110 & \begin{array}{l}100 \\ \mathrm{f} 1(\mathrm{MA})\end{array}\end{array}$

I
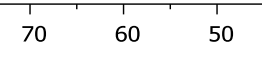

$40 \quad 30$

$20 \quad 10 \quad 0$

tert-Butyl ((5-(fluoromethyl)isoxazol-3-yl)methyl)carbamate (18f) ${ }^{13} \mathrm{C}$ NMR 

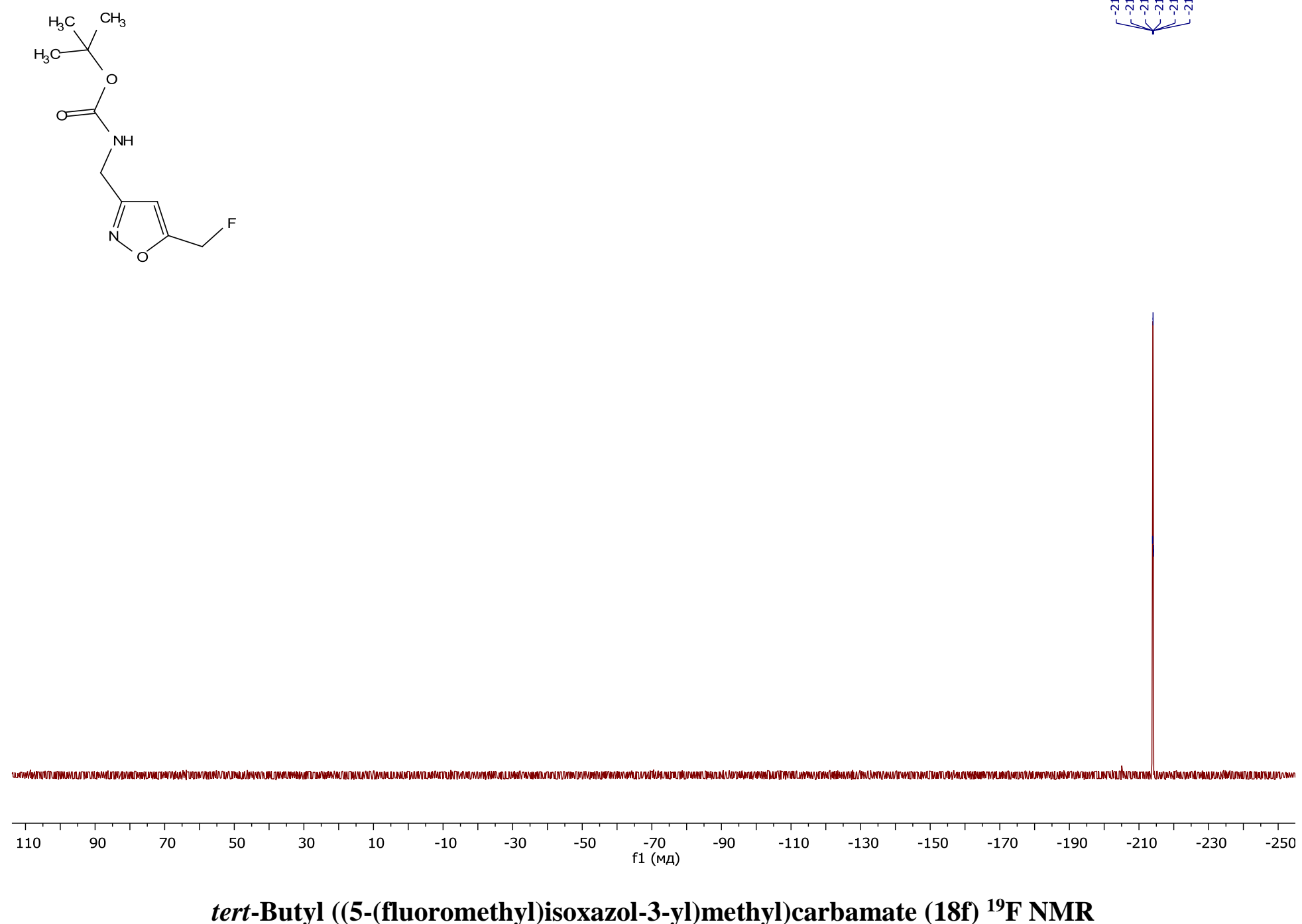


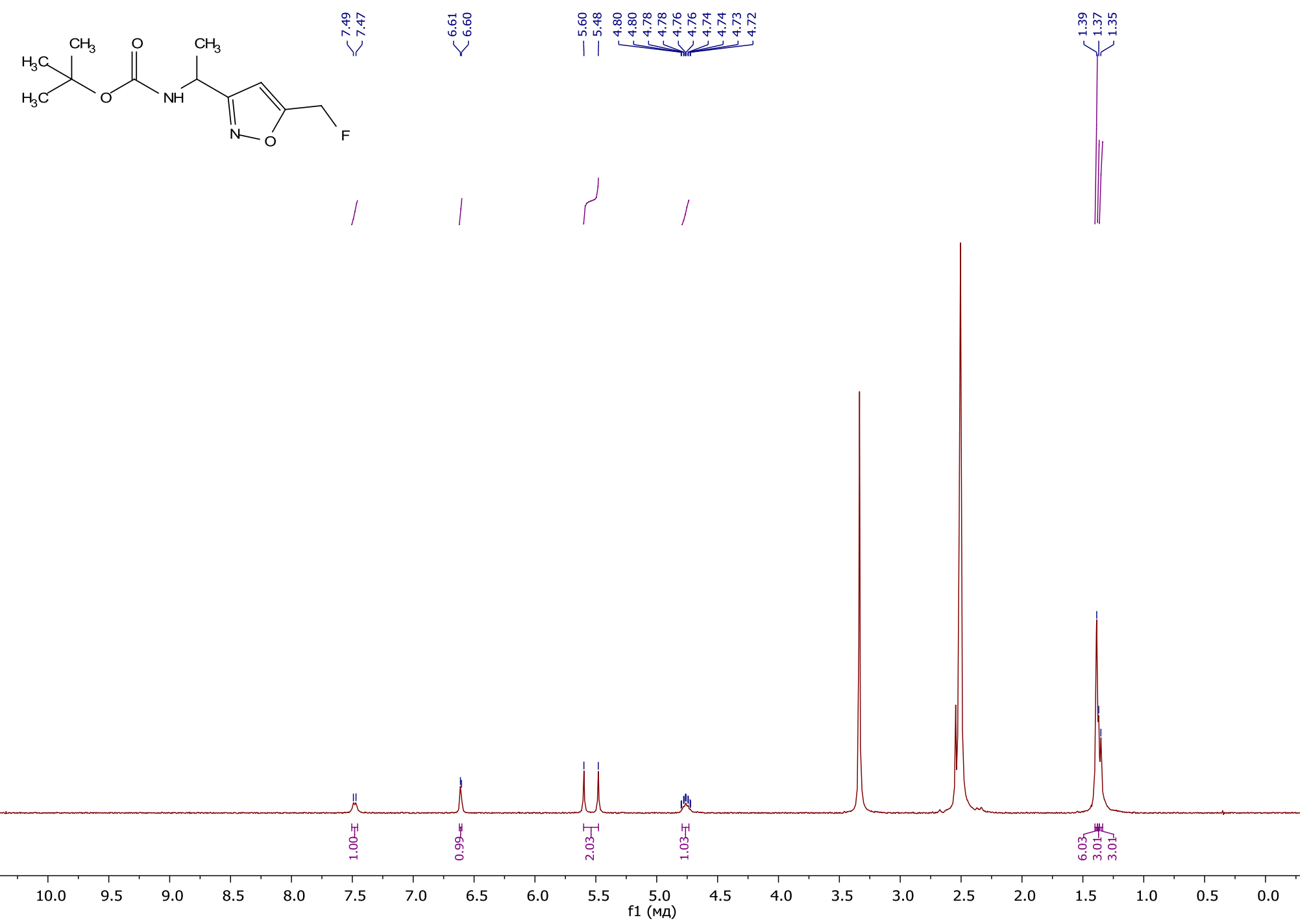

tert-Butyl (1-(5-(fluoromethyl)isoxazol-3-yl)ethyl)carbamate (18g or 18h) ${ }^{1}$ H NMR 

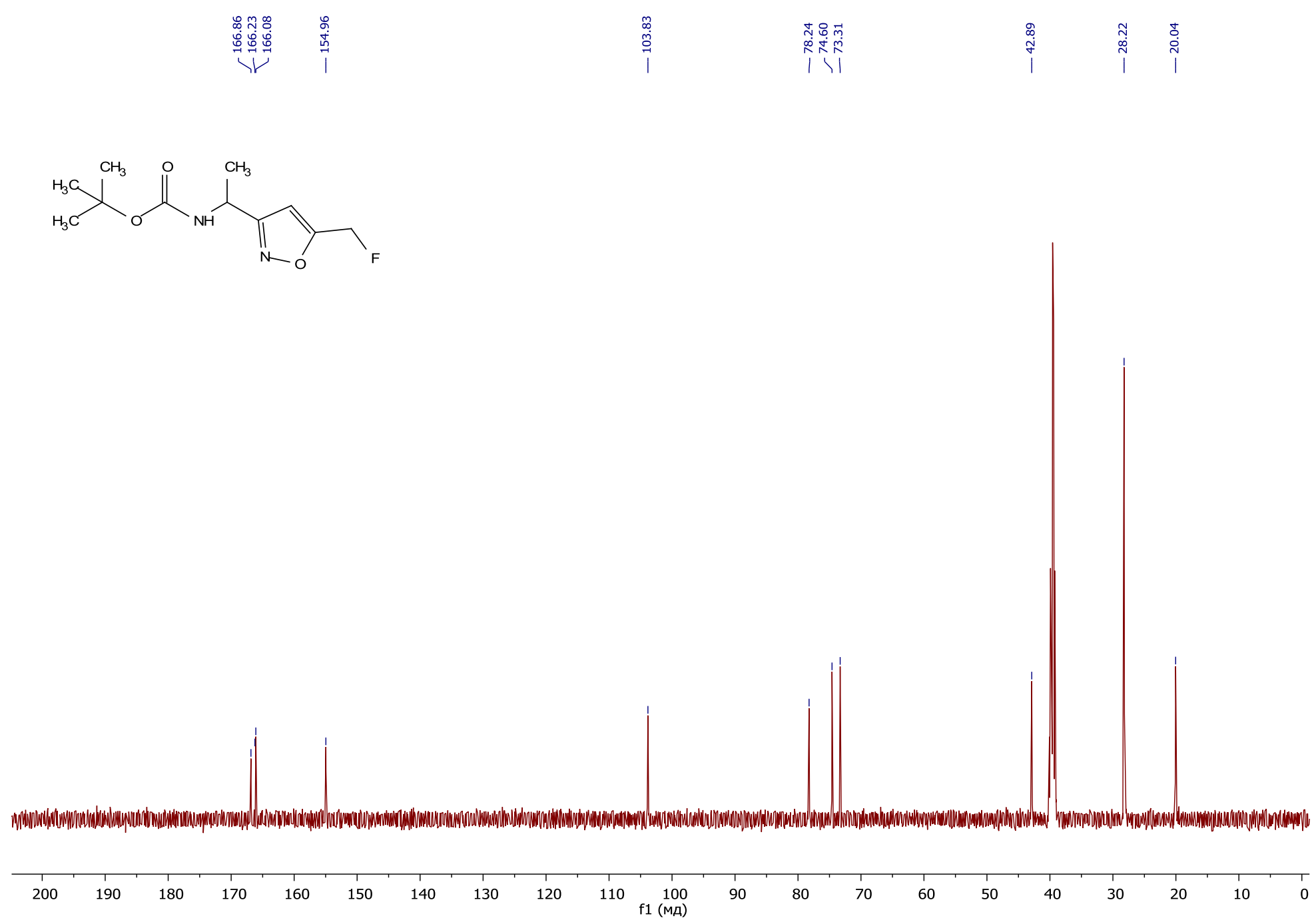
tert-Butyl (1-(5-(fluoromethyl)isoxazol-3-yl)ethyl)carbamate (18g or 18h) ${ }^{13} \mathrm{C}$ NMR 

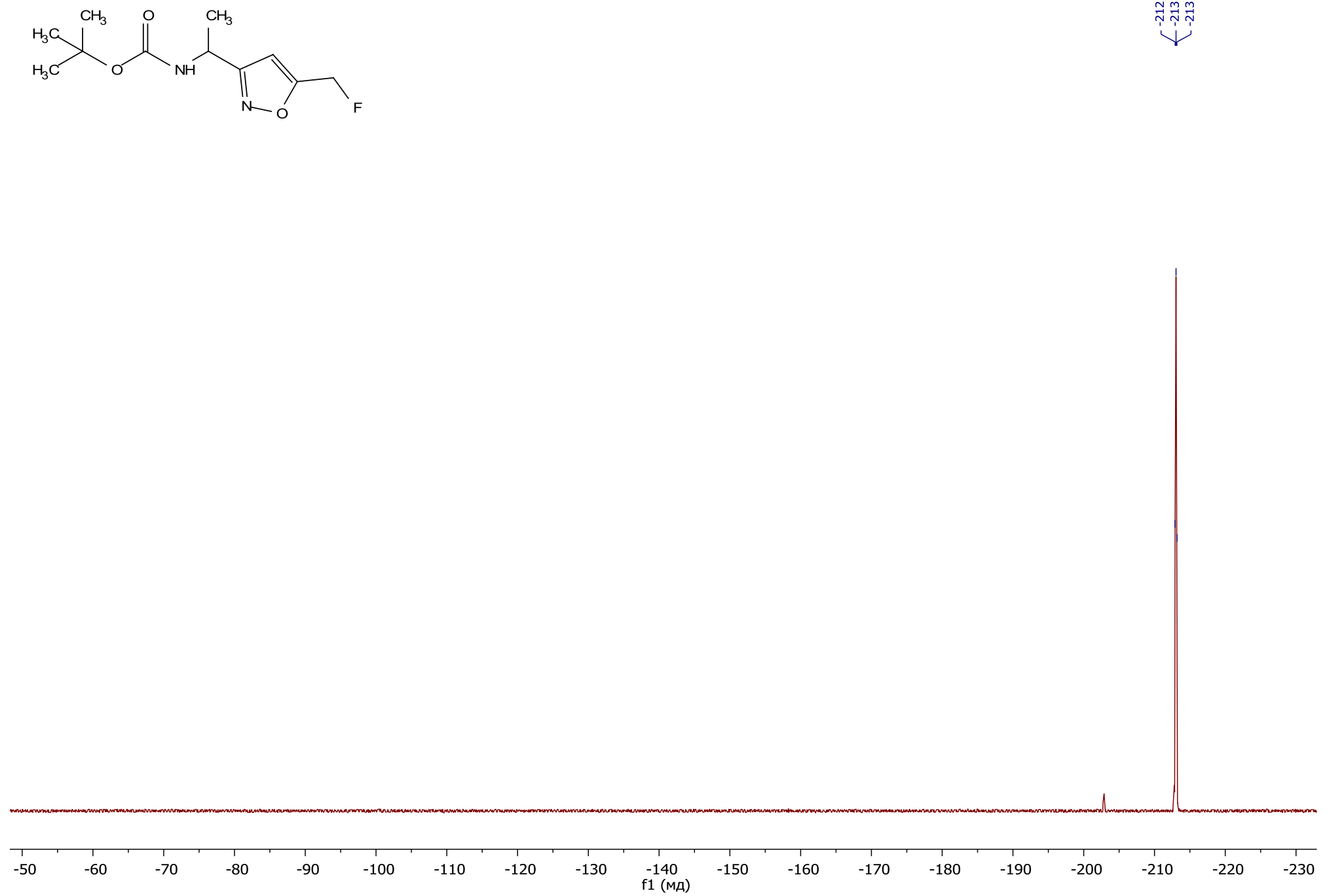

tert-Butyl (1-(5-(fluoromethyl)isoxazol-3-yl)ethyl)carbamate (18g or $18 \mathrm{~h}){ }^{19} \mathrm{~F}$ NMR 

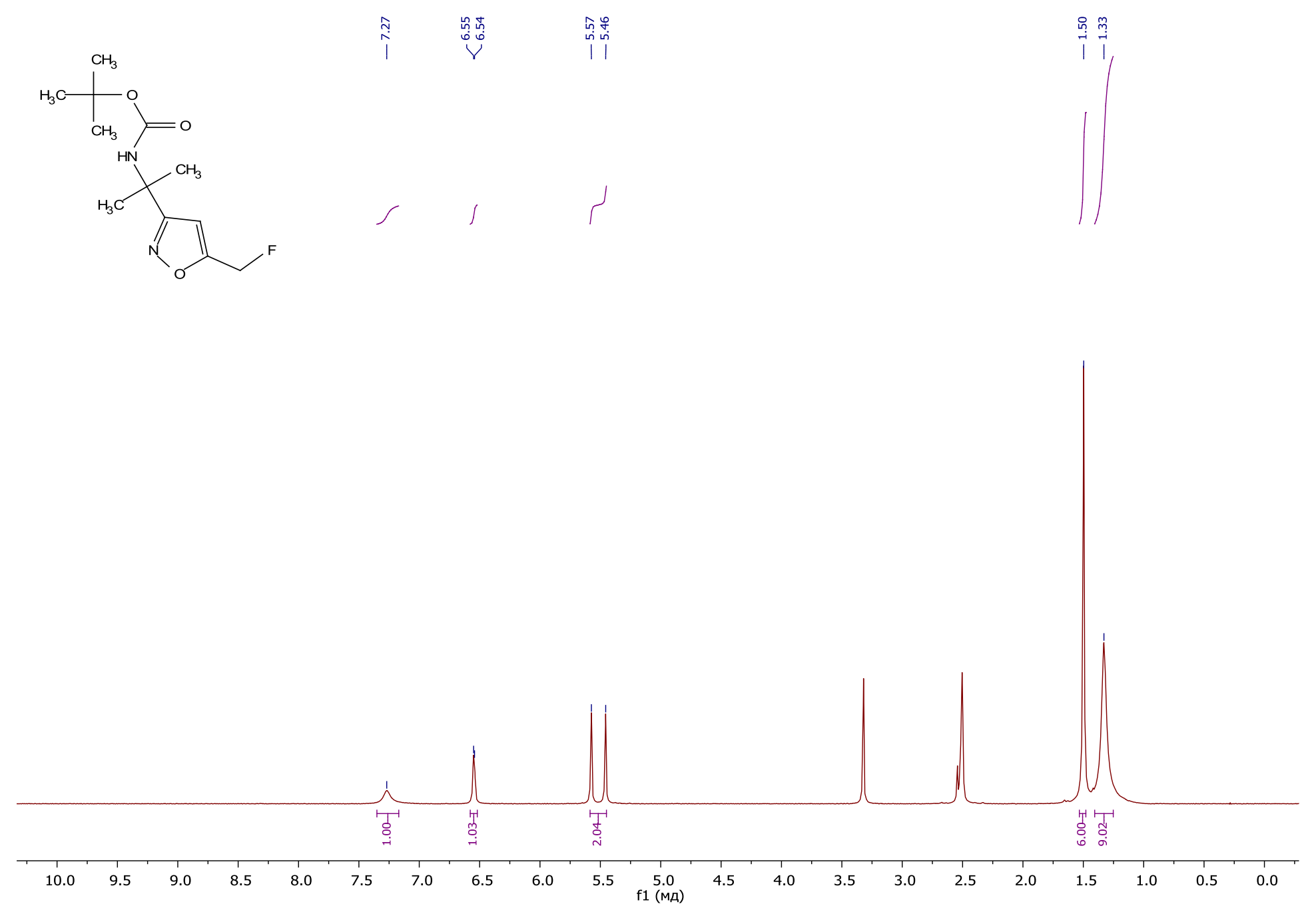

tert-Butyl (2-(5-(fluoromethyl)isoxazol-3-yl)propan-2-yl)carbamate (18i) ${ }^{1} \mathrm{H}$ NMR 

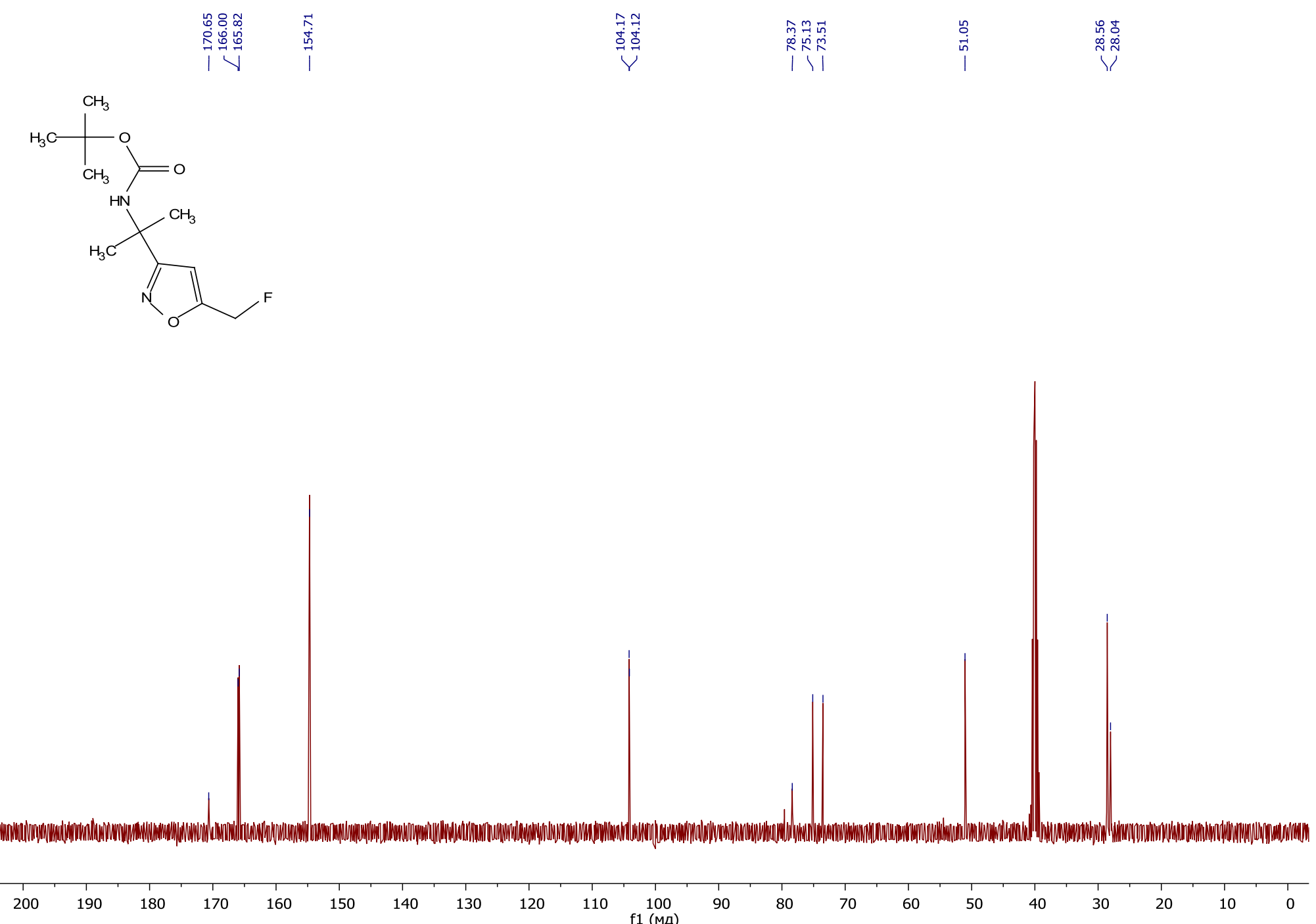

tert-Butyl (2-(5-(fluoromethyl)isoxazol-3-yl)propan-2-yl)carbamate (18i) ${ }^{13} \mathrm{C}$ NMR 


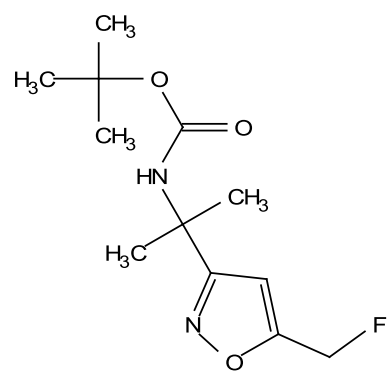

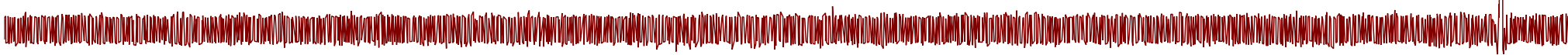

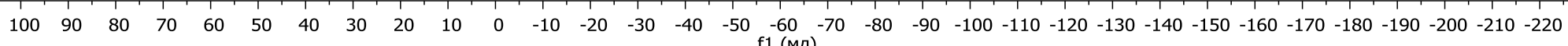
tert-Butyl (2-(5-(fluoromethyl)isoxazol-3-yl)propan-2-yl)carbamate (18i) ${ }^{19}$ F NMR 

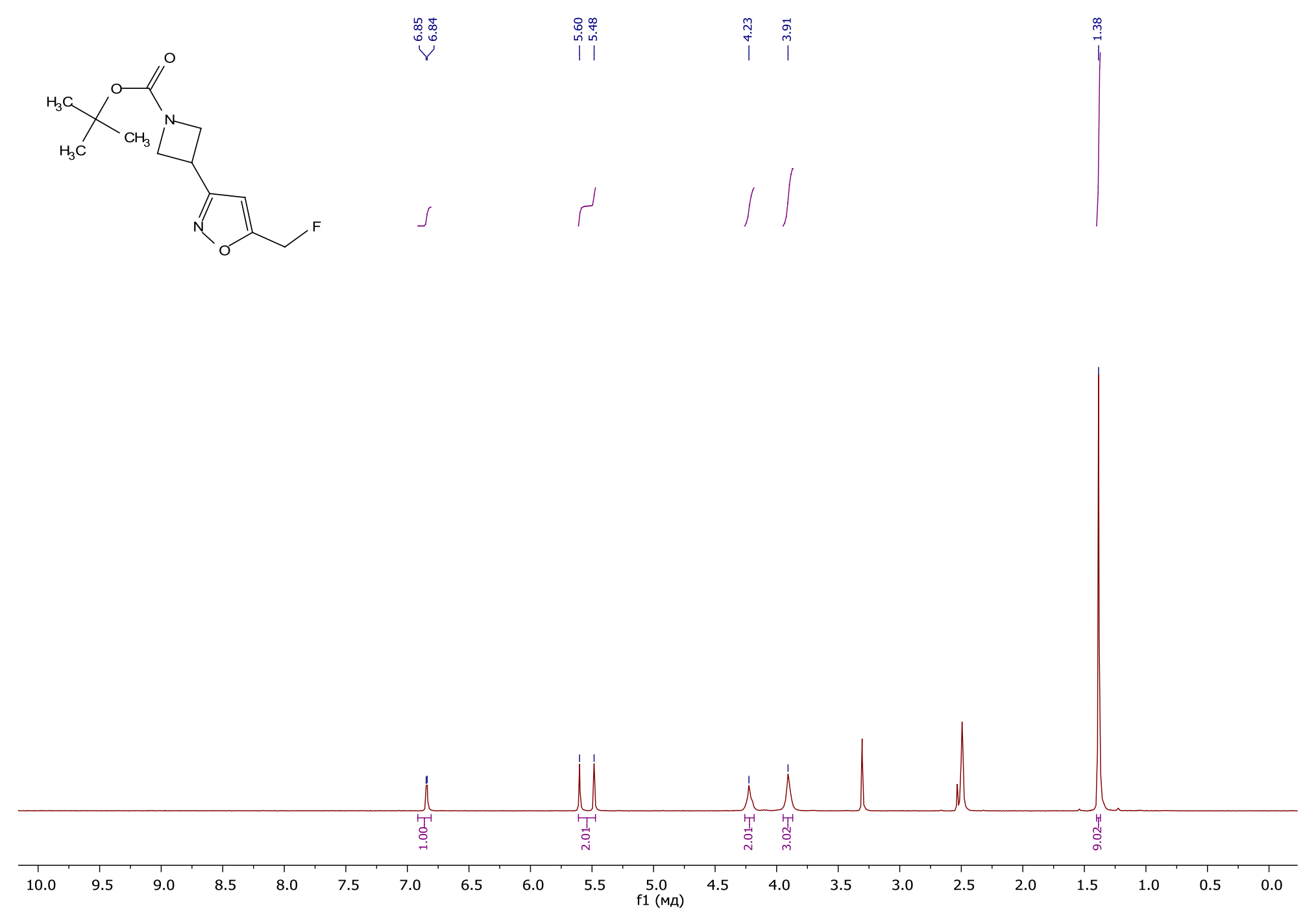

tert-Butyl 3-(5-(fluoromethyl)isoxazol-3-yl)azetidine-1-carboxylate (18j) ${ }^{1}$ H NMR 


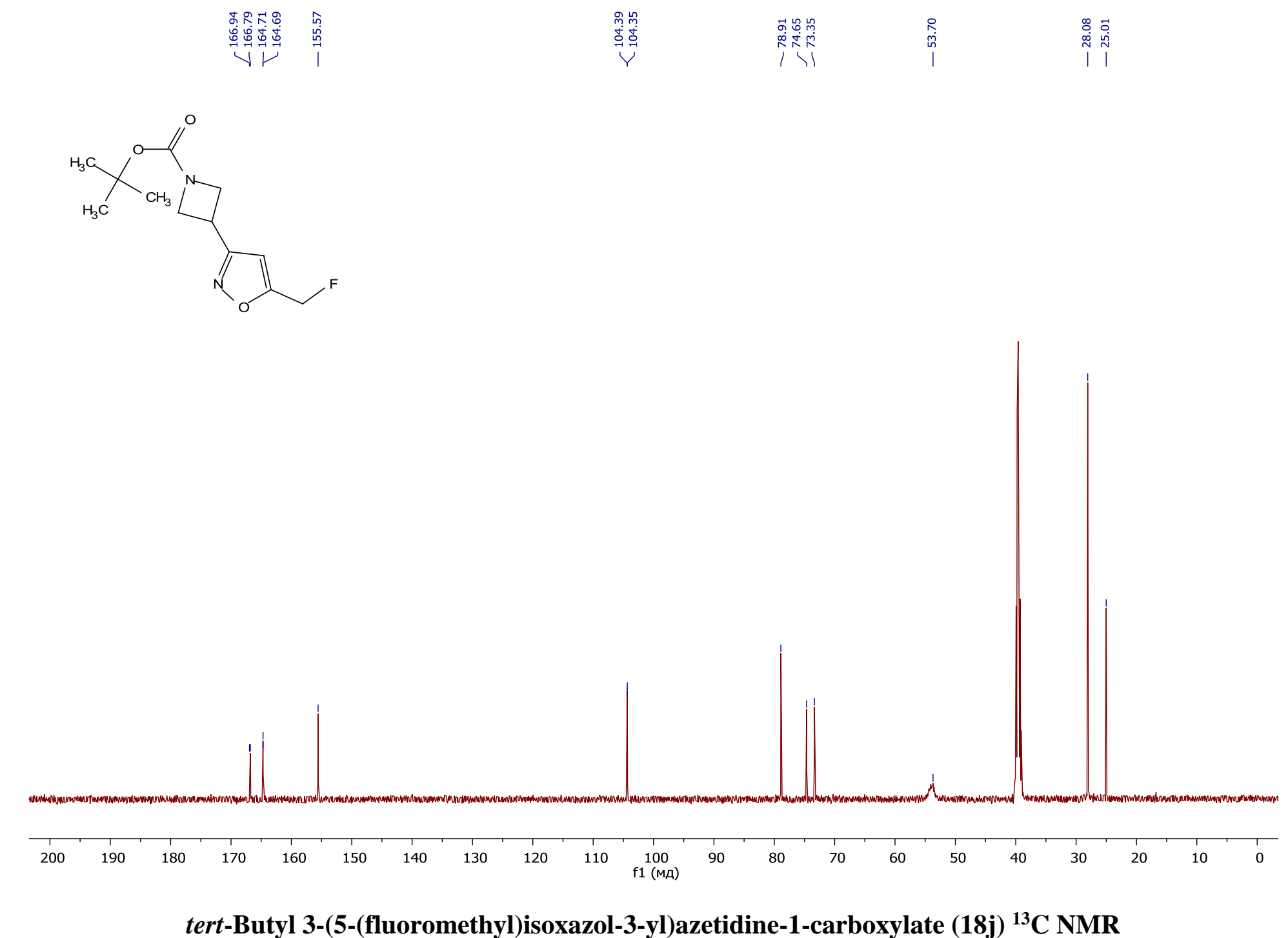




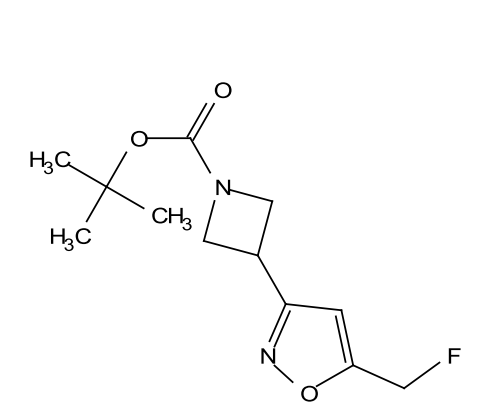

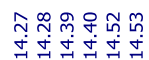
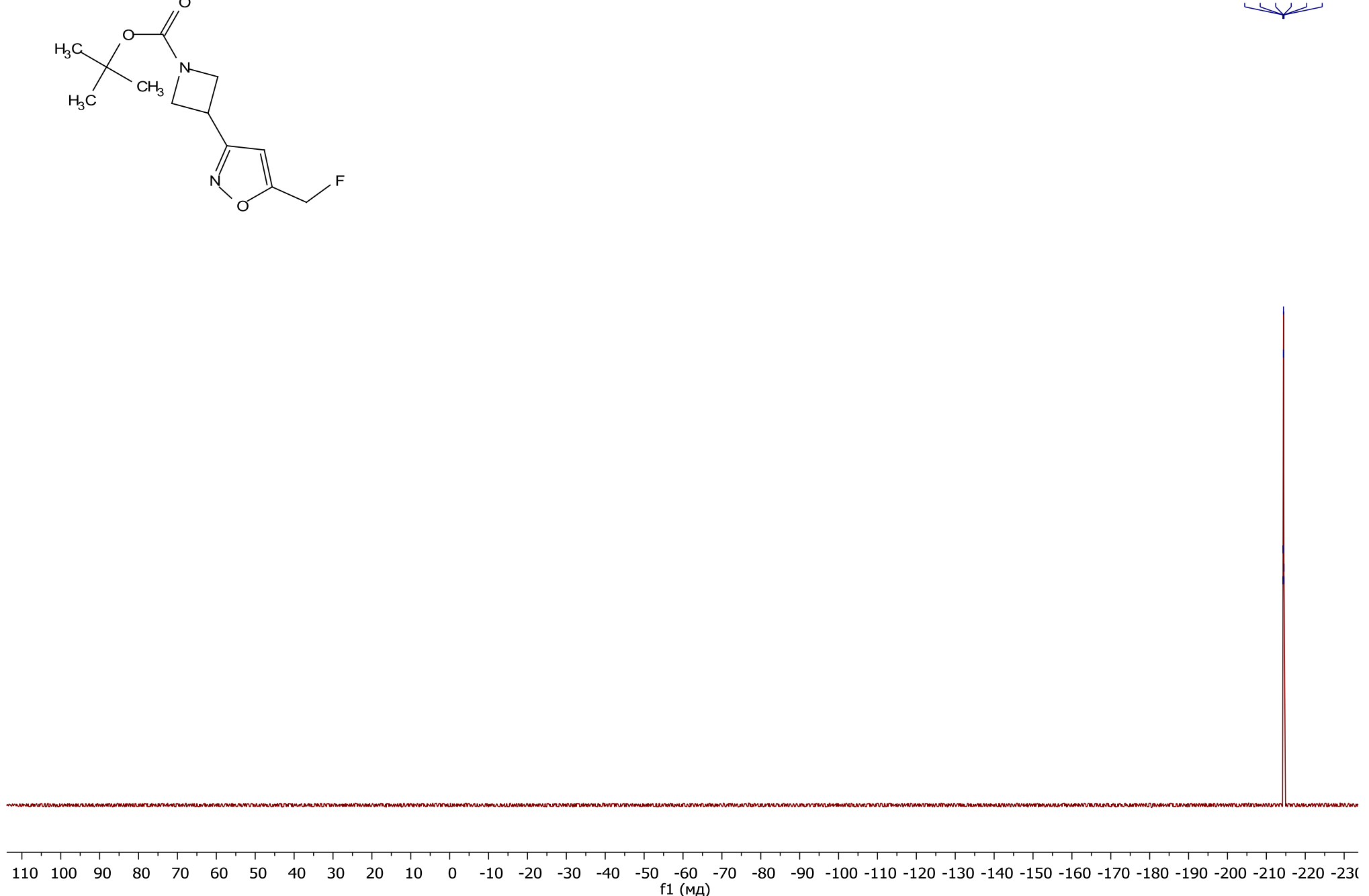

tert-Butyl 3-(5-(fluoromethyl)isoxazol-3-yl)azetidine-1-carboxylate (18j) ${ }^{19}$ F NMR 

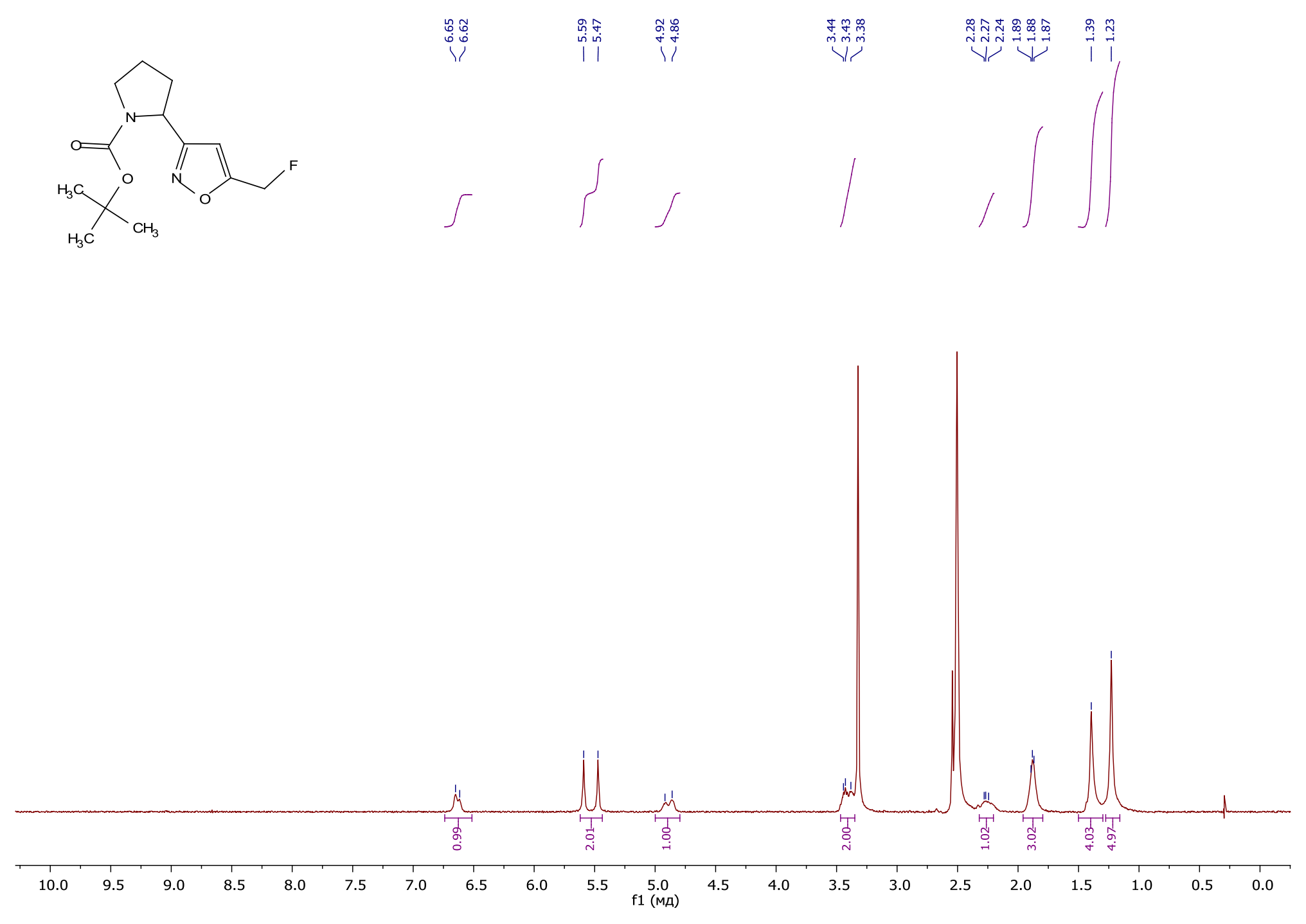

tert-Butyl 2-(5-(fluoromethyl)isoxazol-3-yl)pyrrolidine-1-carboxylate (18k or 18l) ${ }^{1} \mathrm{H}$ NMR 

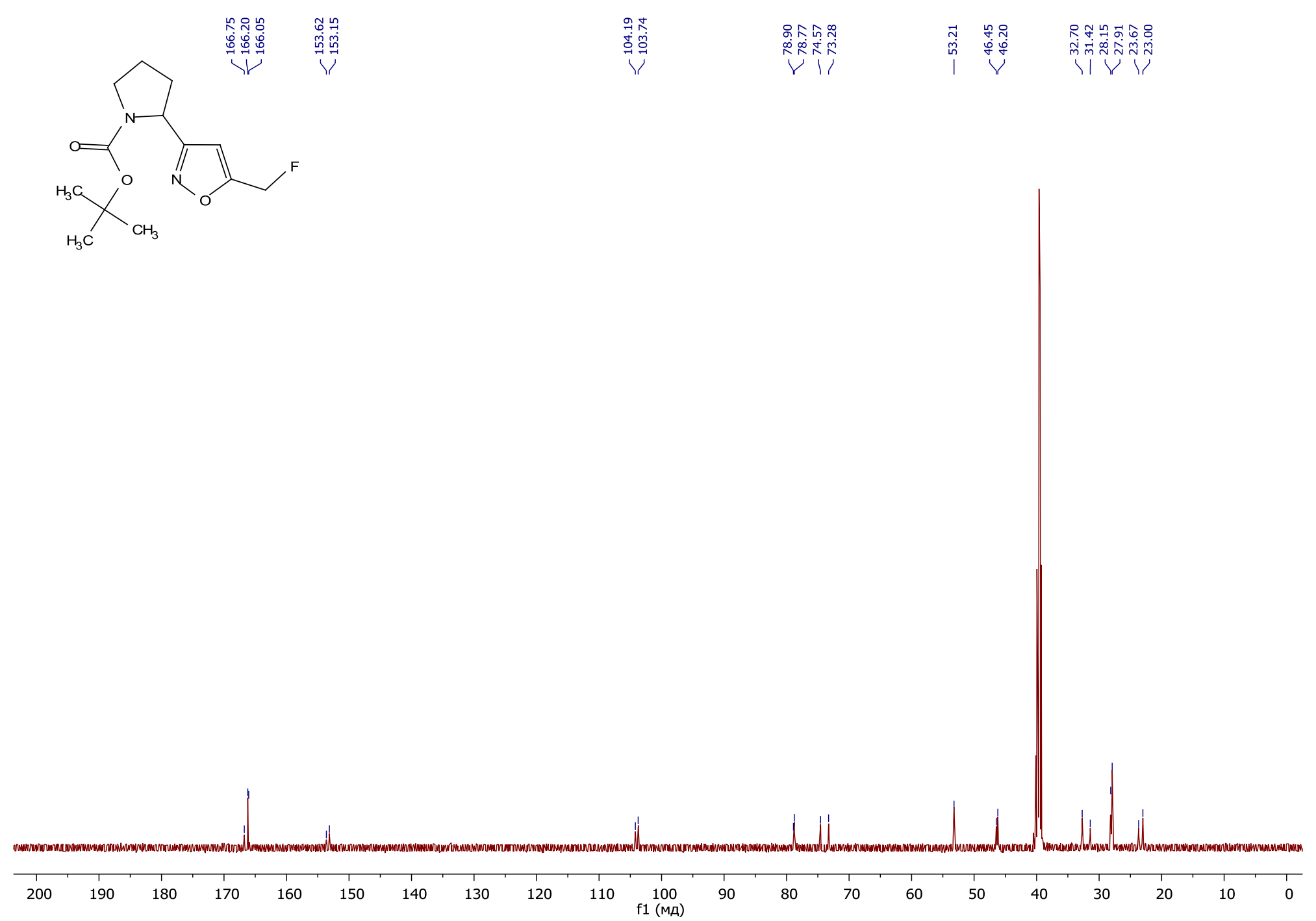

tert-Butyl 2-(5-(fluoromethyl)isoxazol-3-yl)pyrrolidine-1-carboxylate (18k or 18I) ${ }^{13} \mathrm{C} \mathrm{NMR}$ 


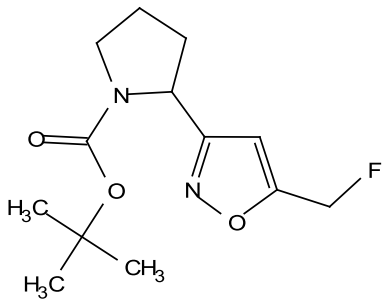



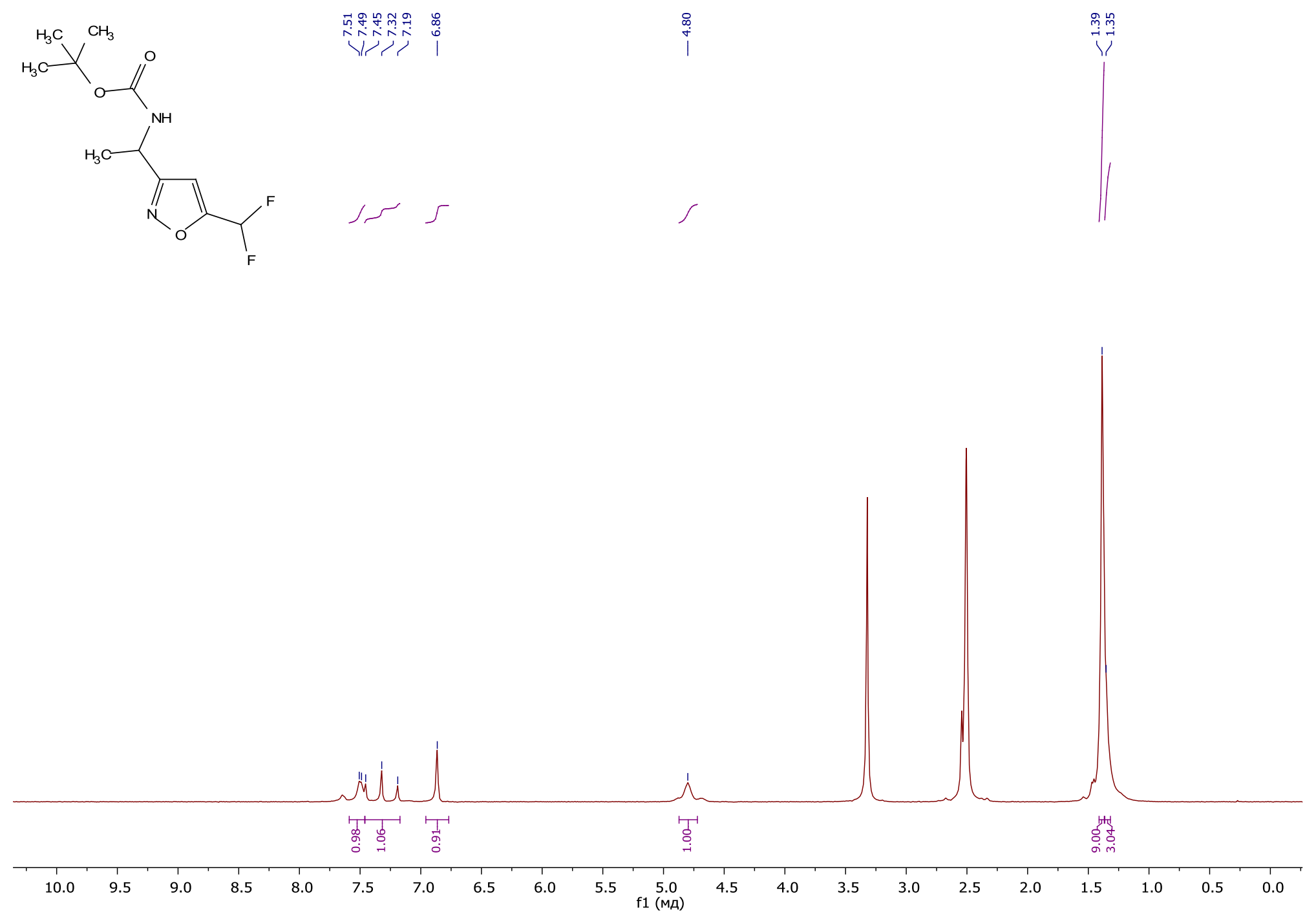

tert-Butyl (1-(5-(difluoromethyl)isoxazol-3-yl)ethyl)carbamate (19g or 19h) ${ }^{1}$ H NMR 


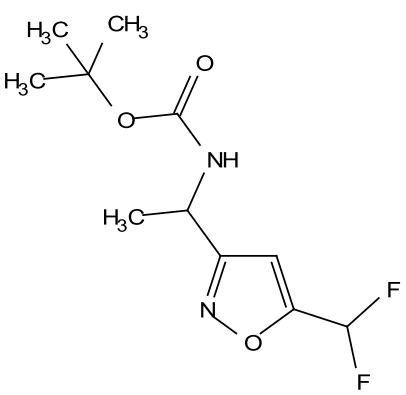

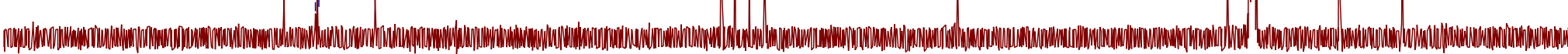

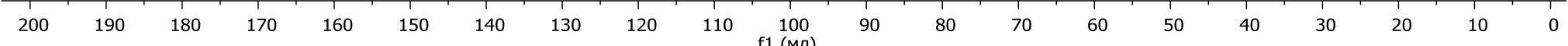

tert-Butyl (1-(5-(difluoromethyl)isoxazol-3-yl)ethyl)carbamate (19g or 19h) ${ }^{13} \mathrm{C}$ NMR 

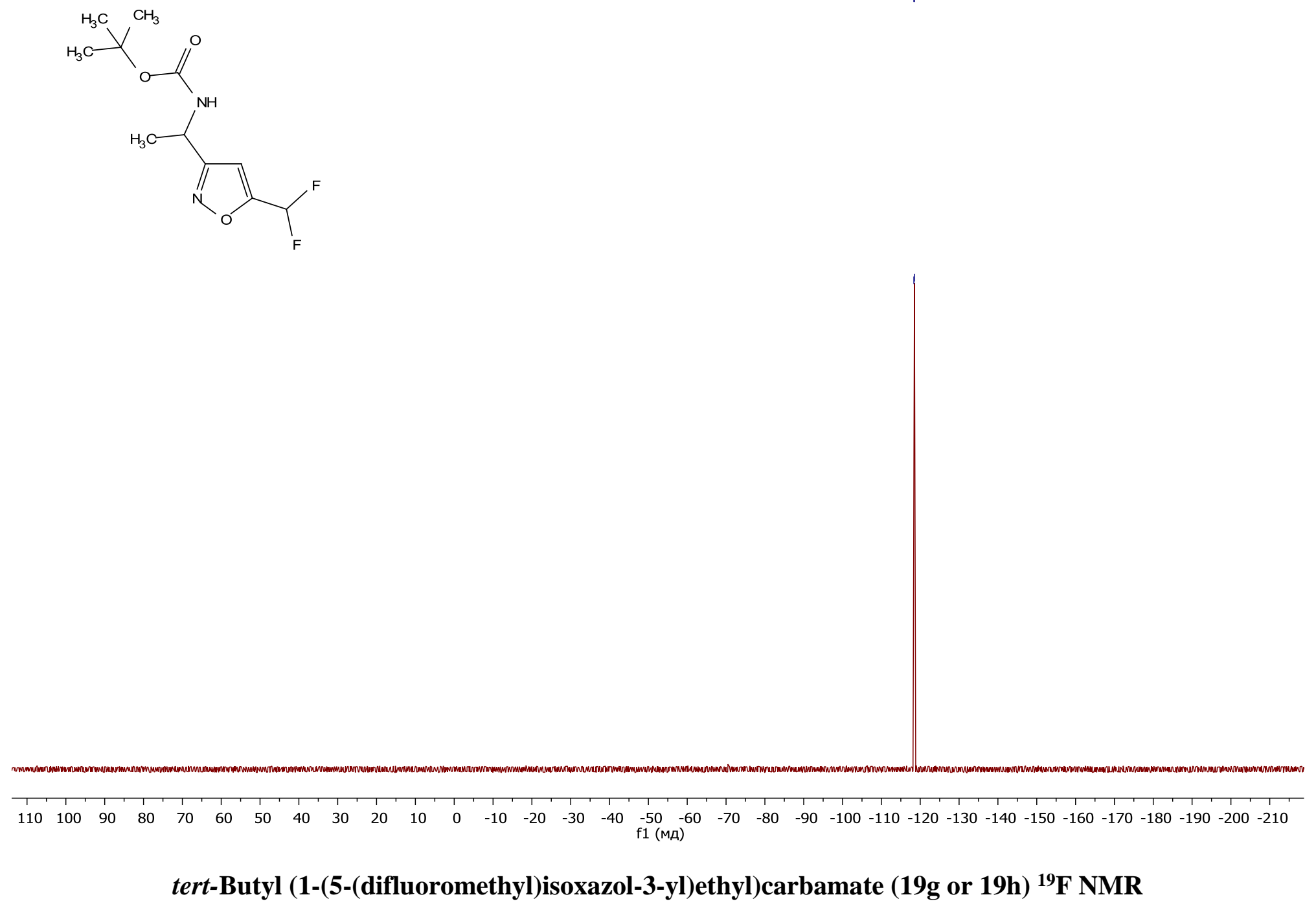


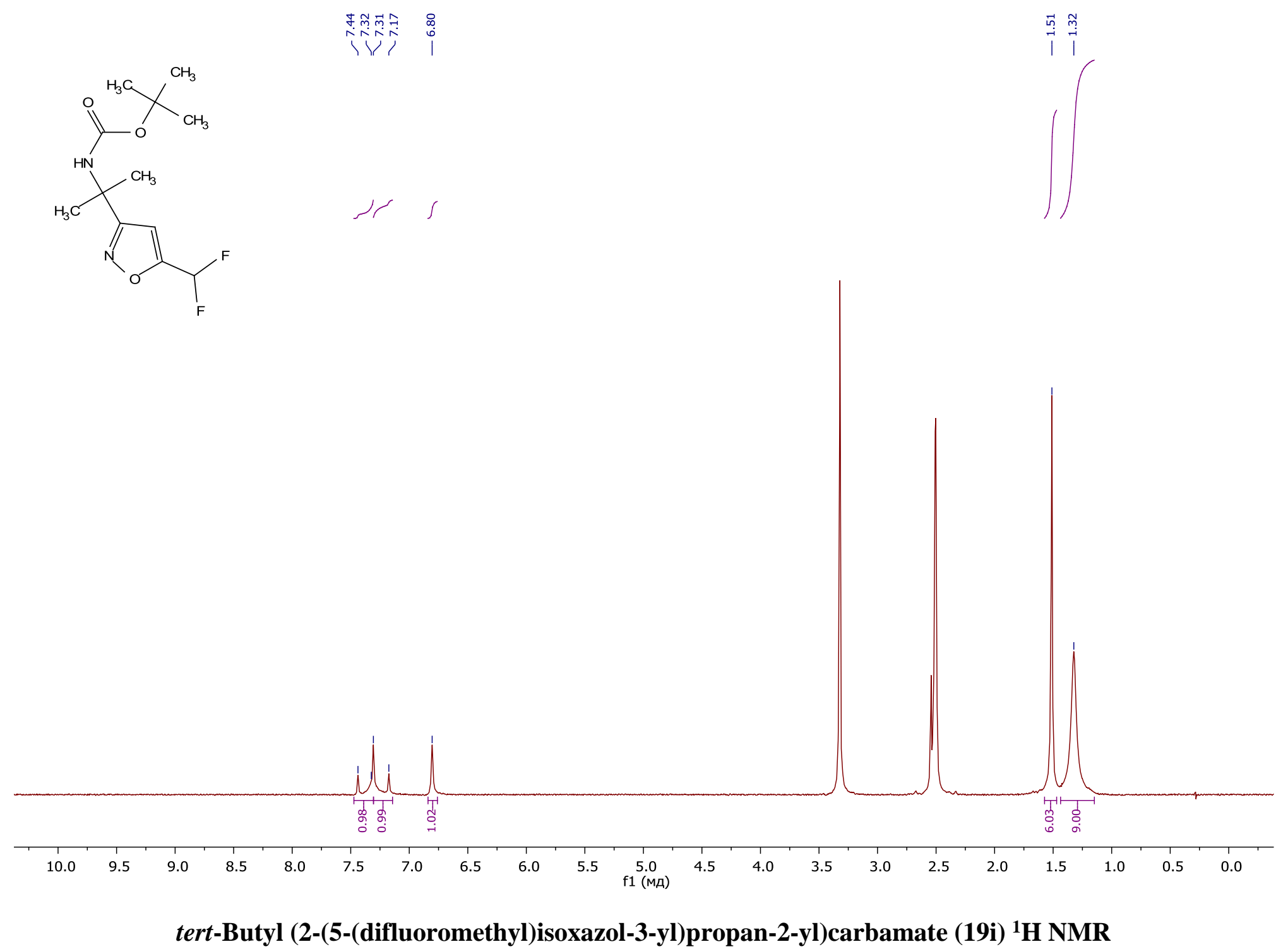



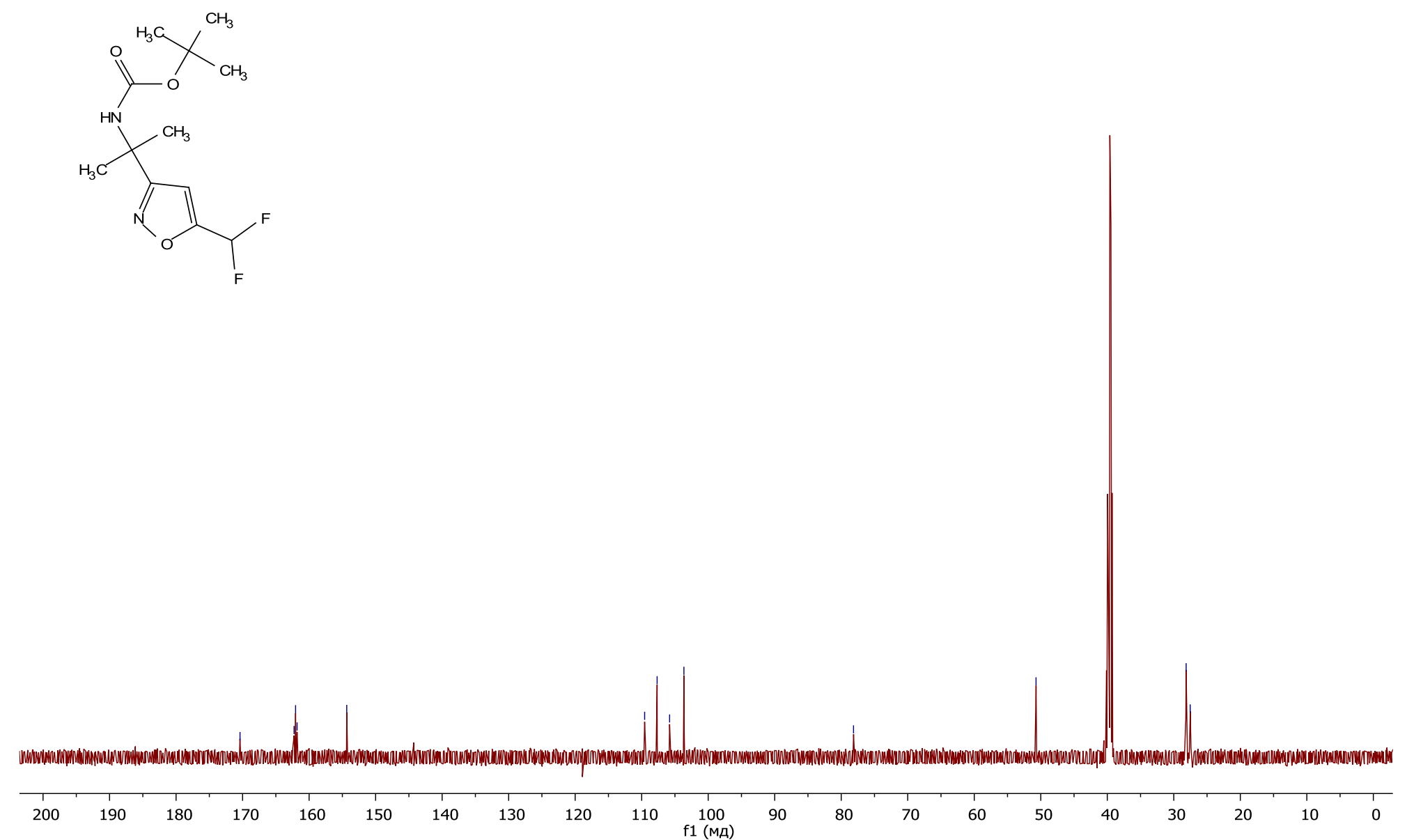

tert-Butyl (2-(5-(difluoromethyl)isoxazol-3-yl)propan-2-yl)carbamate (19i) ${ }^{13}$ C NMR 


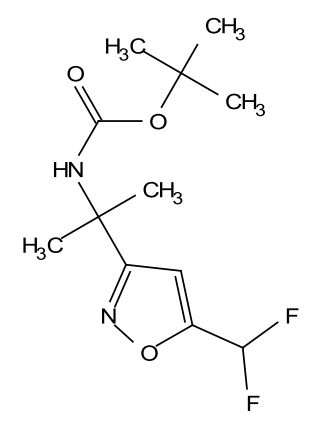

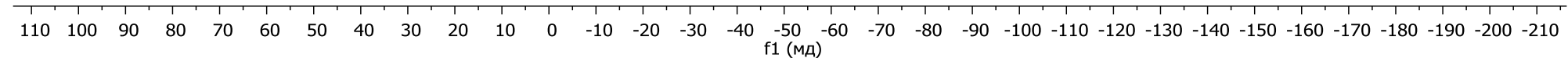

tert-Butyl (2-(5-(difluoromethyl)isoxazol-3-yl)propan-2-yl)carbamate (19i) ${ }^{19}$ F NMR 

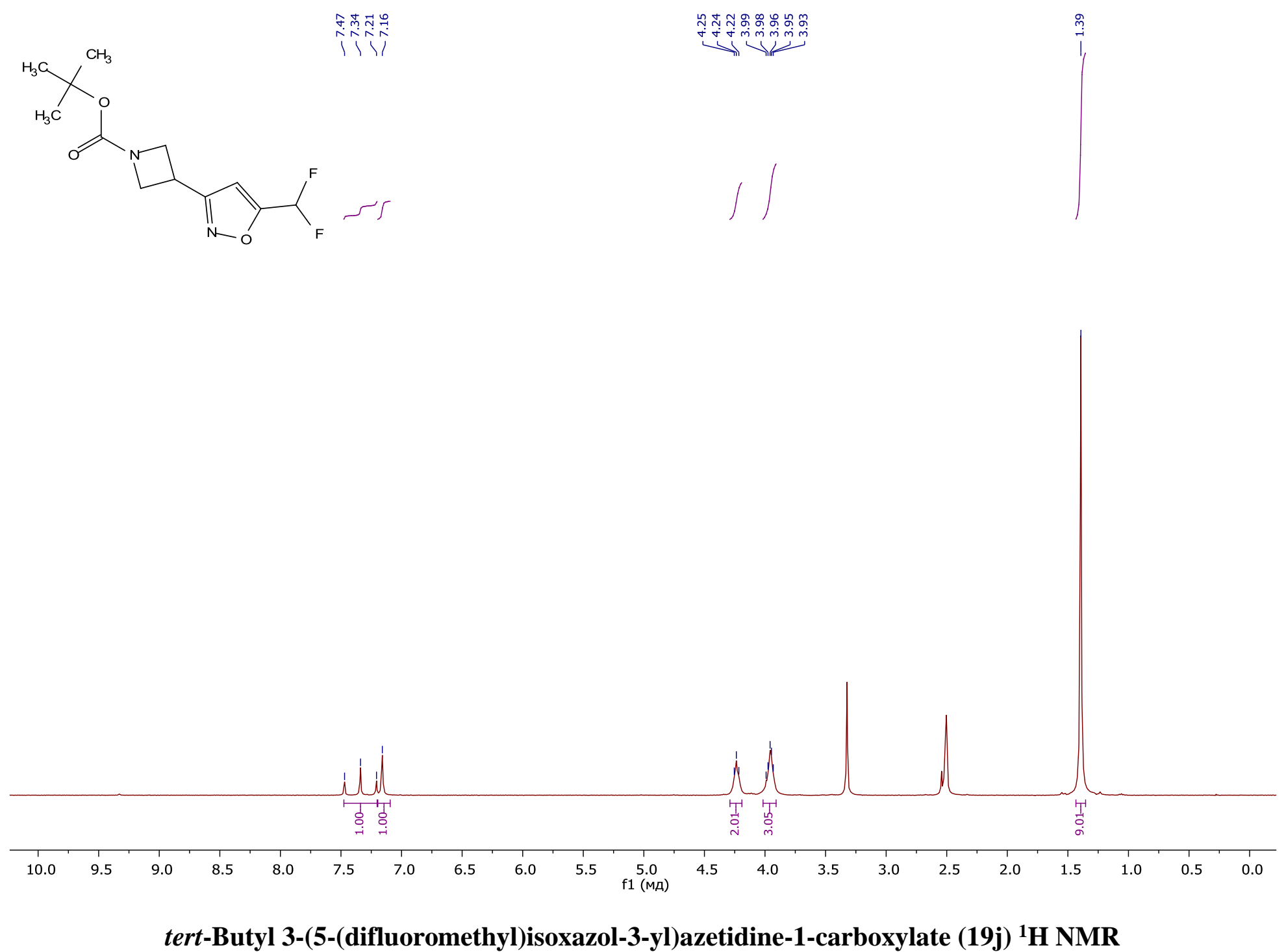


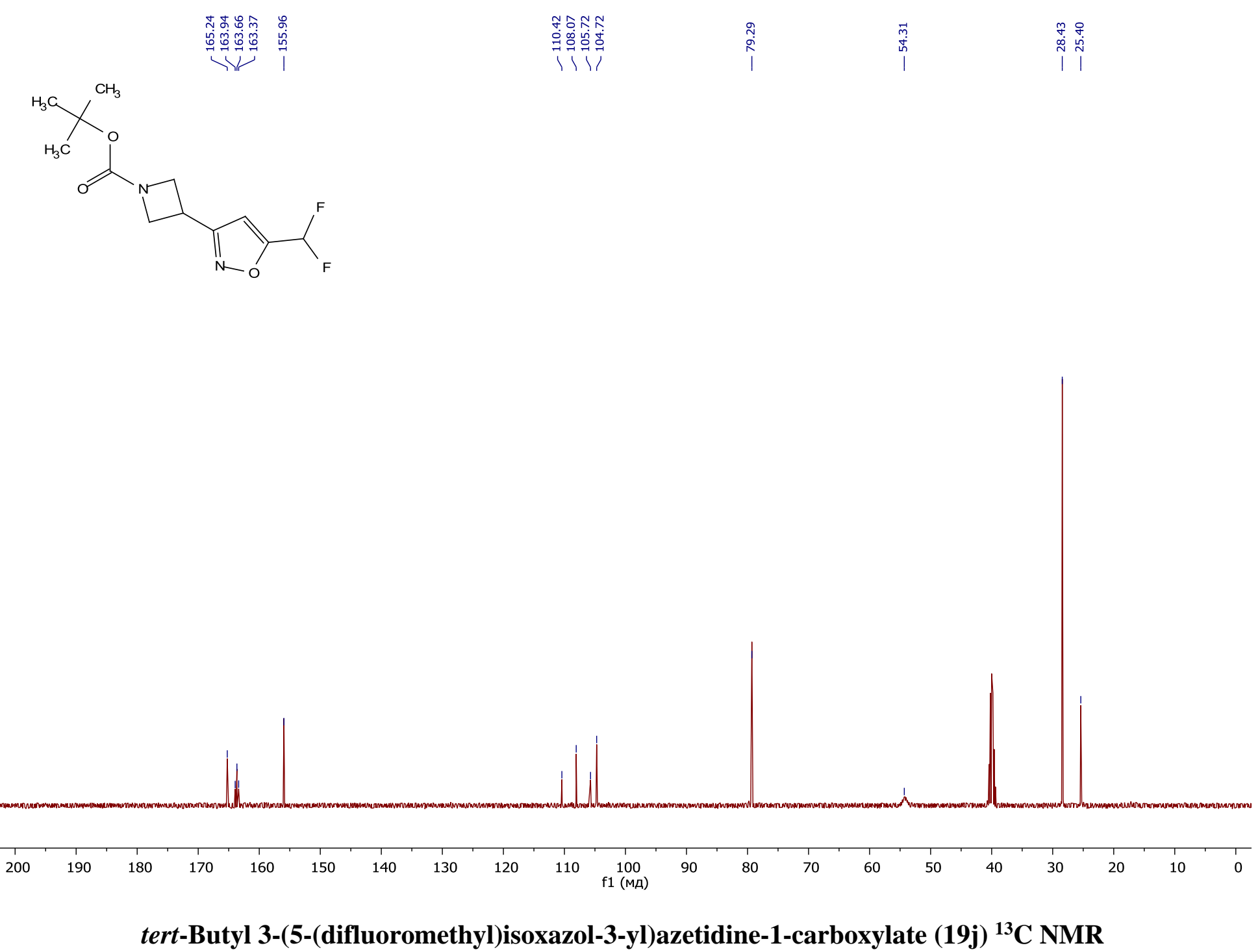



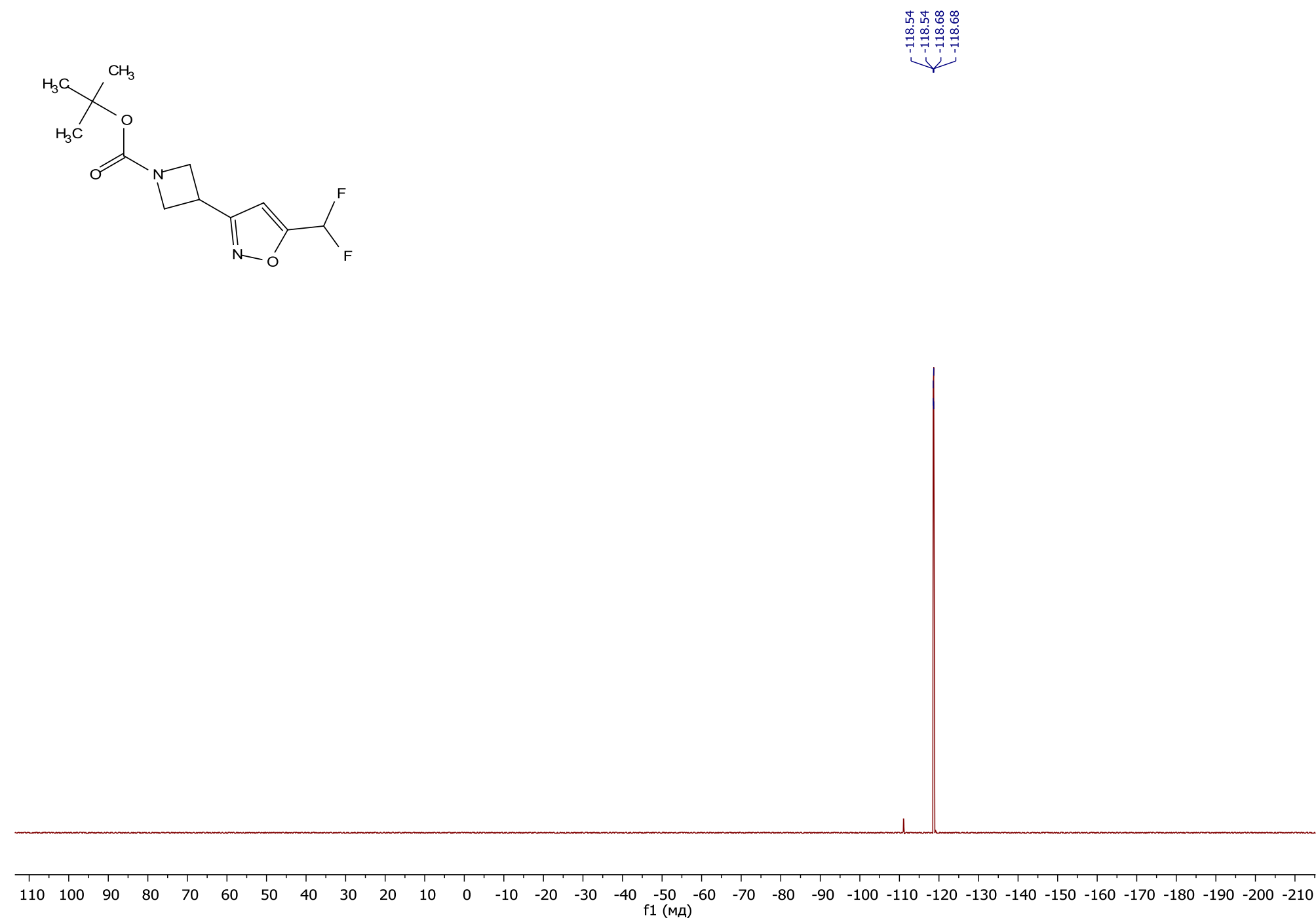

tert-Butyl 3-(5-(difluoromethyl)isoxazol-3-yl)azetidine-1-carboxylate (19j) ${ }^{19}$ F NMR 

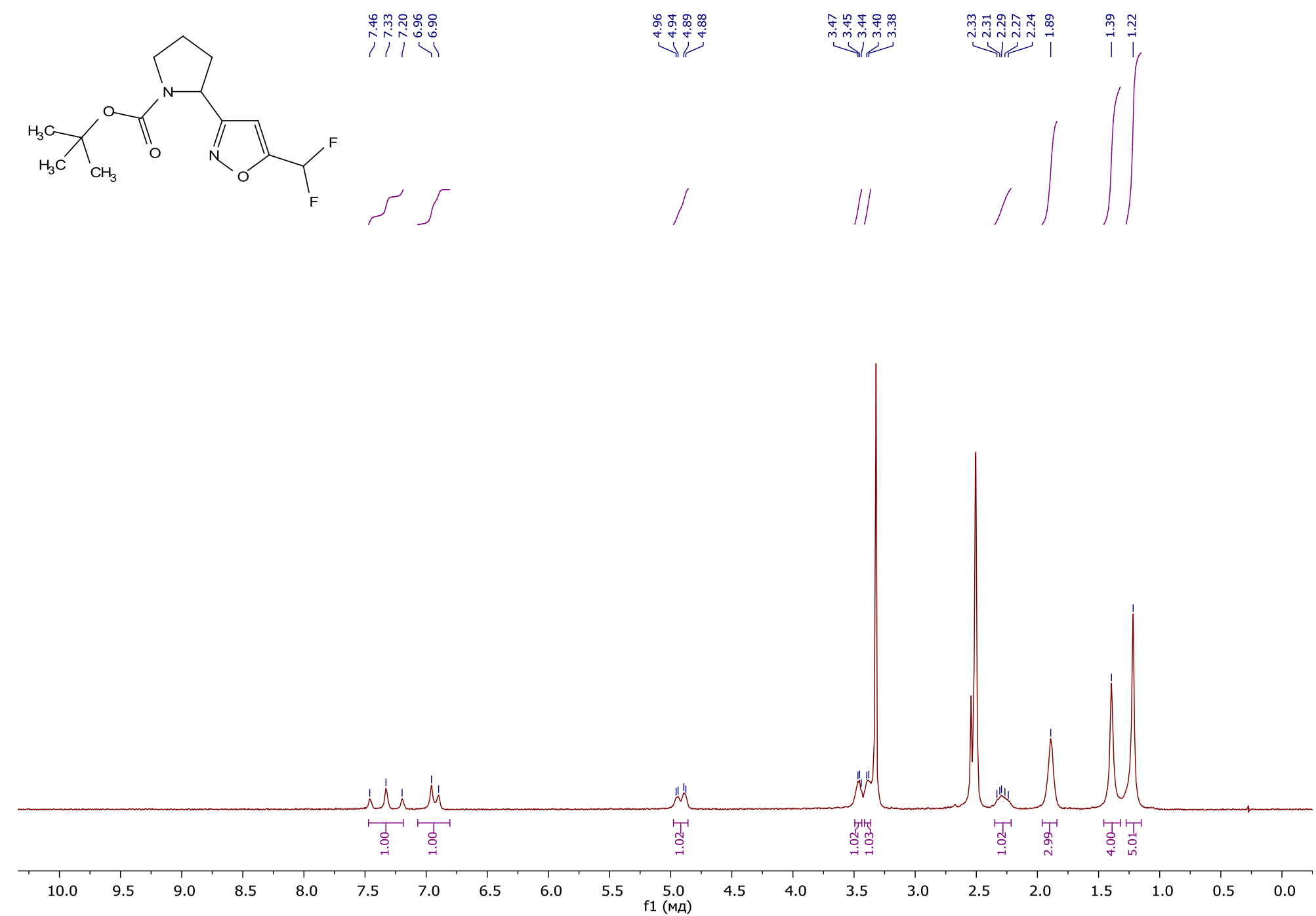

tert-Butyl 2-(5-(difluoromethyl)isoxazol-3-yl)pyrrolidine-1-carboxylate (19k or 19l) ${ }^{1} \mathrm{H}$ NMR 


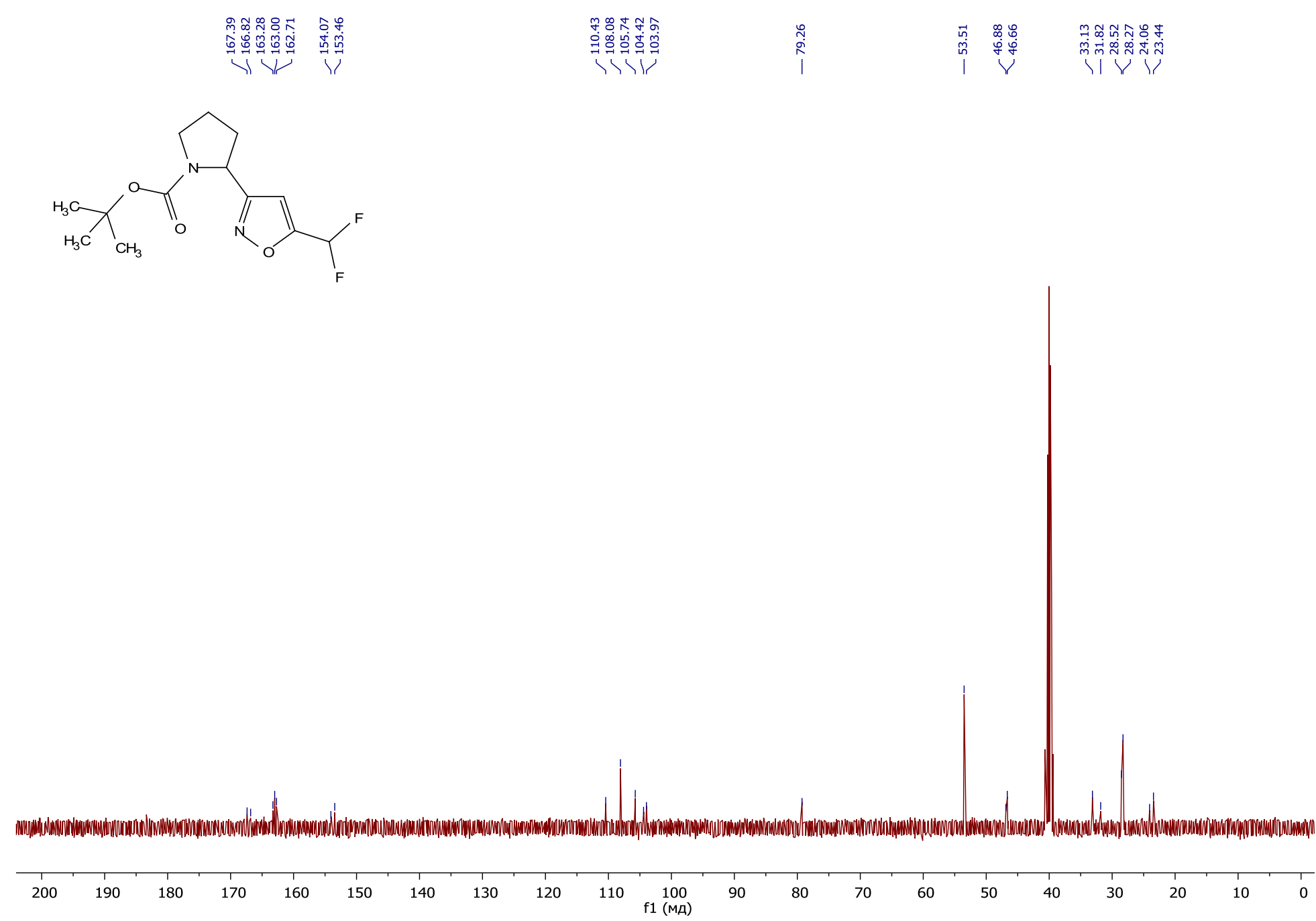

tert-Butyl 2-(5-(difluoromethyl)isoxazol-3-yl)pyrrolidine-1-carboxylate (19k or 19l) ${ }^{13} \mathrm{C}$ NMR 


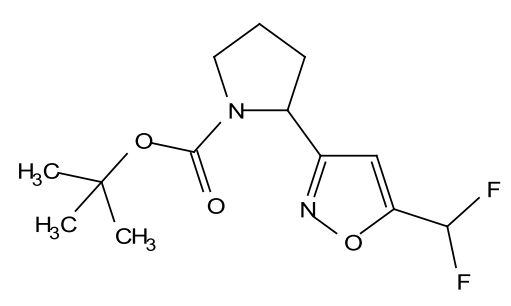

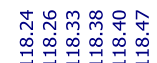

(1)

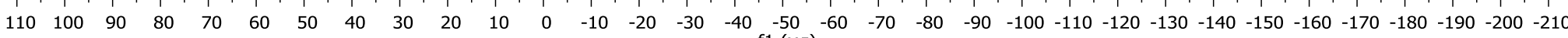

tert-Butyl 2-(5-(difluoromethyl)isoxazol-3-yl)pyrrolidine-1-carboxylate (19k or 19l) ${ }^{19}$ F NMR 


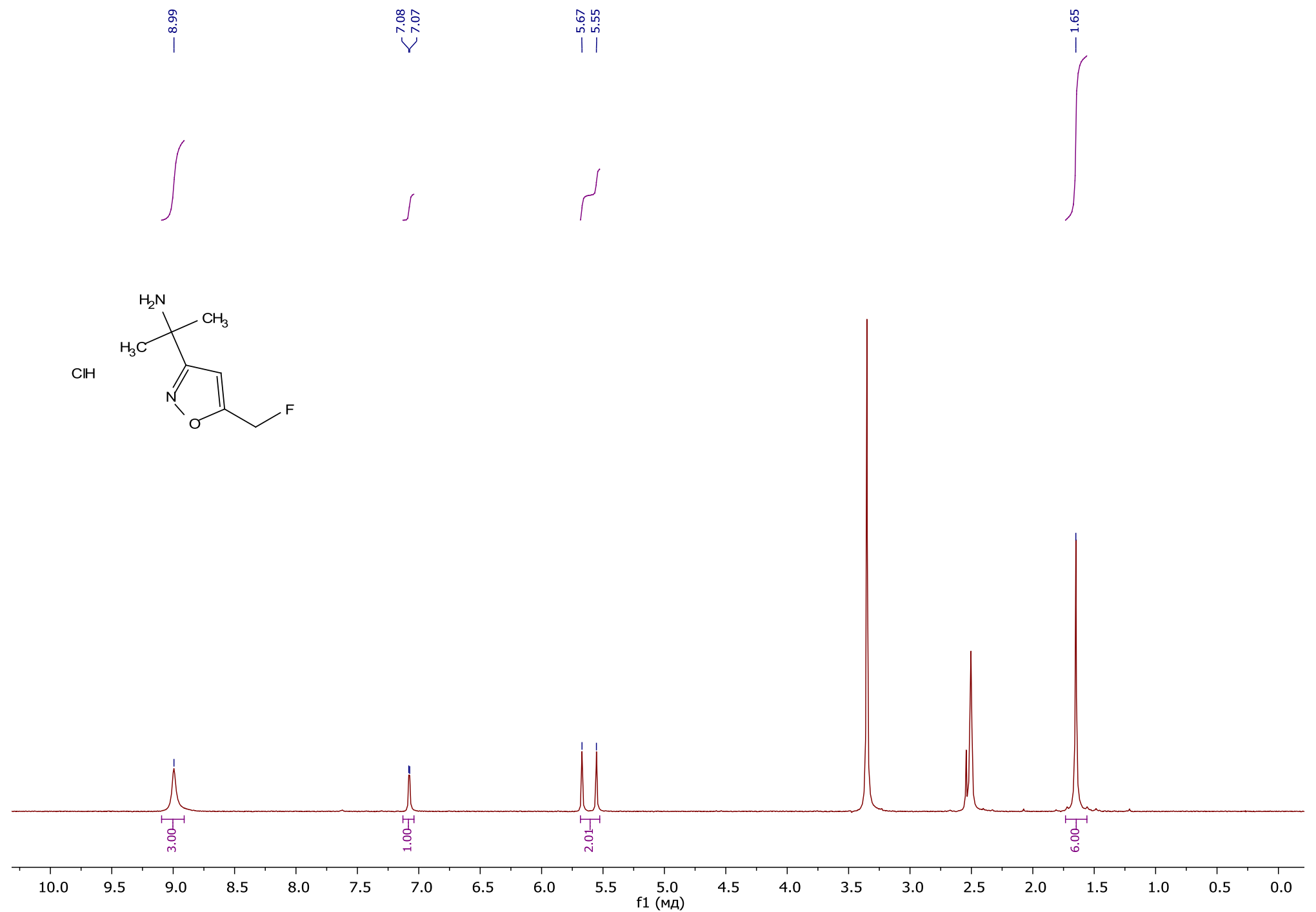

2-(5-(Fluoromethyl)isoxazol-3-yl)propan-2-amine hydrochloride (20i-HCl) ${ }^{1} \mathrm{H}$ NMR 


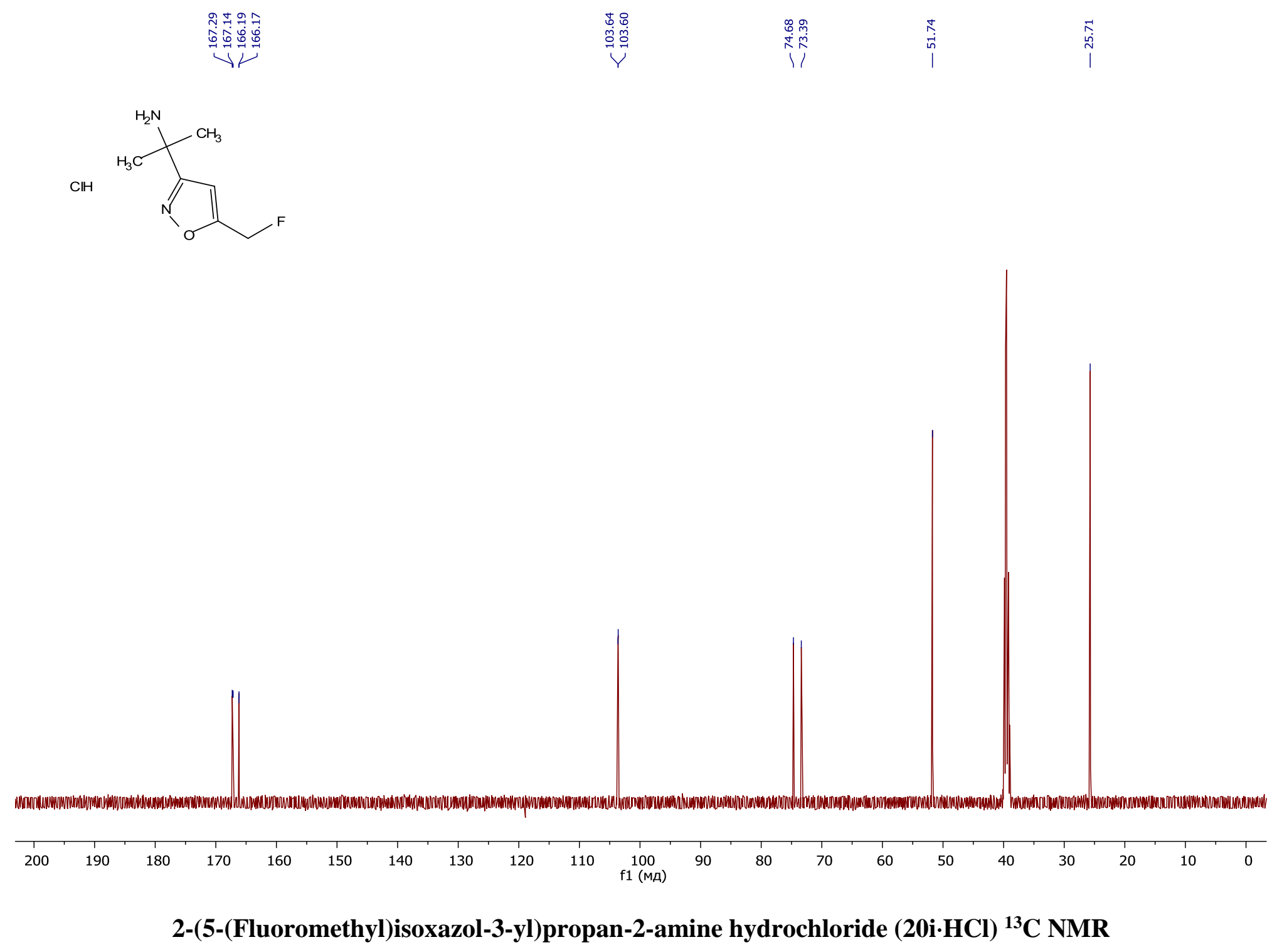




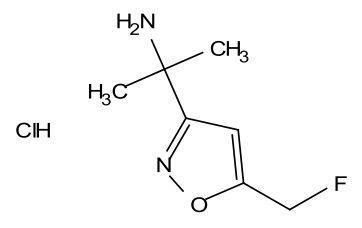

$\mathrm{CIH}$

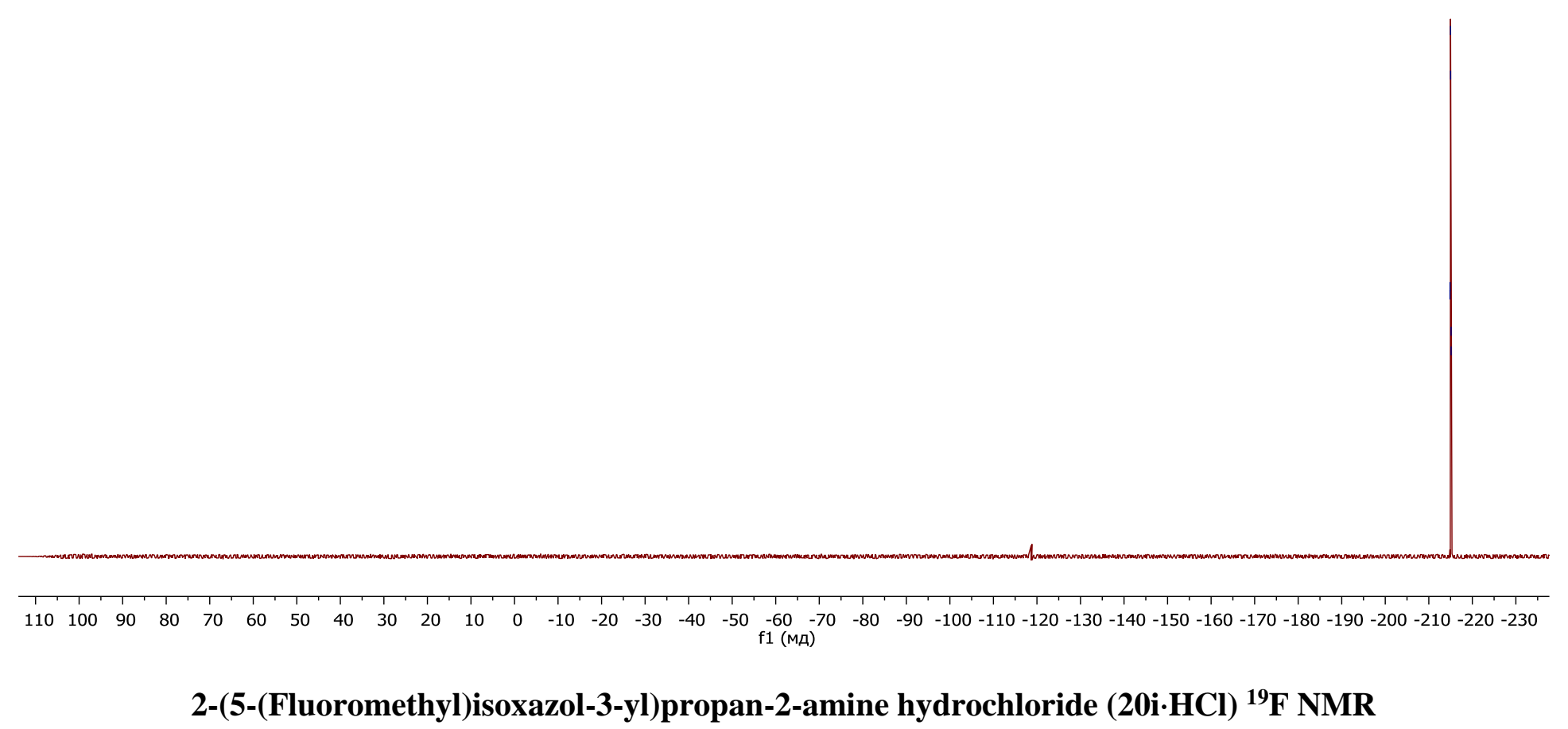



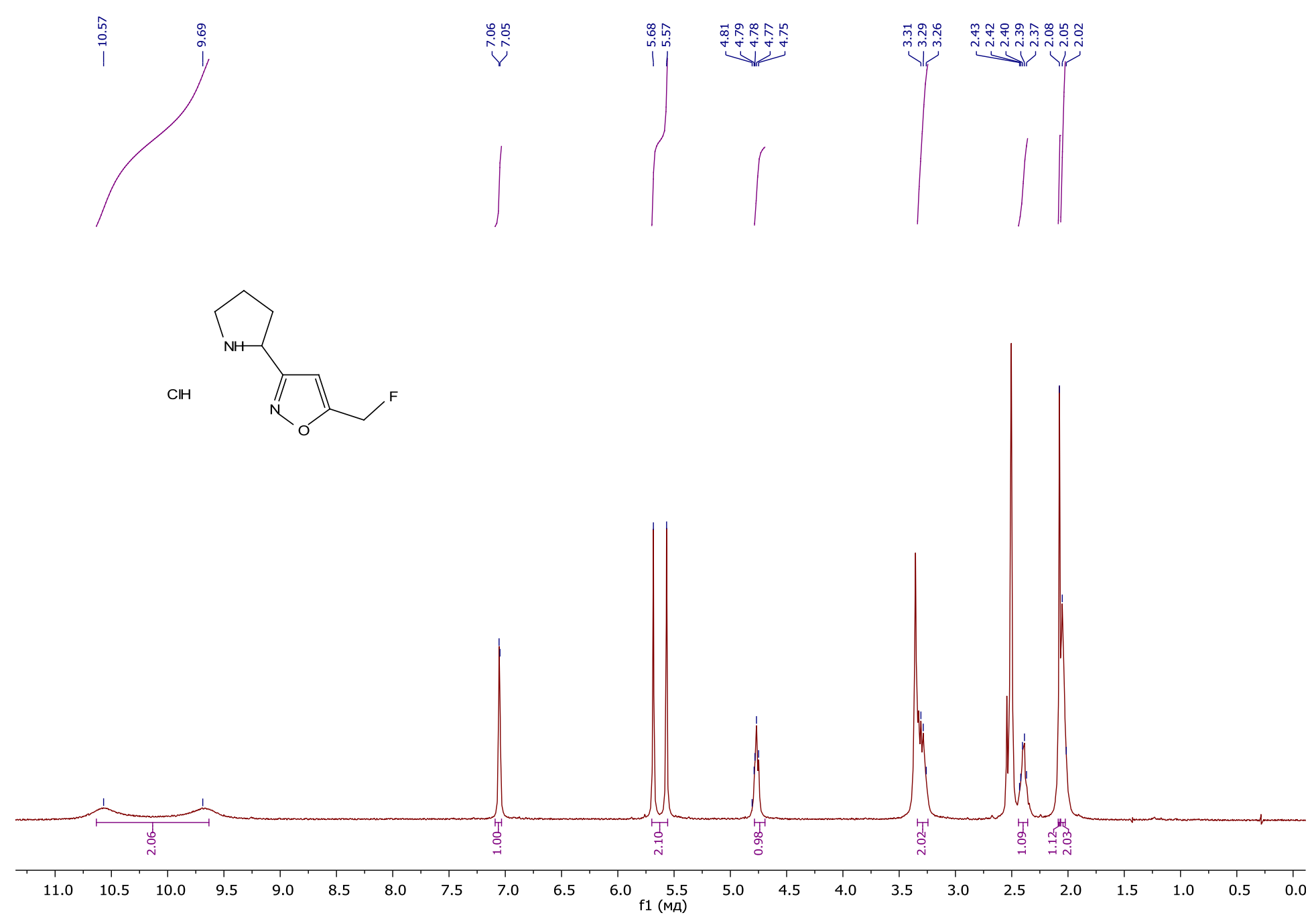

5-(Fluoromethyl)-3-(pyrrolidin-2-yl)isoxazole hydrochloride (20k·HCl or $201 \cdot \mathrm{HCl}$ ) ${ }^{1} \mathrm{H}$ NMR 


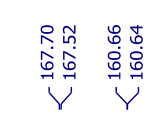

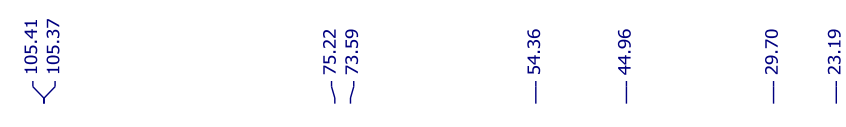

$\mathrm{CH}$
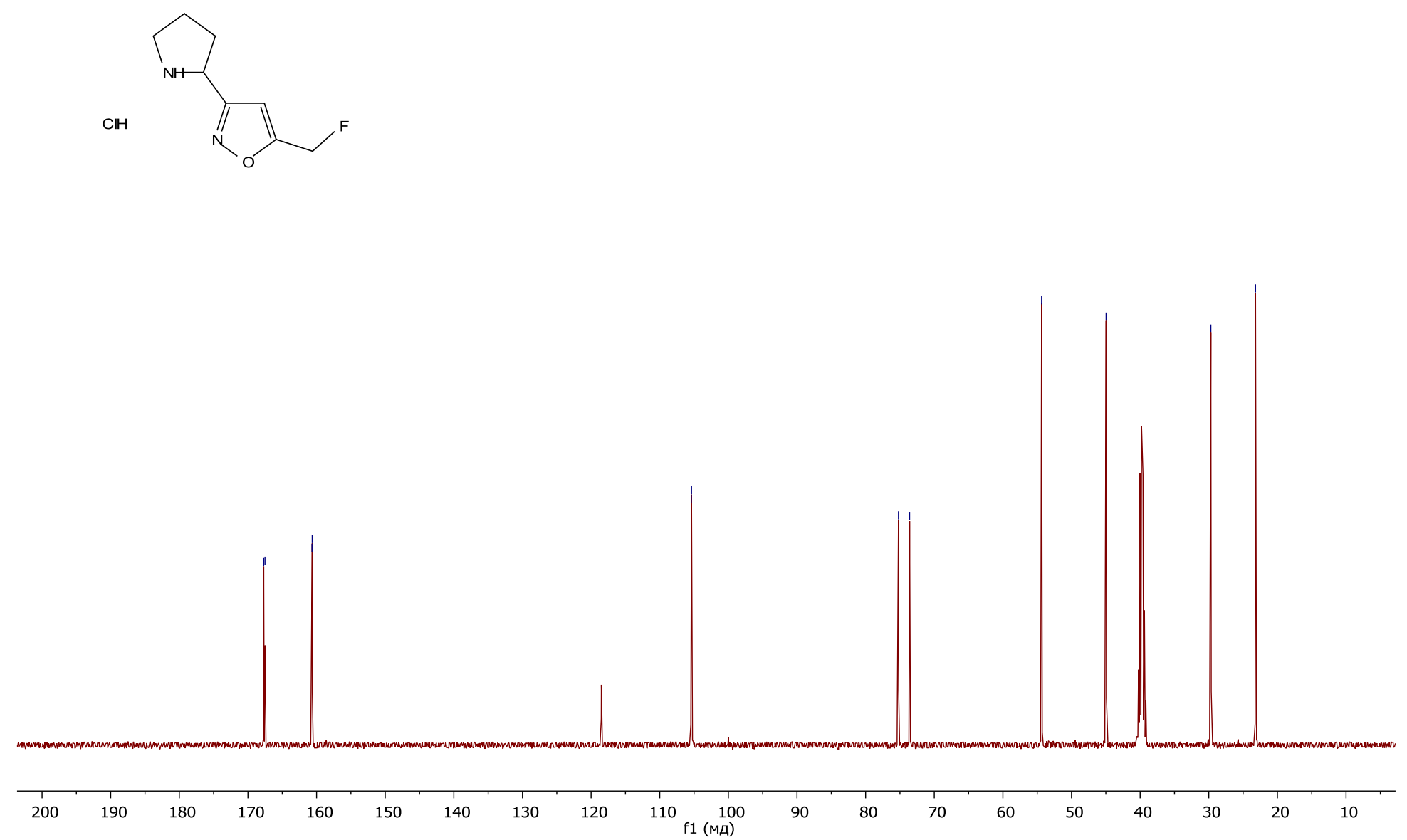

(S)-5-(Fluoromethyl)-3-(pyrrolidin-2-yl)isoxazole hydrochloride (20k·HCl or $201 \cdot \mathrm{HCl}){ }^{13} \mathrm{C}$ NMR 
$\mathrm{ClH}$

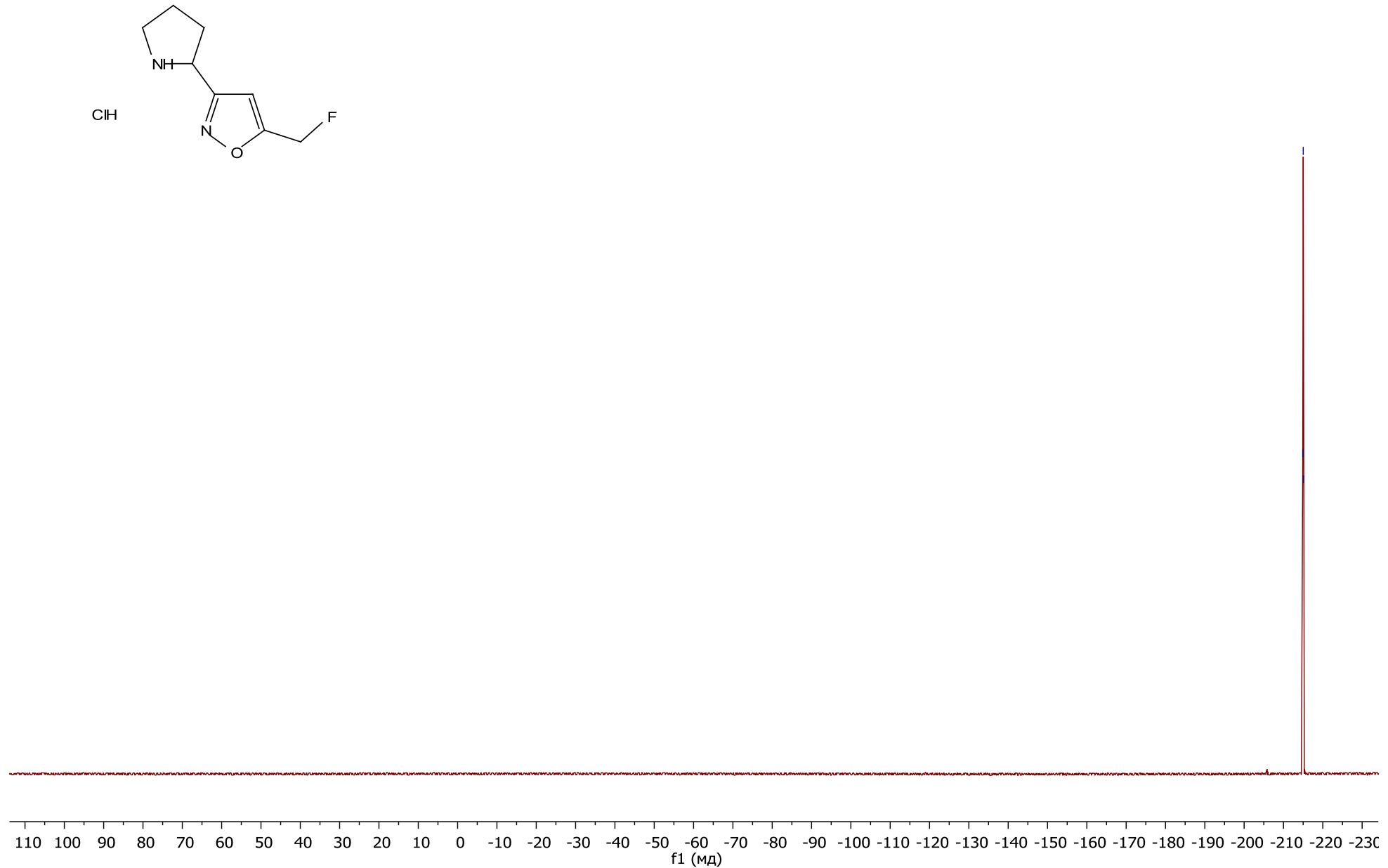

(S)-5-(Fluoromethyl)-3-(pyrrolidin-2-yl)isoxazole hydrochloride (20k·HCl or $201 \cdot \mathrm{HCl}){ }^{19} \mathrm{~F}$ NMR 

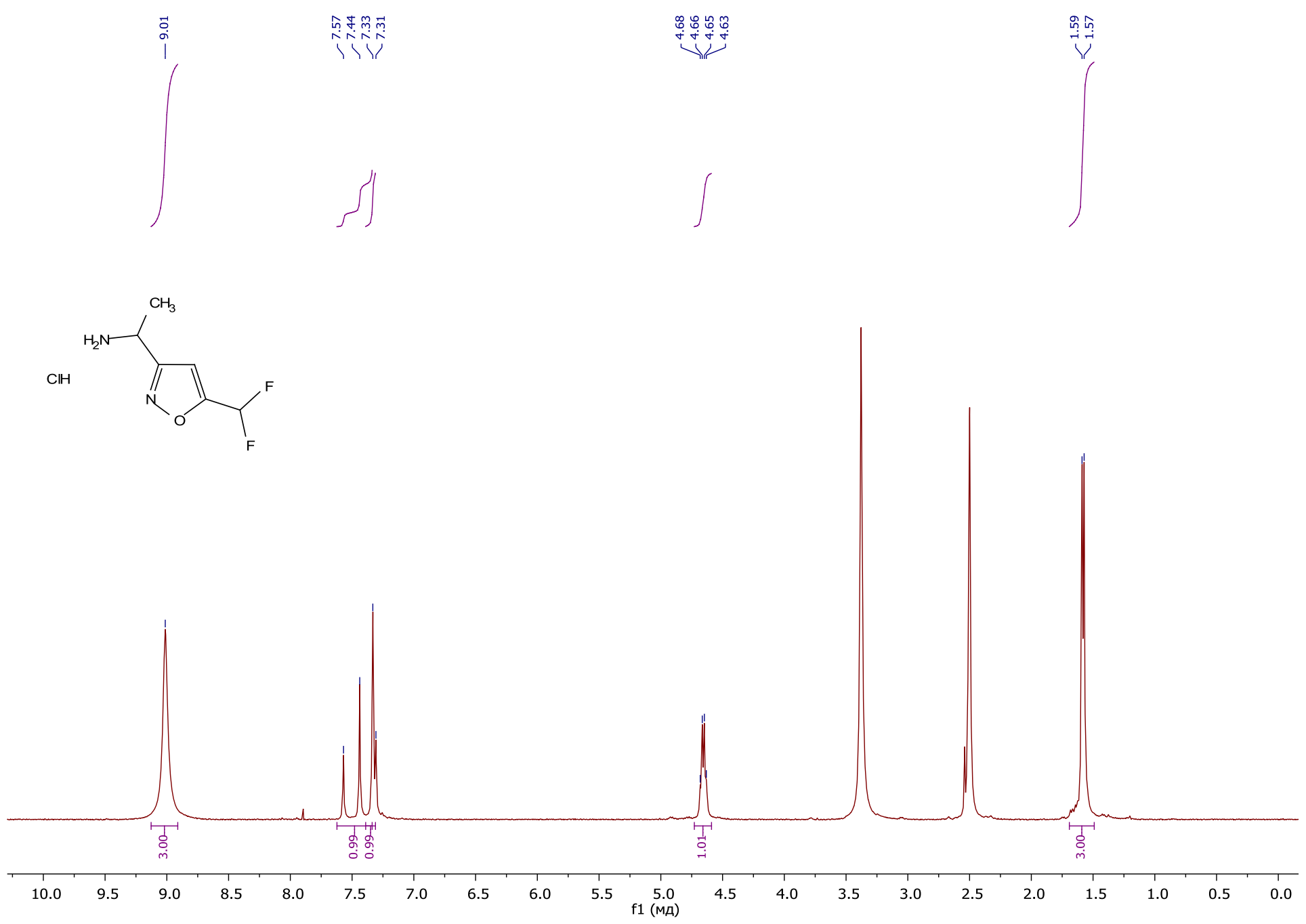

1-(5-(Difluoromethyl)isoxazol-3-yl)ethanamine hydrochloride (21g.HCl or $21 \mathrm{~h} \cdot \mathrm{HCl}){ }^{1} \mathrm{H}$ NMR 


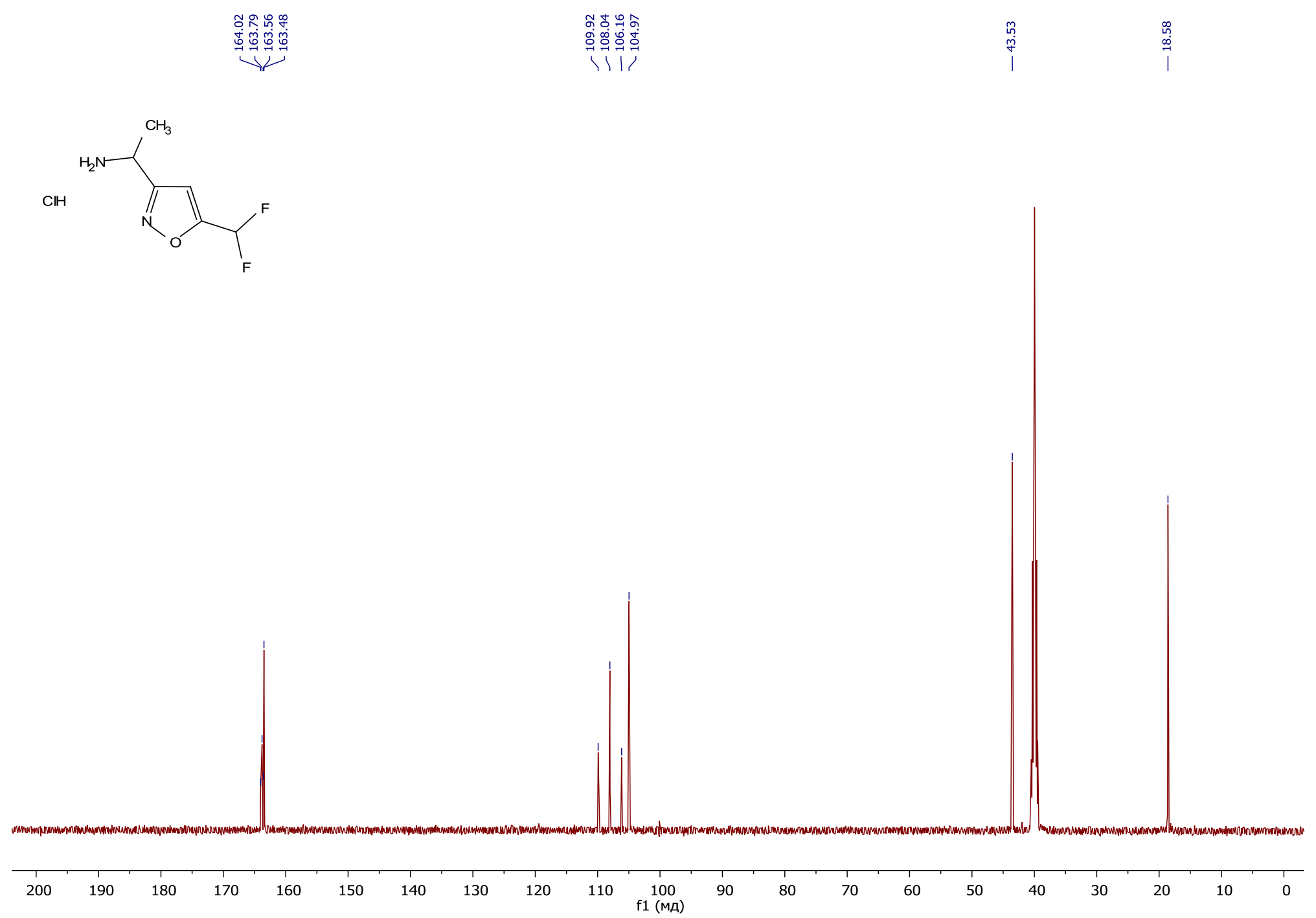

1-(5-(Difluoromethyl)isoxazol-3-yl)ethanamine hydrochloride $(21 \mathrm{~g} \cdot \mathrm{HCl}$ or $21 \mathrm{~h} \cdot \mathrm{HCl}){ }^{13} \mathrm{C} \mathrm{NMR}$ 

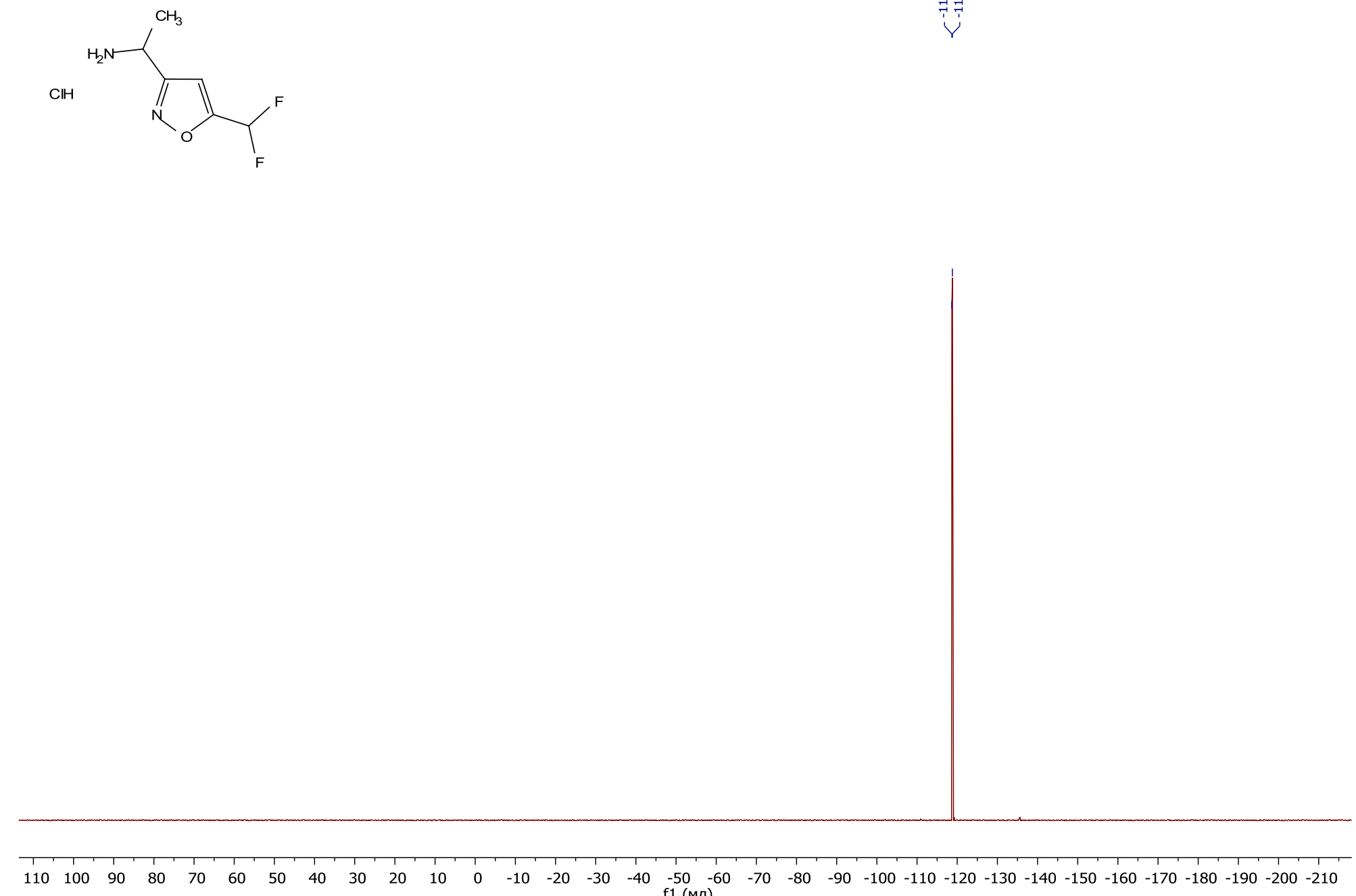

1-(5-(Difluoromethyl)isoxazol-3-yl)ethanamine hydrochloride $(21 \mathrm{~g} \cdot \mathrm{HCl}$ or $21 \mathrm{~h} \cdot \mathrm{HCl}){ }^{19} \mathrm{~F} \mathrm{NMR}$ 


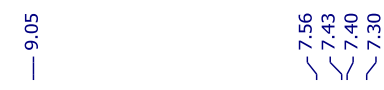

$\int \sqrt{1}$

$\mathrm{ClH}$
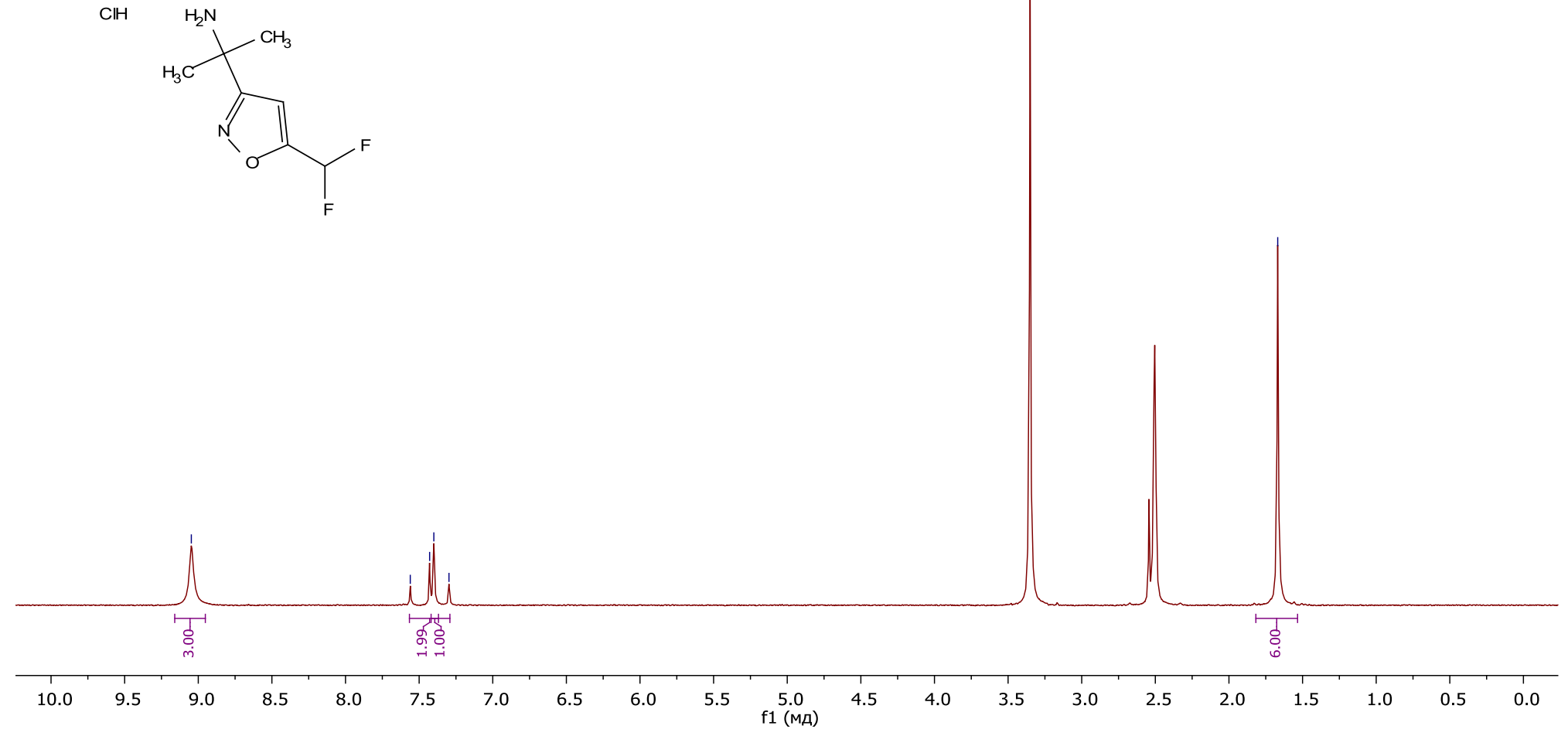

2-(5-(Difluoromethyl)isoxazol-3-yl)propan-2-amine hydrochloride (21i-HCl) ${ }^{1} \mathrm{H}$ NMR 


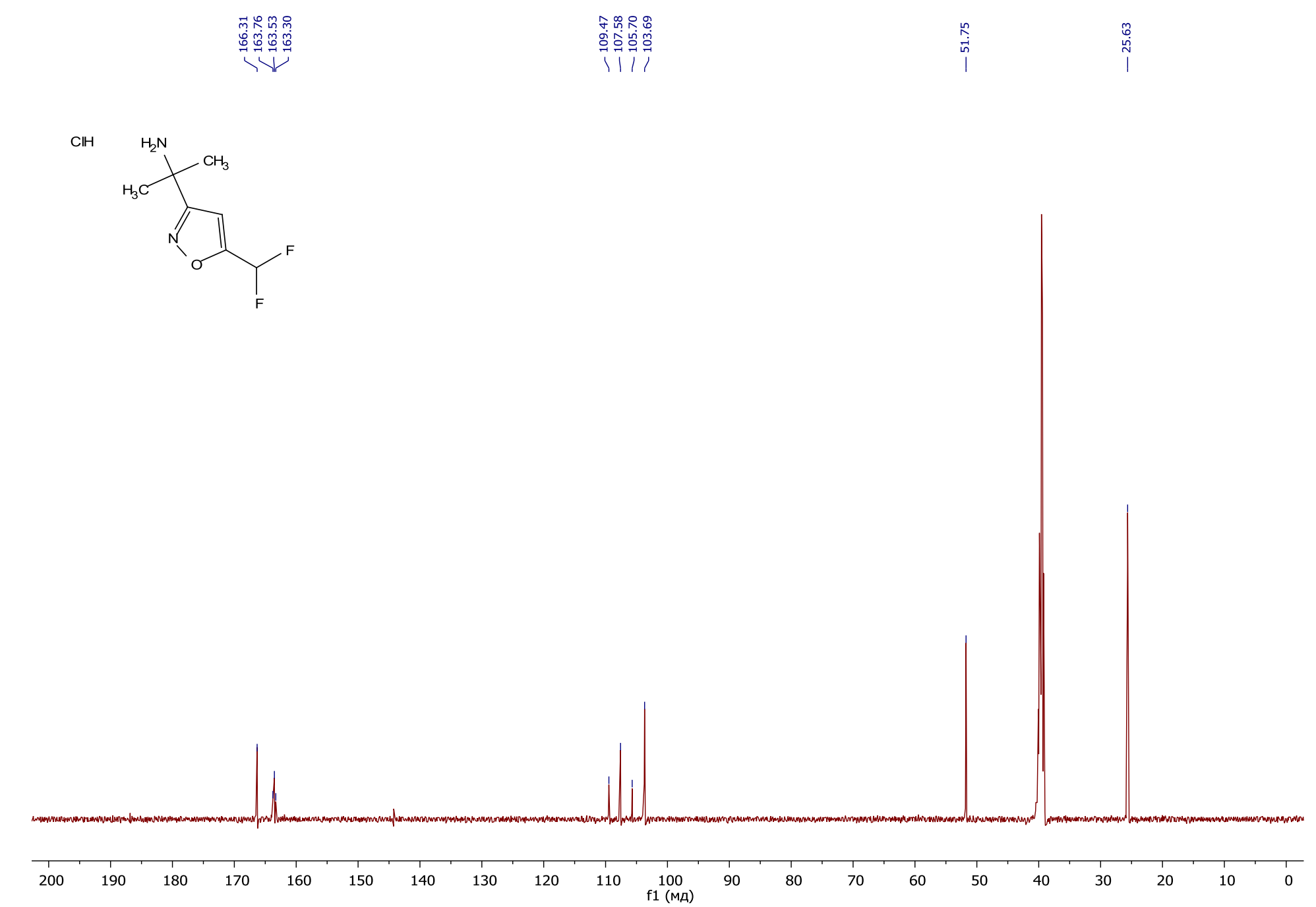

2-(5-(Difluoromethyl)isoxazol-3-yl)propan-2-amine hydrochloride $(21 \mathrm{i} \cdot \mathrm{HCl}){ }^{13} \mathrm{C} \mathrm{NMR}$ 
$\mathrm{ClH}$
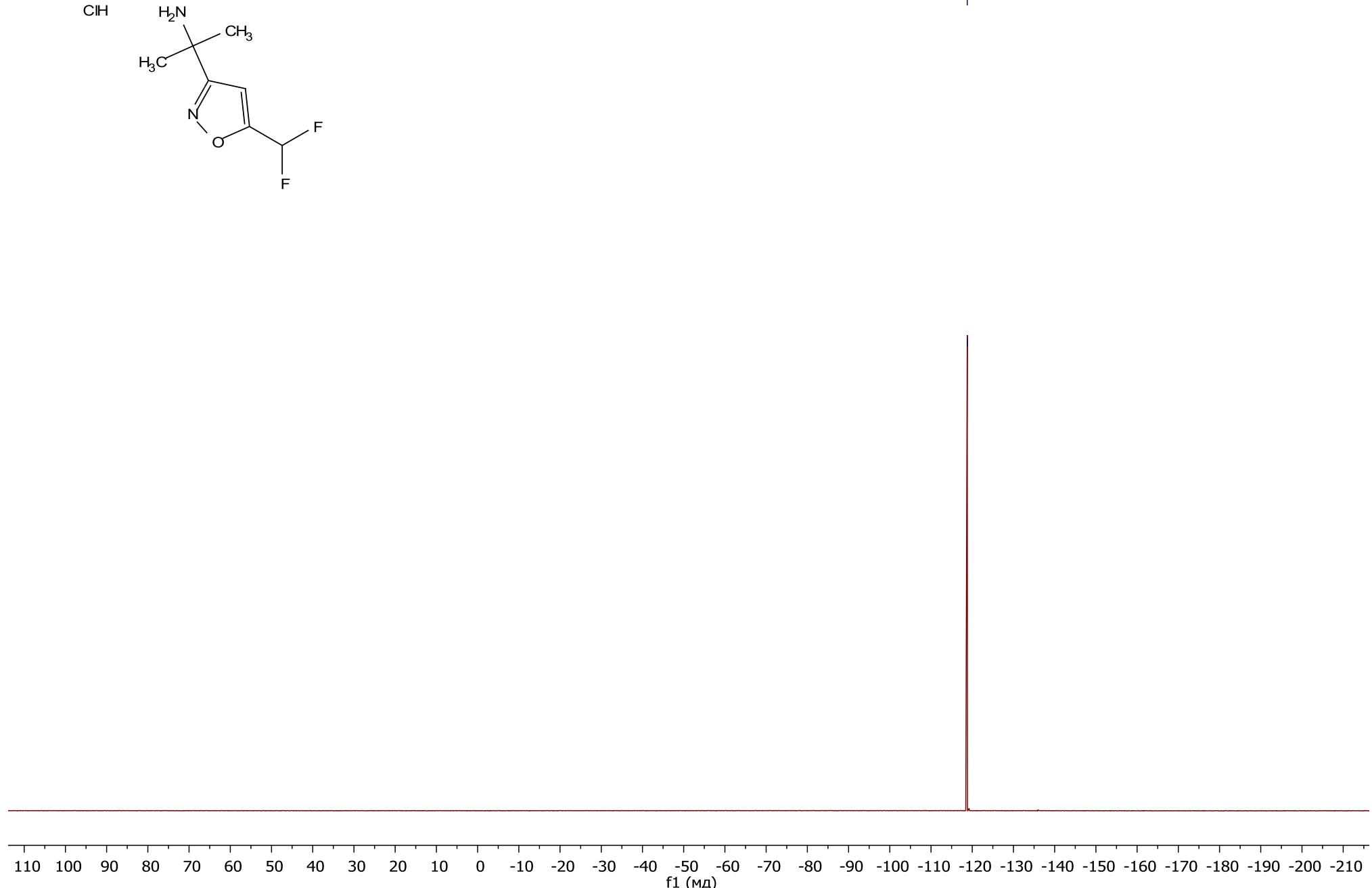

2-(5-(Difluoromethyl)isoxazol-3-yl)propan-2-amine hydrochloride $(21 \mathrm{i} \cdot \mathrm{HCl}){ }^{19} \mathrm{~F}$ NMR 


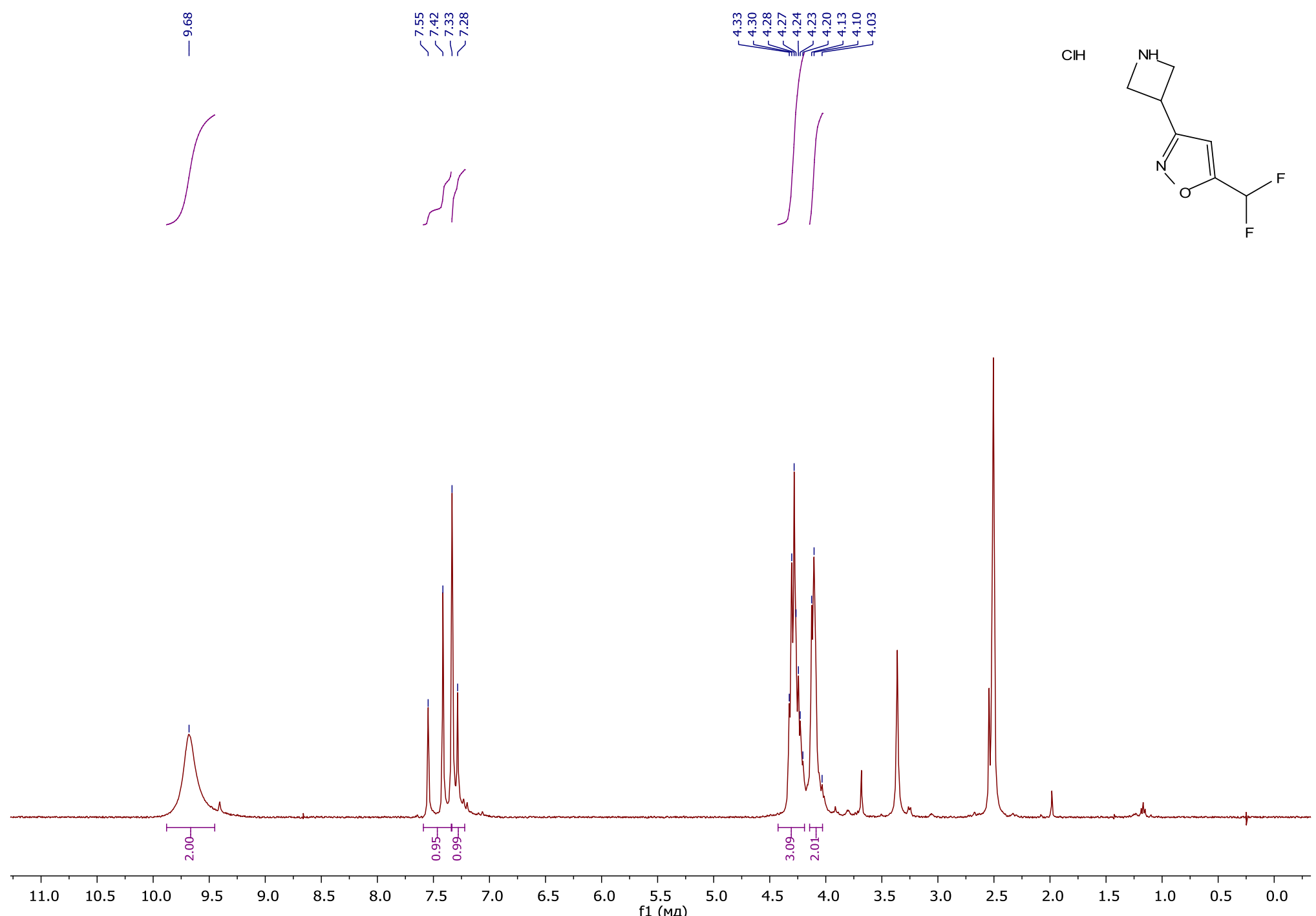

3-(Azetidin-3-yl)-5-(difluoromethyl)isoxazole hydrochloride (21j·HCl) ${ }^{1} \mathrm{H}$ NMR 


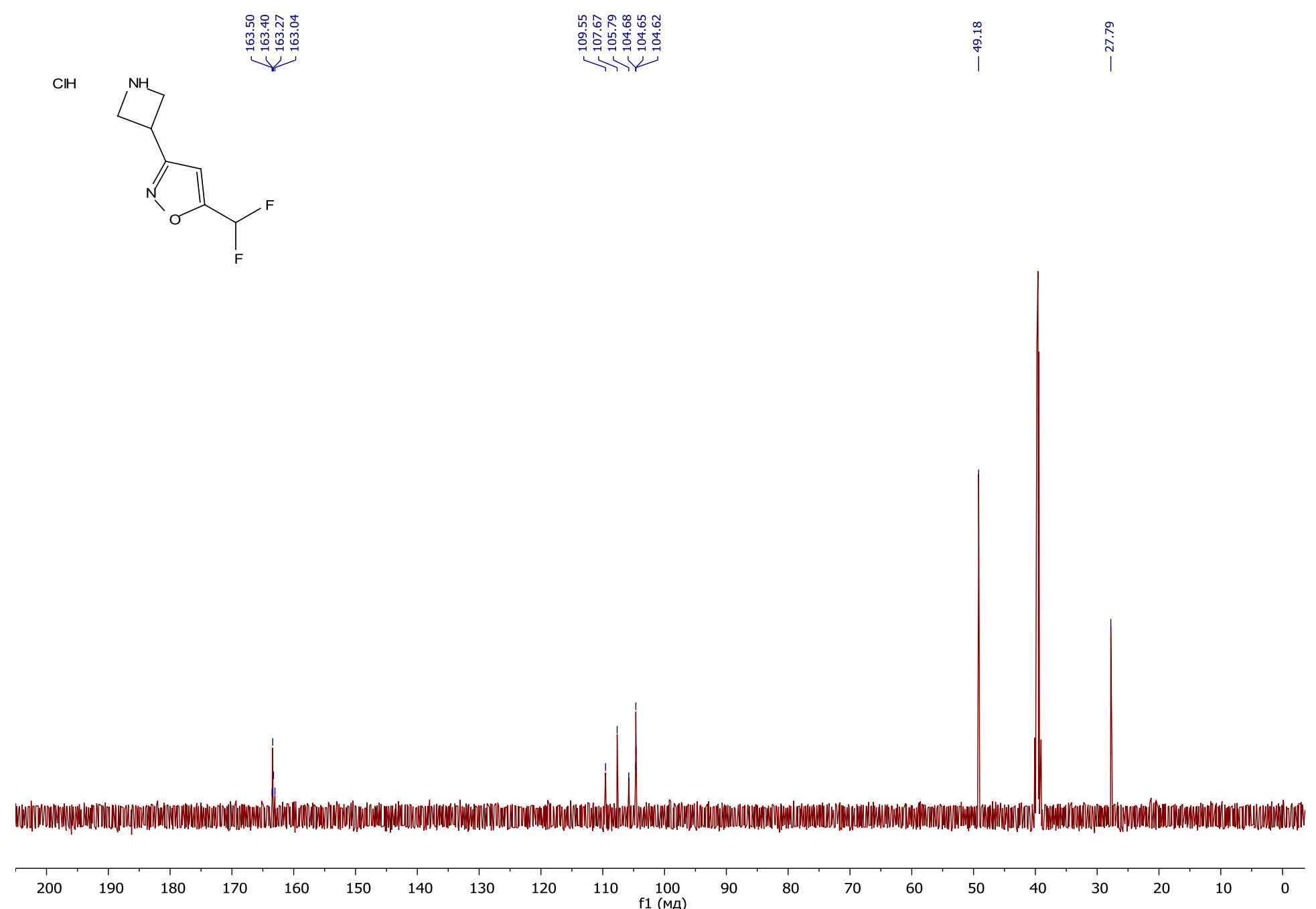

3-(Azetidin-3-yl)-5-(difluoromethyl)isoxazole hydrochloride (21j·HCl) ${ }^{13} \mathrm{C}$ NMR 
$\mathrm{ClH}$
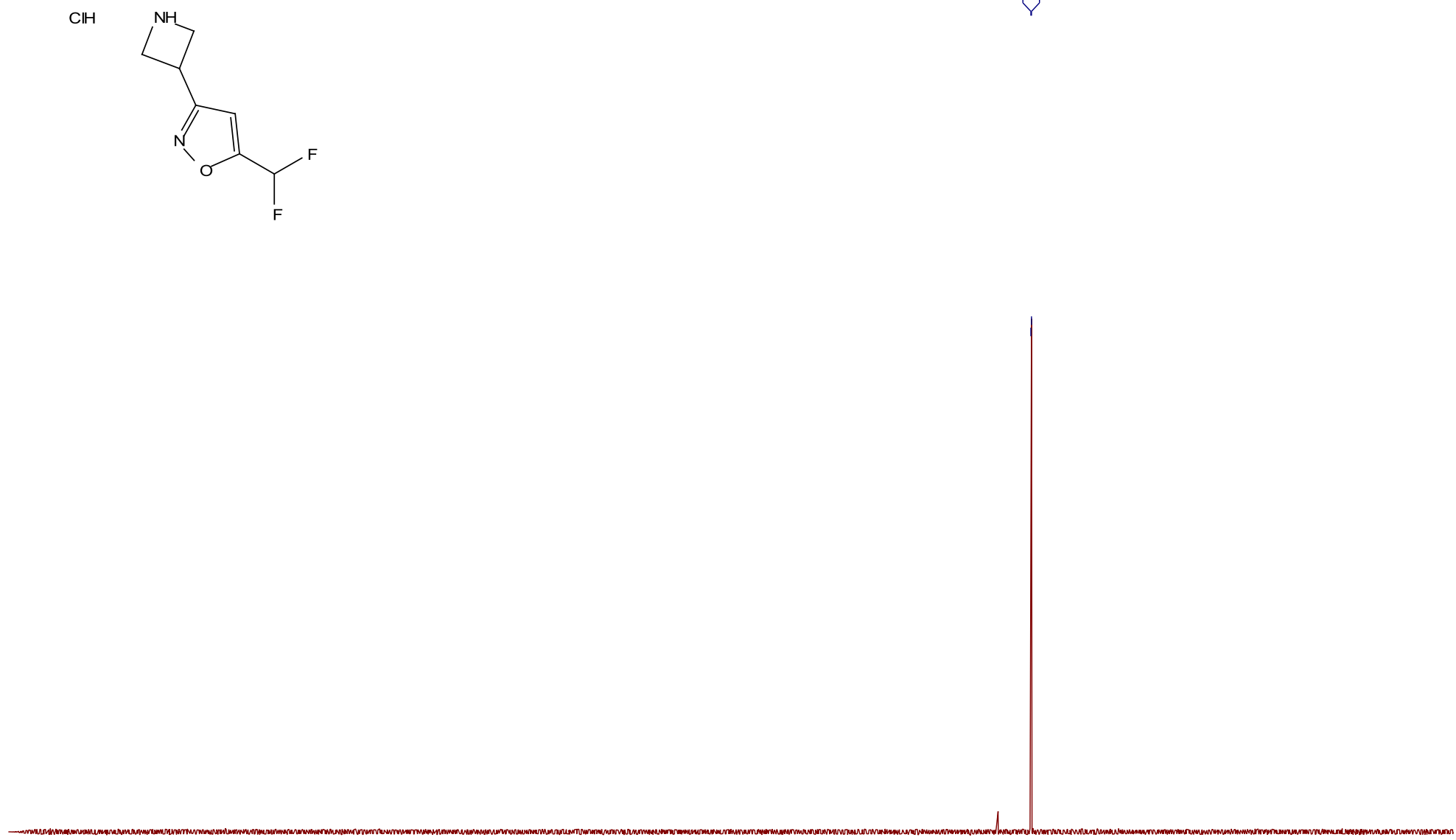

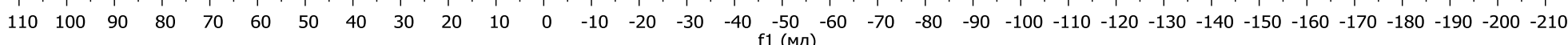
3-(Azetidin-3-yl)-5-(difluoromethyl)isoxazole hydrochloride (21j·HCl) ${ }^{19}$ F NMR 

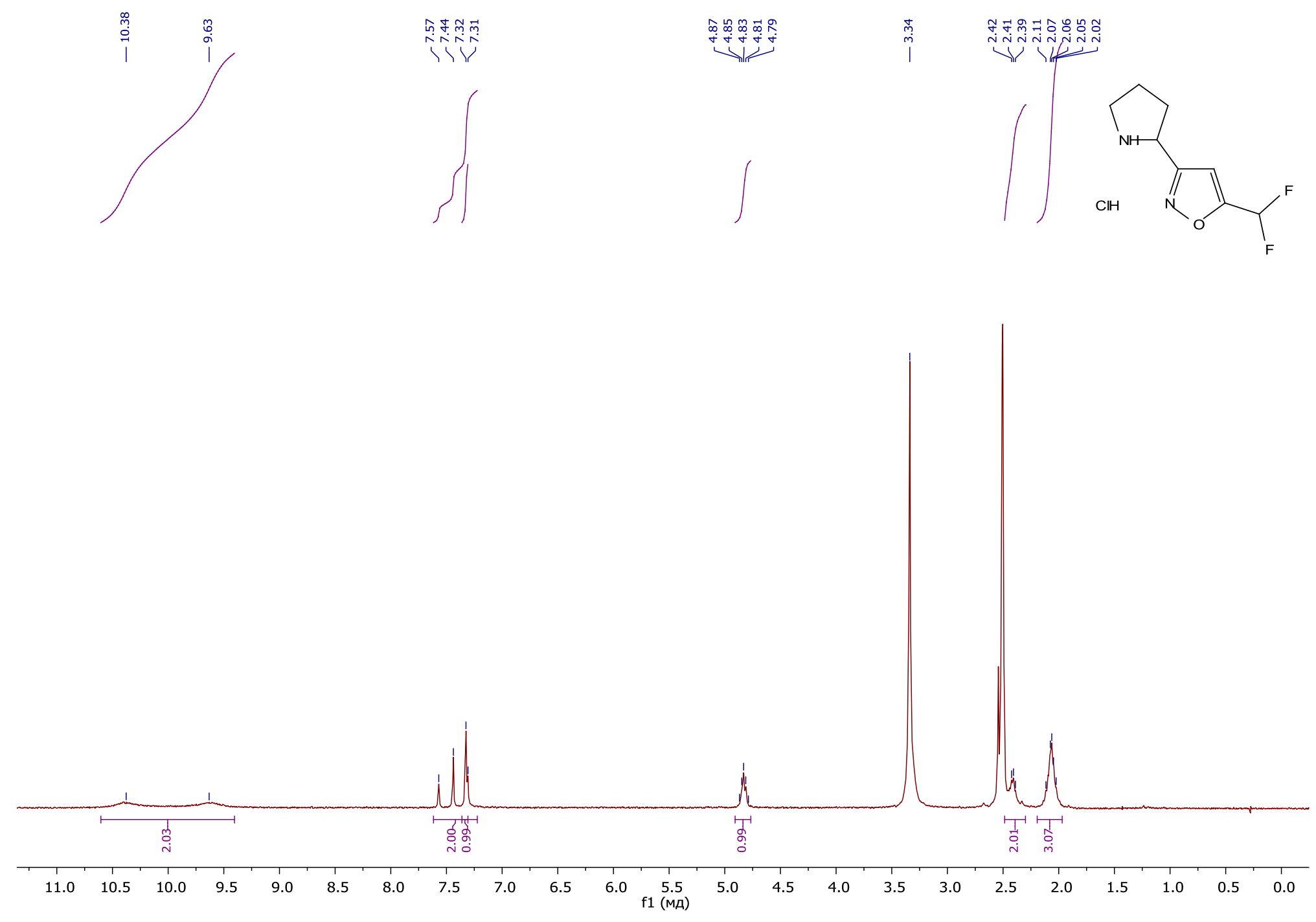

5-(Difluoromethyl)-3-(pyrrolidin-2-yl)isoxazole hydrochloride (21k·HCl or $211 \cdot \mathrm{HCl}){ }^{1} \mathrm{H} \mathrm{NMR}$ 

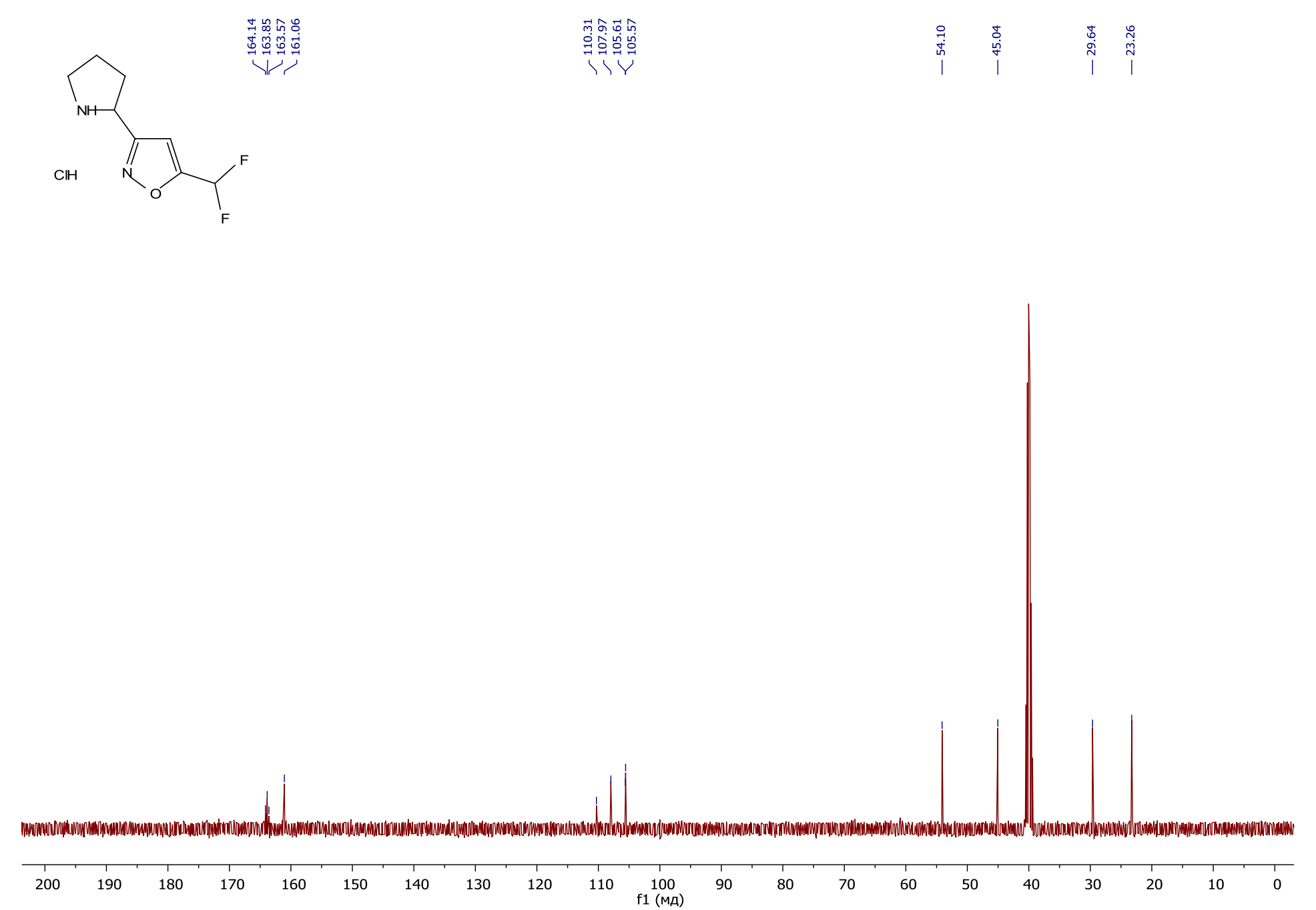

5-(Difluoromethyl)-3-(pyrrolidin-2-yl)isoxazole hydrochloride $(21 \mathrm{k} \cdot \mathrm{HCl}$ or $211 \cdot \mathrm{HCl}){ }^{13} \mathrm{C} \mathrm{NMR}$ 


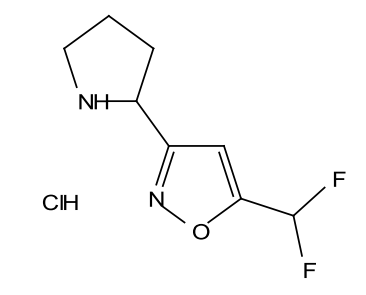

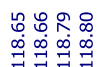

芦

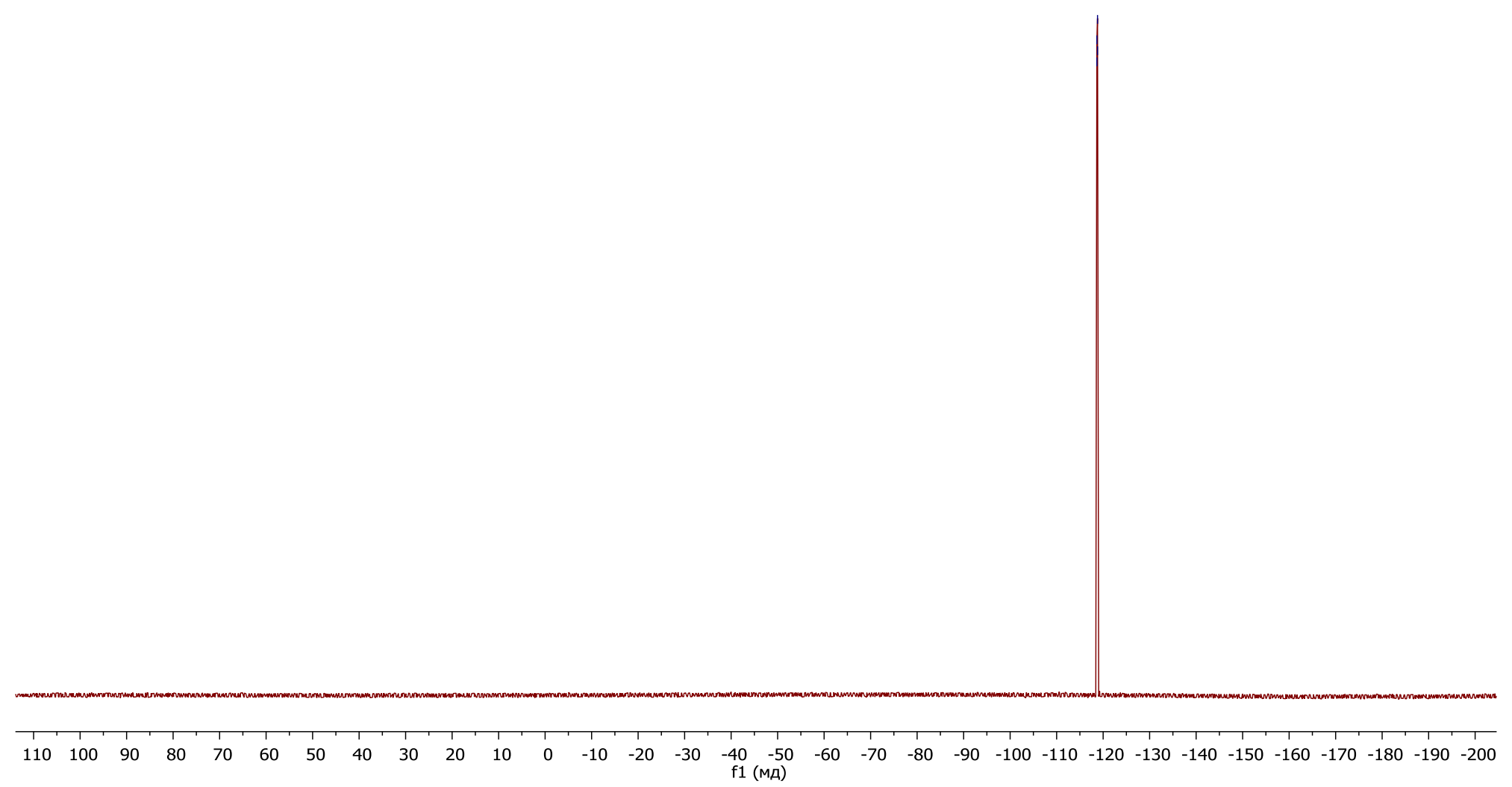

5-(Difluoromethyl)-3-(pyrrolidin-2-yl)isoxazole hydrochloride (21k $\cdot \mathrm{HCl}$ or $211 \cdot \mathrm{HCl}){ }^{19} \mathrm{~F}$ NMR 

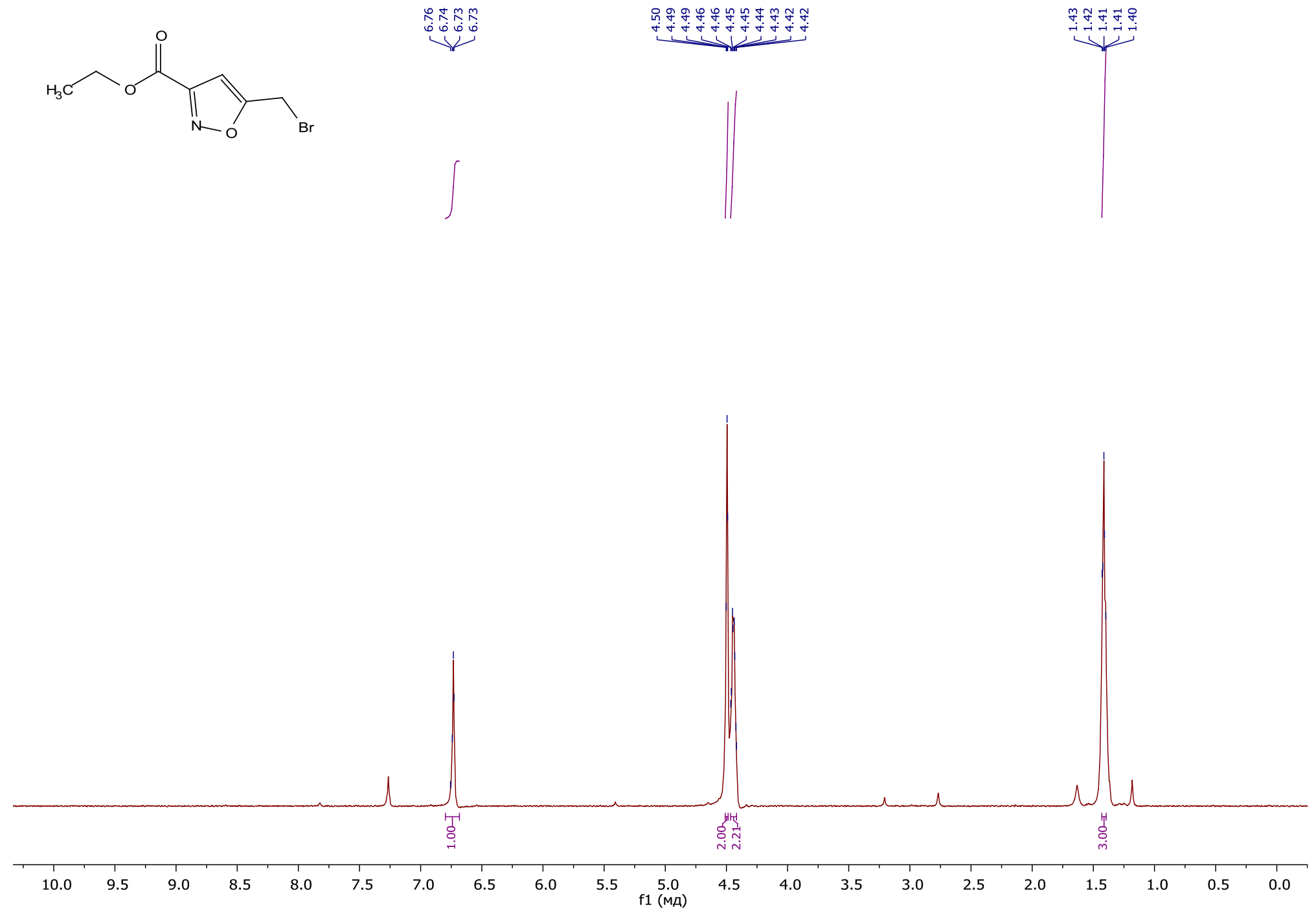

Ethyl 5-(bromomethyl)isoxazole-3-carboxylate (22a) ${ }^{1}$ H NMR 


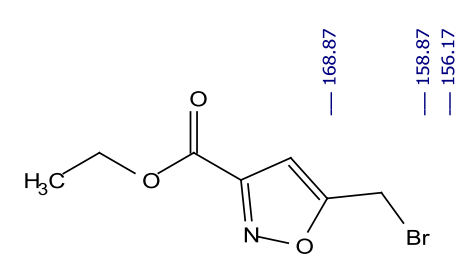

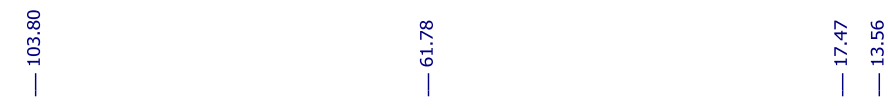

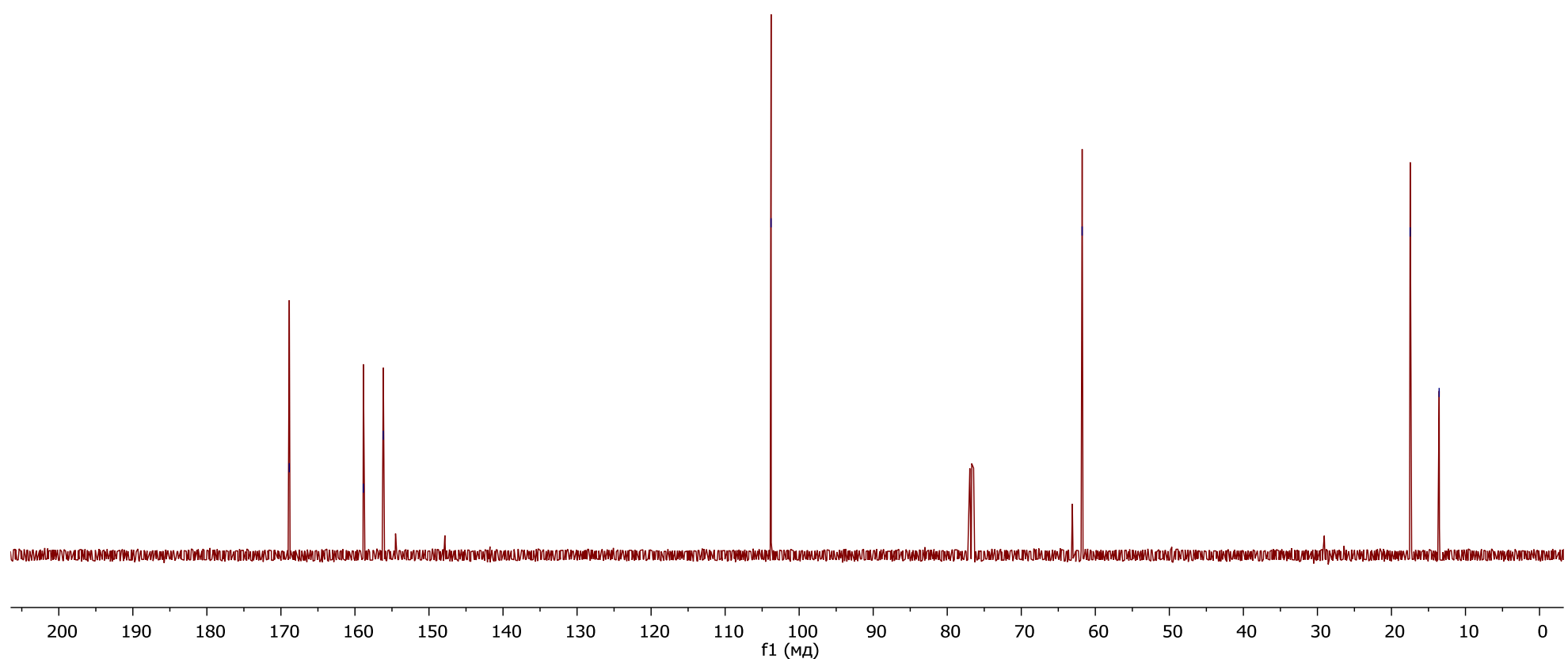

Ethyl 5-(bromomethyl)isoxazole-3-carboxylate (22a) ${ }^{13} \mathrm{C}$ NMR 


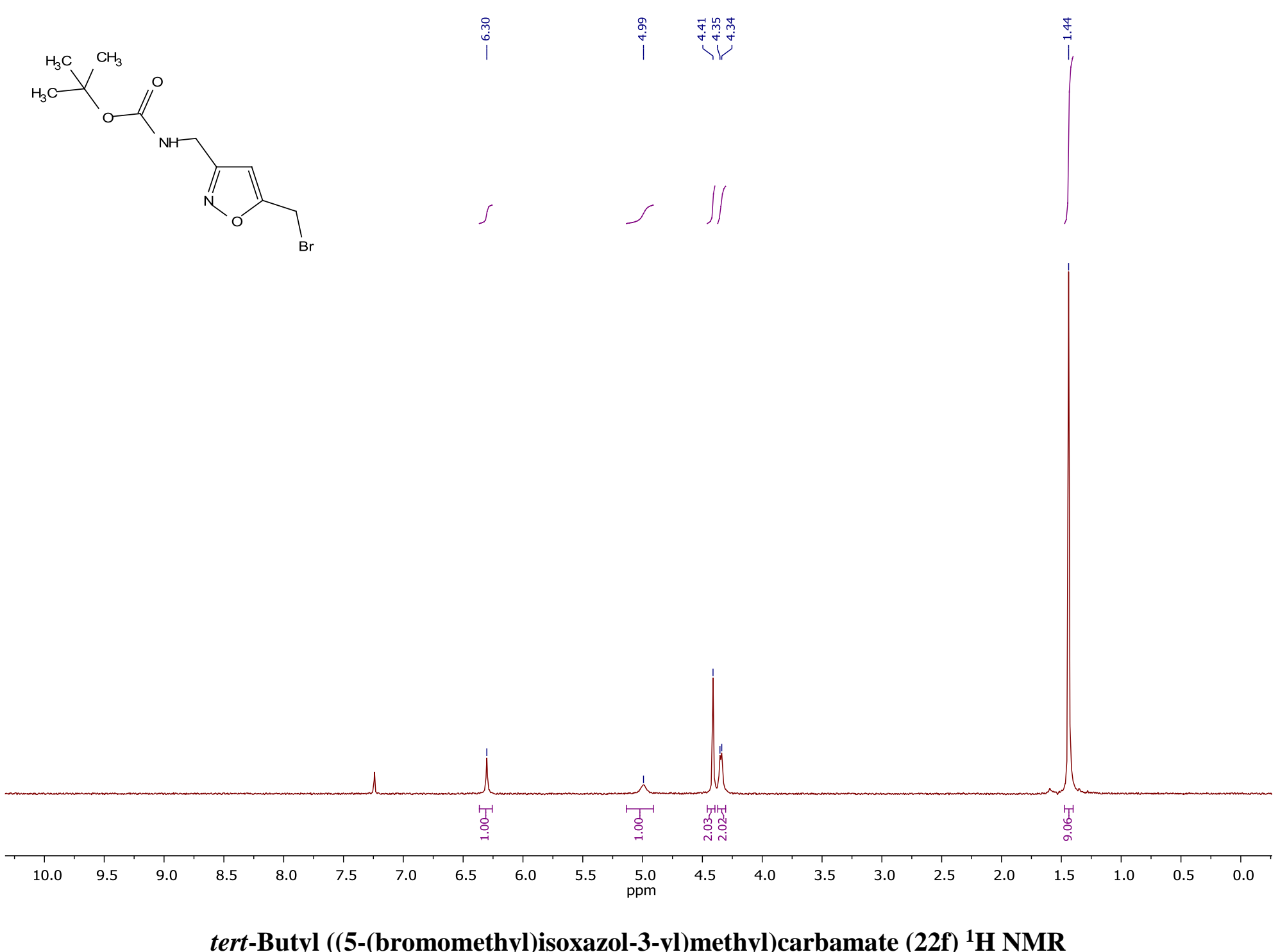

tert-Butyl ((5-(bromomethyl)isoxazol-3-yl)methyl)carbamate (22f) ${ }^{1} \mathrm{H}$ NMR 

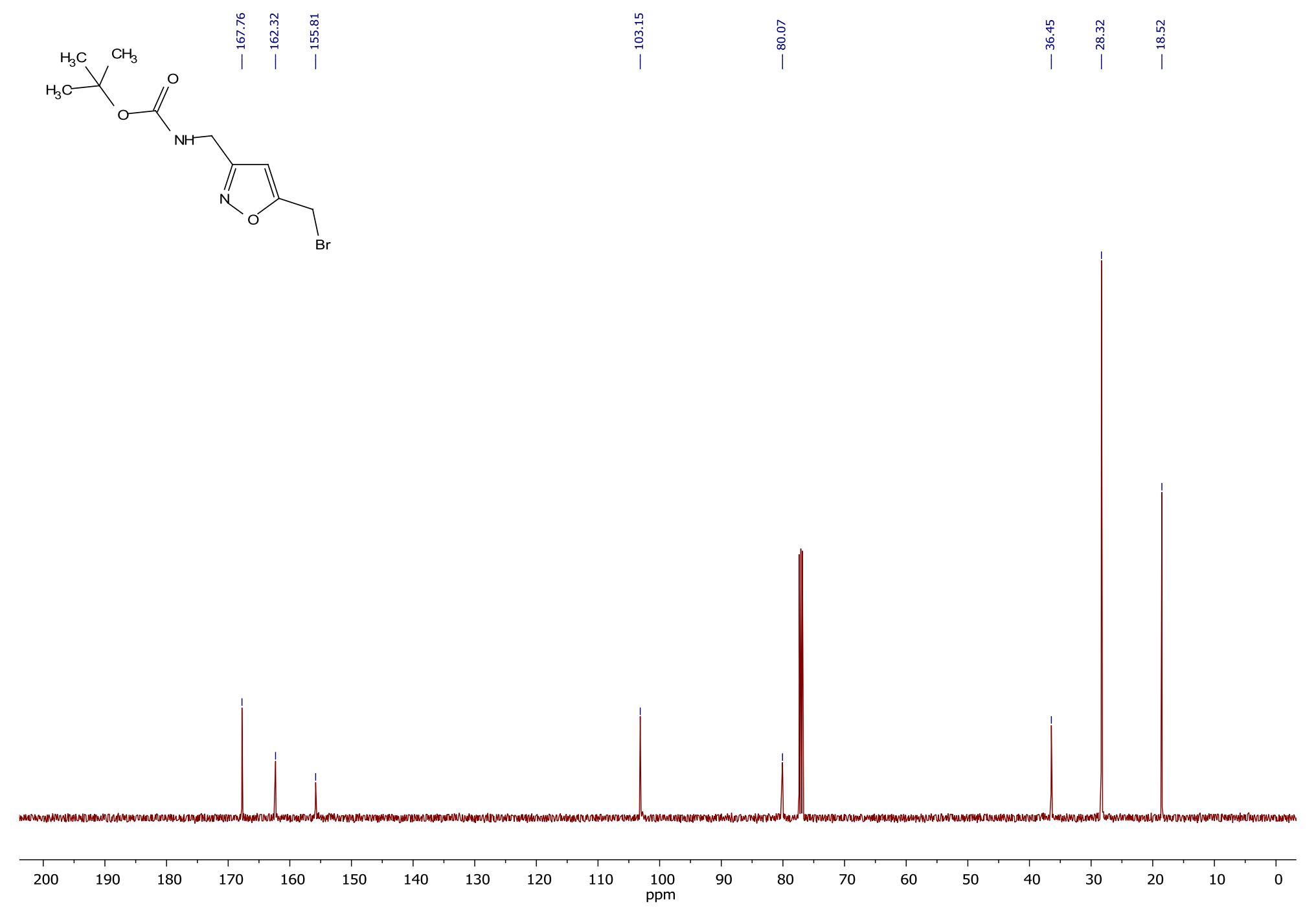

tert-Butyl ((5-(bromomethyl)isoxazol-3-yl)methyl)carbamate (22f) ${ }^{13} \mathrm{C}$ NMR 

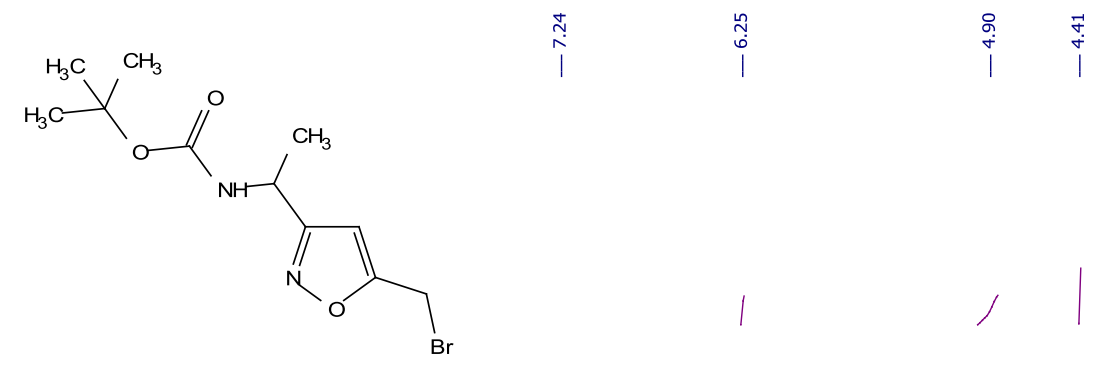

$\mathrm{Br}$

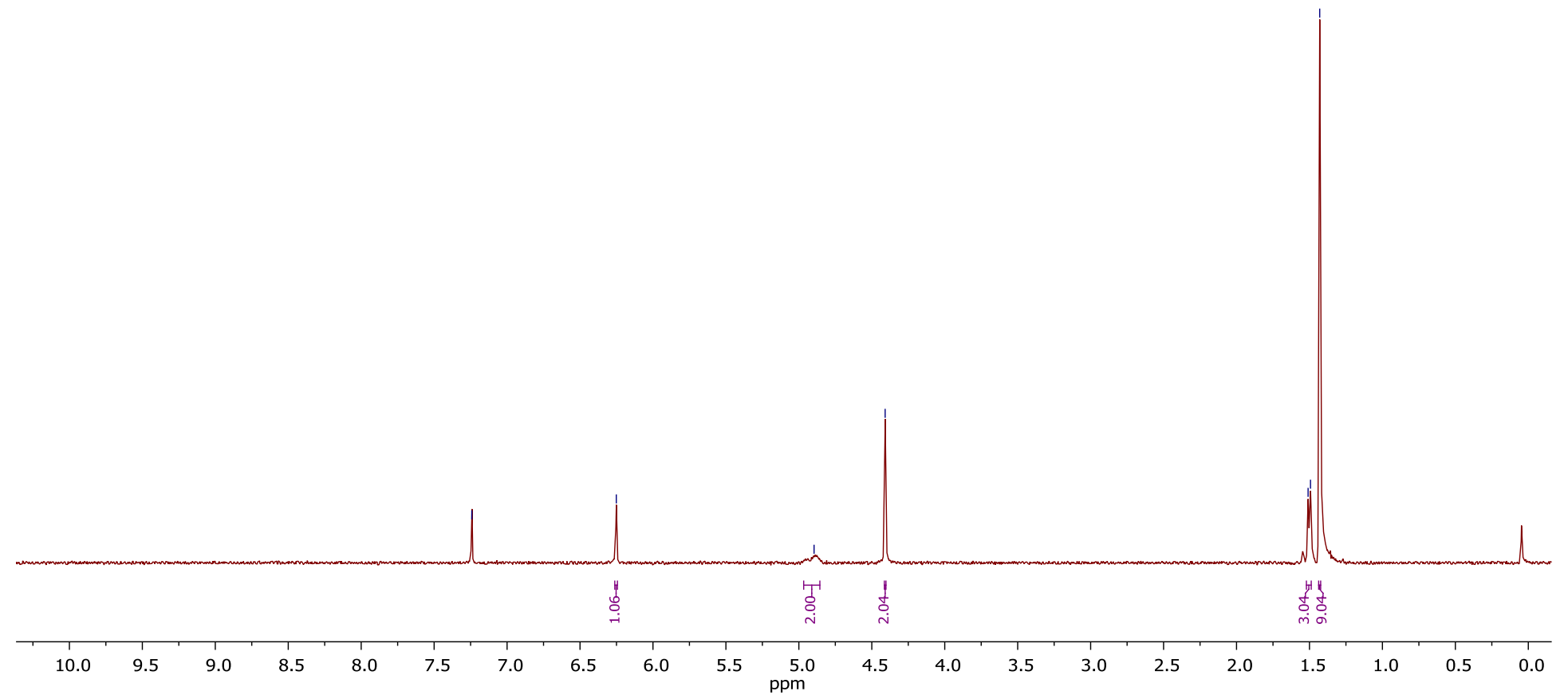

(R)-tert-Butyl (1-(5-(bromomethyl)isoxazol-3-yl)ethyl)carbamate (22g or $22 \mathrm{~h}){ }^{1} \mathrm{H}$ NMR 


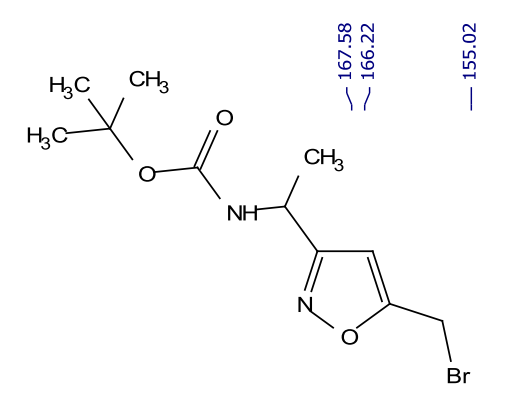

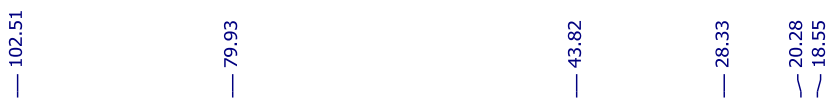

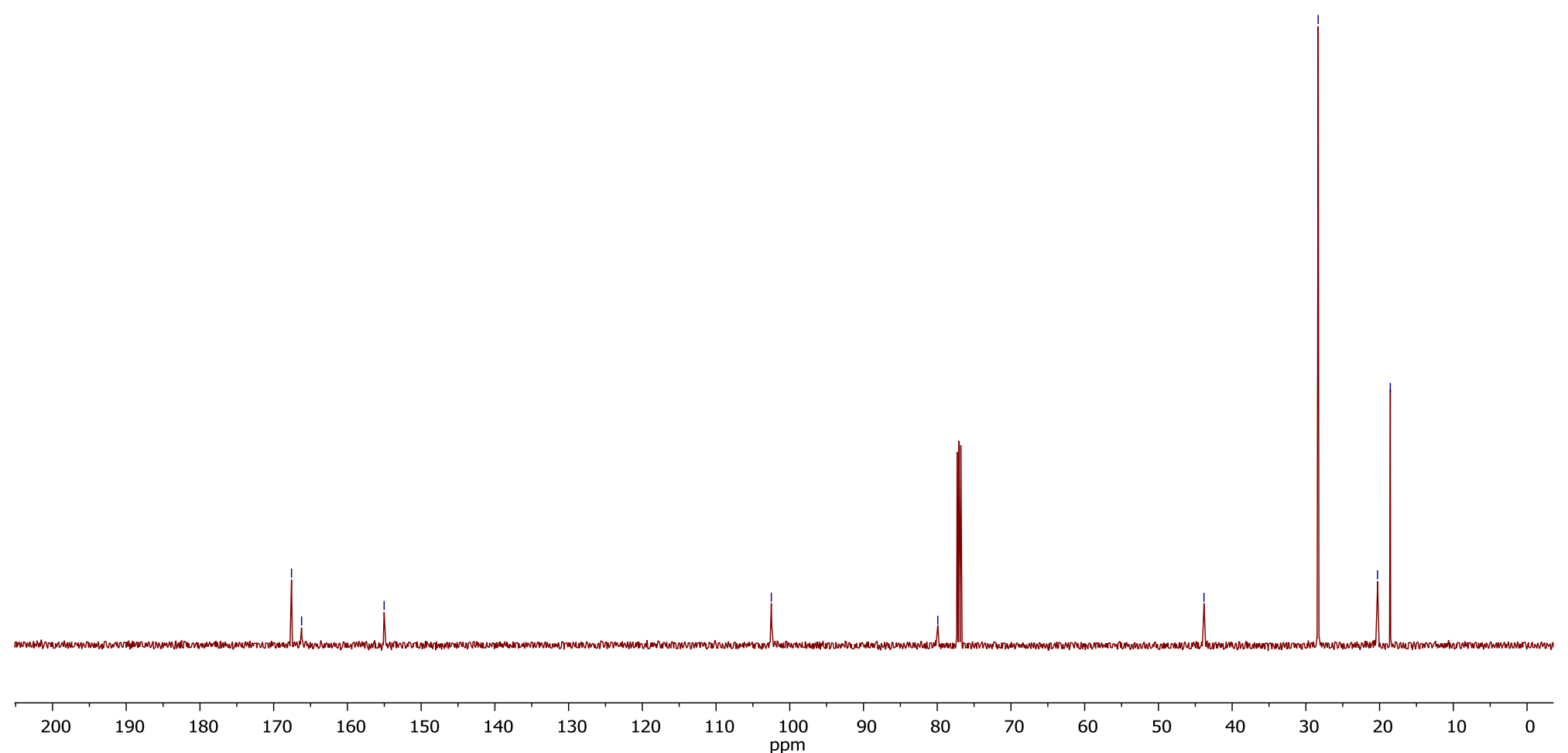

(R)-tert-Butyl (1-(5-(bromomethyl)isoxazol-3-yl)ethyl)carbamate (22g or $22 \mathrm{~h}){ }^{13} \mathrm{C} \mathrm{NMR}$ 


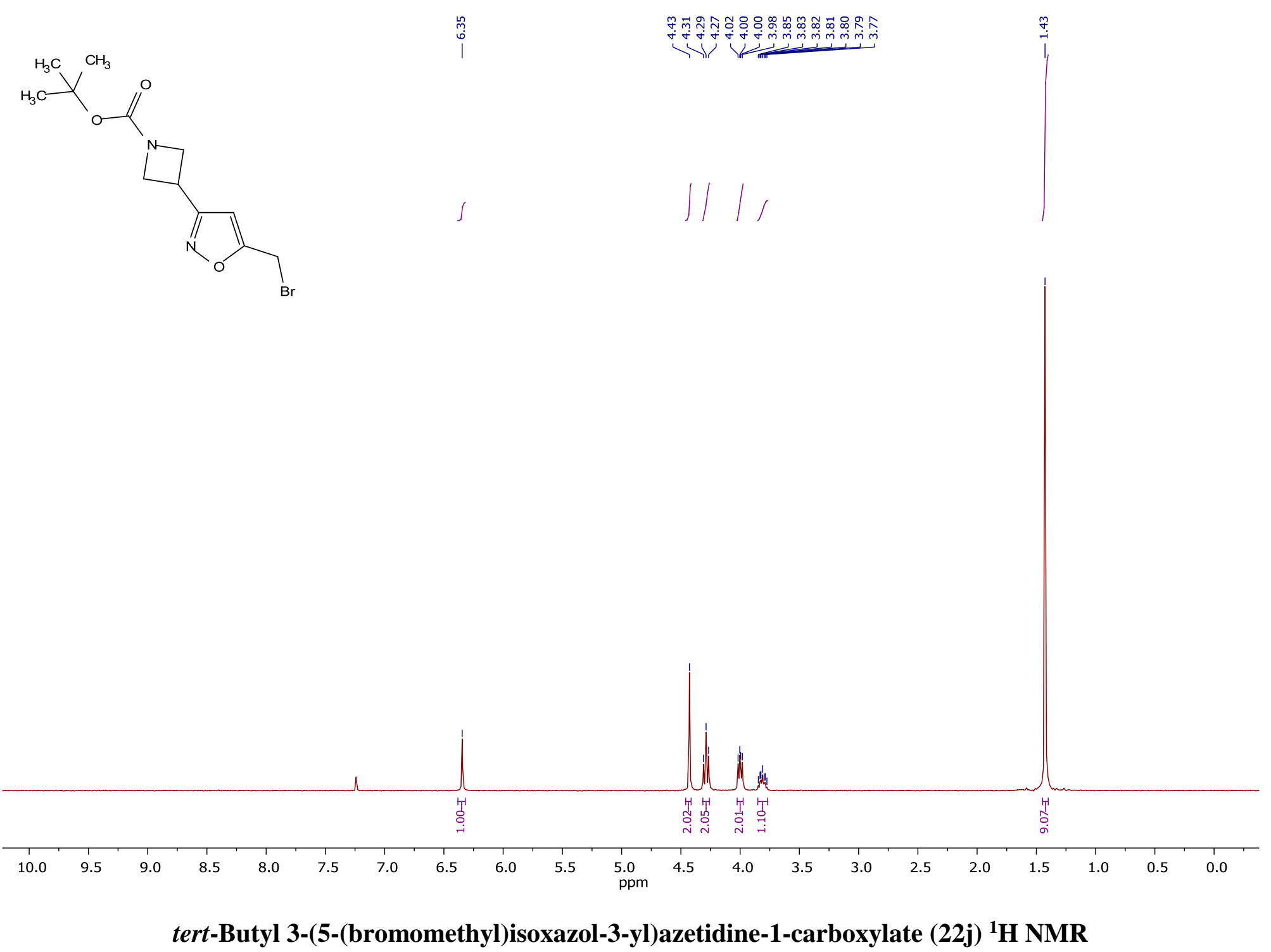



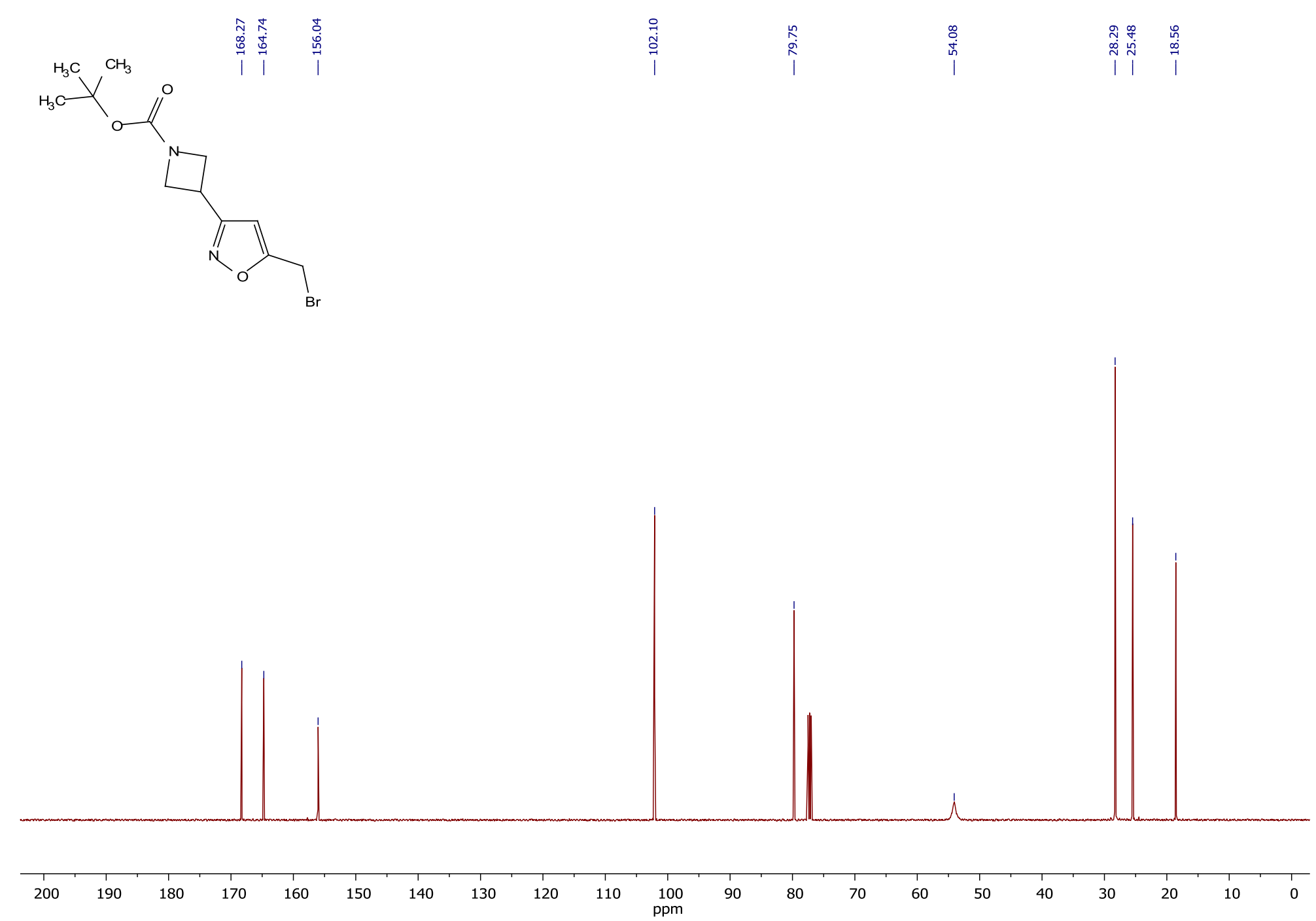

tert-Butyl 3-(5-(bromomethyl)isoxazol-3-yl)azetidine-1-carboxylate (22j) ${ }^{13} \mathrm{C}$ NMR 


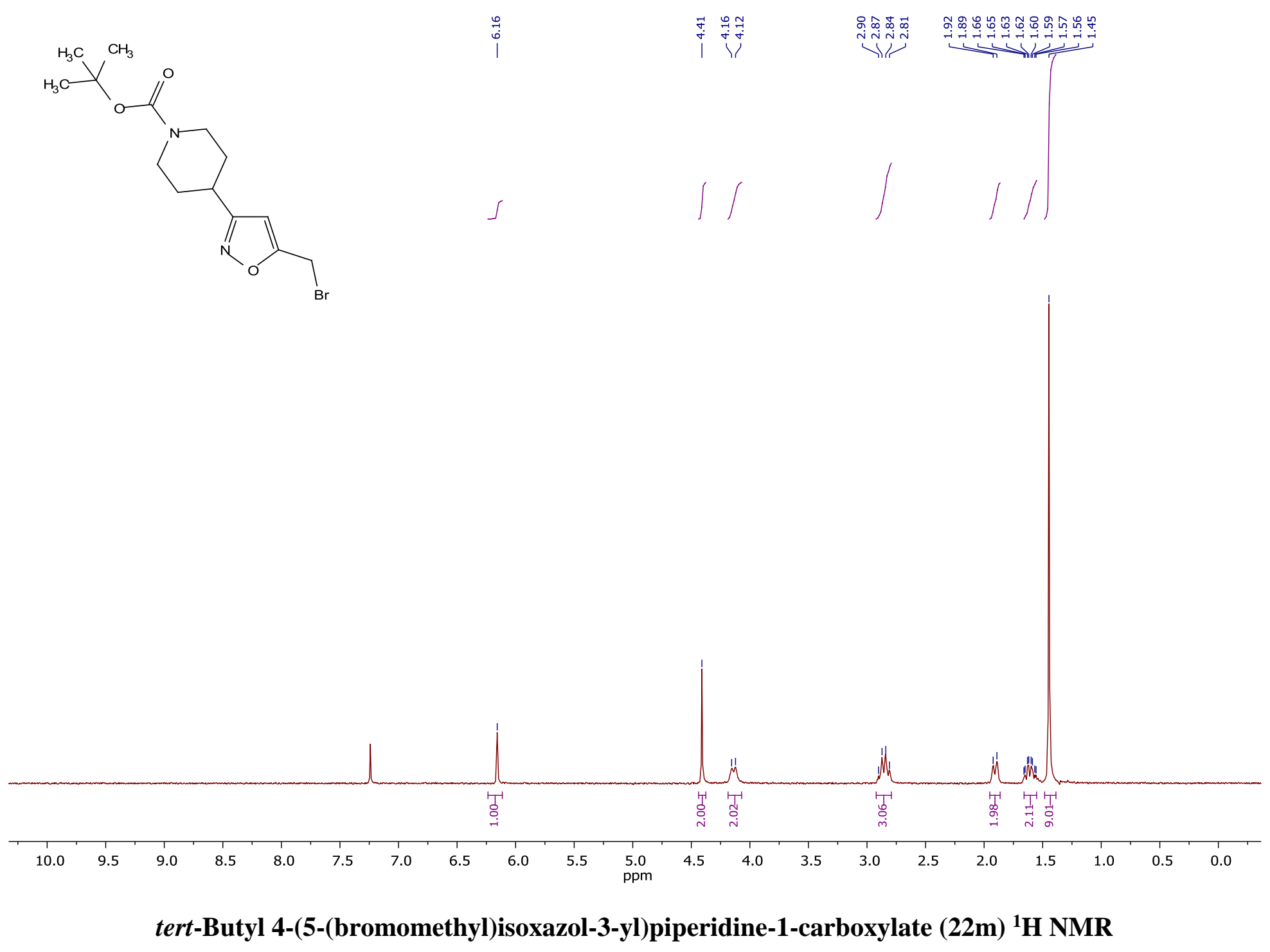




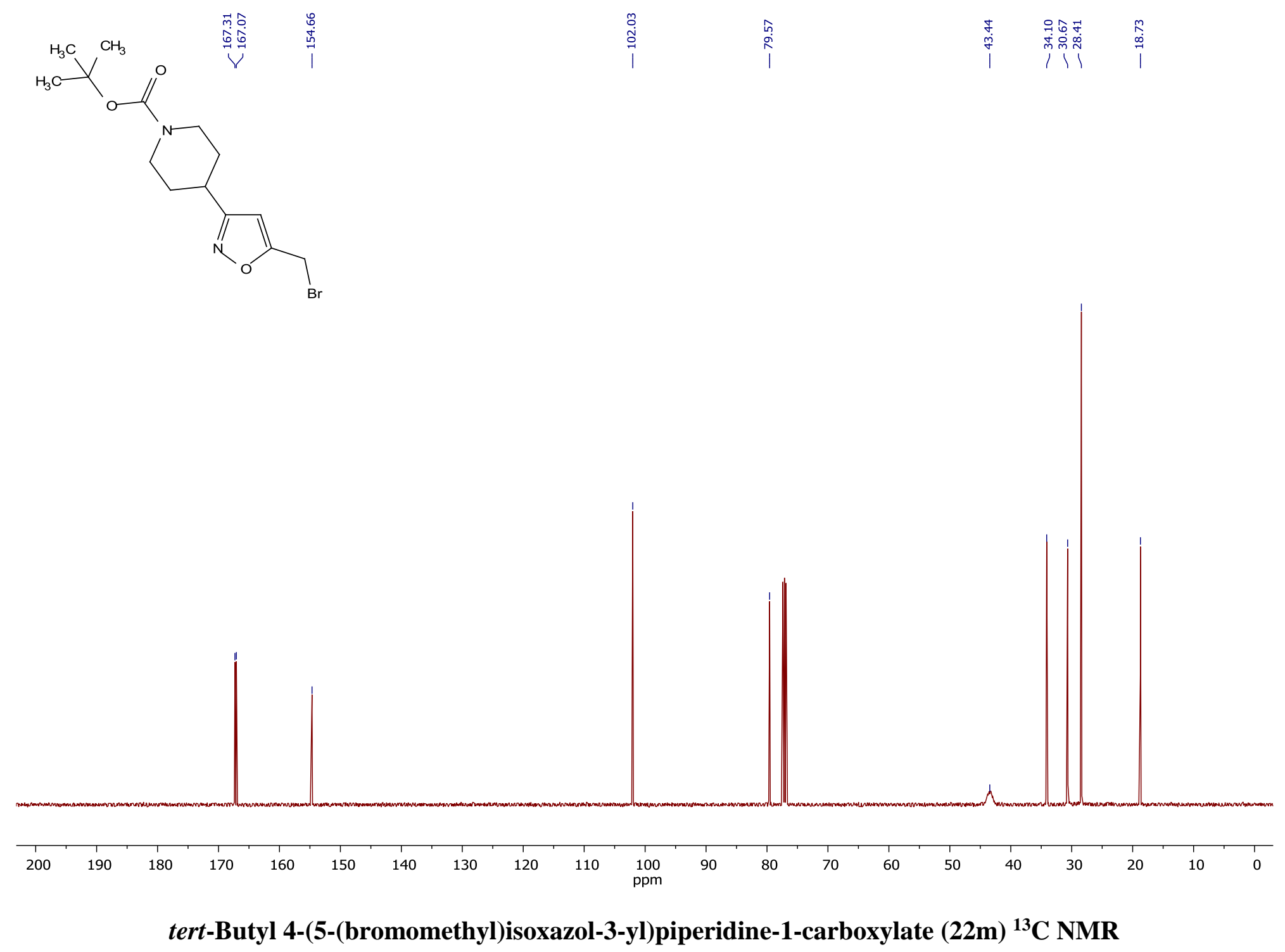



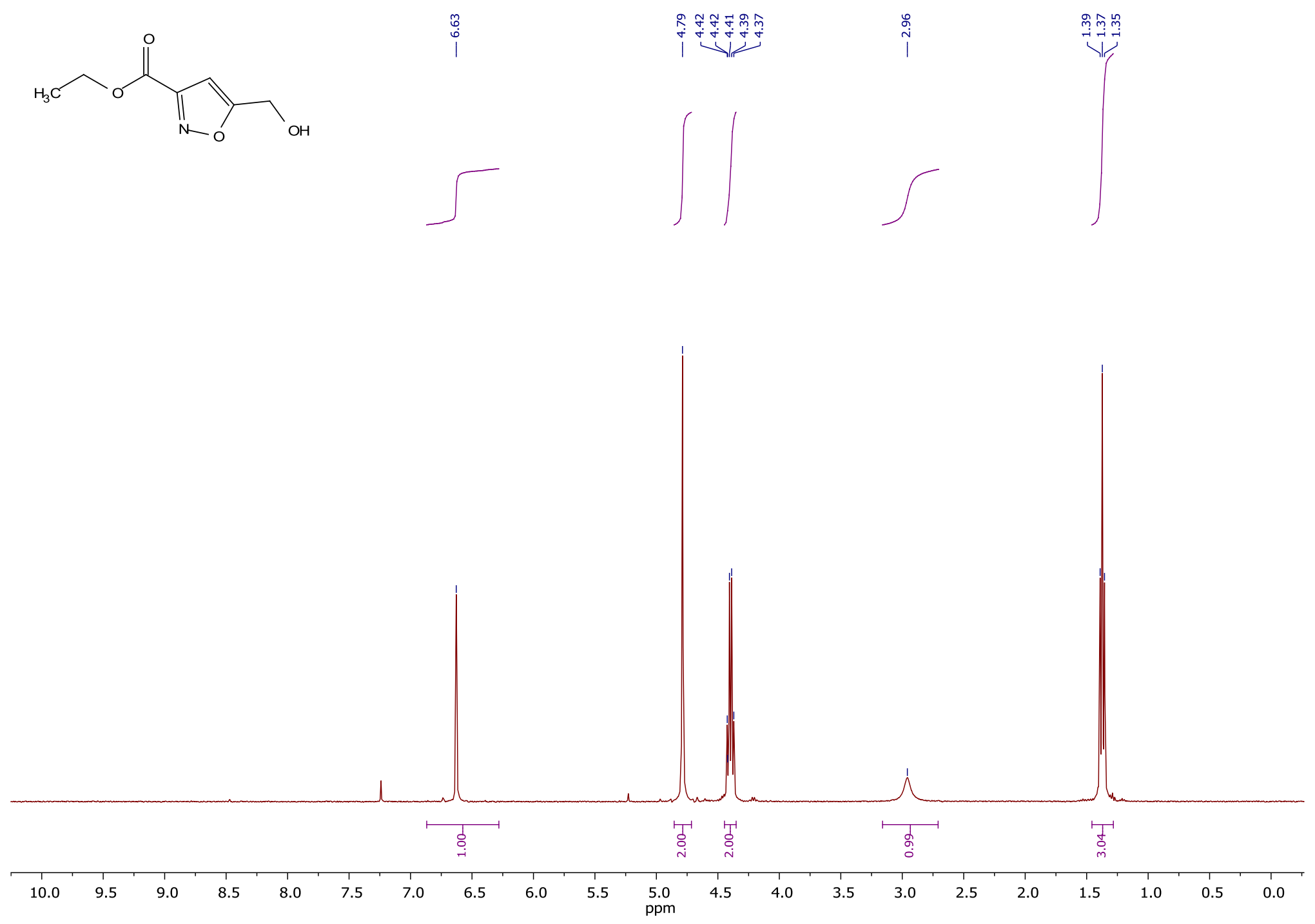

Ethyl 5-(hydroxymethyl)isoxazole-3-carboxylate (23) ${ }^{1}$ H NMR 


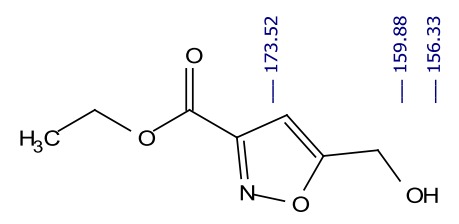

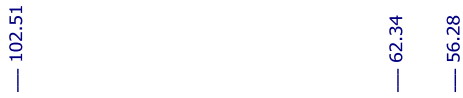

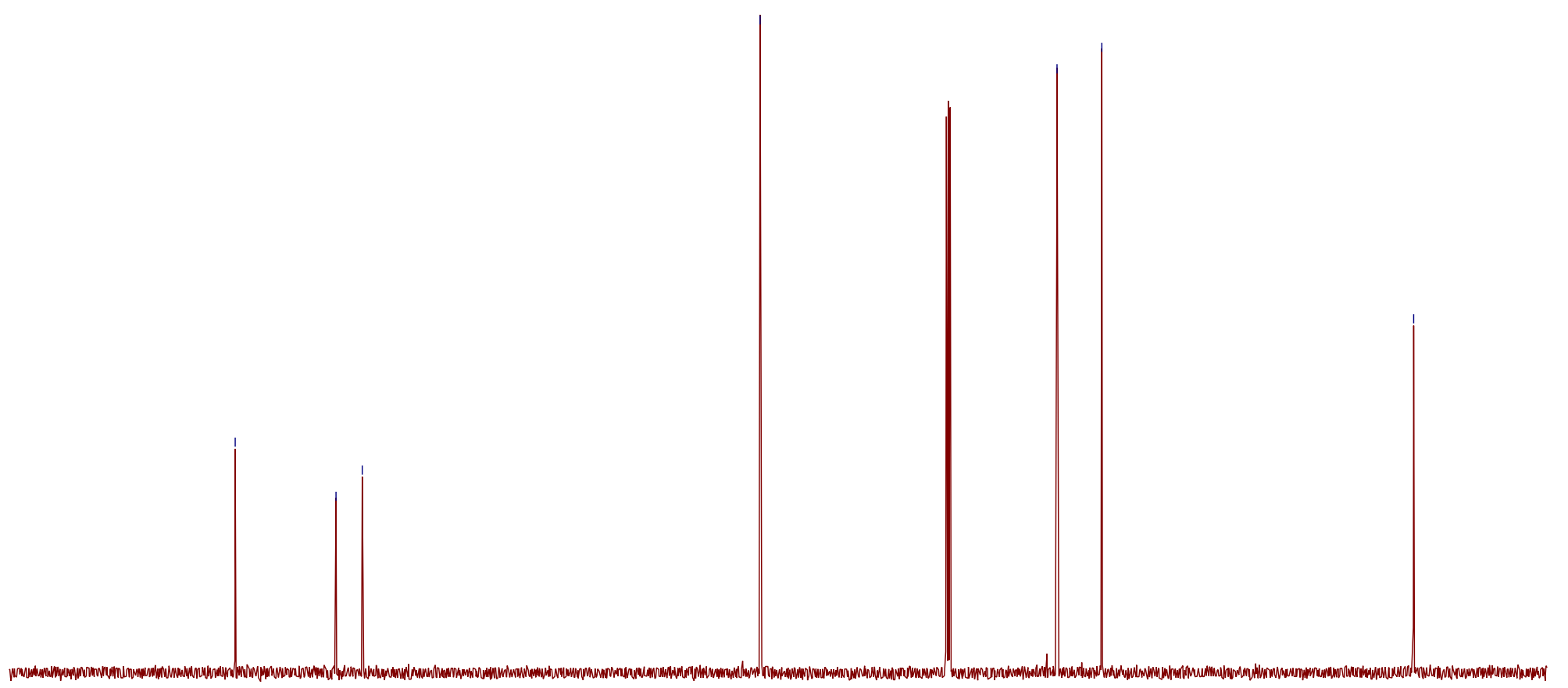

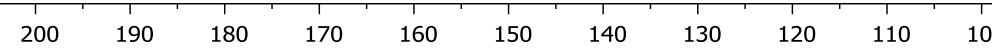

Ethyl 5-(hydroxymethyl)isoxazole-3-carboxylate (23) ${ }^{13}$ C NMR 

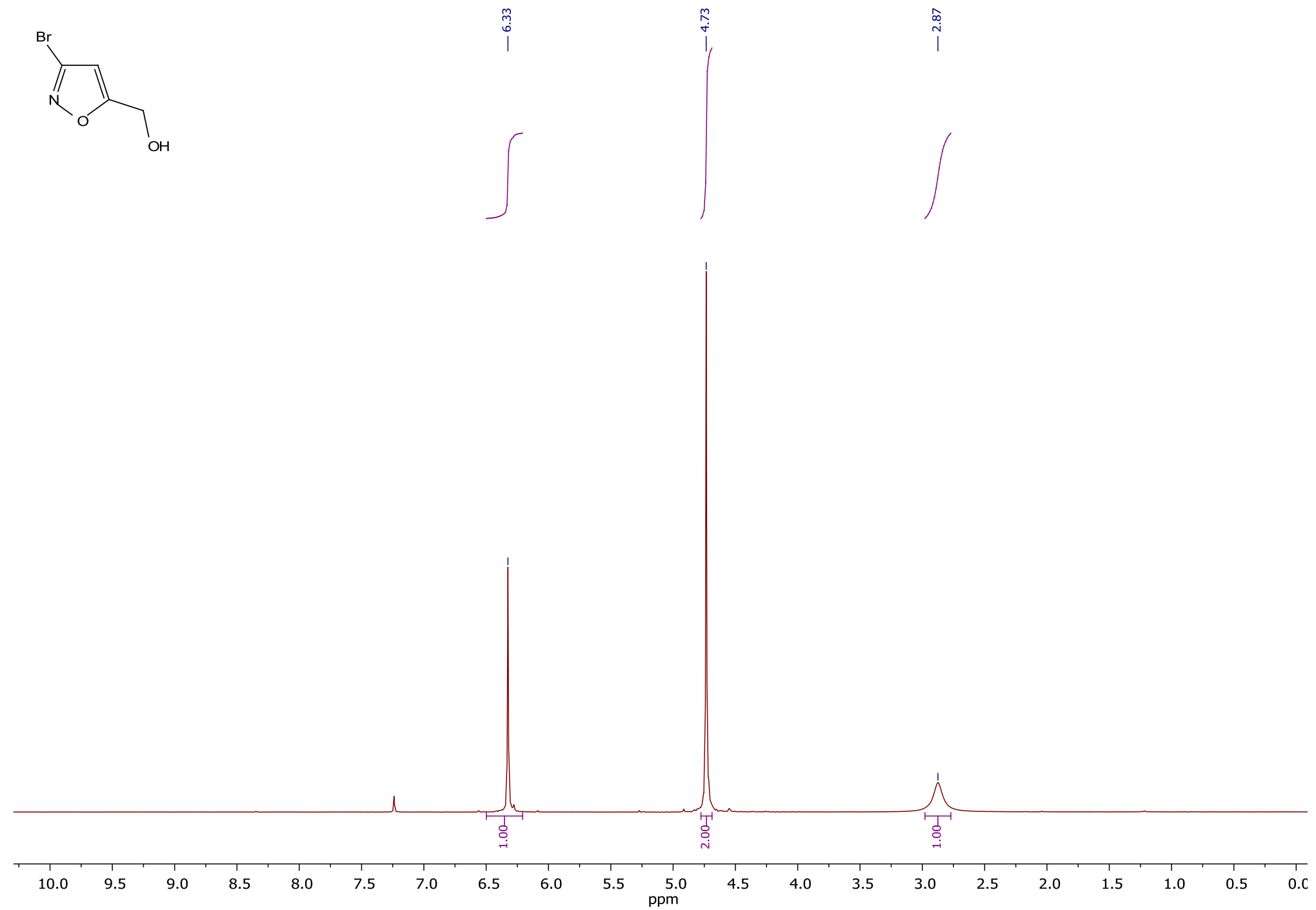

(3-Bromoisoxazol-5-yl)methanol (24) ${ }^{1}$ H NMR 

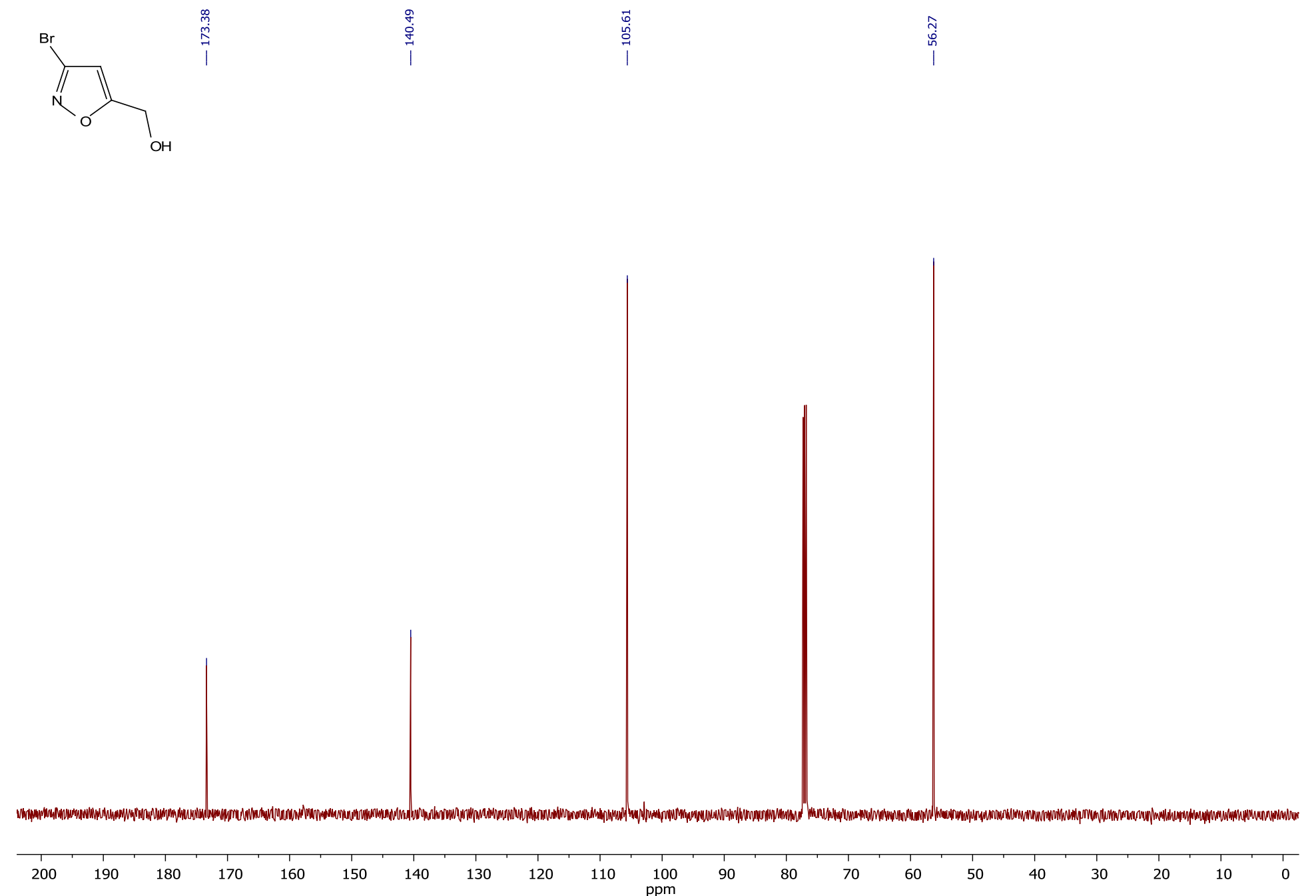

(3-Bromoisoxazol-5-yl)methanol (24) ${ }^{13}$ C NMR 

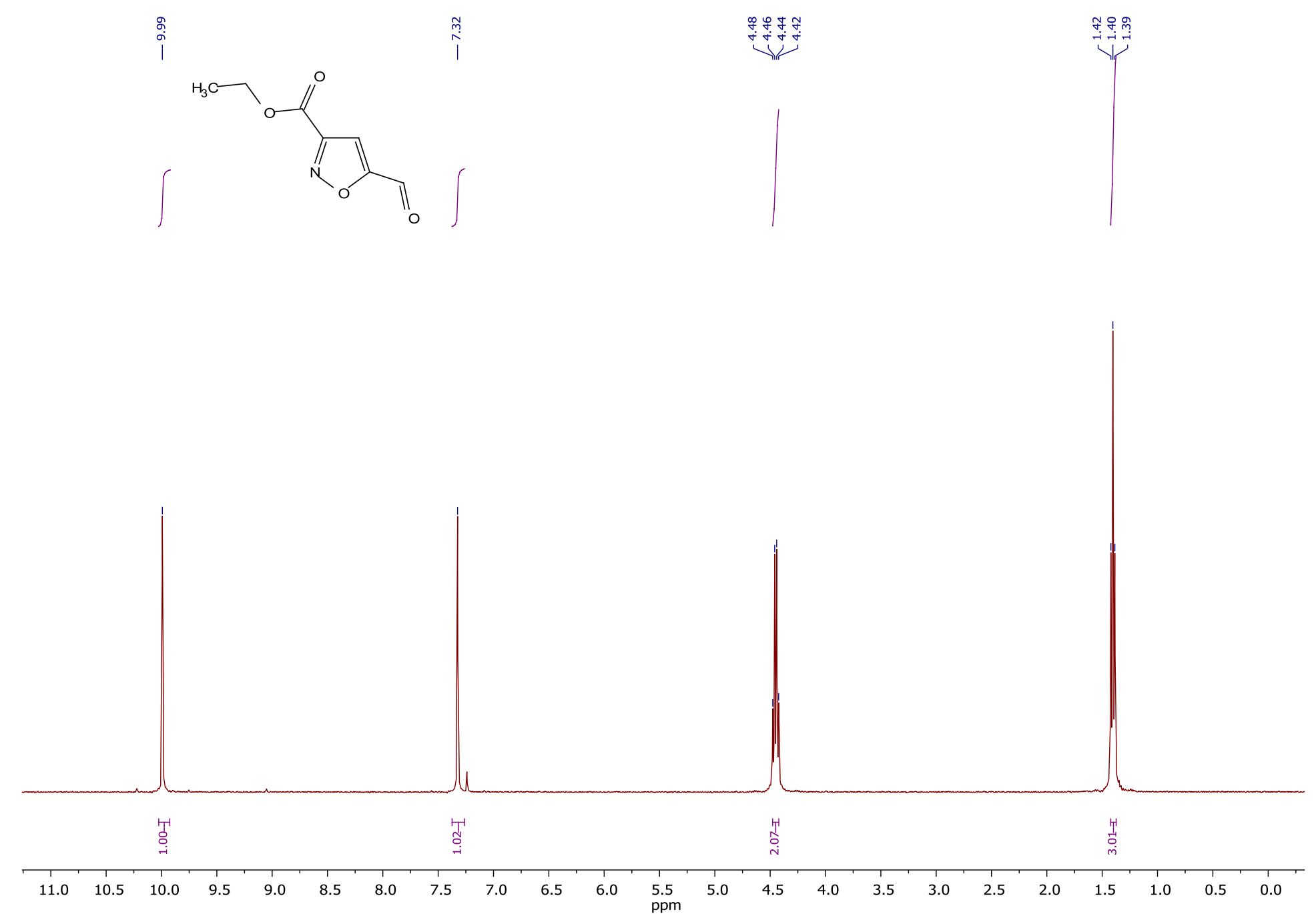

Ethyl 5-formylisoxazole-3-carboxylate (25) ${ }^{1}$ H NMR 

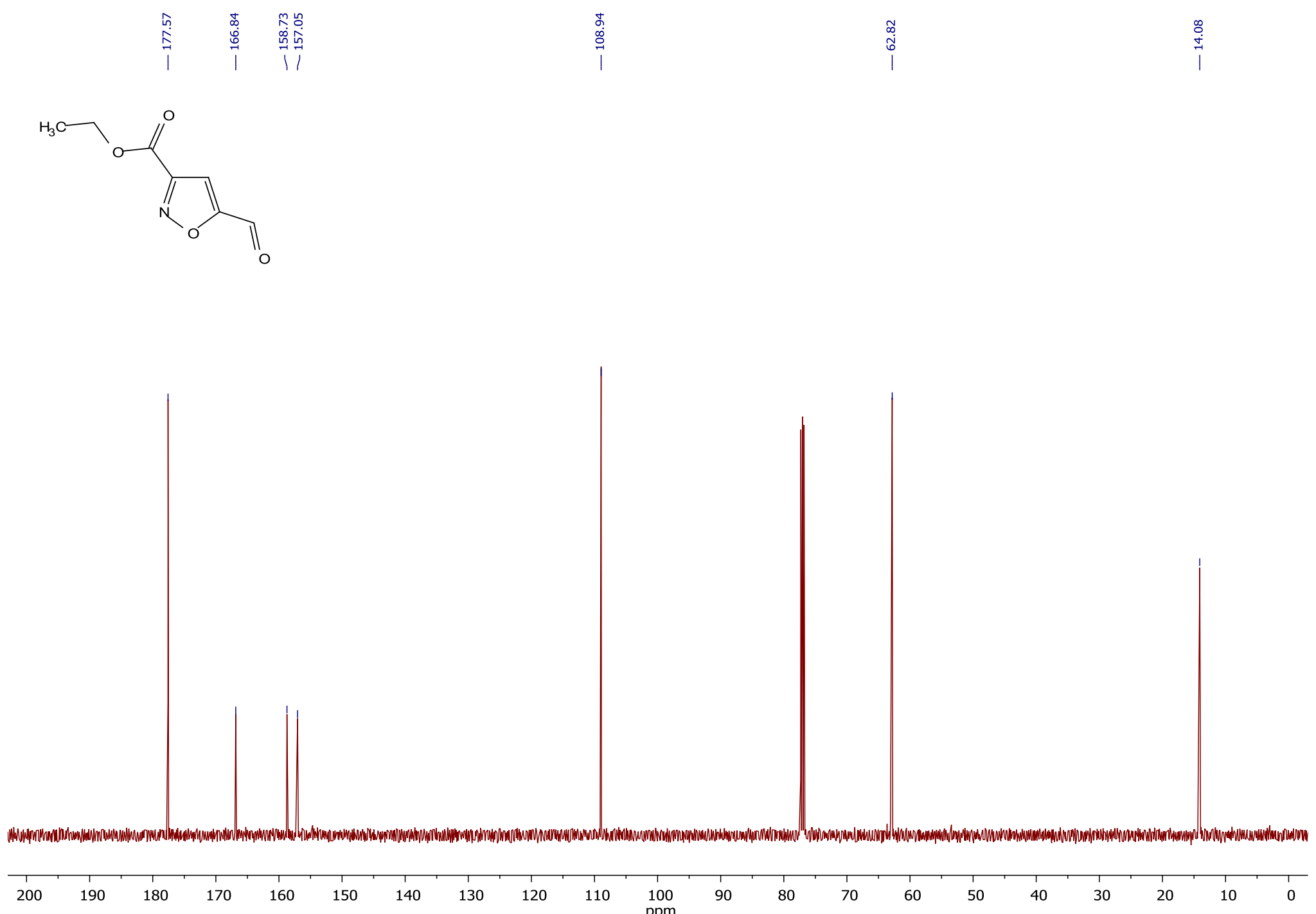

Ethyl 5-formylisoxazole-3-carboxylate (25) ${ }^{13} \mathrm{C}$ NMR 


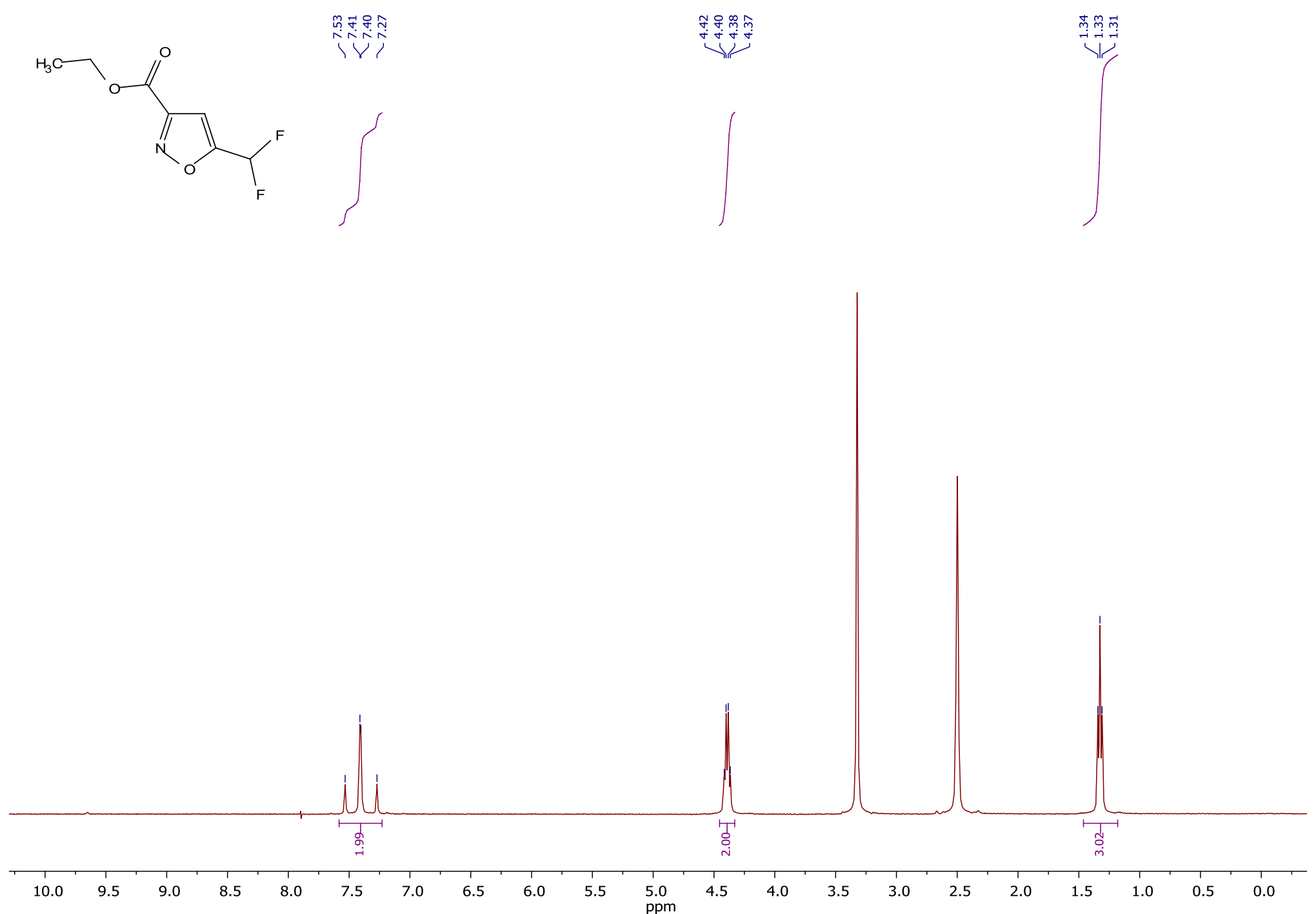

Ethyl 5-(difluoromethyl)isoxazole-3-carboxylate (27) ${ }^{1}$ H NMR 

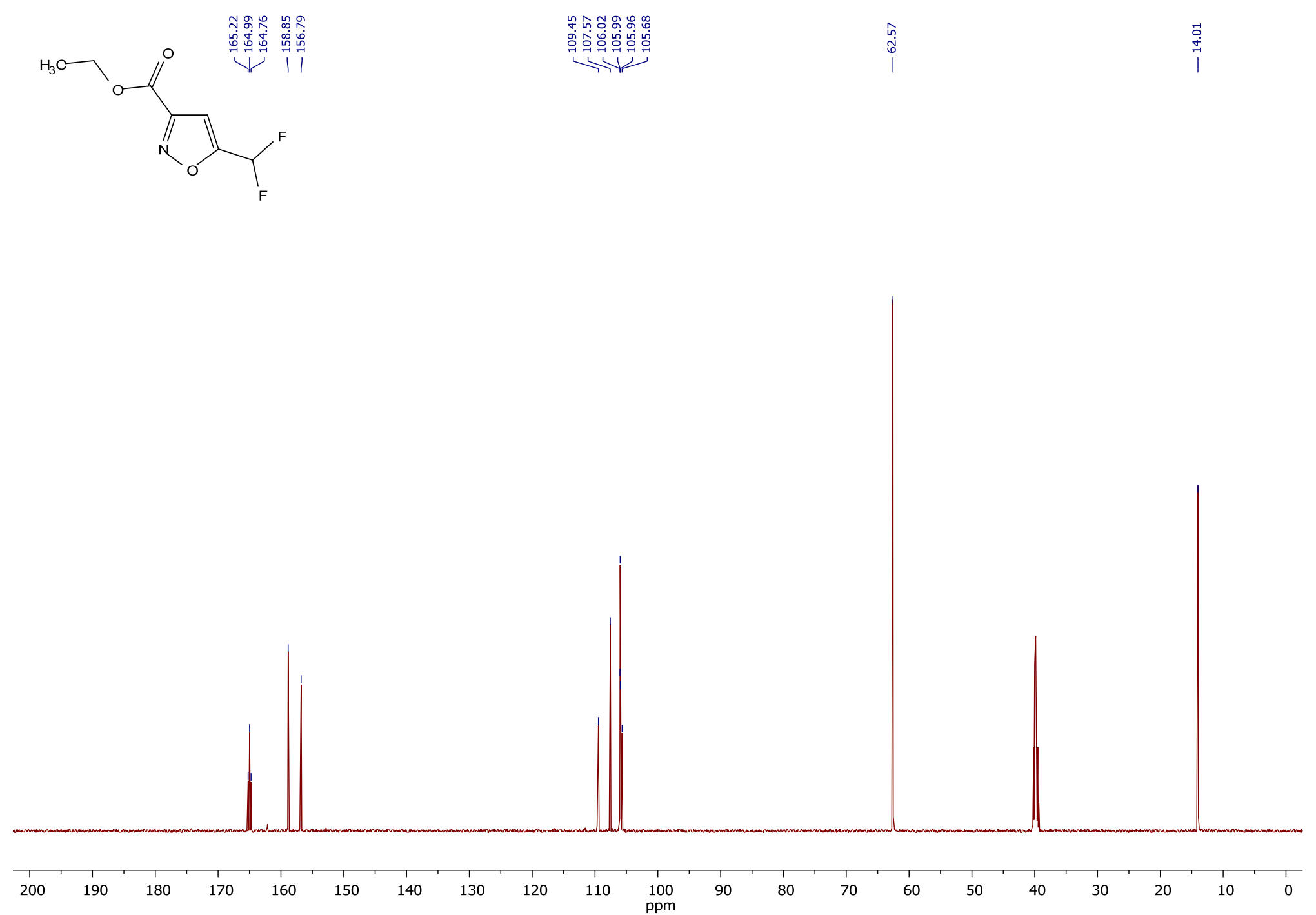

Ethyl 5-(difluoromethyl)isoxazole-3-carboxylate (27) ${ }^{13} \mathrm{C}$ NMR 

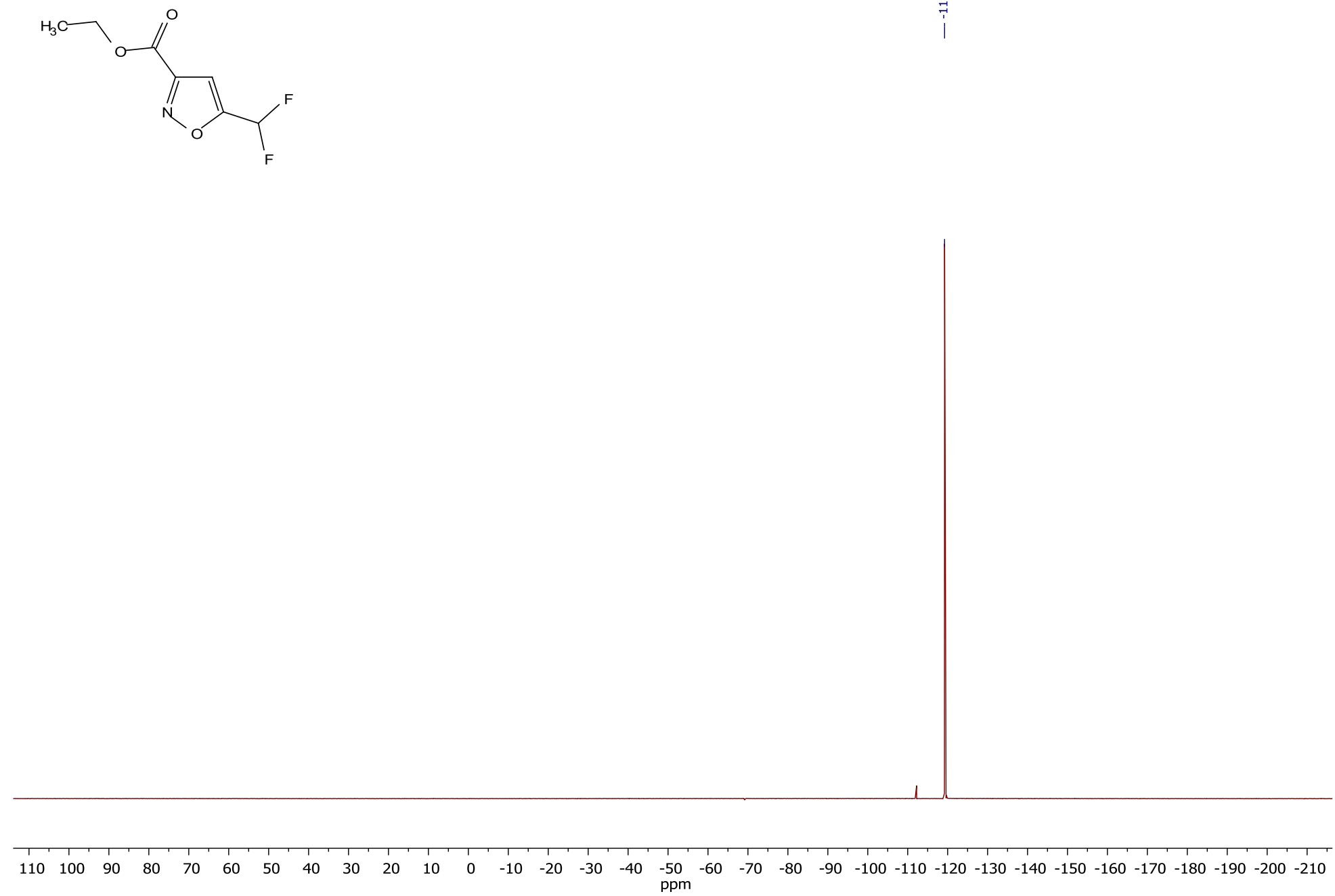

Ethyl 5-(difluoromethyl)isoxazole-3-carboxylate (27) ${ }^{19}$ F NMR 


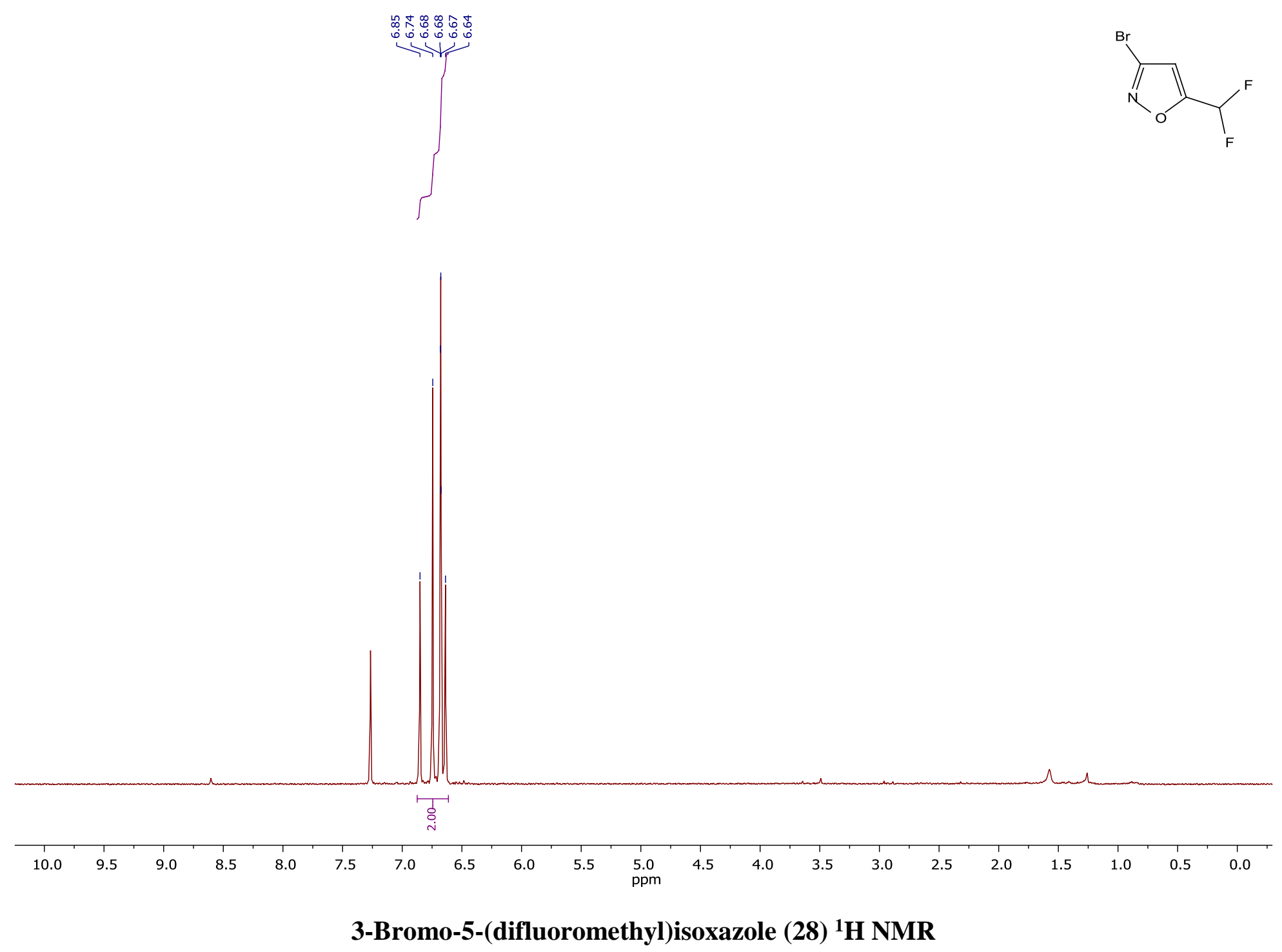




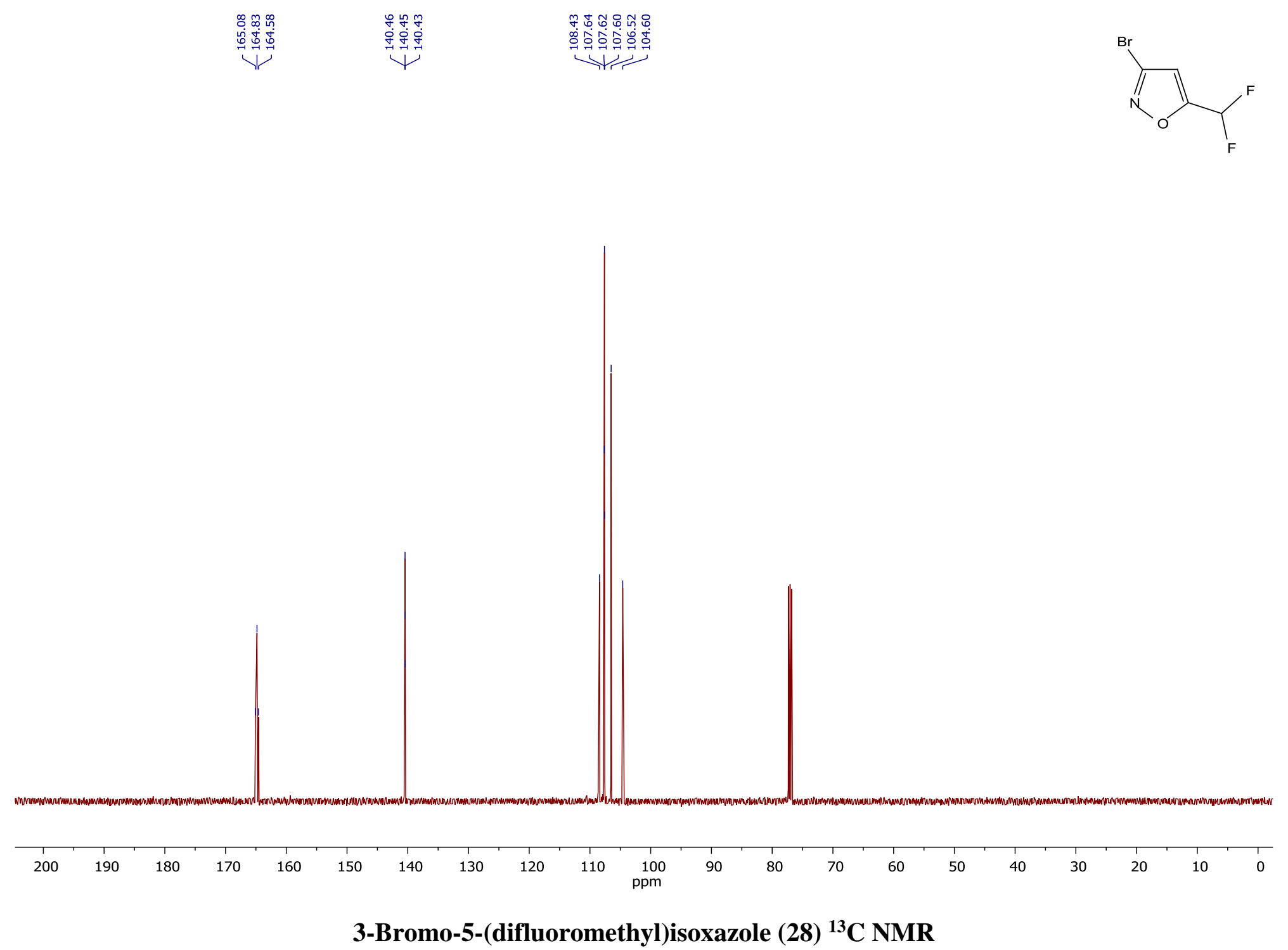



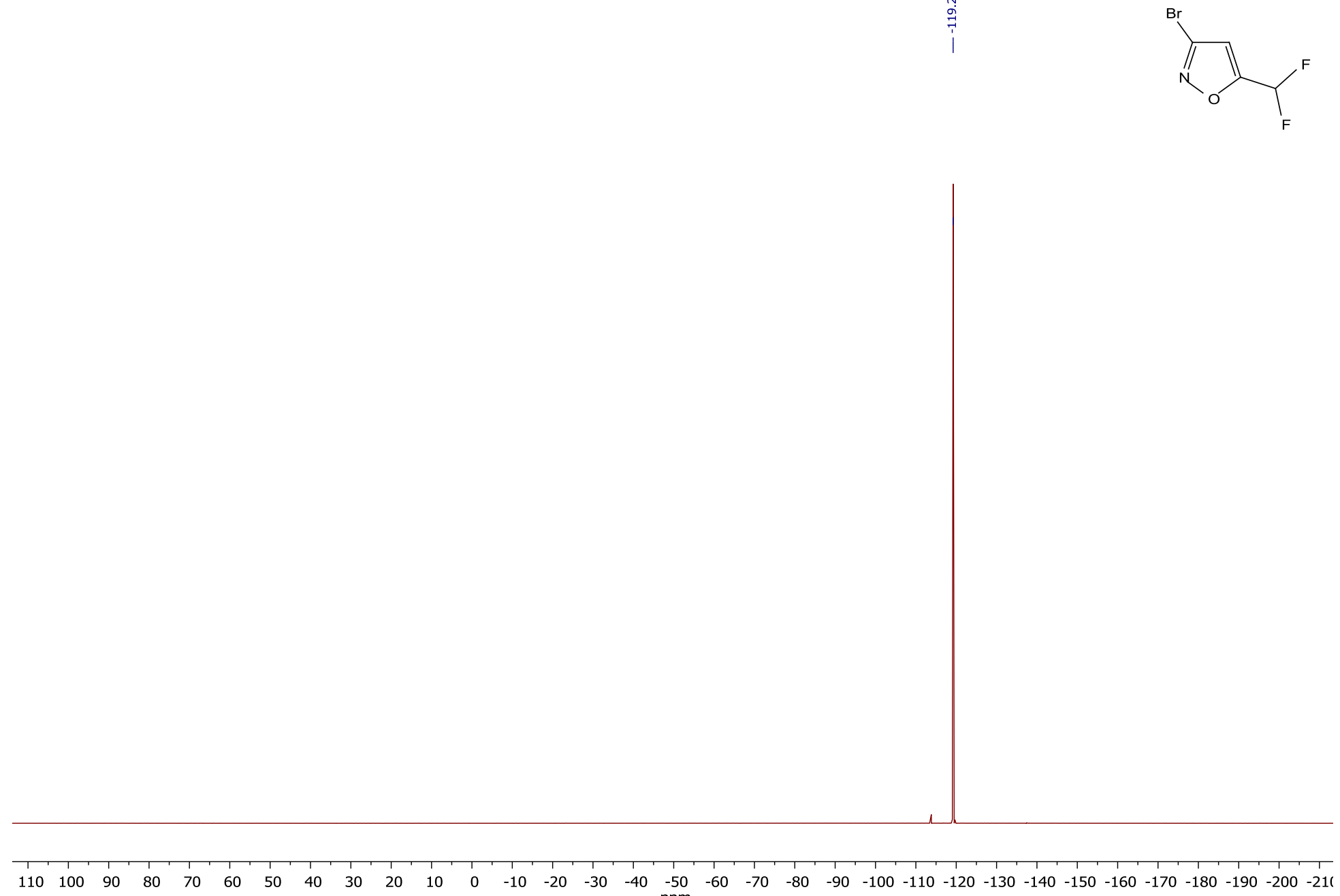

3-Bromo-5-(difluoromethyl)isoxazole (28) ${ }^{19}$ F NMR 


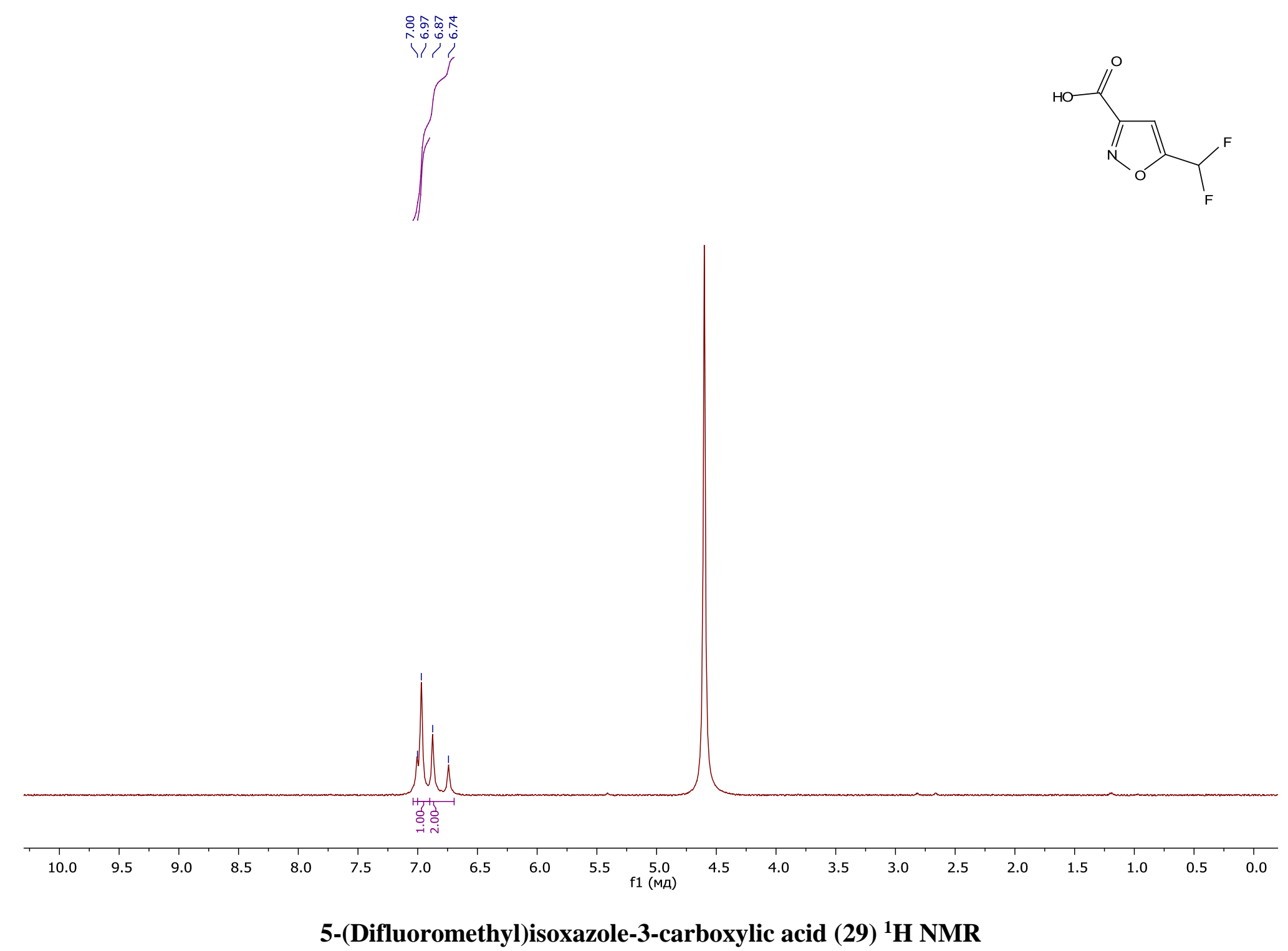




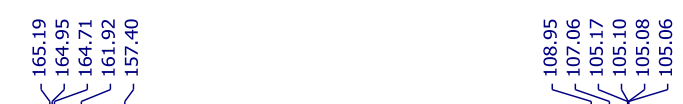

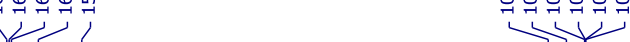
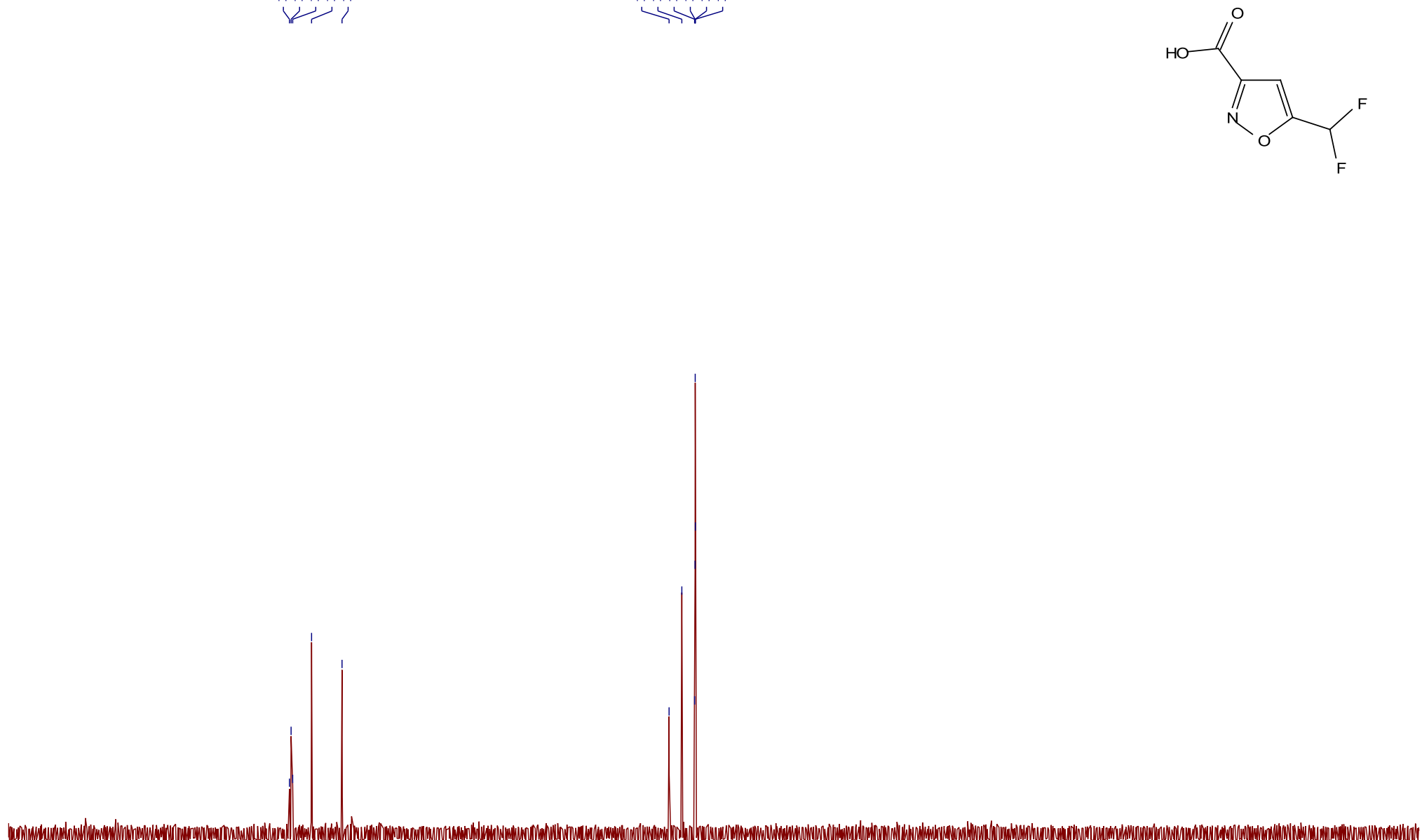

(1)

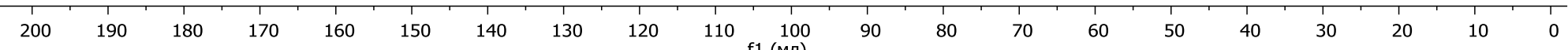

5-(Difluoromethyl)isoxazole-3-carboxylic acid (29) ${ }^{13}$ C NMR 

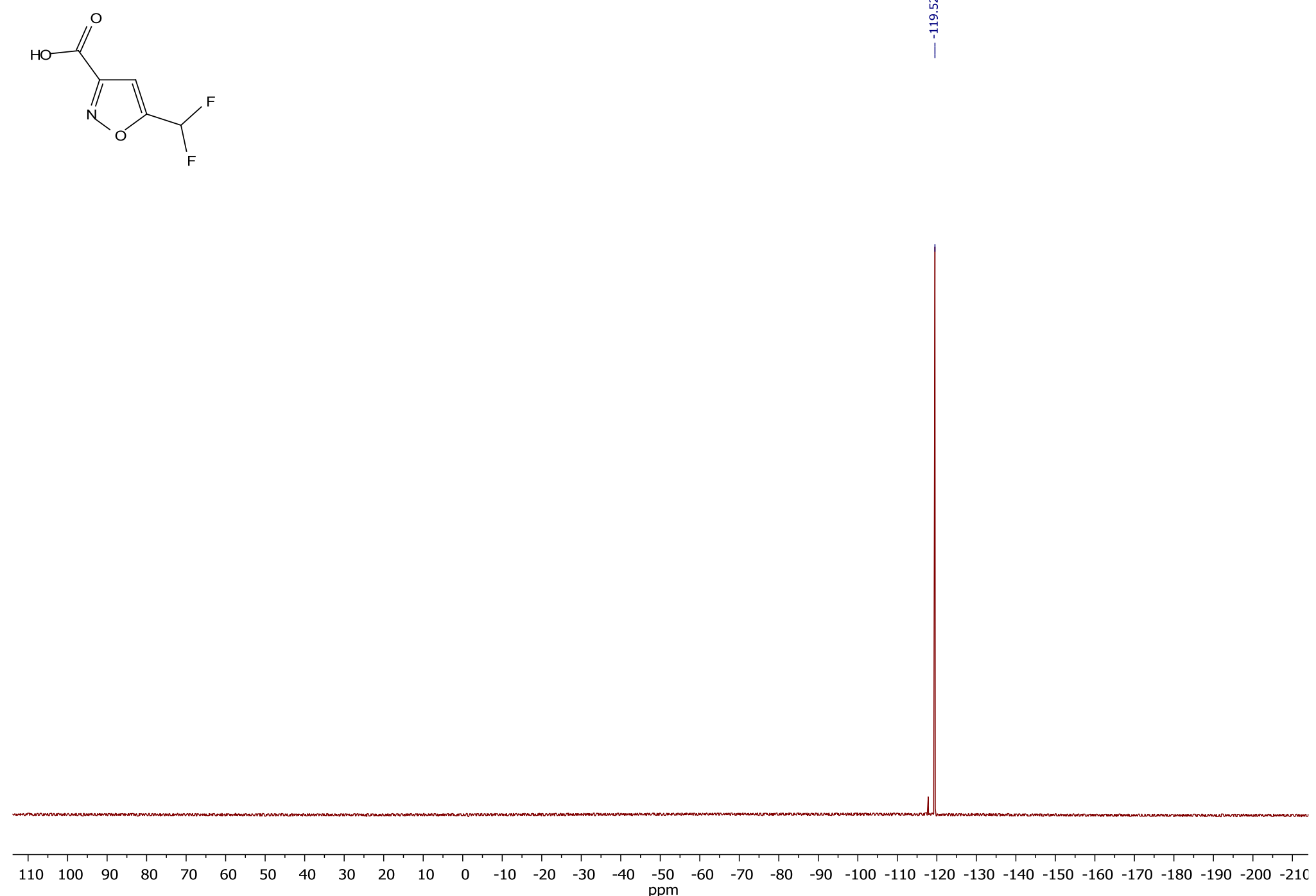

5-(Difluoromethyl)isoxazole-3-carboxylic acid (29) ${ }^{19}$ F NMR 


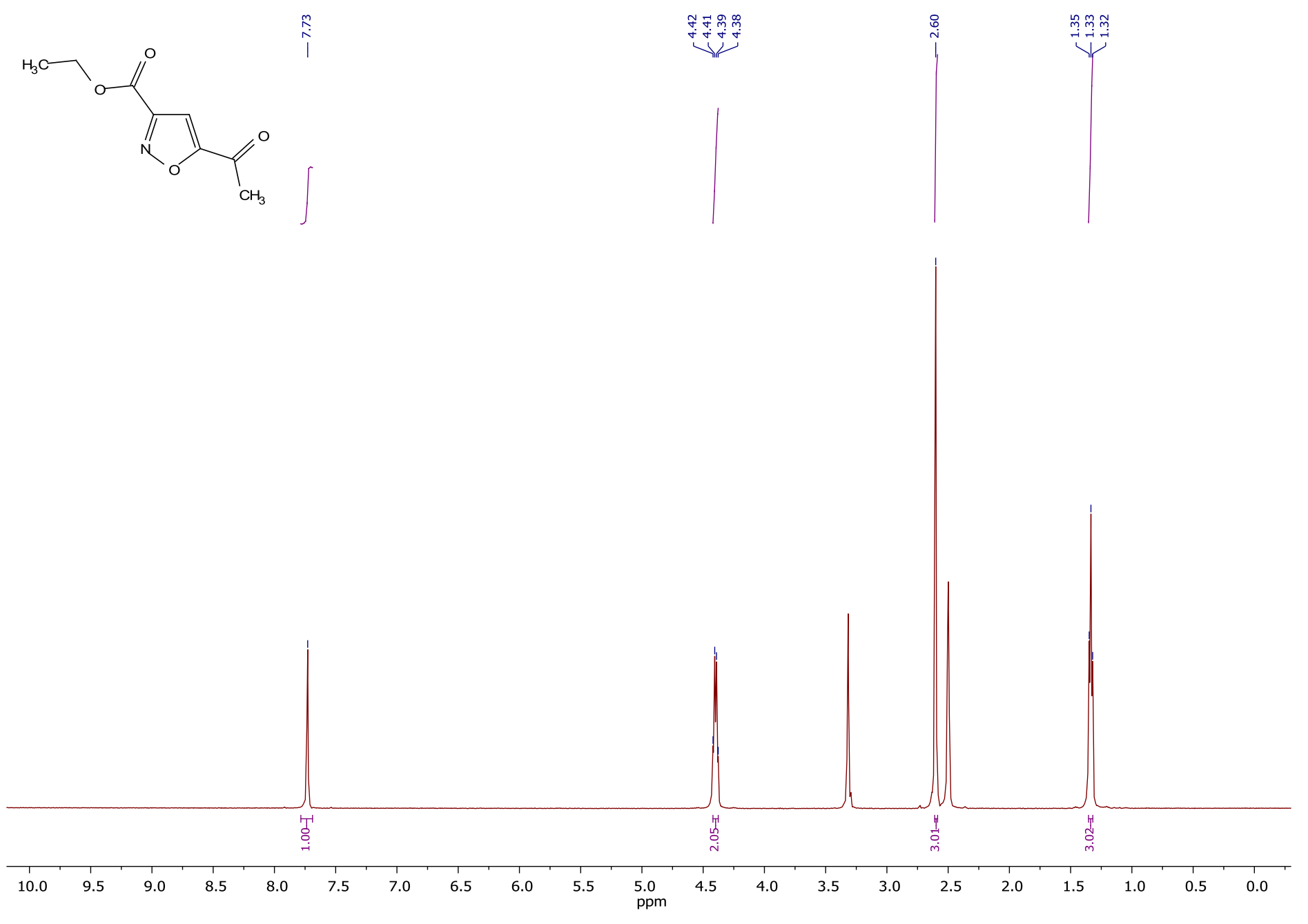

Ethyl 5-acetylisoxazole-3-carboxylate (31) ${ }^{1} \mathrm{H}$ NMR 


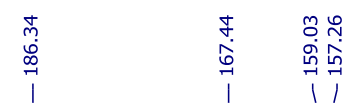
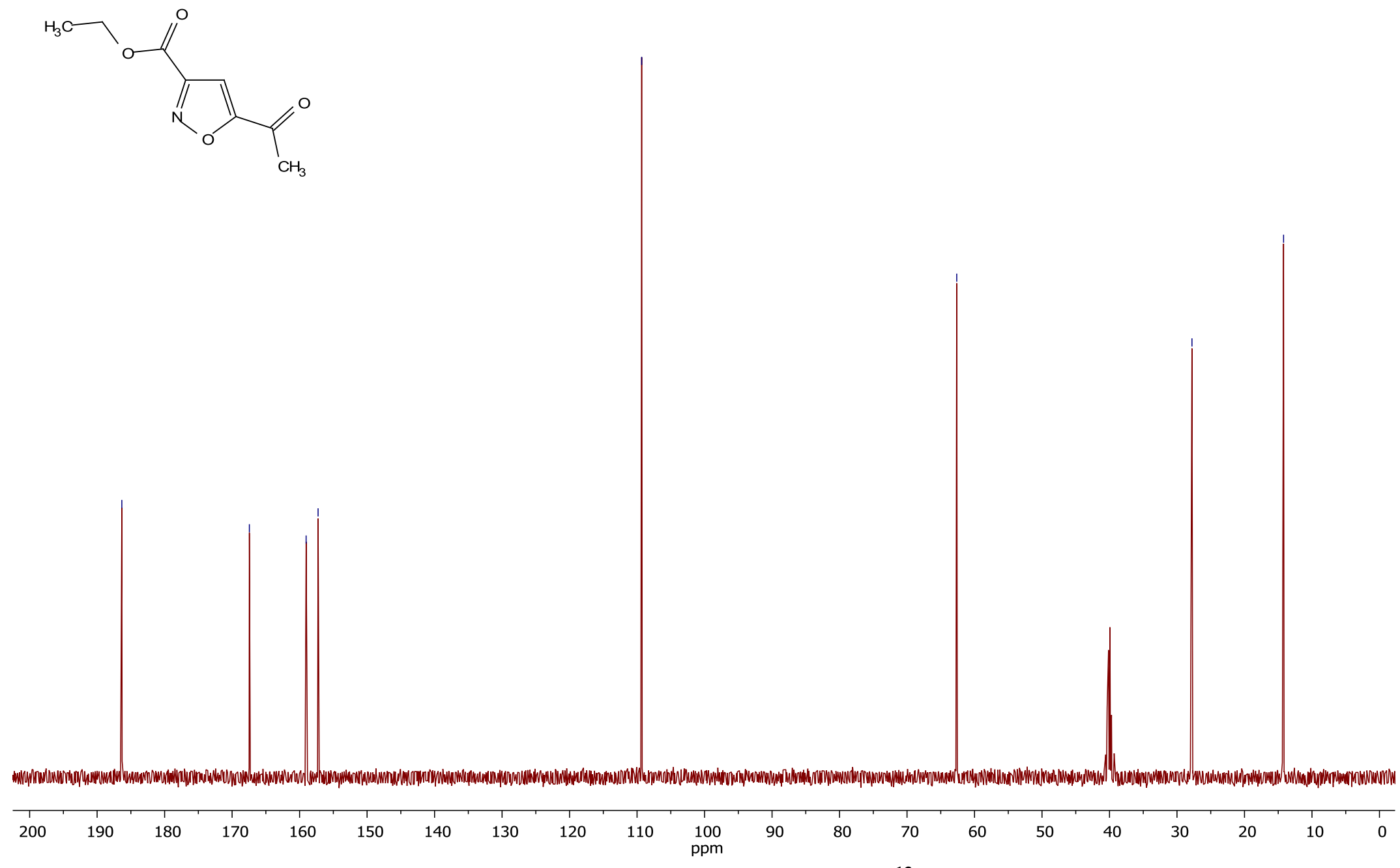

$200 \quad 190$ $180 \quad 170$ 160 $\begin{array}{cccccc}140 & 130 & 120 & 110 & \begin{array}{c}100 \\ \mathrm{ppm}\end{array} \\ \text { Ethyl 5-acetylisoxazole-3-carboxylate (31) } & { }^{\mathbf{1 3}} \mathbf{C} \text { NMR }\end{array}$ 

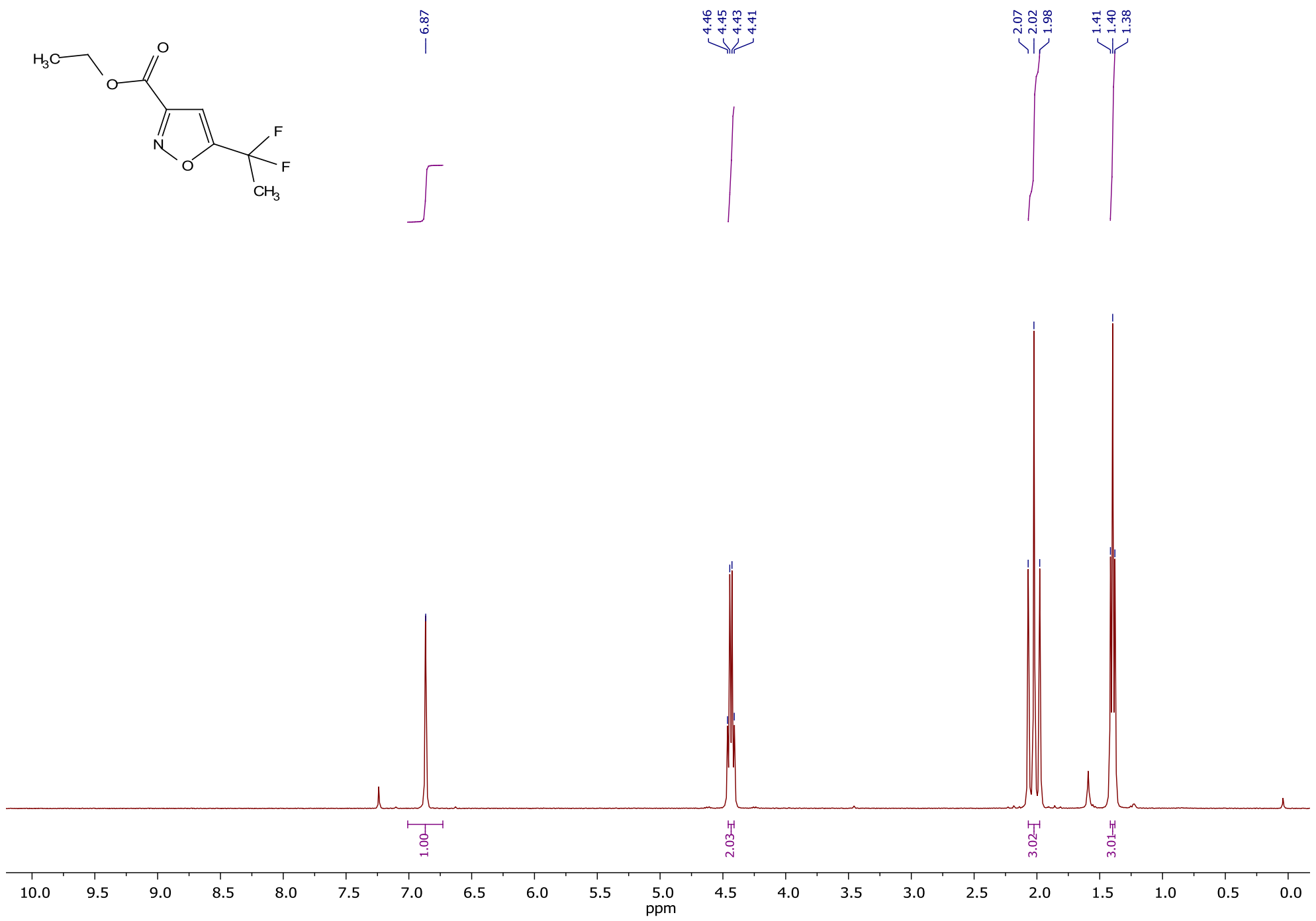

Ethyl 5-(1,1-difluoroethyl)isoxazole-3-carboxylate (32) ${ }^{1} \mathrm{H}$ NMR 


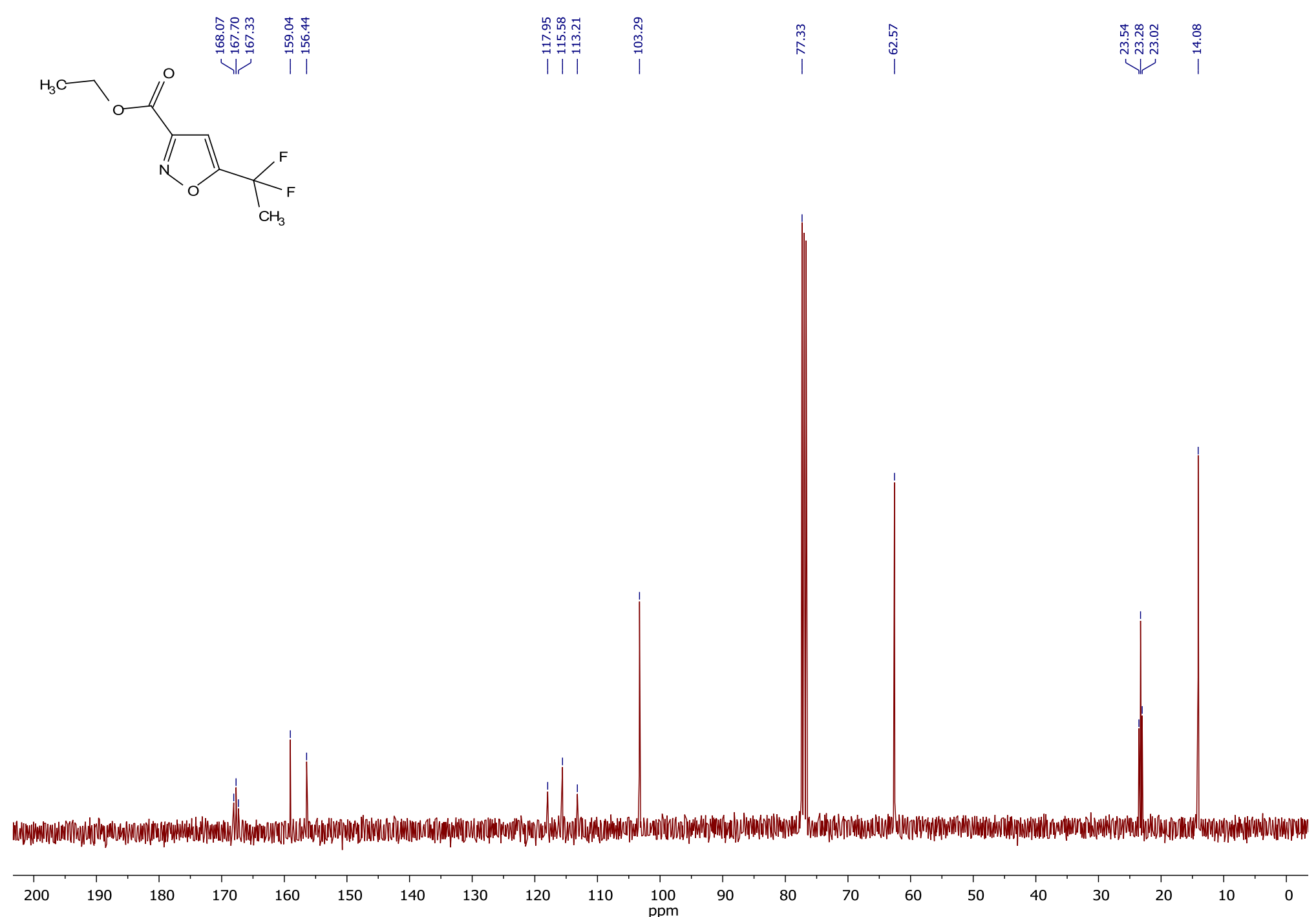

Ethyl 5-(1,1-difluoroethyl)isoxazole-3-carboxylate (32) ${ }^{13} \mathrm{C}$ NMR 

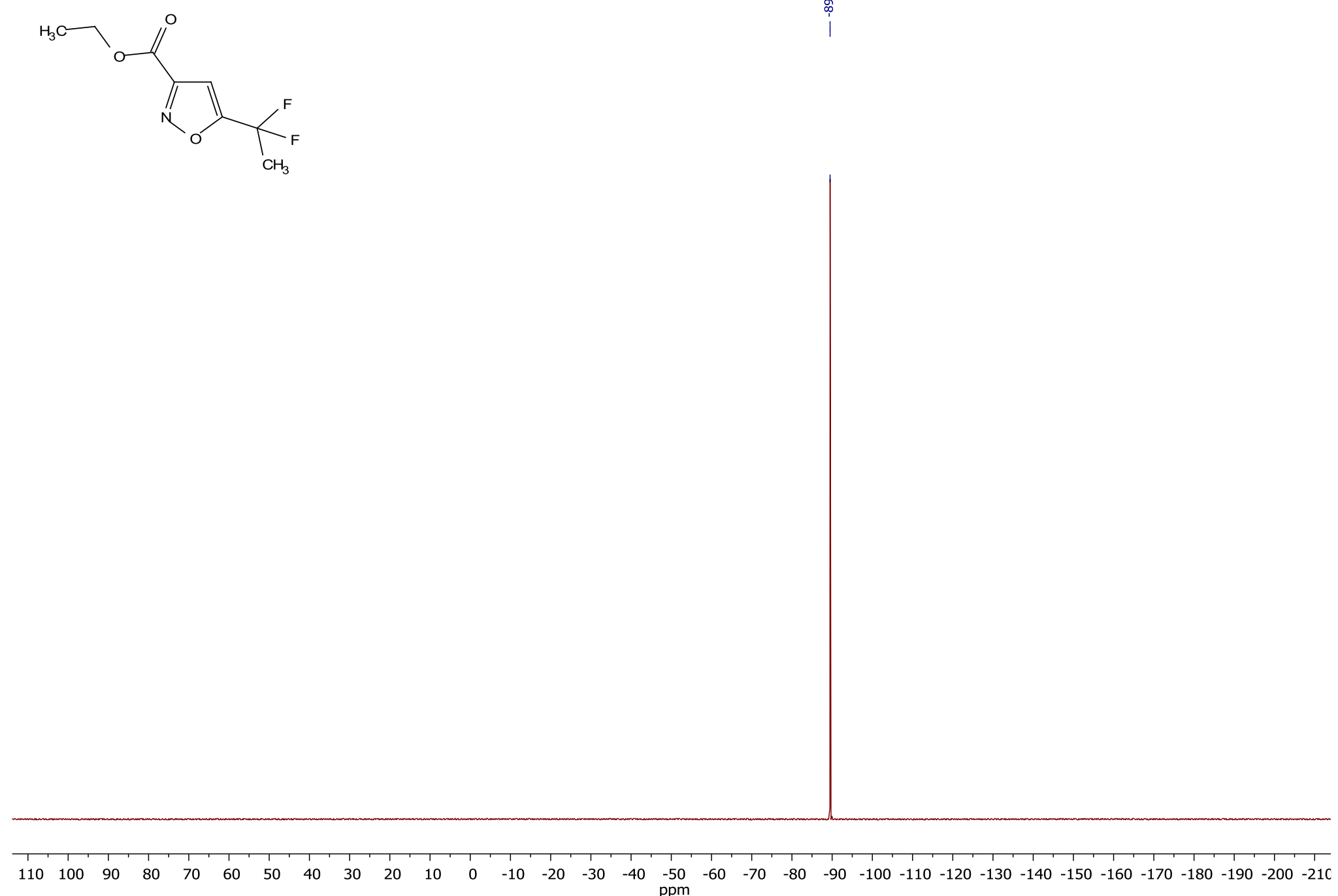

Ethyl 5-(1,1-difluoroethyl)isoxazole-3-carboxylate (32) ${ }^{19}$ F NMR 


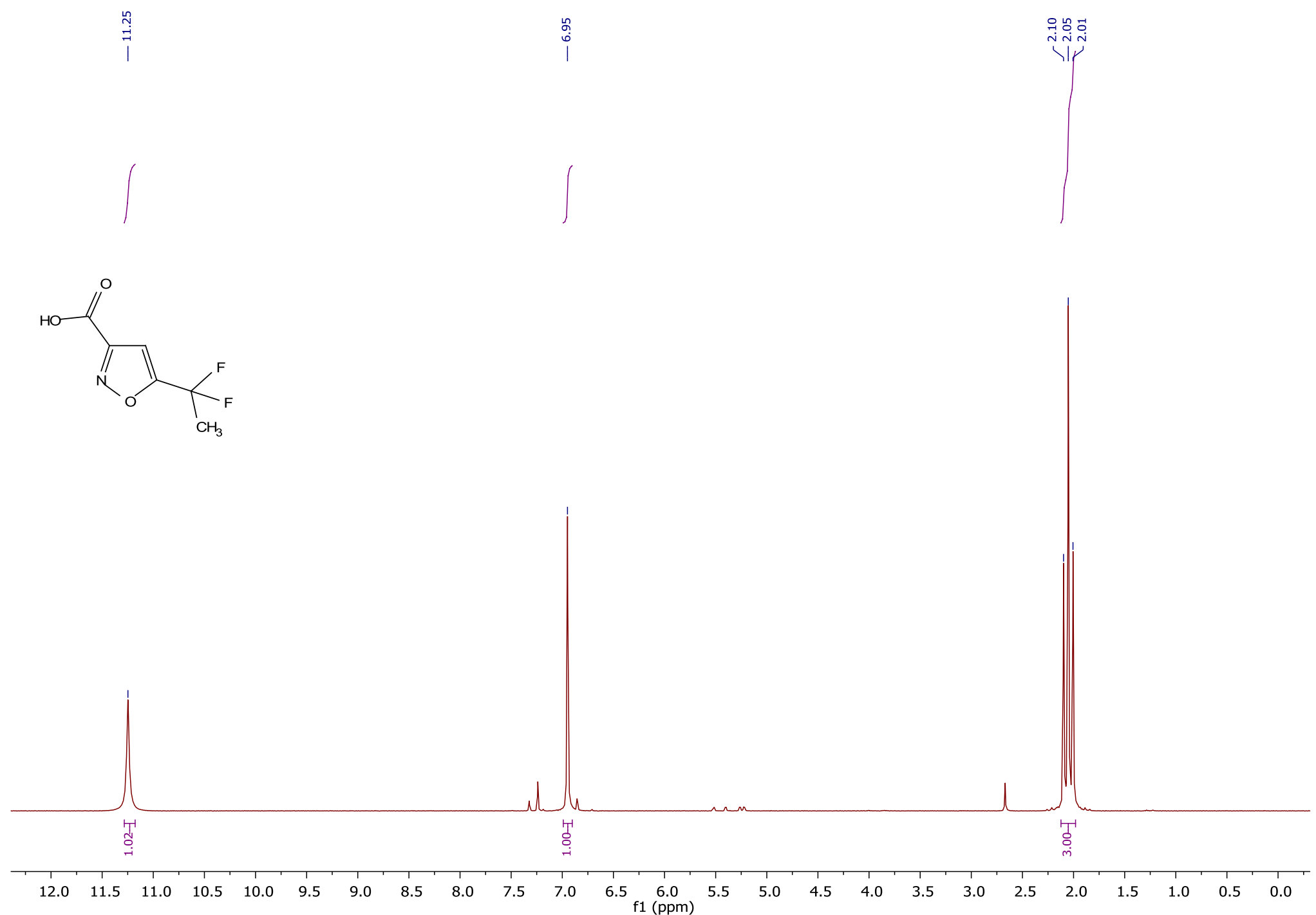

5-(1,1-Difluoroethyl)isoxazole-3-carboxylic acid (33) ${ }^{1}$ H NMR 

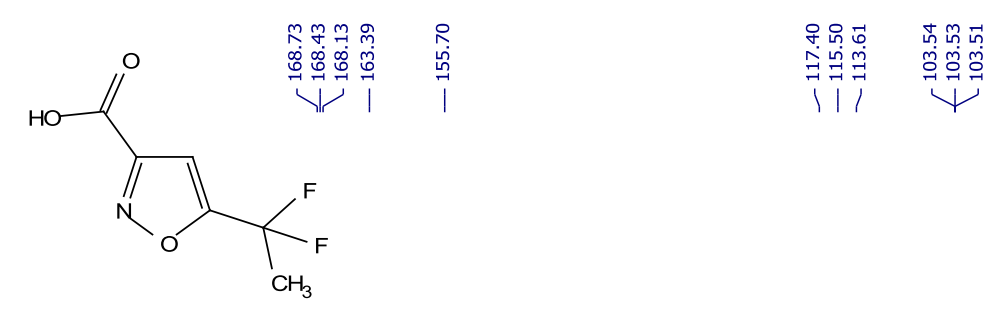

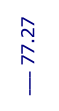

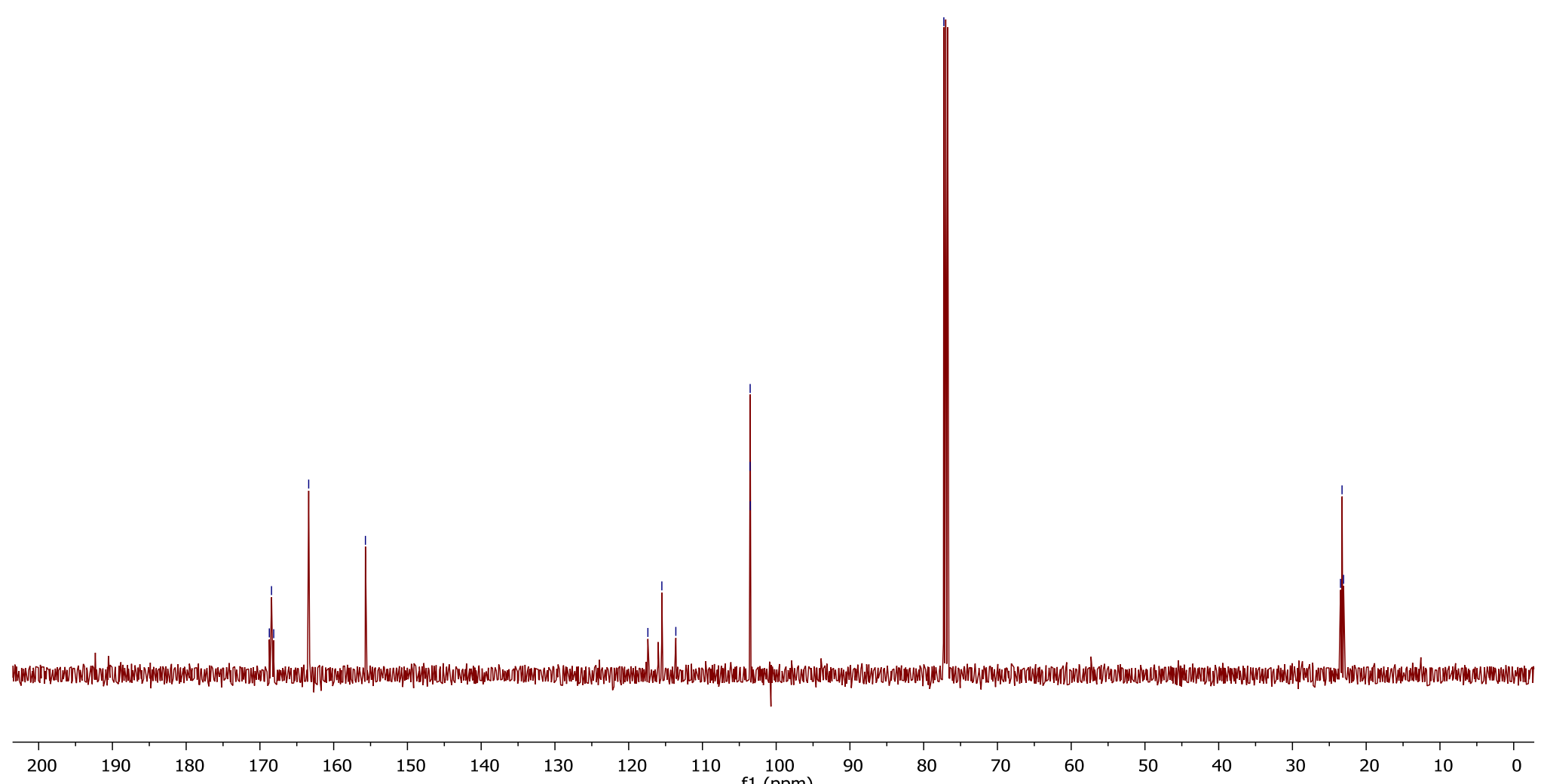

5-(1,1-Difluoroethyl)isoxazole-3-carboxylic acid (33) ${ }^{13} \mathrm{C}$ NMR 

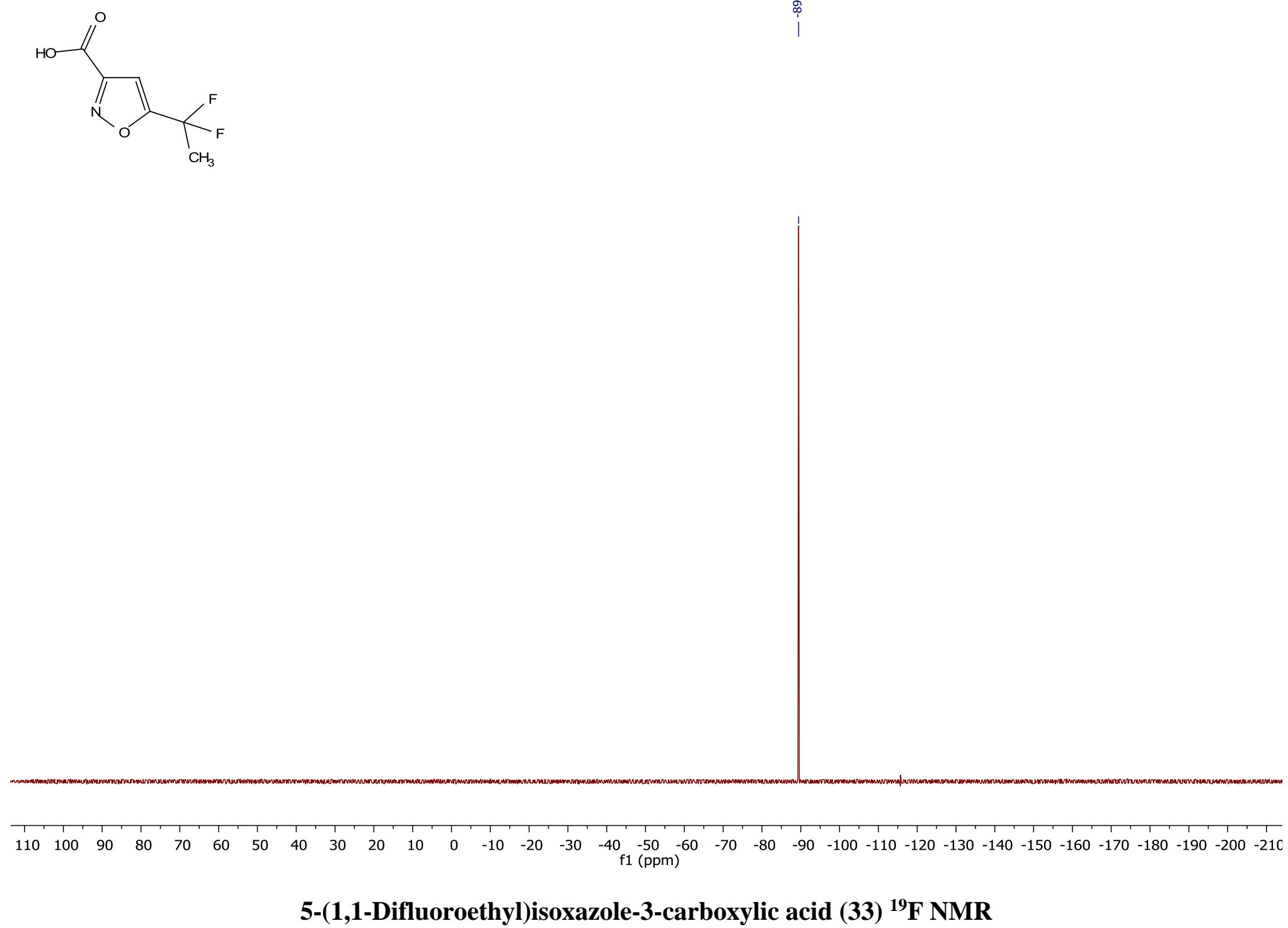

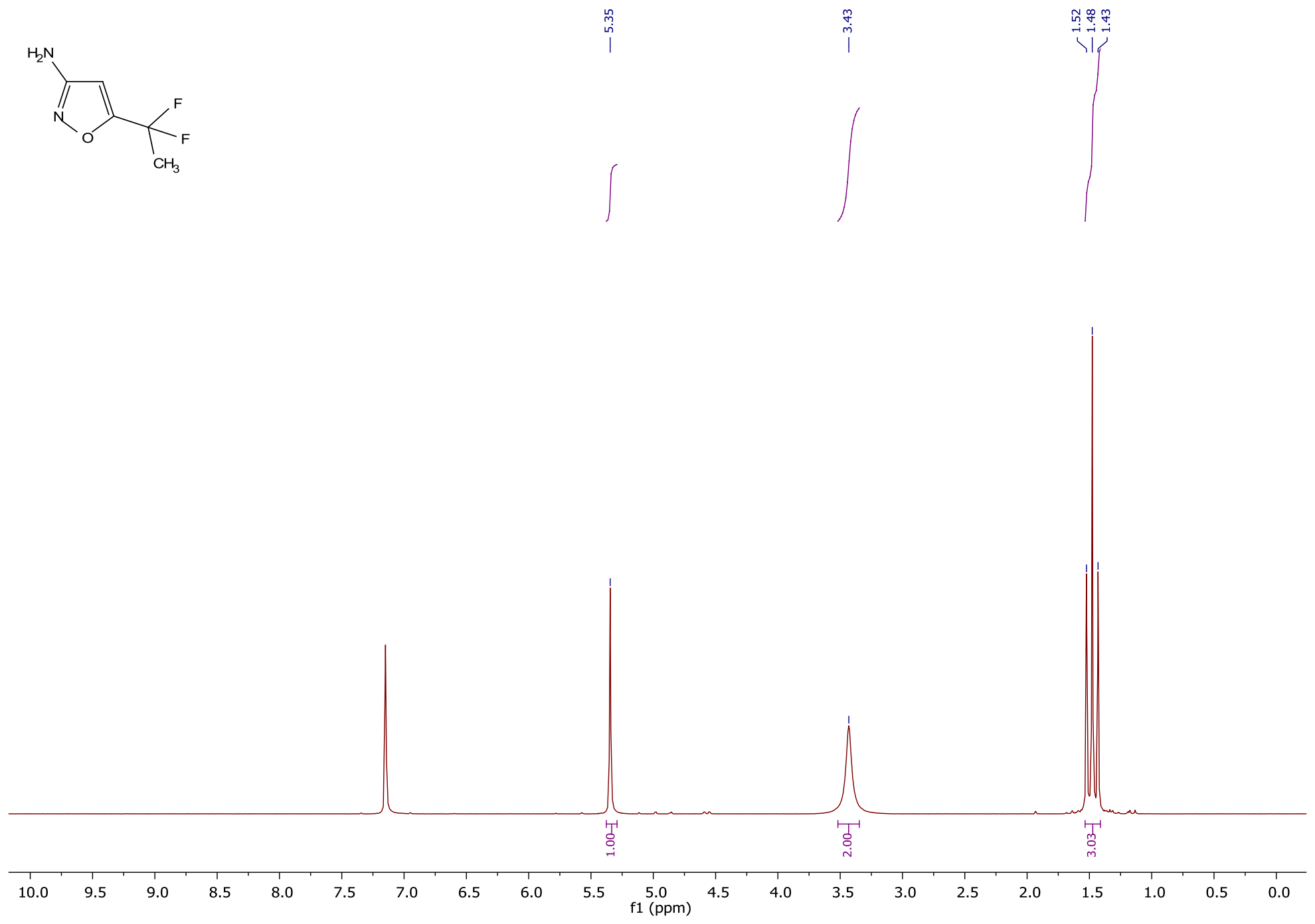

5-(1,1-Difluoroethyl)isoxazol-3-amine (34) ${ }^{1} \mathrm{H}$ NMR 


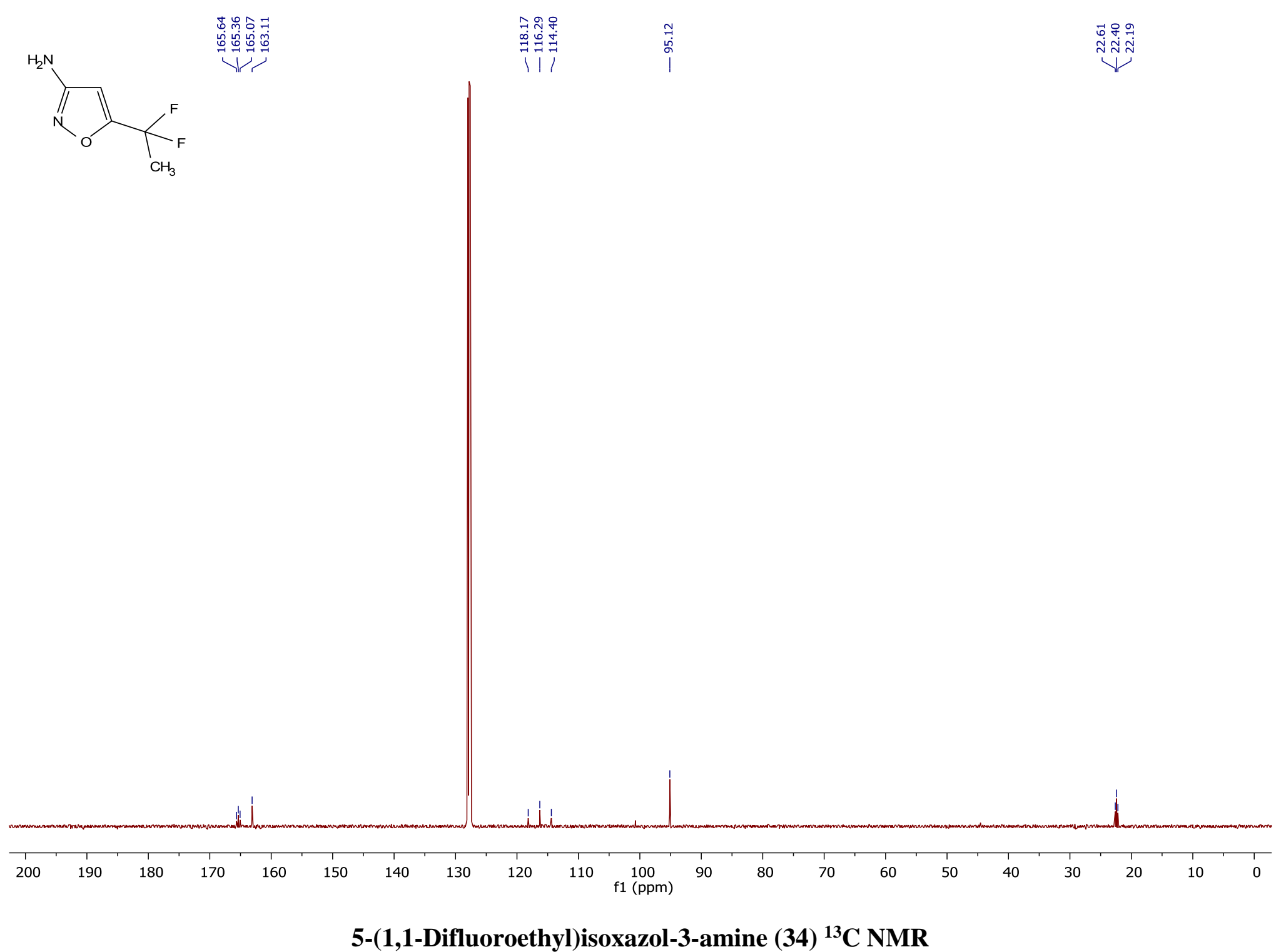




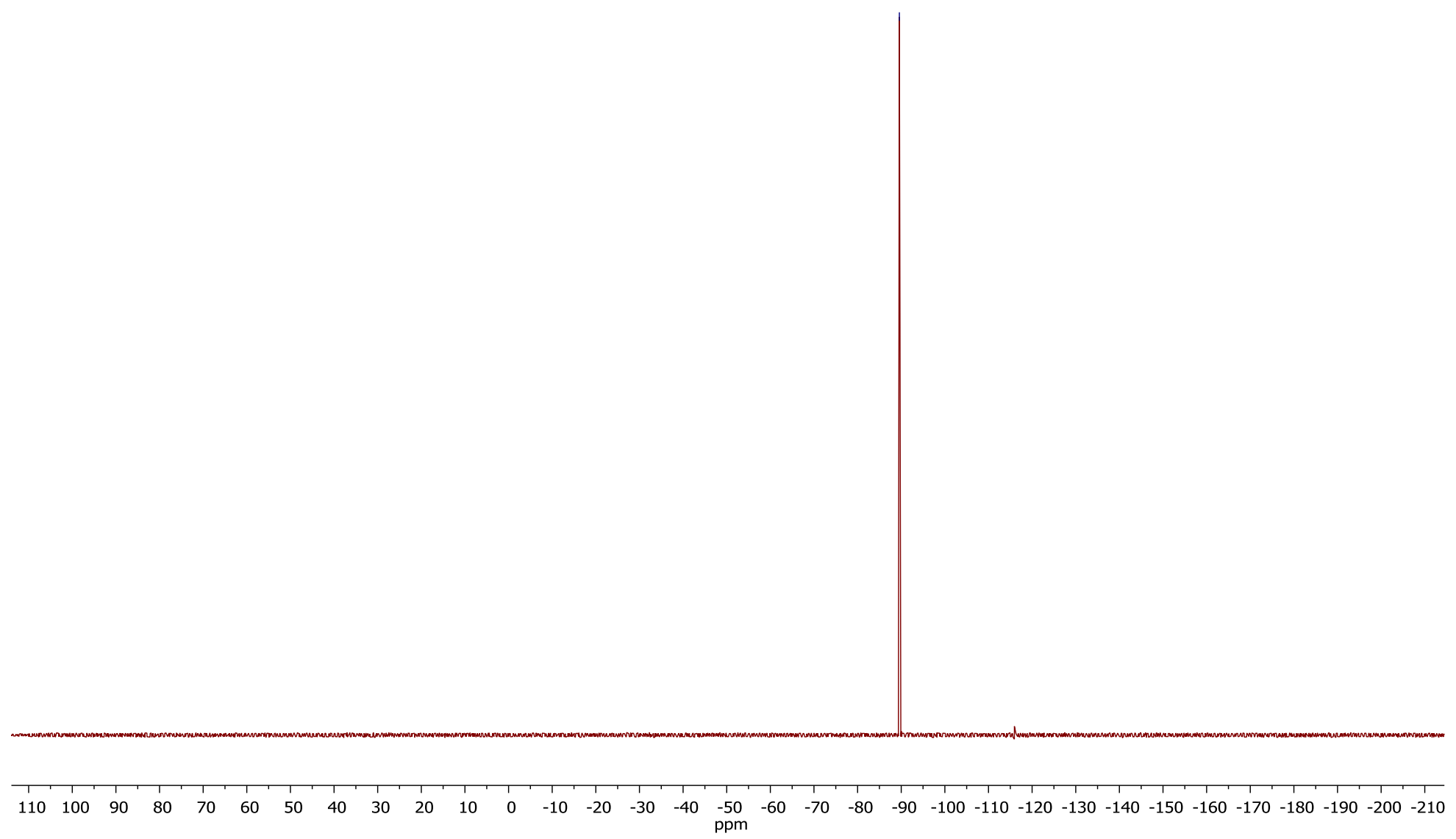

5-(1,1-Difluoroethyl)isoxazol-3-amine (34) ${ }^{19}$ F NMR 

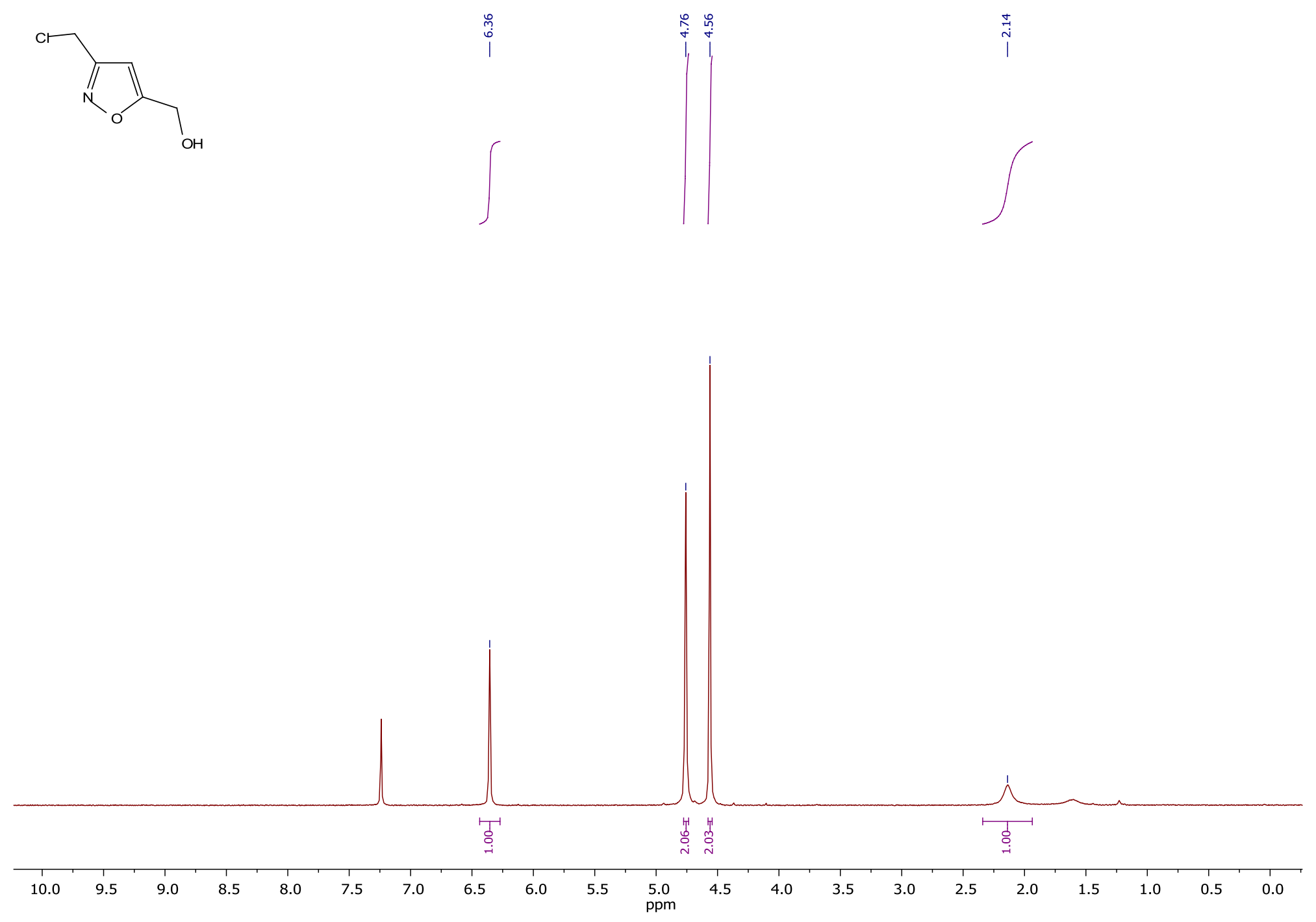

(3-(Chloromethyl)isoxazol-5-yl)methanol (36) ${ }^{1} \mathrm{H}$ NMR 


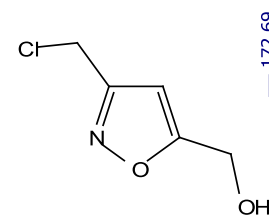

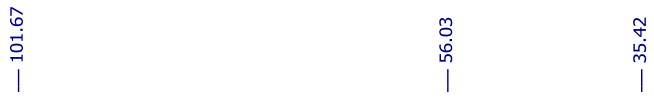

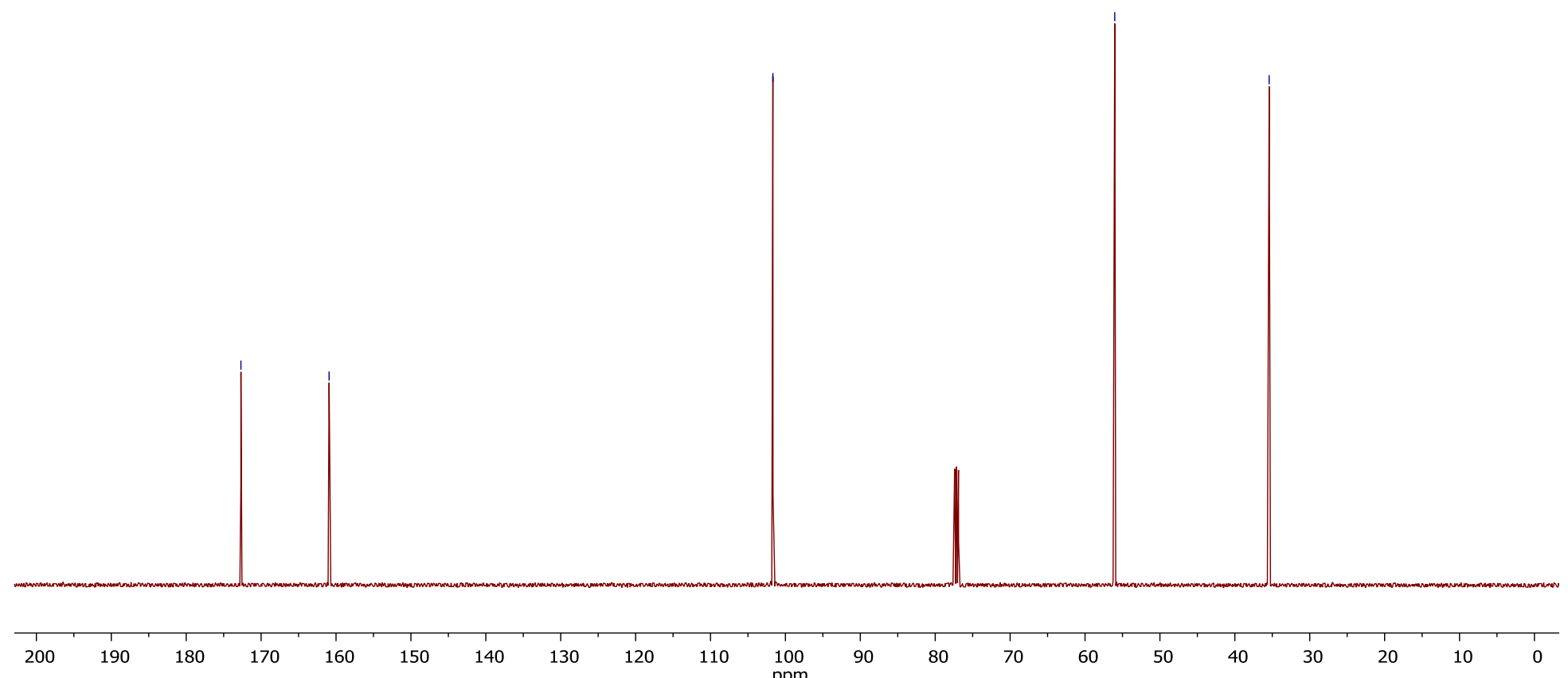

(3-(Chloromethyl)isoxazol-5-yl)methanol (36) ${ }^{13} \mathrm{C}$ NMR 


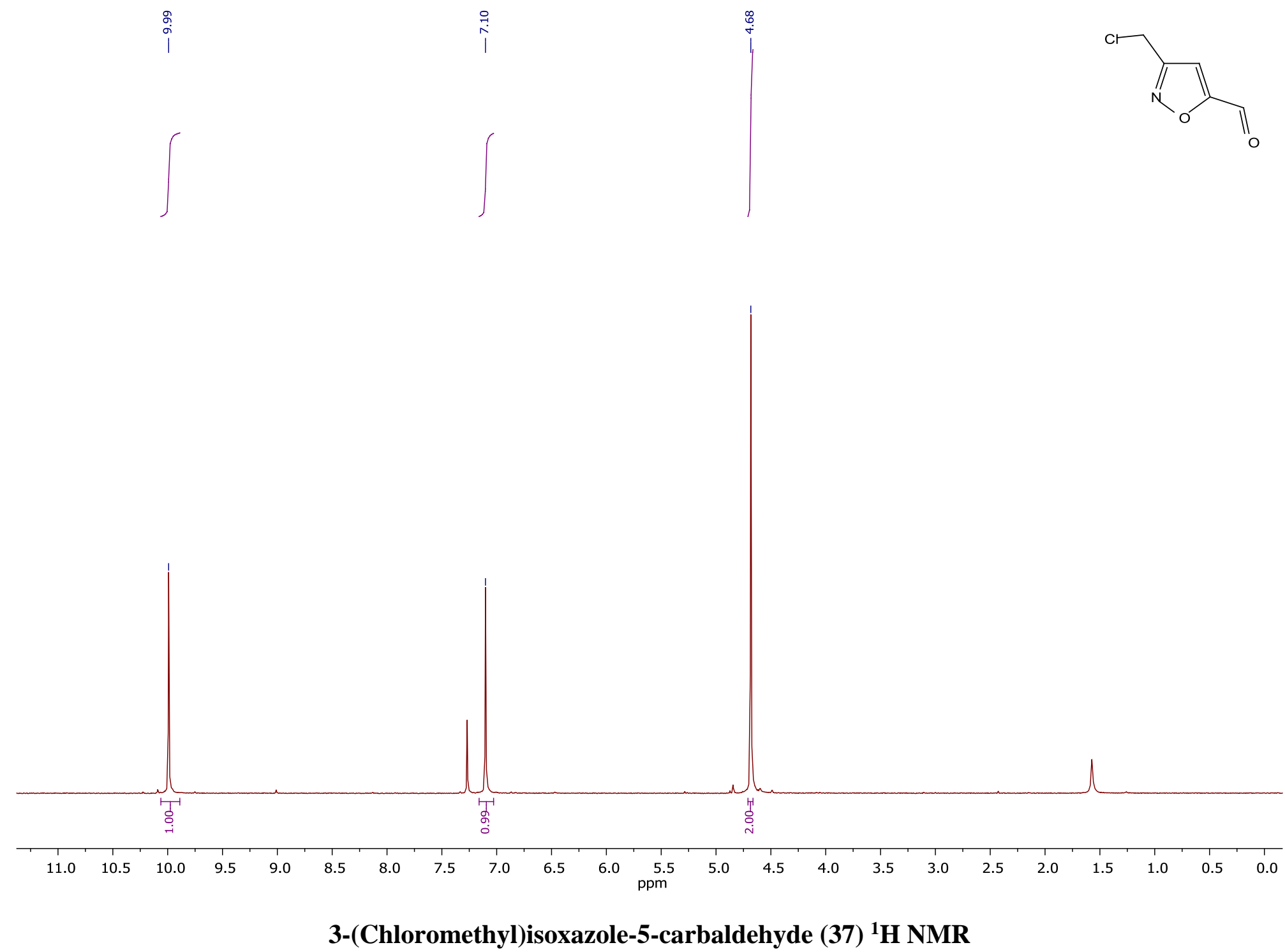



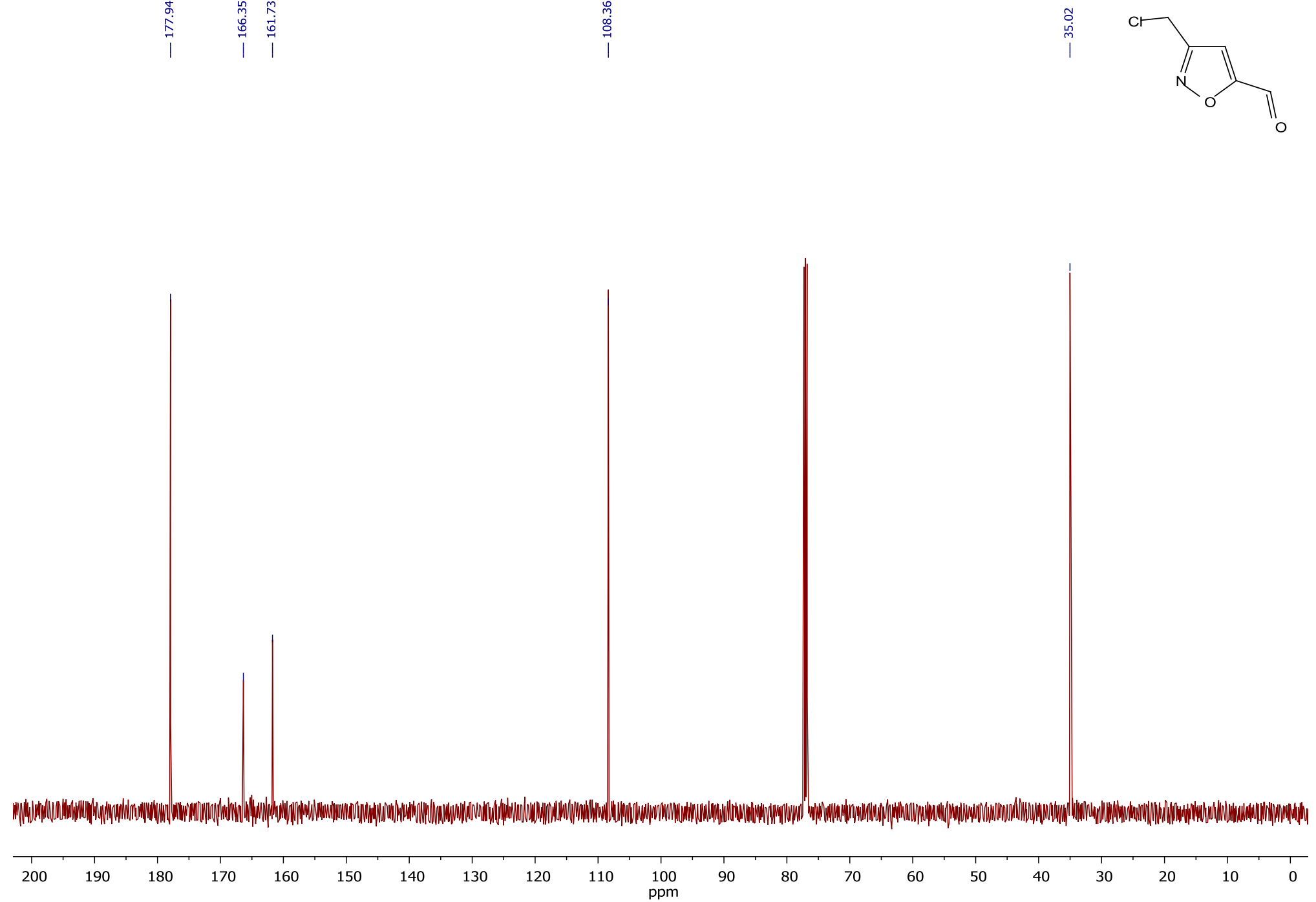

3-(Chloromethyl)isoxazole-5-carbaldehyde (37) ${ }^{13} \mathrm{C}$ NMR 


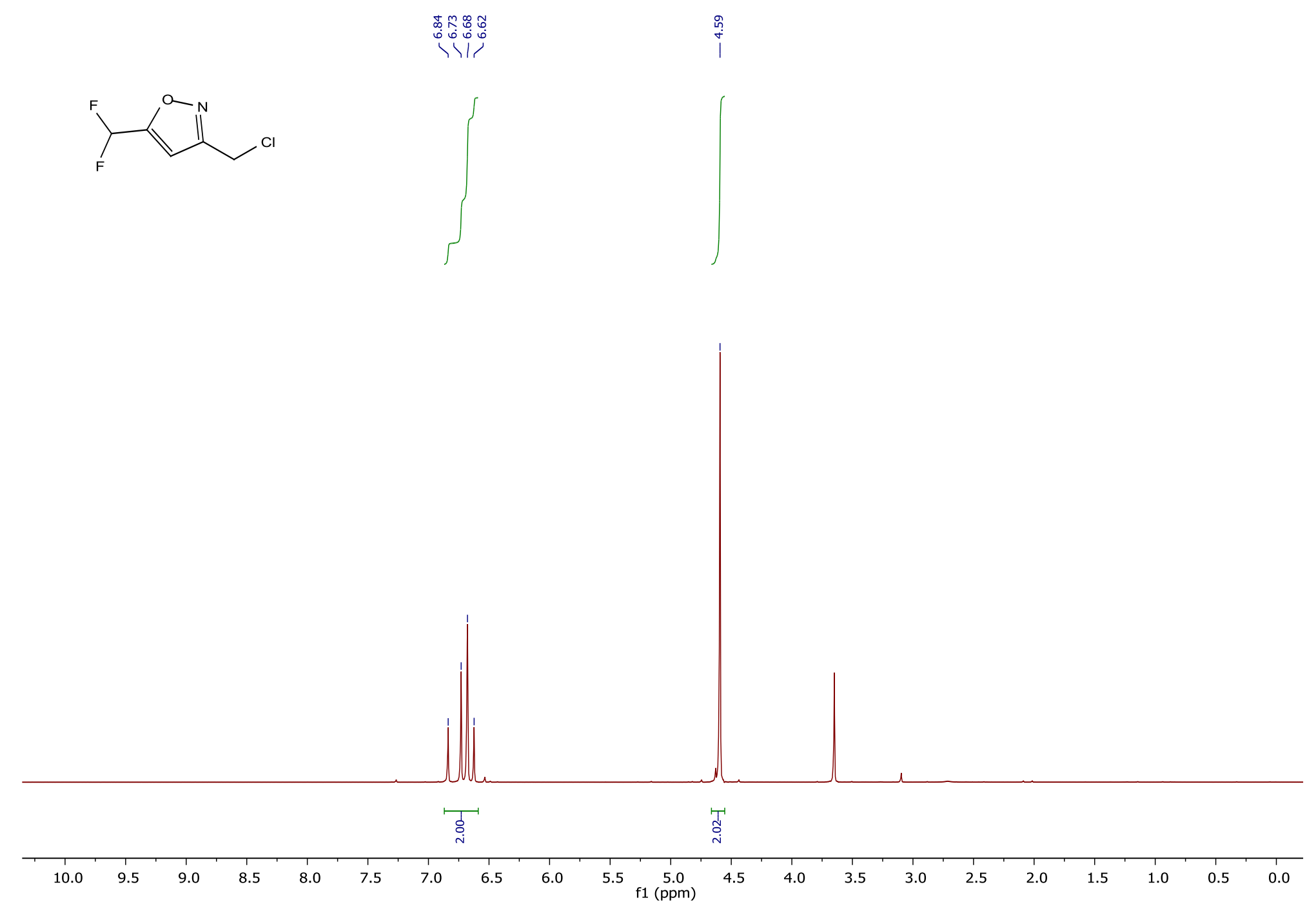

3-(Chloromethyl)-5-(difluoromethyl)isoxazole (38) ${ }^{1}$ H NMR 

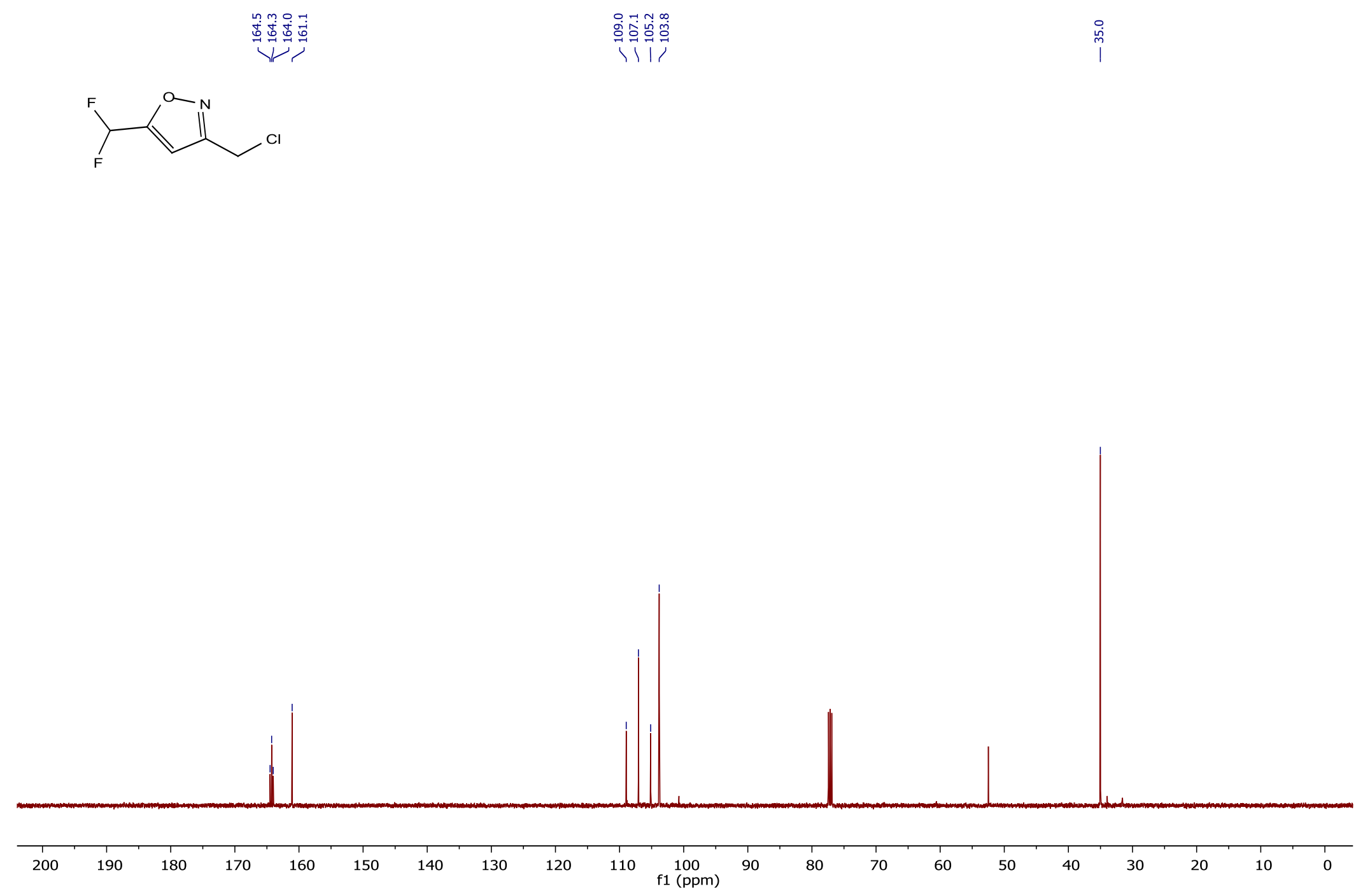

3-(Chloromethyl)-5-(difluoromethyl)isoxazole (38) ${ }^{13} \mathrm{C}$ NMR 

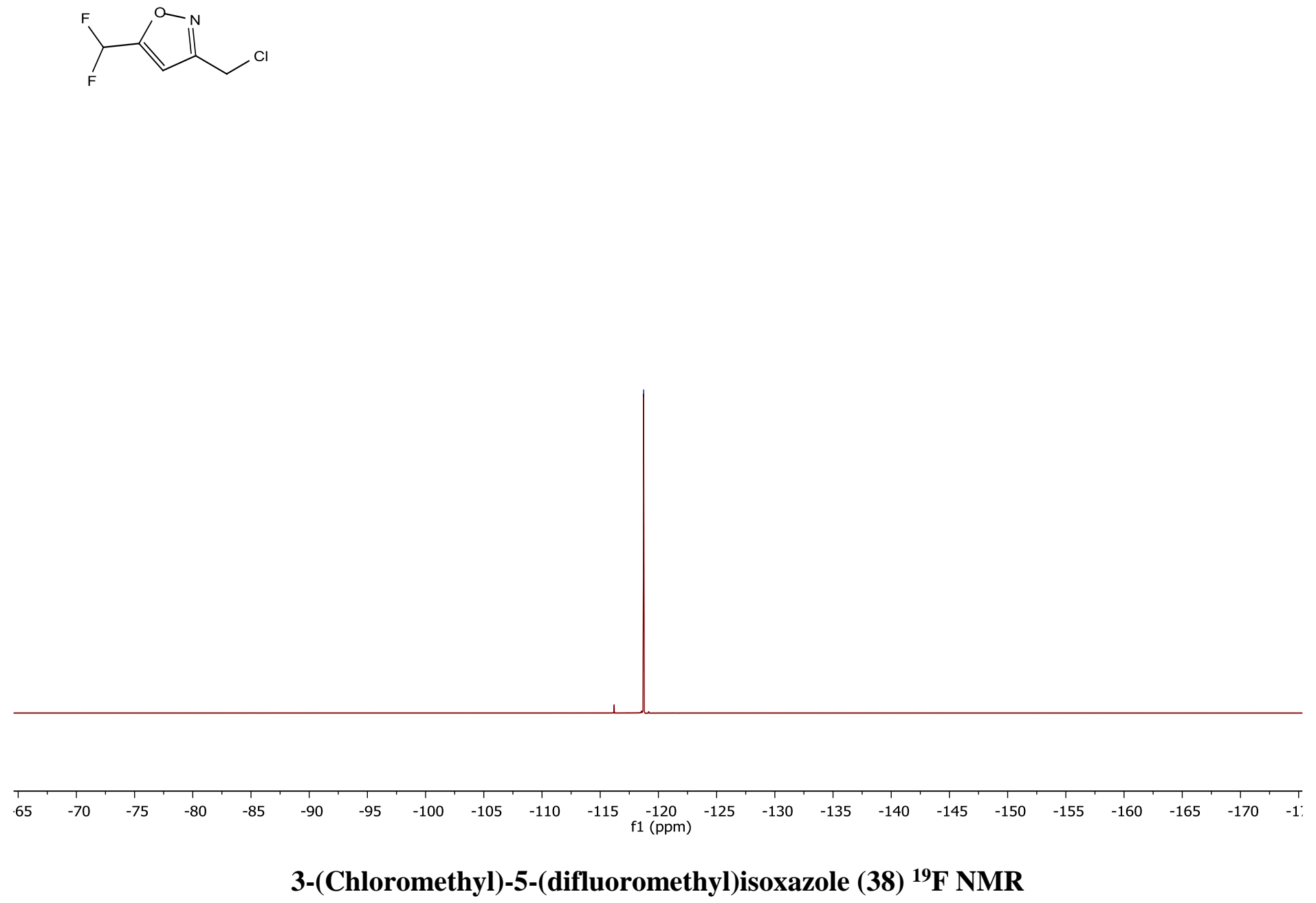

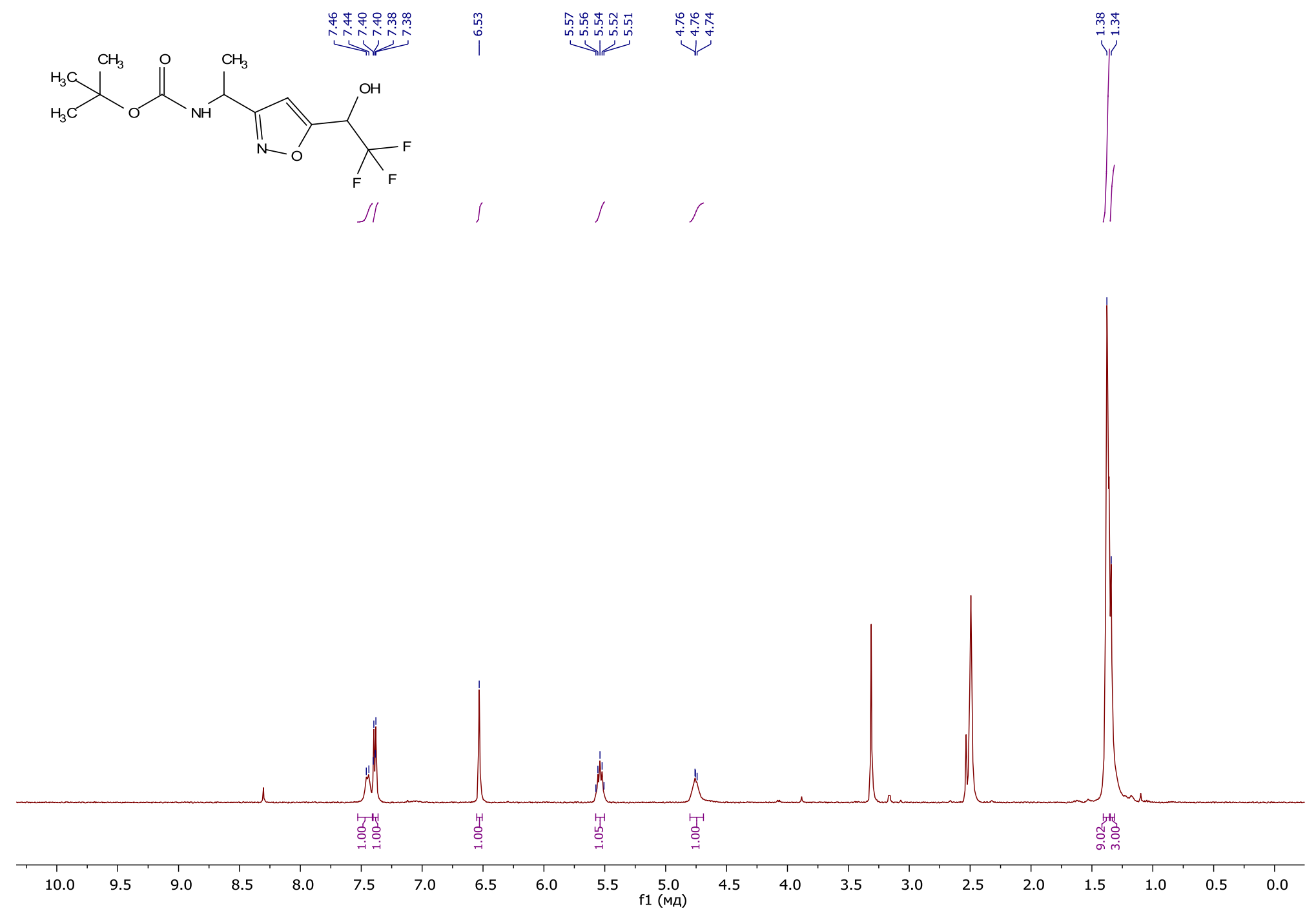

tert-Butyl (1-(5-(2,2,2-trifluoro-1-hydroxyethyl)isoxazol-3-yl)ethyl)carbamate (39g or 39h) ${ }^{1} \mathrm{H}$ NMR 


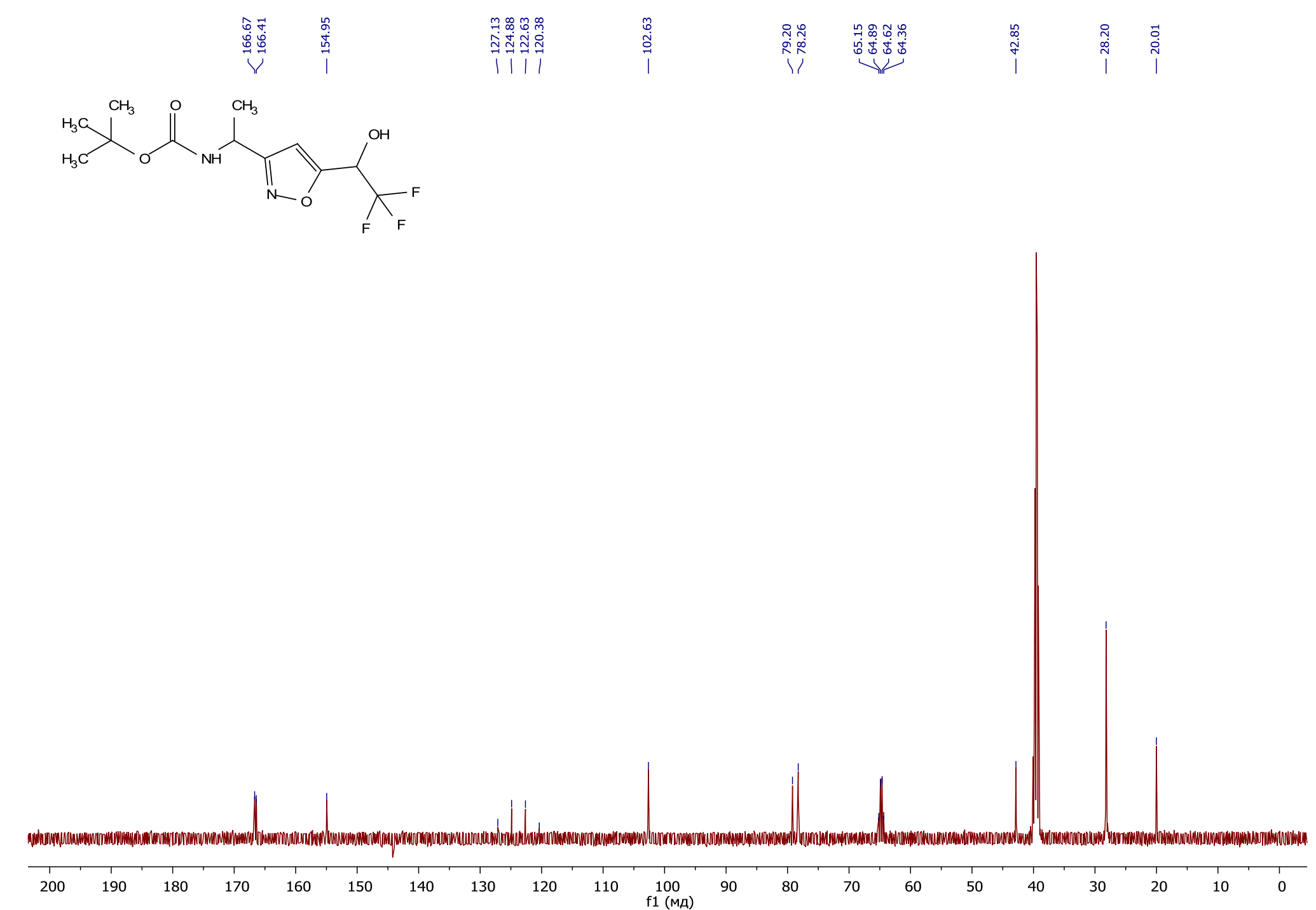

tert-Butyl (1-(5-(2,2,2-trifluoro-1-hydroxyethyl)isoxazol-3-yl)ethyl)carbamate (39g or 39h) ${ }^{13} \mathrm{C}$ NMR 

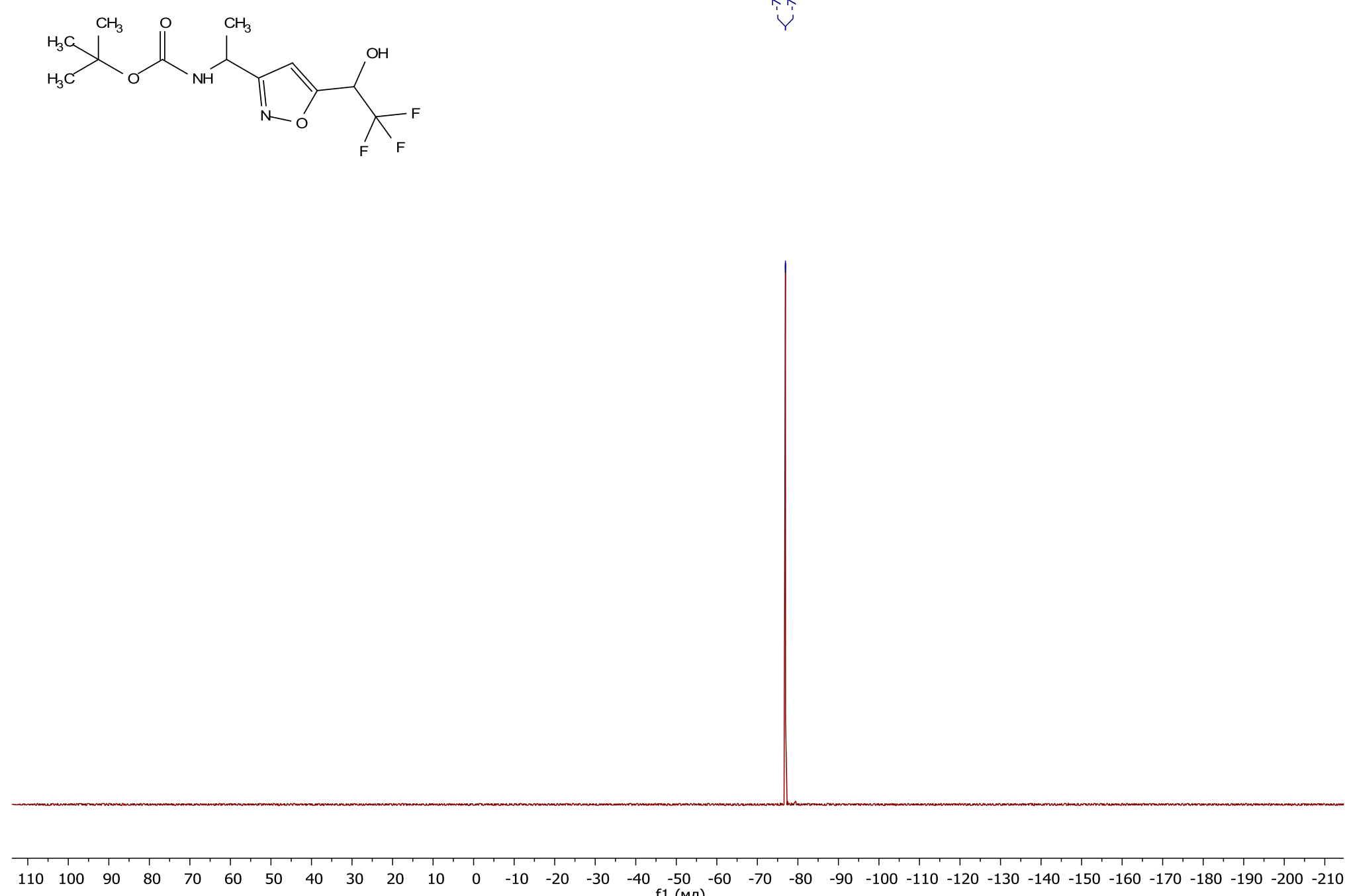

tert-Butyl (1-(5-(2,2,2-trifluoro-1-hydroxyethyl)isoxazol-3-yl)ethyl)carbamate (39g or 39h) ${ }^{19}$ F NMR 

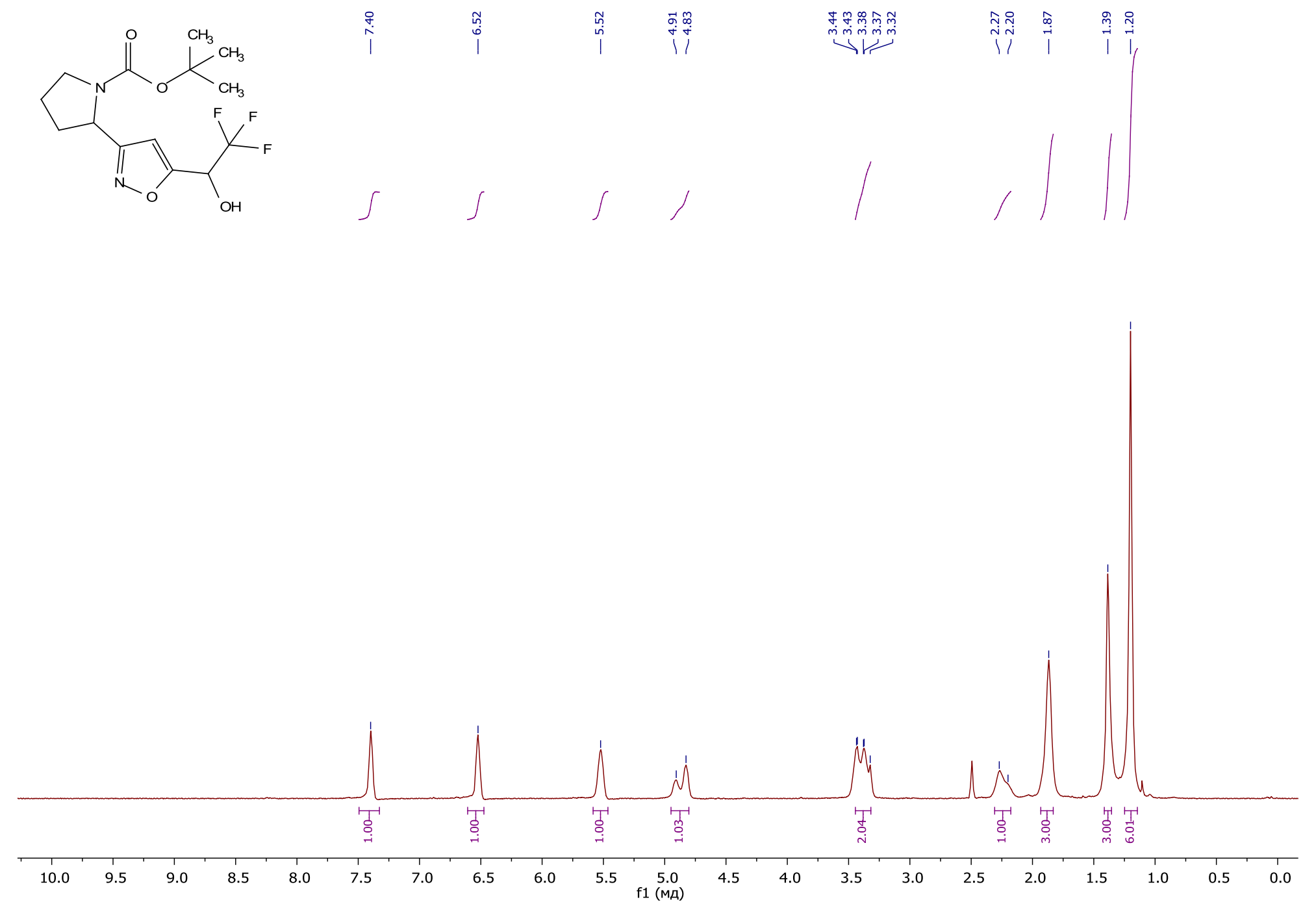

tert-Butyl 2-(5-(2,2,2-trifluoro-1-hydroxyethyl)isoxazol-3-yl)pyrrolidine-1-carboxylate (39k or 39l) ${ }^{1}$ H NMR 


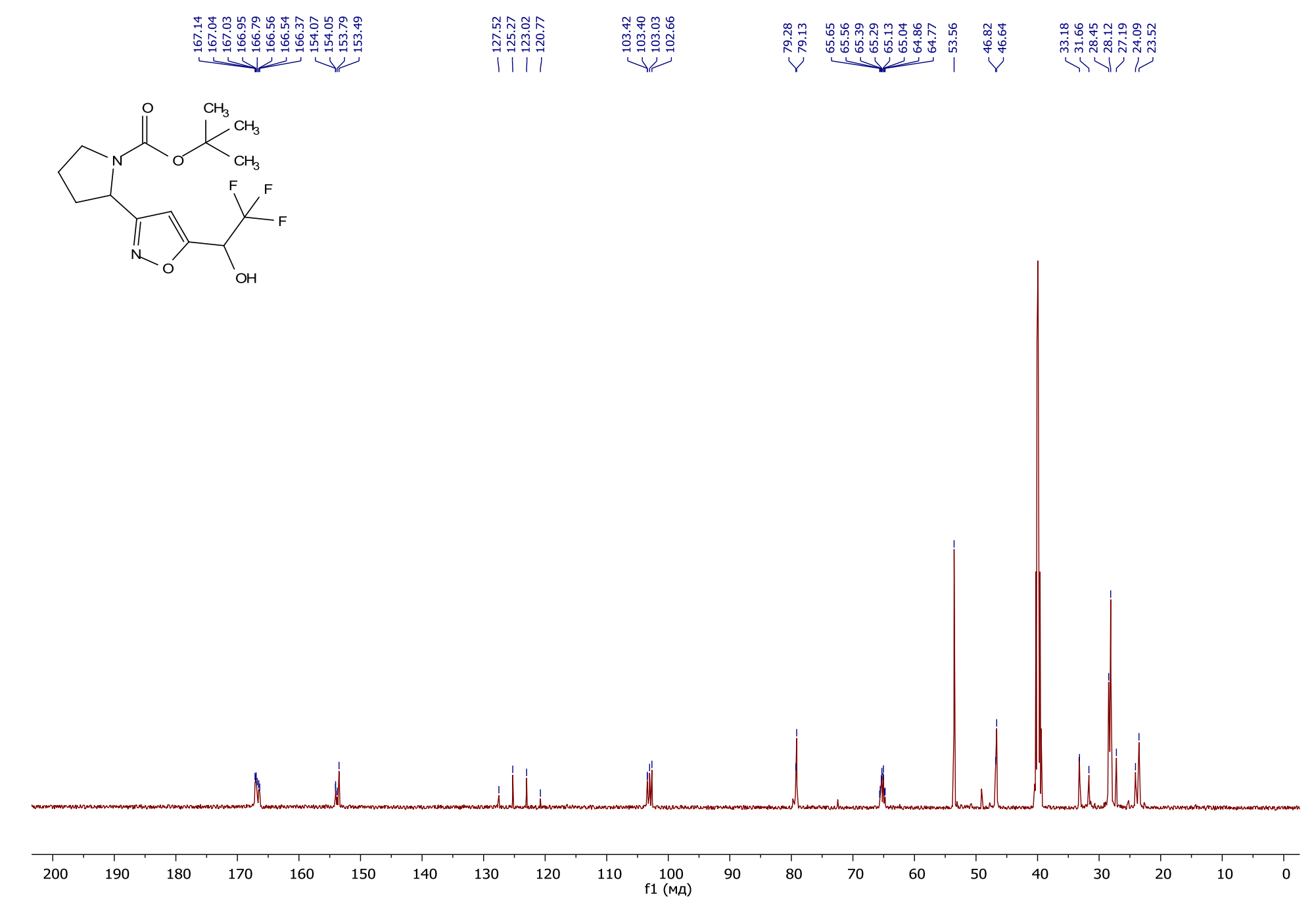

tert-Butyl 2-(5-(2,2,2-trifluoro-1-hydroxyethyl)isoxazol-3-yl)pyrrolidine-1-carboxylate (39k or 39l) ${ }^{13} \mathrm{C}$ NMR 

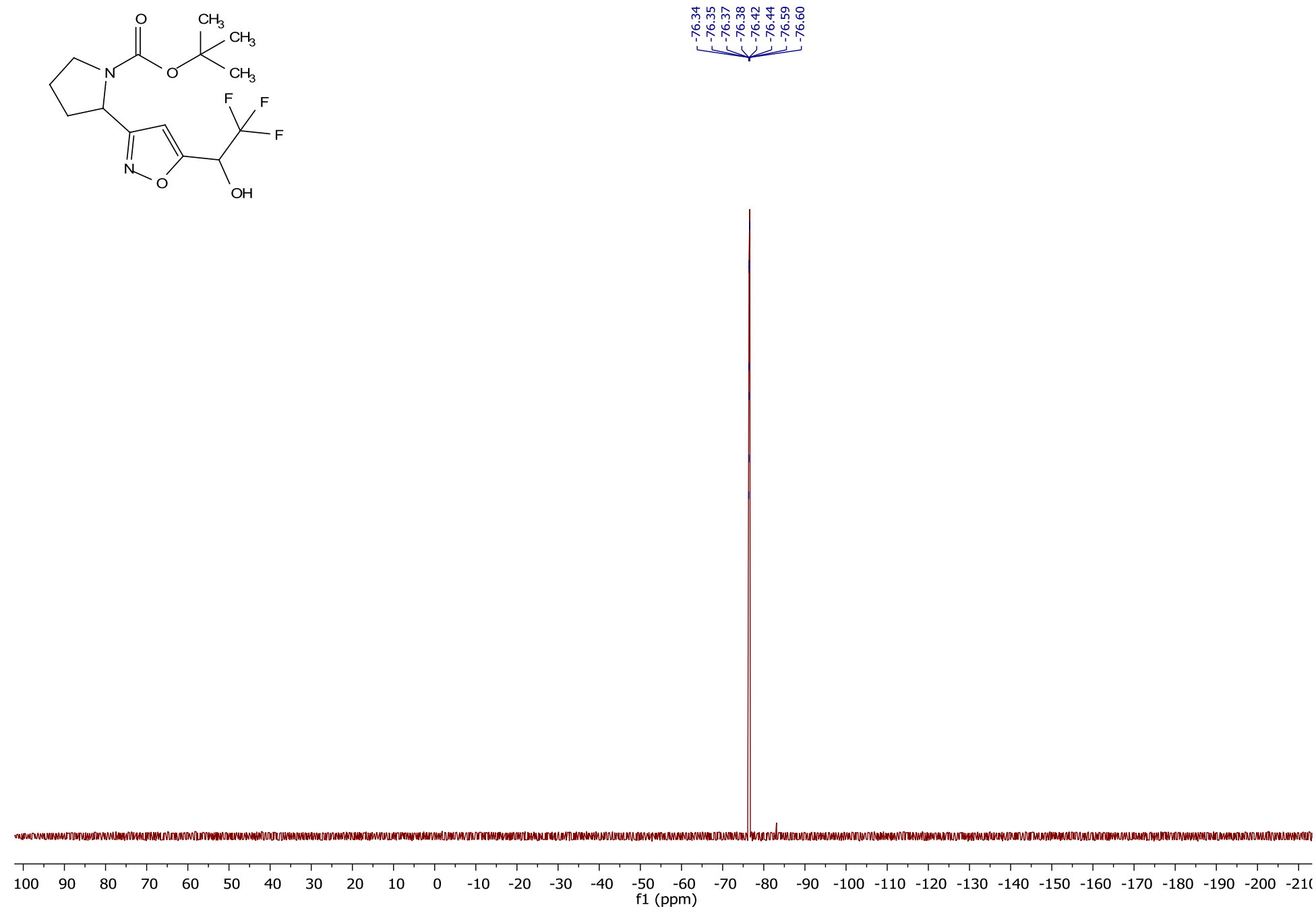

tert-Butyl 2-(5-(2,2,2-trifluoro-1-hydroxyethyl)isoxazol-3-yl)pyrrolidine-1-carboxylate (39k or 39l) ${ }^{19}$ F NMR 

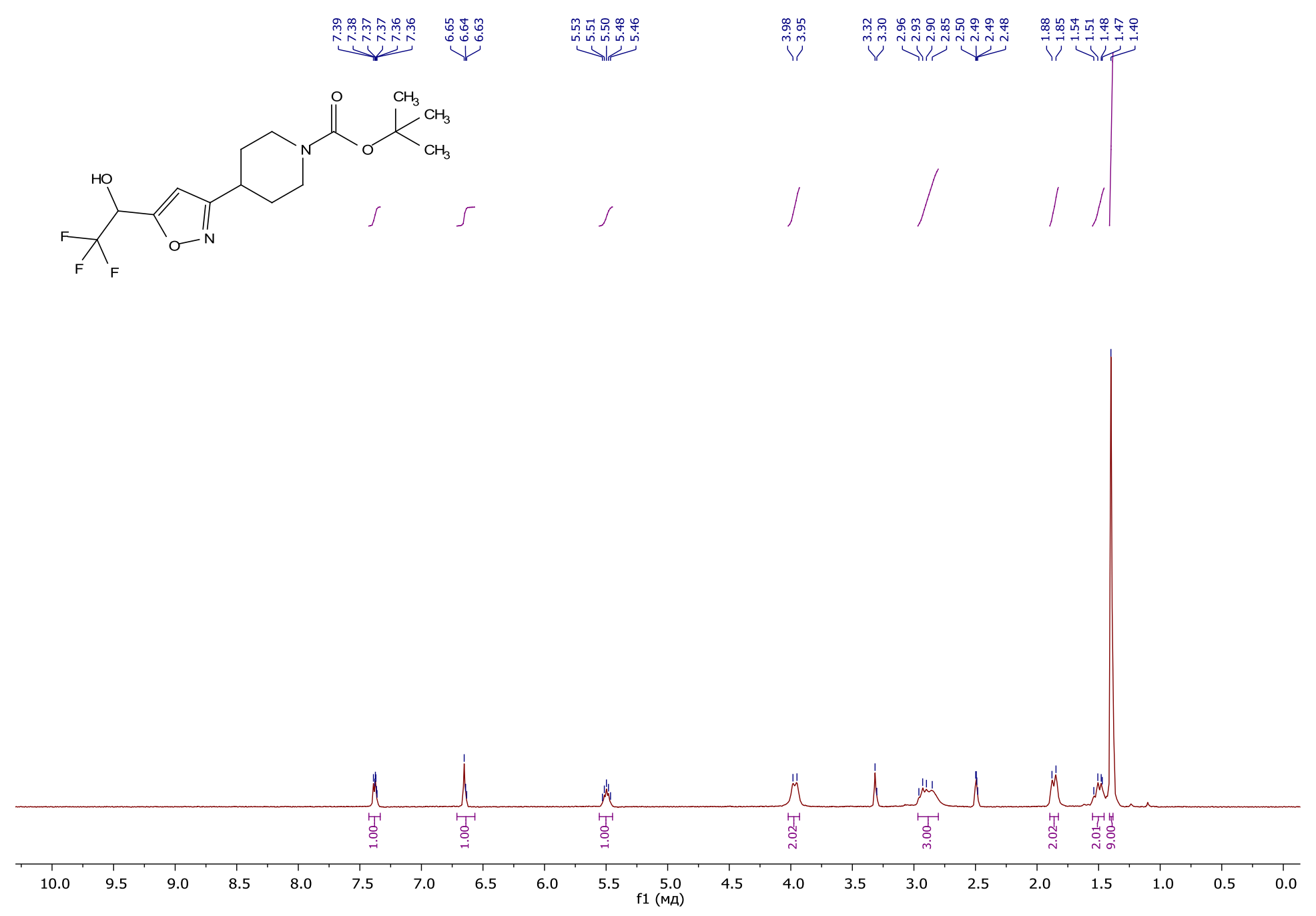

tert-Butyl 4-(5-(2,2,2-trifluoro-1-hydroxyethyl)isoxazol-3-yl)piperidine-1-carboxylate (39m) ${ }^{1}$ H NMR 


\begin{tabular}{|c|c|c|c|c|c|c|}
\hline 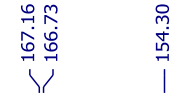 & 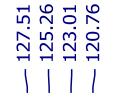 & $\begin{array}{c}\stackrel{2}{n} \\
\stackrel{2}{0} \\
\stackrel{0}{1} \\
\mid\end{array}$ & $\begin{array}{l}\stackrel{\circ}{0} \\
\stackrel{0}{1} \\
1\end{array}$ & 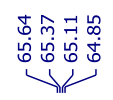 & $\underset{\substack{q \\
\text { g }}}{\mid}$ & 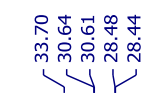 \\
\hline
\end{tabular}
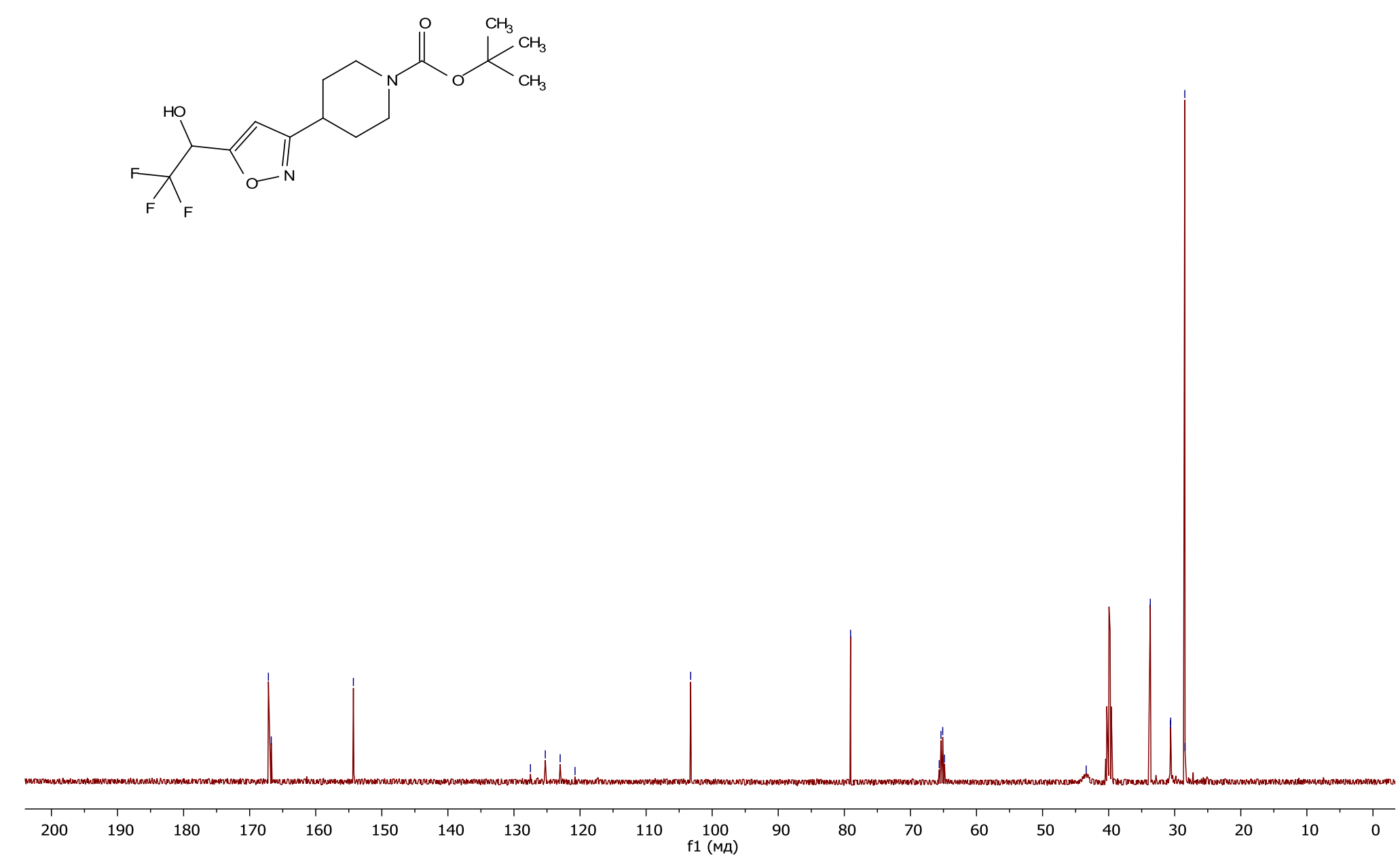

tert-Butyl 4-(5-(2,2,2-trifluoro-1-hydroxyethyl)isoxazol-3-yl)piperidine-1-carboxylate $(39 \mathrm{~m}){ }^{13} \mathrm{C}$ NMR 

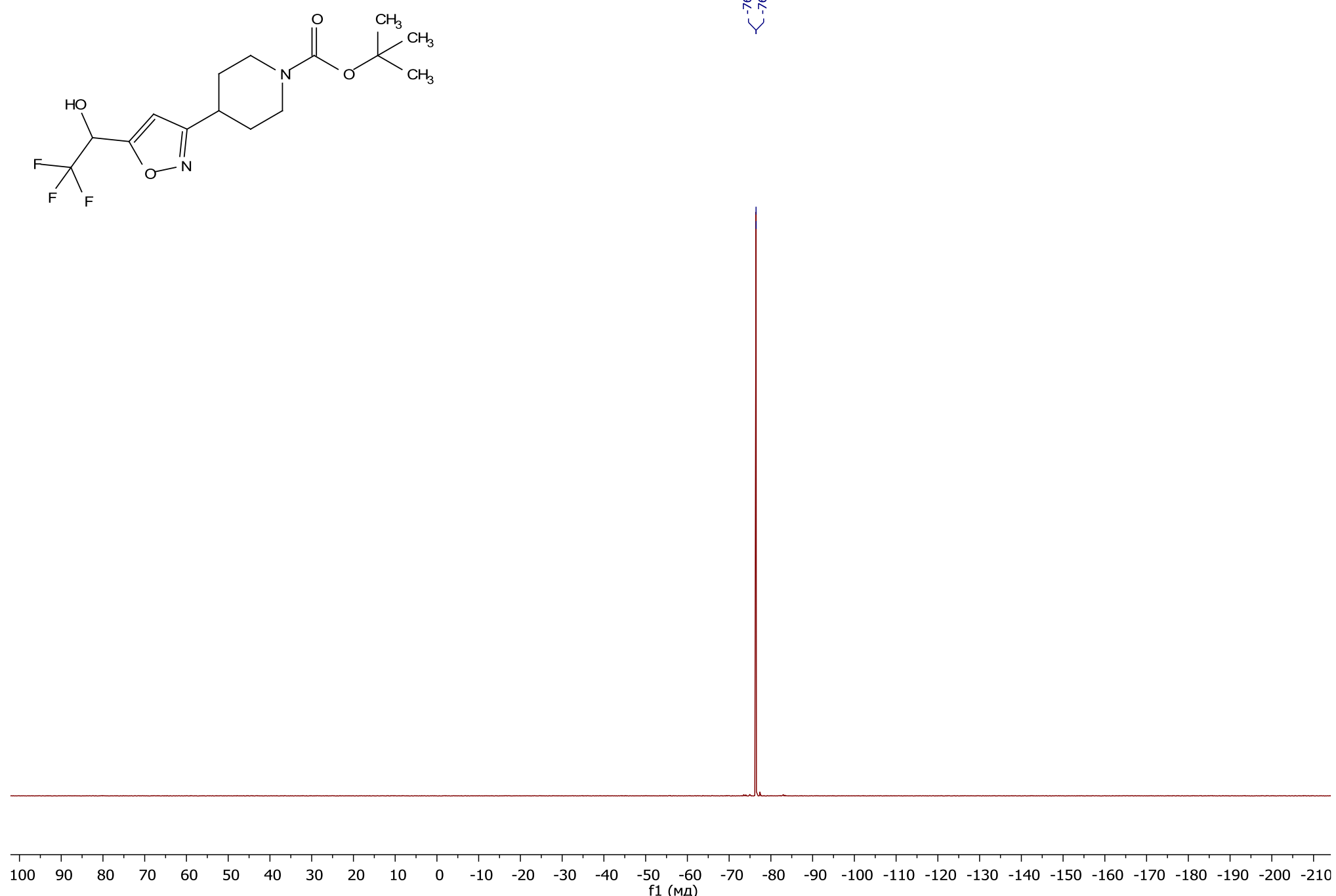

tert-Butyl 4-(5-(2,2,2-trifluoro-1-hydroxyethyl)isoxazol-3-yl)piperidine-1-carboxylate (39m) ${ }^{19}$ F NMR 

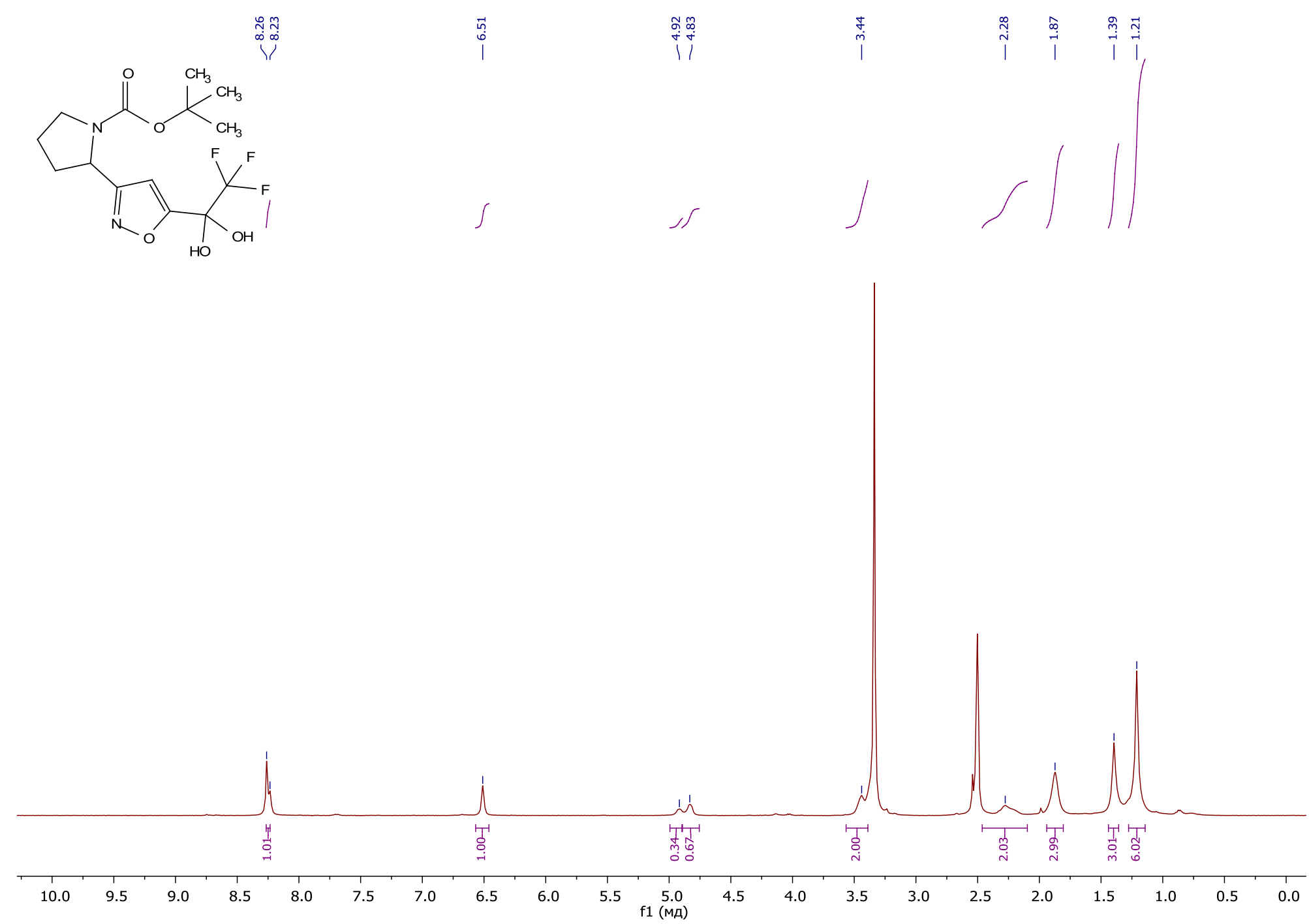

tert-Butyl 2-(5-(2,2,2-trifluoro-1,1-dihydroxyethyl)isoxazol-3-yl)pyrrolidine-1-carboxylate (40k or 40l) ${ }^{1} \mathrm{H}$ NMR 

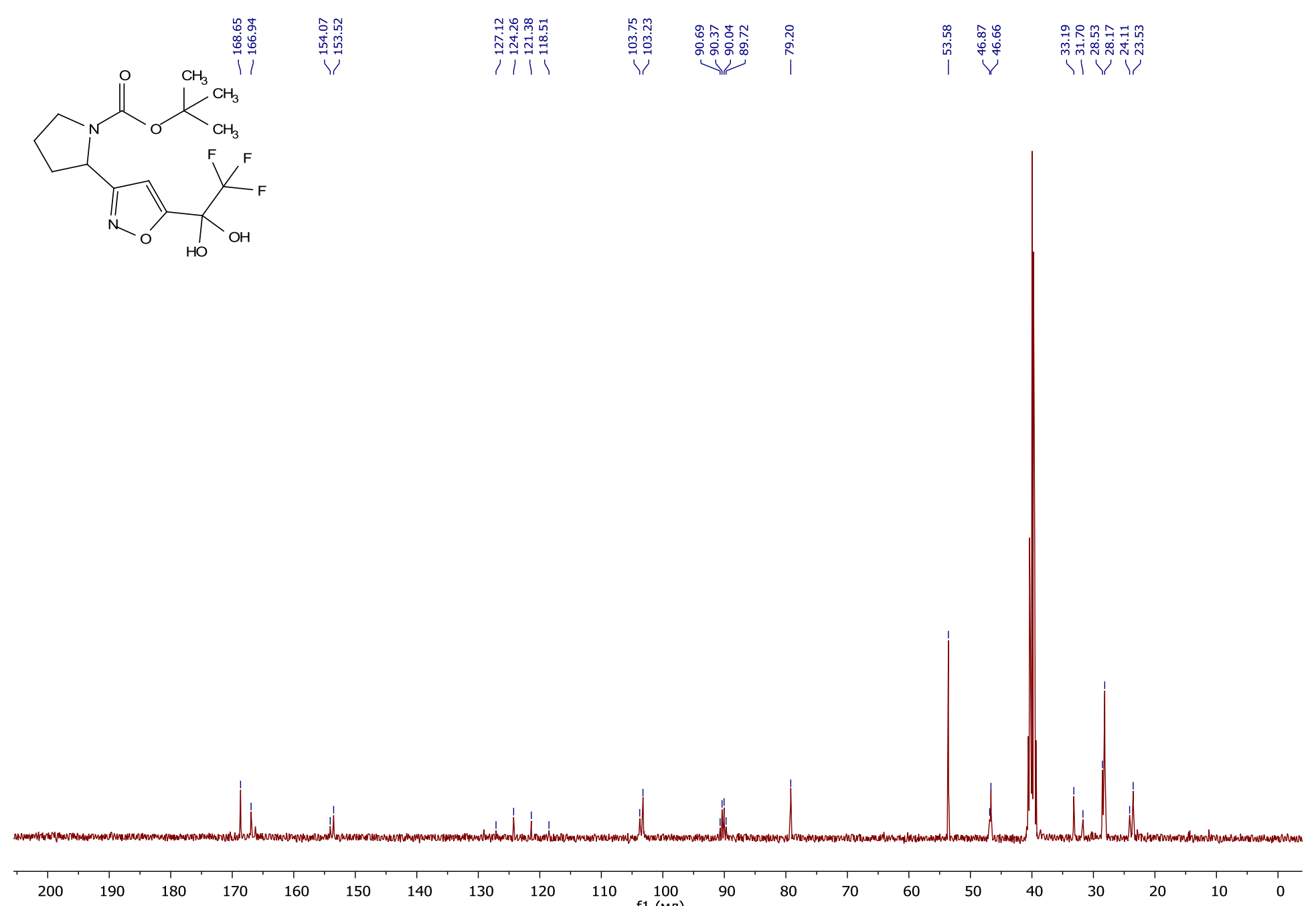

tert-Butyl 2-(5-(2,2,2-trifluoro-1,1-dihydroxyethyl)isoxazol-3-yl)pyrrolidine-1-carboxylate (40k or 40I) ${ }^{13} \mathrm{C}$ NMR 

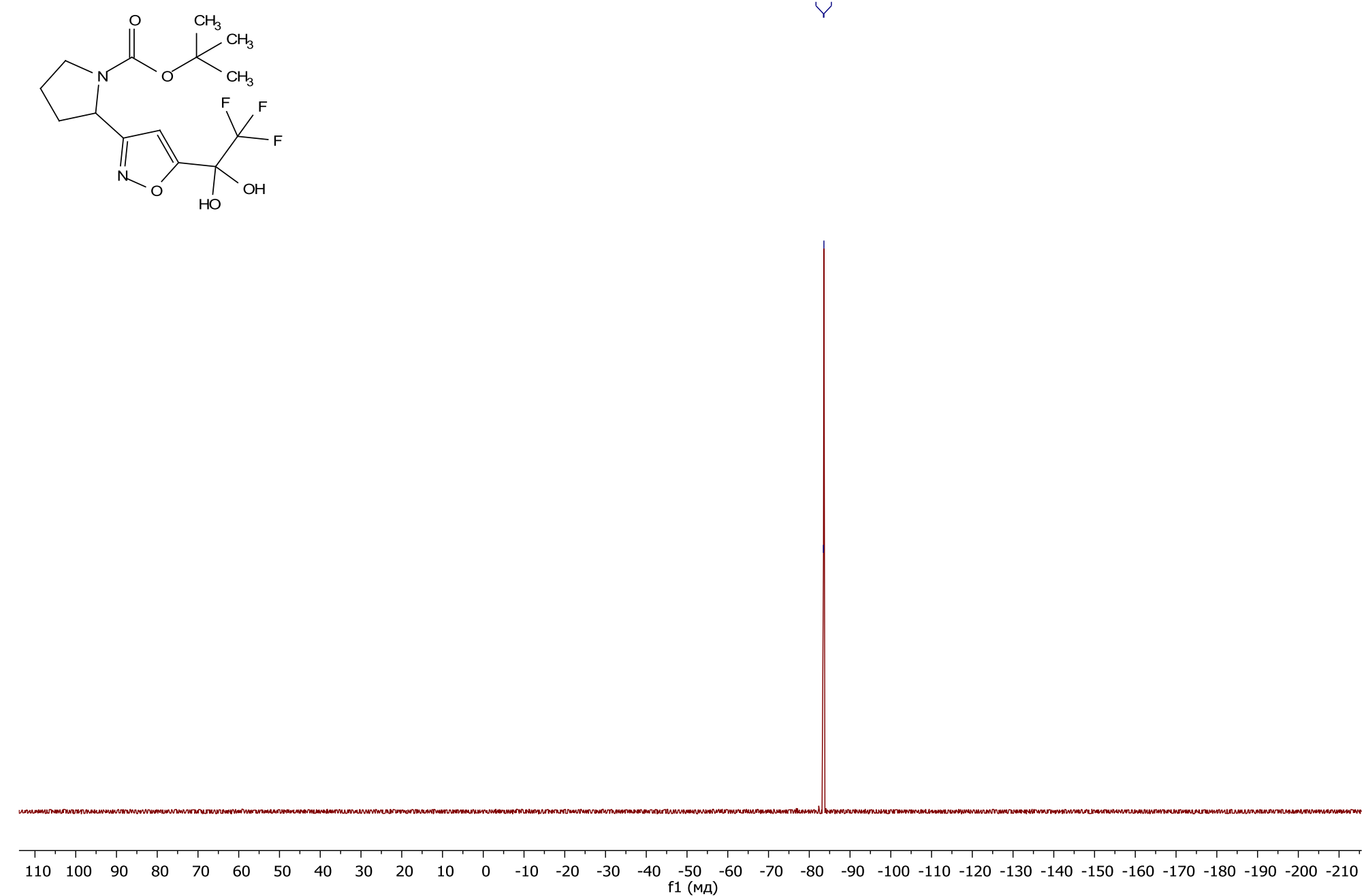

tert-Butyl 2-(5-(2,2,2-trifluoro-1,1-dihydroxyethyl)isoxazol-3-yl)pyrrolidine-1-carboxylate (40k or 40l) ${ }^{19}$ F NMR 

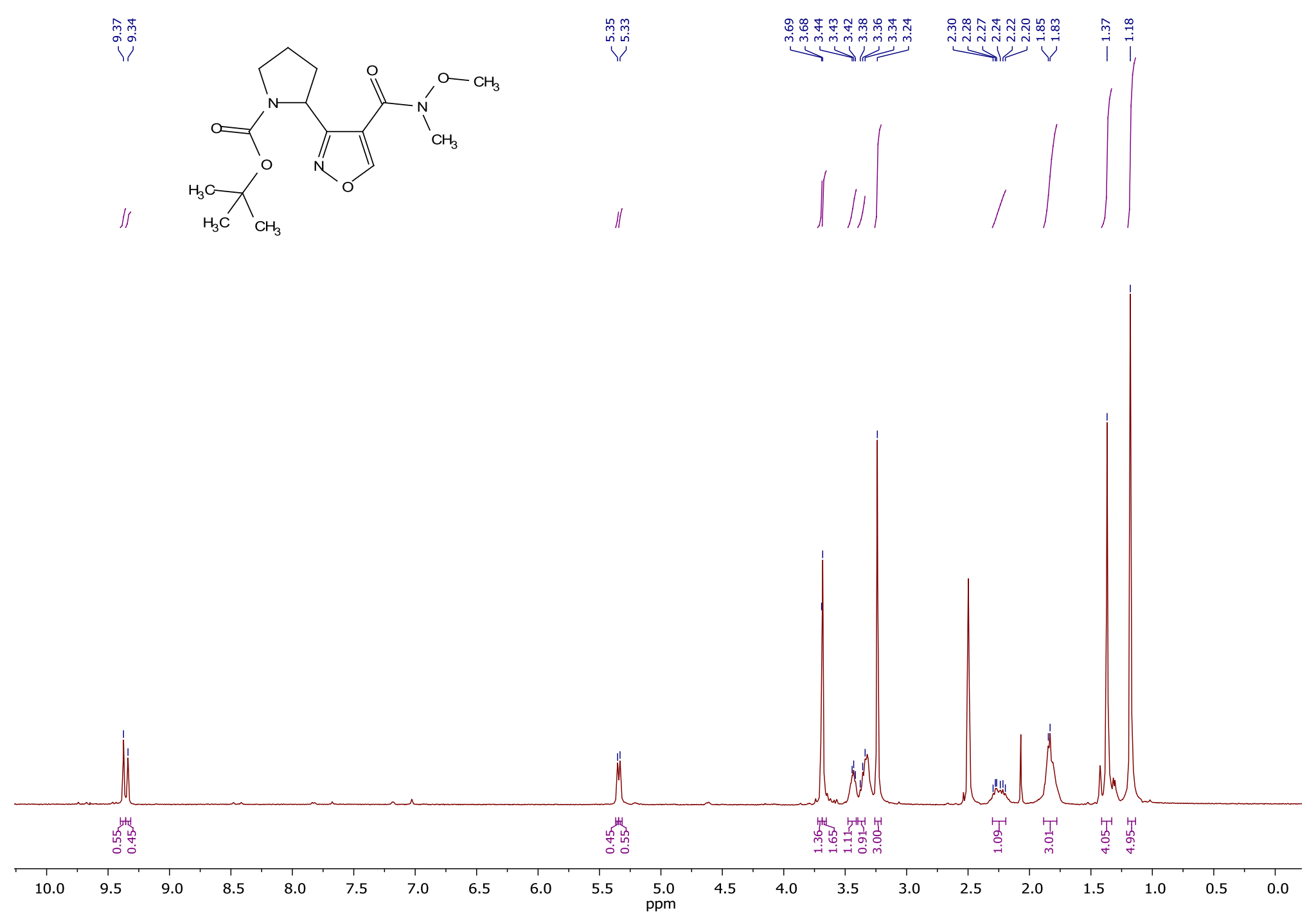

tert-Butyl 2-(4-(methoxy(methyl)carbamoyl)isoxazol-3-yl)pyrrolidine-1-carboxylate (42a or 42b) ${ }^{1} \mathrm{H}$ NMR 


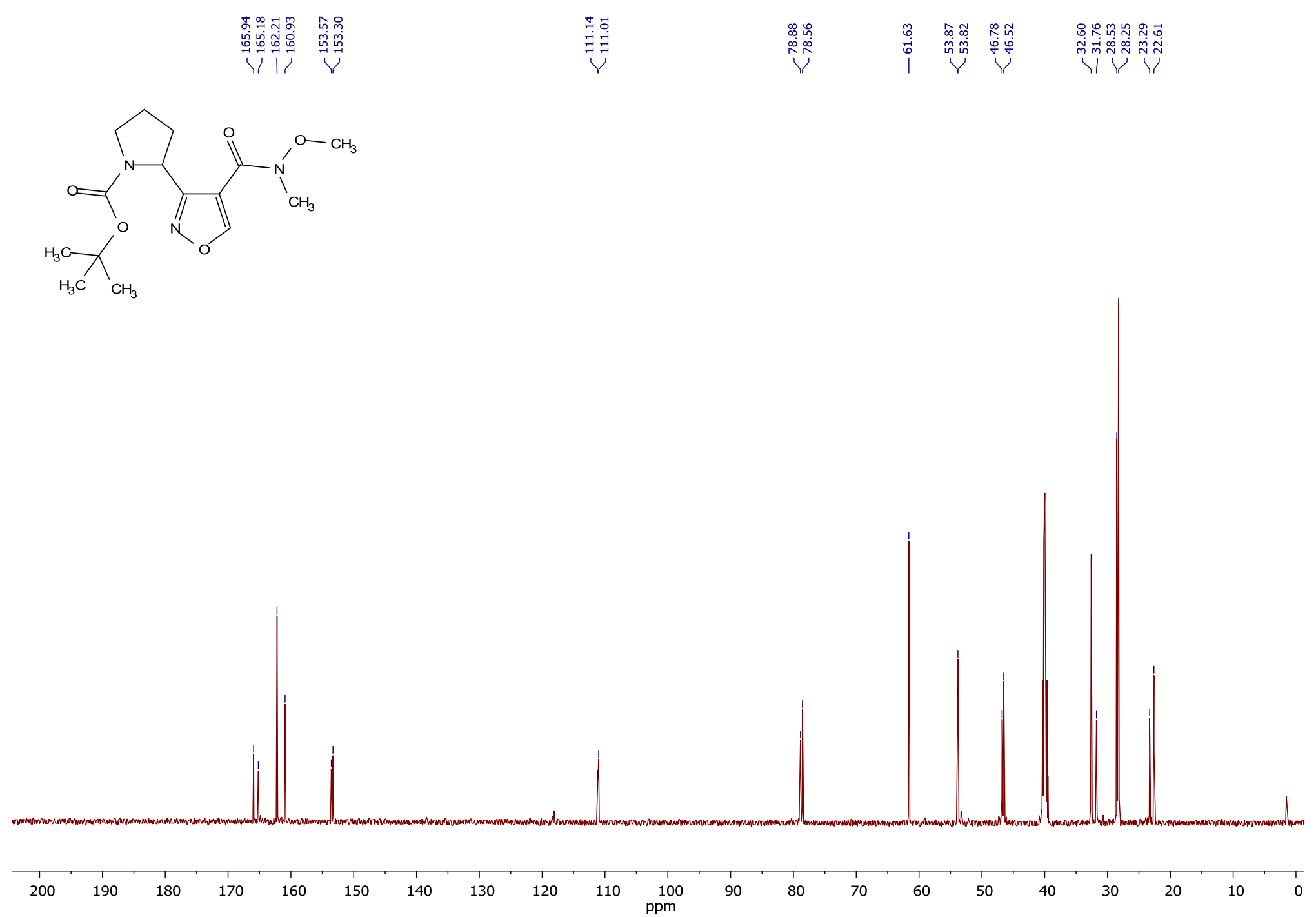

tert-Butyl 2-(4-(methoxy(methyl)carbamoyl)isoxazol-3-yl)pyrrolidine-1-carboxylate (42a or 42b) ${ }^{13} \mathrm{C}$ NMR 

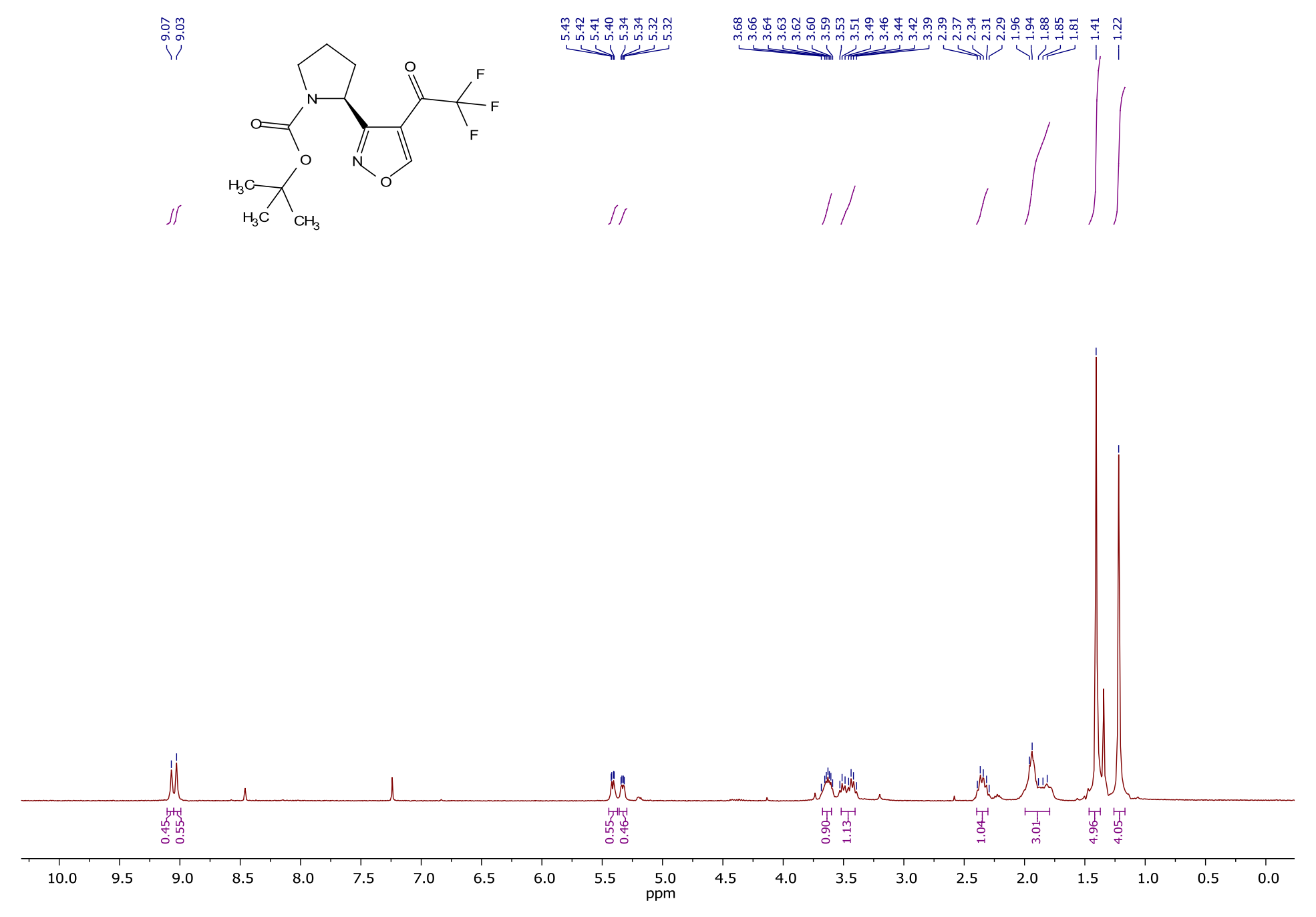

tert-Butyl 2-(4-(2,2,2-trifluoroacetyl)isoxazol-3-yl)pyrrolidine-1-carboxylate (43a or 43b) ${ }^{1} \mathrm{H}$ NMR 


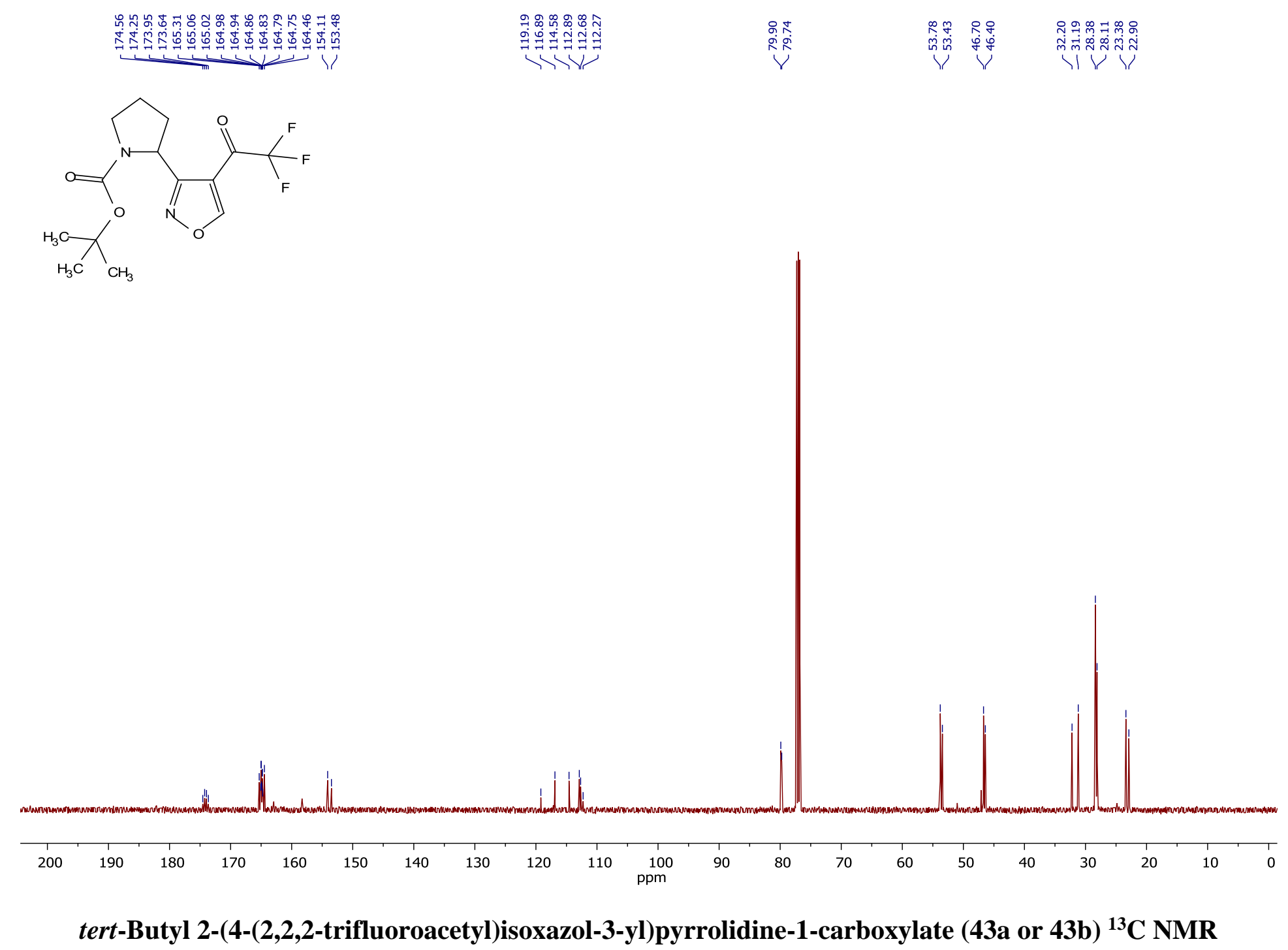



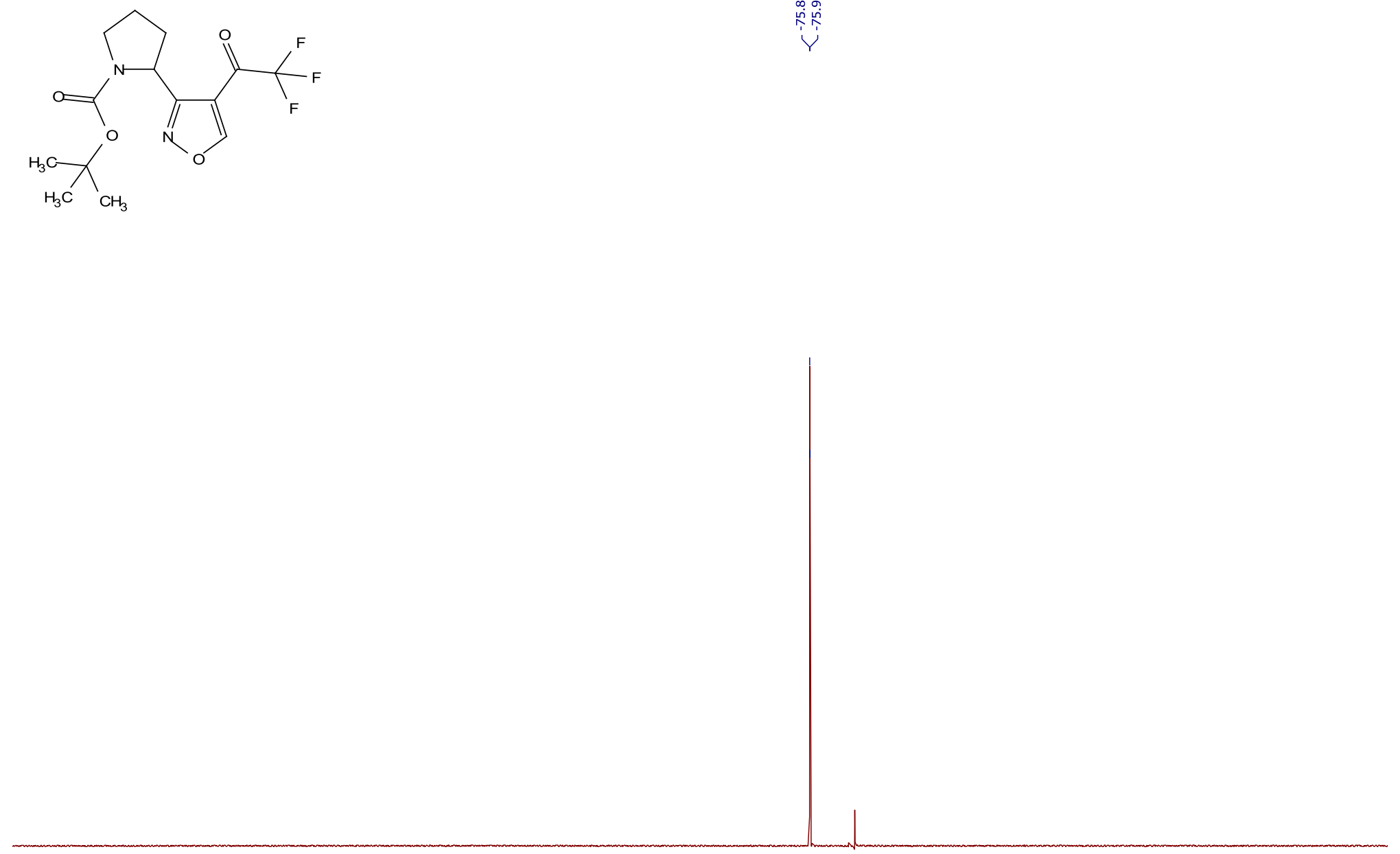

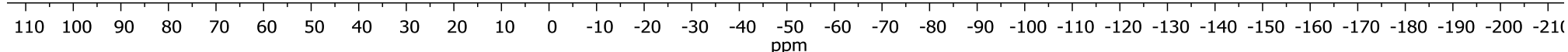
tert-Butyl 2-(4-(2,2,2-trifluoroacetyl)isoxazol-3-yl)pyrrolidine-1-carboxylate (43a or 43b) ${ }^{19} \mathrm{~F}$ NMR 

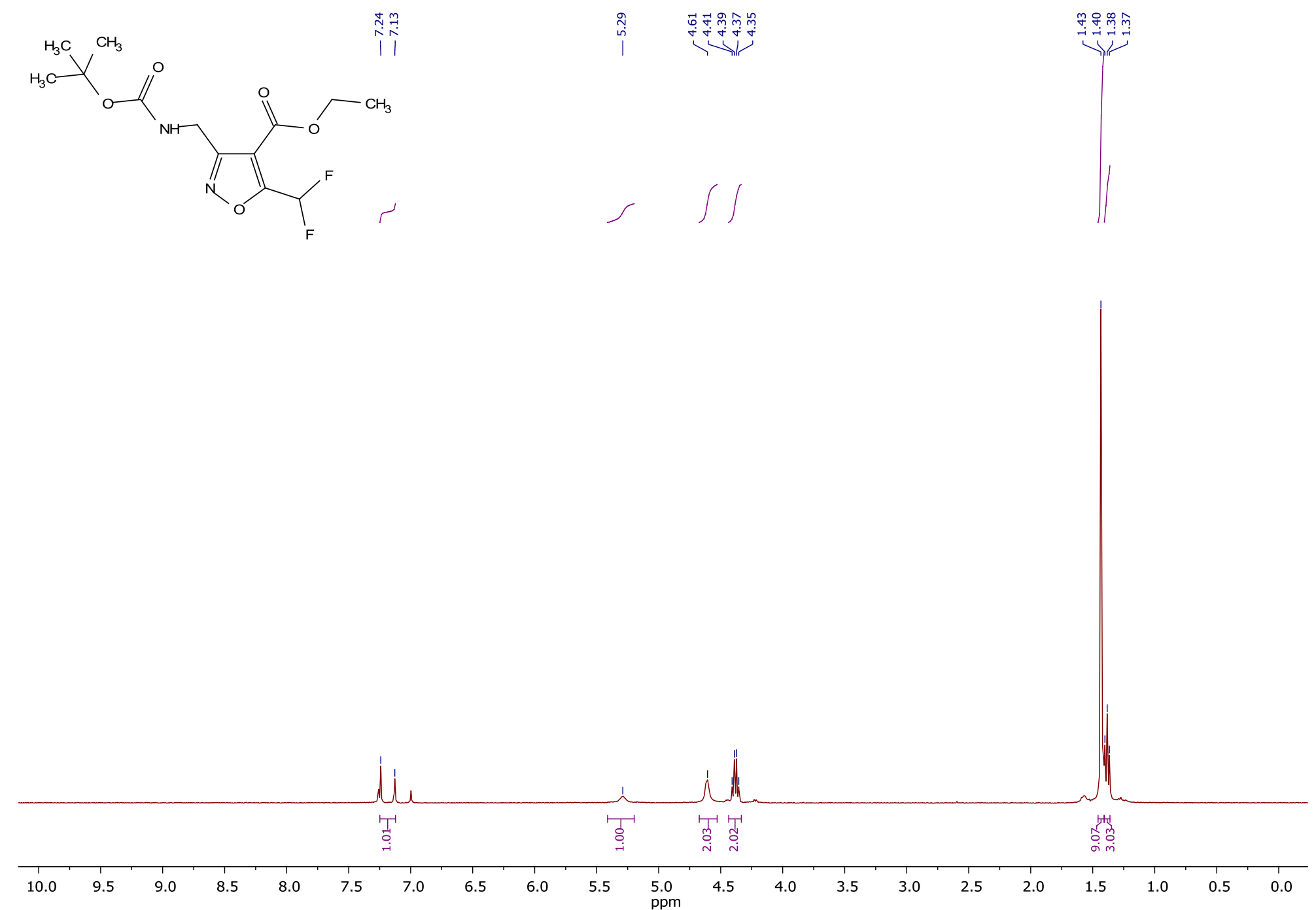

Ethyl 3-(((tert-butoxycarbonyl)amino)methyl)-5-(difluoromethyl)-isoxazole-4-carboxylate (48f) ${ }^{1}$ H NMR 


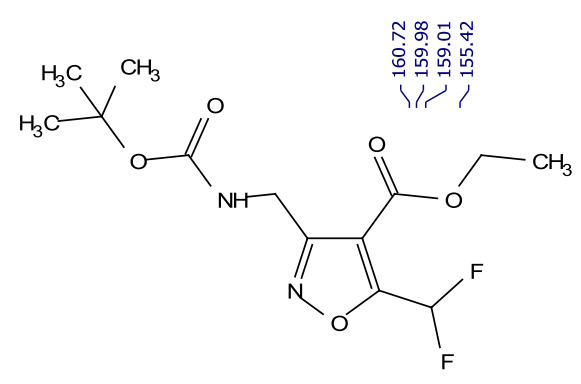

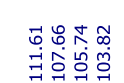

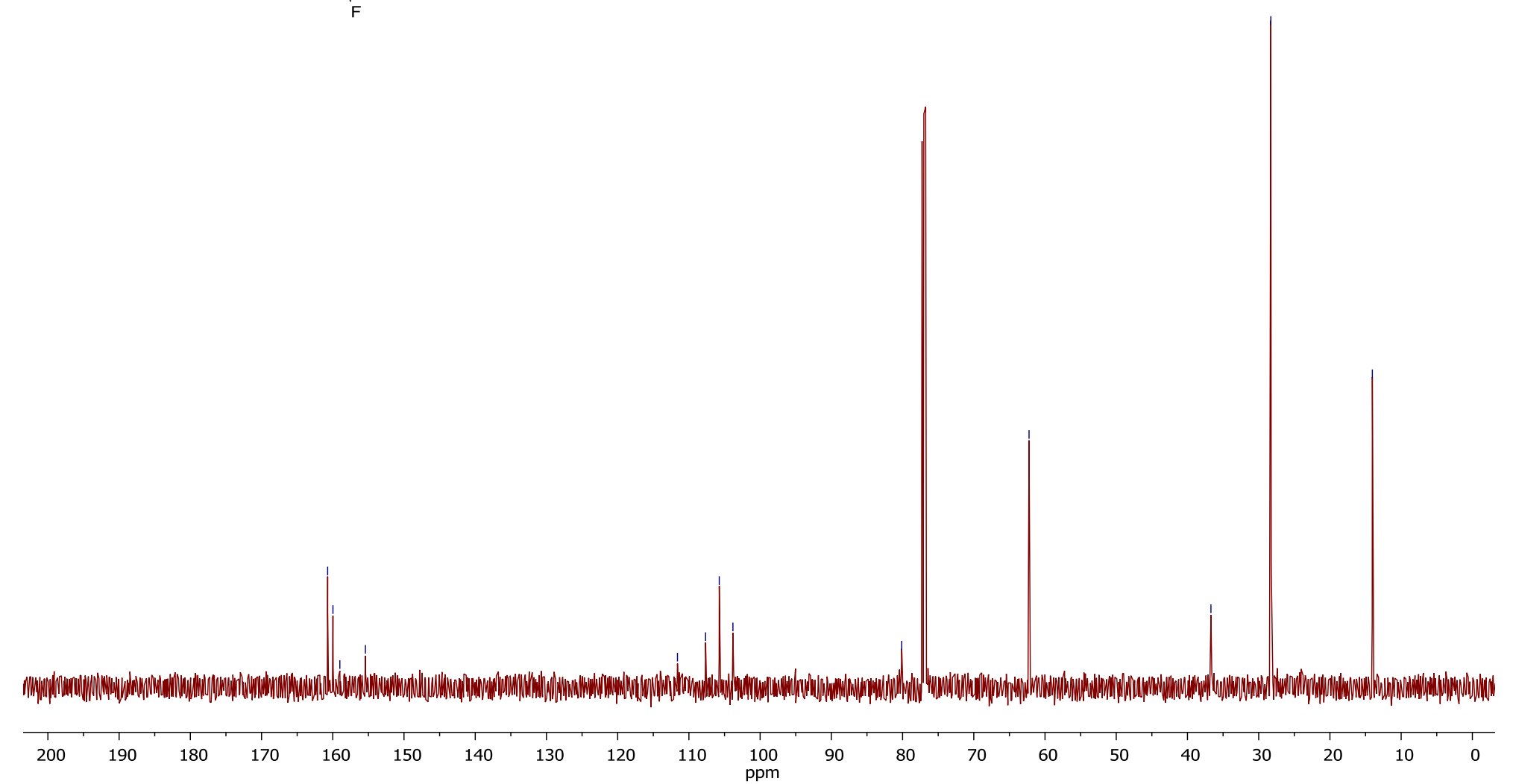

Ethyl 3-(((tert-butoxycarbonyl)amino)methyl)-5-(difluoromethyl)-isoxazole-4-carboxylate (48f) ${ }^{13}$ C NMR 

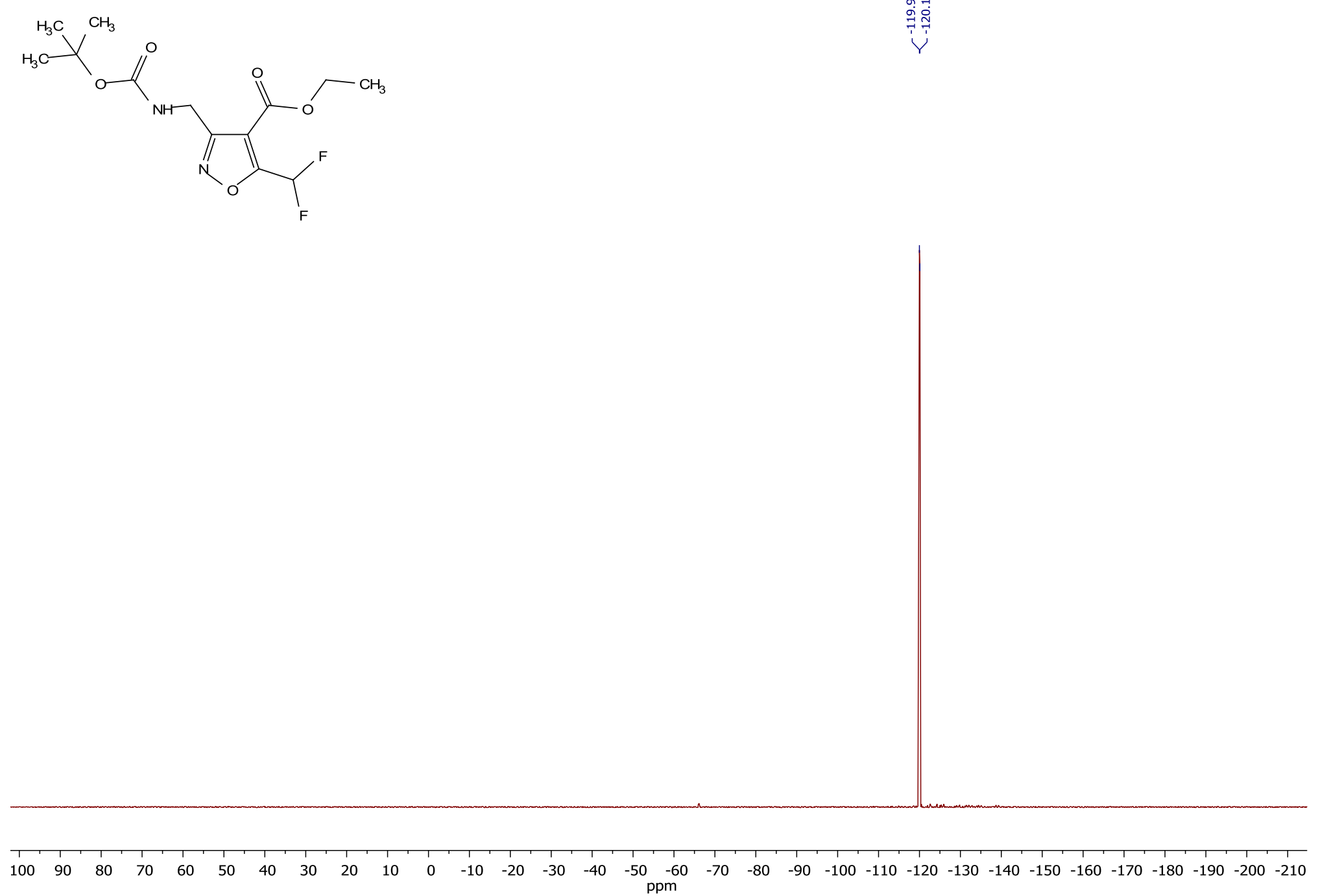

Ethyl 3-(((tert-butoxycarbonyl)amino)methyl)-5-(difluoromethyl)-isoxazole-4-carboxylate (48f) ${ }^{19}$ F NMR 

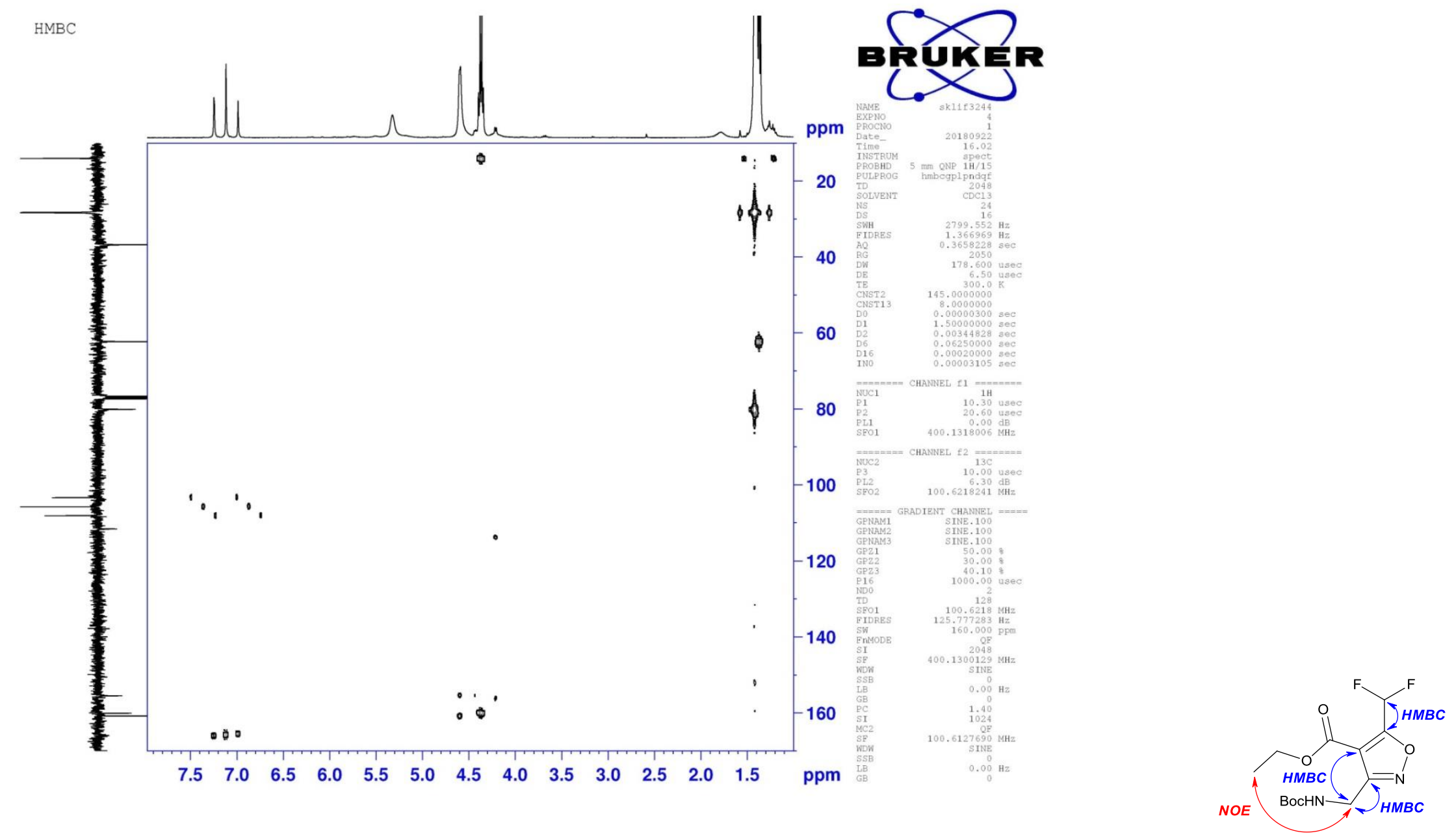

Ethyl 3-(((tert-butoxycarbonyl)amino)methyl)-5-(difluoromethyl)-isoxazole-4-carboxylate (48f) HMBC 

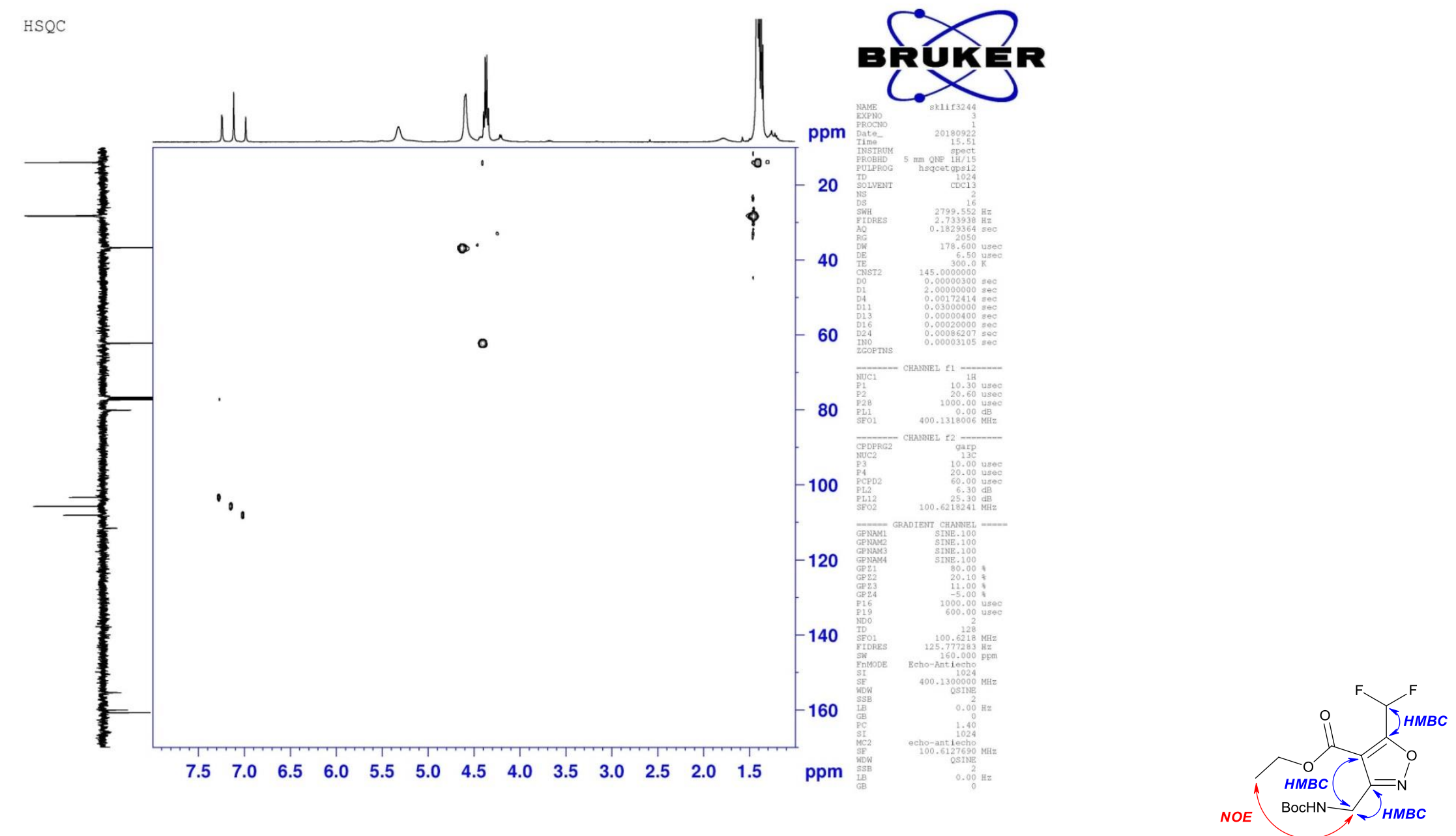

Ethyl 3-(((tert-butoxycarbonyl)amino)methyl)-5-(difluoromethyl)-isoxazole-4-carboxylate (48f) HSQC 

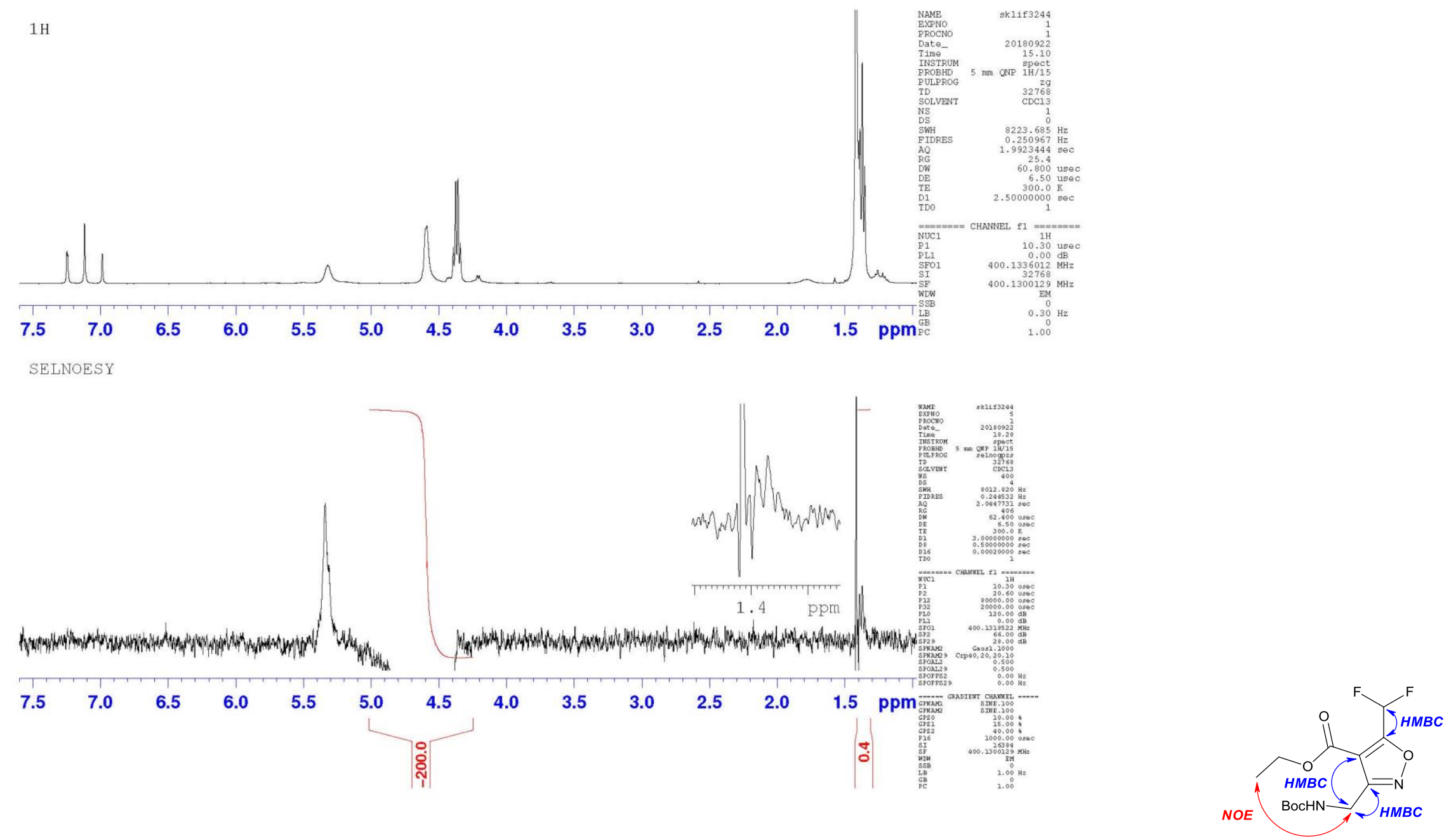

Ethyl 3-(((tert-butoxycarbonyl)amino)methyl)-5-(difluoromethyl)-isoxazole-4-carboxylate (48f) SELNOESY 


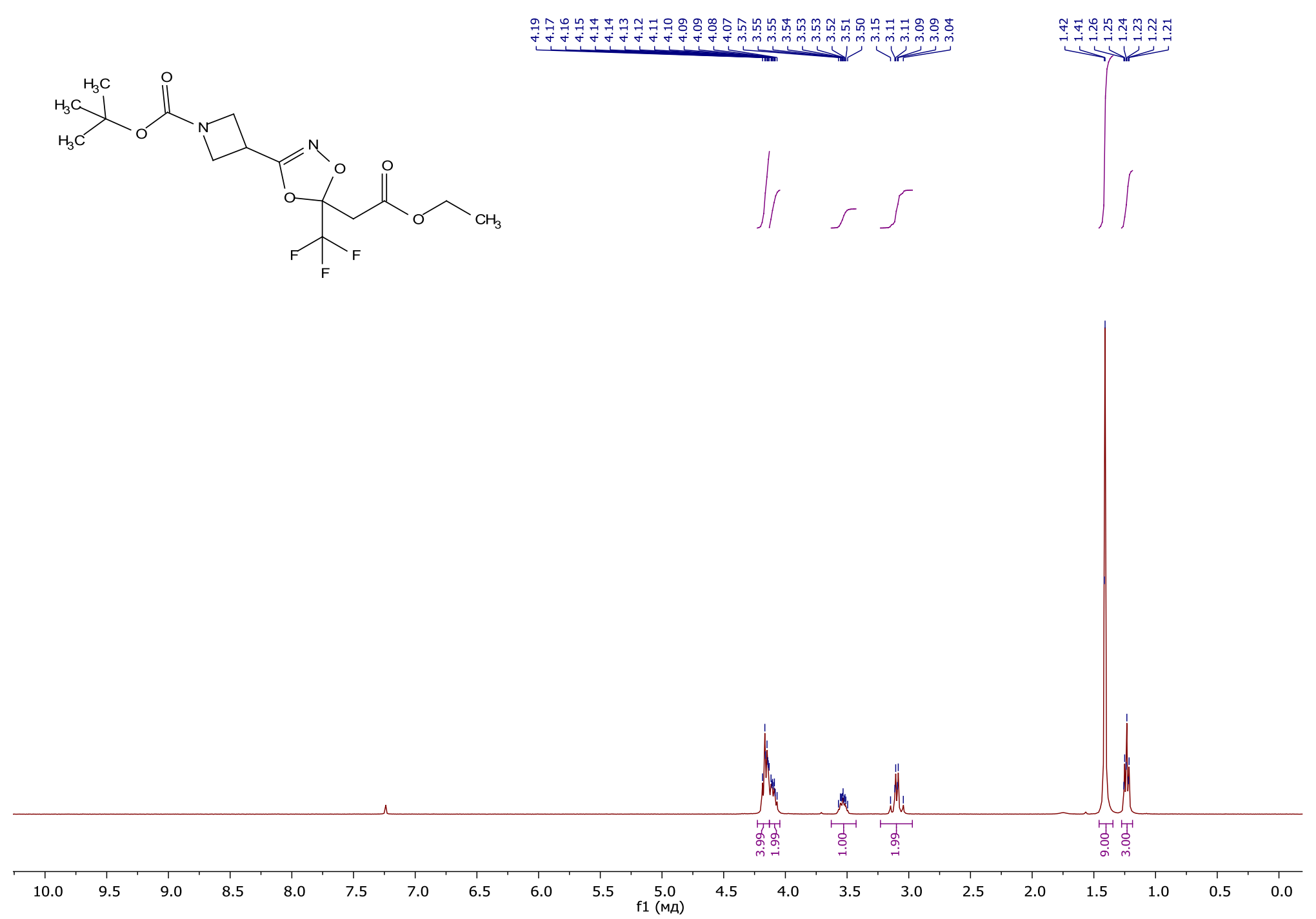

tert-Butyl 3-(5-(2-ethoxy-2-oxoethyl)-5-(trifluoromethyl)-1,4,2-dioxazol-3-yl)azetidine-1-carboxylate (50j) ${ }^{1} \mathrm{H}$ NMR 


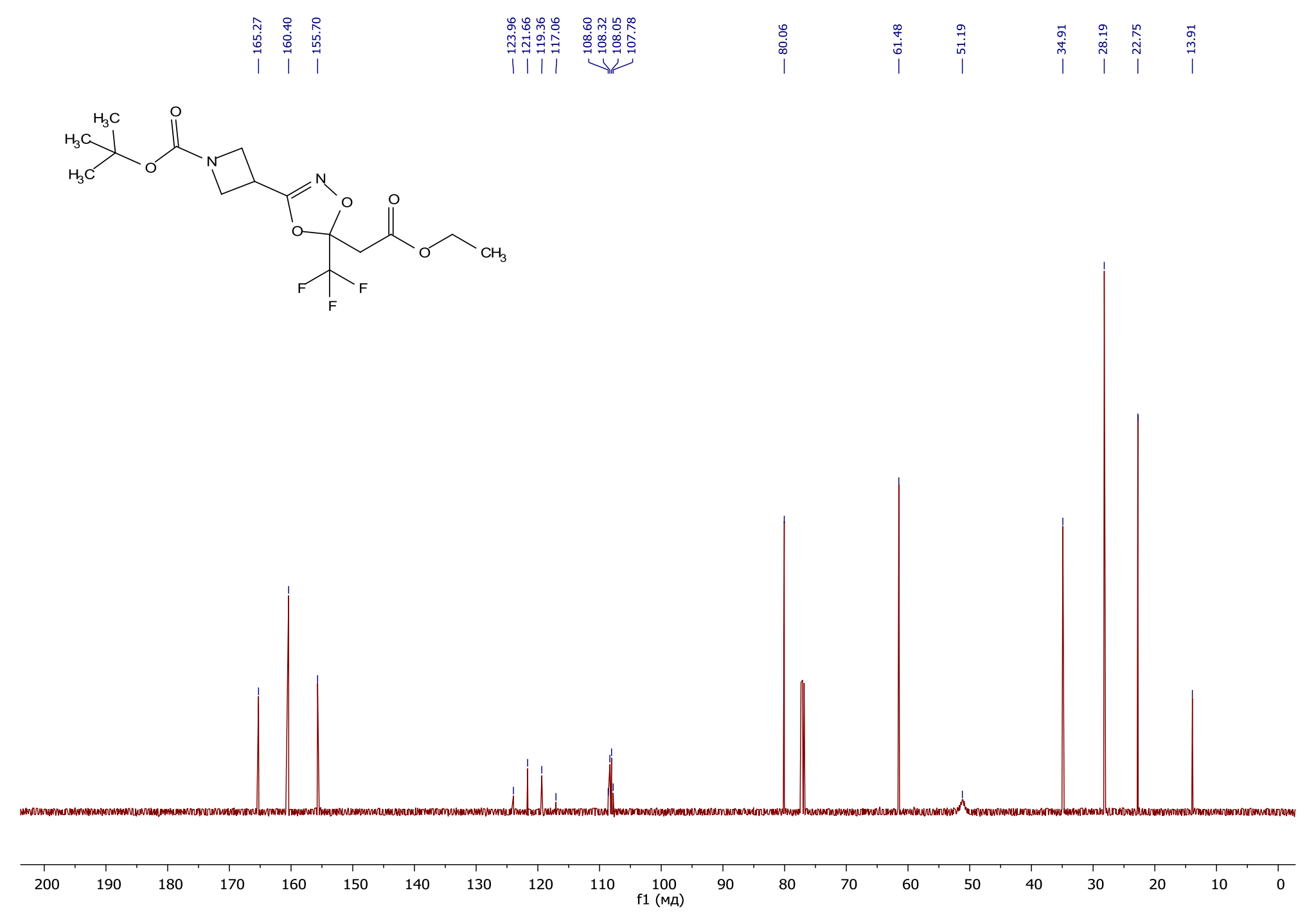

tert-Butyl 3-(5-(2-ethoxy-2-oxoethyl)-5-(trifluoromethyl)-1,4,2-dioxazol-3-yl)azetidine-1-carboxylate (50j) ${ }^{13} \mathrm{C}$ NMR 


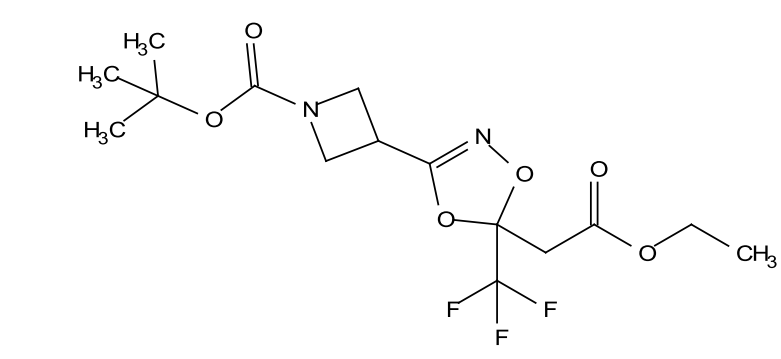

|

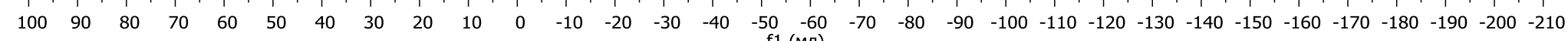
tert-Butyl 3-(5-(2-ethoxy-2-oxoethyl)-5-(trifluoromethyl)-1,4,2-dioxazol-3-yl)azetidine-1-carboxylate (50j) ${ }^{19}$ F NMR 

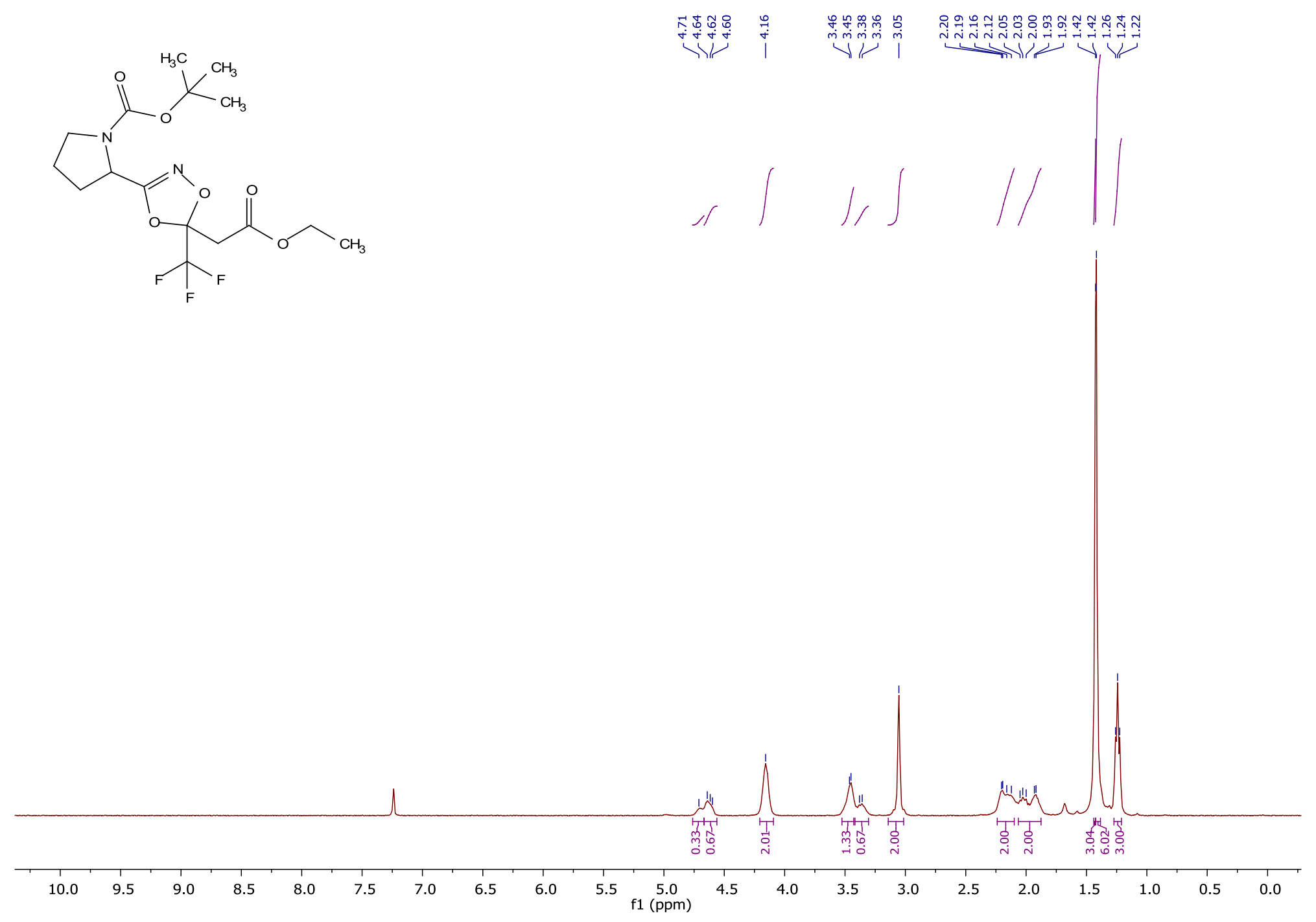

tert-Butyl 2-(5-(2-ethoxy-2-oxoethyl)-5-(trifluoromethyl)-1,4,2-dioxazol-3-yl)pyrrolidine-1-carboxylate (50k or 501) ${ }^{1} \mathrm{H}$ NMR 

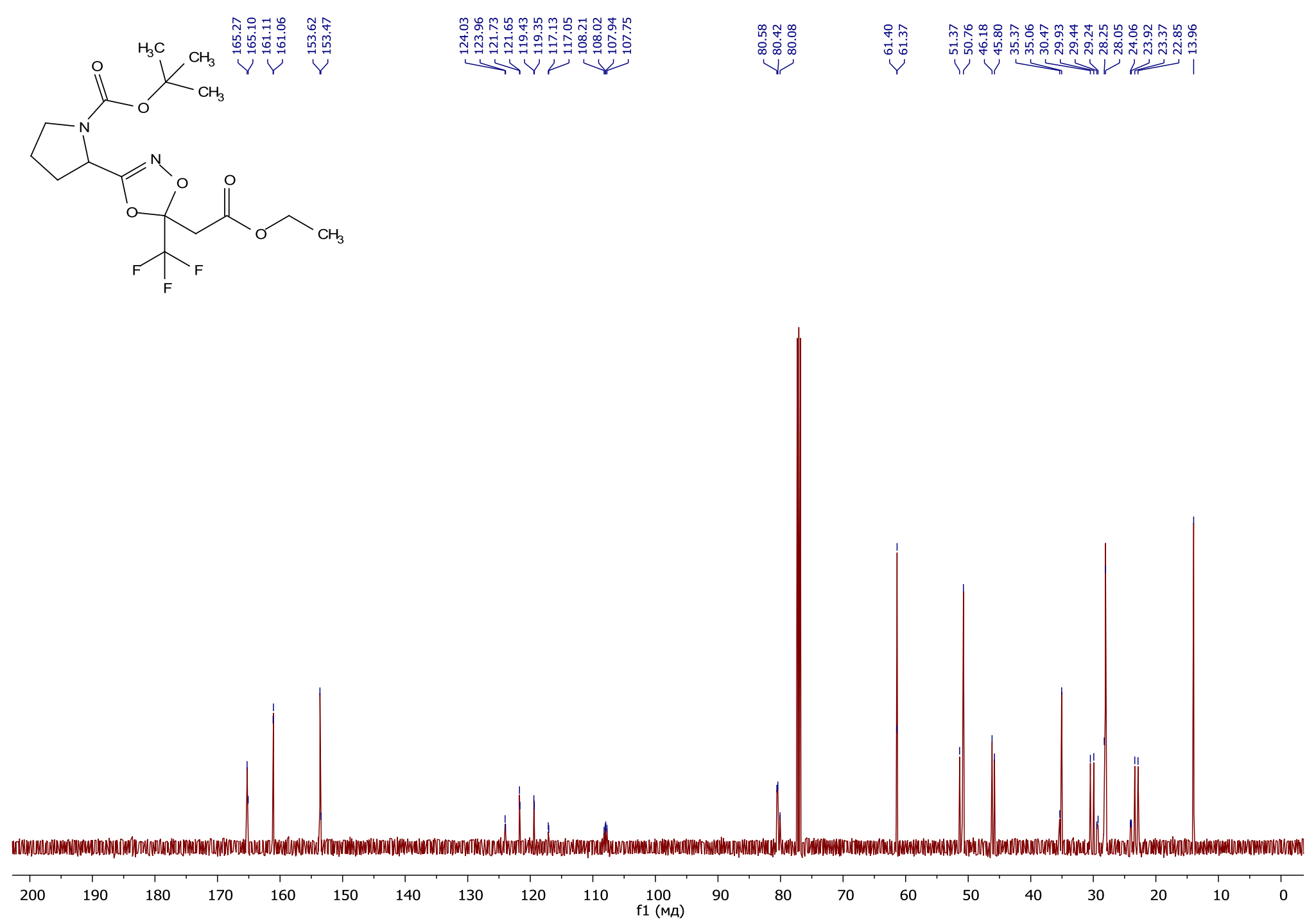

tert-Butyl 2-(5-(2-ethoxy-2-oxoethyl)-5-(trifluoromethyl)-1,4,2-dioxazol-3-yl)pyrrolidine-1-carboxylate (50k or 501) ${ }^{13} \mathrm{C}$ NMR 

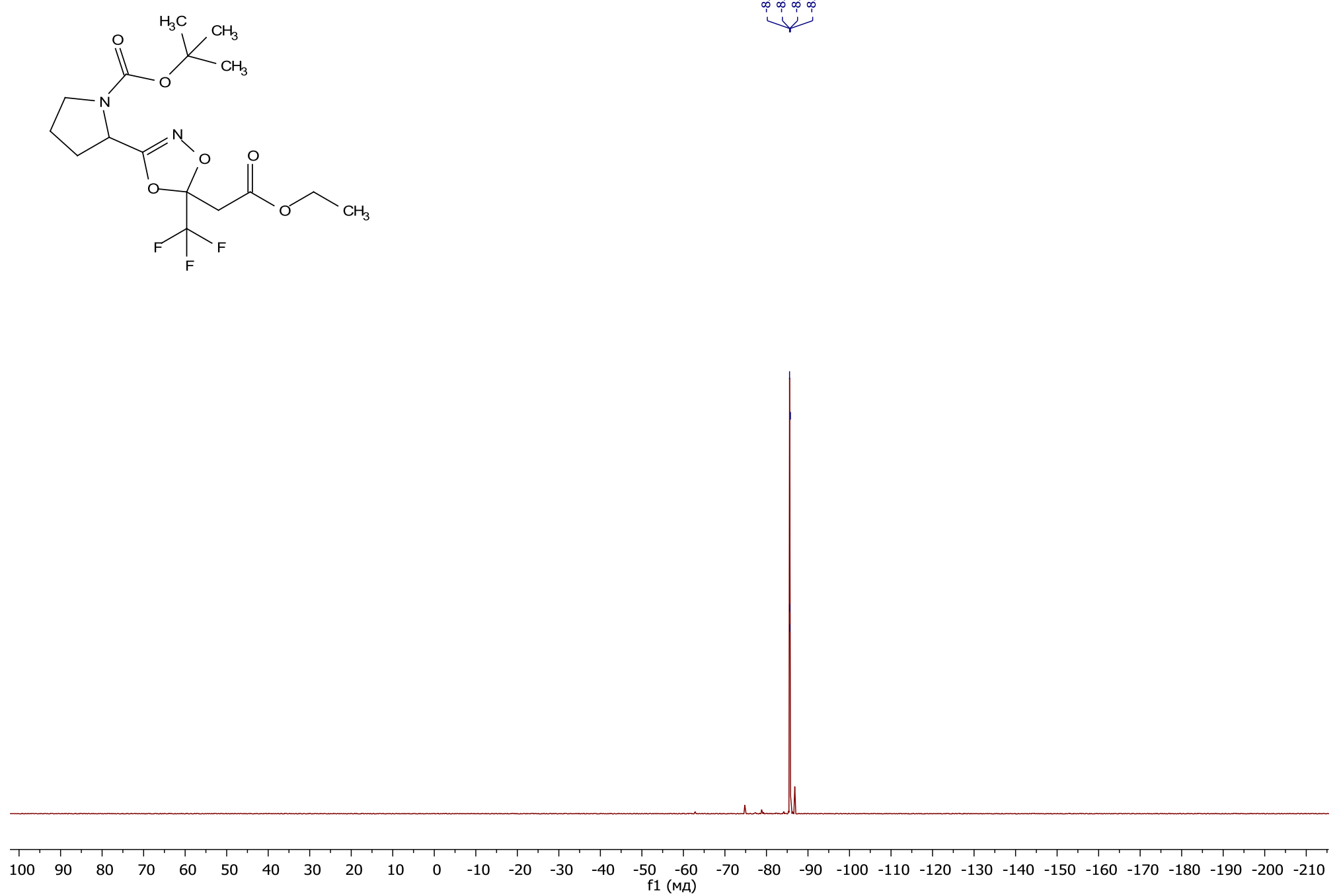

tert-Butyl 2-(5-(2-ethoxy-2-oxoethyl)-5-(trifluoromethyl)-1,4,2-dioxazol-3-yl)pyrrolidine-1-carboxylate (50k or 501) ${ }^{19} \mathrm{~F}$ NMR 

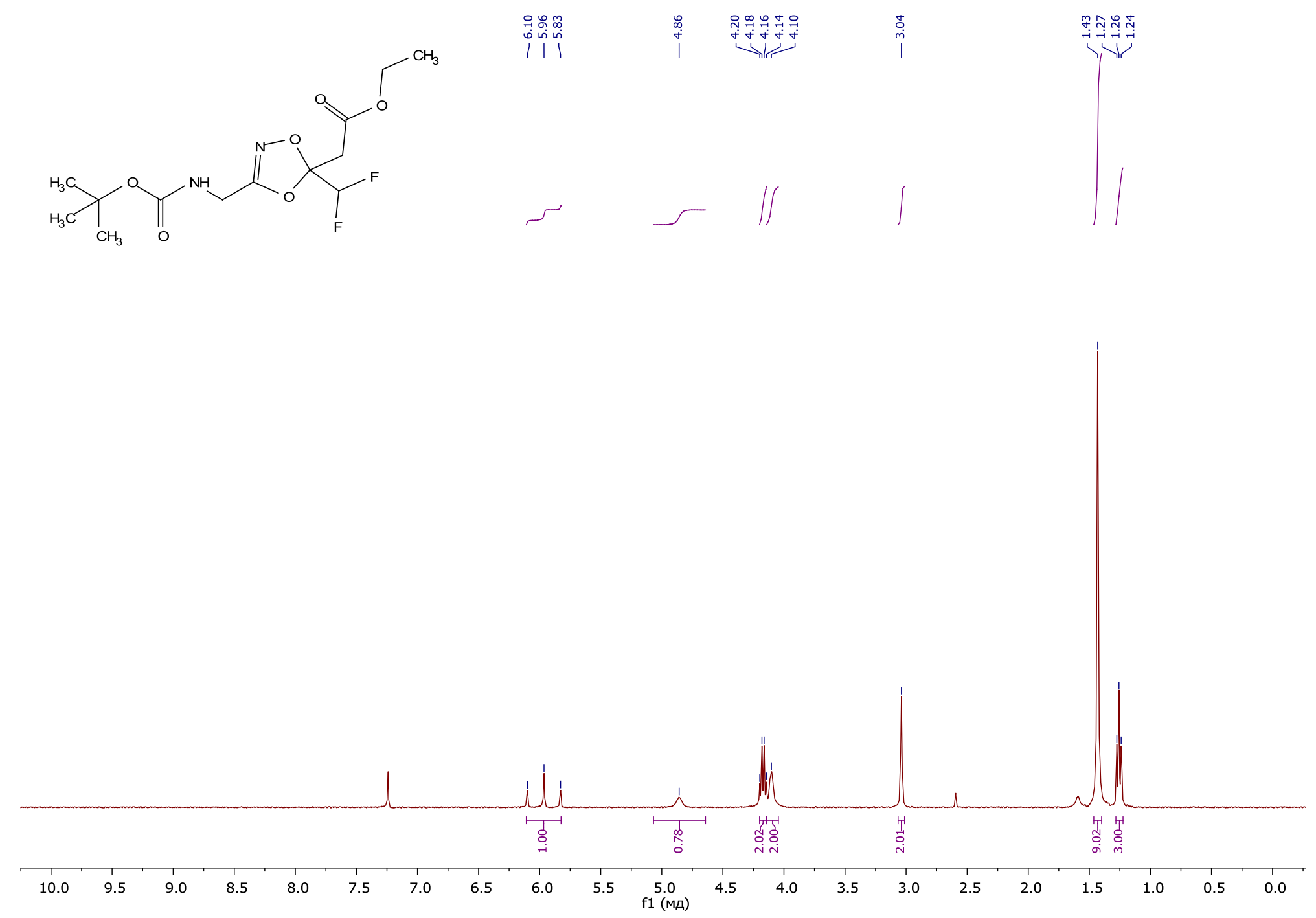

Ethyl 2-(3-(((tert-butoxycarbonyl)amino)methyl)-5-(difluoromethyl)-1,4,2-dioxazol-5-yl)acetate (51f) ${ }^{1}$ H NMR 

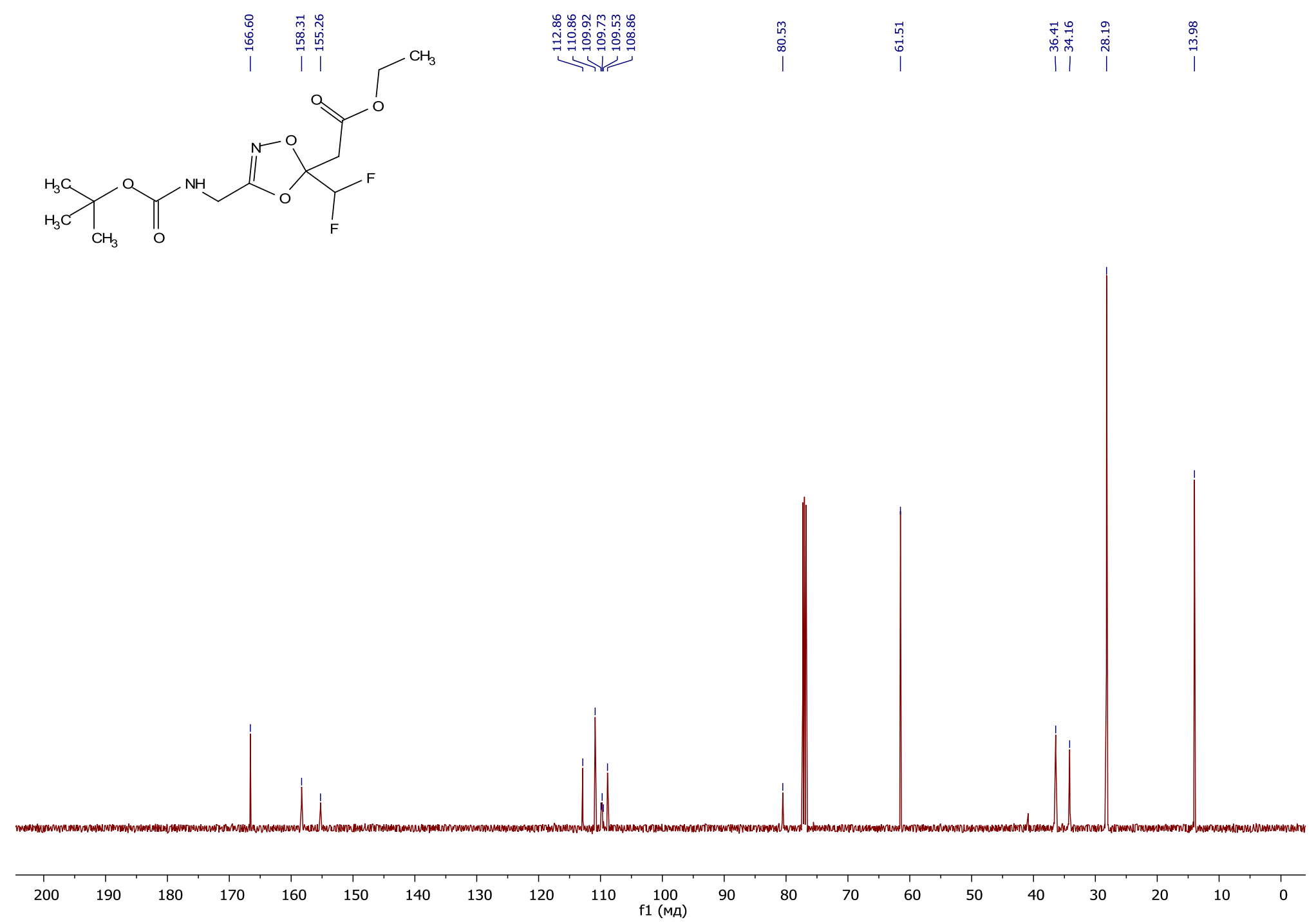

Ethyl 2-(3-(((tert-butoxycarbonyl)amino)methyl)-5-(difluoromethyl)-1,4,2-dioxazol-5-yl)acetate (51f) ${ }^{13} \mathrm{C}$ NMR 


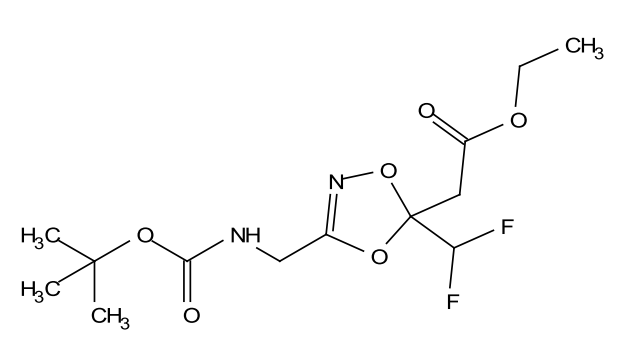

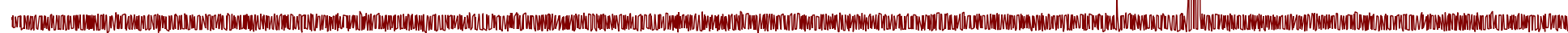

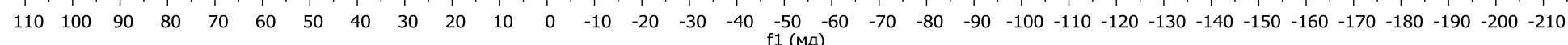

Ethyl 2-(3-(((tert-butoxycarbonyl)amino)methyl)-5-(difluoromethyl)-1,4,2-dioxazol-5-yl)acetate (51f) ${ }^{19}$ F NMR 

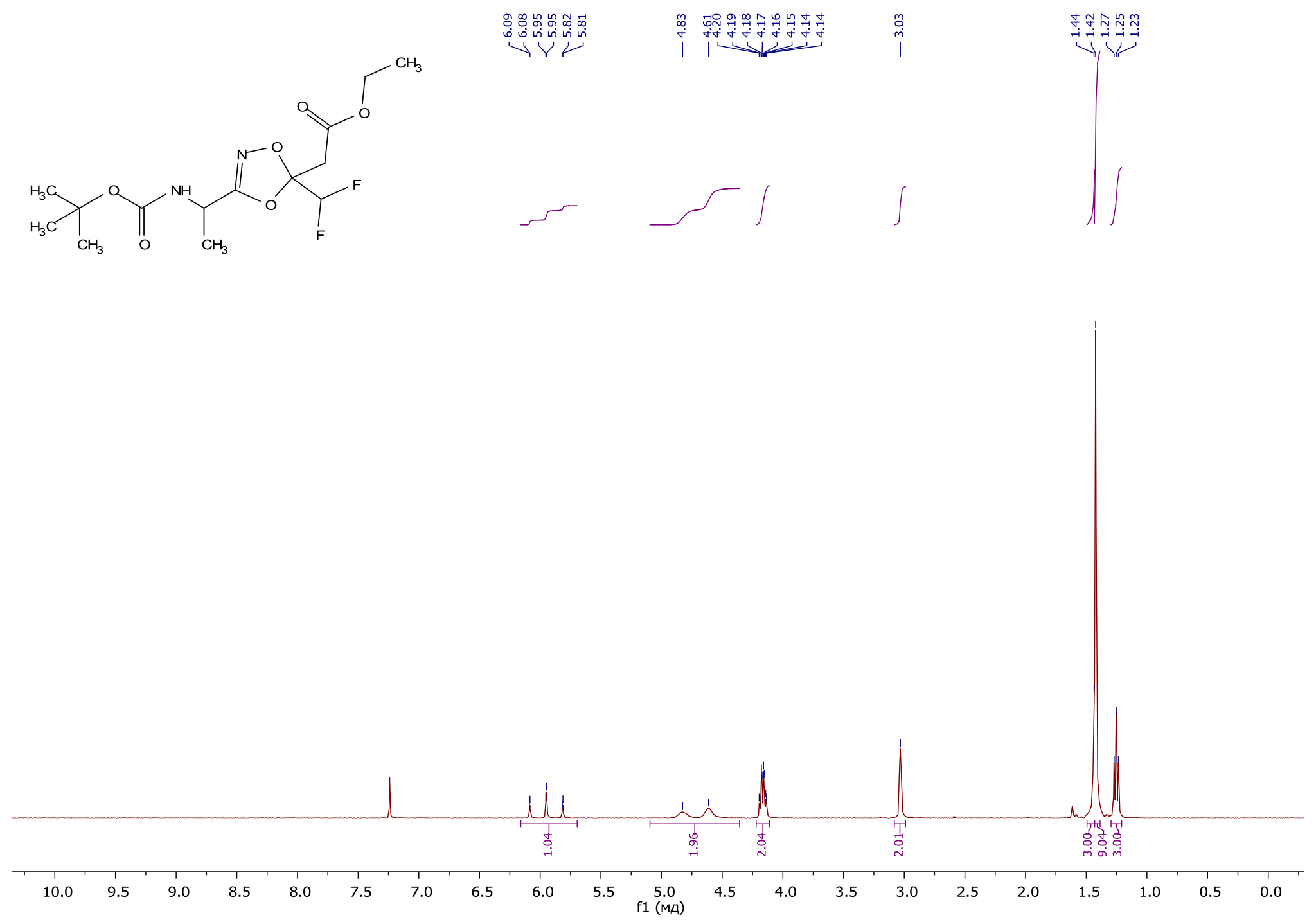

Ethyl 2-(3-(1-((tert-butoxycarbonyl)amino)ethyl)-5-(difluoromethyl)-1,4,2-dioxazol-5-yl)acetate (51g or 51h) ${ }^{1} \mathrm{H}$ NMR 

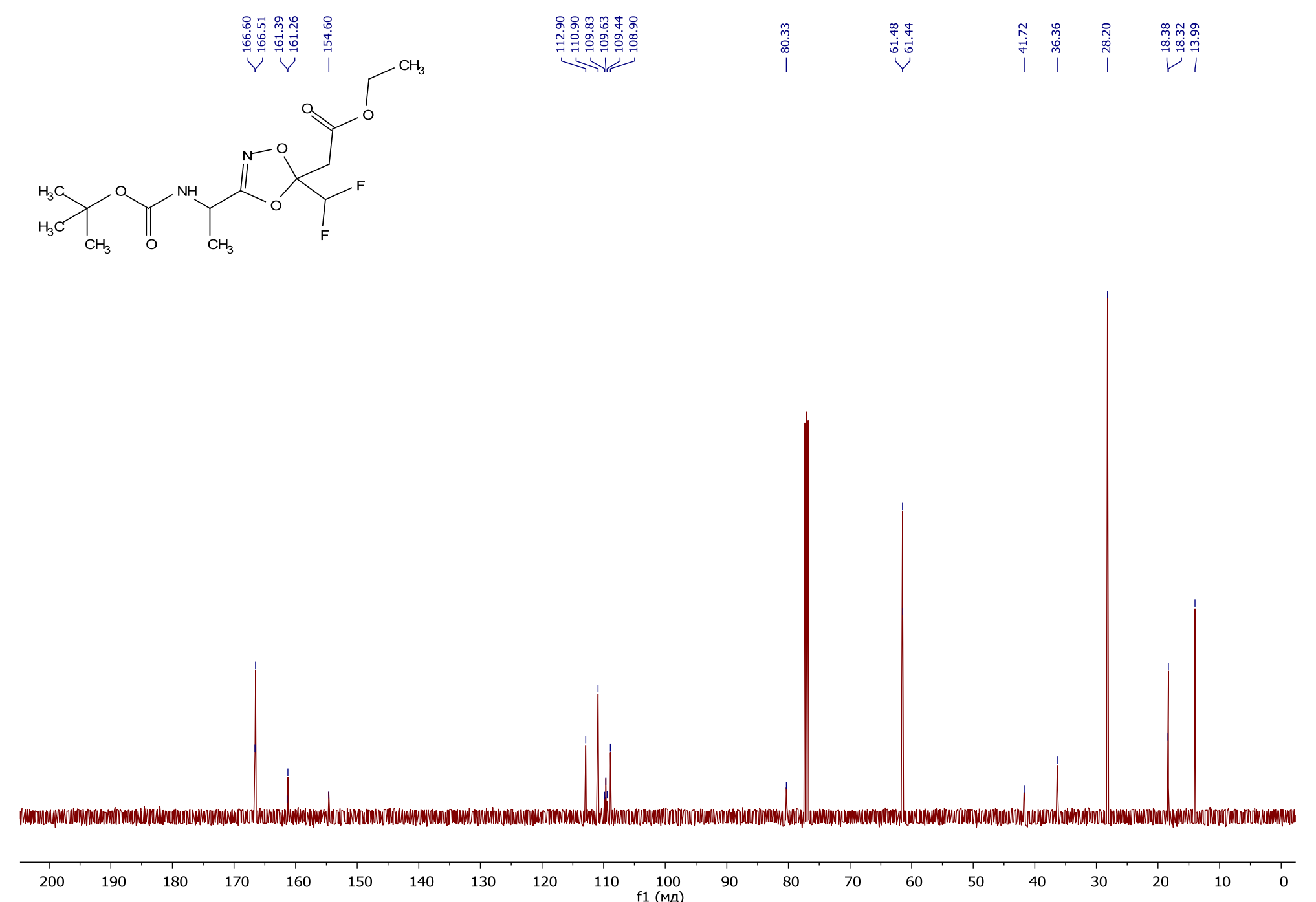

Ethyl 2-(3-(1-((tert-butoxycarbonyl)amino)ethyl)-5-(difluoromethyl)-1,4,2-dioxazol-5-yl)acetate (51g or 51h) ${ }^{13} \mathrm{C}$ NMR 


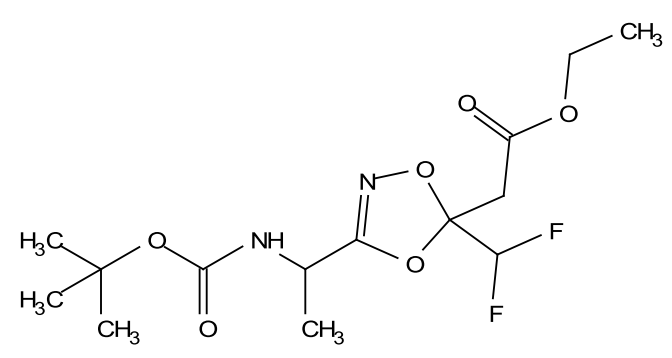



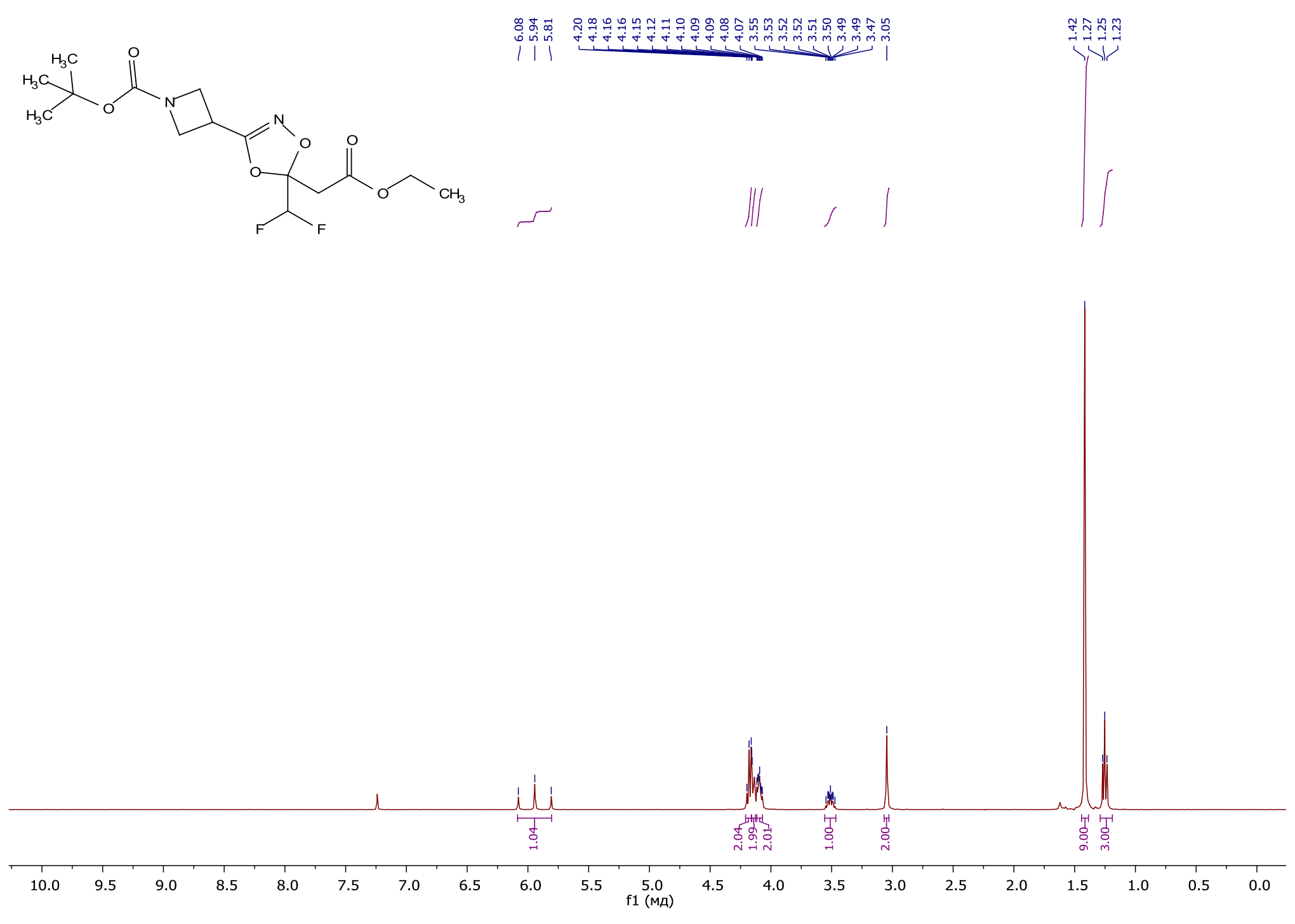

tert-Butyl 3-(5-(difluoromethyl)-5-(2-ethoxy-2-oxoethyl)-1,4,2-dioxazol-3-yl)azetidine-1-carboxylate (51j) ${ }^{1} \mathrm{H}$ NMR 

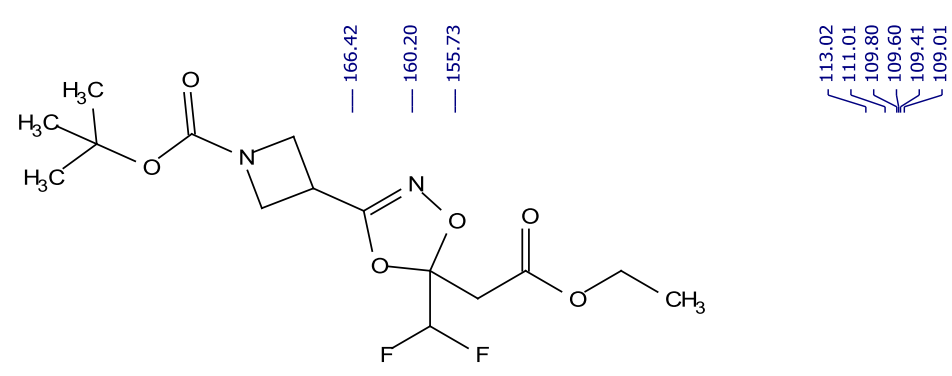

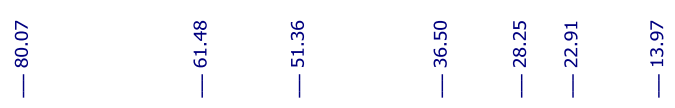

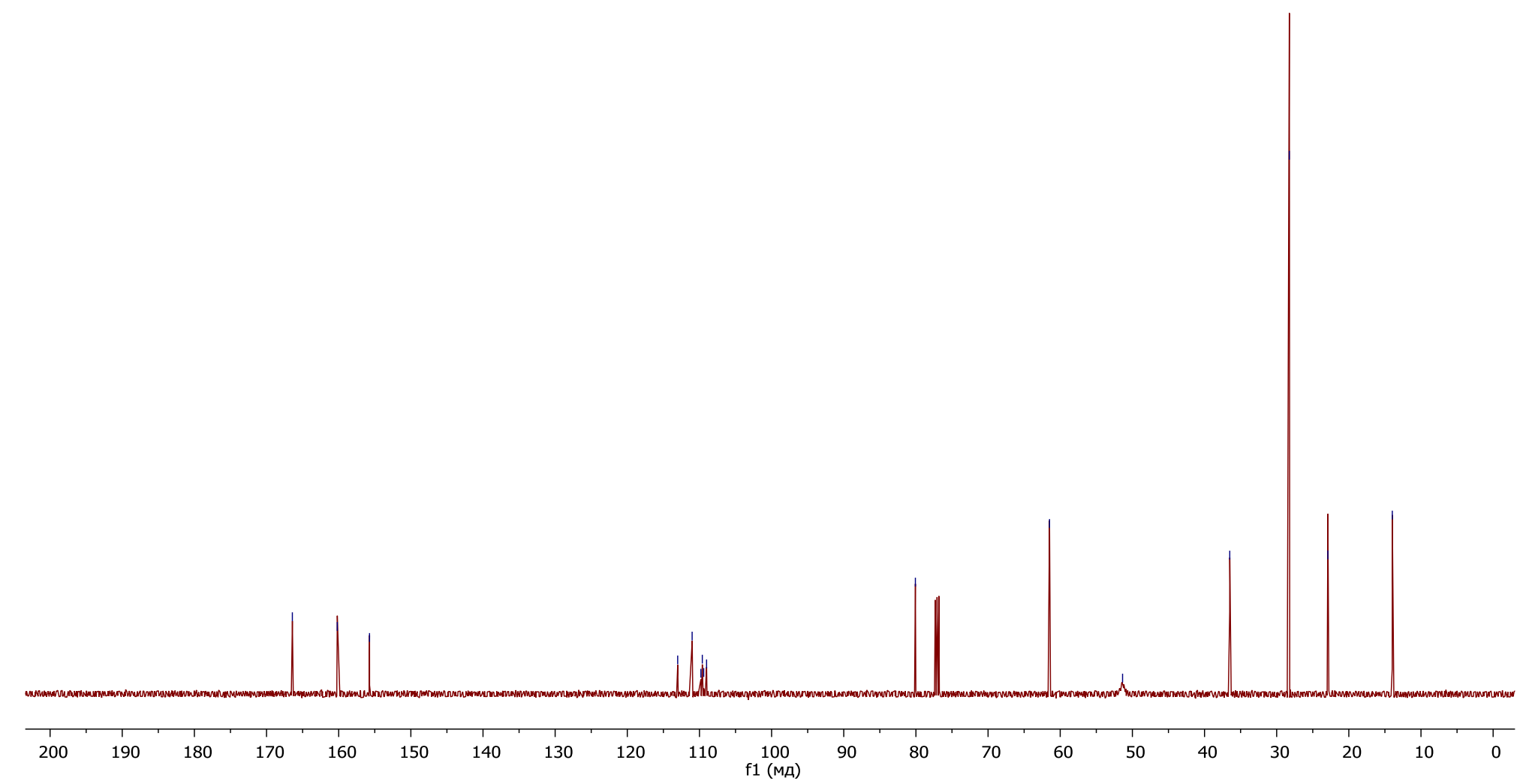

tert-Butyl 3-(5-(difluoromethyl)-5-(2-ethoxy-2-oxoethyl)-1,4,2-dioxazol-3-yl)azetidine-1-carboxylate (51j) ${ }^{13} \mathrm{C}$ NMR 


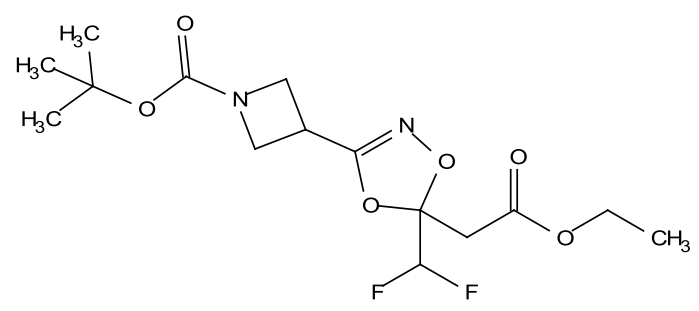

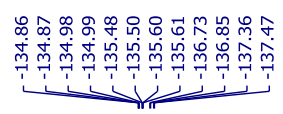

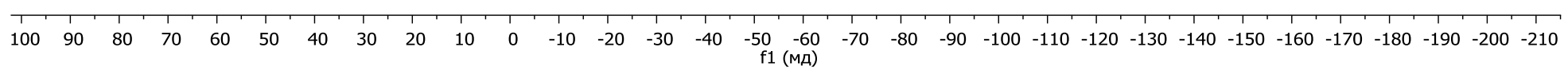

tert-Butyl 3-(5-(difluoromethyl)-5-(2-ethoxy-2-oxoethyl)-1,4,2-dioxazol-3-yl)azetidine-1-carboxylate (51j) ${ }^{19}$ F NMR

S232 


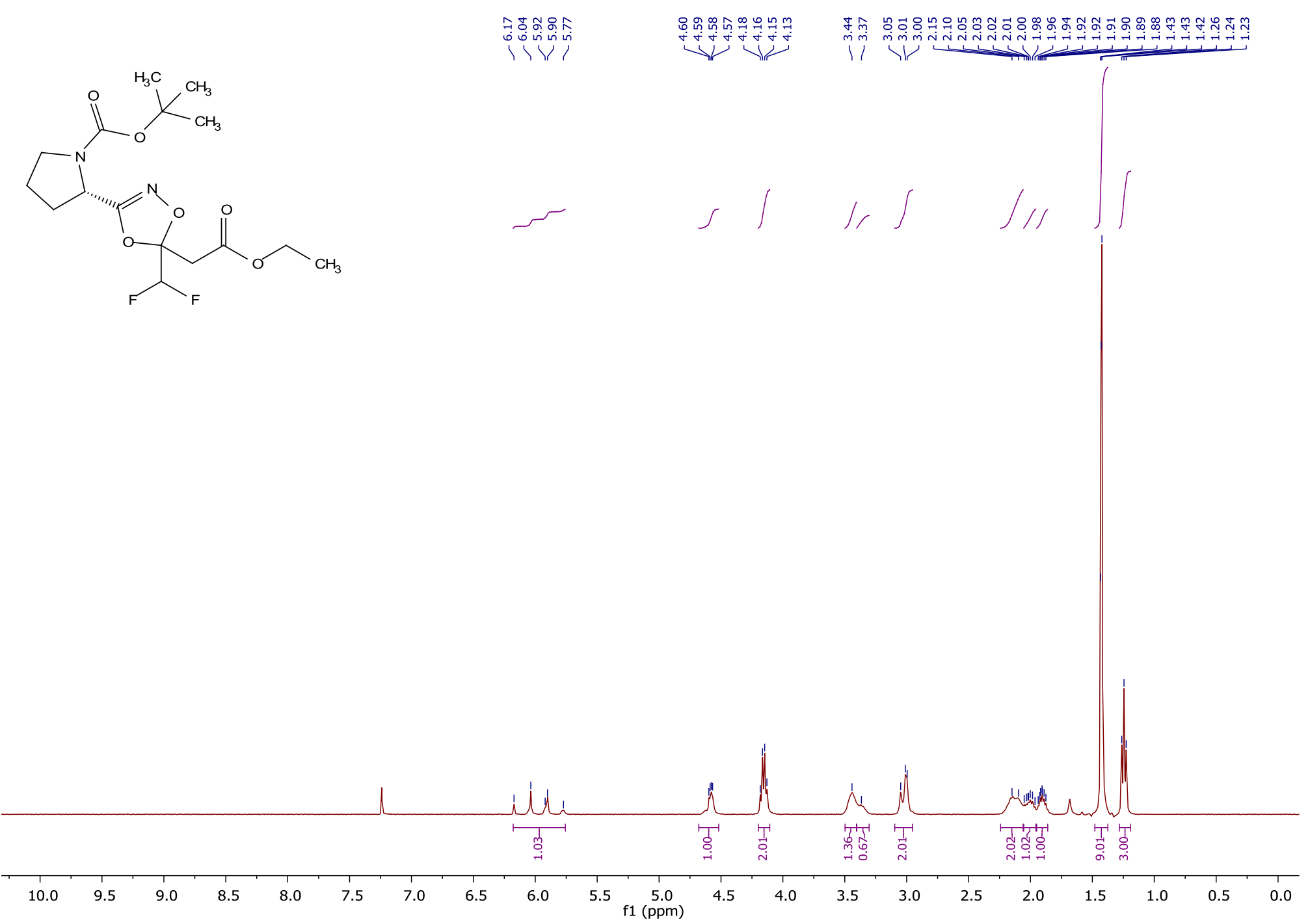

tert-Butyl 2-(5-(difluoromethyl)-5-(2-ethoxy-2-oxoethyl)-1,4,2-dioxazol-3-yl)pyrrolidine-1-carboxylate (51k or 511) ${ }^{1} \mathrm{H}$ NMR 


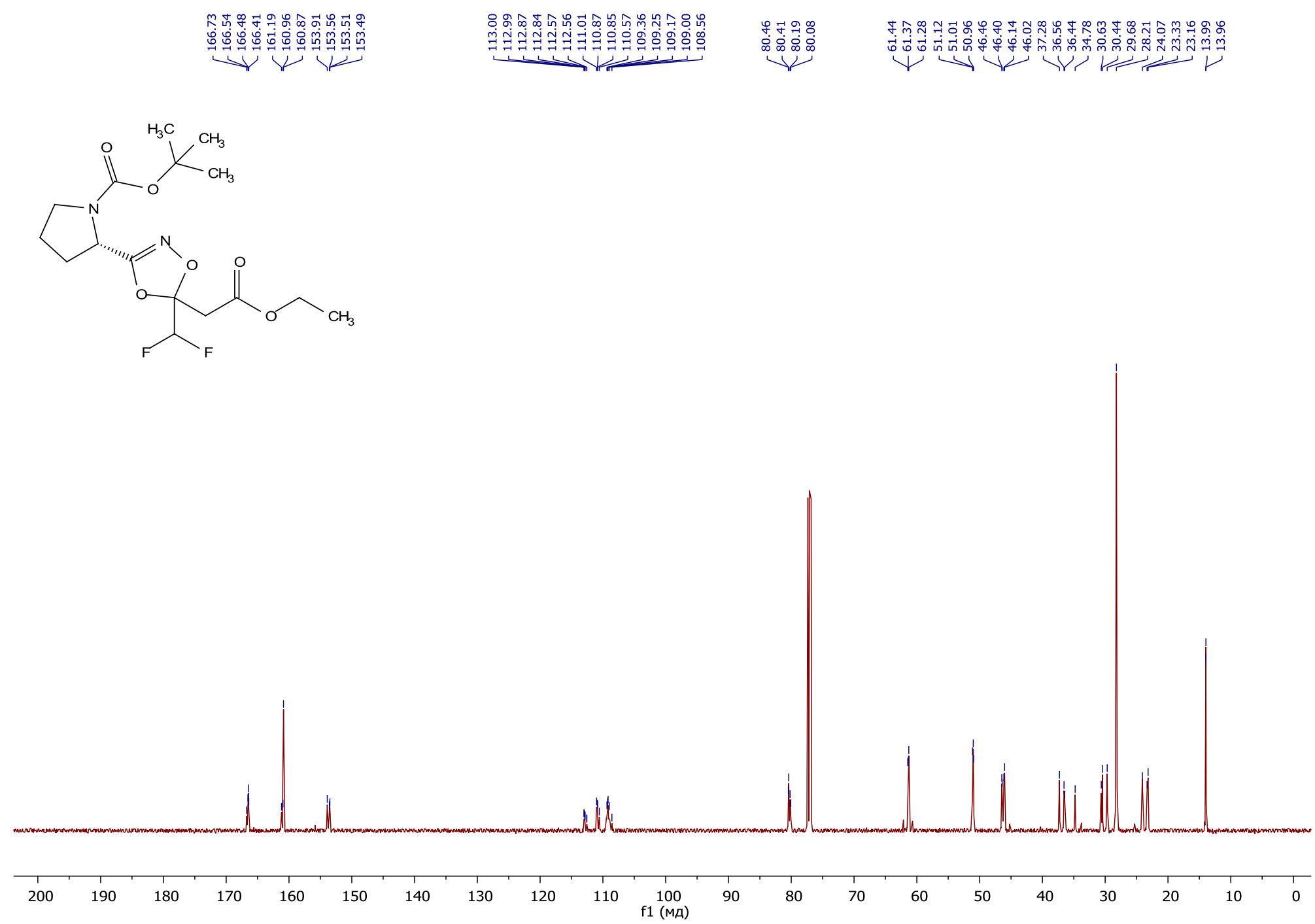

tert-Butyl 2-(5-(difluoromethyl)-5-(2-ethoxy-2-oxoethyl)-1,4,2-dioxazol-3-yl)pyrrolidine-1-carboxylate (51k or 511) ${ }^{13} \mathrm{C}$ NMR 


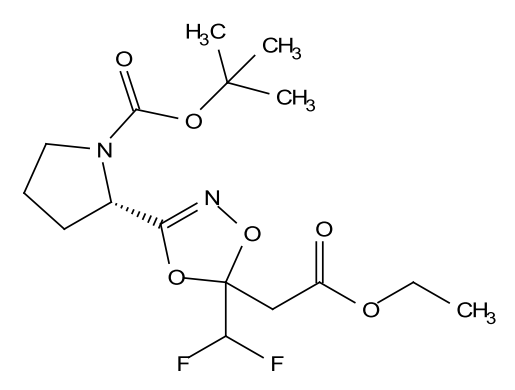

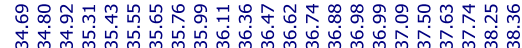

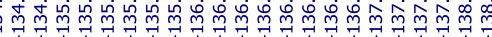

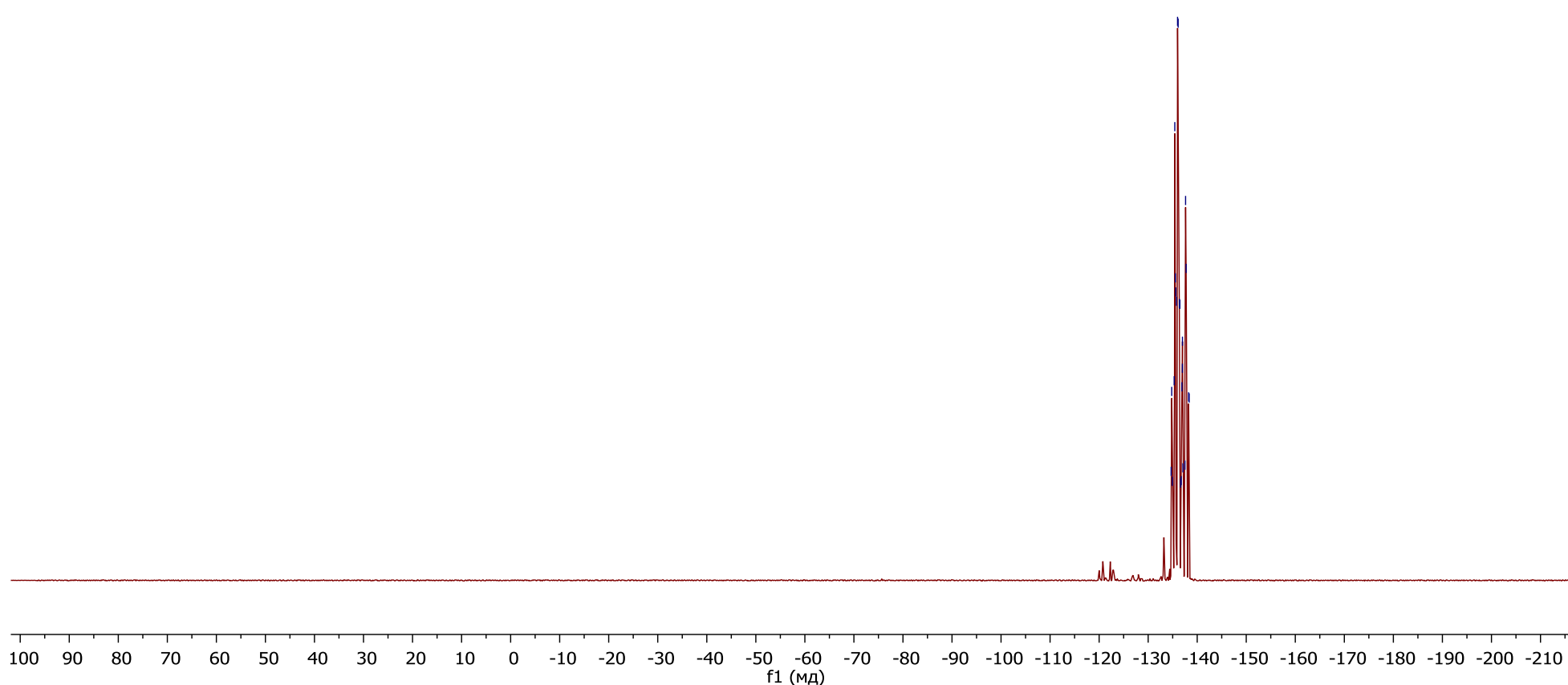

tert-Butyl 2-(5-(difluoromethyl)-5-(2-ethoxy-2-oxoethyl)-1,4,2-dioxazol-3-yl)pyrrolidine-1-carboxylate (51k or 51I) ${ }^{19}$ F NMR 

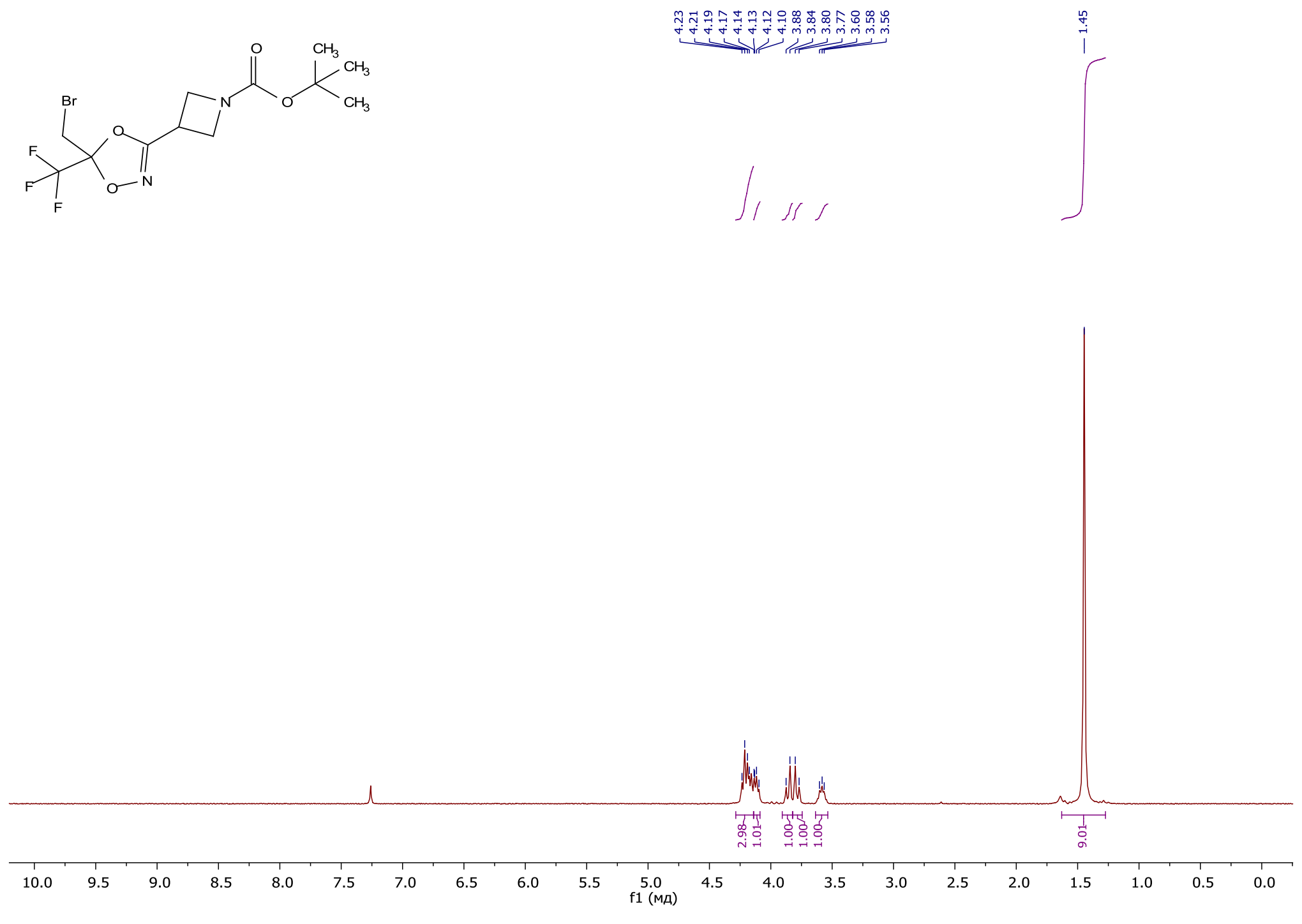

tert-Butyl 3-(5-(bromomethyl)-5-(trifluoromethyl)-1,4,2-dioxazol-3-yl)azetidine-1-carboxylate (52j) ${ }^{1} \mathrm{H}$ NMR 

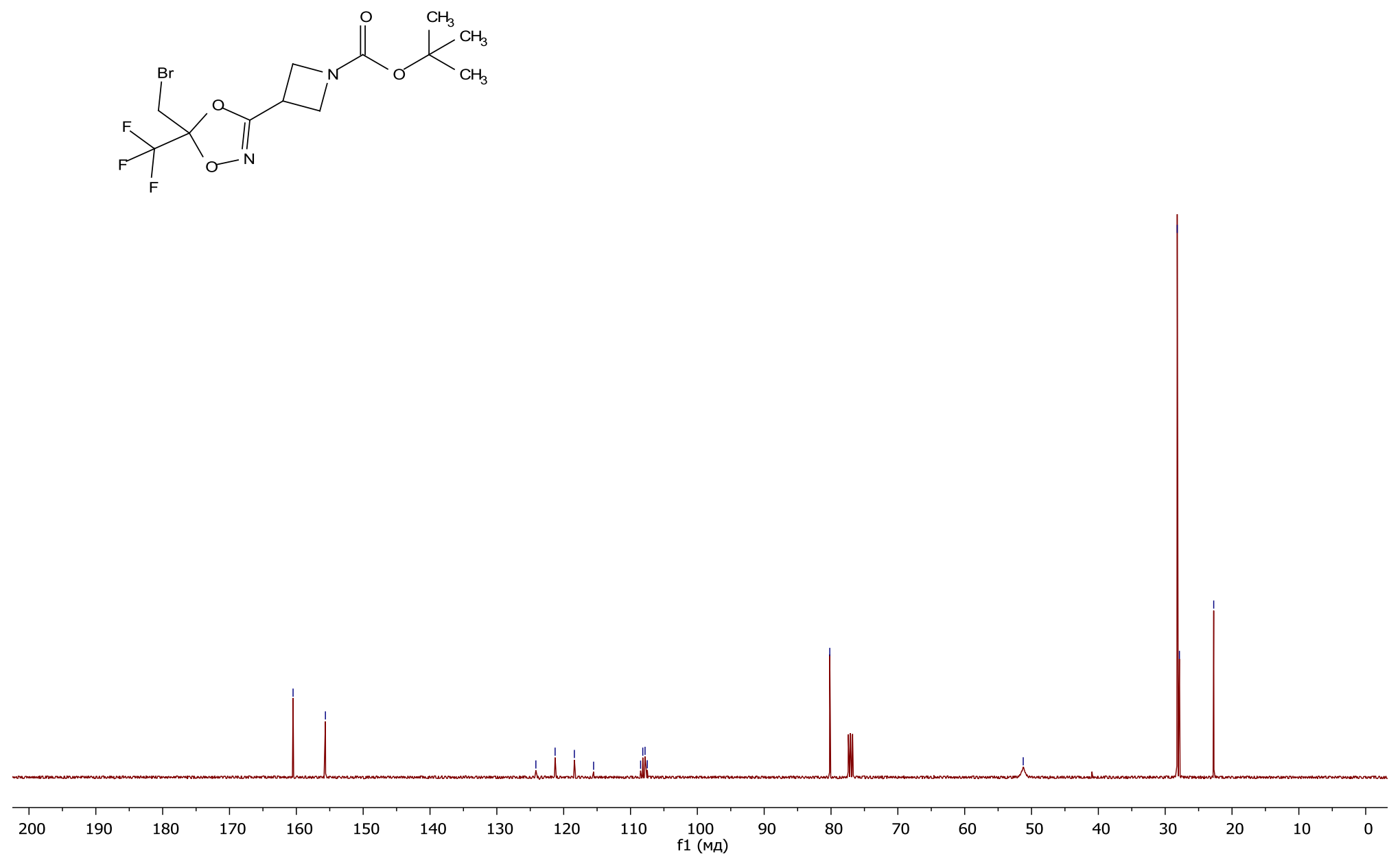

tert-Butyl 3-(5-(bromomethyl)-5-(trifluoromethyl)-1,4,2-dioxazol-3-yl)azetidine-1-carboxylate (52j) ${ }^{13} \mathrm{C}$ NMR 

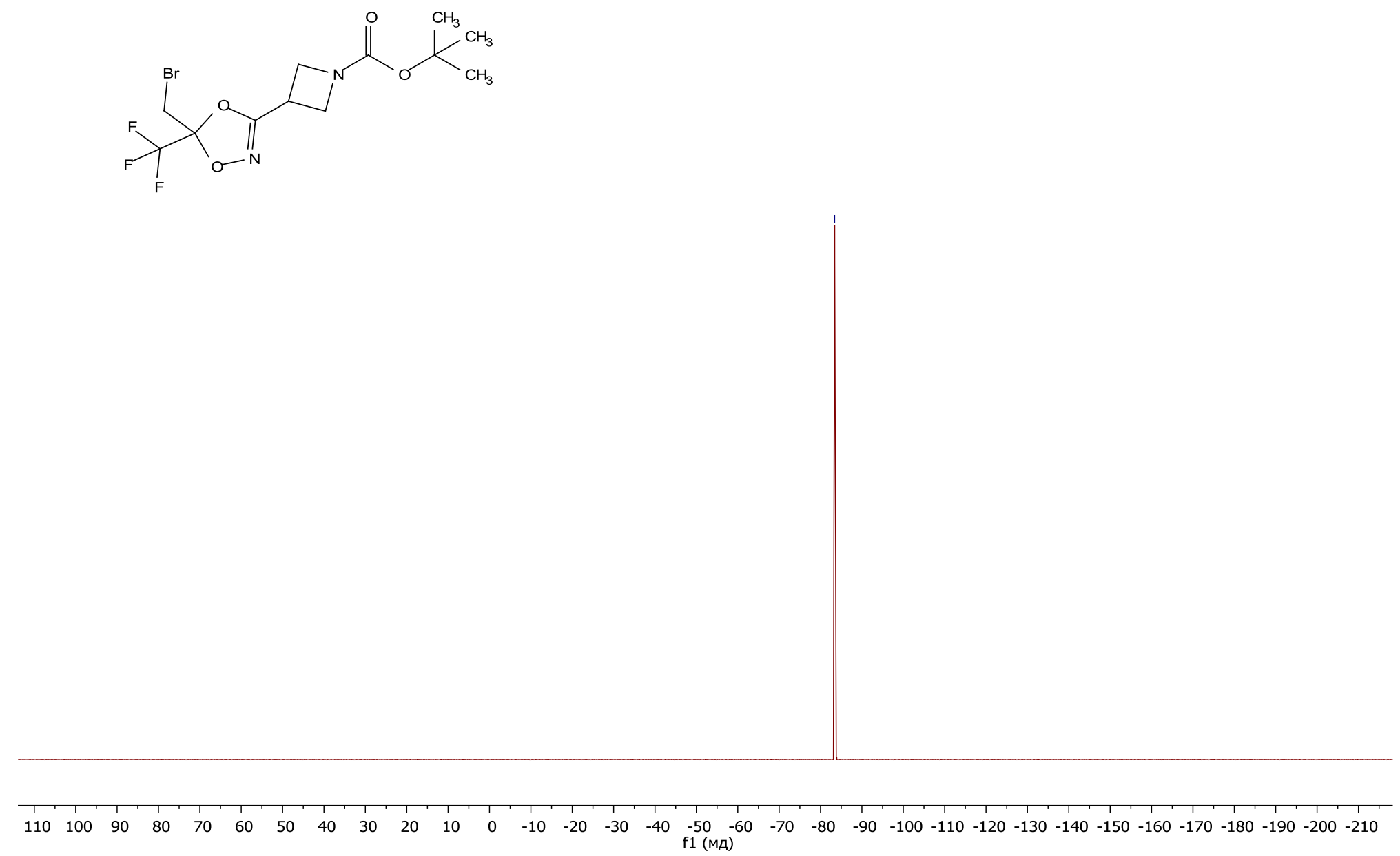

tert-Butyl 3-(5-(bromomethyl)-5-(trifluoromethyl)-1,4,2-dioxazol-3-yl)azetidine-1-carboxylate (52j) ${ }^{19}$ F NMR 

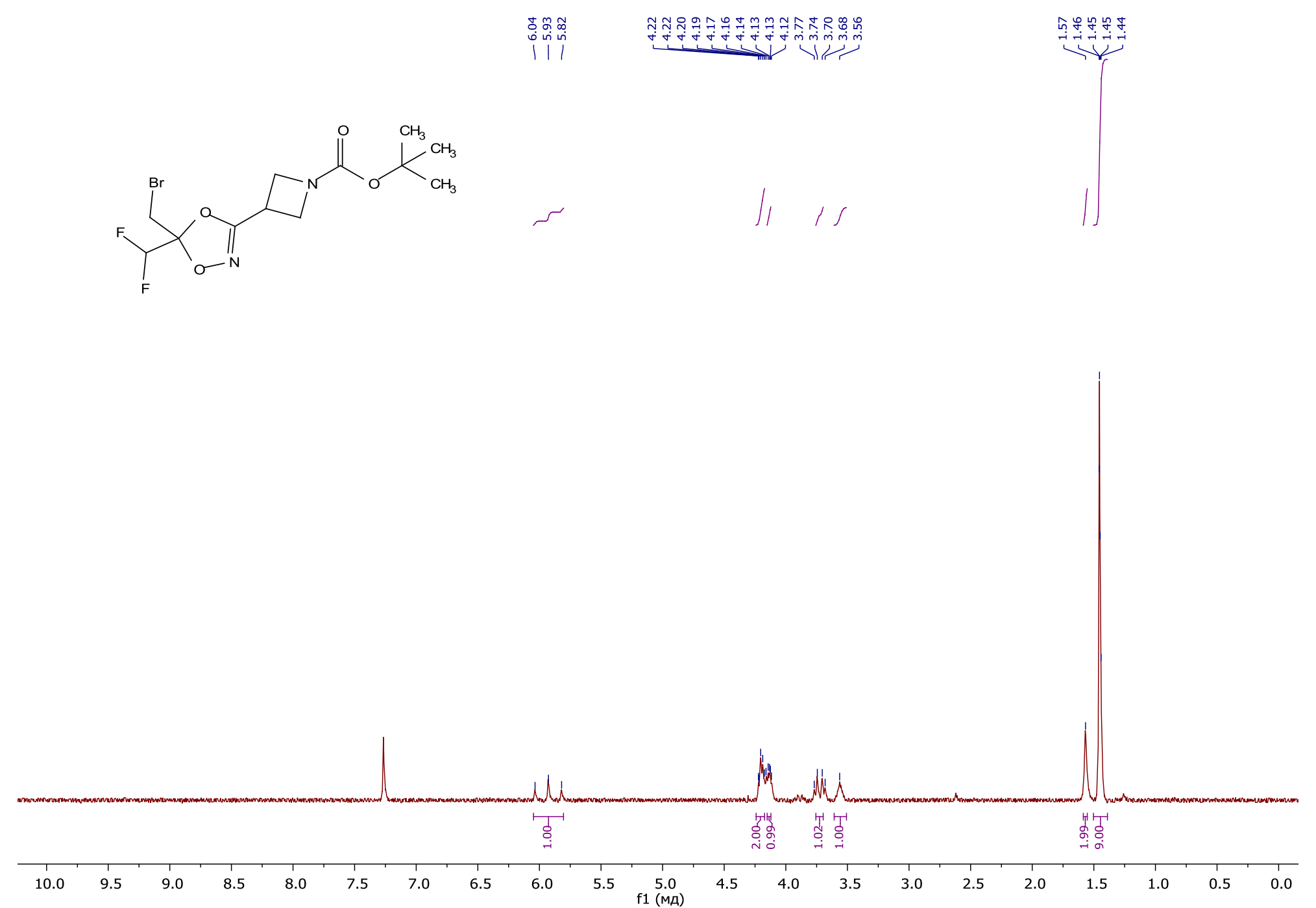

tert-Butyl 3-(5-(bromomethyl)-5-(difluoromethyl)-1,4,2-dioxazol-3-yl)azetidine-1-carboxylate (53j) ${ }^{1}$ H NMR 


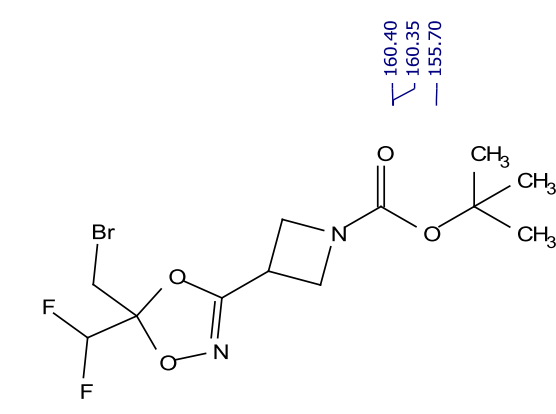

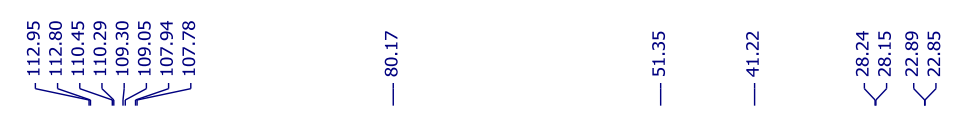

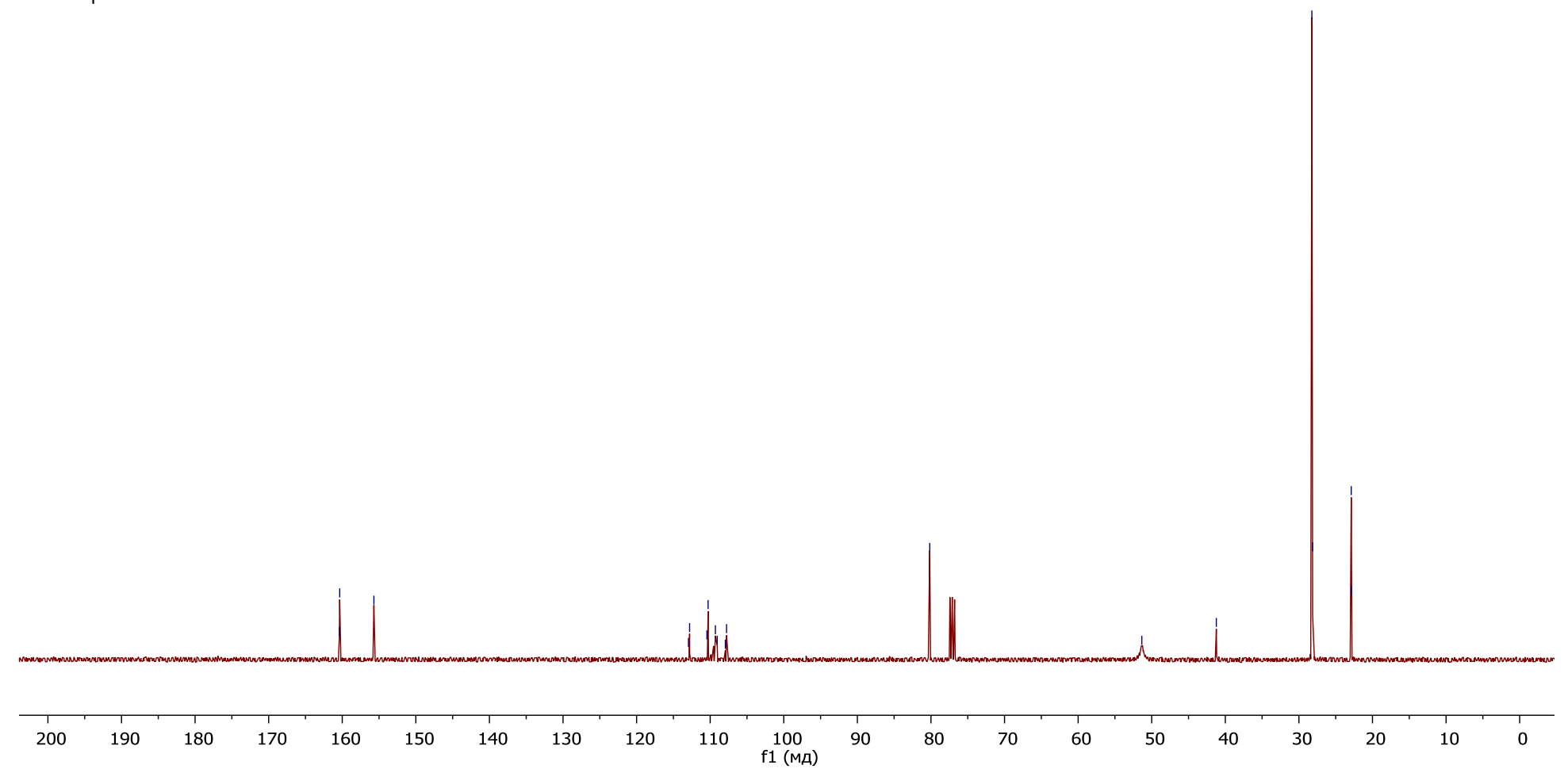

tert-Butyl 3-(5-(bromomethyl)-5-(difluoromethyl)-1,4,2-dioxazol-3-yl)azetidine-1-carboxylate (53j) ${ }^{13} \mathrm{C}$ NMR 

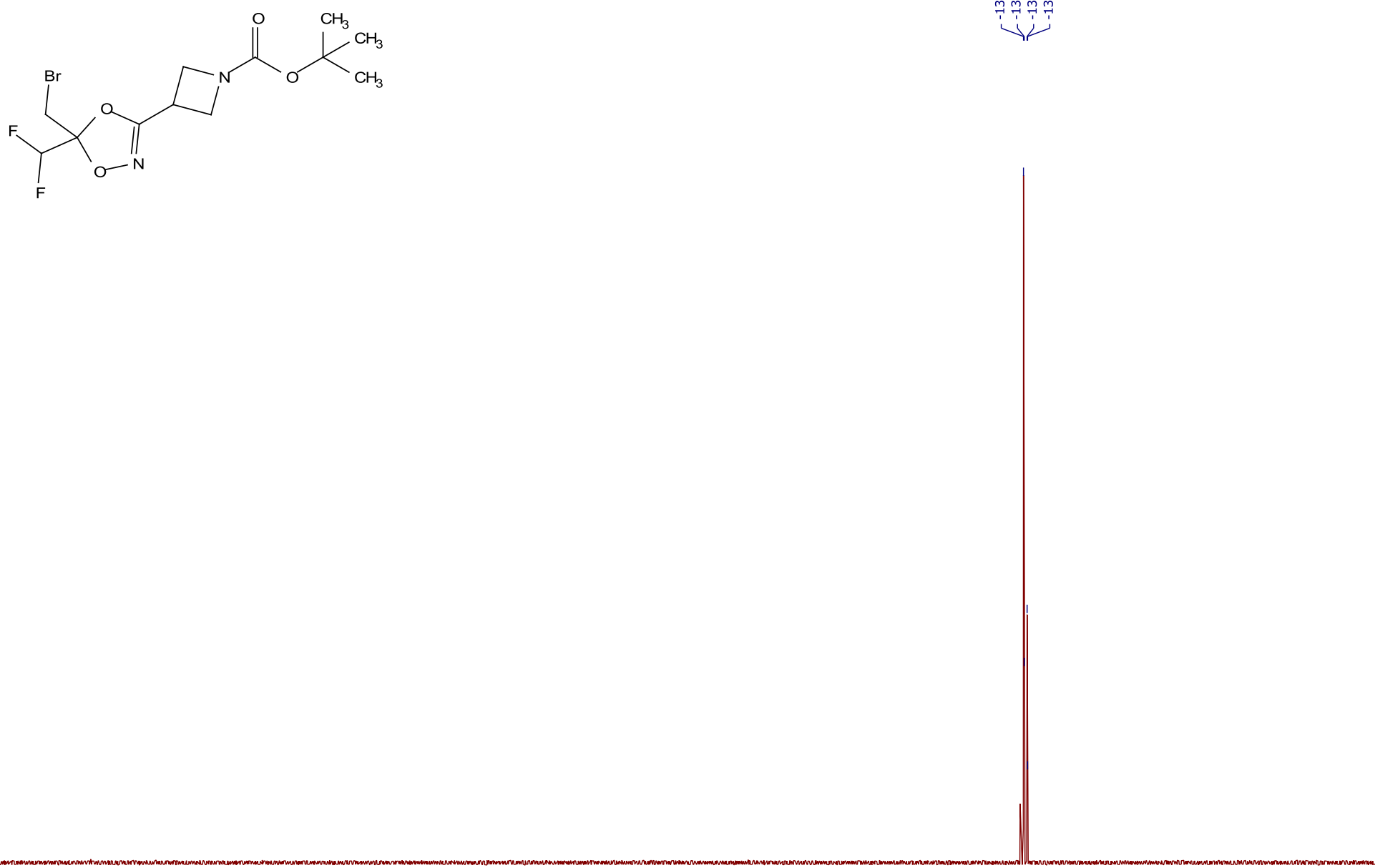

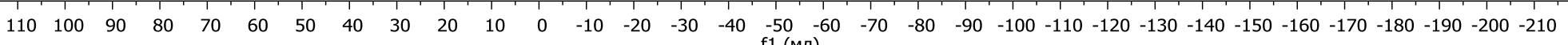
tert-Butyl 3-(5-(bromomethyl)-5-(difluoromethyl)-1,4,2-dioxazol-3-yl)azetidine-1-carboxylate (53j) ${ }^{19}$ F NMR 

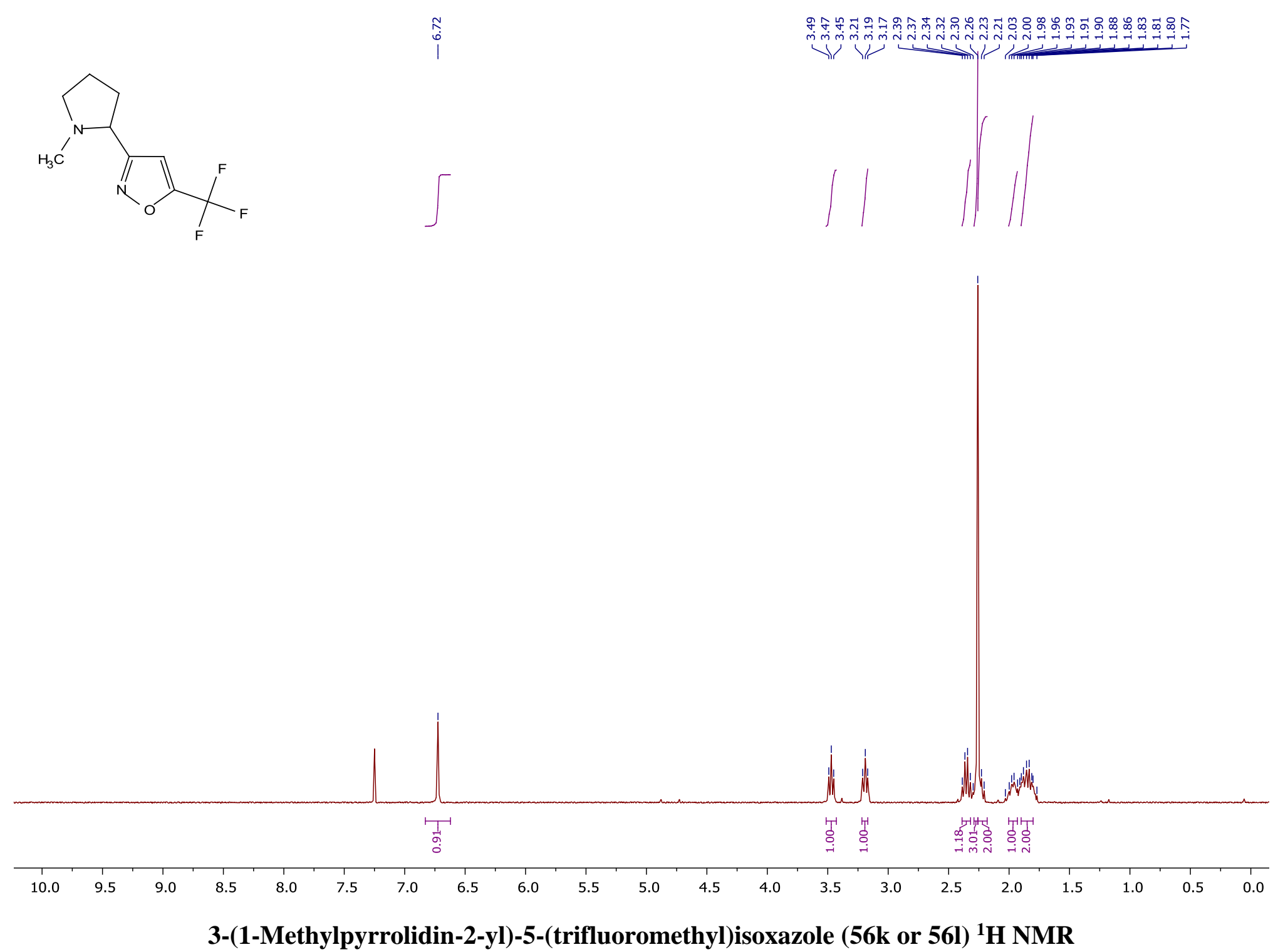


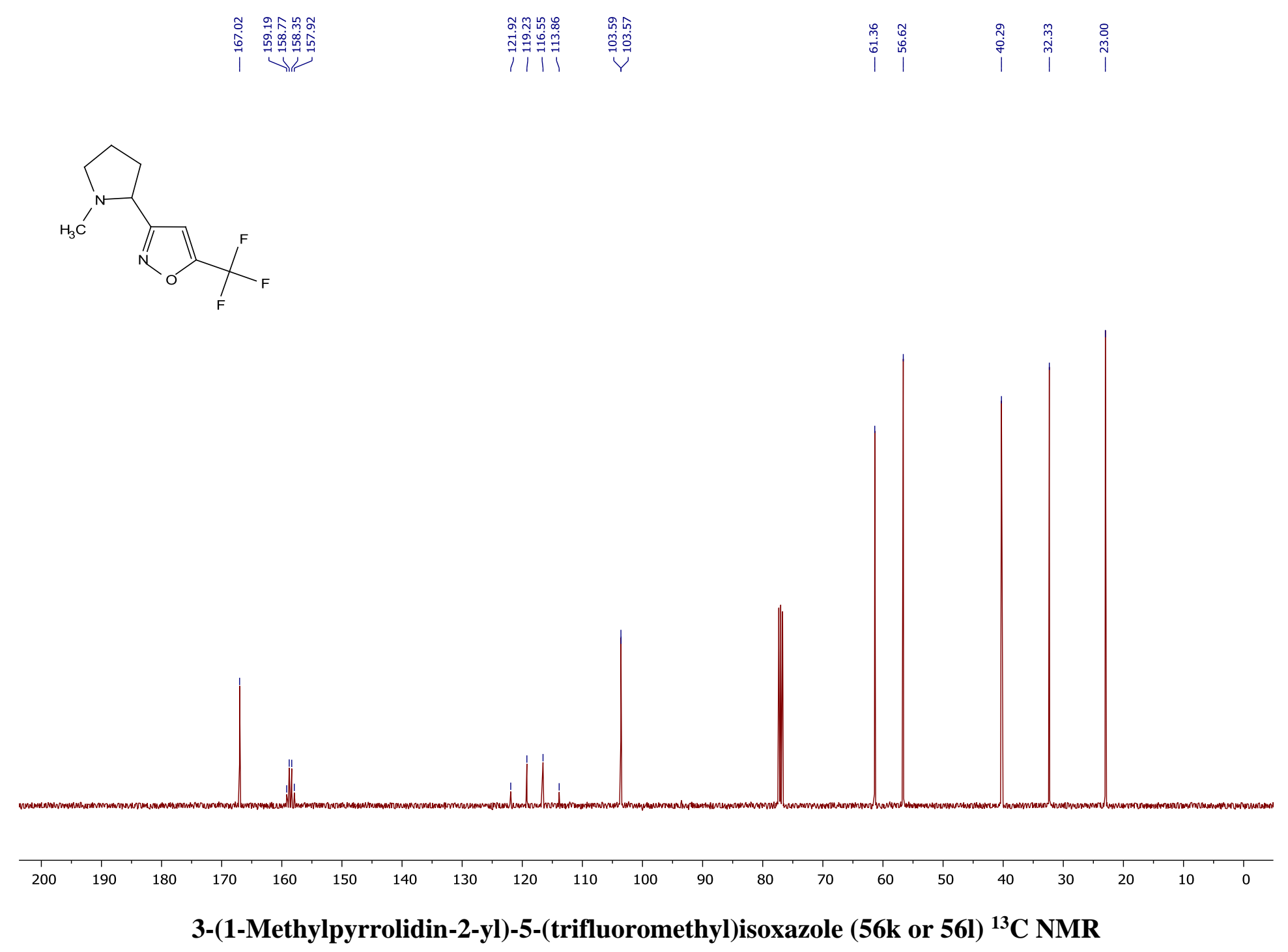




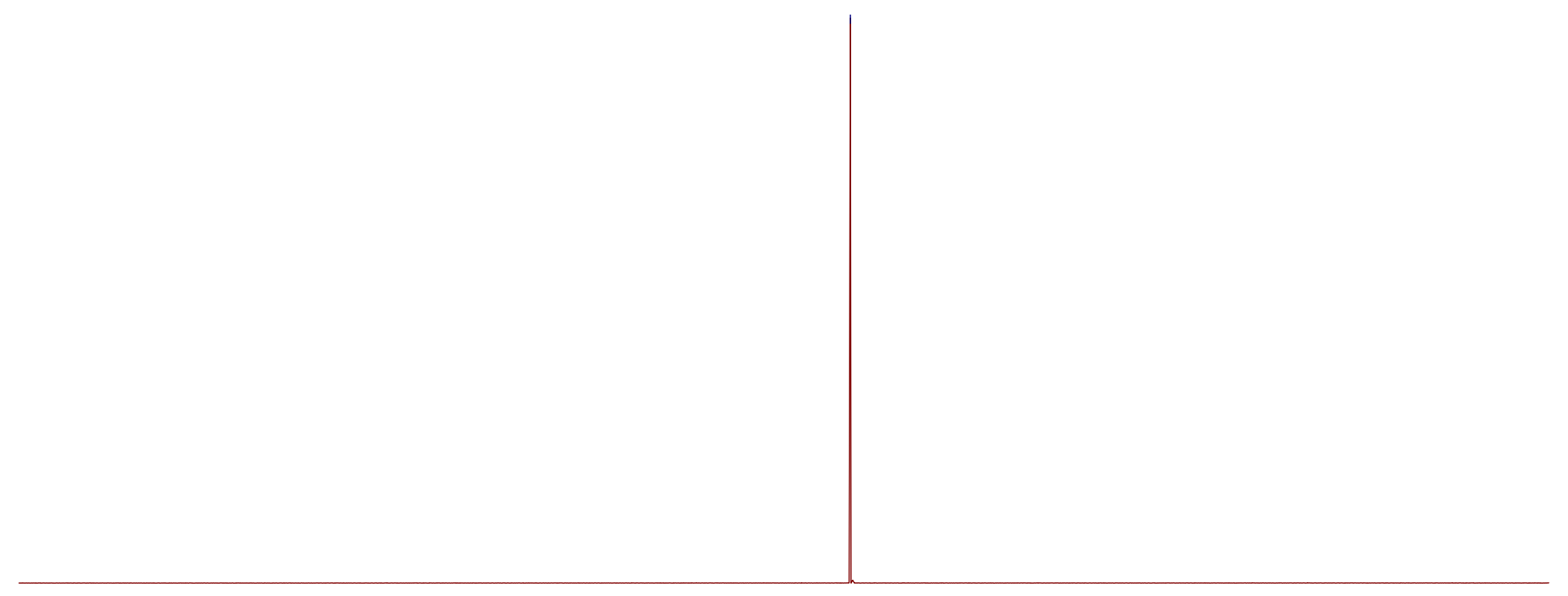



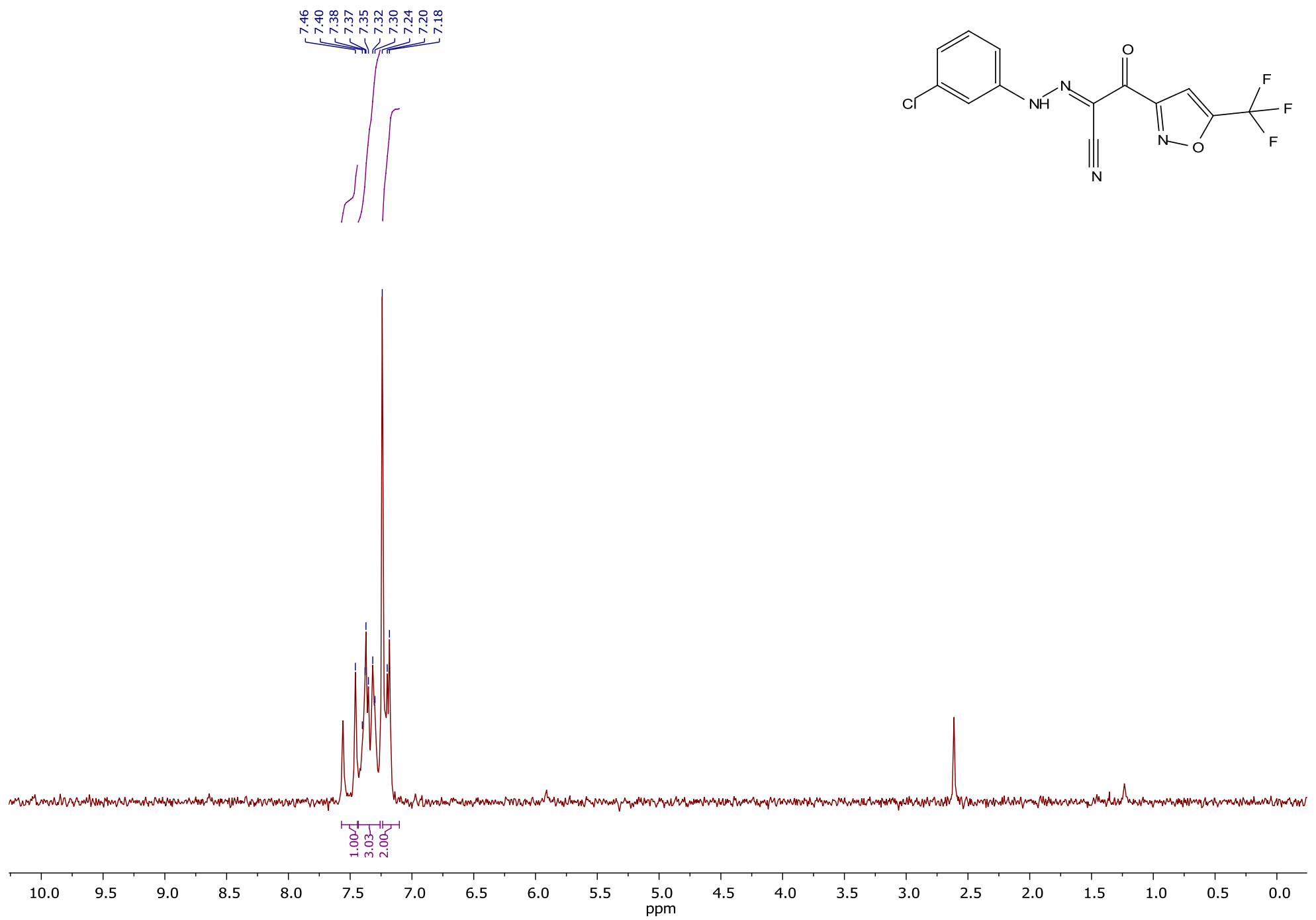

$N^{\prime}$-(3-Chlorophenyl)-2-oxo-2-(5-(trifluoromethyl)isoxazol-3-yl)aceto-hydrazonoyl cyanide (57) ${ }^{1}$ H NMR 


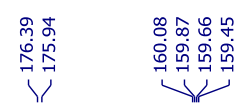

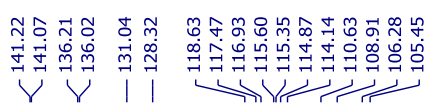
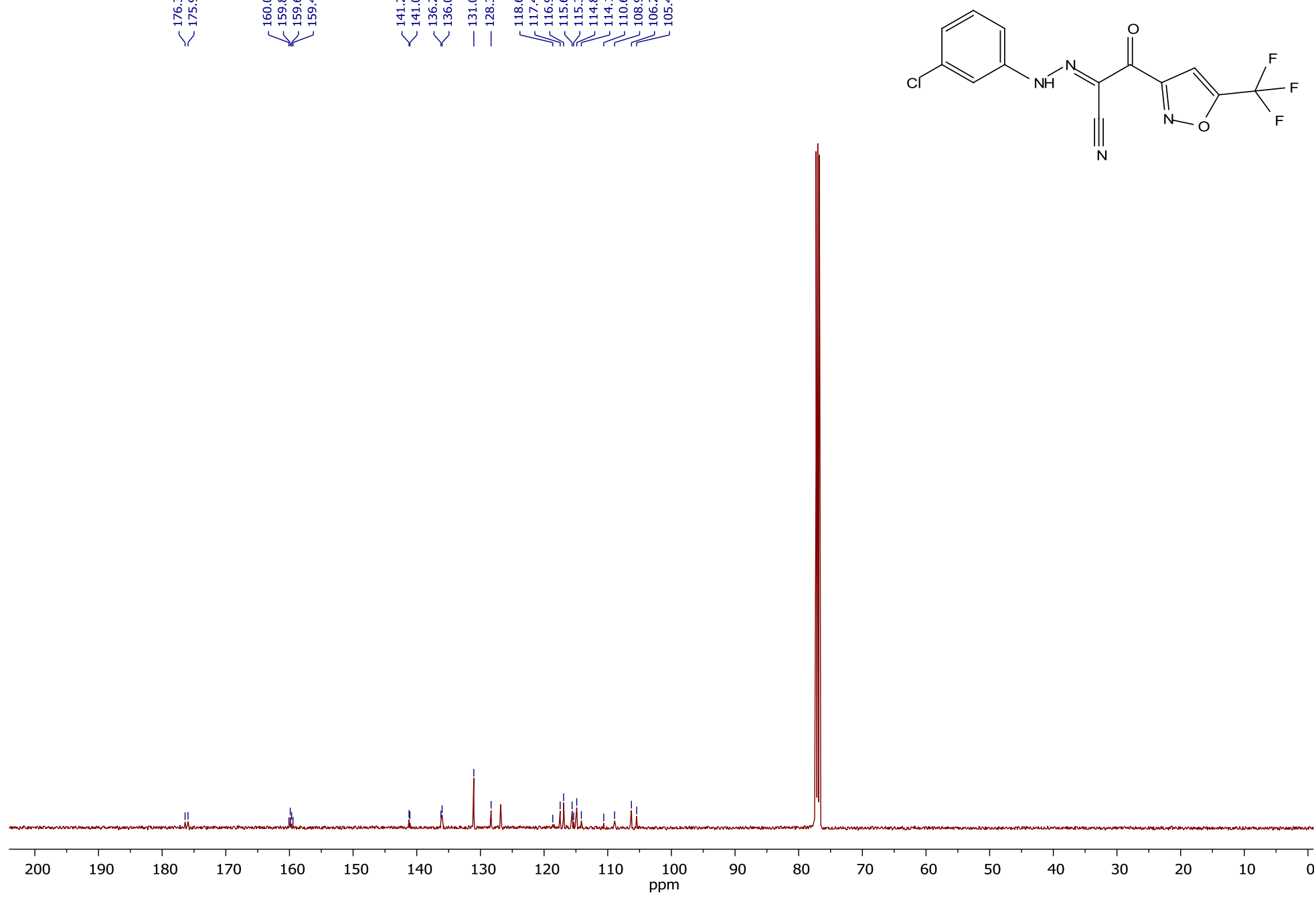

$N^{\prime}$-(3-Chlorophenyl)-2-oxo-2-(5-(trifluoromethyl)isoxazol-3-yl)aceto-hydrazonoyl cyanide (57) ${ }^{13} \mathrm{C}$ NMR

S246 


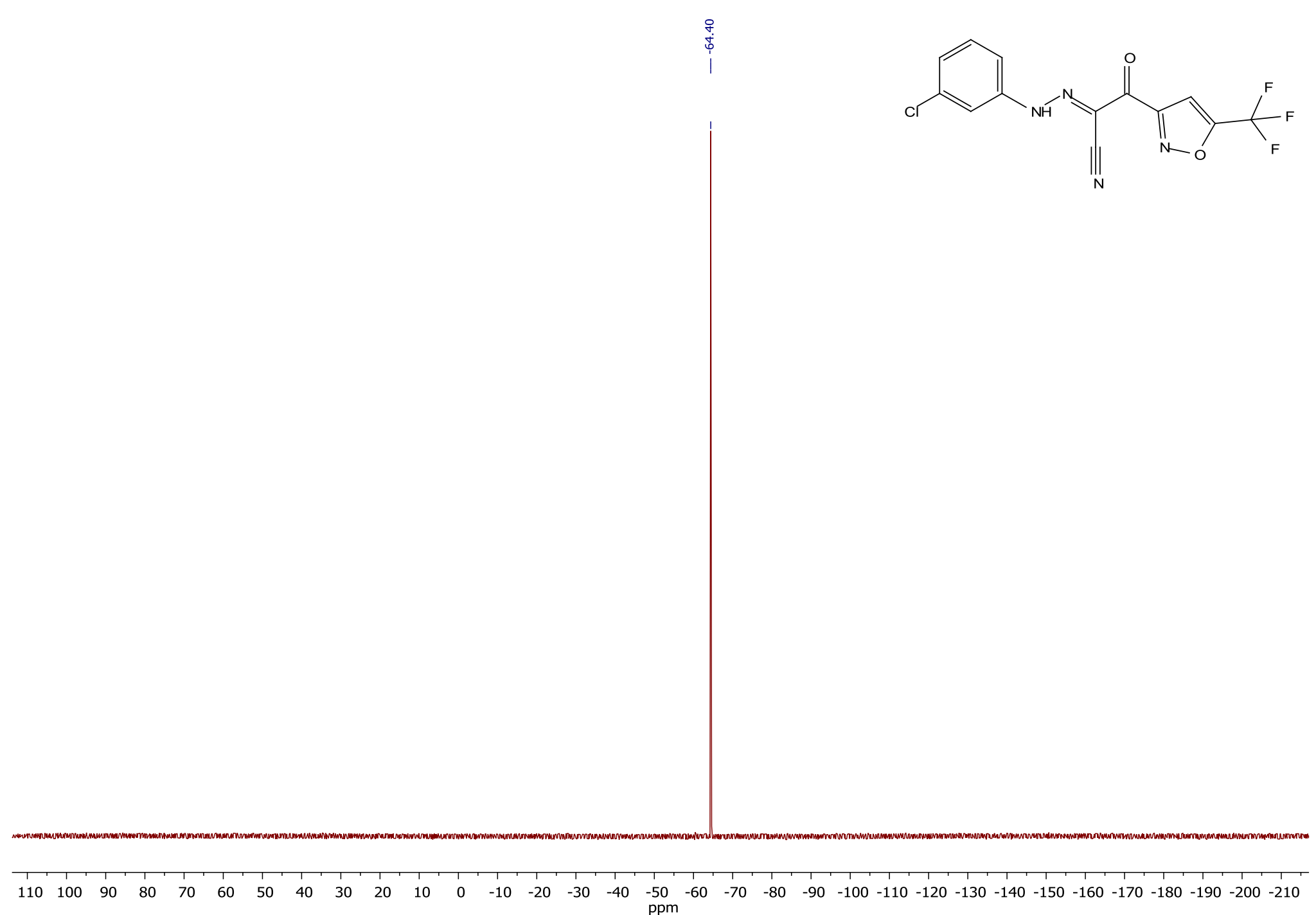

$N^{\prime}$-(3-Chlorophenyl)-2-oxo-2-(5-(trifluoromethyl)isoxazol-3-yl)aceto-hydrazonoyl cyanide (57) ${ }^{19}$ F NMR 


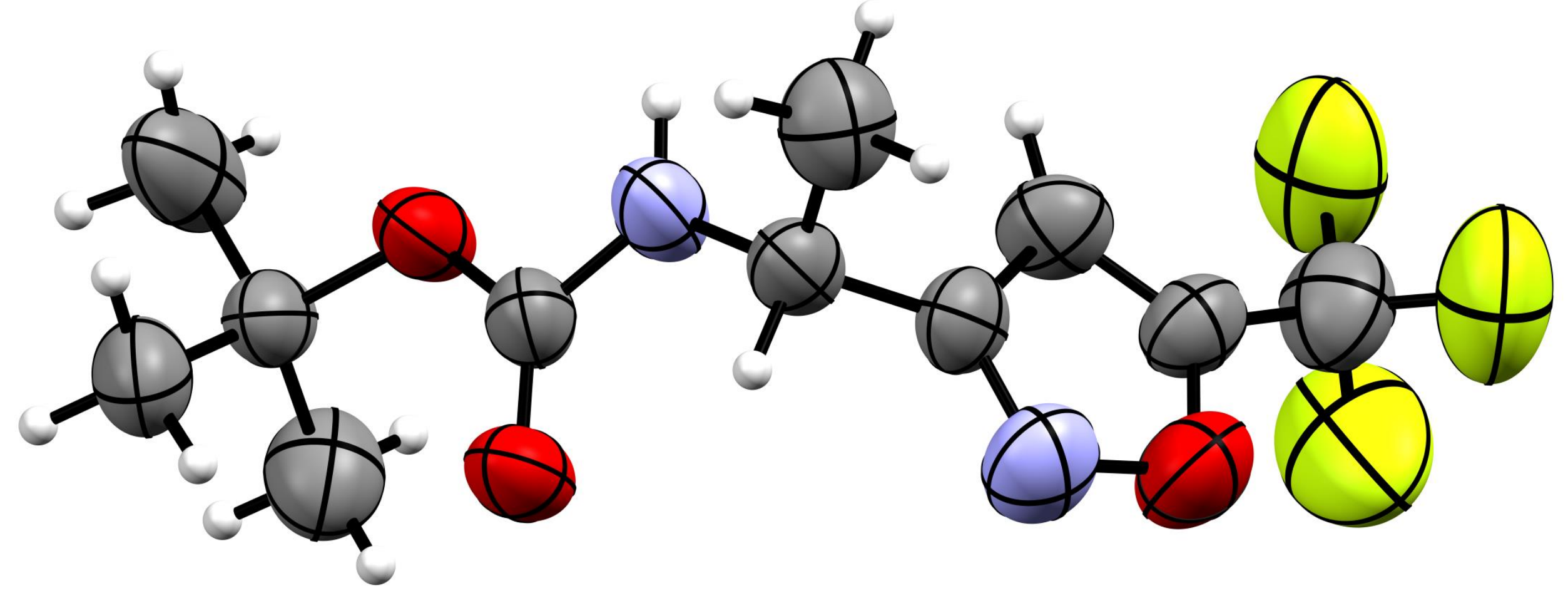

Molecular structure of the compound $3 \mathrm{~h}$

(Thermal ellipsoids are shown at 50\% probability level) 


\section{X-Ray diffraction studies of $3 \mathrm{~h}$}

The crystals for X-ray diffraction studies were obtained by slow evaporation of solution in hexanes - EtOAc. X-Ray diffraction studies were performed on an automatic diffractometer (graphite monochromated $\mathrm{MoK}_{\alpha}$ radiation, CCD-detector, $\omega$-scanning, $2 \theta_{\max }=60^{\circ}$ ). The structure was solved by direct method using SHELXTL package.

The crystals of $\mathbf{3 h}\left(\mathrm{C}_{11} \mathrm{H}_{15} \mathrm{~F}_{3} \mathrm{~N}_{2} \mathrm{O}_{3}\right)$ are monoclinic. At $293 \mathrm{~K} \boldsymbol{a}=5.2476(5), \boldsymbol{b}=18.201(2), \boldsymbol{c}=14.843(2)$ $\AA, \beta=90.1(2)^{\circ}, V=1417.6(3) \AA^{3}, M_{r}=280.25, Z=4$, space group $P 2_{1}, d_{\text {calc }}=1.313 \mathrm{~g} / \mathrm{cm}^{3}, \mu\left(\mathrm{MoK}_{\alpha}\right)=0.120$ $\mathrm{mm}^{-1}, \mathrm{~F}(000)=584$. Intensity of 5392 reflections (5253 independent, $\mathrm{R}_{\mathrm{int}}=0.043$ ) were measured on an automatic «Xcalibur 3» diffractometer (graphite monochromated $\mathrm{MoK}_{\alpha}$ radiation, CCD-detector $\Theta / 2 \Theta$ scaning, $2 \Theta_{\max }=50^{\circ}$.

Final atomic coordinates, geometrical parameters and crystallographic data have been deposited with the Cambridge Crystallographic Data Centre, 12 Union Road, Cambridge, CB2 1EZ, UK (fax: +44 1223 336033; e-mail: deposit@ccdc.cam.ac.uk) and are available on request quoting the deposition number CCDC 1945950 (3h). 


\section{Computational part}

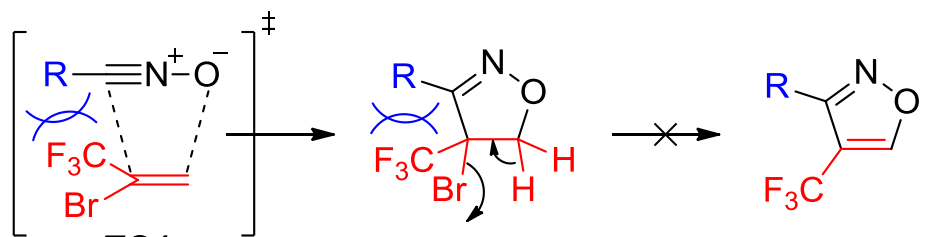

TS1 unfavorable $\mathbf{7 b}$ and $7 f$ (not observed) $\mathbf{8 b}$ and $\mathbf{8 f}$ (not observed)

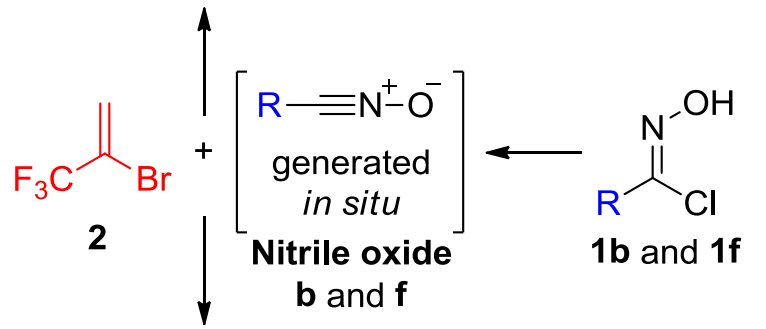

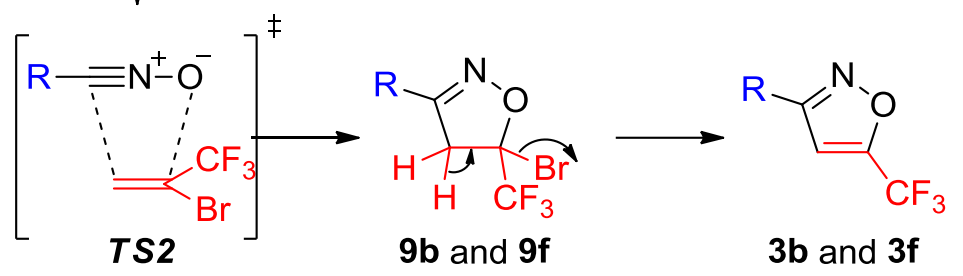

Scheme S1. [3+2] cycloaddition reaction

$$
\text { 1b, 3b, 7b, 9b- R = Ph }
$$

1f, 3f, 7f, 9f $-\mathrm{R}=\mathrm{CH}_{2} \mathrm{NHBoc}$

The formation of the final reaction products evidently determined by structures of intermediates $\mathbf{7}$ and 9 and transition state TS. In order to elucidate the observed regioselectivity, the reaction was studied using quantum chemical (RI-BP86) calculations, modeling the preferable formation of derivatives, 9b and 9 f compared to the isomeric $\mathbf{7 f}$ and $\mathbf{7 b}$ in the considered cyclization reaction. As the studied structures includes sterically crowded $t$-Bu group (9f) or aromatic moiety, e.g. 3-phenyl (9b) disposed to interactions with large contributions of electron dispersion effects. This can significantly influence the $\Delta \mathrm{E}$ and $\Delta \mathrm{G}$ values computed using conventional DFT methods. Taking this circumstance into account, the structures corresponding to local minima were additionally optimized using Grimme's RI-B97-D, having a dispersion correction.

The calculation results predict negative energies of formation (both $\Delta \mathrm{E}$ and $\Delta \mathrm{G}$ ) for cyclic products of the both types. Nevertheless, the formation $\mathbf{9 b}$ and $\mathbf{9 f}$ seems to be more favorable compared to the isomeric $\mathbf{7 b}$ and $7 \mathbf{f}$ (Tables S1, S2 for more detail). Therefore, the calculations predict the formation of the products of type $\mathbf{9 b}$ and $9 \mathrm{f}$ in the thermodynamic controlled cyclization reaction. Taking dispersion interactions into account using the RI-B97-D functional slightly reduces the $\Delta \mathrm{E}$ and $\Delta \mathrm{G}$ negative values (Figures $\mathrm{S} 1$ and $\mathrm{S} 2$ ).

Formation of the corresponding transition state TS2-f and TS2-b geometries are shown in Figures S3,S4. The corresponding activation energy values are by 3.9-4.3 $\mathrm{kcal} / \mathrm{mol}$ lower than the isomeric TS1-f and TS1-b and hence will favour the almost exclusive formation of cyclization products $9 \mathbf{b}$ and $9 f$ observed in the experiment. The moderate activation energy magnitudes estimated at the DFT level of approximation ( $\Delta \mathrm{G} 23.0$ and $25.6 \mathrm{kcal} / \mathrm{mol}$ for $9 \mathbf{b}$ and $9 f$, respectively) agree well with rather soft conditions used in the experiment. Thus, in the studied reaction and for the given set of the substituents the both thermodynamic and kinetic factors synchronously direct the process towards the same type of products. 


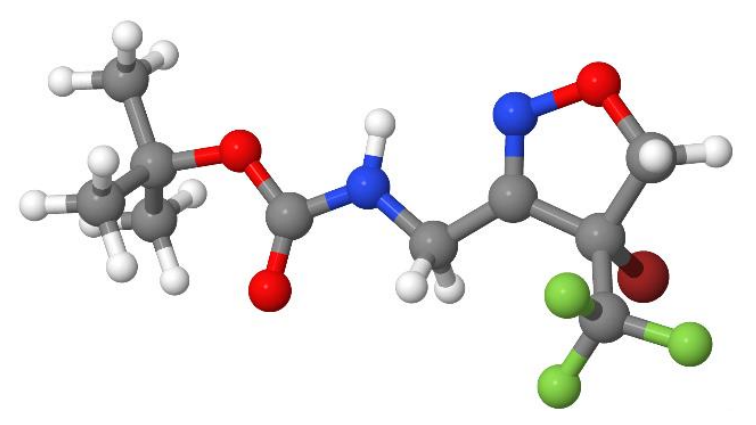

a $(-14.7 \mathrm{kcal} / \mathrm{mol})$

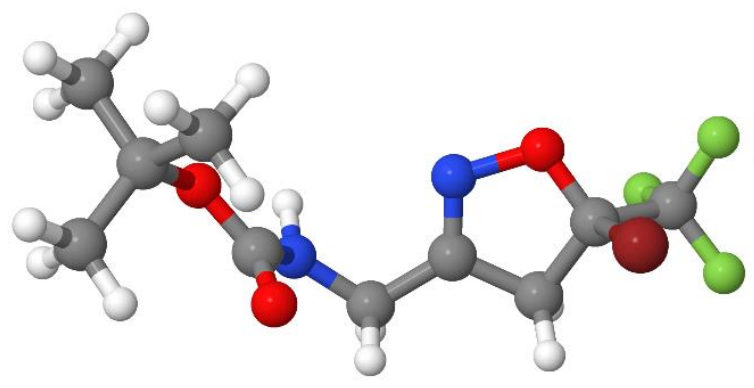

b (-18.6)

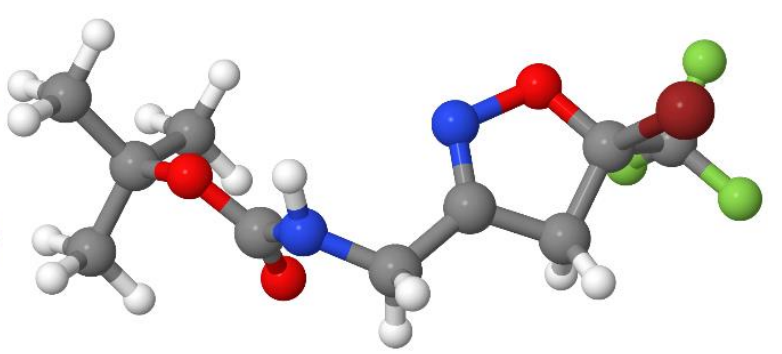

c $(-18.8)$

Figure S1. Optimized (RI-B97-D) structures of $7 f$ (a) and 9f (b,c, two most favored conformations). In parenthesis relative $\Delta \mathrm{G}$ values are given (in $\mathrm{kcal} / \mathrm{mol}$ relative to the free reactants).

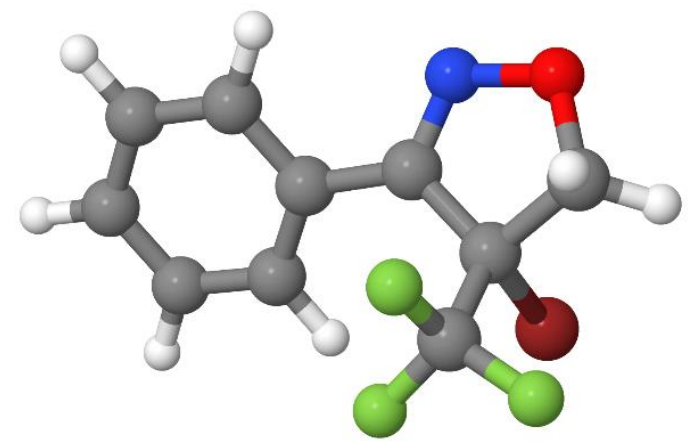

a (-8.6)

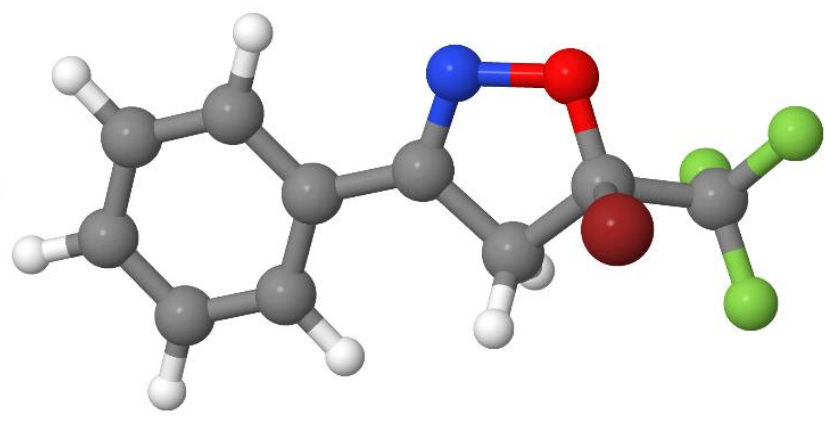

b (-16.1)

Figure S2. Optimized (RI-B97-D) structures of $7 \mathrm{~b}$ (a) and 9b (b). In parenthesis relative $\Delta \mathrm{G}$ values are given (in $\mathrm{kcal} / \mathrm{mol}$ relative to the free reactants).

Relative stability of is driven obviously by the similar electronic and steric factors determining higher stability for $\mathbf{9 b}$ and $9 \mathrm{f}$ relative to their regioisomers. The steric factor is probably of importance. This is best seen by comparing the conformations of TS1-b and TS2-b (Figure S4). In the latter TS structure the phenyl moiety is efficiently conjugated with the CNO triangle, whereas in the former one the aromatic ring is orthogonal to it. Noteworthy, the located TS2 structures evidence the rather synchronic 3+2-addition: the $\mathrm{C}-$ $\mathrm{C}$ and $\mathrm{C}-\mathrm{O}$ distances corresponding to the newly formed bonds are comparable (2.176 and $2.525 \AA$ for TS2-f and 2.196 and $2.489 \AA$ for TS2-b). 


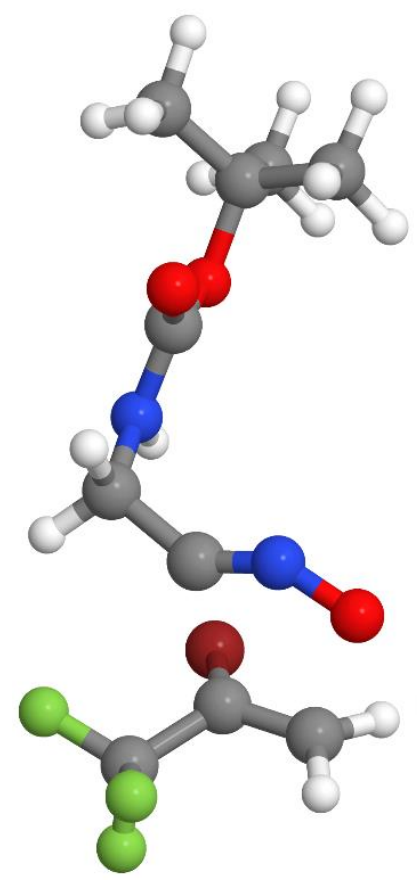

a (26.7)

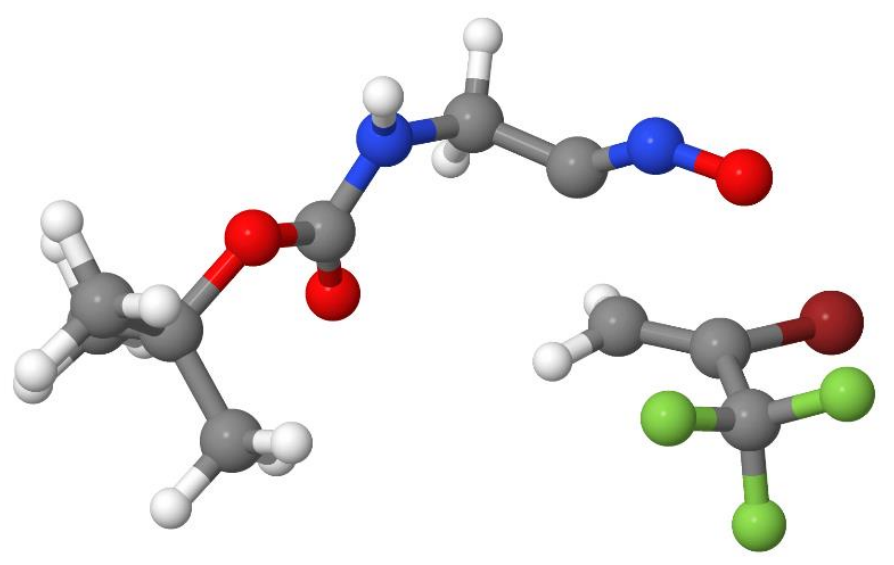

b (23.0)

Figure S3. Optimized (RI-BP86) structures of TS1-f (a) and TS2-f (b). In parenthesis relative $\Delta \mathrm{G}$ values are given (in $\mathrm{kcal} / \mathrm{mol}$ relative to the free reactants).

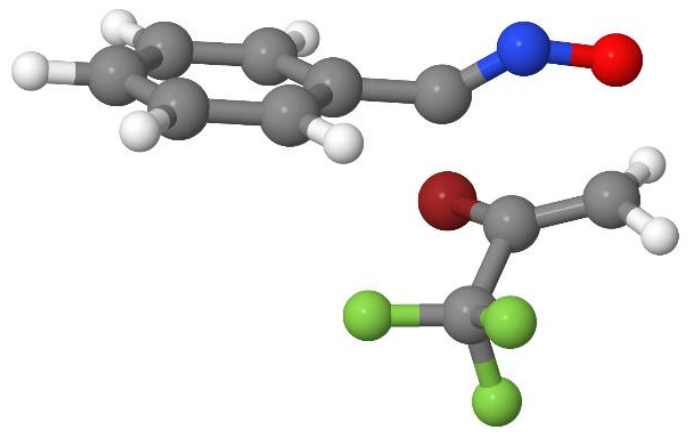

a (29.5)

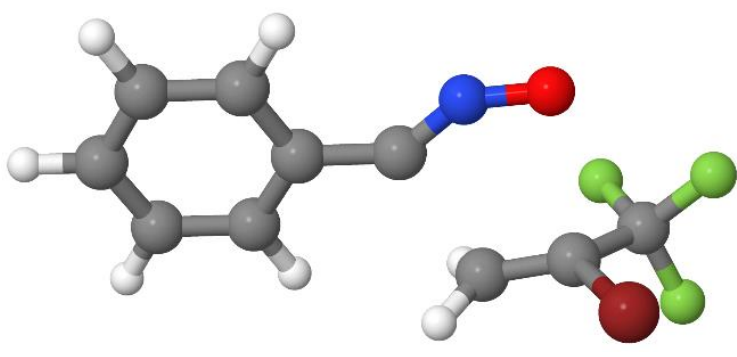

b (25.6)

Figure S4. Optimized (RI-BP86) structures of TS1-b (a) and TS2-b (b). In parenthesis relative $\Delta G$ values are given (in $\mathrm{kcal} / \mathrm{mol}$ relative to the free reactants). 
Table S1. Calculated (RI-B97-D/TZVP) total energy values (E), zero-point energy corrections (ZPE), thermal corrections to Gibbs free energy (TCGFE), corrected energy magnitudes (E+ZPE and E+TCGFE), reaction energies (DE and DG) and lowest vibration

frequencies $(\Delta \Delta \delta$ for the local minima structures)

\begin{tabular}{|c|c|c|c|c|c|c|c|c|}
\hline Structure & E, Hartree & ZPE, Hartree & E+ZPE, Hartree & $\Delta \mathrm{E}, \mathrm{kcal} / \mathrm{mol}^{\mathrm{a}}$ & TCGFE, Hartree & E+TCGFE, Hartree & $\Delta \mathrm{G}, \mathrm{kcal} / \mathrm{mol}^{\mathrm{a}}$ & $\Delta \delta, \mathrm{cm}^{-1}$ \\
\hline Nitrile oxide f & -608.916049 & 0.190158 & -608.725891 & - & 0.146326 & -608.769722 & - & 23.4 \\
\hline 2 & -2990.437402 & 0.045804 & -2990.391598 & - & 0.012398 & -2990.425005 & - & 49.0 \\
\hline $7 f-1$ & -3599.403194 & 0.241397 & -3599.161797 & -27.80 & 0.187366 & -3599.215827 & -13.24 & 17.0 \\
\hline $7 f-2$ & -3599.402911 & 0.241392 & -3599.161519 & -27.63 & 0.187720 & -3599.215190 & -12.84 & 12.4 \\
\hline $9 f-1$ & -3599.407194 & 0.240845 & -3599.166349 & -30.66 & 0.186102 & -3599.221092 & -16.54 & 8.1 \\
\hline $9 f-2$ & -3599.407926 & 0.240901 & -3599.167025 & -31.08 & 0.186490 & -3599.221436 & -16.76 & 11.9 \\
\hline $9 f-3$ & -3599.407188 & 0.240852 & -3599.166336 & -30.65 & 0.186079 & -3599.221109 & -16.55 & 9.3 \\
\hline $9 f-4$ & -3599.407927 & 0.240893 & -3599.167033 & -31.09 & 0.186067 & -3599.221859 & -17.03 & 9.8 \\
\hline Nitrile oxide b & -399.500589 & 0.100068 & -399.400521 & - & 0.065370 & -399.435218 & - & 20.9 \\
\hline $7 \mathbf{b}$ & -3389.981476 & 0.151588 & -3389.829888 & -23.70 & 0.108033 & -3389.873443 & -8.30 & 42.6 \\
\hline $9 b$ & -3389.989231 & 0.151116 & -3389.838115 & -28.86 & 0.106563 & -3389.882668 & -14.08 & 27.8 \\
\hline
\end{tabular}

${ }^{\mathrm{a}}$ Calculated relative to the isolated starting materials 
Table S2. Calculated (RI-BP86/TZVP) total energy values (E), zero-point energy corrections (ZPE), thermal corrections to Gibbs free energy (TCGFE), corrected energy magnitudes (E+ZPE and E+TCGFE), reaction energies (DE and DG) and lowest vibration

frequencies $(\Delta \Delta \delta$ for the local minima and transition state structures)

\begin{tabular}{|c|c|c|c|c|c|c|c|c|}
\hline & E, Hartree & ZPE, Hartree & E+ZPE, Hartree & $\Delta \mathrm{E}, \mathrm{kcal} / \mathrm{mol}^{\mathrm{a}}$ & TCGFE. Hartree & E+TCGFE, Hartree & $\Delta \mathrm{G}, \mathrm{kcal} / \mathrm{mol}^{\mathrm{a}}$ & $\Delta \delta, \mathrm{cm}^{-1}$ \\
\hline Nitrile oxide $f$ & -609.346877 & 0.188252 & -609.158625 & - & 0.143596 & -609.203281 & - & 15.4 \\
\hline 2 & -2989.657297 & 0.045566 & -2989.611731 & - & 0.012154 & -2989.645143 & - & 51.6 \\
\hline $7 f-1$ & -3599.055210 & 0.238680 & -3598.816531 & -28.98 & 0.183413 & -3598.871798 & -14.67 & 7.3 \\
\hline $7 f-2$ & -3599.053587 & 0.239001 & -3598.814586 & -27.75 & 0.184357 & -3598.869229 & -13.06 & 10.90 \\
\hline TS1f-1 & -3598.982476 & 0.234380 & -3598.748097 & 13.97 & 0.178286 & -3598.804190 & 27.76 & -353.0 \\
\hline TS1f-2 & -3598.984323 & 0.234764 & -3598.749559 & 13.05 & 0.178385 & -3598.805938 & 26.66 & -361.4 \\
\hline TS1f-3 & -3598.985124 & 0.234756 & -3598.750368 & 12.54 & 0.179151 & -3598.805973 & 26.64 & -358.3 \\
\hline 9f-1 & -3599.061350 & 0.238504 & -3598.822846 & -32.94 & 0.183283 & -3598.878067 & -18.60 & 9.4 \\
\hline $9 f-2$ & -3599.061710 & 0.238611 & -3598.823099 & -33.10 & 0.183295 & -3598.878415 & -18.82 & 9.5 \\
\hline $9 f-3$ & -3599.061349 & 0.238476 & -3598.822873 & -32.96 & 0.183116 & -3598.878233 & -18.71 & 8.3 \\
\hline $9 f-4$ & -3599.061713 & 0.238634 & -3598.823080 & -33.08 & 0.183550 & -3598.878164 & -18.66 & 10.7 \\
\hline TS2f-1 & -3598.988803 & 0.234713 & -3598.754090 & 10.21 & 0.177802 & -3598.811001 & 23.48 & -332.5 \\
\hline TS2f-2 & -3598.986097 & 0.234538 & -3598.751558 & 11.80 & 0.176427 & -3598.809669 & 24.32 & -334.2 \\
\hline TS2f-3 & -3598.986105 & 0.234558 & -3598.751547 & 11.80 & 0.176766 & -3598.809338 & 24.53 & -334.6 \\
\hline TS2f-4 & -3598.988475 & 0.234632 & -3598.753843 & 10.36 & 0.176709 & -3598.811766 & 23.00 & -331.0 \\
\hline Nitrile oxide b & -399.808274 & 0.099618 & -399.708656 & 0.00 & 0.065195 & -399.743079 & 0.00 & 29.3 \\
\hline $7 b$ & -3389.508641 & 0.150587 & -3389.358055 & -23.64 & 0.106654 & -3389.401987 & -8.64 & 39.9 \\
\hline TS1k & -3389.440572 & 0.146163 & -3389.294409 & 16.30 & 0.099425 & -3389.341147 & 29.54 & -358.1 \\
\hline $9 b$ & -3389.519232 & 0.150179 & -3389.369052 & -30.54 & 0.105309 & -3389.413922 & -16.13 & 25.3 \\
\hline TS2k & -3389.446144 & 0.146032 & -3389.300113 & 12.72 & 0.098781 & -3389.347363 & 25.64 & -321.7 \\
\hline
\end{tabular}

${ }^{\mathrm{a}}$ Calculated relative to the isolated starting materials 
Table S3. Atom coordinates for the optimized [RI-BP86 (left) and RI-B97-D (right)] structures.

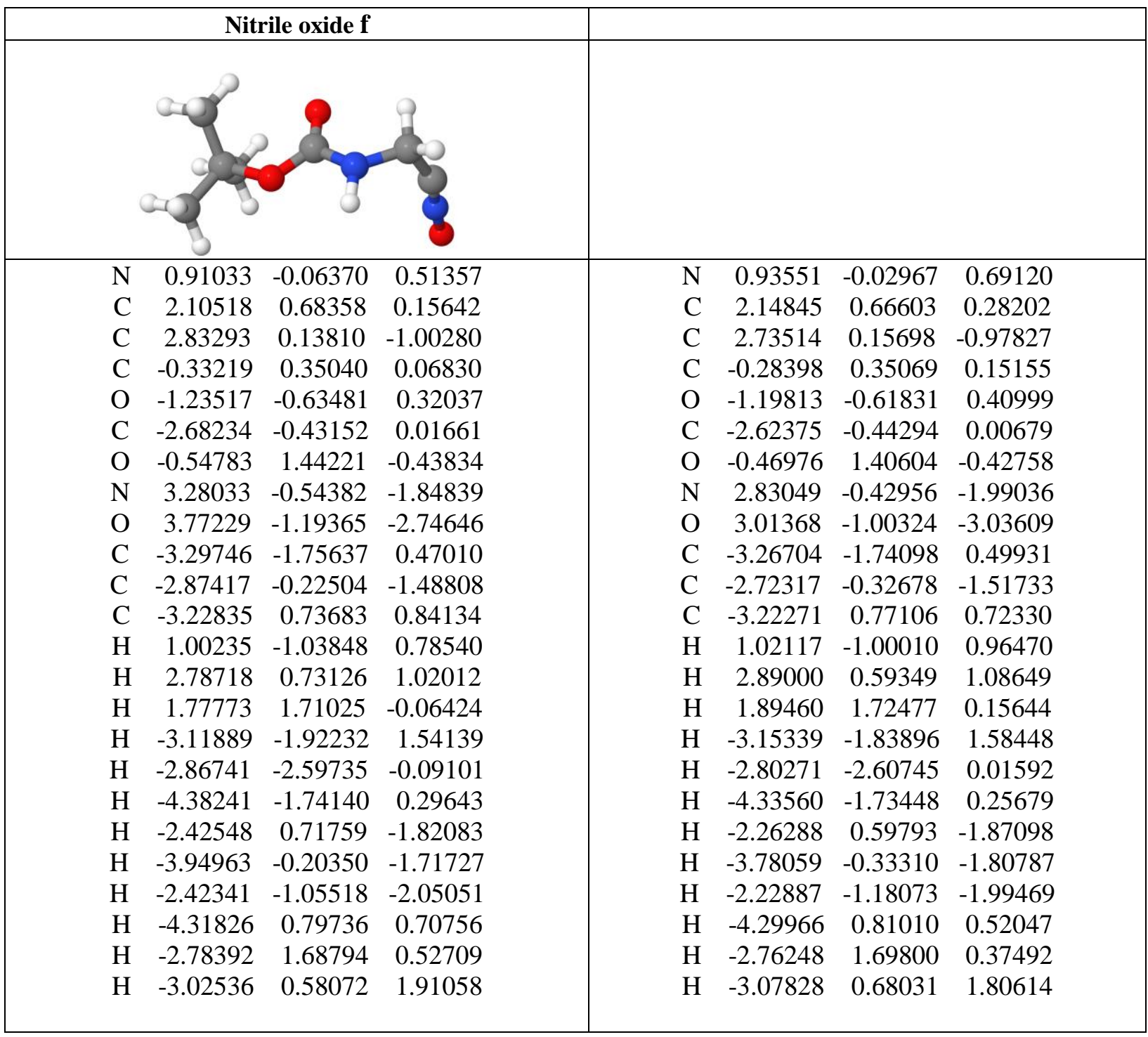

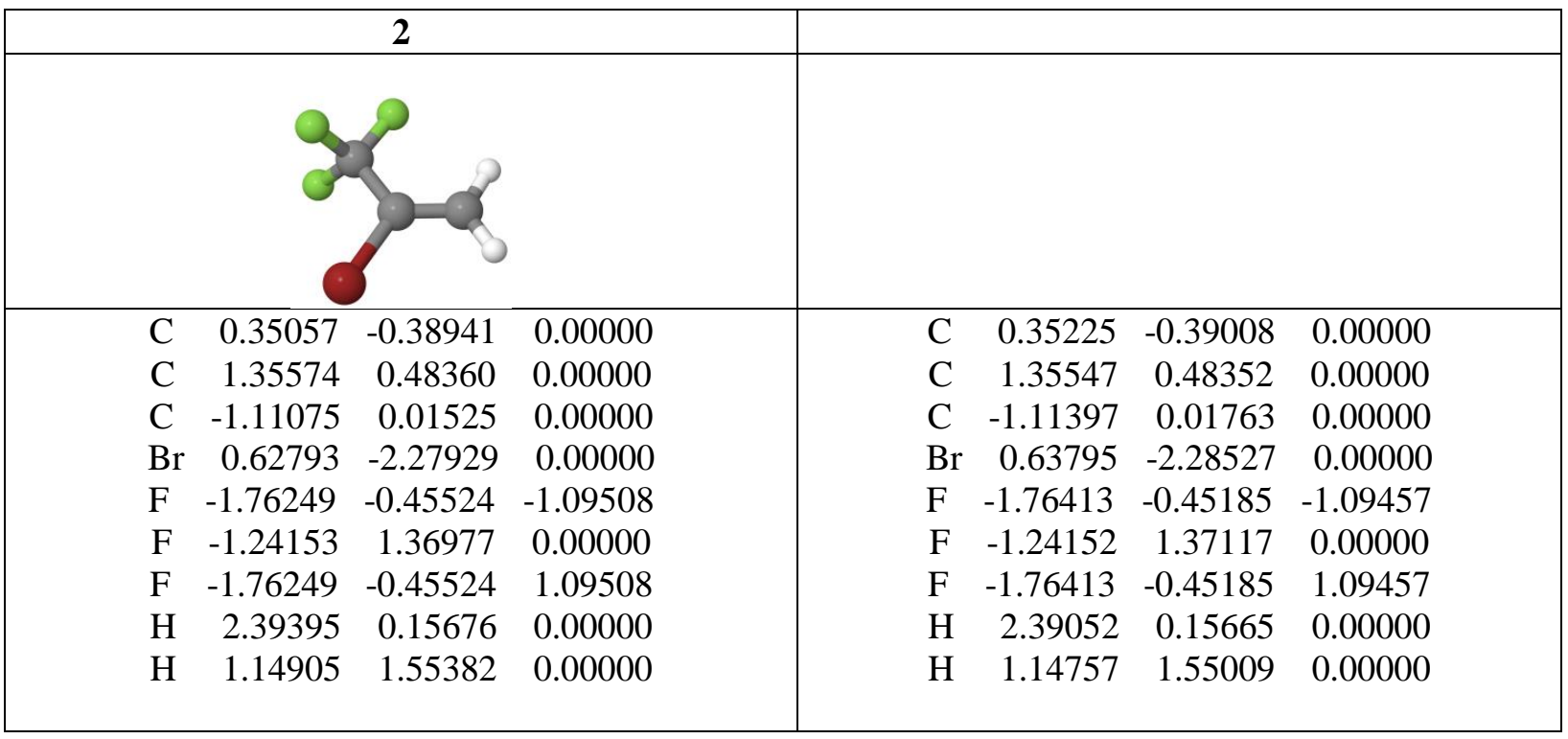




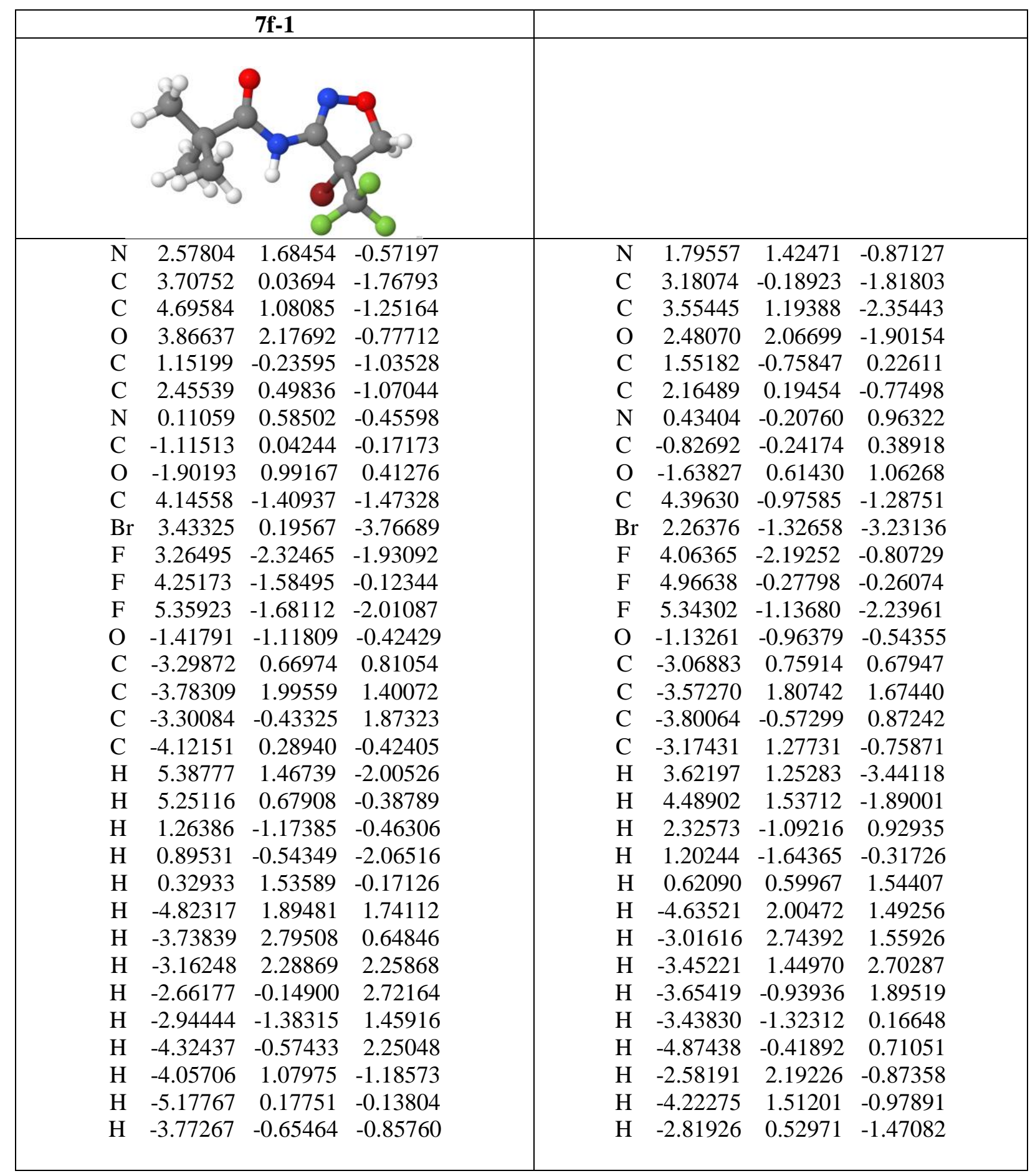

7f-2 


\begin{tabular}{|c|c|c|c|c|c|c|c|}
\hline $\mathrm{N}$ & 3.07637 & -0.41508 & 1.13326 & $\mathrm{~N}$ & 2.96426 & -0.55657 & 1.33003 \\
\hline $\mathrm{C}$ & 1.67152 & 1.35243 & 0.56789 & $\mathrm{C}$ & 1.62315 & 1.12268 & 0.44530 \\
\hline $\mathrm{C}$ & 3.05626 & 1.57787 & -0.03251 & $\mathrm{C}$ & 3.01175 & 1.17990 & -0.18901 \\
\hline $\mathrm{O}$ & 3.89734 & 0.56719 & 0.58819 & $\mathrm{O}$ & 3.81773 & 0.27685 & 0.61744 \\
\hline $\mathrm{C}$ & 0.77744 & -0.92477 & 1.73228 & $\mathrm{C}$ & 0.63911 & -0.85544 & 1.99408 \\
\hline $\mathrm{C}$ & 1.83510 & -0.04759 & 1.10365 & $\mathrm{C}$ & 1.74017 & -0.15566 & 1.23110 \\
\hline $\mathrm{N}$ & -0.15243 & -1.52838 & 0.79524 & $\mathrm{~N}$ & -0.35563 & -1.50995 & 1.15782 \\
\hline $\mathrm{C}$ & -0.00882 & -2.83110 & 0.37207 & $\mathrm{C}$ & -0.08043 & -2.72136 & 0.55934 \\
\hline $\mathrm{O}$ & -0.99722 & -3.10588 & -0.52880 & $\mathrm{O}$ & -1.06346 & -2.99776 & -0.34261 \\
\hline $\mathrm{C}$ & 0.53847 & 1.58913 & -0.44426 & $\mathrm{C}$ & 0.49546 & 1.20893 & -0.60134 \\
\hline $\mathrm{Br}$ & 1.32717 & 2.59103 & 2.14117 & $\mathrm{Br}$ & 1.34394 & 2.65648 & 1.77246 \\
\hline $\mathrm{F}$ & -0.69256 & 1.37679 & 0.08946 & $\mathrm{~F}$ & -0.74210 & 1.20030 & -0.04836 \\
\hline $\mathrm{F}$ & 0.67300 & 0.73888 & -1.49950 & $\mathrm{~F}$ & 0.57244 & 0.14333 & -1.44355 \\
\hline $\mathrm{F}$ & 0.56293 & 2.84985 & -0.93842 & $\mathrm{~F}$ & 0.60521 & 2.32801 & -1.35427 \\
\hline $\mathrm{O}$ & 0.86202 & -3.59348 & 0.76609 & $\mathrm{O}$ & 0.88363 & -3.41410 & 0.83180 \\
\hline $\mathrm{C}$ & -1.10779 & -4.46141 & -1.13417 & $\mathrm{C}$ & -1.03705 & -4.26444 & -1.12121 \\
\hline $\mathrm{C}$ & -2.32569 & -4.31126 & -2.04789 & $\mathrm{C}$ & -2.29819 & -4.15091 & -1.98115 \\
\hline $\mathrm{C}$ & -1.36958 & -5.50366 & -0.04306 & $\mathrm{C}$ & -1.12829 & -5.46612 & -0.17500 \\
\hline $\mathrm{C}$ & 0.15053 & -4.77486 & -1.94878 & $\mathrm{C}$ & 0.21850 & -4.31164 & -1.99876 \\
\hline $\mathrm{H}$ & 3.48866 & 2.56321 & 0.16422 & $\mathrm{H}$ & 3.47493 & 2.16697 & -0.18664 \\
\hline $\mathrm{H}$ & 3.03350 & 1.37781 & -1.11654 & $\mathrm{H}$ & 2.96921 & 0.77484 & -1.20978 \\
\hline $\mathrm{H}$ & 0.22019 & -0.33435 & 2.47601 & $\mathrm{H}$ & 0.12761 & -0.12130 & 2.62758 \\
\hline $\mathrm{H}$ & 1.29744 & -1.74071 & 2.25051 & $\mathrm{H}$ & 1.10760 & -1.60807 & 2.63382 \\
\hline $\mathrm{H}$ & -0.92005 & -0.98640 & 0.41306 & $\mathrm{H}$ & -1.10225 & -0.95720 & 0.76307 \\
\hline $\mathrm{H}$ & -2.52046 & -5.25920 & -2.56865 & $\mathrm{H}$ & -2.39180 & -5.04020 & -2.61466 \\
\hline $\mathrm{H}$ & -2.15281 & -3.52971 & -2.80052 & $\mathrm{H}$ & -2.24588 & -3.26511 & -2.62370 \\
\hline $\mathrm{H}$ & -3.21834 & -4.04426 & -1.46553 & $\mathrm{H}$ & -3.18892 & -4.07467 & -1.34795 \\
\hline $\mathrm{H}$ & -2.24770 & -5.22050 & 0.55509 & $\mathrm{H}$ & -2.00410 & -5.36697 & 0.47680 \\
\hline $\mathrm{H}$ & -0.50384 & -5.61005 & 0.62020 & $\mathrm{H}$ & -0.22989 & -5.54816 & 0.44012 \\
\hline $\mathrm{H}$ & -1.57656 & -6.47710 & -0.51138 & $\mathrm{H}$ & -1.24048 & -6.38190 & -0.76794 \\
\hline $\mathrm{H}$ & 0.33512 & -3.98282 & -2.68873 & $\mathrm{H}$ & 0.27958 & -3.40807 & -2.61668 \\
\hline $\mathrm{H}$ & 0.00719 & -5.72091 & -2.49122 & $\mathrm{H}$ & 0.15992 & -5.18211 & -2.66329 \\
\hline $\mathrm{H}$ & 1.02904 & -4.87045 & -1.30088 & $\mathrm{H}$ & 1.11970 & -4.39031 & -1.38728 \\
\hline
\end{tabular}




\begin{tabular}{|c|c|c|c|c|c|c|c|}
\hline $\mathrm{N}$ & 2.28038 & 1.35889 & -2.66794 & $\mathrm{~N}$ & 1.72803 & -0.21953 & -3.61486 \\
\hline $\mathrm{O}$ & 3.48871 & 1.35211 & -2.90772 & $\mathrm{O}$ & 2.69756 & -0.49445 & -4.32377 \\
\hline $\mathrm{C}$ & 1.38141 & 0.63848 & -2.26633 & $\mathrm{C}$ & 1.36122 & -0.23729 & -2.45373 \\
\hline $\mathrm{C}$ & -0.08981 & 0.68776 & -2.09293 & $\mathrm{C}$ & 0.11381 & -0.07151 & -1.65310 \\
\hline $\mathrm{N}$ & -0.50465 & 1.19986 & -0.80321 & $\mathrm{~N}$ & -0.78785 & -1.20650 & -1.71899 \\
\hline $\mathrm{C}$ & -1.48810 & 0.57390 & -0.06765 & $\mathrm{C}$ & -1.77993 & -1.24950 & -2.67964 \\
\hline $\mathrm{O}$ & -1.66966 & 1.26389 & 1.09212 & $\mathrm{O}$ & -2.32075 & -2.49732 & -2.69717 \\
\hline $\mathrm{O}$ & -2.08222 & -0.43028 & -0.43415 & $\mathrm{O}$ & -2.10841 & -0.28961 & -3.36380 \\
\hline $\mathrm{C}$ & -2.68772 & 0.81517 & 2.08506 & $\mathrm{C}$ & -3.48155 & -2.80675 & -3.58154 \\
\hline $\mathrm{C}$ & -2.54268 & 1.86044 & 3.19239 & $\mathrm{C}$ & -3.75419 & -4.27618 & -3.25593 \\
\hline $\mathrm{C}$ & -2.33255 & -0.58166 & 2.60111 & $\mathrm{C}$ & -3.07888 & -2.63668 & -5.04882 \\
\hline $\mathrm{C}$ & -4.08502 & 0.86946 & 1.46138 & $\mathrm{C}$ & -4.67698 & -1.93037 & -3.19861 \\
\hline $\mathrm{H}$ & -0.50591 & -0.32703 & -2.18380 & $\mathrm{H}$ & -0.42896 & 0.80262 & -2.04400 \\
\hline $\mathrm{H}$ & -0.50473 & 1.28289 & -2.92599 & $\mathrm{H}$ & 0.38582 & 0.12979 & -0.61081 \\
\hline $\mathrm{H}$ & -0.08842 & 2.04780 & -0.43212 & $\mathrm{H}$ & -0.48366 & -2.08832 & -1.31445 \\
\hline $\mathrm{H}$ & -3.24969 & 1.64235 & 4.00490 & $\mathrm{H}$ & -4.60264 & -4.63848 & -3.85294 \\
\hline $\mathrm{H}$ & -2.75487 & 2.86757 & 2.80772 & $\mathrm{H}$ & -3.99890 & -4.40025 & -2.19207 \\
\hline $\mathrm{H}$ & -1.52461 & 1.85067 & 3.60522 & $\mathrm{H}$ & -2.87658 & -4.89524 & -3.48759 \\
\hline $\mathrm{H}$ & -1.30521 & -0.59687 & 2.99192 & $\mathrm{H}$ & -2.18662 & -3.23810 & -5.27439 \\
\hline $\mathrm{H}$ & -2.42385 & -1.33338 & 1.80919 & $\mathrm{H}$ & -2.87156 & -1.58707 & -5.28527 \\
\hline $\mathrm{H}$ & -3.01375 & -0.84960 & 3.42202 & $\mathrm{H}$ & -3.89781 & -2.98829 & -5.69333 \\
\hline $\mathrm{H}$ & -4.28577 & 1.87114 & 1.05468 & $\mathrm{H}$ & -4.90433 & -2.03686 & -2.12820 \\
\hline $\mathrm{H}$ & -4.83714 & 0.66028 & 2.23610 & $\mathrm{H}$ & -5.56091 & -2.25313 & -3.76782 \\
\hline $\mathrm{H}$ & -4.19169 & 0.12933 & 0.66037 & $\mathrm{H}$ & -4.48392 & -0.87477 & -3.42073 \\
\hline $\mathrm{C}$ & 2.67584 & -1.24216 & -2.05821 & $\mathrm{C}$ & 3.27393 & -1.15212 & -1.63356 \\
\hline $\mathrm{C}$ & 3.91181 & -0.74506 & -2.39033 & $\mathrm{C}$ & 4.03278 & -1.25110 & -2.77604 \\
\hline $\mathrm{C}$ & 2.36156 & -1.57927 & -0.61193 & $\mathrm{C}$ & 3.62703 & -0.10913 & -0.59005 \\
\hline $\mathrm{Br}$ & 1.73418 & -2.36776 & -3.33037 & $\mathrm{Br}$ & 2.52874 & -2.77820 & -0.85675 \\
\hline $\mathrm{F}$ & 1.03378 & -1.63670 & -0.34675 & $\mathrm{~F}$ & 2.60348 & 0.17513 & 0.26330 \\
\hline $\mathrm{F}$ & 2.90842 & -0.64688 & 0.22146 & F & 3.99087 & 1.05763 & -1.19016 \\
\hline $\mathrm{F}$ & 2.88487 & -2.78699 & -0.25371 & $\mathrm{~F}$ & 4.67454 & -0.50374 & 0.18742 \\
\hline $\mathrm{H}$ & 4.32708 & -0.88412 & -3.38429 & $\mathrm{H}$ & 4.12227 & -2.19049 & -3.31402 \\
\hline $\mathrm{H}$ & 4.58275 & -0.40442 & -1.60324 & $\mathrm{H}$ & 4.74003 & -0.45993 & -3.01973 \\
\hline
\end{tabular}




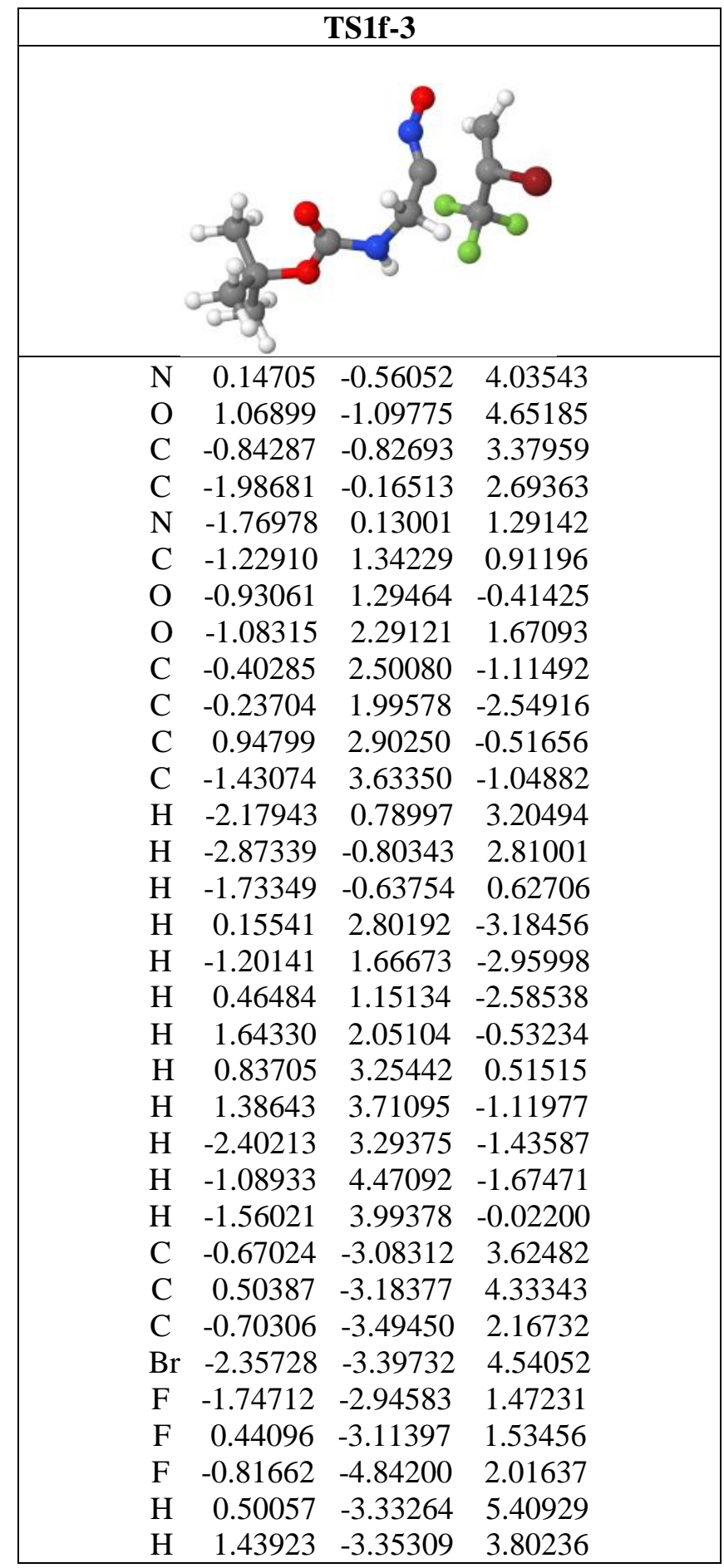

\section{9f-1}




\begin{tabular}{|c|c|c|c|c|c|c|c|}
\hline $\mathrm{N}$ & 1.70354 & 1.17428 & 1.42233 & $\mathrm{~N}$ & 1.35865 & 0.99579 & 1.41245 \\
\hline $\mathrm{C}$ & 3.18716 & -0.58555 & 1.86101 & $\mathrm{C}$ & 3.03510 & -0.61436 & 1.70315 \\
\hline $\mathrm{C}$ & 3.76987 & 0.73314 & 2.37401 & $\mathrm{C}$ & 3.34447 & 0.62737 & 2.54548 \\
\hline $\mathrm{O}$ & 2.96232 & 1.74766 & 1.84233 & $\mathrm{O}$ & 2.46531 & 1.61994 & 2.10937 \\
\hline $\mathrm{C}$ & 0.70075 & -1.00821 & 1.02735 & $\mathrm{C}$ & 0.74878 & -1.17349 & 0.46954 \\
\hline $\mathrm{C}$ & 1.81875 & -0.10464 & 1.45461 & $\mathrm{C}$ & 1.66606 & -0.23028 & 1.20709 \\
\hline $\mathrm{N}$ & -0.51148 & -0.30930 & 0.67190 & $\mathrm{~N}$ & -0.54211 & -0.62088 & 0.12159 \\
\hline $\mathrm{C}$ & -1.70197 & -0.62616 & 1.29072 & $\mathrm{C}$ & -1.58139 & -0.72787 & 1.03454 \\
\hline $\mathrm{O}$ & -2.64503 & 0.28062 & 0.92042 & $\mathrm{O}$ & -2.56712 & 0.13133 & 0.67841 \\
\hline $\mathrm{O}$ & -1.85330 & -1.59074 & 2.03096 & $\mathrm{O}$ & -1.58625 & -1.50821 & 1.97123 \\
\hline $\mathrm{C}$ & -4.04488 & 0.16373 & 1.41910 & $\mathrm{C}$ & -3.82412 & 0.21585 & 1.47460 \\
\hline $\mathrm{C}$ & -4.72558 & 1.36288 & 0.75688 & $\mathrm{C}$ & -4.61223 & 1.29642 & 0.73095 \\
\hline $\mathrm{C}$ & -4.06856 & 0.29518 & 2.94436 & $\mathrm{C}$ & -3.50513 & 0.65957 & 2.90575 \\
\hline $\mathrm{C}$ & -4.66286 & -1.15144 & 0.93669 & $\mathrm{C}$ & -4.56100 & -1.12633 & 1.43141 \\
\hline $\mathrm{Br}$ & 3.68474 & 0.77176 & 4.40832 & $\mathrm{Br}$ & 2.99805 & 0.20848 & 4.52559 \\
\hline $\mathrm{C}$ & 5.23487 & 1.00860 & 1.96011 & $\mathrm{C}$ & 4.79607 & 1.15644 & 2.41099 \\
\hline $\mathrm{F}$ & 5.66962 & 2.21133 & 2.37131 & $\mathrm{~F}$ & 5.00379 & 2.28187 & 3.11042 \\
\hline $\mathrm{F}$ & 5.33167 & 0.96954 & 0.60065 & $\mathrm{~F}$ & 5.04956 & 1.42239 & 1.09901 \\
\hline $\mathrm{F}$ & 6.06341 & 0.05371 & 2.44953 & $\mathrm{~F}$ & 5.68762 & 0.21932 & 2.81104 \\
\hline $\mathrm{H}$ & 3.17903 & -1.37936 & 2.61502 & $\mathrm{H}$ & 3.05814 & -1.54557 & 2.27142 \\
\hline $\mathrm{H}$ & 3.73929 & -0.94767 & 0.97697 & $\mathrm{H}$ & 3.73231 & -0.69960 & 0.85729 \\
\hline $\mathrm{H}$ & 0.44524 & -1.70646 & 1.84155 & $\mathrm{H}$ & 0.57662 & -2.05534 & 1.09861 \\
\hline $\mathrm{H}$ & 1.07127 & -1.63233 & 0.19027 & $\mathrm{H}$ & 1.26677 & -1.51933 & -0.43795 \\
\hline $\mathrm{H}$ & -0.44242 & 0.59837 & 0.21995 & $\mathrm{H}$ & -0.54609 & 0.21967 & -0.44206 \\
\hline $\mathrm{H}$ & -5.78488 & 1.39686 & 1.04728 & $\mathrm{H}$ & -5.57608 & 1.45381 & 1.22797 \\
\hline $\mathrm{H}$ & -4.66679 & 1.28804 & -0.33766 & $\mathrm{H}$ & -4.79632 & 0.99303 & -0.30551 \\
\hline $\mathrm{H}$ & -4.24854 & 2.30194 & 1.06914 & $\mathrm{H}$ & -4.05897 & 2.24182 & 0.72904 \\
\hline $\mathrm{H}$ & -3.56865 & 1.22296 & 3.25730 & $\mathrm{H}$ & -2.91757 & 1.58462 & 2.89173 \\
\hline $\mathrm{H}$ & -3.57442 & -0.55698 & 3.42436 & $\mathrm{H}$ & -2.94609 & -0.11131 & 3.43992 \\
\hline $\mathrm{H}$ & -5.11202 & 0.33758 & 3.28942 & $\mathrm{H}$ & -4.44441 & 0.85297 & 3.43772 \\
\hline $\mathrm{H}$ & -4.58139 & -1.23558 & -0.15655 & $\mathrm{H}$ & -4.71788 & -1.43802 & 0.39219 \\
\hline $\mathrm{H}$ & -5.73038 & -1.16980 & 1.20063 & $\mathrm{H}$ & -5.54121 & -1.01177 & 1.90966 \\
\hline $\mathrm{H}$ & -4.17188 & -2.01462 & 1.40006 & $\mathrm{H}$ & -3.99683 & -1.89902 & 1.95769 \\
\hline
\end{tabular}

\section{9f-2}




\begin{tabular}{|c|c|c|c|c|c|c|c|}
\hline $\mathrm{N}$ & -1.92601 & 1.45467 & 0.37789 & $\mathrm{~N}$ & -1.68135 & 1.26261 & 0.45087 \\
\hline $\mathrm{C}$ & -3.70714 & 0.16159 & -0.42534 & $\mathrm{C}$ & -3.51957 & 0.12120 & -0.44297 \\
\hline $\mathrm{C}$ & -4.16565 & 1.60197 & -0.19565 & $\mathrm{C}$ & -3.95983 & 1.47495 & 0.11666 \\
\hline $\mathrm{O}$ & -3.14497 & 2.22143 & 0.53380 & $\mathrm{O}$ & -2.89156 & 1.94329 & 0.87654 \\
\hline $\mathrm{C}$ & -1.19423 & -0.70555 & -0.48438 & $\mathrm{C}$ & -1.02035 & -0.65323 & -0.91439 \\
\hline $\mathrm{C}$ & -2.23429 & 0.33049 & -0.16077 & $\mathrm{C}$ & -2.03045 & 0.27655 & -0.28755 \\
\hline $\mathrm{N}$ & 0.12870 & -0.40105 & 0.00538 & $\mathrm{~N}$ & 0.33489 & -0.50235 & -0.42932 \\
\hline $\mathrm{C}$ & 0.67963 & -1.15411 & 1.02283 & $\mathrm{C}$ & 0.67504 & -1.11527 & 0.76782 \\
\hline $\mathrm{O}$ & 1.82164 & -0.55121 & 1.44584 & $\mathrm{O}$ & 1.84212 & -0.59279 & 1.21463 \\
\hline $\mathrm{O}$ & 0.20249 & -2.20542 & 1.43274 & $\mathrm{O}$ & 0.01873 & -2.00483 & 1.28321 \\
\hline $\mathrm{C}$ & 2.65484 & -1.17072 & 2.51593 & $\mathrm{C}$ & 2.45809 & -1.08181 & 2.48085 \\
\hline $\mathrm{C}$ & 3.80332 & -0.16962 & 2.65201 & $\mathrm{C}$ & 3.71548 & -0.21655 & 2.59045 \\
\hline $\mathrm{C}$ & 3.17033 & -2.53536 & 2.05117 & $\mathrm{C}$ & 2.82298 & -2.56325 & 2.34536 \\
\hline $\mathrm{C}$ & 1.85165 & -1.26146 & 3.81623 & $\mathrm{C}$ & 1.51059 & -0.82017 & 3.65580 \\
\hline $\mathrm{Br}$ & -4.39699 & 2.53417 & -1.99796 & $\mathrm{Br}$ & -4.31857 & 2.76247 & -1.45559 \\
\hline $\mathrm{C}$ & -5.49208 & 1.75822 & 0.58487 & $\mathrm{C}$ & -5.23033 & 1.43718 & 1.00465 \\
\hline $\mathrm{F}$ & -5.80250 & 3.04422 & 0.82052 & $\mathrm{~F}$ & -5.53769 & 2.63828 & 1.51764 \\
\hline $\mathrm{F}$ & -5.38292 & 1.12526 & 1.78627 & $\mathrm{~F}$ & -5.02244 & 0.57696 & 2.03788 \\
\hline $\mathrm{F}$ & -6.51781 & 1.17721 & -0.08487 & $\mathrm{~F}$ & -6.29123 & 0.97549 & 0.30113 \\
\hline $\mathrm{H}$ & -3.94857 & -0.22011 & -1.42263 & $\mathrm{H}$ & -3.85439 & -0.06451 & -1.46455 \\
\hline $\mathrm{H}$ & -4.14233 & -0.52011 & 0.32562 & $\mathrm{H}$ & -3.86664 & -0.70105 & 0.19975 \\
\hline $\mathrm{H}$ & -1.18898 & -0.85105 & -1.58083 & $\mathrm{H}$ & -1.04101 & -0.50310 & -2.00343 \\
\hline $\mathrm{H}$ & -1.48317 & -1.67125 & -0.03853 & $\mathrm{H}$ & -1.33719 & -1.68541 & -0.72300 \\
\hline $\mathrm{H}$ & 0.49832 & 0.53629 & -0.12795 & $\mathrm{H}$ & 0.79136 & 0.38304 & -0.60835 \\
\hline $\mathrm{H}$ & 4.50558 & -0.51161 & 3.42498 & $\mathrm{H}$ & 4.26737 & -0.48633 & 3.49788 \\
\hline $\mathrm{H}$ & 3.42399 & 0.82046 & 2.93962 & $\mathrm{H}$ & 3.44776 & 0.84426 & 2.64396 \\
\hline $\mathrm{H}$ & 4.34949 & -0.07285 & 1.70364 & $\mathrm{H}$ & 4.36635 & -0.37307 & 1.72330 \\
\hline $\mathrm{H}$ & 3.69596 & -2.44028 & 1.09018 & $\mathrm{H}$ & 3.45247 & -2.71809 & 1.46138 \\
\hline $\mathrm{H}$ & 2.35006 & -3.25367 & 1.94144 & $\mathrm{H}$ & 1.92614 & -3.18049 & 2.26116 \\
\hline $\mathrm{H}$ & 3.88353 & -2.92585 & 2.79175 & $\mathrm{H}$ & 3.38880 & -2.87478 & 3.23160 \\
\hline $\mathrm{H}$ & 1.45026 & -0.27500 & 4.08891 & $\mathrm{H}$ & 1.21608 & 0.23529 & 3.67508 \\
\hline $\mathrm{H}$ & 2.51263 & -1.59665 & 4.62887 & $\mathrm{H}$ & 2.03062 & -1.05264 & 4.59287 \\
\hline $\mathrm{H}$ & 1.02252 & -1.97175 & 3.72259 & $\mathrm{H}$ & 0.61507 & -1.44054 & 3.58282 \\
\hline
\end{tabular}

\section{9f-3}




\begin{tabular}{|c|c|c|c|c|c|c|c|}
\hline $\mathrm{N}$ & 2.12847 & -0.93964 & -1.27367 & $\mathrm{~N}$ & 2.05041 & -0.75768 & -1.60919 \\
\hline $\mathrm{O}$ & 2.48138 & -1.92062 & -0.27264 & $\mathrm{O}$ & 2.58503 & -1.86668 & -0.84482 \\
\hline $\mathrm{C}$ & 0.26944 & -0.37007 & -2.73701 & $\mathrm{C}$ & -0.08980 & 0.02804 & -2.48618 \\
\hline $\mathrm{C}$ & 0.95693 & -1.22603 & -1.71577 & $\mathrm{C}$ & 0.79436 & -0.95553 & -1.76112 \\
\hline $\mathrm{N}$ & 1.07850 & 0.72466 & -3.21834 & $\mathrm{~N}$ & 0.60633 & 1.14223 & -3.09176 \\
\hline $\mathrm{C}$ & 1.29042 & 0.88866 & -4.57048 & $\mathrm{C}$ & 1.07838 & 1.00869 & -4.38951 \\
\hline $\mathrm{O}$ & 2.21858 & 1.86509 & -4.75499 & $\mathrm{O}$ & 1.97422 & 1.99610 & -4.63315 \\
\hline $\mathrm{O}$ & 0.70212 & 0.25351 & -5.43757 & $\mathrm{O}$ & 0.69632 & 0.15126 & -5.16748 \\
\hline $\mathrm{C}$ & 2.62215 & 2.27627 & -6.12966 & $\mathrm{C}$ & 2.63337 & 2.11108 & -5.96488 \\
\hline $\mathrm{C}$ & 3.65429 & 3.37017 & -5.85038 & $\mathrm{C}$ & 3.54763 & 3.32358 & -5.77555 \\
\hline $\mathrm{C}$ & 1.41409 & 2.84258 & -6.88077 & $\mathrm{C}$ & 1.58266 & 2.38152 & -7.04619 \\
\hline $\mathrm{C}$ & 3.26309 & 1.09410 & -6.86178 & $\mathrm{C}$ & 3.45231 & 0.84852 & -6.25116 \\
\hline $\mathrm{C}$ & 0.32622 & -2.41991 & -1.04804 & $\mathrm{C}$ & 0.28254 & -2.20723 & -1.09843 \\
\hline $\mathrm{C}$ & 1.55231 & -2.96911 & -0.31525 & $\mathrm{C}$ & 1.62278 & -2.87543 & -0.77546 \\
\hline $\mathrm{Br}$ & 2.32424 & -4.53924 & -1.35720 & $\mathrm{Br}$ & 2.01964 & -4.31688 & -2.18295 \\
\hline $\mathrm{C}$ & 1.29220 & -3.44869 & 1.13246 & $\mathrm{C}$ & 1.69636 & -3.53591 & 0.62575 \\
\hline $\mathrm{F}$ & 0.39928 & -4.46849 & 1.15137 & $\mathrm{~F}$ & 0.76824 & -4.51448 & 0.74437 \\
\hline $\mathrm{F}$ & 0.75226 & -2.42526 & 1.85366 & F & 1.41733 & -2.59642 & 1.57226 \\
\hline $\mathrm{F}$ & 2.41537 & -3.84492 & 1.75390 & $\mathrm{~F}$ & 2.90646 & -4.04852 & 0.89362 \\
\hline $\mathrm{H}$ & -0.68013 & -0.01078 & -2.29322 & $\mathrm{H}$ & -0.84745 & 0.39745 & -1.77832 \\
\hline $\mathrm{H}$ & -0.01201 & -0.97653 & -3.61379 & $\mathrm{H}$ & -0.62457 & -0.50453 & -3.28207 \\
\hline $\mathrm{H}$ & 1.73218 & 1.17104 & -2.58083 & $\mathrm{H}$ & 1.17340 & 1.71725 & -2.48184 \\
\hline $\mathrm{H}$ & 4.04144 & 3.76773 & -6.79887 & $\mathrm{H}$ & 4.09784 & 3.51556 & -6.70355 \\
\hline $\mathrm{H}$ & 4.49726 & 2.97021 & -5.27042 & $\mathrm{H}$ & 4.26804 & 3.13888 & -4.97133 \\
\hline $\mathrm{H}$ & 3.20201 & 4.19659 & -5.28508 & $\mathrm{H}$ & 2.95925 & 4.21308 & -5.52502 \\
\hline $\mathrm{H}$ & 0.94832 & 3.65351 & -6.30257 & $\mathrm{H}$ & 0.97933 & 3.25614 & -6.77646 \\
\hline $\mathrm{H}$ & 0.66639 & 2.06510 & -7.07409 & $\mathrm{H}$ & 0.92669 & 1.51860 & -7.17823 \\
\hline $\mathrm{H}$ & 1.74658 & 3.25851 & -7.84311 & $\mathrm{H}$ & 2.09140 & 2.59166 & -7.99471 \\
\hline $\mathrm{H}$ & 4.09030 & 0.67842 & -6.26885 & $\mathrm{H}$ & 4.14225 & 0.65057 & -5.42295 \\
\hline $\mathrm{H}$ & 3.67283 & 1.44017 & -7.82200 & $\mathrm{H}$ & 4.04065 & 1.00335 & -7.16358 \\
\hline $\mathrm{H}$ & 2.53027 & 0.30324 & -7.05734 & $\mathrm{H}$ & 2.80203 & -0.01750 & -6.38998 \\
\hline $\mathrm{H}$ & -0.44875 & -2.08969 & -0.33536 & $\mathrm{H}$ & -0.26114 & -1.95097 & -0.17784 \\
\hline $\mathrm{H}$ & -0.12286 & -3.13791 & -1.74195 & $\mathrm{H}$ & -0.36714 & -2.81315 & -1.73198 \\
\hline
\end{tabular}

\section{9f-4}




\begin{tabular}{|c|c|c|c|c|c|c|c|}
\hline $\mathrm{N}$ & 2.14370 & 1.87939 & 0.57399 & $\mathrm{~N}$ & 1.83434 & 1.96894 & 0.51452 \\
\hline $\mathrm{O}$ & 3.27354 & 1.30110 & 1.27182 & $\mathrm{O}$ & 2.77433 & 1.33717 & 1.42358 \\
\hline $\mathrm{C}$ & 0.55315 & 1.29534 & -1.17931 & $\mathrm{C}$ & 0.69030 & 1.48865 & -1.59040 \\
\hline $\mathrm{C}$ & 1.71426 & 1.01026 & -0.26813 & $\mathrm{C}$ & 1.62053 & 1.15430 & -0.44989 \\
\hline $\mathrm{N}$ & -0.12744 & 2.53962 & -0.91222 & $\mathrm{~N}$ & -0.11940 & 2.67206 & -1.39316 \\
\hline $\mathrm{C}$ & -1.41034 & 2.53011 & -0.40236 & $\mathrm{C}$ & -1.26535 & 2.55950 & -0.61927 \\
\hline $\mathrm{O}$ & -1.74817 & 3.79577 & -0.04109 & $\mathrm{O}$ & -1.68704 & 3.79903 & -0.27256 \\
\hline $\mathrm{O}$ & -2.11103 & 1.52781 & -0.32889 & $\mathrm{O}$ & -1.79676 & 1.49566 & -0.34804 \\
\hline $\mathrm{C}$ & -3.10348 & 4.09251 & 0.50501 & $\mathrm{C}$ & -2.91579 & 3.98068 & 0.55157 \\
\hline $\mathrm{C}$ & -3.02735 & 5.59909 & 0.75831 & $\mathrm{C}$ & -2.98709 & 5.50154 & 0.70486 \\
\hline $\mathrm{C}$ & -3.31779 & 3.32808 & 1.81413 & $\mathrm{C}$ & -2.73468 & 3.30210 & 1.91300 \\
\hline $\mathrm{C}$ & -4.17071 & 3.76672 & -0.54324 & $\mathrm{C}$ & -4.13568 & 3.44890 & -0.20683 \\
\hline $\mathrm{C}$ & 2.47308 & -0.29026 & -0.24679 & $\mathrm{C}$ & 2.35039 & -0.15589 & -0.32178 \\
\hline $\mathrm{C}$ & 3.66857 & 0.13577 & 0.60584 & $\mathrm{C}$ & 3.32308 & 0.22196 & 0.79658 \\
\hline $\mathrm{Br}$ & 5.25968 & 0.53502 & -0.61052 & $\mathrm{Br}$ & 5.14377 & 0.71453 & -0.04075 \\
\hline $\mathrm{C}$ & 4.13282 & -0.89850 & 1.65831 & $\mathrm{C}$ & 3.56654 & -0.88240 & 1.85740 \\
\hline $\mathrm{F}$ & 4.52999 & -2.04932 & 1.06154 & $\mathrm{~F}$ & 4.09325 & -1.98944 & 1.28241 \\
\hline $\mathrm{F}$ & 3.08773 & -1.20627 & 2.47623 & $\mathrm{~F}$ & 2.37423 & -1.23825 & 2.40734 \\
\hline $\mathrm{F}$ & 5.13705 & -0.43915 & 2.42386 & $\mathrm{~F}$ & 4.37779 & -0.47797 & 2.84666 \\
\hline $\mathrm{H}$ & -0.19851 & 0.49512 & -1.08172 & $\mathrm{H}$ & 0.01687 & 0.63741 & -1.74607 \\
\hline $\mathrm{H}$ & 0.92188 & 1.25911 & -2.22148 & $\mathrm{H}$ & 1.28994 & 1.60091 & -2.50512 \\
\hline $\mathrm{H}$ & 0.42535 & 3.37813 & -0.75624 & $\mathrm{H}$ & 0.36879 & 3.55550 & -1.31849 \\
\hline $\mathrm{H}$ & -3.98130 & 5.95586 & 1.17095 & $\mathrm{H}$ & -3.86641 & 5.76834 & 1.30185 \\
\hline $\mathrm{H}$ & -2.82775 & 6.14112 & -0.17625 & $\mathrm{H}$ & -3.06835 & 5.98313 & -0.27573 \\
\hline $\mathrm{H}$ & -2.22861 & 5.83279 & 1.47546 & $\mathrm{H}$ & -2.09135 & 5.87864 & 1.21016 \\
\hline $\mathrm{H}$ & -2.50366 & 3.54216 & 2.52119 & $\mathrm{H}$ & -1.81364 & 3.65495 & 2.39077 \\
\hline $\mathrm{H}$ & -3.36560 & 2.24728 & 1.64005 & $\mathrm{H}$ & -2.68975 & 2.21635 & 1.80700 \\
\hline $\mathrm{H}$ & -4.26252 & 3.65302 & 2.27406 & $\mathrm{H}$ & -3.58235 & 3.56313 & 2.55791 \\
\hline $\mathrm{H}$ & -3.95629 & 4.29134 & -1.48538 & $\mathrm{H}$ & -4.19771 & 3.91497 & -1.19707 \\
\hline $\mathrm{H}$ & -5.15192 & 4.10621 & -0.18056 & $\mathrm{H}$ & -5.04378 & 3.70369 & 0.35270 \\
\hline $\mathrm{H}$ & -4.22182 & 2.68928 & -0.73667 & $\mathrm{H}$ & -4.08181 & 2.36438 & -0.32219 \\
\hline $\mathrm{H}$ & 1.87208 & -1.06114 & 0.26569 & $\mathrm{H}$ & 1.64633 & -0.93071 & 0.01530 \\
\hline $\mathrm{H}$ & 2.75386 & -0.66860 & -1.23502 & $\mathrm{H}$ & 2.83892 & -0.49697 & -1.23570 \\
\hline
\end{tabular}




\begin{tabular}{|c|c|c|c|c|c|c|c|}
\hline $\mathrm{N}$ & -1.53666 & -1.76823 & 1.21838 & $\mathrm{~N}$ & 0.21921 & 0.67531 & 2.10535 \\
\hline $\mathrm{O}$ & -2.57170 & -1.21098 & 1.54188 & $\mathrm{O}$ & -0.58347 & 1.51758 & 2.46554 \\
\hline $\mathrm{C}$ & 0.59989 & -2.60153 & 0.11317 & $\mathrm{C}$ & 1.45054 & -1.32228 & 1.11379 \\
\hline $\mathrm{C}$ & -0.70447 & -1.92044 & 0.34383 & $\mathrm{C}$ & 0.36692 & -0.40631 & 1.56875 \\
\hline $\mathrm{N}$ & 1.76232 & -1.88540 & 0.61176 & $\mathrm{~N}$ & 2.07326 & -0.95158 & -0.14544 \\
\hline $\mathrm{C}$ & 2.34370 & -0.88884 & -0.14830 & $\mathrm{C}$ & 3.20851 & -0.15932 & -0.14824 \\
\hline $\mathrm{O}$ & 3.27978 & -0.25195 & 0.59908 & $\mathrm{O}$ & 3.44679 & 0.25856 & -1.41883 \\
\hline $\mathrm{O}$ & 2.04689 & -0.66668 & -1.31646 & $\mathrm{O}$ & 3.87458 & 0.07663 & 0.84969 \\
\hline $\mathrm{C}$ & 4.12034 & 0.83334 & 0.00797 & $\mathrm{C}$ & 4.66175 & 1.06806 & -1.73552 \\
\hline $\mathrm{C}$ & 5.00920 & 1.23071 & 1.18723 & $\mathrm{C}$ & 4.52282 & 1.28317 & -3.24324 \\
\hline $\mathrm{C}$ & 3.23364 & 2.00193 & -0.42824 & $\mathrm{C}$ & 5.92192 & 0.26247 & -1.41020 \\
\hline $\mathrm{C}$ & 4.95575 & 0.27025 & -1.14416 & $\mathrm{C}$ & 4.60871 & 2.40055 & -0.98415 \\
\hline $\mathrm{H}$ & 0.73583 & -2.73495 & -0.96823 & $\mathrm{H}$ & 1.04281 & -2.34046 & 1.03920 \\
\hline $\mathrm{H}$ & 0.56977 & -3.60049 & 0.57241 & $\mathrm{H}$ & 2.24741 & -1.33183 & 1.87251 \\
\hline $\mathrm{H}$ & 1.92967 & -1.86039 & 1.61386 & $\mathrm{H}$ & 1.51997 & -0.96470 & -0.99760 \\
\hline $\mathrm{H}$ & 5.69252 & 2.03545 & 0.88253 & $\mathrm{H}$ & 5.36879 & 1.87902 & -3.61303 \\
\hline $\mathrm{H}$ & 5.60892 & 0.37584 & 1.52854 & $\mathrm{H}$ & 3.59258 & 1.81940 & -3.47588 \\
\hline $\mathrm{H}$ & 4.40182 & 1.59103 & 2.02879 & $\mathrm{H}$ & 4.51574 & 0.32188 & -3.77519 \\
\hline $\mathrm{H}$ & 2.60176 & 2.33861 & 0.40582 & $\mathrm{H}$ & 5.90019 & -0.70985 & -1.92300 \\
\hline $\mathrm{H}$ & 2.59348 & 1.72417 & -1.27283 & $\mathrm{H}$ & 6.02170 & 0.09707 & -0.33151 \\
\hline $\mathrm{H}$ & 3.87057 & 2.84491 & -0.73347 & $\mathrm{H}$ & 6.80563 & 0.81335 & -1.76372 \\
\hline $\mathrm{H}$ & 5.54037 & -0.59779 & -0.80741 & $\mathrm{H}$ & 3.66715 & 2.92595 & -1.19850 \\
\hline $\mathrm{H}$ & 5.65989 & 1.04024 & -1.49145 & $\mathrm{H}$ & 5.43847 & 3.03940 & -1.32016 \\
\hline $\mathrm{H}$ & 4.32195 & -0.02887 & -1.98671 & $\mathrm{H}$ & 4.69675 & 2.25187 & 0.09785 \\
\hline $\mathrm{C}$ & -1.50903 & -0.63492 & -1.21830 & $\mathrm{C}$ & -1.71440 & -0.91572 & 1.19246 \\
\hline $\mathrm{C}$ & -2.52279 & 0.10739 & -0.68211 & $\mathrm{C}$ & -2.47793 & 0.18981 & 1.45274 \\
\hline $\mathrm{Br}$ & -2.19583 & 1.84975 & 0.02887 & $\mathrm{Br}$ & -2.95923 & 1.39505 & 0.05143 \\
\hline $\mathrm{C}$ & -3.98234 & -0.22059 & -0.90271 & $\mathrm{C}$ & -3.27650 & 0.34503 & 2.72726 \\
\hline $\mathrm{F}$ & -4.78405 & 0.10303 & 0.13424 & F & -3.41199 & 1.62187 & 3.14077 \\
\hline F & -4.14242 & -1.55106 & -1.15907 & $\mathrm{~F}$ & -2.70563 & -0.36951 & 3.73934 \\
\hline $\mathrm{F}$ & -4.46747 & 0.45028 & -1.99658 & $\mathrm{~F}$ & -4.54505 & -0.15146 & 2.56389 \\
\hline $\mathrm{H}$ & -0.52125 & -0.20042 & -1.37214 & $\mathrm{H}$ & -1.50024 & -1.18415 & 0.15898 \\
\hline $\mathrm{H}$ & -1.77786 & -1.49136 & -1.83923 & $\mathrm{H}$ & -1.69774 & -1.71733 & 1.93246 \\
\hline
\end{tabular}

\begin{tabular}{|c|c|c|}
\hline TS2f-3 & TS2f-4 & \\
\hline & & \\
\hline
\end{tabular}




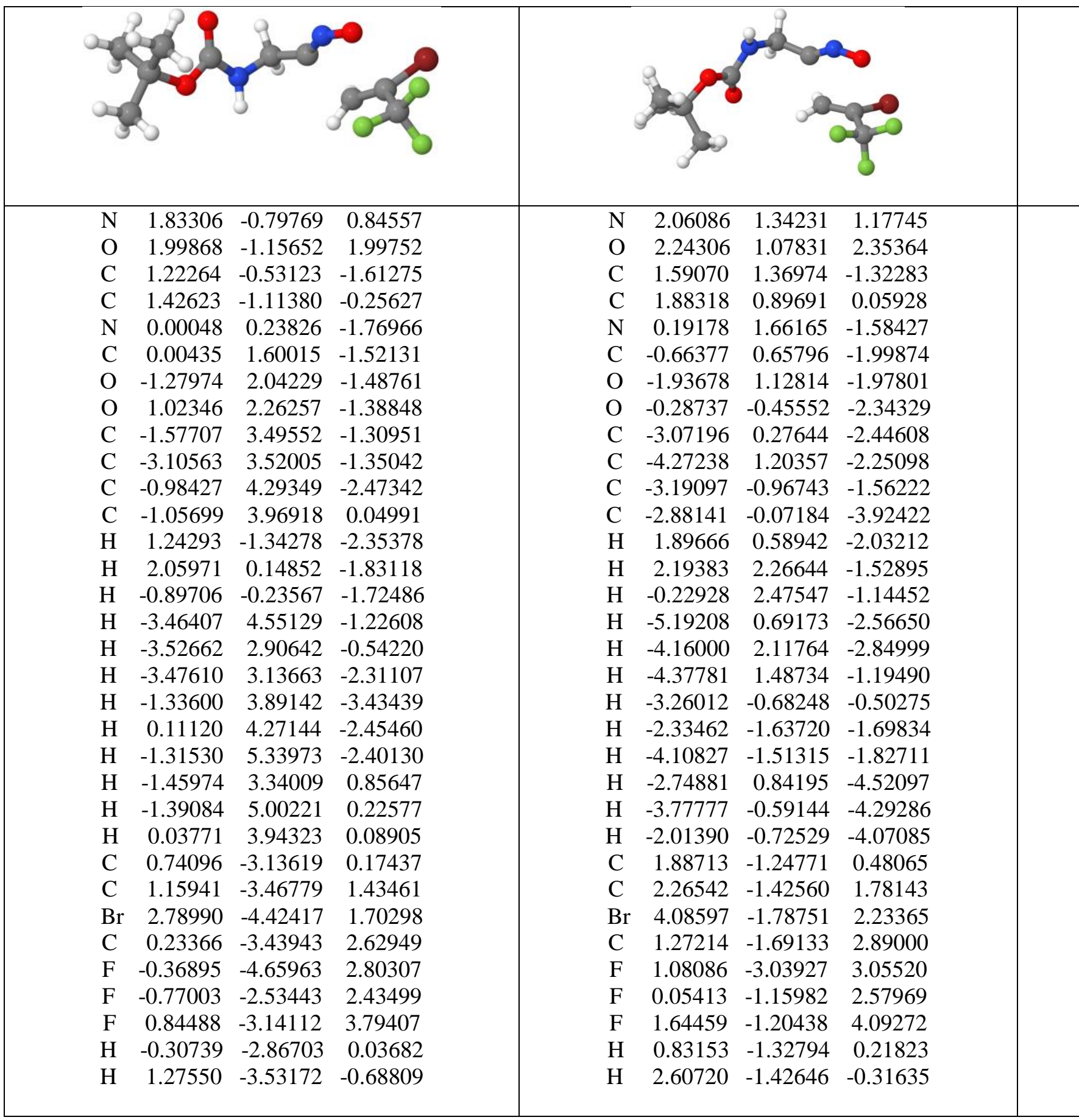




\begin{tabular}{|c|c|c|c|c|c|c|c|}
\hline $\mathrm{C}$ & 0.32480 & -0.91668 & 0.05786 & $\mathrm{C}$ & 0.32528 & -0.91656 & 0.05759 \\
\hline $\mathrm{C}$ & 1.40971 & -1.18970 & 0.93398 & $\mathrm{C}$ & 1.41042 & -1.18951 & 0.93353 \\
\hline $\mathrm{N}$ & 2.30705 & -1.41518 & 1.65803 & $\mathrm{~N}$ & 2.30425 & -1.41478 & 1.65659 \\
\hline $\mathrm{O}$ & 3.23433 & -1.64825 & 2.40637 & $\mathrm{O}$ & 3.22721 & -1.64725 & 2.40277 \\
\hline $\mathrm{C}$ & -2.06481 & -0.87555 & -0.39070 & $\mathrm{C}$ & -2.06431 & -0.87587 & -0.38979 \\
\hline $\mathrm{C}$ & -1.82114 & -0.37648 & -1.67514 & $\mathrm{C}$ & -1.82057 & -0.37668 & -1.67453 \\
\hline $\mathrm{C}$ & -0.50580 & -0.14721 & -2.09414 & $\mathrm{C}$ & -0.50515 & -0.14715 & -2.09421 \\
\hline $\mathrm{C}$ & 0.56358 & -0.41256 & -1.24069 & $\mathrm{C}$ & 0.56458 & -0.41245 & -1.24083 \\
\hline $\mathrm{C}$ & -1.00611 & -1.14590 & 0.47443 & $\mathrm{C}$ & -1.00522 & -1.14616 & 0.47522 \\
\hline $\mathrm{H}$ & -3.08853 & -1.05609 & -0.05910 & $\mathrm{H}$ & -3.08523 & -1.05586 & -0.05921 \\
\hline $\mathrm{H}$ & -2.65401 & -0.16681 & -2.34775 & $\mathrm{H}$ & -2.65131 & -0.16765 & -2.34512 \\
\hline $\mathrm{H}$ & -0.31037 & 0.24183 & -3.09465 & $\mathrm{H}$ & -0.31087 & 0.24082 & -3.09204 \\
\hline $\mathrm{H}$ & 1.58912 & -0.23461 & -1.56517 & $\mathrm{H}$ & 1.58712 & -0.23493 & -1.56465 \\
\hline $\mathrm{H}$ & -1.19379 & -1.53476 & 1.47556 & $\mathrm{H}$ & -1.19215 & -1.53392 & 1.47355 \\
\hline
\end{tabular}




\begin{tabular}{|c|c|c|c|c|c|c|c|}
\hline $\mathrm{N}$ & 0.81474 & 2.59033 & -1.30630 & $\mathrm{~N}$ & 0.82600 & 2.60483 & -1.30586 \\
\hline $\mathrm{C}$ & 2.18364 & 0.94701 & -0.37401 & $\mathrm{C}$ & 2.17739 & 0.94657 & -0.38260 \\
\hline $\mathrm{C}$ & 2.85948 & 2.31440 & -0.28502 & $\mathrm{C}$ & 2.86571 & 2.30855 & -0.28159 \\
\hline $\mathrm{O}$ & 2.05708 & 3.18403 & -1.12414 & $\mathrm{O}$ & 2.07144 & 3.18590 & -1.12207 \\
\hline $\mathrm{C}$ & -0.42331 & 0.56618 & -0.95791 & $\mathrm{C}$ & -0.42318 & 0.58006 & -0.96062 \\
\hline C & 0.81547 & 1.35989 & -0.87778 & C & 0.81393 & 1.37502 & -0.88350 \\
\hline C & 2.23780 & 0.20735 & 0.97873 & $\mathrm{C}$ & 2.21877 & 0.19920 & 0.97015 \\
\hline $\mathrm{Br}$ & 3.11444 & -0.21443 & -1.75205 & $\mathrm{Br}$ & 3.10517 & -0.21377 & -1.78311 \\
\hline $\mathrm{F}$ & 1.76575 & -1.05343 & 0.93607 & $\mathrm{~F}$ & 1.73781 & -1.05542 & 0.92179 \\
\hline $\mathrm{F}$ & 1.49989 & 0.89218 & 1.89825 & $\mathrm{~F}$ & 1.47905 & 0.89018 & 1.88168 \\
\hline $\mathrm{F}$ & 3.51431 & 0.15620 & 1.44655 & $\mathrm{~F}$ & 3.49073 & 0.14052 & 1.44388 \\
\hline $\mathrm{C}$ & -1.66022 & 1.23529 & -0.85279 & $\mathrm{C}$ & -1.66465 & 1.24113 & -0.85689 \\
\hline $\mathrm{C}$ & -2.85671 & 0.52950 & -0.95998 & $\mathrm{C}$ & -2.85601 & 0.52544 & -0.95706 \\
\hline $\mathrm{C}$ & -2.84299 & -0.85464 & -1.16521 & $\mathrm{C}$ & -2.83211 & -0.85994 & -1.15506 \\
\hline $\mathrm{C}$ & -1.62154 & -1.52589 & -1.26696 & $\mathrm{C}$ & -1.60558 & -1.52283 & -1.25455 \\
\hline $\mathrm{C}$ & -0.41826 & -0.82557 & -1.16439 & $\mathrm{C}$ & -0.40776 & -0.81266 & -1.15770 \\
\hline $\mathrm{H}$ & 3.89336 & 2.33515 & -0.64129 & $\mathrm{H}$ & 3.89878 & 2.32062 & -0.62964 \\
\hline $\mathrm{H}$ & 2.80211 & 2.69857 & 0.74705 & $\mathrm{H}$ & 2.80221 & 2.68267 & 0.74981 \\
\hline $\mathrm{H}$ & -1.66619 & 2.31198 & -0.68279 & $\mathrm{H}$ & -1.68040 & 2.31517 & -0.69282 \\
\hline $\mathrm{H}$ & -3.80565 & 1.06106 & -0.87259 & $\mathrm{H}$ & -3.80621 & 1.04867 & -0.87093 \\
\hline $\mathrm{H}$ & -3.78066 & -1.40738 & -1.24252 & $\mathrm{H}$ & -3.76321 & -1.41851 & -1.22739 \\
\hline $\mathrm{H}$ & -1.60155 & -2.60422 & -1.43232 & $\mathrm{H}$ & -1.57769 & -2.59907 & -1.41220 \\
\hline $\mathrm{H}$ & 0.52480 & -1.35976 & -1.26866 & $\mathrm{H}$ & 0.53559 & -1.33852 & -1.25378 \\
\hline
\end{tabular}




\begin{tabular}{|c|c|c|c|c|c|c|c|}
\hline $\mathrm{N}$ & 0.33931 & 2.21007 & -0.61588 & $\mathrm{~N}$ & 0.34548 & 2.20658 & -0.67077 \\
\hline $\mathrm{C}$ & 1.65587 & 0.40645 & 0.09564 & $\mathrm{C}$ & 1.64836 & 0.41356 & 0.08755 \\
\hline $\mathrm{C}$ & 2.48973 & 1.68492 & 0.05812 & $\mathrm{C}$ & 2.48144 & 1.69270 & 0.04154 \\
\hline $\mathrm{O}$ & 1.71087 & 2.63447 & -0.61815 & $\mathrm{O}$ & 1.72200 & 2.62157 & -0.67115 \\
\hline $\mathrm{C}$ & -0.97719 & 0.25650 & -0.10651 & $\mathrm{C}$ & -0.97884 & 0.26433 & -0.12111 \\
\hline $\mathrm{C}$ & 0.29129 & 0.98383 & -0.20328 & $\mathrm{C}$ & 0.28800 & 0.99218 & -0.23248 \\
\hline $\mathrm{C}$ & -2.19964 & 0.89781 & -0.39800 & $\mathrm{C}$ & -2.20950 & 0.90645 & -0.37523 \\
\hline $\mathrm{C}$ & -3.39755 & 0.19449 & -0.31364 & $\mathrm{C}$ & -3.40334 & 0.19780 & -0.27564 \\
\hline $\mathrm{C}$ & -3.40253 & -1.15574 & 0.06174 & $\mathrm{C}$ & -3.39587 & -1.15840 & 0.07824 \\
\hline $\mathrm{C}$ & -2.19753 & -1.79906 & 0.35365 & $\mathrm{C}$ & -2.18257 & -1.80249 & 0.33332 \\
\hline $\mathrm{C}$ & -0.99192 & -1.09884 & 0.27242 & $\mathrm{C}$ & -0.98115 & -1.09711 & 0.23688 \\
\hline $\mathrm{Br}$ & 2.85814 & 2.32231 & 1.95938 & $\mathrm{Br}$ & 2.78953 & 2.37310 & 1.95921 \\
\hline $\mathrm{C}$ & 3.85116 & 1.56544 & -0.66572 & $\mathrm{C}$ & 3.86424 & 1.54811 & -0.64412 \\
\hline $\mathrm{F}$ & 4.50242 & 2.73884 & -0.73709 & $\mathrm{~F}$ & 4.52916 & 2.71094 & -0.71889 \\
\hline $\mathrm{F}$ & 3.64647 & 1.12179 & -1.93816 & $\mathrm{~F}$ & 3.68387 & 1.08352 & -1.91125 \\
\hline $\mathrm{F}$ & 4.65020 & 0.66024 & -0.04821 & $\mathrm{~F}$ & 4.63173 & 0.64475 & 0.01094 \\
\hline $\mathrm{H}$ & 1.72323 & -0.11814 & 1.05334 & $\mathrm{H}$ & 1.70228 & -0.10120 & 1.04669 \\
\hline $\mathrm{H}$ & 1.95646 & -0.28558 & -0.70887 & $\mathrm{H}$ & 1.95425 & -0.28117 & -0.70680 \\
\hline $\mathrm{H}$ & -2.19135 & 1.94864 & -0.68794 & $\mathrm{H}$ & -2.21328 & 1.95871 & -0.64604 \\
\hline $\mathrm{H}$ & -4.33703 & 0.70099 & -0.54023 & $\mathrm{H}$ & -4.34653 & 0.70345 & -0.47190 \\
\hline $\mathrm{H}$ & -4.34466 & -1.70208 & 0.12779 & $\mathrm{H}$ & -4.33214 & -1.70716 & 0.15668 \\
\hline $\mathrm{H}$ & -2.19365 & -2.84960 & 0.64782 & $\mathrm{H}$ & -2.16918 & -2.85468 & 0.60954 \\
\hline $\mathrm{H}$ & -0.05852 & -1.61434 & 0.50397 & $\mathrm{H}$ & -0.04438 & -1.61215 & 0.43697 \\
\hline
\end{tabular}




\begin{tabular}{|lrrrrrrrr|}
\hline & & & \\
\hline
\end{tabular}

